Advances in Experimental Medicine and Biology 1311

Anne Le Editor

The Heterogeneity

of Cancer

Metabolism

Second Edition 


\section{Advances in Experimental Medicine and Biology}

\section{Volume 1311}

\section{Series Editors}

Wim E. Crusio, Institut de Neurosciences Cognitives et Intégratives d'Aquitaine, CNRS and University of Bordeaux,

Pessac Cedex, France

Haidong Dong, Departments of Urology and Immunology, Mayo Clinic, Rochester, MN, USA

Heinfried H. Radeke, Institute of Pharmacology \& Toxicology, Clinic of the Goethe University Frankfurt Main, Frankfurt am Main, Hessen, Germany Nima Rezaei, Research Center for Immunodeficiencies, Children's Medical Center, Tehran University of Medical Sciences, Tehran, Iran Junjie Xiao, Cardiac Regeneration and Ageing Lab, Institute of Cardiovascular Science, School of Life Science, Shanghai University, Shanghai, China 
Advances in Experimental Medicine and Biology provides a platform for scientific contributions in the main disciplines of the biomedicine and the life sciences. This series publishes thematic volumes on contemporary research in the areas of microbiology, immunology, neurosciences, biochemistry, biomedical engineering, genetics, physiology, and cancer research. Covering emerging topics and techniques in basic and clinical science, it brings together clinicians and researchers from various fields.

Advances in Experimental Medicine and Biology has been publishing exceptional works in the field for over 40 years, and is indexed in SCOPUS, Medline (PubMed), Journal Citation Reports/Science Edition, Science Citation Index Expanded (SciSearch, Web of Science), EMBASE, BIOSIS, Reaxys, EMBiology, the Chemical Abstracts Service (CAS), and Pathway Studio.

2019 Impact Factor: 2.4505 Year Impact Factor: 2.324

More information about this series at http://www.springer.com/series/5584 
Anne Le

Editor

\section{The Heterogeneity of Cancer Metabolism}

Second Edition

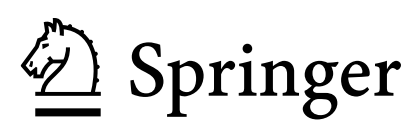




\section{Editor}

Anne Le

Department of Pathology and Oncology

Johns Hopkins University School of Medicine

Baltimore, MD, USA

Department of Chemical and Biomolecular Engineering, Johns Hopkins University Whiting School of Engineering, Baltimore, MD, USA

This book is an open access publication.

ISSN 0065-2598

ISSN 2214-8019 (electronic)

Advances in Experimental Medicine and Biology

$$
\begin{aligned}
& \text { ISBN 978-3-030-65767-3 ISBN 978-3-030-65768-0 (eBook) } \\
& \text { https://doi.org/10.1007/978-3-030-65768-0 }
\end{aligned}
$$

(C) The Editor(s) (if applicable) and The Author(s) 2021

Open Access This book is licensed under the terms of the Creative Commons Attribution 4.0 International License (http://creativecommons.org/licenses/by/4.0/), which permits use, sharing, adaptation, distribution and reproduction in any medium or format, as long as you give appropriate credit to the original author(s) and the source, provide a link to the Creative Commons license and indicate if changes were made.

The images or other third party material in this book are included in the book's Creative Commons license, unless indicated otherwise in a credit line to the material. If material is not included in the book's Creative Commons license and your intended use is not permitted by statutory regulation or exceeds the permitted use, you will need to obtain permission directly from the copyright holder.

The use of general descriptive names, registered names, trademarks, service marks, etc. in this publication does not imply, even in the absence of a specific statement, that such names are exempt from the relevant protective laws and regulations and therefore free for general use.

The publisher, the authors, and the editors are safe to assume that the advice and information in this book are believed to be true and accurate at the date of publication. Neither the publisher nor the authors or the editors give a warranty, expressed or implied, with respect to the material contained herein or for any errors or omissions that may have been made. The publisher remains neutral with regard to jurisdictional claims in published maps and institutional affiliations.

This Springer imprint is published by the registered company Springer Nature Switzerland AG The registered company address is: Gewerbestrasse 11, 6330 Cham, Switzerland 
This book is dedicated to my colleague and mentor, Dr. Arthur J. L. Cooper. 


\section{Foreword}

According to the Center for Disease Control (CDC) in 2018, there were 599,274 cancer deaths in the USA; 283,721 were among females and 315,553 were among males (out of an estimated total US population of 328 million in 2018) [1]. Worldwide in 2020 there were an estimated 10.1 million new cases of cancer in males and 5.5 million cancer-related deaths; in females estimated new cases were 9.2 million and 4.4. million deaths [2]. Lung cancer is currently the leading cause of all cancer deaths (25.1\% of all cases), but for females breast cancer is currently the leading cause of cancer deaths (15.5\%) [2]. According to an April 4th, 2020 report of the International Agency for Research on Cancer (IARC), cancer is now the most common form of death worldwide ahead of heart disease. The report suggests that many cancers can be prevented, and better diagnoses and treatment could improve outcomes [3]. The World Health Organization (WHO) warns that if current trends continue, the world will see a greater than $100 \%$ increase in annual cancer deaths over the next 40 years - from about 9-10 million deaths in 2020 to about 19 million deaths in 2060 [4]. The greatest increase (81\%) in new cases will occur in low- and middle-income countries, where survival rates are currently lowest. This is double (40\%) the new cancer cases that are expected in highincome countries [4]. The report outlines several steps to save millions of lives worldwide, including (1) controlling tobacco use (currently responsible for $25 \%$ of all cancer deaths), (2) vaccinating against hepatitis B to prevent liver cancer, (3) eliminating cervical cancer by vaccinating against HPV, (4) improving screening and treatment, and (5) implementing high-impact cancer management interventions that bring value for money, and ensure access to palliative care, including pain relief. The report notes that in highincome countries there has been a marked decline (by approximately 20\%) in premature deaths due to cancer between the years 2000 and 2015. This is presumably due to outstanding research into cancer causes and the development of improved treatments. On the other hand, the report notes that the situation is much less promising in low-income countries where the reduction in cancer mortality during this period was only $5 \%$. Much of this discrepancy is due to inadequate methods for diagnosis and treatment in lowincome countries versus high-income countries [4].

What can be done to improve the diagnoses of cancer and the survival rate, especially in low-income countries? Obviously, early diagnosis and improved diagnostic accuracy are vitally important and I will return to this point later. President Richard Nixon in his January 1971 State of the Union Address to 
the Nation declared war on cancer signing the National Cancer Act into law. This act gave the National Cancer Institute (NCI) unique autonomy within the National Institutes of Health (NIH) with special budgetary authority in the fight against cancer [5]. Many years later, President Barack Obama, having lost his mother to ovarian cancer, picked up the cause and called for an aggressive war on the disease. In his final State of the Union message in 2016 President Obama tasked his vice president—Joe Biden-to spearhead an initiative to cure cancer: "Last year, Vice President Biden said that with a new moonshot, America can cure cancer," Obama said, noting that Biden had worked with Congress to add resources for the NIH. Obama stated in part "For the loved ones we've all lost, for the family, we can still save, let's make America the country that cures cancer once and for all" [6]. There is no question of the enormous contributions of the NIH (and many other governmentsponsored and private funding agencies in the USA and worldwide) that have helped biomedical researchers understand how cancers arise and how to develop appropriate treatments. The physics of what was required to land a man on the moon was well understood in 1969 (the year of the first successful moon landing). However, no such unique understanding pertains to cancer. We now know that every type of cancer is different and can arise from a multitude of disparate inherited or acquired mutations, and that even within a single type of cancer, individual cancer cells may have varying mutational and phenotypic profiles. These considerations pose enormous challenges for the early diagnosis of many cancers and for appropriate interventions to prevent cancer metastases.

What options are available to minimize the risk of cancer? Obvious lifestyle changes that can help minimize the risk of cancer include eschewing smoking, maintaining a healthy diet, maintaining a recommended body-mass index, exercising regularly, avoiding excessive sunlight, acquiring appropriate vaccinations, and avoiding risky lifestyles [7]. Regular health checkups, including screening for colon, breast, and skin cancers, can help detect cancers at an early enough stage to improve treatment outcomes, especially among those with increased risk. But what sorts of treatments are available once a patient has been diagnosed with cancer? The American Cancer Society lists seven possible treatment options, namely, surgery, chemotherapy, radiation therapy, targeted therapy, immunotherapy, stem cell or bone marrow transplant, and hormone therapy [8]. The NCI provides a similar list of treatment options, but also includes a category entitled precision medicine [9]. Targeted therapy and precision medicine are relatively new concepts regarding cancer treatment options. Targeted therapy is aimed at the changes in cancer cells that help them grow, divide, and spread [9]. On the other hand, precision medicine helps doctors select treatments that are most likely to help patients based on genetic or other characteristics of their disease [9]. Some authors have suggested that a distinction between precision medicine and personalized medicine is useful and not just semantics. Nevertheless, according to the National Research Council, "personalized medicine" is an older term with a meaning similar to "precision medicine." There was concern that the word "personalized" could be misinterpreted to imply that treatments and preventions are being developed uniquely for each individual; in precision 
medicine, the focus is on identifying which approaches will be effective for which patients based on genetic, environmental, and lifestyle factors. The Council therefore preferred the term "precision medicine" to "personalized medicine" [10]. Accordingly, I will use the term precision medicine here. Dr. Anne Le in her capacity as Editor of "Heterogeneity of Cancer Metabolism" and contributor to each chapter in this book makes the convincing case that one way to treat cancers is to understand the varied metabolic "tricks" that enable cancers not only to survive in an often hostile environment but to thrive and multiply. Once these metabolic tricks are uncovered, physicians should be able to use this knowledge to "tailor" interventions to precisely target the unique metabolic profiles of each type of cancer. Dr. Le has done an admirable job of "knitting" together the various ways by which cancers use metabolic pathways to thrive, and she suggests ways in which this knowledge can be used for the design of treatment options.

To understand how precision therapy is intertwined with the theme of the current book it is worth stepping back more than ninety years when the acclaimed German Scientist Otto Warburg noticed that many cancer cells exhibit a unique metabolism, which has since become known as the "Warburg effect." Warburg noted that cancer cells "ferment" glucose to lactate even in the presence of adequate oxygen rather than fully oxidize it to $\mathrm{CO}_{2}$ [e.g., 11]. The energy available from glycolytic conversion of one equivalent of glucose to two equivalents of lactate is much less than that available via complete oxidation of one equivalent of glucose to $\mathrm{CO}_{2}$ via the mitochondrial tricarboxylic acid (TCA) cycle. Nevertheless, cancer cells can afford such wastefulness because they can obtain ample glucose from the circulation-in a sense parasitizing the body. Warburg believed that the mitochondria and the TCA cycle in cancer cells are somewhat defective [12]. However, the formation of two equivalents of lactate (empirical formula $\mathrm{C}_{3} \mathrm{H}_{6} \mathrm{O}_{3}$ ) from glucose (empirical formula $\mathrm{C}_{6} \mathrm{H}_{12} \mathrm{O}_{6}$ ) and no change in NAD+ and $\mathrm{NADH}$ status will result in no net biomass formation [13]. Thus, in order to support growth and proliferation (i.e., increase biomass) the TCA cycle in cancer cells remains intact but has been preempted to serve the cancer cell's unique properties. It appears that the TCA cycle in cancer cells is "rewired" to provide an effective "anaplerosis machine" that can provide building blocks for cancer cell growth and proliferation, and thereby contribute to the increase in biomass [e.g., 13]. The Warburg effect, although not entirely forgotten since its discovery, was mentioned only cursorily in Biochemistry texts for many years. However, interest in the Warburg effect in cancers has burgeoned in recent decades as evidenced by the thousands of articles a year published on the subject since the year 2000 [13]. An excellent background to the Warburg effect is provided in Chap. 1.

It is now recognized that the amino acid glutamine has a special role in cancer metabolism and that many cancer cells exhibit "glutamine addiction" making use of a process known as glutaminolysis. Glutamine is the most abundant amino acid in the body [14] and is readily converted to $\alpha$-ketoglutarate by glutaminolysis, while at the same time contributing nitrogen for DNA and polyamine syntheses. This $\alpha$-ketoglutarate can enter the TCA cycle for the anaplerotic production of energy and/or for the provision of carbon for the 
construction of important small-molecular-weight biomolecules and more complex biomolecules. Several excellent reviews of glutamine addiction in cancers have been published [e.g., 15, 16] including one written by Dr. Le [17]. Glutaminolysis in cancer cells is described in Chap. 2.

Given the importance of glutamine addiction to the survival of many cancers it is not surprising that several clinical trials are ongoing targeting the glutaminolysis pathway. The advantages and disadvantages of current strategies that are designed to inhibit glutaminolysis either directly (e.g., by inhibiting glutaminase, an important enzyme contributing to glutaminolysis) or indirectly (e.g., by inhibiting glutamate dehydrogenase or aspartate aminotransferase) as cancer therapies are well documented in Chap. 2. Three glutaminase 1 inhibitors are currently under investigation as possible anticancer agents, namely CB-839 (Talglenasat; a potent inhibitor of glutaminase C splice variant), BPTES (bis-2-(5-phenylacetamido-1,3,4-thiadiazol-2-yl) ethyl sulfide; an allosteric inhibitor of glutaminase 1) and 968 (an allosteric inhibitor of glutaminase $\mathrm{C}$ splice variant). In fact, $\mathrm{CB}-839$ is in current clinical trials (see chapter 2; Glutamine metabolism in cancer). Li et al. suggest methods by which the effectiveness of glutaminase inhibitors as anticancer agents may be improved, perhaps in combination with other drugs (e.g., metformin).

Another aspect of glutamine addiction relates to fatty acid/lipid synthesis and metabolism. As noted by Cheng et al "Reprogramming of lipid metabolism is a newly recognized hallmark of [cancer] malignancy" [18]. It is well known that glucose is a major source of acetyl carbon for the synthesis of fatty acids and cholesterol. Cholesterol and fatty acids are important components of cell membranes, and fatty acids (but not cholesterol) are also an important energy source. Cholesterol and fatty acids must either be taken up from the circulation or synthesized de novo in rapidly dividing cancer cells. Pyruvate carbon obtained through glycolysis enters the TCA cycle as acetyl units via the pyruvate dehydrogenase complex (PDHC) reaction. During de novo fatty acid synthesis, the acetyl units derived from pyruvate are incorporated into citrate via the TCA cycle in mitochondria. This citrate then exits the mitochondria to the cytosol where it acts as a precursor for acetyl-CoA and hence for both fatty acids and cholesterol synthesis. However, during "times of plenty" (e.g., after a fat-laden meal) fatty acids can be taken up from the circulation by cancer cells and stored, later to be oxidized to produce energy as needed. Thus, cancer cells can "fine-tune" their needs to synthesize and store fatty acids or to metabolize them as an effective energy source as dictated by the needs of the cell. But as mentioned above, lactate is a preferred end product of glycolysis (rather than pyruvate) in many cancer cells. As a result, pyruvate carbon entry into the TCA cycle may become limiting in cancer cells. Thus, glutamine "steps in" once again. Glutaminolysis provides anaplerotic $\alpha$-ketoglutarate to the TCA cycle which in turn can be used for the synthesis of fatty acids. As noted throughout the book, many cancers upregulate the glutaminase pathway and also the transporter principally involved in glutamine uptake (SLC1 A5/ ASCT2). Thus, as discussed by Dr. Le and associates, an inhibitor of the glutaminolysis pathway(s) or transporter would not only interfere with energy production in cancer cells but also interfere with lipid and cholesterol synthesis. 
Dr. Le and her associates have emphasized the importance of metabolic reservoirs that help cancers survive during "lean times." One such reservoir is glycogen that can be a source of glucose when necessary. Not surprisingly, inhibitors of glycogen synthesis or breakdown are being considered as anticancer agents. N-Acetyl-aspartyl-glutamate (NAAG) is a well-documented neurotransmitter, but Dr. Le and her associates have found that it also has an unexpected role in cancer cell metabolism, namely as a metabolic reservoir of aspartate and glutamate. These amino acids can then be readily converted to the TCA cycle components oxaloacetate and $\alpha$-ketoglutarate, respectively. As noted above, cancers generate large amounts of lactate through glycolysis as an, albeit inefficient, source of ATP. Thus, not surprisingly, selective inhibitors of lactate dehydrogenase are also being considered as potential anticancer agents. However, Dr. Le has suggested that the generated lactate may not be just a waste product of inefficient glycolysis but may have a role as a metabolic reservoir of pyruvate, the carbon of which can then enter the TCA cycle via the PDHC. The concept of metabolic reservoir cycles, their importance to cancer cells, and potential for disruption by anticancer agents is exceptionally well covered in this book.

An important factor in a cancer cell's survival is its microenvironment. Recently there has been considerable interest and research into the role of cancer-associated fibroblasts (CAF) as cancer promoters (pCAF) [e.g., 19]. CAF may also act to restrain cancer growth (rCAF). As noted by Miyai et al. [20] in a recent review "CAF promote cancer progression through pleiotropic mechanisms, including the secretion of soluble factors and extracellular matrix, physical interactions with cancer cells, and the regulation of angiogenesis, immunity and metabolism." The authors provide a review of rCAF and discuss how the findings with these cells "can be exploited to develop novel anticancer therapies in the future." An interesting property of CAF is their ability to shed exosomes to the microenvironment containing macromolecules that can aid in cancer survival and promote metastasis and chemoresistance [e.g., 21]. Dr. Le and her associates discuss CAF from a number of perspectives, including their role in immunosuppression, but in the current treatise emphasize the role of CAF in providing metabolic support for their associated cancers. They also provide an excellent overview of how CAF may be an "indirect" target for cancer therapy, in particular targeting glutaminolysis in the CAF cells that contributes to the survival of the associated cancer.

Finally, Dr. Le and her associates discuss cancer in relation to, e.g., diabetes, neurological diseases, and heart disease. She and her associates posit that, although there are very obvious differences between these diseases and cancer, there are similarities in their observed metabolic derangements. Thus, lessons learned from treatments of these noncancerous diseases may provide clues as to better treatment of cancers and vice versa. One final point I wish to make is that although contributions of metabolic processes such as glutaminolysis are beginning to be understood in terms of cancer survival in broad outlines, as emphasized throughout the current book each cancer exhibits a unique metabolic profile and even within a given cancer the metabolic profile may vary with time and position within the cancer. It is anticipated that metabolomics will play an increasingly important role in understanding these metabolic nuances. 
In summary, I hope that I have convinced the interested reader that understanding how cancer cells adapt metabolically to their often hostile environment will provide new insights for designing cancer treatments, perhaps in conjunction with existing conventional therapies. Dr. Le is to be commended not only for her obvious enthusiasm for the topic but also for her work as a leading expert in the field of cancer metabolism. In returning full circle to my introductory statements, I anticipate that her work will contribute, through evermore increasingly sophisticated and sensitive metabolomic analyses methods, to realizing the goal of precision medicine. This will help not only in the invention of treatments designed for the early detection of cancer but also for effective treatments for cancers that have already been diagnosed, especially for the more devastating cancers such as pancreatic cancer. Finally, it is hoped that these considerations will eventually lead to a relatively inexpensive methodology that will lessen the cancer burden, not only in the more developed nations but also in the less developed nations.

Croton-on-Hudson, NY, USA

Arthur J. L. Cooper

April 14, 2021

\section{References}

1. Retrieved from https://www.cdc.gov/cancer/dcpc/research/update-on-cancer-deaths/ index.htm.

2. Sung H., Ferlay J., Siegel R. L., Laversanne M., Soerjomataram I., Jemal A., \& Bray F. (2021). Global cancer statistics 2020: GLOBOCAN estimates of incidence and mortality worldwide for 36 cancers in 185 countries. CA: A Cancer Journal for Clinicians, 0:1-41.

3. Retrieved from https://www.occupationalcancer.ca/2020/iarc-releases-the-worldcancer-report-cancer-research-for-cancerprevention/\#: :text=On $\% 20 \mathrm{February} \% 20$ $4 \% 2 \mathrm{C} \% 202020 \% 2 \mathrm{C} \% 20$ the $\% 20$ International $\% 20$ Agency $\% 20$ for, of $\% 20$ occupational $\% 20$ cancer $\% 20$ are $\% 20$ reviewed $\% 20$ in $\% 20$ Chapter $\% 202.10$.

4. Retrieved from https://www.who.int/news/item/04-02-2020-who-outlines-stepstosave-7-million-lives-from-cancer.

5. Retrieved from https://dtp.cancer.gov/timeline/flash/milestones/M4_Nixon.htm.

6. Retrieved from https://time.com/4178412/cancer-state-of-the-union-barack-obamasotu/.

7. Retrieved from https://www.mayoclinic.org/healthy-lifestyle/adult-health/in-depth/ cancer-prevention/art-20044816.

8. Retrieved from https://www.cancer.org/treatment/treatments-and-side-effects/treatment-types.html.

9. Retrieved from https://www.cancer.gov/about-cancer/treatment/types.

10. Retrieved from https://medlineplus.gov/genetics/understanding/precisionmedicine/ precisionvspersonalized/

11. Warburg, O., Wind, F., \& Negelein, E. (1927). The metabolism of tumors in the body. Journal of General Physiology, 8, 519-530.

12. Warburg, O. (1956). On the origin of cancer cells. Science, 123, 309-314.

13. Liberti, M. V., \& Locasale, J. W. (2016). The Warburg effect: How does it benefit cancer cells? TIBS, 41, 211-218.

14. Cruzat, V., Macedo Rogero, M., Noel Keane, K., Curi, R., \& Newsholme, P. (2018). Glutamine: metabolism and immune function, supplementation and clinical translation. Nutrients, 10, 1564. 
15. Chen, L., \& Cui, H. (2015). Targeting glutamine induces apoptosis: A cancer therapy approach. International Journal of Molecular Sciences, 16, 22830-22855.

16. Altman, B. J., Stine, Z. E., \& Dang, C. V. (2016). From Krebs to clinic: Glutamine metabolism to cancer therapy. Nature Reviews Cancer, 16, 619-634.

17. Li, T., \& Le, A. (2018). Glutamine metabolism in cancer. Advances in Experimental Medicine and Biology, 1063, 13-32.

18. Cheng, C., Geng, F., Cheng, X., \& Guo, D. (2018). Lipid metabolism reprogramming and its potential targets in cancer. Cancer Communications, 38, 1-14.

19. Altorki, N. K., Markowitz, G. J., Gao, D., Port, J. L., Saxena, A., Stiles, B., et al. (2019). The lung microenvironment: an important regulator of tumour growth and metastasis. Nature Reviews Cancer, 19, 9-31.

20. Miyai, Y., Esaki, N., Takahashi, M., \& Enomoto, A. (2020). Cancer-associated fibroblasts that restrain cancer progression: Hypotheses and perspectives. Cancer Science, 111, 1047-1057.

21. Hu, J. L., Wang, W., Lan, X. L., Zeng, Z. C., Liang, Y. S., Yan Y. R., et al. CAFs secreted exosomes promote metastasis and chemotherapy resistance by enhancing cell stemness and epithelial-mesenchymal transition in colorectal cancer. Molecular Cancer, 18, 91. 


\section{Preface}

Genetic alterations in cancer, in addition to being the fundamental drivers of tumorigenesis, can give rise to a variety of metabolic adaptations that allow cancer cells to survive and proliferate in diverse tumor microenvironments. This metabolic flexibility is different from normal cellular metabolic processes and leads to heterogeneity in cancer metabolism within the same cancer type or even within the same tumor. In this book, the authors delve into the complexity and diversity of cancer metabolism and highlight how understanding the heterogeneity of cancer metabolism is fundamental to the development of effective metabolism-based therapeutic strategies for cancer treatment. Deciphering how cancer cells utilize various nutrient resources will enable researchers and clinicians to pair specific chemotherapeutic agents with patients who are most likely to respond with positive outcomes, allowing for more cost-effective and personalized cancer treatment.

This book is the second edition and contains all the contents from the first edition with the addition of new information with unique features that make the book distinctive. This book has four major parts:

Part I: Basic Metabolism of Cancer Cells

Part II: Heterogeneity of Cancer Metabolism

Part III: Relationship Between Cancer Cells and Cancer-Associated Fibroblasts

Part IV: The Metabolic Interplay Between Cancer and Other Diseases.

This book is designed for cancer metabolism researchers, cancer biologists, any other researchers, physicians, epidemiologists, health care professionals of various disciplines, policymakers, marketing and economic strategists, etc. It is also designed for teaching undergraduate and graduate students.

The metabolic pathways and their regulations mentioned in this book serve as examples to illustrate the heterogeneity of cancer metabolism and are non-inclusive.

Baltimore, MD

Anne Le 


\section{Acknowledgments}

This book was made possible by members of the Le Cancer Metabolism Research Laboratory (http://pathology.jhu.edu/lelab/index.cfm). We thank the outstanding support of the Associate Editor, Mrs. Larissa Albright, at Springer Nature Publisher. We especially thank Dr. Arthur J. L. Cooper for his editing and insightful suggestions. Special thanks to Michel Soudée for his helpful editing. Figures were created using BioRender.com.

This work was supported by the Sol Goldman Pancreatic Cancer Research Fund, NIH grants R01-CA193895, and R01-CA112314 (to A.L.). 


\section{Contents}

Part I Basic Metabolism of Cancer Cells

Glucose Metabolism in Cancer: The Warburg Effect and Beyond . . 3 Sminu Bose, Cissy Zhang, and Anne Le

Glutamine Metabolism in Cancer . . . . . . . . . . . . . . . 17

Ting Li, Christopher Copeland, and Anne Le

The Heterogeneity of Lipid Metabolism in Cancer . . . . . . . . . . . 39

Joshua K. Park, Nathan J. Coffey, Aaron Limoges, and Anne Le

Part II Heterogeneity of Cancer Metabolism

The Multifaceted Glioblastoma: From Genomic Alterations to Metabolic Adaptations . . . . . . . . . . . . . . . . . . . . . . . . . 59

Addison Quinones and Anne Le

The Intricate Metabolism of Pancreatic Cancers . . . . . . . . . . 77

Felipe Camelo and Anne Le

The Heterogeneity of Breast Cancer Metabolism . . . . . . . . . . . 89

Jessica Tan and Anne Le

Non-Hodgkin Lymphoma Metabolism . .

Brian James Kirsch, Shu-Jyuan Chang,

Michael James Betenbaugh, and Anne Le

The Heterogeneity Metabolism of Renal Cell Carcinomas.

Mohammadreza Zarisfi, Tu Nguyen, Jessie R. Nedrow,

and Anne Le

The Heterogeneity of Liver Cancer Metabolism

Javier Salazar and Anne Le

Different Tumor Microenvironments Lead to Different

Metabolic Phenotypes. . . . . . . . . . . . . . . . . . 137

Marjorie Justine Antonio, Cissy Zhang, and Anne Le

The Intratumoral Heterogeneity of Cancer Metabolism

Karim Nabi and Anne Le 
Cancer Stem Cell Metabolism 161

Fidelia B. Alvina, Arvin M. Gouw, and Anne Le

Metabolism of Immune Cells in the Tumor Microenvironment . . . . 173

Jin G. Jung and Anne Le

\section{Part III Relationship Between Cancer Cells and Cancer-Associated Fibroblasts}

Metabolic Relationship Between Cancer-Associated

Fibroblasts and Cancer Cells. .

Christos Sazeides and Anne Le

Targeting Metabolic Cross Talk Between Cancer Cells and Cancer-Associated Fibroblasts

Jin G. Jung and Anne Le

Part IV The Metabolic Interplay Between Cancer and Other Diseases

Diabetes and Cancer: The Epidemiological and Metabolic Associations

Cissy Zhang and Anne Le

Bridging the Metabolic Parallels Between Neurological

Diseases and Cancer

Shenghao Guo, Yanni Gu, Jiayin Qu, and Anne Le

Metabolic Intersection of Cancer and Cardiovascular

Diseases: Opportunities for Cancer Therapy .

Giang Hoang, Kiet Nguyen, and Anne Le

Index. 


\section{Editor and Contributors}

\section{About the Editor}

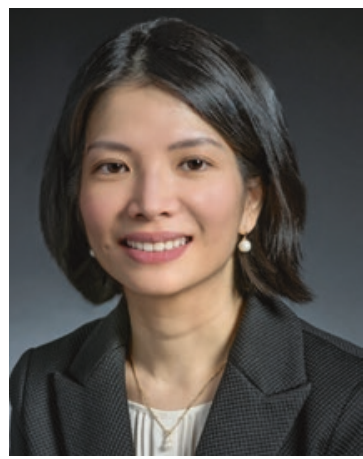

Anne Le studied at the Paris Descartes University, Cochin Port-Royal School of Medicine, in France where she obtained a Habilitation degree (https:// en.wikipedia.org/ wiki/Habilitation), the highest academic qualification a scholar can achieve in Europe. After her clinical training at Henri Poincaré University Hospital, Nancy, in France, she started her postdoctoral research fellowship at the Johns Hopkins University School of Medicine in 2007. Since 2011, Dr. Le has been an independent investigator, who has yielded a number of contributions to the field of cancer metabolism, demonstrated by her publication record as a pioneer in the field. She has published in the best journals, such as Cell Metabolism and the Proceedings of the National Academy of Sciences of the United States of America. Dr. Le has been invited to present her work at several annual American Association for Cancer Research meetings, the most prestigious international meeting for cancer research scientists and professionals, as well as by the National Cancer Institute, and universities in France, Monaco, Japan, and Taiwan. Research media, such as Science Daily, American Association for the Advancement of Science (AAAS), Business Insider, ALN ${ }^{\circledR}$ Magazine, among many others, have written about her work. Dr. Le is highly respected and sought after for her strong proficiency in judging the work and ideas of her peers. She is regularly invited to serve on review panels by prestigious organizations such as the National Institutes of Health and the US Department of Defense. She is frequently asked by highly cited scientific journals to review manuscripts submitted to their journals. 


\section{Contributors}

Fidelia B. Alvina University of Wisconsin-Madison, Madison, WI, USA

Marjorie Justine Antonio Department of Pathology, Johns Hopkins University School of Medicine, Baltimore, MD, USA

Michael James Betenbaugh Department of Chemical and Biomolecular Engineering, Johns Hopkins University Whiting School of Engineering, Baltimore, MD, USA

Sminu Bose Division of Hematology and Oncology, Department of Medicine, Columbia University Medical Center, New York, NY, USA

Felipe Camelo MD Program, Weill Cornell Medicine, New York, NY, USA

Shu Jyuan Chang Graduate Institute of Medicine, College of Medicine, Kaohsiung Medical University, Kaohsiung, Taiwan

Nathan J. Coffey National Institute on Alcohol Abuse and Alcoholism, National Institutes of Health, Bethesda, MD, USA

Christopher Copeland Massachusetts Institute of Technology, Cambridge, MA, USA

Arvin M. Gouw Division of Oncology, Departments of Medicine and Pathology, Stanford University School of Medicine, Stanford, CA, USA

Shenghao Guo Department of Pathology, Johns Hopkins University School of Medicine, Baltimore, MD, USA

Department of Biomedical Engineering, Johns Hopkins University Whiting School of Engineering, Baltimore, MD, USA

Yanni Gu Program of Neuroscience, Johns Hopkins University Krieger School of Arts and Sciences, Baltimore, MD, USA

Giang Hoang Department of Pathology, Johns Hopkins University School of Medicine, Baltimore, MD, USA

Department of Biomedical Engineering, Johns Hopkins University Whiting School of Engineering, Baltimore, MD, USA

Jin G. Jung Department of Pathology, Johns Hopkins University School of Medicine, Baltimore, MD, USA

Brian James Kirsch Department of Pathology, Johns Hopkins University School of Medicine, Baltimore, MD, USA

Department of Chemical and Biomolecular Engineering, Johns Hopkins University Whiting School of Engineering, Baltimore, MD, USA

Anne Le Department of Pathology and Oncology, Johns Hopkins University School of Medicine, Baltimore, MD, USA

Department of Chemical and Biomolecular Engineering, Johns Hopkins University Whiting School of Engineering, Baltimore, MD, USA 
Aaron Limoges Department of Biological Sciences, Columbia University, New York, NY, USA

Ting Li Massachusetts Institute of Technology, Cambridge, MA, USA

Karim Nabi Department of Pathology, Johns Hopkins University School of Medicine, Baltimore, MD, USA

Jessie R. Nedrow Department of Radiology, Johns Hopkins University School of Medicine, Baltimore, MD, USA

Kiet Nguyen Department of Chemistry and Biology, Emory University, Atlanta, GA, USA

Tu Nguyen University of California, Los Angeles (UCLA) David Geffen School of Medicine, Los Angeles, CA, USA

Joshua K. Park Icahn School of Medicine at Mount Sinai, New York, NY, USA

Addison Quinones Icahn School of Medicine at Mount Sinai, New York, NY, USA

Jiayin Qu Program of Neuroscience, Johns Hopkins University Krieger School of Arts \& Sciences, Baltimore, MD, USA

Department of Applied Mathematics and Statistics, Johns Hopkins University Whiting School of Engineering, Baltimore, MD, USA

Javier Salazar Universidad Nacional Mayor de San Marcos, Lima, Peru

Christos Sazeides Icahn School of Medicine at Mount Sinai, New York, NY, USA

Jessica Tan Wayne State University School of Medicine, Detroit, MI, USA

Mohammadreza Zarisfi Department of Radiology, Johns Hopkins University School of Medicine, Baltimore, MD, USA

Cissy Zhang Department of Pathology, Johns Hopkins University School of Medicine, Baltimore, MD, USA

Department of Biology, Johns Hopkins University Krieger School of Arts \& Sciences, Baltimore, MD, USA 
Part I

Basic Metabolism of Cancer Cells 


\title{
Glucose Metabolism in Cancer: The Warburg Effect and Beyond
}

\author{
Sminu Bose, Cissy Zhang, and Anne Le
}

\begin{abstract}
Keywords
Glucose metabolism - Warburg effect .

Glycogenolysis · Gluconeogenesis · Cancer

metabolism
\end{abstract}

$\begin{array}{ll}\text { ATP } & \text { Adenosine triphosphate } \\ \text { CP-320626 } & \text { 5-Chloro- } N \text {-[(2S)-3-(4-fluorophenyl)- } \\ & \begin{array}{l}\text { 1-(4-hydroxypiperidin-1-yl)- } \\ \text { 1-oxopropan-2-yl]-1H-indole- } \\ \text { 2-carboxamide }\end{array} \\ & \text { Cancer stem-cell-like cell } \\ \text { CSC } & \text { Dihydroxyacetone phosphate } \\ \text { DHAP } & \text { Fructose-1,6-bisphosphatase } \\ \text { F1,6-BP } & \text { Fructose-2,6-bisphosphate } \\ \text { F2,6-BP } & \text { Fructose-6-phosphate } \\ \text { F6P } & \text { Fluoro-2-deoxy-d-glucose } \\ \text { FDG } & 3 \text {-Dihydroxy-6-methy 1-7- } \\ \text { FX-11 } & \text { phenylmethyl-4-propylnaphtha- } \\ & \text { lene-1-carboxylic acid } \\ \text { G1P } & \text { Glucose-1-phosphate } \\ \text { G6P } & \text { Glucose-6-phosphate } \\ \text { GAPDH } & \text { Glyceraldehyde 3-phosphate de- } \\ & \text { hydrogenase }\end{array}$

Division of Hematology and Oncology, Department of Medicine, Columbia University Medical Center, New York, NY, USA

GBE

GLUT

GSK2

GSK3 $\beta$

GYS1

HIF- $1 \alpha$

HK2

LDHA mTOR

NAD

$\mathrm{NADPH}$

PCK1

1,4-Alpha-glucan branching enzyme Glucose transporter

Glycogen synthase kinase 2

Glycogen synthase kinase $3 \beta$

Krieger School of Arts and Sciences,

Glycogen synthase 1

Baltimore, MD, USA

A. Le $(\bowtie)$

Department of Pathology and Oncology, Johns

Hopkins University School of Medicine,

Baltimore, MD, USA

Department of Chemical and Biomolecular Engineering, Johns Hopkins University Whiting

School of Engineering, Baltimore, MD, USA
Hypoxia-inducible factor $1 \alpha$

Hexokinase 2

Lactate dehydrogenase A

Mechanistic target of rapamycin

Nicotinamide adenine dinucleotide

Nicotinamide adenine dinucleotide phosphate

Phosphoenolpyruvate carboxykinase 1

e-mail: annele@jhmi.edu 


$\begin{array}{ll}\text { PCK2 } & \begin{array}{l}\text { Phosphoenolpyruvate carboxyki- } \\ \text { nase 2 }\end{array} \\ \text { PET } & \begin{array}{l}\text { Positron-emission tomography } \\ \text { PFK }\end{array} \\ \text { PFKFB3 } & \begin{array}{l}\text { Phosphofructokinase } \\ \text { 6-Phosphofructo-2-kinase/fruc- } \\ \text { tose-2,6-biphosphatase 3 }\end{array} \\ \text { PGM } & \text { Phosphoglucomutase } \\ \text { PI3K } & \text { Phosphoinositide 3-kinase } \\ \text { PPP } & \text { Pentose phosphate pathway } \\ \text { PPP1R3C } & \text { Protein phosphatase 1 regulatory } \\ & \text { subunit 3C } \\ \text { ROS } & \text { Reactive oxygen species } \\ \text { SCLC } & \text { Small-cell lung cancer } \\ \text { TCA } & \text { Tricarboxylic acid } \\ \text { TIGAR } & \text { TP53-induced glycolysis and } \\ & \text { apoptosis regulator } \\ \text { TP53 } & \text { Tumor protein 53 } \\ \text { UGP2 } & \text { UTP:glucose-1-P uridylyltransfer- } \\ & \text { ase 2 } \\ \text { VHL } & \text { Von Hippel-Lindau }\end{array}$

\section{Key Points}

- Tumor cells exhibit an upregulation in glycolysis, glycogen metabolism, and gluconeogenesis as opposed to normal cells.

- Several oncogenes and tumor suppressors drive the metabolic reprogramming underlying the Warburg effect and other changes in glucose metabolism.

- There is heterogeneity in glucose metabolism across tumor types as well as within the tumor microenvironment.

- Numerous therapies targeting glucose metabolism have been developed but have yet to show success in clinical trials.

\section{Introduction}

Otto Warburg observed a peculiar phenomenon in 1924, unknowingly laying the foundation for the field of cancer metabolism. While his contemporaries hypothesized that tumor cells derived the energy required for uncontrolled replication from proteolysis and lipolysis, Warburg instead found them to rapidly consume glucose, converting it to lactate even in the presence of oxygen
[1]. The significance of this finding, later termed the Warburg effect, went unnoticed by the broader scientific community at that time. The field of cancer metabolism lay dormant for almost a century awaiting advances in molecular biology and genetics, which would later open the doors to new cancer therapies $[2,3]$.

\section{The Warburg Effect}

\subsection{Otto Warburg's Early Studies of Normal Cellular Respiration}

Warburg began his forays into research studying the oxygen consumption of sea urchin eggs, finding that the rate of respiration increased severalfold after fertilization. He went on to further describe two processes that were crucial to cellular glucose metabolism: respiration and fermentation [4].

Most differentiated cells metabolize glucose through the tricarboxylic acid (TCA) cycle under aerobic conditions. They then undergo oxidative phosphorylation to generate ATP (between 32 and 34 ATP molecules per glucose molecule) [5] (Fig. 1). While glycolysis produces two net molecules of ATP per one molecule of glucose, the majority of ATP production occurs during the TCA cycle and oxidative phosphorylation. During these latter steps of respiration, the pyruvate molecule produced in glycolysis undergoes a series of reactions in the presence of oxygen. Without the presence of oxygen, cells undergo fermentation or anaerobic glycolysis, shunting the resultant pyruvate molecules to lactate production.

\subsection{The Warburg Effect Is a Prominent Feature of Cancer Cell Metabolism}

In 1927, Warburg studied the processes of respiration and fermentation in tumor cells. According to normal cellular respiration, glucose is converted to pyruvate, which then enters the TCA cycle to undergo oxidative phosphorylation in 


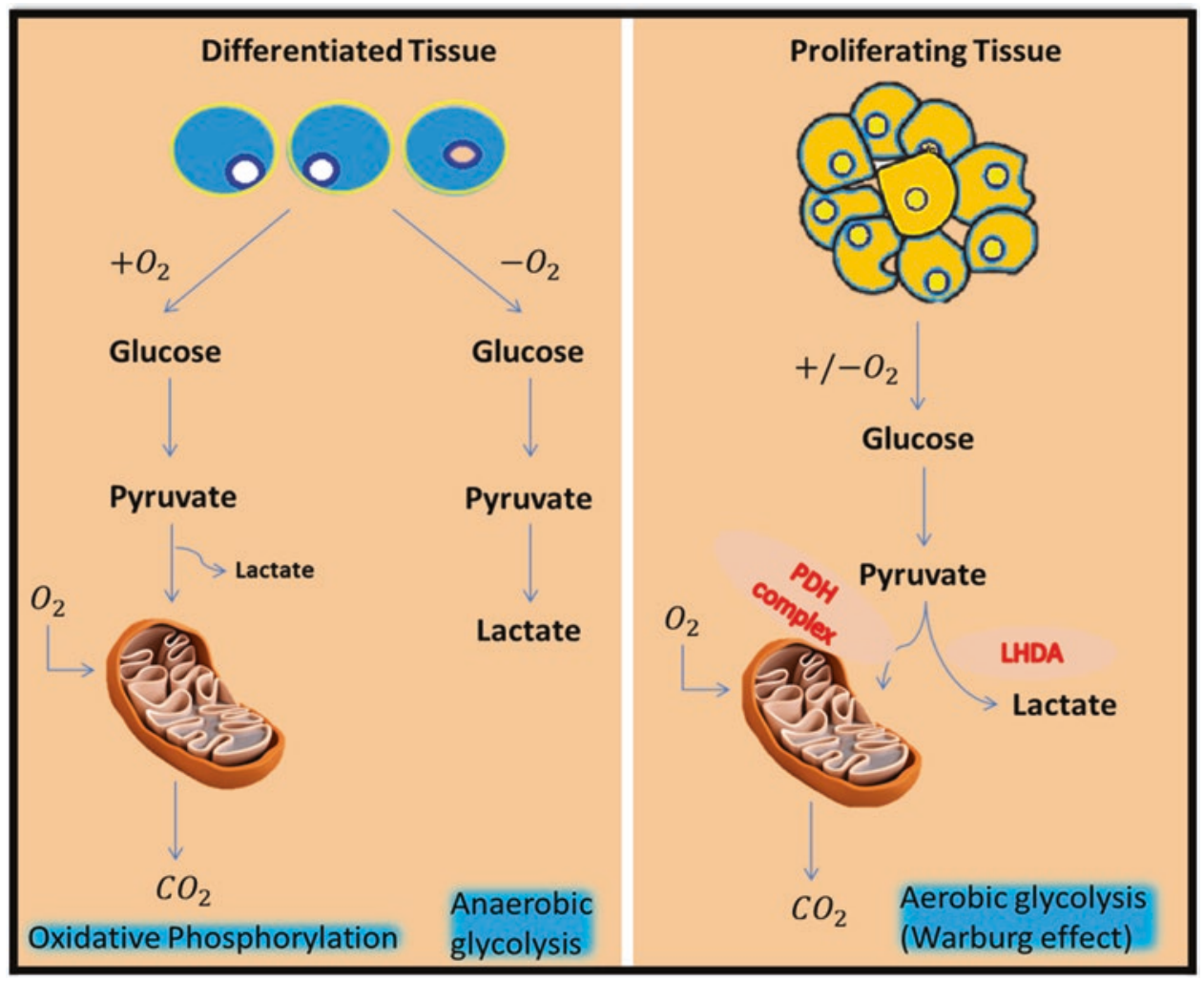

Fig. 1 Respiration in normal differentiated tissue (left) in contrast with the Warburg effect in proliferating tissue (right)

the presence of oxygen, and there should be minimal lactate production. However, in his in vivo and ex vivo studies, Warburg observed an increased glucose uptake and increased lactic acid production in tumor cells as compared to normal cells, even in the presence of oxygen [6]. This phenomenon, the metabolism of glucose to lactate despite the presence of adequate oxygen, is called the Warburg effect or aerobic glycolysis (Fig. 1).

For Warburg, several questions remained unanswered, including why cancer cells would inefficiently shunt glucose-derived pyruvate to lactate production instead of to the TCA cycle, which would result in significantly higher ATP production. Warburg hypothesized that the lactate production in cancer cells was due to the impairment of oxidative phosphorylation caused by mitochondrial damage [7].

There was a debate surrounding this theory with disagreement arising particularly from Sidney Weinhouse, one of Warburg's contemporaries. Using isotope tracing [8], Weinhouse's experiments showed that the rates of oxidative phosphorylation in both normal cells and tumor cells are similar, suggesting that the mitochondria of tumor cells are intact [9]. Rather, tumor cells in oxygen-rich environments utilize both aerobic glycolysis and oxidative phosphorylation to sustain their rapid rates of proliferation. Only in hypoxic environments, such as the tumor core, do the rates of lactic acid production by anaerobic glycolysis overtake oxidative phosphorylation as the primary source of energy [10]. 


\subsection{The Biochemical Nature and Clinical Significance of the Warburg Effect}

Examination of the underlying biochemical processes elucidated possible reasons for why cancer cells paradoxically undergo aerobic glycolysis, a process yielding less ATP than oxidative phosphorylation per cycle. For example, given the inefficiency of ATP production in the Warburg effect, there are likely differences in the kinetics of aerobic glycolysis and oxidative phosphorylation, which have led to cancer cells promoting aerobic glycolysis. Demetrios et al. found that, in the Warburg effect, the flux of glucose to lactate is up to 100 times faster than through the TCA cycle resulting in similar amounts of ATP production over the same time [11]. Even when oxidative phosphorylation is actively continuing, aerobic glycolysis will see much higher glucose flux [12].

To further understand a cancer cell's dependence on aerobic glycolysis, it is necessary to revisit one of the hallmarks of cancer-rapid proliferation supported by strong anabolism. Tumor cells need not only ATP but also anabolic metabolism to accumulate a large amount of biomass to sustain their growth. The Warburg effect via multiple glycolytic intermediates provides a carbon source which contributes to the nucleotide, fatty acid, and amino acid synthesis pathways [13]. For example, glucose-6-phosphate (G6P) becomes partially oxidized via the pentose phosphate pathway (PPP) to generate NADPH and nucleotide components. In addition to the PPP, NADPH is also generated with the shunting of 3-phosphoglycerate out of the glycolytic pathway and into the serine and glycine biosynthesis pathway [14]. NADPH is a reducing equivalent, which is then further used for lipid biosynthesis $[15,16]$. In addition, phospholipid biosynthesis is enabled by the conversion of dihydroxyacetone phosphate (DHAP) to glycerol-3-phosphate [17], and fructose-6-phosphate (F6P) enters the hexosamine pathway to support protein posttranslational modification [18].

Two other clinically significant hallmarks of cancer, the evasion of apoptotic cell death and the ability to metastasize, may provide additional reasons behind the upregulation of aerobic glycolysis in cancer. Anoikis is a type of apoptosis that is a consequence of reactive oxygen species (ROS) accumulation in the setting of the detachment of a cell from the extracellular matrix [19]. When this detachment happens for a cancer cell, however, anoikis is inhibited because the Warburg effect reduces mitochondrial ROS production by decreasing the flow of pyruvate into oxidative phosphorylation [20]. Resistance to apoptosis in the setting of matrix detachment is essential to the metastatic spread of tumor cells.

The Warburg effect has clinical utility as well. One ubiquitous application is the use of positron-emission tomography (PET) imaging in oncology, which has become indispensable in the detection of tumors and the monitoring of the response of existing cancer to therapeutic intervention. PET is an exploitation of the high rate of glycolysis in cancer cells as it uses a radiolabeled glucose analog, $\left[{ }^{18} \mathrm{~F}\right]$ fluoro-2deoxy-d-glucose (FDG), which accumulates in tumor cells due to their rapid uptake of glucose. Another developing application of the Warburg effect is the use of gene expression profiles linked to glycolysis to determine prognosis. Tools in both lung adenocarcinoma and triplenegative breast cancer have shown that glycolytic phenotypes are generally associated with worse patient survival [21-23].

\subsection{Metabolic and Genetic Reprogramming Underlying the Warburg Effect}

With current advances in genetics and molecular biology, much of the past several decades of cancer research have been consumed by characterizing the genetic alterations, which lead to the development of cancers. However, cancer cells need not only a genetic switch but also metabolic building blocks and energy sources to undergo rapid proliferation. The recognition of the importance of energy sources allowed for the resurgence of cancer metabolism as a field that is closely related to tumor genetics. It is now understood that the metabolic reprogramming 
underlying the Warburg effect is driven by several oncogenes and tumor suppressors.

Some of the identified oncogenes, namely protein kinase B (PKB/AKT), phosphoinositide 3-kinase (PI3K), Ras, and Von Hippel-Lindau (VHL), act via the protein hypoxia-inducible factor $1 \alpha(\mathrm{HIF}-1 \alpha)$, resulting in the non-hypoxic expression of HIF-1 $\alpha$. In normal cells, HIF- $1 \alpha$ becomes stabilized in a hypoxic environment to form a transcription factor involved in promoting glycolysis and suppressing oxidative phosphorylation [24]. HIF-1 $\alpha$, when present, upregulates glucose transporter 1 (GLUT1) to promote the retention of glucose inside cells in addition to upregulating hexokinase 2 (HK2), the enzyme which catalyzes the first committed step of glycolysis [25]. Typically, when oxygen is present, HIF-1 $\alpha$ degrades in a concentration-dependent manner. In tumor cells, however, even in the presence of oxygen, high AKT and mechanistic target of rapamycin (mTOR) oncogenic activity promote HIF-1 $\alpha$ expression, leading to persistent transcription of the enzymes driving glycolysis and lactate production.

Other oncogenic pathways have been found to work independently of HIF- $1 \alpha$ to promote aerobic glycolysis as well, namely the activation of oncogenes such as MYC, Ras, and AKT and the deactivation of tumor suppressors such as TP53. Like HIF-1 $\alpha$, MYC directly upregulates GLUT and HK2. The loss of TP53 function also upregulates GLUT expression. Additionally, TP53 deactivation indirectly leads to increased glycolysis. Without TP53 expression, TP53-induced glycolysis and apoptosis regulator (TIGAR), a protein, which causes shunting of glucose to the PPP, is no longer upregulated, resulting in a greater flux of glucose through the glycolytic pathway [26].

\section{$3 \quad$ Heterogeneity in Glucose Metabolism}

Aerobic glycolysis is not consistent across tumor types or even within a single tumor's microenvironment (Fig. 2). Examination of different tumor types revealed that the balance between aerobic glycolysis and oxidative phosphorylation could be very different. In a study evaluating the variability of metabolic gene expression across multiple different tumor types, it was found that there was an upregulation of genes related to oxidative phosphorylation in ovarian, lymphoma [27], leukemia, and lung cancers, whereas the opposite was true in thyroid, colon, pancreatic [28], and renal cancers $[29,30]$. It is thought that the variable activation of different oncogenes such as $R A S, A K T$, and $\mathrm{c}-M Y C$ is the driver behind these differences [31-35].

These differences in metabolism can be seen even in cells within the same tumor [36, 37]. Sometimes, these differences result from variations in the tumor microenvironment leading to metabolic flexibility, the ability of cancer cells to change their bioenergetic pathways according to available nutrients [38, 39]. One important resource is oxygen, which can vary significantly with the aberrant vascularization of tumors. As demonstrated in HeLa cells in hypoxia, there was an observed decrease in ATP derived from oxidative phosphorylation to just $29 \%$ compared to $79 \%$ in normoxia [40]. However, in a study by Le et al., it was shown that a subpopulation of cancer cells under hypoxia still exhibited expressions of genes related to mitochondrial function and maintained their oxidative phosphorylation and tumorigenicity [37]. These results suggest that respiration, even when there is an oxygen shortage, may be necessary for tumorigenicity, which does not depend on the Warburg effect alone and is not reduced as a result of the maintenance of respiration under hypoxic conditions. There may also be differences rooted in the type of tumor cells within the microenvironment-cancer stem-cell-like cells (CSCs) versus more differentiated tumor cells. A recent study found that $80 \%$ showed high levels of glucose uptake, and $20 \%$ showed low levels of glucose uptake [41]. This may have been due to the presence of both CSCs and differentiated cells within the studied population. Similarly, studies of small-cell lung cancer (SCLC) cells showed that the CSC subpopulation was metabolically less active and preferred oxidative phosphorylation rather than glycolysis to fulfill energy requirements [42]. 


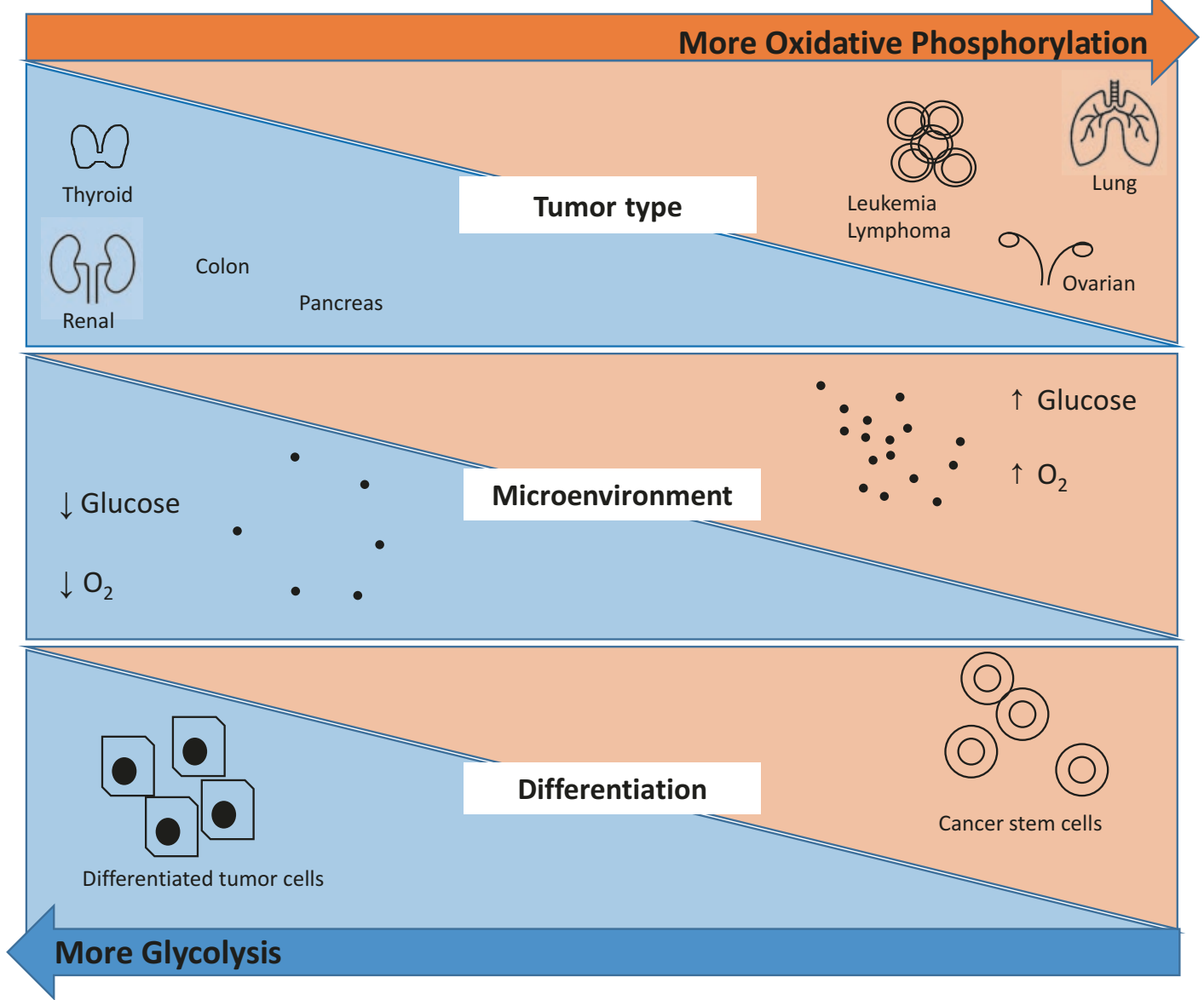

Fig. 2 Heterogeneity in cancer glucose metabolism with respect to tumor type, tumor microenvironment, and differentiation

\section{The Role of Glycogen Metabolism and Gluconeogenesis in Tumor Growth}

\subsection{Glycogen Metabolism Is Upregulated in Several Cancers}

Glycolysis is not the only component of glucose metabolism, which plays a significant role in tumor growth. Glycogenolysis, the process by which glycogen is converted to glucose-1-phosphate (G1P) and then to G6P to enter the glycolytic pathway, provides another energy source for tumors in the face of nutrient stress (Fig. 2).
Glycogen metabolism, although studied far less than glycolysis by cancer researchers, is upregulated in many cancer types, including renal, breast, bladder, uterine, ovarian, skin, and brain cancers. However, the glycogen content of cancer cells was found to be not associated with the rate of replication [43]. Renal cell carcinoma, which classically has clear cells on histology, appears this way due to high glycogen content.

Advances in tumor genetics have allowed for the characterization of tumor-suppressor genes and oncogenes, which have driven these changes in glycogen metabolism in tumor cells. The overexpression of the oncogene Rab25 has been demonstrated as a driver in increasing cellular glycogen stores via the AKT pathway [44]. In bladder cancer, the glycogen debranching enzyme 
AGL has been identified as a tumor suppressor. Additionally, deactivation of AGL leads to the accumulation of abnormal glycogen stores and promotes tumorigenesis in xenograft models [45]. Given this, Guo-Min Shen and colleagues studied glycogen metabolism in the setting of hypoxia. It was noted that glycogen accumulated in breast cancer cells after 24 and $48 \mathrm{~h}$ under hypoxia due to HIF- $1 \alpha$ induction of protein phosphatase 1 regulatory subunit 3C (PPP1R3C), a glycogen synthase [46]. Later studies demonstrated that glycogen synthesis promotes cancer cell survival in the setting of hypoxic conditions [47]. Both glycogenolysis and glycogen synthesis enzymes appear to be upregulated by tumor cells with HIF- $1 \alpha$ dependence, including UTP:glucose-1-P uridylyltransferase 2 (UGP2), phosphoglucomutase (PGM), 1,4-alpha-glucan branching enzyme (GBE), glycogen synthase 1 (GYS1), and PPP1R3C [48]. In vivo studies of suppression of glycogen synthase kinase 2 (GSK2) activity demonstrated a reduction in prostate tumor growth [49]. Glycogen metabolism is an important target of therapy given that cancer cells can utilize glycogen as an energy source even during nutrient deficiency due to poor angiogenesis $[50,51]$.

\subsection{Upregulation of Gluconeogenic Enzymes in Cancer}

Gluconeogenesis is the process of generating glucose from carbon substrates that are not carbohydrates. There are two gluconeogenic enzymes that play important roles in cancer metabolism: phosphoenolpyruvate carboxykinase 1 (PCK1) and phosphoenolpyruvate carboxykinase 2 (PCK2). It has been demonstrated that TP53 inhibits both enzymes, meaning that the loss of TP53 upregulates these enzymes and gluconeogenesis [52]. It was also observed that the inhibition of mTOR in hepatocellular carcinoma and renal cell carcinoma cells directs the glycolytic flux towards lactate and gluconeogenesis with resultant tumor cell death via the downregulation of PCK1 [53].

\section{Success and Failures of Targeting Glucose Metabolism for Cancer Therapy}

\subsection{Therapies Targeting Glycolysis and the Warburg Effect}

As discussed previously, over the latter half of the twentieth century, advances in molecular biology and the identification of oncogenes and tumor suppressors drew the attention of much of the anticancer therapeutic efforts. It is true that genetic alterations drive uncontrolled replication in cancer cells, but it is also important to recognize that a cancer cell is still dependent on nutrient availability. In the past two decades, there has been an upsurge in efforts to exploit the addiction of cancer cells to glucose and the Warburg effect for cancer treatment [54]. Several enzymes in the glycolytic pathway have been targeted, some showing tumoricidal effects in vitro and in vivo (Fig. 3). Unfortunately, there has been little clinical success given that glycolysis is crucial to the glucose metabolism of normal cells as well. Thus, the focus should be on targeting those elements of aerobic glycolysis, which are more upregulated in cancer.

Glucose transporters (GLUT1-4) are upregulated in tumor cells by MYC and HIF- $1 \alpha$. Previous attempts with small-molecule inhibitors of GLUT1 have seen in vitro tumoricidal effects in a renal cell carcinoma cell line [55] and hepatocellular carcinoma cell line [56]. However, GLUT1 is a prevalent glucose transporter in normal cells as well, which would likely preclude clinical success. Homozygous Glut1 deletion is embryonically lethal in mice, and heterozygous deletion causes impaired motor activity and seizures [57]. A GLUT1 inhibitor called silibinin failed to demonstrate any reduction in prostatespecific antigen, a well-known biomarker for prostate cancer, in a phase I clinical trial and was associated with significant side effects [58].

Hexokinase phosphorylates glucose to glucose-6-phosphate in the first committed step of glycolysis. Hexokinase 2 (HK2) is mostly 


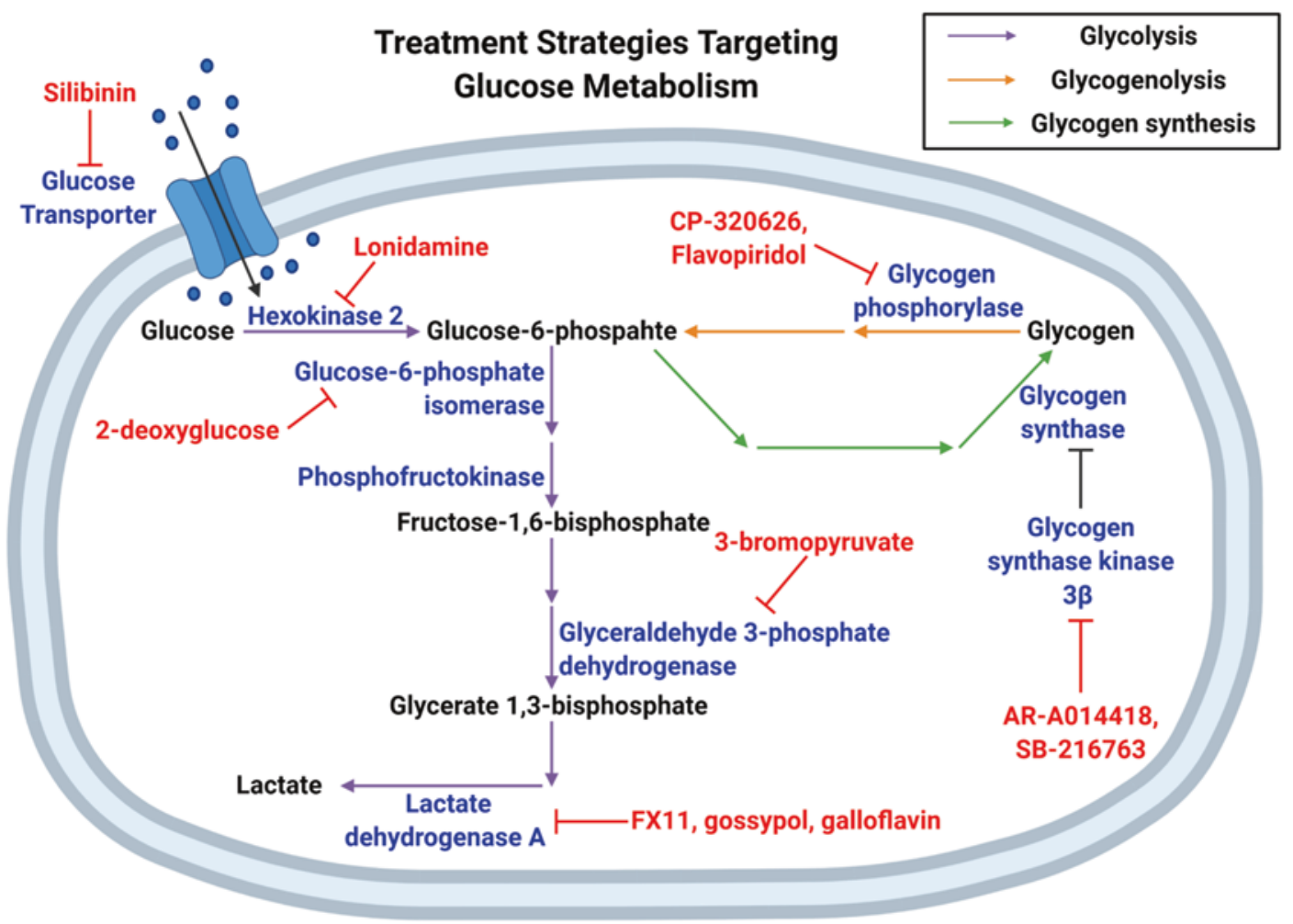

Fig. 3 Current targets of cancer therapies directed at glucose metabolism

expressed in cancer cells and is the primary hexokinase to function in tumors, so it is another potential therapeutic target. Experiments in which HK2 was systemically deleted have shown to be well tolerated in mice [59]. A glucose analog that competitively inhibits G6P isomerase in order to inhibit the phosphorylation of glucose, 2-deoxyglucose, has been studied in a phase I clinical trial in combination with radiation therapy with good toleration in glioblastoma multiforme [60, 61]. However, a HK inhibitor called lonidamine failed to show any benefit in two phase III randomized clinical trials [58].

Phosphofructokinase (PFK) is the enzyme which catalyzes the second committed step in glycolysis, the conversion of fructose-6-phosphate to fructose-1,6-bisphosphate (F1,6-BP). Although inhibiting PFK directly is not possible since it is crucial to glycolysis in normal cells, it may be feasible to target it indirectly. PFK is strongly allosterically activated by fructose2,6-bisphosphate (F2,6-BP). F2,6-BP is activated by another protein, 6-phosphofructo-2-kinase/ fructose-2,6-biphosphatase 3 (PFKFB3), a target of HIF-1 $\alpha$. Attenuation of glycolysis was achieved in in vitro and in vivo studies with a small-molecule PFKFB3 inhibitor called 3-(3-pyridinyl)-1-(4-pyridinyl)-2-propen-1-one (3PO) [62]. PFKFB3 inhibitors were also shown to reduce tumor angiogenesis [63].

Glyceraldehyde 3-phosphate dehydrogenase (GAPDH) converts glyceraldehyde 3-phosphate to glycerate 1,3-bisphosphate with the production of NADH and is a promising target for antiglycolytic therapy given the role of NADH in biosynthesis. The small-molecule pyruvate ana$\log$, 3-bromopyruvate, is a nonselective inhibitor of GAPDH and has been shown to inhibit tumor oxidative phosphorylation and glycolysis with good preclinical efficacy [64, 65]. However, there is concern for toxicities such as burning sensation 
with intravenous infusion and there are no ongoing clinical trials with this compound [66].

In seeking a target that was more unique to cancer cell metabolism and central to the Warburg effect, Le et al. focused on lactate dehydrogenase A (LDHA) which reciprocally mediates the redox-coupled conversion between lactate with NAD+ and pyruvate with NADH [67, 68]. Elevated expression level of LDHA is a hallmark of many types of tumors, including squamous head and neck cancer, colorectal cancer, and nonsmall cell lung cancer [69-71]. By perturbing the $\mathrm{NADH} / \mathrm{NAD}+$ ratio, a small-molecular inhibitor of LDHA called FX-11 was shown to increase reactive oxygen species in tumor cells with subsequent cell death in not only in vitro studies but also pancreatic and lymphoma xenografts [72-74].

Several other LDHA inhibitors, such as gossypol, galloflavin, and $N$-hydroxyindole-based inhibitors, were tested in preclinical settings [72, 75-78]. Among them, gossypol (AT-101), a nonselective inhibitor of $\mathrm{LDH}$, was tested in phase I and phase II clinical trials targeting glioblastoma (NCT00390403, NCT00540722), small-cell lung cancer, and prostate cancer [79, 80]. Despite active investigations for developing LDH inhibitors, there is still a clinical need for highly selective and efficient LDH inhibitors, as gossypol shows off-target effects such as the inhibition of NADH-dependent enzymes (e.g., GAPDH) [77]. Although compounds targeting lactate metabolism have not yet been approved, it is clear that LDH-targeting strategies are promising approaches for cancer therapy.

On a macro level, dietary changes to limit glucose availability to tumor cells have also been studied. For example, ketogenic therapy, a diet with severe carbohydrate restriction, has been shown to sensitize gliomas and glioblastoma to chemoradiation therapy, reduce oxidative stress, and downregulate angiogenic proteins [81]. The success of this therapy may lie in the relative metabolic inflexibility of neuronal cells and their addiction to glucose.

\subsection{Therapies Targeting Glycogenolysis and Glycogen Synthesis Have Shown Promising Results}

Significantly fewer therapies targeting glycogen metabolism have been developed (Fig. 3). Lee et al. inhibited glycogen phosphorylase in a pancreatic cell line with a compound called CP-320626, leading to tumor cell death with no effect on normal human fibroblasts [82]. Flavopiridol, another glycogen phosphorylase inhibitor, had safe and modest efficacy in clinical trials with prostate cancer, renal cell carcinoma, and colorectal carcinoma [83-85]. However, flavopiridol is also a cyclin-dependent kinase inhibitor [86], so it is uncertain whether the antitumor effects were purely from glycogen phosphorylase inhibition. More recently, inhibition of glycogen synthase kinase (GSK) $3 \beta$ by AR-A014418 and SB-216763 in an esophageal squamous cell carcinoma cell line has resulted in attenuated tumor growth and induced apoptosis; thus GSK3 $\beta$ has emerged as a potential target [87]. Similar results were shown in sarcoma cell lines [88]. Given these promising results, further investigation of glycogenolysis and glycogen synthesis-targeting agents is warranted.

\section{Conclusion}

Currently, there are several challenges to metabolic cancer therapies. First, an understanding of the heterogeneity of metabolic phenotypes is only beginning to be established. Metabolic phenotypes likely vary based on tissue of origin, tumor microenvironment, primary versus metastatic tumors, and mutational differences. Second, there are limitations in translating in vivo mouse studies to clinical trials, as is evidenced by the lack of success in advancing metabolic inhibitors through clinical trials up until this point. Third, there is the potential for metabolic inhibitors to be overcome by the adaptation of tumors to new energy sources as well as their inherent metabolic flexibility. 
With renewed interest in cancer metabolism, the development of metabolic inhibitors will continue to grow, and it may be most effective to combine these therapies with other modalities of therapy in order to increase efficacy.

\section{References}

1. Warburg, O. (1924). Über den stoffwechsel der carcinomzelle. Naturwissenschaften, 1924, 1131-1137.

2. Dang, C. V., et al. (2011). Therapeutic targeting of cancer cell metabolism. Journal of Molecular Medicine (Berlin), 89(3), 205-212.

3. Hirschey, M. D., et al. (2015). Dysregulated metabolism contributes to oncogenesis. Seminars in Cancer Biology, 35(Suppl), S129-S150.

4. Warburg, O. (1928). Chemical constitution of respiration ferment. Science, 68(1767), 437-443.

5. Cooper, G. M., \& Hausman, R. E. (2009). The cell: A molecular approach (Sinauer Associates) (Vol. 5). Washington, DC: ASM Press, xix, 820 p.

6. Warburg, O., Wind, F., \& Negelstein, E. (1927). The metabolism of tumors in the body. Journal of General Physiology, 8(6), 519-530.

7. Warburg, O. (1956). On the origin of cancer cells. Science, 123(3191), 309-314.

8. Hoang, G., Udupa, S., \& Le, A. (2019). Application of metabolomics technologies toward cancer prognosis and therapy. International Review of Cell and Molecular Biology, 347, 191-223.

9. Weinhouse, S. (1951). Studies on the fate of isotopically labeled metabolites in the oxidative metabolism of tumors. Cancer Research, 11, 585-591.

10. Hay, N. (2016). Reprogramming glucose metabolism in cancer: Can it be exploited for cancer therapy? Nature Reviews. Cancer, 16, 635-649.

11. Demetrius, L., \& Tuszynski, J. A. (2010). Quantum metabolism explains the allometric scaling of metabolic rates. Journal of the Royal Society Interface, 7(44), 507-514.

12. Pfeiffer, T., Schuster, S., \& Bonhoeffer, S. (2001). Cooperation and competition in the evolution of ATPproducing pathways. Science, 292(5516), 504-507.

13. Liberti, M. V., \& Locasale, J. W. (2016). The Warburg effect: How does it benefit cancer cells? Trends in Biochemical Sciences, 41(3), 211-218.

14. Locasale, J. W., et al. (2011). Phosphoglycerate dehydrogenase diverts glycolytic flux and contributes to oncogenesis. Nature Genetics, 43(9), 869-874.

15. Jiang, P., Du, W., \& Wu, M. (2014). Regulation of the pentose phosphate pathway in cancer. Protein \& Cell, 5(8), 592-602.

16. Park, J. K., et al. (2021). The heterogeneity of lipid metabolism in cancer. Advances in Experimental Medicine and Biology, 1311, https://doi. org/10.1007/978-3-030-65768-0_3
17. Pavlova, N. N., \& Thompson, C. B. (2016). The emerging hallmarks of cancer metabolism. Cell Metabolism, 23(1), 27-47.

18. Itkonen, H. M., et al. (2013). O-GlcNAc transferase integrates metabolic pathways to regulate the stability of c-MYC in human prostate cancer cells. Cancer Research, 73(16), 5277-5287.

19. Lu, J. (2019). The Warburg metabolism fuels tumor metastasis. Cancer Metastasis Reviews, 38(1-2), 157-164.

20. Kamarajugadda, S., et al. (2012). Glucose oxidation modulates anoikis and tumor metastasis. Molecular and Cellular Biology, 32(10), 1893-1907.

21. Li, C., et al. (2020). Identification of a prognosis-associated signature associated with energy metabolism in triple-negative breast cancer. Oncology Reports, 44(3), 819-837.

22. Zhang, L., Zhang, Z., \& Yu, Z. (2019). Identification of a novel glycolysis-related gene signature for predicting metastasis and survival in patients with lung adenocarcinoma. Journal of Translational Medicine, 17(1), 423.

23. Tan, J., \& Le, A. (2021). The heterogeneity of breast cancer metabolism. Advances in Experimental Medicine and Biology, 1311, https://doi. org/10.1007/978-3-030-65768-0_6.

24. Semenza, G. L. (2010). HIF-1: Upstream and downstream of cancer metabolism. Current Opinion in Genetics \& Development, 20(1), 51-56.

25. Christofk, H. R., Vander Heiden, M. G., Harris, M. H., Ramanathan, A., Gerszten, R. E., Wei, R., Fleming, M. D., Schreiber, S. L., \& Cantley, L. C. (2008). The M2 splice isoform of pyruvate kinase is important for cancer metabolism and tumour growth. Nature, 452(7184), 230-233.

26. Levine, A. J., \& Puzio-Kuter, A. (2010). The control of the metabolic switch in cancers by oncogenes and tumor suppressor genes. Science, 330(6009), 1340-1344.

27. Kirsch, B. J., et al. (2021). Non-Hodgkin lymphoma metabolism. Advances in Experimental Medicine and Biology, 1311, https://doi. org/10.1007/978-3-030-65768-0_7

28. Camelo, F., \& Le, A. (2021). The intricate metabolism of pancreatic cancers. Advances in Experimental Medicine and Biology, 1311, https:// doi.org/10.1007/978-3-030-65768-0_5

29. Zarisfi, M., et al. (2021). The heterogeneity metabolism of renal cell carcinomas. Advances in Experimental Medicine and Biology, 1311, https:// doi.org/10.1007/978-3-030-65768-0_8

30. Hu, J., et al. (2013). Heterogeneity of tumor-induced gene expression changes in the human metabolic network. Nature Biotechnology, 31(6), 522-529.

31. Elstrom, R. L., et al. (2004). AKT stimulates aerobic glycolysis in cancer cells. Cancer Research, 64(11), 3892-3899.

32. Gough, D. J., et al. (2009). Mitochondrial STAT3 supports Ras-dependent oncogenic transformation. Science, 324(5935), 1713-1716. 
33. Kim, J. W., et al. (2006). HIF-1-mediated expression of pyruvate dehydrogenase kinase: A metabolic switch required for cellular adaptation to hypoxia. Cell Metabolism, 3(3), 177-185.

34. Dang, C. V., Le, A., \& Gao, P. (2009). MYC-induced cancer cell energy metabolism and therapeutic opportunities. Clinical Cancer Research, 15(21), 6479-6483.

35. Le, A., \& Dang, C. V. (2013). Studying Myc's role in metabolism regulation. Methods in Molecular Biology, 1012, 213-219.

36. Nabi, K., \& Le, A. (2021). The intratumoral heterogeneity of cancer metabolism. Advances in Experimental Medicine and Biology, 1311, https:// doi.org/10.1007/978-3-030-65768-0_11

37. Le, A., et al. (2014). Tumorigenicity of hypoxic respiring cancer cells revealed by a hypoxia-cell cycle dual reporter. Proceedings of the National Academy of Sciences of the United States of America, 111(34), 12486-12491.

38. Jose, C., Bellance, N., \& Rossignol, R. (2011). Choosing between glycolysis and oxidative phosphorylation: A tumor's dilemma? Biochimica et Biophysica Acta, 1807(6), 552-561.

39. Antonio, M. J., Zhang, C., \& Le, A. (2021). Different tumor microenvironments lead to different metabolic phenotypes. Advances in Experimental Medicine and Biology, 1311, https:// doi.org/10.1007/978-3-030-65768-0_10

40. Rodriguez-Enriquez, S., et al. (2010). Oxidative phosphorylation is impaired by prolonged hypoxia in breast and possibly in cervix carcinoma. The International Journal of Biochemistry \& Cell Biology, 42(10), 1744-1751.

41. Xue, M., et al. (2015). Chemical methods for the simultaneous quantitation of metabolites and proteins from single cells. Journal of the American Chemical Society, 137(12), 4066-4069.

42. Gao, C., et al. (2016). Cancer stem cells in small cell lung cancer cell line H446: Higher dependency on oxidative phosphorylation and mitochondrial substrate-level phosphorylation than non-stem cancer cells. PLoS One, 11(5), e0154576.

43. Rousset, M., Zweibaum, J., \& Fogh, J. (1981). Presence of glycogen and growth-related variations in 58 cultured human tumor cell lines of various tissue origins. Cancer Research, 41(3), 1165-1170.

44. Cheng, K. W., et al. (2012). Rab25 increases cellular ATP and glycogen stores protecting cancer cells from bioenergetic stress. EMBO Molecular Medicine, 4(2), 125-141.

45. Guin, S., et al. (2014). Role in tumor growth of a glycogen debranching enzyme lost in glycogen storage disease. Journal of the National Cancer Institute, 106, 5.

46. Shen, G.-M., et al. (2010). Hypoxia-inducible factor 1 -mediated regulation of PPP1R3C promotes glycogen accumulation in human MCF-7 cells under hypoxia. FEBS Letters, 584(20), 4366-4372.
47. Pelletier, J., et al. (2012). Glycogen synthesis is induced in hypoxia by the hypoxia-inducible factor and promotes cancer cell survival. Frontiers in Oncology, 2, 18-18.

48. Zois, C. E., Favaro, E., \& Harris, A. L. (2014). Glycogen metabolism in cancer. Biochemical Pharmacology, 92(1), 3-11.

49. Zhu, Q., et al. (2011). Suppression of glycogen synthase kinase 3 activity reduces tumor growth of prostate cancer in vivo. The Prostate, 71(8), 835-845.

50. Ros, S., \& Schulze, A. (2012). Linking glycogen and senescence in cancer cells. Cell Metabolism, 16(6), 687-688.

51. Elgogary, A., et al. (2016). Combination therapy with BPTES nanoparticles and metformin targets the metabolic heterogeneity of pancreatic cancer. Proceedings of the National Academy of Sciences of the United States of America, 113(36), E5328-E5336.

52. Zhang, P., et al. (2014). Tumor suppressor p53 cooperates with SIRT6 to regulate gluconeogenesis by promoting FoxO1 nuclear exclusion. Proceedings of the National Academy of Sciences of the United States of America, 111(29), 10684-10689.

53. Khan, M., Biswas, D., Ghosh, M., Mandloi, S., Chakrabarti, S., \& Chakrabarti, P. (2015). mTORC2 controls cancer cell survival by modulating gluconeogenesis. Cell Death Discovery, 1, 15016.

54. Dang, C. V., et al. (2011). Therapeutic targeting of cancer cell metabolism. Journal of Molecular Medicine, 89(3), 205-212.

55. Chan, D. A., et al. (2011). Targeting GLUT1 and the Warburg effect in renal cell carcinoma by chemical synthetic lethality. Science Translational Medicine, 3, 94.

56. Amann, T., \& Hellerbrand, C. (2009). GLUT1 as a therapeutic target in hepatocellular carcinoma. Expert Opinion on Therapeutic Targets, 13(12), 1411-1427.

57. Marin-Valencia, I., et al. (2012). GLUT1 deficiency (G1D): Epilepsy and metabolic dysfunction in a mouse model of the most common human phenotype. Neurobiology of Disease, 48(1), 92-101.

58. Sborov, D. W., Haverkos, B. M., \& Harris, P. J. (2015). Investigational cancer drugs targeting cell metabolism in clinical development. Expert Opinion on Investigational Drugs, 24(1), 79-94.

59. Heikkinen, S., et al. (1999). Hexokinase ii-deficient: Mice prenatal death of homozygotes without disturbances in glucose tolerance in heterozygotes. Journal of Biological Chemistry, 274(32), 22517-22523.

60. Dwarakanath, B. S., et al. (2009). Clinical studies for improving radiotherapy with 2-deoxy-Dglucose: Present status and future prospects. Journal of Cancer Research and Therapeutics, 5(Suppl 1), S21-S26.

61. Quinones, A., \& Le, A. (2021). The multifaceted glioblastoma: From genomic alterations to metabolic adaptations. Advances in Experimental Medicine and Biology, 1311, https://doi. org/10.1007/978-3-030-65768-0_4 
62. Clem, B., et al. (2008). Small-molecule inhibition of 6-phosphofructo-2-kinase activity suppresses glycolytic flux and tumor growth. Molecular Cancer Therapeutics, 7(1), 110-120.

63. Schoors, S., et al. (2014). Partial and transient reduction of glycolysis by PFKFB3 blockade reduces pathological angiogenesis. Cell Metabolism, 19(1), 37-48.

64. Pereira da Silva, A. P., et al. (2009). Inhibition of energy-producing pathways of HepG2 cells by 3-bromopyruvate. The Biochemical Journal, 417(3), 717-726.

65. Chapiro, J., et al. (2014). Systemic delivery of microencapsulated 3-bromopyruvate for the therapy of pancreatic cancer. Clinical Cancer Research, 20(24), 6406-6417.

66. El Sayed, S. M. (2018). Enhancing anticancer effects, decreasing risks and solving practical problems facing 3-bromopyruvate in clinical oncology: 10 years of research experience. International Journal of Nanomedicine, 13, 4699-4709.

67. Wu, S., et al. (2017). Risk factors of post-operative severe hyperlactatemia and lactic acidosis following laparoscopic resection for pheochromocytoma. Scientific Reports, 7(1), 403.

68. Doherty, J. R., \& Cleveland, J. L. (2013). Targeting lactate metabolism for cancer therapeutics. The Journal of Clinical Investigation, 123(9), 3685-3692.

69. Koukourakis, M. I., et al. (2005). Lactate dehydrogenase 5 (LDH5) relates to up-regulated hypoxia inducible factor pathway and metastasis in colorectal cancer. Clinical \& Experimental Metastasis, 22(1), 25-30.

70. Koukourakis, M. I., et al. (2003). Lactate dehydrogenase-5 (LDH-5) overexpression in non-small-cell lung cancer tissues is linked to tumour hypoxia, angiogenic factor production and poor prognosis. British Journal of Cancer, 89(5), 877-885.

71. Koukourakis, M. I., et al. (2009). Lactate dehydrogenase 5 expression in squamous cell head and neck cancer relates to prognosis following radical or postoperative radiotherapy. Oncology, 77(5), 285-292.

72. Le, A., et al. (2010). Inhibition of lactate dehydrogenase A induces oxidative stress and inhibits tumor progression. Proceedings of the National Academy of Sciences of the United States of America, 107(5), 2037-2042.

73. Dutta, P., et al. (2013). Evaluation of LDH-A and glutaminase inhibition in vivo by hyperpolarized 13C-pyruvate magnetic resonance spectroscopy of tumors. Cancer Research, 73(14), 4190-4195.

74. Rajeshkumar, N. V., et al. (2015). Therapeutic targeting of the Warburg effect in pancreatic cancer relies on an absence of p53 function. Cancer Research, 75(16), 3355-3364.
75. Granchi, C., et al. (2011). Discovery of $\mathrm{N}$-hydroxyindole-based inhibitors of human lactate dehydrogenase isoform A (LDH-A) as starvation agents against cancer cells. Journal of Medicinal Chemistry, 54(6), 1599-1612.

76. Manerba, M., et al. (2012). Galloflavin (CAS 56880-9): A novel inhibitor of lactate dehydrogenase. ChemMedChem, 7(2), 311-317.

77. Vander Jagt, D. L., Deck, L. M., \& Royer, R. E. (2000). Gossypol: Prototype of inhibitors targeted to dinucleotide folds. Current Medicinal Chemistry, 7(4), 479-498.

78. Yu, Y., et al. (2001). Selective active site inhibitors of human lactate dehydrogenases A4, B4, and C4. Biochemical Pharmacology, 62(1), 81-89.

79. Schelman, W. R., et al. (2014). A phase I study of AT-101 with cisplatin and etoposide in patients with advanced solid tumors with an expanded cohort in extensive-stage small cell lung cancer. Investigational New Drugs, 32(2), 295-302.

80. Sonpavde, G., et al. (2012). Randomized phase II trial of docetaxel plus prednisone in combination with placebo or AT-101, an oral small molecule Bcl-2 family antagonist, as first-line therapy for metastatic castration-resistant prostate cancer. Annals of Oncology, 23(7), 1803-1808.

81. Poff, A., et al. (2019). Targeting the Warburg effect for cancer treatment: Ketogenic diets for management of glioma. Seminars in Cancer Biology, 56, 135-148.

82. Lee, W.-N. P., et al. (2004). Metabolic sensitivity of pancreatic tumour cell apoptosis to glycogen phosphorylase inhibitor treatment. British Journal of Cancer, 91(12), 2094-2100.

83. Aklilu, M., et al. (2003). Phase II study of flavopiridol in patients with advanced colorectal cancer. Annals of Oncology, 14(8), 1270-1273.

84. Van Veldhuizen, P. J., et al. (2005). A phase II study of flavopiridol in patients with advanced renal cell carcinoma: Results of Southwest Oncology Group Trial 0109. Cancer Chemotherapy and Pharmacology, $56(1), 39-45$.

85. Liu, G., et al. (2004). A Phase II trial of flavopiridol (NSC \#649890) in patients with previously untreated metastatic androgen-independent prostate cancer. Clinical Cancer Research, 10(3), 924-928.

86. Shapiro, G. I. (2004). Preclinical and clinical development of the cyclin-dependent kinase inhibitor flavopiridol. Clinical Cancer Research, 10(12 Pt 2), 4270s-4275s.

87. Bolidong, D., et al. (2020). Potential therapeutic effect of targeting glycogen synthase kinase 3 beta in esophageal squamous cell carcinoma. Scientific Reports, 10(1), 11807.

88. Abe, K., et al. (2020). Glycogen synthase kinase 3 beta as a potential therapeutic target in synovial sarcoma and fibrosarcoma. Cancer Science, 111(2), 429-440. 
Open Access This chapter is licensed under the terms of the Creative Commons Attribution 4.0 International License (http://creativecommons.org/licenses/by/4.0/), which permits use, sharing, adaptation, distribution and reproduction in any medium or format, as long as you give appropriate credit to the original author(s) and the source, provide a link to the Creative Commons license and indicate if changes were made.

The images or other third party material in this chapter are included in the chapter's Creative Commons license, unless indicated otherwise in a credit line to the material. If material is not included in the chapter's Creative Commons license and your intended use is not permitted by statutory regulation or exceeds the permitted use, you will need to obtain permission directly from the copyright holder. 


\title{
Glutamine Metabolism in Cancer
}

\author{
Ting Li, Christopher Copeland, and Anne Le
}

Keywords
Glutamine metabolism $\cdot$ Glutamine addiction
- Targeting glutamine metabolism $\cdot$
Transaminase upregulation $\cdot$ Targeting amino
acid synthesis

Abbreviations

2HG

2-Hydroxyglutaric acid

Acetyl-CoA Acetyl coenzyme A

ASS

$\mathrm{CAF}$

ccRCC

ECG

EGCG

ETC

FDG-PET

Argininosuccinate synthetase

Cancer-associated fibroblast

Clear cell renal cell carcinoma

Epicatechin gallate

Epigallocatechin gallate

Electron transport chain

Fluorodeoxyglucose-positronemission tomography

$\mathrm{FH}$

GBM

Fumarate hydratase

Glioblastoma multiforme
T. Li · C. Copeland

Massachusetts Institute of Technology,

Cambridge, MA, USA

A. Le $(\bowtie)$

Department of Pathology and Oncology, Johns

Hopkins University School of Medicine,

Baltimore, MD, USA

Department of Chemical and Biomolecular Engineering, Johns Hopkins University Whiting School of Engineering, Baltimore, MD, USA

e-mail: annele@jhmi.edu
GDH

GLS

GOT

GPNA

GPT

HIF

IDH

IDO

IL

NK

NMR

PEG

PHD

PLGA

PPP

PSAT

RCC

SDH

SHMT

SIRM

Glutamate dehydrogenase

Glutaminase

Glutamic-oxaloacetic transaminase

L- $\gamma$-Glutamyl- $p$-nitroanilide

Glutamic-pyruvate transaminase

Hypoxia-inducible factor

Isocitrate dehydrogenase

Indoleamine-2,3-dioxygenase

Interleukin

Natural killer (cell)

Nuclear magnetic resonance

Poly(ethylene glycol)

Prolyl 4-hydroxylases

Poly(lactic-co-glycolic acid)

Pentose phosphate pathway

Phosphoserine aminotransferase

Renal cell carcinomas

Succinate dehydrogenase

Serine hydroxymethyltransferase

Stable isotope-resolved metabolomics

TAM Tumor-associated macrophage

TCA Tricarboxylic acid

TDO Tryptophan-2,3-dioxygenase

TGF $\beta \quad$ Transforming growth factor beta

TNBC Triple-negative breast cancer

$\mathrm{TNF} \alpha \quad$ Tumor necrosis factor alpha

$\mathrm{T}_{\text {reg }}$

TXNIP Thioredoxin-interacting protein

UTR Untranslated region

$\alpha$-KG $\quad \alpha$-Ketoglutarate 


\section{Key Points}

- Glutamine addiction and dysregulation of the TCA cycle are characteristic features of glutamine metabolism in cancers.

- Glutamine metabolism can be targeted by inhibiting glutaminolysis, employing combination therapies, suppressing c-MYC expression, inhibiting GDH, depleting the glutamine supply, inhibiting glutamine uptake, and exploiting glutamine analogs.

- Transaminase upregulation and targeting of amino acid synthesis have the potential for cancer therapy.

- The metabolic reprogramming of cancers provides them with alternative sources of glutamate: via $\mathrm{N}$-acetyl-aspartyl-glutamate (NAAG) and via glutaminase II pathway.

- Glutamine metabolism in the tumor microenvironment can impact the development of cancers.

\section{Introduction}

Metabolism is a fundamental process for all cellular functions. For decades, there has been growing evidence of a relationship between metabolism and malignant cell proliferation. Unlike normal differentiated cells, cancer cells have reprogrammed metabolism in order to fulfill their energy requirements. These cells display crucial modifications in many metabolic pathways, such as glycolysis and glutaminolysis, which include the tricarboxylic acid (TCA) cycle, the electron transport chain (ETC), and the pentose phosphate pathway (PPP) [1]. Since the discovery of the Warburg effect, it has been shown that the metabolism of cancer cells plays a critical role in cancer survival and growth. More recent research suggests that the involvement of glutamine in cancer metabolism is more significant than previously thought. Glutamine, a nonessential amino acid with both amine and amide functional groups, is the most abundant amino acid circulating in the bloodstream [2]. This chapter discusses the characteristic features of glutamine metabolism in cancers and the thera- peutic options to target glutamine metabolism for cancer treatment.

\section{Characteristic Features of Glutamine Metabolism in Cancer}

\subsection{Dysregulation of the TCA Cycle}

This section focuses on abnormalities within the TCA cycle, also known as the citric acid cycle or Krebs cycle (Fig. 1), that alter cancer cell metabolism. The TCA cycle is the central metabolic hub of the cell; it acts as a common pathway for the catabolism of many different sugars, fatty acids, and amino acids [3]. It also generates electrons that fuel oxidative phosphorylation, a process that produces a majority of the energy used by normoxic cells [3].

Under aerobic conditions, pyruvate formed as a product of glycolysis goes through oxidative decarboxylation, a process that removes a carboxyl group as $\mathrm{CO}_{2}$ to form acetyl coenzyme A (acetyl-CoA), the typical starting molecule of the TCA cycle [4]. The TCA cycle takes place within the mitochondrial matrix [5]. The steps of the TCA cycle are as follows: (1) citrate synthase facilitates the condensation of oxaloacetate and acetyl-CoA to form citrate, (2) the enzyme aconitase then converts citrate to isocitrate, (3) isocitrate is further oxidatively decarboxylated by isocitrate dehydrogenase (IDH), (4) the resulting compound $\alpha$-ketoglutarate $(\alpha-\mathrm{KG})$ is transformed into succinyl-CoA, (5) succinyl-CoA is then further converted to succinate by succinyl-CoA synthetase, (6) the succinate dehydrogenase (SDH) complex catalyzes the oxidation of succinate to fumarate, (7) fumarate is hydrated to malate by fumarate hydratase (FH), and finally (8) malate is then oxidized to oxaloacetate by malate dehydrogenase-initiating the cycle once again [6] (Fig. 1).

Mutations of TCA cycle genes have been linked to familial cancer types [6]. Recent research has found that mutations in the TCA cycle enzymes SDH, FH, and IDH resulted in a 


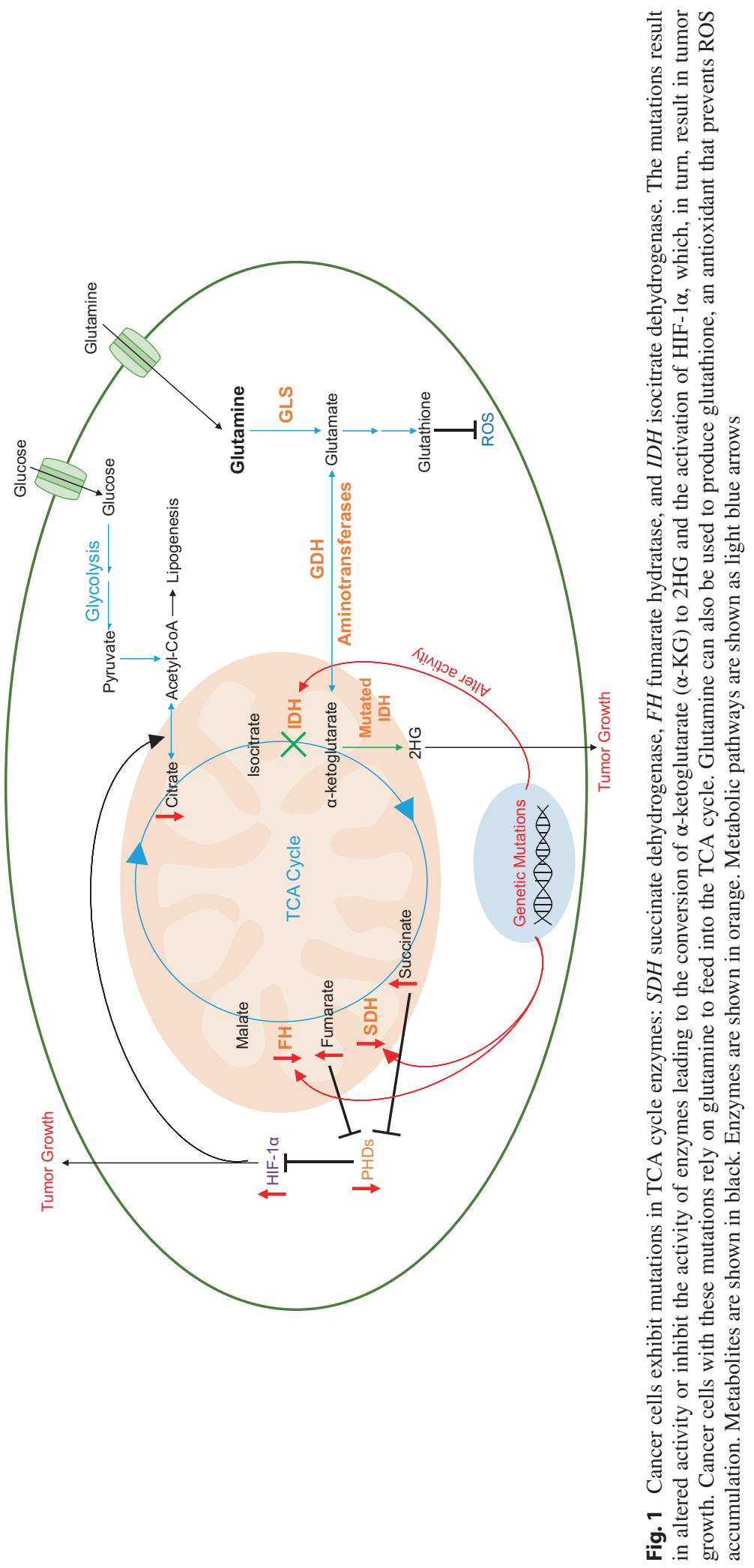


dysfunction of the TCA cycle and defects in mitochondrial metabolism in a wide range of human cancers $[7,8]$. The SDH complex (also known as mitochondrial complex II) is the only membrane-bound enzyme of the TCA cycle and consists of four subunits: SDHA, SDHB, SDHC, and SDHD. SDHA and SDHB are catalytic subunits that protrude into the mitochondrial matrix, while SDHC and SDHD are anchored to the inner membrane [9]. The SDH enzyme plays an essential role in tumor suppression. Heterozygous mutations in SDH genes cause complete inactivation of the protein function and are associated with hereditary paragangliomas and pheochromocytomas [10-12]. Tumors exhibiting SDH mutations are more aggressive and usually proliferate at a much faster rate than normal cells [9]. In addition to these cancers, several other neoplasms have been associated with mutations in SDH subunits, including renal cell carcinoma (RCC), neuroblastoma, gastrointestinal stromal tumors, thyroid cancer, and testicular seminoma [13-15].

Similar to SDH, FH mutations occur throughout the genome. Research has indicated an association between heterozygous FH mutations and uterine fibroids, hereditary leiomyomatosis, and papillary renal cell cancer [16]. Additionally, loss of the wild-type allele in these cancers resulted in the absence of FH enzymatic activity. FH acts as a tumor suppressor in these cancers, and its reduced expression leads to the accumulation of the transcription factor hypoxia-inducible factor- $1 \alpha$ $(\mathrm{HIF}-1 \alpha)[1,17]$. High levels of fumarate accumulate and act as oncometabolites, which often result in dysregulation of cellular functions in SDH- or FH-deficient cells [1]. Both accumulated succinate and fumarate are potent inhibitors of prolyl 4-hydroxylases (PHDs). PHDs are negative regulators of HIF-1 $\alpha$, a transcriptional factor that is upregulated under hypoxic conditions when tumor cells are deprived of adequate oxygen supplies. Impaired PHD activity leads to HIF- $1 \alpha$ activation under normoxia, a condition known as pseudohypoxia [6]. Pseudohypoxia, in turn, facilitates tumor cell growth.

Similar to the metabolic consequences of $\mathrm{SDH}$ and FH mutations, mutations in the IDH enzyme also result in dysfunction of the TCA cycle. There are three isoforms of the enzyme IDH. IDH1 is found in the cytoplasm and peroxisomes; IDH2 and IDH3 are localized in the mitochondrial matrix. IDH3 is the primary form of IDH in the TCA cycle, whose function is to convert isocitrate to $\alpha-\mathrm{KG}$. Genomic analysis has identified mutations in either IDH1 or IDH2 in the vast majority of grade II and III gliomas as well as glioblastoma multiforme (GBM) patient samples [18]. The abnormal expression and activity of IDHs result in the loss of the enzyme's ability to catalyze the conversion of isocitrate to $\alpha-\mathrm{KG}$; instead, it gains a new ability to facilitate the NADPHdependent reduction of $\alpha-K G$ to $\mathrm{D}-2-$ hydroxyglutaric acid (D-2HG), an oncometabolite. Subsequently, excess accumulation of $2 \mathrm{HG}$ contributes to the formation of malignant gliomas [19]. The discovery that IDH1 and IDH2 mutations are nearly all missense mutations localized to residues R132 and R172, respectively, provides a promising biomarker for cancer diagnosis and possibly gene therapy $[20,21]$. It was found that cells harboring IDH1-R132 and IDH2-R172 mutations in patients with acute myeloid leukemia gained the ability to convert $\alpha-\mathrm{KG}$ to D-2HG [22].

\subsection{Glutamine Addiction}

In addition to glucose, proliferating cancer cells also rely on glutamine as a major source of energy and building blocks, with glutamine feeding into the TCA cycle. This condition is known as glutamine addiction. Glutamine is one of the most abundant nonessential amino acids in the bloodstream (produced by the human body and thus not an essential part of the diet) and contributes to virtually every biosynthetic pathway in proliferating cells. Moreover, it acts as a nitrogen donor in purine and pyrimidine synthesis as well as a precursor for protein and glutathione biosynthesis [23]. Many tumor cells are reliant on exogenous glutamine and have been reported to die in its absence [24]. 
Since glutamine-derived $\alpha-K G$ fuels the TCA cycle, cancer cells can employ glutaminolysis to sustain the biosynthesis of many essential molecules. In RCCs that were either ETC or TCA cycle deficient, it was found that cancer cells relied on the reductive carboxylation of glutamine-derived citrate to produce acetyl-CoA and other precursors to TCA cycle metabolites. Acetyl-CoA is a necessary intermediate for the synthesis of lipids, and without it, cancer cells are not viable. Furthermore, TCA cycle intermediates are needed to synthesize other essential cellular building blocks. Thus, cells can become utterly dependent upon glutaminolysis as a result of genetic alterations affecting oxidative mitochondrial function [25]. A study by Gameiro et al. found that the transcription factor HIF expression maintained a low level of intracellular citrate to maintain adequate lipogenesis. Therefore, the von Hippel-Lindau (VHL)deficient RCC cells that constitutively express HIF- $1 \alpha$ and/or HIF- $2 \alpha$ become heavily dependent on glutamine for proliferation [26, 27].

Glutamine addiction was also found to occur in glioma cells that possess a recurrent mutation of IDH1. As mentioned in the section above, IDH1 catalyzes typically the conversion of isocitrate to $\alpha-\mathrm{KG}$, but the mutant isoform converts $\alpha-\mathrm{KG}$ into D-2HG instead, which has been shown to inhibit cellular differentiation through epigenetic alterations [19]. Due to the mutant IDH1 function, glioma cells become increasingly dependent upon glutamine-derived $\alpha-K G$ production. Thereby cancer cells exhibiting glutamine addiction rely on glutaminase (GLS), an enzyme that converts glutamine to glutamate, which in turn is converted to $\alpha-\mathrm{KG}$ for survival. The inhibition of GLS suppresses the growth of glioma cells with IDH1 mutations by decreasing the availability of glutamine-derived $\alpha-\mathrm{KG}$ [28].

Further evidence suggests that the dependence of specific cancer cells on glutamine may be more profound than previously thought. Fluorodeoxyglucose-positron-emission tomography (FDG-PET) scanning, a clinical imaging technique, can detect cancers based on areas of increased glucose uptake. Specifically, ${ }^{18}$ F-FDGPET imaging exploits increased glucose transport and hexokinase II activities in proliferating tumor tissues to locate high levels of ${ }^{18} \mathrm{~F}-\mathrm{FDG}$, which can be visualized by PET images. However, some cancers are invisible to PET scans and are deemed PET negative. These PET-negative cancers must rely on alternative metabolic substrates for their primary source of energy, namely glutamine [29]. Researchers have used $5-{ }^{11} \mathrm{C}-(2 S)$-glutamine and ${ }^{18} \mathrm{~F}-(2 S, 4 R) 4$-fluoroglutamine as glutamine-based PET imaging agents, where ${ }^{18} \mathrm{~F}$ is preferred because it has 5.5 times the half-life of ${ }^{11} \mathrm{C}[30$, 31]. Both tracers are useful tools for probing in vivo metabolism of glutamine and monitoring radiation effects in cancer patients.

Cancer cells use precursors derived from the TCA cycle intermediates to synthesize proteins, lipids, and nucleic acids. In order to maintain mitochondrial activity, these cells must compensate for lost TCA cycle intermediates caused by their metabolic diversions [32]. This process of replenishing metabolic intermediates is known as anaplerosis [33]. Glutamine provides mitochondrial anaplerosis because of its role as a nitrogen and carbon donor to the cells [32]. It traverses the cell membrane through amino acid transporters, ASCT2 (alanine, serine, cysteine transporter 2), and system N transporter SN2 [34]. Once it enters the cytoplasm, glutamine is hydrolyzed to glutamate and ammonia $\left(\mathrm{NH}_{3}\right)$ via GLS (Fig. 2) [24].

Glutamate can be further catabolized through the TCA cycle (via conversion to $\alpha \mathrm{KG}$ ) or serve as a substrate for glutathione synthesis. $\alpha-\mathrm{KG}$ formation can be catalyzed by either glutamate dehydrogenase (GDH) or aminotransferases. Alternatively, glutamate can be further converted to glutathione by glutathione cysteine ligase and glutathione synthetase. Glutathione is an antioxidant vital to a cell's immune defense, nutrient metabolism, and cellular functions [35]. It also plays an important role in the neutralization of mitochondrial reactive oxygen species (ROS). ROS are by-products of oxygen metabolism, the concentration of which plays a vital role in tumor proliferation, growth, apoptosis, and metastasis. Studies have found that ROS acts as a "doubleedged sword" in some cancers; moderately elevated levels of ROS facilitate carcinogenesis while excessive levels can trigger apoptosis by causing 


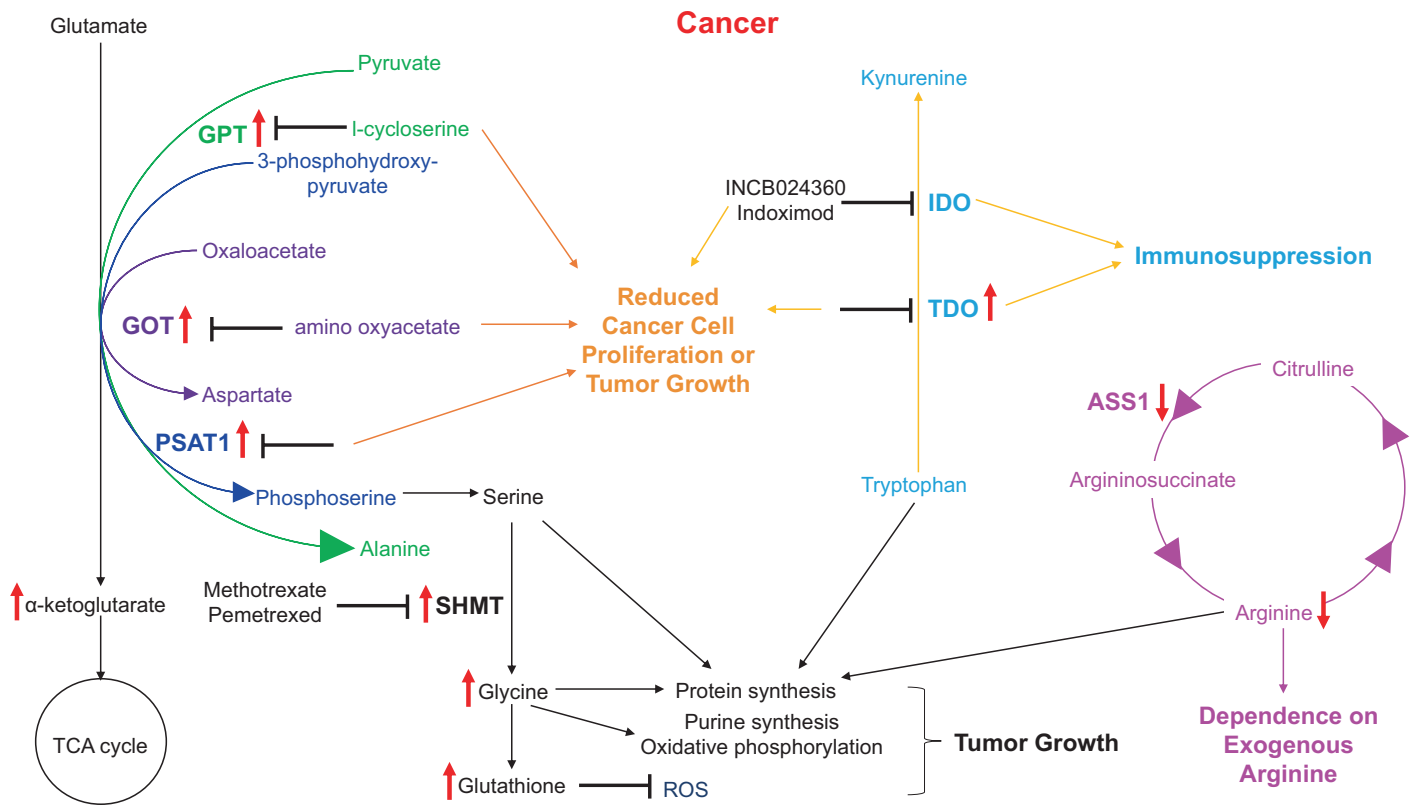

Fig. 2 Glutamate can be catalyzed via three different pathways by the aminotransferases GPT, GOT, and PSAT1, all of which yield $\alpha$-ketoglutarate $(\alpha-K G)$ and a different amino acid, alanine, aspartate, and phosphoserine, respectively. These enzymes are upregulated in cancer, which results in increased $\alpha-K G$ production. Inhibition of the enzymes results in reduced cancer cell proliferation or tumor growth. Phosphoserine can enter serine and glycine metabolism, where the enzyme SHMT is also upregulated in cancer. This results in increased production of glycine, which supports protein and purine synthesis and oxidative phosphorylation, thus leading to tumor growth. Elevated level of glycine further leads to increased level of glutathione, which mediates oxidative stress and also leads to tumor growth. Tryptophan metabolism is linked to the regulation of antitumor immune responses, where the enzymes IDO and TDO can lead to immunosuppression. Inhibition of IDO or TDO leads to reduced tumor growth. Some cancers have reduced ASS1 which leads to decreased arginine level, causing these cancers to rely on exogenous arginine supply. GPT glutamic-pyruvate transaminase, GOT glutamic-oxaloacetic transaminase, PSAT1 phosphoserine aminotransferase 1, ASS1 argininosuccinate synthetase 1, IDO indoleamine-2,3-dioxygenase, $T D O$ tryptophan-2,3-dioxygenase, SHMT serine hydroxymethyltransferase

damage to DNA and other macromolecules [36]. The dual role of ROS is also evident in the early and late stages of cancer. At precancerous and neoplastic stages, ROS tends to promote proliferation by inducing oxidative damage and base-pair mutations. In the late stages, however, increased energy need is correlated with an increase in ROS production [37]. Therefore, for a ROS-mediated therapeutic approach to be effective, this therapy must limit ROS production when ROS would induce proliferation, and it must promote ROS production when ROS would induce apoptosis.
The inhibition of glutamine metabolism has been linked to ROS overproduction, which can devastate cancer cells [38, 39]. Mitochondrial glutamine metabolism is a significant anaplerotic step in tumorigenesis. It is often enhanced in cancer cells with increased levels of TCA cycle metabolites [32]. Therefore, inhibiting GLS could effectively starve cancer cells of the glutamine essential to their survival and prevent further glutathione synthesis, thus increasing cancer cell exposure to excessive ROS levels [40]. 


\subsection{The Metabolic}

Reprogramming of Cancers

Provides Them

with Alternative Sources

of Glutamate: Via $\mathrm{N}$-Acetyl-

Aspartyl-Glutamate (NAAG) and via the Glutaminase II

Pathway

Two recent studies have uncovered alternative sources of glutamate in cancer. The first study identified $\mathrm{N}$-acetyl-aspartyl-glutamate (NAAG), which can be hydrolyzed to glutamate when needed by the oncogenic cells via glutamate carboxypeptidase II (GCP II) [41, 42], suggesting that GCPII is a viable target for cancer therapy, either alone or in combination with glutaminase inhibition. The second study demonstrated that upon the inhibition of glutaminase I pathway (the conversion of glutamine to glutamate by GLS), pancreatic cancer cells utilized the glutaminase II pathway to generate glutamate via glutamine transaminase K (GTK) [43]. Knocking down the expression of GTK inhibited the growth of pancreatic cancer cells in vitro and tumorigenesis in vivo. The uncovering of the alternative sources of glutamate in cancer opens up new strategic approaches. Specifically, these studies suggest a combination therapy of GLS1 and GCPII and/or GTK inhibition for pancreatic cancer therapy.

\section{$3 \quad$ Targeting Glutamine Metabolism for Cancer Therapy}

Due to its central role in many cancers, glutaminolysis is becoming an increasingly prominent target for cancer therapy. Mammalian cells express two isoforms of GLS: kidney-type GLS1 and liver-type GLS2. GLS1 is more broadly expressed in normal tissue, while GLS2 is mainly present in the liver, brain, pituitary gland, and pancreas [44]. Both encode a mitochondrial GLS, which catalyzes the hydrolysis of glutamine to glutamate [45, 46]. Studies have shown that $c-M Y C$, a multifaceted transcriptional factor, upregulates glutamine importers and GLS1 expression and that $\mathrm{p} 53$, a tumor-suppressor gene/protein, upregulates GLS2 expression [46-48].

Using stable isotope-resolved metabolomics (SIRM) studies [49], Le et al. also reported the persistence of glutamine oxidation via the TCA cycle under hypoxia. SIRM studies track metabolic transformations using stable isotope labeling and analyze the metabolic products using nuclear magnetic resonance (NMR) and mass spectrometry (MS) at different time points. Using a human Burkitt lymphoma model P493 cell line carrying an inducible $M Y C$ vector, the group showed the coexistence of oxidative and aerobic glycolysis. Thus, inhibition of GLS induced oxidative stress and diminished ATP levels in hypoxic cells [39]. It was also found that glutamine metabolism supports cellular bioenergetics and redox homeostasis for proliferation under both aerobic and hypoxic conditions. P493 cells exhibited low glutathione levels and high ROS production under inhibition of GLS and hypoxia. Furthermore, glutaminederived glutathione production was sustained under hypoxia as a coping method under high ROS levels [39]. These results again suggest that glutamine metabolism, specifically through GLS enzyme, is a promising target for cancer therapy.

The TP53 gene codes for a tumor-suppressor protein known to trigger cell cycle arrest, apoptosis, or senescence in response to a variety of cellular dysfunctions, including DNA damage, oncogene activation, and hypoxia [50]. It is one of the most frequently mutated genes among all cancers. However, recent studies have discovered TP53's additional roles in regulating energy metabolism and antioxidant defense mechanisms [51, 52]. GLS2 is a $p 53$ target gene that plays an important role in mediating the tumor-suppressant properties of $p 53$. GLS2 increases intracellular levels of glutamate and $\alpha-\mathrm{KG}$, thus leading to enhanced mitochondrial respiration and ATP production. It also leads to increased cellular glutathione levels and thus decreased ROS levels [46]. Hu et al. demonstrated that $p 53$ increased GLS2 expression under both stressed and nonstressed conditions - enhancing glutamate levels, mitochondrial respiration rates, and glutathione levels while decreasing ROS levels. Furthermore, 
Table 1 Current therapeutic strategies and their mechanism of action in targeting glutamine metabolism

\begin{tabular}{l|l}
\hline Classification & Drug \\
\hline \multirow{2}{*}{ GLS inhibitors } & CB-839 (Telaglenastat) \\
\cline { 2 - 2 } & BPTES \\
\cline { 2 - 2 } Glutamine depletion & L-As \\
\cline { 2 - 2 } ASCT2 inhibitors & Phenylbutyrate \\
\hline & $\begin{array}{l}\text { L- } \gamma \text {-Glutamyl-p-nitroanilide } \\
\text { (GPNA) }\end{array}$ \\
\cline { 2 - 2 } & V-9302 \\
\cline { 2 - 2 } & $\gamma$-FBP \\
\hline Glutamine mimetics & Acivicin \\
\cline { 2 - 2 } & Azaserine \\
\cline { 2 - 2 } & $\begin{array}{l}\text { 6-Diazo-5-oxo-L-norleucine } \\
\text { (DON) }\end{array}$ \\
\hline GDH inhibitors & R162 \\
\cline { 2 - 2 } & EGCG \\
\hline & ECG \\
\hline $\begin{array}{l}\text { Aminotransferase } \\
\text { inhibitors }\end{array}$ & AOA \\
\hline
\end{tabular}

the GLS2 gene promoter contains a $p 53$ consensus DNA-binding element whose expression was induced in response to oxidative stress $[1,46]$. Hu's findings suggest that GLS2 may be a mediator to $p 53$ 's role in energy metabolism and antioxidant defense, ultimately contributing to its tumor suppression abilities.

Due to its crucial role in energy regulation and biosynthesis, targeting glutamine metabolism has the potential to affect a broad spectrum of cancers. In addition to GLS inhibition, the role of oncogenes and tumor suppressors in regulating glutamine metabolism makes it a promising venture for therapeutic strategies. However, while many drugs have been synthesized to target glutamine metabolism from its initial transport into the cell to its conversion to $\alpha-\mathrm{KG}$, most are still in preclinical stages (Table 1) [44].

\subsection{Inhibition of Glutaminolysis by GLS Inhibitors}

The most straightforward approach for targeting glutaminolysis is the inhibition of GLS, which catalyzes the hydrolysis of glutamine to glutamate. The kidney isoform, GLS1 (or GLS), is found in many primary tumors, while the liver isoform, GLS2, is less often expressed in cancers [53]. Inhibiting GLS can starve cancer cells by blocking the synthesis of glutamate and thus prevent $\alpha-\mathrm{KG}$ from feeding the TCA cycle. After nuclear factor kappa B (NF-kB) activates glutaminase $\mathrm{C}$ isoform (GAC), an alternatively spliced isoform of GLS1, via phosphorylation, NF-kB itself is activated by Rho GTPases. Alteration of Rho GTPases by small-molecule inhibitors showed a significant decrease in GAC activity in human breast cancer cells [54]. The decrease in GAC activity caused breast cancer cells to stop proliferating and reduced their ability to invade surrounding cells [54]. Potent therapeutic GLS inhibitors, such as bis-2-(5-phenylacetamido-1,2,4-thiadiazol-2-yl)ethyl sulfide (BPTES) and its analogs such as CB-839, are being actively investigated in GLS-targeting studies [38, 54-58].

BPTES allosterically inhibits GLS1 by altering the conformation of the enzyme and has been proven in many studies to inhibit cancer cell growth in vitro and slow tumor growth in vivo $[39,56,59]$. While BPTES produces formidable results in vitro, higher concentrations are needed to achieve the same effect in vivo. Due to its low solubility, BPTES tends to precipitate at high concentrations, posing a challenge to the physiological delivery of the drug in clinical trials [60]. A solution was proposed by Elgogary et al. with an emulsification method that encapsulated BPTES into biodegradable nanoparticles coated with poly(ethylene glycol) (PEG) and poly(lacticcoglycolic acid) (PLGA) to improve nanoparticle circulation time in the blood. This process enhanced the efficacy of BPTES in vivo by improving its solubility and increasing tumor drug exposure [38].

The only GLS inhibitor to have entered clinical trials is commonly referred to as CB-839 or commercially as Telaglenastat. CB-839 is a highly potent allosteric inhibitor that completed phase I clinical trials in March 2019 in patients with advanced RCC (NCT02071862) and has in 2020 moved to phase II clinical trials in patients with non-small cell lung cancer (NCT04265534). Phase I trials have been completed for hematological tumors (NCT02071888) and leukemias (NCT02071927), although no clinical data is pub- 
licized. CB-839 has shown efficacy in the treatment of triple-negative breast cancer (TNBC), showing a marked decrease in glutamine consumption, glutamate production, and levels of glutathione and other TCA cycle intermediates [55]. Elevated GLS expression is also associated with different cancer types, including glioblastoma and pancreatic cancers, thereby encouraging investigation of more targets should current clinical trials prove successful [28, 61].

Taken together, glutamine dependency may be a particular metabolic vulnerability of cancer cells, and glutaminolysis-targeting strategies could be promising approaches for glutamineaddicted cancer therapy.

\subsection{Combination Therapy}

The heterogeneity of cancer metabolism, even within the same tumor [62], poses many challenges for potential drug therapies. Hence, the use of combination therapies to target multiple metabolic pathways to suppress tumor growth may be most effective, especially in identifying cases that induce synthetic lethality, where two drugs induce cell death in combination, but not individually. Glutamine's role in cellular functions makes GLS inhibition an ideal candidate for combination therapy. In their study, Elgogary et al. found that combination therapy of BPTES and metformin produced better results in pancreatic tumors than monotherapy of either drug alone. Metformin is an FDA-approved drug for the treatment of type 2 diabetes that inhibits glycolysis and glycogen synthesis. In this case, BPTES targets glutamine metabolism, and metformin targets glucose metabolism, resulting in an optimal reduction of tumor development [38]. Other treatments that are synthetically lethal with the inhibition of GLS include GLUT2q1 inhibition, mTOR inhibition, and ATF4 activation [44].

In recent years, researchers have increasingly leveraged combination therapies for the treatment of a variety of cancers (Table 2) [63]. Parlati et al. combined CB-839 with pomalidomide to target myeloma models [64]. Momcilovic et al. treated lung cancer with the synergistic
Table 2 Current combination therapeutic strategies employing CB-839 and BPTES on various cancer types and their results, non-exhaustive [63]

\begin{tabular}{|c|c|c|}
\hline $\begin{array}{l}\text { Tumor xenograft } \\
\text { cell line and type }\end{array}$ & Treatments & $\begin{array}{l}\text { Tumor } \\
\text { growth } \\
\text { inhibition }\end{array}$ \\
\hline \multirow{3}{*}{$\begin{array}{l}\text { RPMI-8226 } \\
\text { (myeloma) [38, } \\
64]\end{array}$} & CB-839 & $64 \%$ \\
\hline & Pomalidomide & $46 \%$ \\
\hline & $\begin{array}{l}\text { CB- } \\
839+\text { pomalidomide }\end{array}$ & $97 \%[64]$ \\
\hline \multirow{3}{*}{$\begin{array}{l}\text { H1650 (lung) } \\
{[64,65]}\end{array}$} & CB-839 & $26 \%$ \\
\hline & Erlotinib & $66 \%$ \\
\hline & CB-839 + erlotinib & $89 \%[65]$ \\
\hline \multirow{3}{*}{$\begin{array}{l}\text { JIMT-1 (breast) } \\
{[55,65]}\end{array}$} & CB-839 & $54 \%$ \\
\hline & Paclitaxel & $73 \%$ \\
\hline & CB-839 + paclitaxel & $100 \%[55]$ \\
\hline \multirow{3}{*}{$\begin{array}{l}\text { Caki-1 (RCC) } \\
{[55,66]} \\
\text { DU145 } \\
\text { (prostate) [67] }\end{array}$} & $\begin{array}{l}\text { CB- } \\
839+\text { cabozantinib }\end{array}$ & $\begin{array}{l}\text { *Precise } \\
\text { data not } \\
\text { shown [66] }\end{array}$ \\
\hline & $\begin{array}{l}\text { CB- } \\
839+\text { everolimus }\end{array}$ & $\begin{array}{l}\text { *Precise } \\
\text { data not } \\
\text { shown }\end{array}$ \\
\hline & $\begin{array}{l}\text { CB- } \\
839+\text { talazoparib }\end{array}$ & $\begin{array}{l}\text { *Precise } \\
\text { data not } \\
\text { shown [67] }\end{array}$ \\
\hline $\begin{array}{l}\text { MCF-7 (breast) } \\
{[66,67]} \\
\text { HCT-116 } \\
\text { (colon) }[67]\end{array}$ & $\begin{array}{l}\text { CB- } \\
839+\text { palbociclib }\end{array}$ & $\begin{array}{l}\text { *Precise } \\
\text { data not } \\
\text { shown [67] }\end{array}$ \\
\hline \multirow{3}{*}{$\begin{array}{l}\text { JH094 } \\
\text { (pancreatic) }\end{array}$} & BPTES & $* 50 \%$ \\
\hline & Metformin & $* 20 \%$ \\
\hline & BPTES + metformin & $* 65 \%[38]$ \\
\hline
\end{tabular}

combination of CB-839 and erlotinib, demonstrating significant reductions in glucose and glutamine uptake [68]. Emberley et al. explored the various combinations of CB-839 with CDK4/6 and PARP inhibitors in colorectal carcinoma (CRC), TNBC, ovarian cancer, and nonsmall cell lung carcinoma [67].

\subsection{Knockdown of c-MYC}

In a study by Wise et al., $c-M Y C$ expression was found to activate the transcription of key regulatory genes required for glutamine uptake and metabolism by selectively binding to the promoter regions of glutamine transporters ASCT2 and $\mathrm{SN} 2$. As a result, $c-M Y C$ induced reprogramming of mitochondrial metabolism by diverting glucose away from the TCA cycle and leaving 
cells susceptible to glutamine addiction to sustain anaplerosis. Moreover, $c-M Y C$-transformed cells were found to be sensitive to GDH inhibitors. These results suggest that glutamine addiction may be a direct consequence of $c-M Y C$ activation [48]. Gao et al. found that $c-M Y C$ expression induced the expression of mitochondrial GLS in human P493 lymphoma B cells and PC3 prostate cancer cells by suppressing microRNAs miR-23a and miR-23b, which target the GLS $3^{\prime}$ untranslated region (UTR) seed sequence [1, 47]. Liu et al. established a direct link between glutamine and proline via MYC-induced proline biosynthesis from glutamine, suggesting novel therapeutic strategies [69]. Overall, these results may be exploited for cancer therapy using inhibitors of enzymes involved in glutamine metabolism or therapeutics that inhibit the transcriptional properties of $c-M Y C$.

The transcriptional factor $M Y C$ is an essential growth regulator that is overexpressed in most cancers, and hence is a highly sought-after target for cancer therapies $[70,71]$. Niu et al. found that suppressing $c-M Y C$ expression resulted in reduced cell growth, colony formation, and tumor formation in nasopharyngeal carcinoma cell lines both in vitro and in vivo [72]. Using RNA interference (RNAi)-mediated silencing of the $c-M Y C$ gene, Zhang et al. showed that the downregulation of $c-M Y C$ induced apoptosis in vitro and suppressed the growth of colon cancer cells in vivo [73].

Other attempts at directly or indirectly targeting $M Y C$ have included knockdown, protein/protein and DNA interaction inhibitors, and translation and expression regulation [74]. Direct inhibition of $M Y C$ expression can be achieved by using G-quadruplex stabilizers that prevent $M Y C$ transcription or antisense oligonucleotides and siRNAs that prevent MYC translation [75-78]. Small-molecule protein/protein interaction inhibitors have also been used to interfere with $M Y C$ transcriptional activation $[79,80]$. Indirect inhibition methods encompass blocking transcription, hindering mRNA translation, and targeting regulators of MYC protein stability [81, 82]. The most advanced methods currently in clinical trials or commercially approved employ indirect targeting by immunotherapies, which focus on immune components required for $M Y C$-driven tumors or checkpoints that are altered in $M Y C$ driven tumors [83, 84].

\subsection{Inhibition of Glutamate Dehydrogenase (GDH)}

Inhibiting the oxidative deamination of glutamate to $\alpha-K G$ has devastating effects on cancer cells comparable to inhibiting glutaminolysis $[61,85]$. This process is catalyzed by GDH, which can be inhibited by the preclinical compounds R162, epigallocatechin gallate (EGCG), and epicatechin gallate (ECG) [44, 86]. Using perifusion assays, Li et al. showed that EGCG and ECG blocked GDH activity by binding to the allosteric regulator ADP's binding site [87].

Furthermore, it was demonstrated that polyphenols such as hexachlorophene and bithionol might inhibit GDH function. These inhibitors work by restricting enzyme movement, either by forming ring barriers around the enzyme or by wedging between the enzyme's subunits. Currently, polyphenols have been shown to inhibit lung, colon, and prostate adenocarcinoma growth in xenograft models [88]. These compounds also had significant effects on glioblastoma, colon, lung, and prostate adenocarcinoma cell proliferation [89]. Additionally, it was found that GDH inhibition through siRNA resulted in a marked decrease in the proliferation of glioblastoma cells that were glutamine dependent $[18,90]$.

\subsection{Inhibiting the TCA Cycle by Depleting Glutamine, $\alpha$-Ketoglutarate, and Asparagine}

One of the earlier means of suppressing glutamine metabolism arose from reducing the amount of available glutamine itself. Ollenschläger et al. found that the abundance of glutamine in the body dropped precipitously by giving L-asparaginase to patients with acute myeloid leukemia. 
Asparaginase catalyzes the removal of the amide nitrogen from asparagine to form aspartic acid. The administration of asparaginase also depleted stored levels of glutamine [91]. When applied in cell culture, asparaginase inhibited cell growth and induced cell death in pancreatic cancer cells. The effect of asparaginase can largely be reversed through the reintroduction of small amounts of glutamine [92].

Studies of acute lymphoblastic leukemia indicated that asparaginase activity correlated with glutamine depletion in the bloodstream and improved treatment outcomes [93, 94]. Furthermore, cancer cells, with a deficiency of asparagine synthase (ASNS), an enzyme that generates asparagine, such as acute lymphoblastic leukemia (ALL), must use asparagine from the blood [95]. In 1979, Ertel et al. treated ALL patients with asparaginase, which exhausted the asparagine supply in the blood. This treatment re-induced remission in up to $60 \%$ of cases [96]. ALL can upregulate ASNS to restore intracellular asparagine levels and satisfy their asparagine demand [97]. However, some studies show that ASNS levels may not impact the sensitivity of ALL to asparaginase treatments in all cases [98]. The diverse metabolic phenotypes of malignant cells create many challenges for therapeutic strategies. It seems that a combination of drug therapy targeting both asparagine and glutamine metabolism could be a promising treatment.

Tempol (4-hydroxy-2,2,6,6-tetramethylpiperidine-1-oxyl), a drug previously known to inhibit cancer cell proliferation and increase vulnerability to other cytotoxic agents, has been shown to interfere with glutamine metabolism through inhibition of IDH1/2 and slowing of the TCA cycle [99]. This effect was found both in vitro and in vivo and showed that Tempol might be a powerful therapeutic in combination with other cancer drugs.

Another possible therapeutic in development is phenylbutyrate, a drug that lowers glutamine concentrations in the plasma. It is currently approved by the FDA and has shown clinical improvement in patients with hormone-refractory prostatic carcinoma and GBM [94, 100, 101].

\subsection{Inhibiting Glutamine Uptake}

The $c-M Y C$-activated amino acid transporter ASCT2 (or SLC1A5) is upregulated in many cancers and is involved in controlling glutamine uptake [48, 94]. High levels of ASCT2 are correlated with aggressive tumor growth and short survival time. ASCT2 inhibitors include benzylserine, L- $\gamma$-glutamyl- $p$-nitroanilide (GPNA), and V-9302 [44, 102, 103]. Additionally, Colas et al. found that ASCT2 ligands chloroalanine, aminooxetane3 -carboxylate (AOC), and $\gamma$-FBP also inhibit ASCT2-mediated glutamine uptake in human melanoma cells [104]. Research shows that the inhibition of glutamine importers significantly slowed growth in human colon and lung cancer cells $[105,106]$.

Benzylserine and GPNA are amino acid analogs; however, while they reduce glutamine uptake, they do not exclusively inhibit the ASCT2 function [107]. Their low affinity and specificity make them unsuitable for clinical trials that specifically target glutamine addiction via ASCT2. V-9302 is a more contemporary inhibitor; however, research has indicated that its efficacy was likely unrelated to ASCT2 inhibition, yielding again to the problem of non-specificity [108].

The development of blocking antibodies and antibody-drug conjugates presents an alternative, perhaps a more viable approach to inhibiting glutamine uptake by regulating the ASCT2 transporter [109]. MEDI7247 is the only ASCT2 antibody-drug conjugate currently in phase I clinical trials (NCT03811652, NCT03106428).

\subsection{Using Glutamine Mimetics}

Another means of decreasing the availability of glutamine is the creation of glutamine analogs. Analogs such as 6-diazo-5-oxo-L-norleucine (DON) and acivicin did show cytotoxic effects against several tumor types, including leukemia and colorectal cancers; however, these analogs are no longer clinically available due to patient toxicity [110]. DON prodrugs have shown enhanced cerebrospinal fluid delivery, although toxicity was still observed [111]. 
A few glutamine analogs, namely acivicin, azaserine, and DON, have been extensively researched in an effort to inhibit glutamine metabolism. DON is a substrate analog of glutamine that binds to the active site of human kidney GLS to serve as an inhibition mechanism [112]. However, DON has had difficulty progressing into clinical trials due to concerns regarding its lack of selectivity to GLS and toxicity $[110,113$, 114]. Clinical studies of DON co-administered with PEGylated glutaminase (PEG-PGA) suggested improved efficacy and it holds promise [115]. Similar to DON, acivicin and azaserine are also glutamine analogs that interrupt nucleotide synthesis by inhibiting amidotransferases [110, 116]. All three analogs exhibit excessive side effects and toxicity that have prevented them from reaching or advancing in clinical trials.

\section{Transaminase Upregulation and Targeting Amino Acid Synthesis for Cancer Therapy}

Another means of inhibiting glutaminolysis is to target alanine transaminase through L-cycloserine or aspartate transaminase through the inhibitor amino oxyacetate, which could almost completely halt the growth of breast cancer in xenograft mice $[117,118]$. What is truly promising is that there appears to be little to no toxicity in non-neoplastic cells. The effectiveness of the inhibitor, combined with the lack of toxicity, makes inhibition of aspartate aminotransferase a potentially successful chemotherapeutic target.

Transaminases, also known as aminotransferases, are enzymes that catalyze reactions between amino acids and $\alpha$-keto acids. Specifically, aminotransferases can convert glutamate to $\alpha-\mathrm{KG}$ without producing ammonia. Glutamate acts as a nitrogen donor in these transaminations. Alanine aminotransferase, also known as glutamic-pyruvate transaminase (GPT), and aspartate aminotransferase, also known as glutamic-oxaloacetic transaminase (GOT), are abundantly present in the liver and often serve as markers for liver toxicity. There are three aminotransferase pathways through which glutamate can be transformed to $\alpha-K G$. These key enzymes in these pathways are GPT, GOT, and phosphoserine aminotransferase 1 (PSAT1) - each of which produces a different amino acid by-product in addition to $\alpha-K G$. As illustrated in Fig. 2, GPT transfers nitrogen from glutamate to pyruvate to produce alanine and $\alpha-K G$. GOT transfers nitrogen from glutamate to oxaloacetate to produce aspartate and $\alpha-K G$. PSAT1 transfers nitrogen from glutamate to 3-phosphohydroxypyruvate to produce phosphoserine and $\alpha-K G$ [44]. PSAT1 is also involved in the serine synthesis pathway, which is essential for many breast cancers [119]. Serine is essential for the synthesis of proteins necessary for cell proliferation. PSAT1 expression has recently been demonstrated to be upregulated in cancers in many studies [44]. Possemato et al. found that serine pathway flux is augmented in some breast cancer cell lines and that suppression of PSAT1 inhibited proliferation of these cells in addition to causing a significant reduction of $\alpha-K G$ [120]. In a study by Son et al., aspartate aminotransferases were demonstrated to be vital in maintaining redox homeostasis in pancreatic ductal adenocarcinoma (PDAC) cells. Furthermore, oncogenic mutant KRAS activity was found to upregulate the expression of aminotransferases, hence yielding high ROS levels and slowing tumor growth in vivo [85, 116, 121]. Taken together, these works suggest that targeting the amino acid synthesis pathway may be another effective strategy for cancer therapy.

Apart from glutamine, many other amino acids play important roles in tumorigenesis, namely arginine, tryptophan, serine, and glycine. Arginine is a precursor for the synthesis of proteins, urea, and various signaling molecules [122]. Although arginine is considered a nonessential amino acid, many cancer cells are dependent upon arginine for proliferation. Argininosuccinate synthetase 1 (ASS1) catalyzes the conversion of citrulline into argininosuccinate in the arginine synthesis pathway. Loss or suppression of ASS1 in osteosarcoma cells resulted in the depletion of arginine. Studies have shown that ASS1 acts as a tumor suppressor because cells with low ASS1 expression could not grow in an environment without arginine [123, 124]. 
Tryptophan is linked to the regulation of antitumor immune responses [125]. Figure 2 shows that it can be degraded to kynurenine via two enzymes: indoleamine-2,3-dioxygenase (IDO) and tryptophan-2,3-dioxygenase (TDO). IDO activity commonly leads to the suppression of the immune system [126]. Dendritic cells (immune system cells that present antigens to $\mathrm{T}$ cells) expressing IDO can limit tryptophan supply to T cells in the extracellular matrix, thus limiting T-cell response to tumor growth [127]. Studies have shown that mice transfected with IDO-induced cells developed large tumors and exhibited poor survival, while mice transfected with IDO-negative cells showed no signs of tumor development [128]. To further support this finding, immunohistochemical staining for IDO expression revealed a correlation between high IDO expression and low levels of immune cells $\mathrm{CD}^{+}, \mathrm{CD}^{+}$, and $\mathrm{CD}^{+} 7^{+}$[129]. This, in turn, can be correlated with aggressive tumor progression and poor survival in cancer patients with high IDO expression [129].

There are currently four IDO inhibitors under clinical development and more in preclinical testing [130]. In 2013, Beatty et al. studied the effects of the small-molecule IDO inhibitor INCB024360 treatment in 52 cancer patients. The drug was well tolerated by patients and successfully inhibited more than $90 \%$ of IDO activity when administered twice a day. Results showed stable disease conditions in $30 \%$ of patients [131]. Because INCB024360 was well tolerated, it has the potential to be potent as either a monotherapy or part of combination therapy. Phase II clinical trials of this inhibitor have been completed for patients with ovarian cancer and myelodysplastic syndrome (NCT01822691, NCT01685255). Combinatorial therapies with IDO inhibitors and cancer vaccines have also shown progress. A phase I study of indoximod, another IDO inhibitor, in combination with docetaxel, an antimitotic chemotherapy drug, showed stable or partially stable disease conditions in more than $50 \%$ of patients [132]. Other combinatorial therapies being tested in the clinic include INCB024360 and MK3475, an immune checkpoint inhibitor [130].
Other than IDO, cancer cells can also use TDO, an immunosuppressive enzyme, to avoid immune destruction. TDO is abundantly present in melanomas, bladder carcinomas, and hepatocarcinomas. Similar to IDO, the use of TDO inhibitors prevents the growth of TDO-expressing tumor cells [133]. There are several other enzymes that cancer cells exploit for immune tolerance; hence, targeting tryptophan metabolism with combinatorial approaches may yield optimal therapies [123].

The serine and glycine biosynthesis pathways are interconnected. They both provide methyl groups for the one-carbon pool that supports purine and pyrimidine synthesis in proliferating cancer cells [134]. Research has shown that phosphoglycerate dehydrogenase (PHGDH), the enzyme that catalyzes the first reaction in the serine synthesis pathway, was highly upregulated in metastatic breast cancer and was correlated to short patient survival times $[135,136]$. The gene encoding PHGDH is also amplified in melanoma and breast cancer types [120]. In addition to PHGDH, serine hydroxymethyltransferase (SHMT) is also implicated in tumorigenesis. SHMT catalyzes the conversion of serine to glycine and is regulated by $c-M Y C$, an oncogene that controls the transcription of $15 \%$ of human genes [123, 137, 138]. Glycine is a component of glutathione and is required for regulating cellular redox balance. It also fuels nucleotide biosynthesis and sustains oxidative phosphorylation in mitochondria. Thus, glycine metabolism has been shown to promote rapid tumor proliferation [134, 139, 140]. In an attempt to block glycine biosynthesis, researchers are using antimetabolites (drugs that interfere with the effects of metabolites), methotrexate, and pemetrexed to inhibit SHMT [134, 141]. Since serine and glycine are considered nonessential, their depletion can be tolerated in vivo. Maddocks et al. found that mice fed diets lacking serine and glycine showed a reduction in tumor sizes and survived longer than those fed diets containing the amino acids, indicating that diet regulation may be a potential therapy for investigation [142]. 
Many cancers become dependent on exogenous amino acid supplies to increase de novo synthesis of other amino acids. This characteristic can be exploited for cancer therapy by depleting amino acid supplies, blocking uptake by transporters, and inhibiting biosynthetic enzymes. The identification of novel therapeutic strategies targeting amino acid pathways could allow for the emergence of new drugs and enhance the current therapeutic efficacy.

\section{Glutamine Metabolism in the Tumor Microenvironment}

In addition to its effects on metabolism in cancer cells, glutamine can have relevant effects on the metabolic, and subsequently functional, profiles of cells in a tumor microenvironment [143]. Immune cells are present throughout the tumor microenvironment, and they can heavily influence tumor progression [144]. Natural killer (NK) cells and $\mathrm{T}$ cells are regularly involved in early detection, control, and clearance of tumors [144, 145]. Macrophages, on the other hand, can contribute to a microenvironment conducive to tumor growth, invasion into nearby tissues, and metastasis [146, 147]. In addition, other cell types, such as fibroblasts, play important roles in promoting tumor growth and metastasis, providing growth factors and contributing to extracellular matrix remodeling with matrix metalloproteinases [148, 149]. These cell types elicit unique responses to different metabolites, each of which could enhance or impede a cancer therapy's effectiveness in clearing cancers.

The responses to glutamine that these cell types exhibit add to the vast heterogeneity of possible metabolic schemes that cancers can adopt. Studying these metabolic processes builds upon a well-mapped understanding of cancer metabolism, which, in concert with existing and newly developed therapies, can make an immense difference in a cancer patient's course of treatment.

\subsection{The Role of Glutamine Metabolism in T Cells and NK Cells}

$\mathrm{T}$ cells and NK cells are the cytotoxic effector cells of productive immune response to cancer, but they can be as influenced by a metabolic landscape as cancer cells are. Presnell et al. showed that human $\mathrm{CD}^{+}$cytotoxic $\mathrm{T}$ cells, when stimulated in vitro, produce significantly less interferon- $\gamma$ and tumor necrosis factor- $\alpha$ (TNF $\alpha)$ in a glutamine-deprived environment, compared to a baseline glutamine-supplemented environment [150]. This decrease in cytokine production correlates with a decrease in $\mathrm{CD} 8^{+} \mathrm{T}$-cell effector function when in a low-glutamine environment. Thioredoxin-interacting protein (TXNIP), a suppressor of glucose uptake known to be active in low-glutamine conditions as a glycolytic sensor, was found to be strongly expressed in the glutamine-deprived setting in activated CD8+ T cells [151]. These findings were corroborated in the work presented by Song et al., who showed that ovarian cancer could induce endoplasmic reticulum stress in cytotoxic $\mathrm{T}$ cells, which depleted glutamine transporters in these cells and subsequently led to T-cell dysfunction [152]. This phenotype was rescued by repairing glutamine uptake in these $\mathrm{T}$ cells, further suggesting that glutamine deprivation in the tumor microenvironment serves as a powerful tool for tumor immune evasion.

However, Presnell et al. also showed that NK cells are more resilient against glutamine depletion and still produced interferon- $\gamma$ in a glutamine-limited setting [150]. Additionally, activated NK cells did not show upregulation of TXNIP expression in a low-glutamine environment. This was investigated further, and, when faced with a low-glucose/low-glutamine environment, these cells were shown to rely on fatty acid oxidation or acetate for energy to mount a cytokine response, further highlighting the metabolic resilience of NK cells.

Considering these findings in the greater scheme of cancer treatments and given the explosive development of cancer immunotherapy tech- 
nologies to kick-start T-cell activity, it will be important for clinicians to consider the metabolic implications that different combinations of drugs can have.

\subsection{The Role of Glutamine Metabolism in Tumor- Associated Macrophages}

Macrophages in the tumor microenvironment can define the immune landscape of a tumor, depending on the cytokines that they produce. Macrophages polarize to either M1 macrophages or M2 macrophages, and these phenotypes are dependent on the broader environment that the cells are in [146]. In a cancer setting, M1 macrophages generally promote inflammation and active antitumor immune responses. In contrast, M2 macrophages produce cytokine signals like IL-6 and IL-10 that help cancer cells evade immune surveillance, recruit endothelial cells for angiogenesis, and avoid apoptosis, thus allowing them to proliferate more freely [146, 153].

$\mathrm{Fu}$ et al. showed that, in a microenvironment deprived of glutamine, due to glutamine addiction in clear cell renal cell carcinoma (ccRCC), tumorassociated macrophages (TAMs) promote an immunosuppressive microenvironment [154]. Glutamine-addicted ccRCC tumors deprive the tumor microenvironment of glutamine. This study showed that, in glutamine-deprived microenvironments, TAMs began producing interleukin-23 (IL-23) via HIF1 $\alpha$ activation. IL-23 subsequently activated the immune-suppressive regulatory $\mathrm{T}$ cells $\left(T_{\text {reg }}\right)$ in the tumor microenvironment. Through the production of IL-10 and transforming growth factor-beta (TGF- $\beta$ ), $\mathrm{T}_{\text {regs }}$ suppressed nearby cytotoxic cells, leading to immune evasion for the tumor $[155,156]$. This phenotype was explored in both mouse models and in vitro with an anti-IL-23 antibody to block IL-23 signaling where mice experienced prolonged survival and decreased tumor burden, and $\mathrm{CD} 8^{+}$cells in mice and in vitro showed enhanced cytotoxicity in the IL-23-depleted state [154] (Fig. 3).

This example highlights a direct link between glutamine addiction and immune function in the tumor microenvironment. These findings show that an understanding of cancer metabolism can be used to not only shape metabolically targeted therapies but also hint at therapies against other factors impacting the landscape of the cancer treatment.

\subsection{The Role of Glutamine Metabolism in Cancer- Associated Fibroblasts}

Fibroblasts play a key role in solid tumors, fulfilling important functions like secreting growth factors, remodeling the extracellular matrix, and promoting metastasis [148]. They may also influence the metabolic behaviors of cancer cells. Zhao et al. reported that cancer-associated fibroblasts (CAFs) secreted exosomes that contain metabolites, which are taken up by cancer cells and can alter the metabolic patterns within those cancer cells $[157,158]$. Using an in vitro system of prostate cancer cell lines and patient-derived CAFs, this study visualized exosome uptake and noted that the uptake of these exosomes was followed by a decrease in cancer cell mitochondrial oxidative phosphorylation, concomitantly leading to an increase in glycolysis. Through ${ }^{13} \mathrm{C}_{5^{-}}$ glutamine labeling experiments, they found that these prostate cancer cells also exhibited a greater reliance on glutamine following exosome uptake, with increased levels of $1^{3} \mathrm{C}$-labeled $\mathrm{m}+5 \alpha$-KG and $\mathrm{m}+5$ glutamate indicative of reductive glutamine metabolism for anaplerosis into the TCA cycle. This data suggests that CAFs are capable of shifting the metabolism of cancer cells from mitochondrial dependent to glycolytic dependent and upregulating their glutamine metabolism. These findings open the opportunities for therapy targeting metabolic cross talk between cancer cells and cancer-associated fibroblasts [159].

\section{Conclusion}

Glutaminolysis is a metabolic process that has been shown to play a critical part in a wide variety of cancers. As a result, glutamine metabolism is 


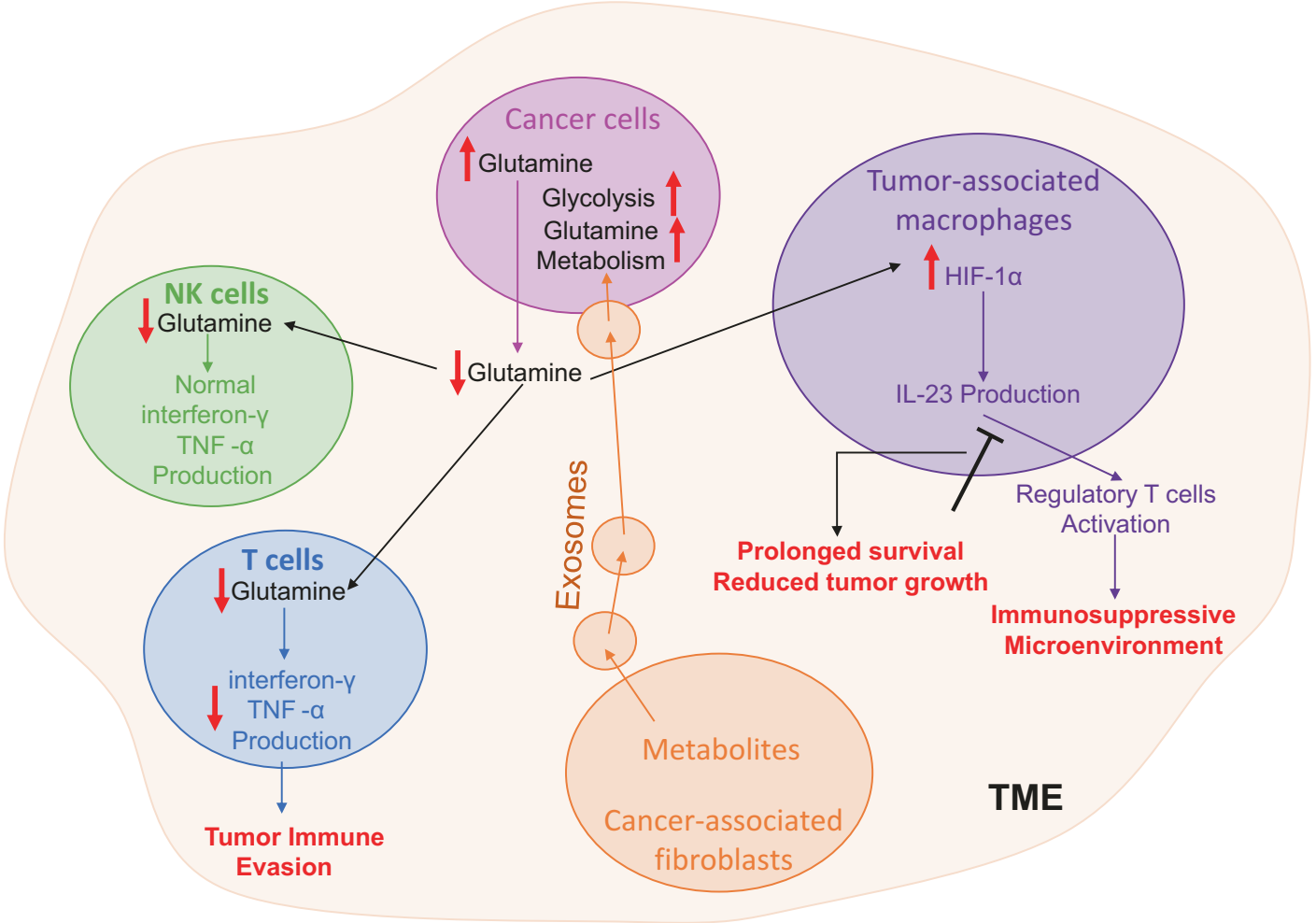

Fig. 3 Cancer cells with glutamine addiction result in a glutamine-deprived tumor microenvironment. Decreased glutamine level results in decreased interferon- $\gamma$ and TNF- $\alpha$ production in T cells leading to tumor immune evasion. However, NK cells are not affected by decreased glutamine level. Decreased glutamine level also results in HIF-1 $\alpha$ activation in tumor-associated macrophages which leads to immunosuppression through IL-23 signaling. Inhibition of IL-23 results in prolonged survival and reduced tumor growth. Cancer-associated fibroblasts in the tumor microenvironment can release exosomes containing metabolites, which when absorbed by the cancer cells can promote glycolysis and glutamine metabolism

an important potential target for cancer therapy. Cancer metabolism is heterogeneous. Just as only some cancers are dependent upon glucose for the TCA cycle, only some cancers will exhibit aberrant glutaminolysis. Even within a single patient, the cancer cells may exhibit vast differences in their dependence on metabolic fuel supplies. This implies that not all cancers will respond in the same manner, or to the same extent, to the inhibition of glutaminolysis. It is important to note that inhibiting glutaminolysis will be more effective on cancers that display glutamine addiction. That being said, there is a huge potential for inhibition of glutaminolysis in cancers [160, 161]. As stated before, genetic alterations, as well as the tumor microenvironment, can influence cancer cells' use of glutaminolysis. Developing and exploring glutaminolysis inhibitors present a strategic course of action toward the goal of finding an effective treatment for the many glutamine-dependent cancers. Inhibitors of GLS, GDH, GCPII, GTK, or other key enzymes could be used in combination with standard chemotherapy treatments to increase their overall effectiveness (Fig. 3).

Currently, the use of SIRM with NMR has been very useful in tracking and examining metabolite usage within certain cancer lines [56]. Increased efforts should be made in the future to use metabolomic technologies for the analysis of different cancers. 


\section{References}

1. Chen, J. Q., \& Russo, J. (2012). Dysregulation of glucose transport, glycolysis, TCA cycle and glutaminolysis by oncogenes and tumor suppressors in cancer cells. Biochimica et Biophysica Acta, 1826(2), 370-384.

2. Scriver, C. R., \& Rosenberg, L. E. (1973). Amino acid metabolism and its disorders. Major Problems in Clinical Pediatrics, 10, 1-478.

3. Berg, J. M., Tymoczko, J. L., \& Stryer, L. (2012). Biochemistry (7th ed.). New York: W.H. Freeman. xxxii, 1054, 43, 41, 48 p.

4. Vander Heiden, M. G., Cantley, L. C., \& Thompson, C. B. (2009). Understanding the Warburg effect: The metabolic requirements of cell proliferation. Science, 324(5930), 1029-1033.

5. Akram, M. (2014). Citric acid cycle and role of its intermediates in metabolism. Cell Biochemistry and Biophysics, 68(3), 475-478.

6. Cardaci, S., \& Ciriolo, M. R. (2012). TCA cycle defects and cancer: When metabolism tunes redox state. International Journal of Cell Biology, 2012, 161837.

7. Cairns, R. A., Harris, I. S., \& Mak, T. W. (2011). Regulation of cancer cell metabolism. Nature Reviews. Cancer, 11(2), 85-95.

8. Laurenti, G., \& Tennant, D. A. (2016). Isocitrate dehydrogenase (IDH), succinate dehydrogenase $(\mathrm{SDH})$, fumarate hydratase $(\mathrm{FH})$ : Three players for one phenotype in cancer? Biochemical Society Transactions, 44(4), 1111-1116.

9. Bardella, C., Pollard, P. J., \& Tomlinson, I. (2011). $\mathrm{SDH}$ mutations in cancer. Biochimica et Biophysica Acta, 1807(11), 1432-1443.

10. Baysal, B. E., et al. (2000). Mutations in SDHD, a mitochondrial complex II gene, in hereditary paraganglioma. Science, 287(5454), 848-851.

11. Baysal, B. E., et al. (2002). Prevalence of SDHB, SDHC, and SDHD germline mutations in clinic patients with head and neck paragangliomas. Journal of Medical Genetics, 39(3), 178-183.

12. Burnichon, N., et al. (2010). SDHA is a tumor suppressor gene causing paraganglioma. Human Molecular Genetics, 19(15), 3011-3020.

13. Cascon, A., et al. (2008). Molecular characterisation of a common SDHB deletion in paraganglioma patients. Journal of Medical Genetics, 45(4), 233-238.

14. Ricketts, C., et al. (2008). Germline SDHB mutations and familial renal cell carcinoma. Journal of the National Cancer Institute, 100(17), 1260-1262.

15. Zantour, B., et al. (2004). A thyroid nodule revealing a paraganglioma in a patient with a new germline mutation in the succinate dehydrogenase B gene. European Journal of Endocrinology, 151(4), 433-438.

16. Tomlinson, I. P., et al. (2002). Germline mutations in $\mathrm{FH}$ predispose to dominantly inherited uterine fibroids, skin leiomyomata and papillary renal cell cancer. Nature Genetics, 30(4), 406-410.
17. Shanmugasundaram, K., et al. (2014). The oncometabolite fumarate promotes pseudohypoxia through noncanonical activation of NF-kappaB signaling. The Journal of Biological Chemistry, 289(35), 24691-24699.

18. Quinones, A., \& Le, A. (2021). The multifaceted glioblastoma: From genomic alterations to metabolic adaptations. Advances in Experimental Medicine and Biology, 1311, https://doi. org/10.1007/978-3-030-65768-0_4

19. Dang, L., et al. (2010). Cancer-associated IDH1 mutations produce 2-hydroxyglutarate. Nature, 465(7300), 966.

20. Parsons, D. W., et al. (2008). An integrated genomic analysis of human glioblastoma multiforme. Science, 321(5897), 1807-1812.

21. Yan, H., et al. (2009). IDH1 and IDH2 mutations in gliomas. The New England Journal of Medicine, 360(8), 765-773.

22. Ward, P. S., et al. (2010). The common feature of leukemia-associated IDH1 and IDH2 mutations is a neomorphic enzyme activity converting alpha-ketoglutarate to 2-hydroxyglutarate. Cancer Cell, 17(3), 225-234.

23. DeBerardinis, R. J., \& Cheng, T. (2010). Q's next: The diverse functions of glutamine in metabolism, cell biology and cancer. Oncogene, 29(3), 313-324.

24. Still, E. R., \& Yuneva, M. O. (2017). Hopefully devoted to Q: Targeting glutamine addiction in cancer. British Journal of Cancer, 116(11), 1375-1381.

25. Mullen, A. R., et al. (2011). Reductive carboxylation supports growth in tumour cells with defective mitochondria. Nature, 481(7381), 385-388.

26. Gameiro, P. A., et al. (2013). In vivo HIF-mediated reductive carboxylation is regulated by citrate levels and sensitizes VHL-deficient cells to glutamine deprivation. Cell Metabolism, 17(3), 372-385.

27. Zarisfi, M., et al. (2021). The heterogeneity metabolism of renal cell carcinomas. Advances in Experimental Medicine and Biology, 1311, https:// doi.org/10.1007/978-3-030-65768-0_8

28. Seltzer, M. J., et al. (2010). Inhibition of glutaminase preferentially slows growth of glioma cells with mutant IDH1. Cancer Research, 70(22), 8981-8987.

29. Ma, W. W., et al. (2009). [18F]fluorodeoxyglucose positron emission tomography correlates with Akt pathway activity but is not predictive of clinical outcome during mTOR inhibitor therapy. Journal of Clinical Oncology, 27(16), 2697-2704.

30. Qu, W., et al. (2012). Preparation and characterization of L-[5-11C]-glutamine for metabolic imaging of tumors. Journal of Nuclear Medicine, 53(1), 98-105.

31. Zhu, L., et al. (2017). Metabolic imaging of glutamine in cancer. Journal of Nuclear Medicine, 58(4), 533-537.

32. Jeong, S. M., et al. (2016). Enhanced mitochondrial glutamine anaplerosis suppresses pancreatic cancer growth through autophagy inhibition. Scientific Reports, 6, 30767. 
33. Owen, O. E., Kalhan, S. C., \& Hanson, R. W. (2002). The key role of anaplerosis and cataplerosis for citric acid cycle function. The Journal of Biological Chemistry, 277(34), 30409-30412.

34. Umapathy, N. S., et al. (2008). Expression and function of system $\mathrm{N}$ glutamine transporters (SN1/ $\mathrm{SN} 2$ or SNAT3/SNAT5) in retinal ganglion cells. Investigative Ophthalmology \& Visual Science, 49(11), 5151-5160.

35. Wu, G., et al. (2004). Glutathione metabolism and its implications for health. The Journal of Nutrition, 134(3), 489-492.

36. Zhang, L., et al. (2016). Reactive oxygen species and targeted therapy for pancreatic cancer. Oxidative Medicine and Cellular Longevity, 2016, 1616781.

37. Assi, M. (2017). The differential role of reactive oxygen species in early and late stages of cancer. American Journal of Physiology. Regulatory, Integrative and Comparative Physiology, 313(6), R646-R653.

38. Elgogary, A., et al. (2016). Combination therapy with BPTES nanoparticles and metformin targets the metabolic heterogeneity of pancreatic cancer. Proceedings of the National Academy of Sciences of the United States of America, 113(36), E5328-E5336.

39. Le, A., et al. (2012). Glucose-independent glutamine metabolism via TCA cycling for proliferation and survival in B cells. Cell Metabolism, 15(1), 110-121.

40. Erickson, J. W., \& Cerione, R. A. (2010). Glutaminase: A hot spot for regulation of cancer cell metabolism? Oncotarget, 1(8), 734-740.

41. Nguyen, T., et al. (2019). Uncovering the role of $\mathrm{N}$-acetyl-aspartyl-glutamate as a glutamate reservoir in cancer. Cell Reports, 27(2), 491-501. e6.

42. Asaka, R., \& Le, A. (2019). Dual role of N-acetylaspartyl-glutamate metabolism in cancer monitor and therapy. Molecular \& Cellular Oncology, 6(5), e1627273.

43. Udupa, S., et al. (2019). Upregulation of the glutaminase II pathway contributes to glutamate production upon glutaminase 1 inhibition in pancreatic cancer. Proteomics, 19(21-22), e1800451.

44. Altman, B. J., Stine, Z. E., \& Dang, C. V. (2016). From Krebs to clinic: Glutamine metabolism to cancer therapy. Nature Reviews. Cancer, 16(11), 749.

45. Colombo, S. L., et al. (2011). Molecular basis for the differential use of glucose and glutamine in cell proliferation as revealed by synchronized HeLa cells. Proceedings of the National Academy of Sciences of the United States of America, 108(52), 21069-21074.

46. Hu, W., et al. (2010). Glutaminase 2, a novel p53 target gene regulating energy metabolism and antioxidant function. Proceedings of the National Academy of Sciences of the United States of America, 107(16), 7455-7460.

47. Gao, P., et al. (2009). c-Myc suppression of miR$23 \mathrm{a} / \mathrm{b}$ enhances mitochondrial glutaminase expression and glutamine metabolism. Nature, 458(7239), 762-765.
48. Wise, D. R., et al. (2008). Myc regulates a transcriptional program that stimulates mitochondrial glutaminolysis and leads to glutamine addiction. Proceedings of the National Academy of Sciences of the United States of America, 105(48), 18782-18787.

49. Hoang, G., Udupa, S., \& Le, A. (2019). Application of metabolomics technologies toward cancer prognosis and therapy. International Review of Cell and Molecular Biology, 347, 191-223.

50. Zilfou, J. T., \& Lowe, S. W. (2009). Tumor suppressive functions of p53. Cold Spring Harbor Perspectives in Biology, 1(5), a001883.

51. Matoba, S., et al. (2006). p53 regulates mitochondrial respiration. Science, 312(5780), 1650-1653.

52. Sablina, A. A., et al. (2005). The antioxidant function of the p53 tumor suppressor. Nature Medicine, 11(12), 1306-1313.

53. Xiang, Y., et al. (2015). Targeted inhibition of tumorspecific glutaminase diminishes cell-autonomous tumorigenesis. The Journal of Clinical Investigation, 125(6), 2293-2306.

54. Wang, J. B., et al. (2010). Targeting mitochondrial glutaminase activity inhibits oncogenic transformation. Cancer Cell, 18(3), 207-219.

55. Gross, M. I., et al. (2014). Antitumor activity of the glutaminase inhibitor CB-839 in triple-negative breast cancer. Molecular Cancer Therapeutics, 13(4), 890-901.

56. Robinson, M. M., et al. (2007). Novel mechanism of inhibition of rat kidney-type glutaminase by bis-2-(5phenylacetamido-1,2,4-thiadiazol-2-yl)ethyl sulfide (BPTES). Biochemical Journal, 406(3), 407-414.

57. Willis, R. C., \& Seegmiller, J. E. (1977). The inhibition by 6-diazo-5-oxo-1-norleucine of glutamine catabolism of the cultured human lymphoblast. Journal of Cellular Physiology, 93(3), 375-382.

58. Zimmermann, S. C., et al. (2016). Allosteric glutaminase inhibitors based on a 1,4-di(5-amino-1,3,4thiadiazol-2-yl)butane scaffold. ACS Medicinal Chemistry Letters, 7(5), 520-524.

59. Elgadi, K. M., et al. (1999). Cloning and analysis of unique human glutaminase isoforms generated by tissue-specific alternative splicing. Physiological Genomics, 1(2), 51-62.

60. Shukla, K., et al. (2012). Design, synthesis, and pharmacological evaluation of bis-2-(5-phenylacetamido-1,2,4-thiadiazol-2-yl)ethyl sulfide 3 (BPTES) analogs as glutaminase inhibitors. Journal of Medicinal Chemistry, 55(23), 10551-10563.

61. Son, J., et al. (2013). Glutamine supports pancreatic cancer growth through a KRAS-regulated metabolic pathway. Nature, 496(7443), 101-105.

62. Nabi, K., \& Le, A. (2021). The intratumoral heterogeneity of cancer metabolism. Advances in Experimental Medicine and Biology, 1311, https:// doi.org/10.1007/978-3-030-65768-0_11

63. Xu, X., et al. (2019). Overview of the development of glutaminase inhibitors: Achievements and future directions. Journal of Medicinal Chemistry, 62(3), 1096-1115. 
64. Parlati, F., et al. (2014). Glutaminase inhibitor CB-839 synergizes with pomalidomide in preclinical multiple myeloma models. Blood, 124(21), 4720-4720.

65. Parlati, F. (2015). CB-839, a selective glutaminase inhibitor, synergizes with signaling pathway inhibitors to produce an anti-tumor effect in cell lines and tumor xenografts. Cancer Research, 75, 4711.

66. Emberley, E., et al. (2017). CB-839, a selective glutaminase inhibitor, has anti-tumor activity in renal cell carcinoma and synergizes with cabozantinib and everolimus, in keystone symposia, tumor metabolism: Mechanisms and targets. Canada: Whistler.

67. Emberley, E. D., et al. (2018). The glutaminase inhibitor CB-839 synergizes with CDK4/6 and PARP inhibitors in pre-clinical tumor models. Cancer Research, 78(13), 3509-3509.

68. Momcilovic, M., et al. (2017). Targeted inhibition of EGFR and glutaminase induces metabolic crisis in EGFR mutant lung cancer. Cell Reports, 18(3), 601-610.

69. Liu, W., et al. (2012). Reprogramming of proline and glutamine metabolism contributes to the proliferative and metabolic responses regulated by oncogenic transcription factor c-MYC. Proceedings of the National Academy of Sciences of the United States of America, 109(23), 8983-8988.

70. Dang, C. V., Le, A., \& Gao, P. (2009). MYC-induced cancer cell energy metabolism and therapeutic opportunities. Clinical Cancer Research, 15(21), 6479-6483.

71. Le, A., \& Dang, C. V. (2013). Studying Myc's role in metabolism regulation. Methods in Molecular Biology, 1012, 213-219.

72. Niu, Z., et al. (2015). Knockdown of c-Myc inhibits cell proliferation by negatively regulating the $\mathrm{Cdk} /$ $\mathrm{Rb} / \mathrm{E} 2 \mathrm{~F}$ pathway in nasopharyngeal carcinoma cells. Acta Biochimica et Biophysica Sinica Shanghai, 47(3), 183-191.

73. Zhang, X., Ge, Y. L., \& Tian, R. H. (2009). The knockdown of c-myc expression by RNAi inhibits cell proliferation in human colon cancer HT-29 cells in vitro and in vivo. Cellular \& Molecular Biology Letters, 14(2), 305-318.

74. Whitfield, J. R., Beaulieu, M. E., \& Soucek, L. (2017). Strategies to inhibit myc and their clinical applicability. Frontiers in Cell and Development Biology, 5, 10.

75. Brooks, T. A., \& Hurley, L. H. (2010). Targeting MYC expression through G-quadruplexes. Genes \& Cancer, 1(6), 641-649.

76. Brown, R. V., et al. (2011). Demonstration that drugtargeted down-regulation of MYC in non-Hodgkin's lymphoma is directly mediated through the promoter G-quadruplex. Journal of Biological Chemistry, 286(47), 41018-41027.

77. Devi, G. R., et al. (2005). In vivo bioavailability and pharmacokinetics of a c-MYC antisense phosphorodiamidate morpholino oligomer, AVI-4126, in solid tumors. Clinical Cancer Research, 11(10), 3930-3938.
78. Tolcher, A. W., et al. (2015). Safety and activity of DCR-MYC, a first-in-class Dicer-substrate small interfering RNA (DsiRNA) targeting MYC, in a phase I study in patients with advanced solid tumors. Journal of Clinical Oncology, 33(15_suppl), 11006-11006.

79. Stellas, D., et al. (2014). Therapeutic effects of an anti-Myc drug on mouse pancreatic cancer. Journal of the National Cancer Institute, 106, 12.

80. Yin, X., et al. (2003). Low molecular weight inhibitors of Myc-Max interaction and function. Oncogene, 22(40), 6151-6159.

81. Farrell, A. S., et al. (2014). Targeting inhibitors of the tumor suppressor PP2A for the treatment of pancreatic cancer. Molecular Cancer Research, 12(6), 924-939.

82. Polivka, J., Jr., \& Janku, F. (2014). Molecular targets for cancer therapy in the PI3K/AKT/mTOR pathway. Pharmacology \& Therapeutics, 142(2), 164-175.

83. Casey, S. C., et al. (2016). MYC regulates the antitumor immune response through CD47 and PD-L1. Science, 352(6282), 227-231.

84. Masso-Valles, D., Jauset, T., \& Soucek, L. (2016). Ibrutinib repurposing: From B-cell malignancies to solid tumors. Oncoscience, 3(5-6), 147-148.

85. Jin, L., Alesi, G. N., \& Kang, S. (2016). Glutaminolysis as a target for cancer therapy. Oncogene, 35(28), 3619-3625.

86. Li, C., et al. (2006). Green tea polyphenols modulate insulin secretion by inhibiting glutamate dehydrogenase. The Journal of Biological Chemistry, 281(15), 10214-10221.

87. Li, C., et al. (2011). Green tea polyphenols control dysregulated glutamate dehydrogenase in transgenic mice by hijacking the ADP activation site. The Journal of Biological Chemistry, 286(39), 34164-34174.

88. Yang, C. S., et al. (2009). Cancer prevention by tea: Animal studies, molecular mechanisms and human relevance. Nature Reviews. Cancer, 9(6), 429-439.

89. Li, M., et al. (2009). Novel inhibitors complexed with glutamate dehydrogenase: Allosteric regulation by control of protein dynamics. The Journal of Biological Chemistry, 284(34), 22988-23000.

90. Yang, C., et al. (2009). Glioblastoma cells require glutamate dehydrogenase to survive impairments of glucose metabolism or Akt signaling. Cancer Research, 69(20), 7986-7993.

91. Ollenschlager, G., et al. (1988). Asparaginaseinduced derangements of glutamine metabolism: The pathogenetic basis for some drug-related side-effects. European Journal of Clinical Investigation, 18(5), 512-516.

92. Wu, M. C., Arimura, G. K., \& Yunis, A. A. (1978). Mechanism of sensitivity of cultured pancreatic carcinoma to asparaginase. International Journal of Cancer, 22(6), 728-733.

93. Grigoryan, R. S., et al. (2004). Changes of amino acid serum levels in pediatric patients with higherrisk acute lymphoblastic leukemia (CCG-1961). In Vivo, 18(2), 107-112. 
94. Lukey, M. J., Wilson, K. F., \& Cerione, R. A. (2013). Therapeutic strategies impacting cancer cell glutamine metabolism. Future Medicinal Chemistry, 5(14), 1685-1700.

95. Nguyen, H. A., Su, Y., \& Lavie, A. (2016). Structural insight into substrate selectivity of Erwinia chrysanthemi L-asparaginase. Biochemistry, 55(8), 1246-1253.

96. Ertel, I. J., et al. (1979). Effective dose of L-asparaginase for induction of remission in previously treated children with acute lymphocytic leukemia: A report from Children's Cancer Study Group. Cancer Research, 39(10), 3893-3896.

97. Panosyan, E. H., et al. (2014). Asparagine depletion potentiates the cytotoxic effect of chemotherapy against brain tumors. Molecular Cancer Research, 12(5), 694-702.

98. Stams, W. A., et al. (2003). Sensitivity to L-asparaginase is not associated with expression levels of asparagine synthetase in $\mathrm{t}(12 ; 21)+$ pediatric ALL. Blood, 101(7), 2743-2747.

99. Ye, S., et al. (2020). The heterocyclic compound Tempol inhibits the growth of cancer cells by interfering with glutamine metabolism. Cell Death \& Disease, 11(5), 312.

100. Darmaun, D., et al. (1998). Phenylbutyrate-induced glutamine depletion in humans: Effect on leucine metabolism. The American Journal of Physiology, 274(5 Pt 1), E801-E807.

101. Thibault, A., et al. (1994). A phase I and pharmacokinetic study of intravenous phenylacetate in patients with cancer. Cancer Research, 54(7), 1690-1694.

102. Schulte, M. L., et al. (2018). Pharmacological blockade of ASCT2-dependent glutamine transport leads to antitumor efficacy in preclinical models. Nature Medicine, 24(2), 194-202.

103. Wahi, K., \& Holst, J. (2019). ASCT2: A potential cancer drug target. Expert Opinion on Therapeutic Targets, 23(7), 555-558.

104. Colas, C., et al. (2015). Ligand discovery for the alanine-serine-cysteine transporter (ASCT2, SLC1A5) from homology modeling and virtual screening. PLoS Computational Biology, 11(10), e1004477.

105. Fuchs, B. C., \& Bode, B. P. (2005). Amino acid transporters ASCT2 and LAT1 in cancer: Partners in crime? Seminars in Cancer Biology, 15(4), 254-266.

106. Hassanein, M., et al. (2013). SLC1A5 mediates glutamine transport required for lung cancer cell growth and survival. Clinical Cancer Research, 19(3), 560-570.

107. Chiu, M., et al. (2017). GPNA inhibits the sodiumindependent transport system $\mathrm{L}$ for neutral amino acids. Amino Acids, 49(8), 1365-1372.

108. Broer, A., Fairweather, S., \& Broer, S. (2018). Disruption of amino acid homeostasis by novel ASCT2 inhibitors involves multiple targets. Frontiers in Pharmacology, 9, 785.

109. Osanai-Sasakawa, A., et al. (2018). An anti-ASCT2 monoclonal antibody suppresses gastric cancer growth by inducing oxidative stress and antibody dependent cellular toxicity in preclinical models. American Journal of Cancer Research, 8(8), 1499-1513.
110. Ahluwalia, G. S., et al. (1990). Metabolism and action of amino acid analog anti-cancer agents. Pharmacology \& Therapeutics, 46(2), 243-271.

111. Rais, R., et al. (2016). Discovery of 6-Diazo-5-oxo1-norleucine (DON) prodrugs with enhanced CSF delivery in monkeys: A potential treatment for glioblastoma. Journal of Medicinal Chemistry, 59(18), 8621-8633.

112. Thangavelu, K., et al. (2014). Structural basis for the active site inhibition mechanism of human kidneytype glutaminase (KGA). Scientific Reports, 4, 3827.

113. Ortlund, E., et al. (2000). Reactions of pseudomonas 7A glutaminase-asparaginase with diazo analogues of glutamine and asparagine result in unexpected covalent inhibitions and suggests an unusual catalytic triad Thr-Tyr-Glu. Biochemistry, 39(6), 1199-1204.

114. Ovejera, A. A., et al. (1979). Efficacy of 6-diazo5-oxo-L-norleucine and N-[N-gamma-glutamyl-6diazo-5-oxo-norleucinyl]-6-diazo-5-oxo-norleucine against experimental tumors in conventional and nude mice. Cancer Research, 39(8), 3220-3224.

115. Mueller, C., et al. (2008). A phase IIa study of PEGylated glutaminase (PEG-PGA) plus 6-diazo5-oxo-L-norleucine (DON) in patients with advanced refractory solid tumors. Journal of Clinical Oncology, 26(15_suppl), 2533-2533.

116. Lukey, M. J., Katt, W. P., \& Cerione, R. A. (2017). Targeting amino acid metabolism for cancer therapy. Drug Discovery Today, 22(5), 796-804.

117. Beuster, G., et al. (2011). Inhibition of alanine aminotransferase in silico and in vivo promotes mitochondrial metabolism to impair malignant growth. The Journal of Biological Chemistry, 286(25), 22323-22330.

118. Thornburg, J. M., et al. (2008). Targeting aspartate aminotransferase in breast cancer. Breast Cancer Research, 10(5), R84.

119. Tan, J., \& Le, A. (2021). The heterogeneity of breast cancer metabolism. Advances in Experimental Medicine and Biology, 1311, https://doi. org/10.1007/978-3-030-65768-0_6

120. Possemato, R., et al. (2011). Functional genomics reveal that the serine synthesis pathway is essential in breast cancer. Nature, 476(7360), 346-350.

121. Camelo, F., \& Le, A. (2021). The intricate metabolism of pancreatic cancers. Advances in Experimental Medicine and Biology, 1311, https:// doi.org/10.1007/978-3-030-65768-0_5

122. Wu, G., \& Morris, S. M., Jr. (1998). Arginine metabolism: Nitric oxide and beyond. Biochemical Journal, 336(Pt 1), 1-17.

123. Ananieva, E. (2015). Targeting amino acid metabolism in cancer growth and anti-tumor immune response. World Journal of Biological Chemistry, 6(4), 281-289.

124. Kobayashi, E., et al. (2010). Reduced argininosuccinate synthetase is a predictive biomarker for the development of pulmonary metastasis in patients with osteosarcoma. Molecular Cancer Therapeutics, 9(3), 535-544. 
125. Grohmann, U., \& Bronte, V. (2010). Control of immune response by amino acid metabolism. Immunological Reviews, 236, 243-264.

126. Godin-Ethier, J., et al. (2011). Indoleamine 2,3-dioxygenase expression in human cancers: Clinical and immunologic perspectives. Clinical Cancer Research, 17(22), 6985-6991.

127. Mellor, A. L., \& Munn, D. H. (1999). Tryptophan catabolism and T-cell tolerance: Immunosuppression by starvation? Immunology Today, 20(10), 469-473.

128. Uyttenhove, C., et al. (2003). Evidence for a tumoral immune resistance mechanism based on tryptophan degradation by indoleamine 2,3-dioxygenase. Nature Medicine, 9(10), 1269-1274.

129. Ino, K., et al. (2008). Inverse correlation between tumoral indoleamine 2,3-dioxygenase expression and tumor-infiltrating lymphocytes in endometrial cancer: Its association with disease progression and survival. Clinical Cancer Research, 14(8), 2310-2317.

130. Moon, Y. W., et al. (2015). Targeting the indoleamine 2,3-dioxygenase pathway in cancer. Journal for ImmunoTherapy of Cancer, 3, 51.

131. Beatty, G. L., et al. (2013). A phase I study of an agonist CD40 monoclonal antibody (CP-870,893) in combination with gemcitabine in patients with advanced pancreatic ductal adenocarcinoma. Clinical Cancer Research, 19(22), 6286-6295.

132. Soliman, H. H., et al. (2016). A phase I study of indoximod in patients with advanced malignancies. Oncotarget, 7(16), 22928-22938.

133. Pilotte, L., et al. (2012). Reversal of tumoral immune resistance by inhibition of tryptophan 2,3-dioxygenase. Proceedings of the National Academy of Sciences of the United States of America, 109(7), 2497-2502.

134. Amelio, I., et al. (2014). Serine and glycine metabolism in cancer. Trends in Biochemical Sciences, 39(4), 191-198.

135. DeBerardinis, R. J. (2011). Serine metabolism: Some tumors take the road less traveled. Cell Metabolism, 14(3), 285-286.

136. Pollari, S., et al. (2011). Enhanced serine production by bone metastatic breast cancer cells stimulates osteoclastogenesis. Breast Cancer Research and Treatment, 125(2), 421-430.

137. Dang, C. V. (2012). MYC on the path to cancer. Cell, 149(1), 22-35.

138. Nikiforov, M. A., et al. (2002). A functional screen for Myc-responsive genes reveals serine hydroxymethyltransferase, a major source of the one-carbon unit for cell metabolism. Molecular and Cellular Biology, 22(16), 5793-5800.

139. di Salvo, M. L., et al. (2013). Glycine consumption and mitochondrial serine hydroxymethyltransferase in cancer cells: The heme connection. Medical Hypotheses, 80(5), 633-636.

140. Jain, M., et al. (2012). Metabolite profiling identifies a key role for glycine in rapid cancer cell proliferation. Science, 336(6084), 1040-1044.
141. Daidone, F., et al. (2011). In silico and in vitro validation of serine hydroxymethyltransferase as a chemotherapeutic target of the antifolate drug pemetrexed. European Journal of Medicinal Chemistry, 46(5), 1616-1621.

142. Maddocks, O. D., et al. (2013). Serine starvation induces stress and p53-dependent metabolic remodelling in cancer cells. Nature, 493(7433), 542-546.

143. Antonio, M. J., Zhang, C., \& Le, A. (2021). Different tumor microenvironments lead to different metabolic phenotypes. Advances in Experimental Medicine and Biology, 1311, https:// doi.org/10.1007/978-3-030-65768-0_10.

144. Binnewies, M., et al. (2018). Understanding the tumor immune microenvironment (TIME) for effective therapy. Nature Medicine, 24(5), 541-550.

145. Larsen, S. K., Gao, Y., \& Basse, P. H. (2014). NK cells in the tumor microenvironment. Critical Reviews in Oncogenesis, 19(1-2), 91-105.

146. Wang, J., et al. (2019). Crosstalk between cancer and immune cells: Role of tumor-associated macrophages in the tumor microenvironment. Cancer Medicine, 8(10), 4709-4721.

147. Wu, L., et al. (2019). Tumor-associated neutrophils in cancer: Going pro. Cancers (Basel), 11, 4.

148. Xing, F., Saidou, J., \& Watabe, K. (2010). Cancer associated fibroblasts (CAFs) in tumor microenvironment. Frontiers in Bioscience (Landmark Ed), 15, 166-179.

149. Weinberg, R. A. (2014). The biology of cancer. New York, NY: Garland Science, Taylor \& Francis Group LLC.

150. Presnell, S. R., et al. (2020). Differential fuel requirements of human NK cells and human CD8 $\mathrm{T}$ cells: Glutamine regulates glucose uptake in strongly activated CD8 T cells. Immunohorizons, 4(5), 231-244.

151. Parikh, H., et al. (2007). TXNIP regulates peripheral glucose metabolism in humans. PLoS Medicine, 4(5), e158.

152. Song, M., et al. (2018). IRE1 $\alpha-X B P 1$ controls T cell function in ovarian cancer by regulating mitochondrial activity. Nature, 562(7727), 423-428.

153. Owen, J. A., et al. (2013). Kuby Immunology. New York: W.H. Freeman.

154. Fu, Q., et al. (2019). Tumor-associated macrophagederived interleukin-23 interlinks kidney cancer glutamine addiction with immune evasion. European Urology, 75(5), 752-763.

155. Viguier, M., et al. (2004). Foxp3 expressing $\mathrm{CD} 4+\mathrm{CD} 25$ (high) regulatory $\mathrm{T}$ cells are overrepresented in human metastatic melanoma lymph nodes and inhibit the function of infiltrating $\mathrm{T}$ cells. Journal of Immunology, 173(2), 1444-1453.

156. Bradley, C. A. (2018). IL-23 links glutamine addiction and immune function. Nature Reviews. Urology, 15(12), 725.

157. Zhao, H., et al. (2016). Tumor microenvironment derived exosomes pleiotropically modulate cancer cell metabolism. eLife, 5, e10250. 
158. Sazeides, C., \& Le, A. (2021). Metabolic relationship between cancerassociated fibroblasts and cancer cells. Advances in Experimental Medicine and Biology, 1311, https://doi. org/10.1007/978-3-030-65768-0_14

159. Jung, J. G., \& Le, A. (2021). Targeting metabolic cross talk between cancer cells and cancerassociated fibroblasts. Advances in Experimental
Medicine and Biology, 1311, https://doi. org/10.1007/978-3-030-65768-0_15.

160. Dang, C. V., et al. (2011). Therapeutic targeting of cancer cell metabolism. Journal of Molecular Medicine (Berlin), 89(3), 205-212.

161. Hirschey, M. D., et al. (2015). Dysregulated metabolism contributes to oncogenesis. Seminars in Cancer Biology, 35(Suppl), S129-S150.

Open Access This chapter is licensed under the terms of the Creative Commons Attribution 4.0 International License (http://creativecommons.org/licenses/by/4.0/), which permits use, sharing, adaptation, distribution and reproduction in any medium or format, as long as you give appropriate credit to the original author(s) and the source, provide a link to the Creative Commons license and indicate if changes were made.

The images or other third party material in this chapter are included in the chapter's Creative Commons license, unless indicated otherwise in a credit line to the material. If material is not included in the chapter's Creative Commons license and your intended use is not permitted by statutory regulation or exceeds the permitted use, you will need to obtain permission directly from the copyright holder. 


\title{
The Heterogeneity of Lipid Metabolism in Cancer
}

\author{
Joshua K. Park, Nathan J. Coffey, Aaron Limoges, \\ and Anne Le
}

\section{Keywords \\ Cancer metabolism - Tumor heterogeneity . Lipid synthesis · Fatty acid oxidation · Fatty acid uptake $\cdot$ Metastasis $\cdot$ Lipidomics}

\begin{tabular}{ll}
\hline Abbreviations \\
4-HNE & $\begin{array}{l}\text { 4-Hydroxy-nonenal } \\
\text { Acetyl-coenzyme A carboxylase }\end{array}$ \\
ACC & $\begin{array}{l}\text { Adenosine triphosphate citrate lyase } \\
\text { ACLY }\end{array}$ \\
ACSL3 & $\begin{array}{l}\text { Acyl-coenzyme A synthetase long- } \\
\text { chain family member } 3\end{array}$ \\
ACSS2 & $\begin{array}{l}\text { Acyl-coenzyme A synthetase short- } \\
\text { chain family member } 2\end{array}$
\end{tabular}

J. K. Park

Icahn School of Medicine at Mount Sinai,

New York, NY, USA

N. J. Coffey

National Institute on Alcohol Abuse and Alcoholism,

National Institutes of Health, Bethesda, MD, USA

\section{A. Limoges}

Department of Biological Sciences, Columbia University, New York, NY, USA

\section{A. Le ( $\square)$}

Department of Pathology and Oncology, Johns Hopkins University School of Medicine,

Baltimore, MD, USA

Department of Chemical and Biomolecular Engineering, Johns Hopkins University Whiting School of Engineering, Baltimore, MD, USA e-mail: annele@jhmi.edu
AMPK Adenosine monophosphateactivated protein kinase

ATP Adenosine triphosphate

BMI Body mass index

BTA Benzene-tricarboxylate

CAV1 Caveolin 1

CD36

CoA

Cluster of differentiation 36 protein Coenzyme A

CPT1 Carnitine palmitoyltransferase 1

CTP Citrate transporter protein

DNA Deoxyribonucleic acid

DNLS De novo lipid synthesis

EMT Epithelial-mesenchymal transition

ERS Endoplasmic reticulum stress

FADH2 Flavin adenine dinucleotide

FAO Fatty acid oxidation

FAS Fatty acid synthase

FATP Fatty acid transport protein

GBM Glioblastoma multiforme

HCC Hepatocellular carcinoma

HFD High-fat diet

HIF-1 $\alpha \quad$ Hypoxia-inducible factor 1-alpha

HMGCR 3-Hydroxy-3-methylglutarylcoenzyme A reductase

IDH Isocitrate dehydrogenase

LD Lipid droplet

LDL Low-density lipoprotein

LPL Lipoprotein lipase

MAPK Mitogen-activated protein kinase

mTOR Mammalian target of rapamycin

mTORC1 Mammalian target of rapamycin complex 1 


\begin{tabular}{|c|c|}
\hline NADH & Nicotinamide adenine dinucleotide \\
\hline NADPH & $\begin{array}{l}\text { Nicotinamide adenine dinucleotide } \\
\text { phosphate }\end{array}$ \\
\hline NSCLC & Non-small cell lung cancer \\
\hline PDAC & Pancreatic ductal adenocarcinoma \\
\hline PE & Phosphatidylethanolamine \\
\hline PIK3CA & $\begin{array}{l}\text { Ph o s ph at i d y lin o s it o } 1-4,5 \text { - } \\
\text { bisphosphate } 3 \text {-kinase catalytic sub- } \\
\text { unit alpha }\end{array}$ \\
\hline PIP2 & $\begin{array}{l}\text { Phosphatidylinositol-4, } \\
\text { 5-bisphosphate }\end{array}$ \\
\hline PTEN & Phosphatase and tensin homolog \\
\hline PUFA & Polyunsaturated fatty acid \\
\hline ROS & Reactive oxygen species \\
\hline SCD & Stearoyl-coenzyme A desaturase \\
\hline TCA & Tricarboxylic acid \\
\hline TG & Triglyceride \\
\hline TME & Tumor microenvironment \\
\hline TSC & Tuberous sclerosis protein \\
\hline$\omega-3 / 6$ & Omega-3/6 fatty acid \\
\hline
\end{tabular}

\section{Key Points}

- Fatty acid synthesis is upregulated in cancer.

- The mitochondrial citrate transporter protein (CTP) protects mitochondrial function in cancer.

- ATP citrate lyase (ACLY) is upregulated in cancer.

- Inhibiting acetyl-CoA carboxylase (ACC) has multifaceted effects on cancer.

- The first fatty acid synthase (FAS) inhibitor, TVB-2640, is in clinical trials for cancer.

- Markers such as cell type, oncogene mutations, expression/activity of lipid synthesis enzymes, and metabolic profiles can be used to predict cancer cell sensitivity to lipid synthesis inhibition.

- The tumor microenvironment influences the sensitivity of cancer cells to lipid synthesis inhibitors.

- The efficacy of inhibiting cholesterol synthesis with adjuvant statins is variable.

- Fatty acid uptake is associated with metastasis.

- Targeting fatty acid oxidation (FAO) for cancer therapy may be achieved by inhibiting carnitine palmitoyltransferase 1 .
- Carnitine palmitoyltransferase 1 (CPT1) inhibitors are now in clinical trials for cancer treatment.

- FAO occurs at peroxisomes, where peroxisome proliferator-activated receptors (PPARs) act as ligand-activated transcription factors.

\section{Introduction}

The study of cancer cell metabolism has traditionally focused on glycolysis and glutaminolysis. However, lipidomic technologies have matured considerably over the last decade and broadened our understanding of how lipid metabolism is relevant to cancer biology [1-3]. Studies now suggest that the reprogramming of cellular lipid metabolism contributes directly to malignant transformation and progression $[4,5]$. For example, de novo lipid synthesis can supply proliferating tumor cells with phospholipid components that comprise the plasma and organelle membranes of new daughter cells $[6,7]$. Moreover, the upregulation of mitochondrial $\beta$-oxidation can support tumor cell energetics and redox homeostasis [8], while lipid-derived messengers can regulate major signaling pathways or coordinate immunosuppressive mechanisms [9-11]. Lipid metabolism has, therefore, become implicated in a variety of oncogenic processes, including metastatic colonization, drug resistance, and cell differentiation [10, 12-16]. However, whether we can safely and effectively modulate the underlying mechanisms of lipid metabolism for cancer therapy is still an open question.

As discussed in previous chapters, inter- and intra-tumoral heterogeneities are major causes of treatment failure in clinical oncology because tumor subclones with either intrinsic or acquired resistance to therapy can be selected by Darwinian mechanisms and allowed to drive disease relapse [17-20]. An alarming number of parameters seem to be capable of inducing this diversity, including (epi)genetic lesions, microenvironmental constraints, stromal interactions, and treatment effects [21-23]. Perhaps unsurprisingly then, translational strategies targeting lipid metabolism have reported mixed or even diverging responses in 
preclinical models of cancer. These results hint at differential tumor cell dependencies on lipids, but we are far from understanding the extent to which this heterogeneity arises. Moreover, how this nonuniformity of lipid metabolism undermines patient treatment is unclear. To better understand the clinical potential of this emerging discipline, we will have to address both the spatial and temporal heterogeneities of cellular lipid metabolism.

Here, we provide a brief synopsis of novel findings on the lipid metabolism of cancer cells, with an emphasis on heterogeneity across and/or within tumors. Given the rapid pace of this field, we focus on central pathways involving fatty acid synthesis, uptake, and oxidation.

\section{Fatty Acid Synthesis Is Upregulated in Cancer}

Endogenous fatty acid synthesis is frequently upregulated in cancer because fatty acids can serve as substrates to produce lipid signaling molecules, modify protein functions through lipidation, synthesize phospholipids for cell membranes, or store energy as triglycerides. The primary source of carbons for fatty acid synthesis in cancer cells comes from glucose. Glucose carbon is incorporated into acetyl-CoA, which then forms citrate in the mitochondria. The mitochondrial citrate transporter protein (CTP) carries citrate from the mitochondria to the cytosol. ATP citrate lyase (ACLY), a key enzyme of de novo fatty acid synthesis (DNLS), cleaves cytosolic citrate into acetyl-CoA and oxaloacetate. Cytosolic acetyl-CoA is used to form fatty acids. Hence, the localization of acetyl-CoA within a cell can determine its metabolic fate.

\subsection{The Mitochondrial Citrate Transporter Protein (CTP) Protects Mitochondrial Function in Cancer}

The Avantaggiati research group has extensively studied the mitochondrial citrate transporter protein $(\mathrm{CTP})$ and demonstrated that CTP plays an important role in preventing mitochondrial damage and preserving its function, such as in cellular bioenergetics [24]. The inhibition of CTP resulted in anti-tumorigenesis in vivo. Although the authors observed a decrease in fatty acid synthesis from glucose due to the suppression of CTP-dependent transport of citrate by a benzene-tricarboxylate analog (BTA), they believe that this effect only played a partial role in tumor reduction because the total FA levels were not drastically affected. Moreover, CTP levels were associated with cancer aggressiveness [24].

\subsection{ATP Citrate Lyase (ACLY) Is Upregulated in Cancer}

ATP citrate lyase (ACLY) is found to be elevated in many types of cancers, including breast [25], lung [26], liver [27], and bladder cancers [28]. Migita et al. found that ACLY expression is significantly higher in human lung adenocarcinoma samples as compared to normal lung tissue. It also correlated with stage, differentiation grade, and a poorer prognosis. ACLY inhibition arrested lung cancer cell growth in vitro and in vivo. ACLY knockdown compromised de novo lipogenesis, but intracellular lipids were increased, suggesting alternative mechanisms of lipid accumulation [26]. A study by Schlichtholz et al. similarly demonstrated upregulations of ACLY, glucose 6-phosphate dehydrogenase, 6-phosphogluconate dehydrogenase, and citrate synthase, which are enzymes involved in fatty acid synthesis, in bladder cancer [28].

\subsection{Multifaceted Effects of Inhibiting Acetyl-CoA Carboxylase (ACC) in Cancer}

After ACLY produces cytosolic acetyl-CoA, the enzyme acetyl-CoA carboxylase (ACC) irreversibly converts acetyl-CoA into malonyl-CoA. Malonyl-CoA is required for fatty acid synthesis and elongation and negatively regulates 
$\beta$-oxidation of long-chain fatty acids by inhibiting the enzyme carnitine palmitoyltransferase 1 (CPT1) [29]. ACC exists as two isozymes (ACC1/2, genes $A C A C A / B$ ) [30]. ACC1 is preferentially expressed in lipogenic cells, such as adipocytes [30]. The two ACC isozymes catalyze the same reaction, and one can compensate for the loss of function of another as malonyl-CoA levels only decrease in hepatocytes if both ACC1 and ACC2 are inhibited [31]. This demonstrates that inhibiting both ACC1 and ACC2 isozymes may be more efficacious than inhibiting either isozyme alone for the treatment of cancer. ACC is now receiving greater attention as a therapeutic target against cancer because the formation of malonyl-CoA catalyzed by ACC is the ratelimiting step of fatty acid synthesis.

The expression of ACC1 is highly enriched in breast [32], prostate [33], liver [34], and renal cancers [35]. The expression of ACC1 also increases with tumor grade in liver cancer, and its overexpression increases liver cancer cell viability while decreasing apoptosis $[34,36]$. ACC1 expression is also prognostic for some cancers. High expression of ACC1 is correlated with worse survival in renal cancer [35]. Inhibition of ACC1 with siRNA reduced cell viability in breast [37] and liver cancers [36]. Furthermore, simultaneous inhibition of both ACC 1 and ACC 2 with a small chemical molecule or siRNA reduced tumor growth in the prostate [38], brain [39], and pancreatic cancers [40].

While ACC inhibition appears to arrest the growth of certain cancer types, it has paradoxically been shown to promote breast cancer invasion and metastasis by promoting epithelial-to-mesenchymal transition (EMT) [41]. ACC-deficient hepatocytes are also more susceptible to diethylnitrosamine-induced hepatocellular carcinoma. ACC-deficient mice exhibited a reduction in hepatic lipogenesis, a decrease in glutathione, and an increase in NADPH [34]. Collectively, these preclinical studies demonstrate the duality of ACC inhibition: it could attenuate tumor growth in some cancer types, but it could also contribute to carcinogenesis or promote metastasis in others.
Long-term regulation of ACC occurs at the level of transcription, while short-term regulation of ACC occurs through allosteric binding and reversible phosphorylation. Short-term regulation allows ACC activity to rapidly adapt to the microenvironment. For instance, AMP-activated protein kinase (AMPK) can inactivate $\mathrm{ACC}$ via phosphorylation (p-ACC). Metformin is a widely prescribed first-line treatment for type 2 diabetes that activates AMPK. Preclinical studies in mice have demonstrated that metformin can reduce cancer growth, in part by increasing p-ACC levels $[42,43]$. There are currently hundreds of clinical trials investigating whether metformin can be repurposed to treat cancer as an adjuvant monotherapy or in combination with other drugs. However, a potential adverse effect of metformin may be an increase in the metastasis of certain cancer types, given that both metastatic breast and lung tumors have increased levels of p-ACC1 [41]. Protein phosphatase 2A (PP2A) can reactivate $\mathrm{p}$-ACC by dephosphorylation. The tumor suppressor known as breast cancer susceptibility gene 1 (BRCA1), which is deactivated primarily in breast and ovarian cancers, prevents dephosphorylation of p-ACC [44]. Cancers with loss-offunction mutations in BRCA1 have increased ACC activity due to less phosphorylation of ACC and thus may be more susceptible to ACC inhibition $[44,45]$.

\subsection{The First Fatty Acid Synthase (FAS) Inhibitor TVB-2640 Is in Clinical Trials for Cancer}

A large number of studies have now documented an increase in the expressions of lipogenic enzymes across several cancers. For instance, Szutowicz et al. revealed that the activity of citrate lyase, an important enzyme in lipogenesis, is elevated in breast carcinoma and fibrocystic disease compared to healthy breast tissue [25]. As such, it is reasonable that key enzymes involved in de novo fatty acid synthesis could be potential targets for cancer therapy. One such enzyme is fatty acid synthase (FAS) encoded by the FASN gene [46]. 
FAS is a multienzyme protein complex that catalyzes the final reactions converting malonylCoA and acetyl-CoA into a saturated long-chain fatty acid composed of 16 carbons known as palmitic acid. Palmitic acid can be used as a precursor to produce lipid signaling molecules, modify protein functions through palmitoylation, store energy as triglycerides, or form structural lipids for cell membranes. NADPH is the reducing agent for fatty acid synthesis, and 14 molecules of NADPH are used to synthesize each molecule of palmitic acid starting with acetyl-CoA. The pentose phosphate pathway (PPP) generates NADPH through the oxidation of glucose into pentose sugars and ribulose-5-phosphate. Overexpression of FASN is usually accompanied by the overexpression of enzymes in the PPP to supply NADPH for fatty acid synthesis. Increased expression of FAS and PPP enzymes is associated with worse survival in renal and breast cancers [35, 47]. Increased FAS expression is also associated with tumor grade in prostate cancer [48]. Inhibition of FAS reduces cell proliferation and increases cell death in human breast [37, 43], prostate [49], and colon cancers [50]. FAS inhibitors can also be used in combination with chemotherapy taxane to improve anticancer efficacy [51]. Colorectal cancer metastasis is also mitigated by FAS inhibition in mice [50]. While inhibition of FAS reduces tumor growth and metastasis in most cancers, it has also been demonstrated to reduce survival rates in mice with lung cancer by increasing metastasis [52], demonstrating how FAS inhibition can sometimes worsen cancer outcomes as seen with ACC inhibition.

The FAS inhibitor TVB-2640 has been tested on cancer patients in clinical trials (Clinical Trial ID: NCT02223247). Inhibiting FAS did not result in severe side effects, and all of the mild side effects were reversible after discontinuation [53]. Moreover, side effects were not worsened by its combined application with the chemotherapy drug paclitaxel [53]. Monotherapy with TVB2640 stabilized cancer progression in three out of six patients with $K R A S$-driven non-small cell lung cancer (NSCLC) [53]. There are now two clinical trials (phase II) testing the efficacy of TVB-2640 in combination with additional che- motherapy drugs for $\mathrm{HER}^{+}$breast cancer (Clinical Trial ID: NCT03179904) and astrocytoma (Clinical Trial ID: NCT03032484). The third clinical trial is a phase I study investigating the pharmacodynamic effects of TVB-2640 in patients who require surgery for colon cancer (Clinical Trial ID: NCT02980029).

\subsection{Which Markers Can Predict Cancer Cell Sensitivity to Lipid Synthesis Inhibition?}

Phosphatidylinositol (3,4,5)-trisphosphate (PIP3) is produced by phosphoinositide 3-kinase (PI3K) activation, which is mutated in many types of cancers [54-56]. PI3K, which is a key regulator of phosphoinositide metabolisms, is considered a potential target in preclinical and clinical settings to suppress advanced solid tumors, including malignant glioma, NSCLC, and breast cancer [57] (NCT00485719, NCT00777699, NCT00704080, NCT00907205, NCT00600275, NCT00876109, and NCT00726583).

Two highly studied intracellular signaling pathways that oncogenes activate to drive tumorigenesis and increase expression of lipid synthesis enzymes are the PI3K/Akt/mammalian target of rapamycin (mTOR) pathway and the RAS/mitogen-activated protein kinase (MAPK) pathway [58, 59]. Constitutive activation of the $\mathrm{PI} 3 \mathrm{~K} / \mathrm{Akt} / \mathrm{mTOR}$ pathway results from activation of receptor tyrosine kinases (RTKs), loss-offunction mutations in the tumor suppressors phosphatase and tensin homolog (PTEN) and tuberous sclerosis protein (TSC), or gain-of-function mutations in phosphatidylinositol-4,5-bisphosphate 3-kinase catalytic subunit alpha (PIK3CA) and AKT1 [60]. The RAS oncogene family including HRAS, KRAS, and NRAS can also activate the $\mathrm{PI} 3 \mathrm{~K} / \mathrm{Akt} / \mathrm{mTOR}$ pathway [61]. Cancer cells that are driven by the overactivation of the PI3K or RAS pathways are more susceptible to inhibitors that target lipid synthesis than cancers that are driven by pathways not associated with lipid synthesis regulation [62, 63]. Inhibitors of ACLY, ACC, and FAS have been shown to be efficacious in cancers with PI3K and RAS pathways [64-67]. 
Cancer cells that overactivate RTKs, such as epidermal growth factor receptor (EGFR, also known as HER) family members $1-4$ and c-MET [6870], are also sensitive to lipid synthesis inhibition because these RTKs activate PI3K and RAS pathways. ACLY and ACC inhibitions are efficacious in HER1- and HER2-driven cancers [39, 66, 7173], and FAS inhibition is efficacious in HER1-, HER2-, and c-MET-driven cancers [68, 74, 75]. Additional oncogenic signaling pathways that may be susceptible to FAS inhibition are MYC, beta-catenin, and steroid-responsive tumors because FAS inhibition downregulates these pathways $[64,76,77]$. Cancers with a loss of function in BRCA1 and p53 are also sensitive to FAS and ACC inhibition [45, 67, 78]. Preclinical studies testing ACLY, ACC, and FAS inhibitors may reveal the oncogenes and RTKs that confer susceptibility to DNLS inhibition and guide the design of future clinical trials. To date, several mTOR/PI3K inhibitors, such as idelalisib, copanlisib, rapamycin, temsirolimus, everolimus, and ridaforolimus, have been approved by the FDA.

Nevertheless, not all cancers with oncogenemediated overactivation of RAS and PI3K pathways appear to be susceptible to lipid synthesis inhibition. For instance, KRAS mutations correlated with FAS inhibition sensitivity in lung cancer cell lines but not in colon cancer cell lines [64], which demonstrates that cancer cell susceptibility to lipid synthesis inhibition is not always driven by oncogenes. In other studies, oncogenes conferred resistance to FAS inhibition. Hepatocytes that are transformed into malignant cancer cells by the overactivation of c-MET and Akt are susceptible to FAS inhibition, but hepatocytes that are transformed by the overactivation of c-MET and Wnt/beta-catenin signaling are unresponsive to FAS inhibition [63]. The c-MET/beta-catenin-driven cancer cells may be unresponsive because beta-catenin activation in hepatocytes reduces FAS expression and lipid synthesis $[63,79,80]$. Interestingly, while beta-catenin decreases lipid synthesis in hepatocytes, beta-catenin signaling can increase lipid synthesis in B-cell lymphoma. Betacatenin-driven B-cell lymphoma is susceptible to FAS inhibition [81]. This demonstrates that cancer cell type is relevant to determining susceptibility to FAS inhibition since oncogenic signaling pathways can result in different phenotypes depending on the cell type. In order for oncogenes to be reliable markers of lipid synthesis inhibition sensitivity, it will be important to consider the cell type of the cancer being discussed.

Of note, protein expression or enzyme activity may be better predictors of susceptibility to lipid synthesis inhibitors than genetic markers. For example, mRNA expression of ACLY, ACC, and FAS may not correlate with protein expression and activity [64, 82]. Increased expression of FAS and ACC at the protein level can occur without an increase in mRNA expression by increased translation of FAS and ACC mRNA via mTOR signaling [82].

Metabolic profiling may be a valuable method for determining susceptibility to FAS inhibition. One study examined 38 pancreatic cancer cell lines and classified them as lipogenic or glycolytic depending on their metabolic profile, which was determined by the amount of lipogenic or glycolytic metabolites [83]. Glycolytic cancer cells were significantly more susceptible to glycolytic inhibitors than those that were lipogenic. However, lipogenic cancer cells were not significantly more susceptible to lipogenic inhibitors, such as FAS inhibitors, than glycolytic cancer cells [83]. Only half of the lipogenic cancer cell lines were sensitive to FAS inhibition, suggesting that broad lipogenic profiling is not an accurate predictor of susceptibility to FAS inhibition. While no single marker is able to definitively predict which cancers are susceptible to lipid synthesis inhibition, using a combination of markers, such as cell type, oncogene mutations, expression/activity of lipid synthesis enzymes, and metabolic profiles, may provide a reliable means to identify cancers that are sensitive to lipid synthesis inhibitors. 


\subsection{Tumor Microenvironment Influences the Sensitivity of Cancer Cells to Lipid Synthesis Inhibitors}

As mentioned in the chapter "Different Tumor Microenvironments Lead to Different Metabolic Phenotypes," TCA cycle activity is reduced under hypoxic conditions, which results in reduced citrate and acetyl-CoA production. However, cancer cells manage to generate acetyl-CoA for fatty acid synthesis by different mechanisms, including reliance on glutamine for citrate synthesis and acetate for acetyl-CoA via acetyl-CoA synthetase (ACSS2). Evidence suggests that ACSS2 expression can be increased to maintain growth under microenvironmental stress, such as hypoxia [84].

Bensaad et al. showed that while DNLS is repressed in hypoxia, lipid droplet accumulation and expression of fatty acid uptake proteins, such as fatty acid-binding protein 3 and 7 (FABP3 and FABP7), are induced by hypoxia-inducible factor 1-alpha (HIF-1 $\alpha)$. Lipid synthesis was restored in cancer cells after reoxygenation or the removal of antiangiogenic therapy [85]. Other studies have corroborated that hypoxic tumor cells may be extraordinarily dependent on fatty acid uptake compared to those in normoxia [86, 87]. However, this can be differentially driven by oncogenic mammalian target of rapamycin complex 1 (mTORC1), RAS, and/or HIF-1 $\alpha$ signaling [8587]. Moreover, triple-negative breast cancer is reliant on lipid droplet-derived substrates for $\beta$-oxidation and ATP generation after hypoxiareoxygenation, whereas glioblastoma multiforme (GBM) is more dependent on glycolytic pathways [85]. This implies that FA uptake is not a universal feature of hypoxic cancer cells; therefore, inhibiting FA uptake may be a strategy for targeting tumor cells in hypoxic microenvironments for certain types of cancers but not others.

The availability of metabolic nutrients can also greatly impact the susceptibility of cancer cells to inhibition of DNLS. FAS expression was observed to be the highest at the edge of tumors, suggesting that DNLS is preferred in cancer cells that are vascularized and have access to oxygen and glucose [88]. Tumors in low-lipid environments increase de novo fatty acid synthesis and thus may demonstrate increased sensitivity to FAS inhibition. The fact that lipoprotein supplementation can override DNLS inhibition emphasizes the importance of nutrient availability and, again, the role of exogenous lipid uptake $[89,90]$. The availability of glucose for glucose-dependent lipogenesis is also important for cancer cell sensitivity to ACLY inhibitors. Low-glucose environments result in cancer cells that are less susceptible to ACLY inhibition because cancer cells can use acetate instead of citrate to produce acetyl-CoA for DNLS [90, 91].

\section{$3 \quad$ Targeting Fatty Acid Elongation}

Once palmitic acid is produced by de novo lipid synthesis, it can be modified by having its fatty acid chain elongated. Elongation of fatty acids is important for creating lipid precursors that are involved in cellular signaling and for producing phospholipids of cell membranes. Fatty acids that consist of 16 carbons or more, such as palmitic acid, are elongated in the smooth endoplasmic reticulum, while fatty acids consisting of fewer than 16 carbons are elongated in the mitochondria. Elongation of fatty acids in the smooth endoplasmic reticulum is regulated by four enzymes. These enzymes elongate fatty acids by using malonyl-CoA. The first step is the ratedetermining reaction regulated by the enzyme $\beta$-ketoacyl-CoA synthase (elongase). There are seven types of elongases in humans, known as ELOVL1-7. ELOVL7 was identified to be overexpressed in prostate cancer, and feeding mice a diet high in long- and very-long-chain fatty acids increased the growth of ELOVL7-expressing tumor cells [92]. Meanwhile, inhibiting ELOVL7 with siRNA attenuated prostate cancer growth [92]. ELOVL1 is another elongase implicated in cancer growth. ELOVL1 was observed to be overexpressed in breast cancer, and inhibition of ELOVL1 with siRNA reduced breast cancer cell viability in some cell lines [37]. 
While inhibiting elongases appears to be a therapeutic strategy for cancer treatment, inhibiting $\mathrm{ACC} 1$ as described previously may be more promising because ACC1 inhibition reduces both fatty acid synthesis and fatty acid elongation, while elongase inhibition only targets elongation. ACC1 inhibition can reduce fatty acid elongation by decreasing the availability of malonyl-CoA [93]. This is suggested by a study in which silencing of ELOVL1 with a silencing efficiency of $70-80 \%$ decreased cell viability by greater than $50 \%$ in one breast cancer cell line while silencing of ACC1 with a lower silencing efficiency of $30 \%$ decreased cell viability by greater than $50 \%$ in two breast cancer cell lines [37]. Neoadjuvant chemotherapy-resistant breast cancer has been associated with increased expression of fatty acid elongation proteins in the mitochondria [94]. Whether inhibiting mitochondrial fatty acid elongation is a potential therapeutic strategy against cancer remains to be determined.

\section{$4 \quad$ The Efficacy of Inhibiting Cholesterol Synthesis with Adjuvant Statins Is Variable}

Another anabolic pathway associated with lipid metabolism is the mevalonate pathway, which synthesizes cholesterol. Cholesterol is a major component of cell membranes, influencing membrane fluidity, and function. It also forms detergent-resistant microdomains called lipid rafts that coordinate the activation of signal transduction pathways. The enzyme 3-hydroxy-3methylglutaryl-CoA reductase (HMGCR) catalyzes the rate-limiting step of cholesterol synthesis. Increased expression of HMGCR and other cholesterol synthesis enzymes is associated with reduced survival rates in breast cancer [95]. HMGCR is the target of a class of cholesterollowering drugs called statins. Numerous epidemiological studies have demonstrated that patients who use statins have a reduced risk of cancer and cancer mortality [96-98]. This has raised the question as to whether statins can improve treatment outcomes in cancer patients.
There are many clinical trials currently investigating whether statins can be prescribed to reduce the progression of cancer.

Results from preclinical studies suggest that the efficacy of statins can be predicted based on the status of gene expression, such as that of HMGCR [99]. Breast cancer cells with overactive HER2 are also sensitive to statins because of HER2 signals through the RAS pathway [100]. On the other hand, estrogen receptor-positive breast cancer cells appear to be less responsive to statins. $M Y C$ is another transcription factor that regulates cholesterol synthesis. Cancers with overactive $M Y C$ have been observed with increased expression of HMGCR and sensitivity to statins [101, 102]. Statins have also been shown to reduce metastasis in colon and ovarian cancer and selectively induce apoptosis in cancer cells [103, 104]. Along with monotherapy of statins being efficacious in preclinical studies, statins are also efficacious in combination therapy by increasing sensitivity to radiation therapy [105].

The ability of statins to bind to HMGCR greatly affects their efficacy. Genetic variations in HMGCR have been found to modify the therapeutic effect that statins have on colorectal cancer [106]. A single-nucleotide polymorphism (SNP) in the HMGCR-statin-binding domain reduced the protective association between statins and colorectal cancer. An in vitro experiment demonstrated that the SNP in the HMGCR gene reduced the ability of statins to inhibit HMGCR and cholesterol synthesis. The anticancer activity of statins is also dependent on the ability of statins to enter cancer cells. For instance, the statin pravastatin was found to inhibit tumor growth preferentially in cancers that express sodiumindependent organic anion transporter protein1B1 (OATP1B1), such as liver cancer, because this transporter is necessary for cellular uptake of pravastatin [107].

While preclinical studies have provided promising results for statins, clinical trials have not been as successful. A phase II clinical trial demonstrated that combining the statin simvastatin with the chemotherapy drug afatinib did not improve treatment efficacy compared to using afatinib in monotherapy [108]. Two additional 
phase II clinical trials found that statins were unable to resensitize cancers harboring KRASactivating mutations to the chemotherapy drugs cetuximab and panitumumab [109, 110].

\section{$5 \quad$ Fatty Acid Uptake Is Associated with Metastasis}

As discussed previously, enhanced lipogenesis is a frequent alteration of lipid metabolism in cancer cells, and therapies targeting it are promising. However, studies show that this strategy can be undermined by the supplementation of exogenous fatty acids, suggesting that extracellular lipids in the microenvironment may functionally substitute for endogenously derived FA [111]. After all, the scavenging of circulating nutrients is another hallmark of cancer cell metabolism [112]. Recently, it was reported that tumors, including those of breast cancer and liposarcoma, may rely on extracellular lipolysis in addition to lipogenesis to fuel cellular lipid requirements [113]. Lipoprotein lipase (LPL) is a rate-limiting enzyme of this mechanism, hydrolyzing circulating triglyceride-rich lipoprotein, such as verylow-density lipoproteins and chylomicrons, into free FAs and monoacylglycerol molecules. Free FAs are then imported into cells by FA transporters such as a cluster of differentiation (CD36) or those of the fatty acid-binding protein (FABP). Both LPL and CD36 expressions have been associated with aggressive cancers, including hepatocellular carcinoma (HCC) and pancreatic ductal adenocarcinoma (PDAC), and negatively correlated with patient prognosis [114-117], but how this phenomenon varies among tumors and whether it can be inhibited for therapeutic effect remain uncertain.

An estimated $90 \%$ of all cancer-related deaths are attributed to metastasis, but the detailed mechanisms of metastasis remain unclear [118]. Recently, metastasis was associated with enhanced lipid metabolism [14, 119, 120]. One study identified an altered gene signature associated with fatty acid uptake (e.g., caveolin 1 (CAV1), CD36) in metastatic tumors across cancer types [121]. Moreover, this genetic signature had a significant effect on patient survival rates, suggesting prominent roles of extracellular fatty acids, specifically on metastatic progression. Corroborating this is a recent report describing abnormally high expression of CD36 in metastasis-initiating oral cancer cells [12]. Treating orthotopic xenografts with CD36neutralizing antibodies inhibited metastasis initiation. These studies suggest that tumor cells of high metastatic potential have an outsized need for FA uptake compared to those displaying less aggressive phenotypes. Interestingly, however, an earlier study observed up to a 100-fold lower expression of CD36 in breast cancer cells with high metastatic potential compared to their less aggressive counterparts [122]. This inconsistency may be due to alternative mechanisms of CD36 related to cell adhesion [123]. Nevertheless, these data support the overarching concept of asymmetrical CD36 expression and fatty acid uptake even within cancers of the same type.

\section{$6 \quad$ Fatty Acid Oxidation Encompasses a Diverse Set of Molecular Mechanisms}

Lipids are important for cancer proliferation not only because of their ability to provide structural support as a component of the cell membrane but also because they can be broken down to provide energy. Lipids can be catabolized after cellular uptake via the $\beta$-oxidation pathway, also known as fatty acid oxidation (FAO). FAO has not been examined as thoroughly as glycolysis or glutaminolysis, but recent advances have shed light on the role of FAO in cancer cells. Recently, lipids were also identified as a carbon source for nucleotide synthesis and histone acetylation in nonmalignant cells, and emerging evidence suggests that these mechanisms are relevant to tumor cells as well $[124,125]$. The tumor microenvironment is often depleted of nutrients like glucose, so cancer cells often rely on FAO to generate ATP. Lipids are energetically dense molecules that cancer cells can exploit as an alternative source of energy. FAO yields ATP and NADPH, which support cellular energetics and redox homeostasis, 
respectively. Several studies have demonstrated that certain malignancies, such as those in the prostate, breast, and lung, and B-cell lymphoma heavily depend on FAO for growth and survival [126-128]. Similarly, acetate is a 2-carbon fatty acid that is avidly oxidized in tumors, including GBM $[129,130]$.

\subsection{Targeting FAO for Cancer Therapy May Be Achieved by Inhibiting Carnitine Palmitoyltransferase 1}

The inhibition of the FAO pathway could prevent cancer progression. An example of this strategy is the inhibition of carnitine palmitoyltransferase 1 (CPT1), which is the rate-limiting enzyme of FAO. CPT1 is a membrane protein that removes an acyl group from a fatty acyl-CoA and attaches the acyl group to carnitine. This results in the formation of acylcarnitine, including palmitoylcarnitine, and thereby facilitates the shuttling of fatty acids, such as palmitate, into the mitochondrial matrix for FAO [124]. There are three subtypes of CPT1. CPT1A is expressed throughout several tissue types, but CPT1B is restricted mostly to muscle tissue. In physiological settings, all isoenzymes are inhibited by malonyl-CoA, but due to the greater binding efficiency of CPT1A to malonyl-CoA, CPT1A is found to be the isoform with the greatest capacity to perform the rate-limiting step of FAO [131]. The third and final isoform of CPT1 is CPT1C, which is normally found only in the brain [132]. However, many cancers also express CPT1C [133]. CPT1C is thought to confer resistance to oxidative stress in many tumors. CPT1C promotes resistance to rapamycin, an mTOR pathway inhibitor [133].

Physiologically, it is crucial to note that successful inhibition of CPT1 is dependent on the source and location of malonyl-CoA. MalonylCoA produced via acetyl-CoA carboxylase 1 (ACC1) is localized in the cytosol and thus will not inhibit CPT1. The malonyl-CoA produced via the mitochondrial ACC 2 enzyme, however, is capable of this inhibitory action. Thus, the relative concentrations of acetyl-CoA to malonylCoA can influence whether the cell is in a state of
FAS or FAO [134]. AMP-activated protein kinase (AMPK) inhibits both ACC1 and ACC2 and increases reactive oxygen species (ROS) in doing so. The increase in ROS leads to depletion of NADPH and induces oxidative stress on the cell, eventually leading to cell death [8]. This finding is in accordance with other studies that have noted the role of AMPK activation in cancer states. For example, metformin exerts anticancer effects and activates AMPK, but in tumors lacking CPT1C, the effect of metformin is less pronounced. This suggests that the action of metformin on AMPK is upstream of its effect on CPT1C [133].

The upregulation of CPT1 in several cancer types makes it a potential therapeutic target [131]. However, this upregulation does not appear to be a universal feature of all tumors, as demonstrated by a recent study showing that, in clear cell renal cell carcinoma (ccRCC), transcriptional repression of CPT1A is mediated by hypoxia-inducible factors (HIF-1 $\alpha$ and HIF-2 $\alpha$ ) [135]. However, conflicting reports regarding the role of HIFs in FAO have also emerged, and one may speculate that this is again due to the heterogeneity of metabolism across cancer subtypes. Although HIFs are known to inhibit FAO, one study performed in liver cancer cells determined that HIF-1 also decreases ROS levels and maintains redox homeostasis, thereby promoting cell proliferation [136]. This effect is thought to be mediated by the action of HIF-1 on medium- and long-chain acyl-CoA dehydrogenases (MCAD and LCAD, respectively). This study further pointed to correlations between decreased LCAD expression and patient mortality rates [136]. Thus, we see that the precise role of HIFs varies across cancer types, and as such, therapies targeting HIF-related pathways may need to be tailored to specific cancers to maximize their impact.

\subsection{CPT1 Inhibitors Are Now in Clinical Trials}

As far as pharmacological interventions for FAO are concerned, some CPT1 inhibitors are being developed for other conditions such as diabetes 
[137]. Therefore, the possibility of repurposing them for cancer therapy is an intriguing possibility requiring further clinical trials [137]. One CPT1 inhibitor, etomoxir, has been difficult to advance through clinical trials due to its toxicity. A clinical study examining etomoxir in healthy adults found elevated levels of transaminases of some patients, and the study had to be terminated early [138]. The issue with etomoxir arises from its inability to distinguish CPT1 across tissue types. However, it has been applied to preclinical studies of breast cancer, where an interesting degree of heterogeneity has been noted. In one study, etomoxir was compared across two triplenegative breast cancer (TNBC) lines. One line expressed high amounts of the oncogenic transcription factor $M Y C$, whereas the other expressed low amounts of $M Y C$. In the high- $M Y C$-expressing line, the application of etomoxir decreased levels of ATP, and this effect was not observed in the low-MYC-expressing line [128]. Furthermore, this effect was observed in no other breast cancer subtypes besides TNBC. This provides further evidence of the ways in which cancer heterogeneity should be appreciated and exploited for the development of viable treatments.

\subsection{FAO for Very-Long-Chain Fatty Acids Occurs at the Peroxisome Where Peroxisome Proliferator- Activated Receptors (PPARs) Act as Ligand-Activated Transcription Factors}

FAO also occurs in peroxisomes. Oxidation at the peroxisome is restricted to very-long-chain fatty acids. The peroxisome breaks these very long chains into smaller chains, which may then be further oxidized in the mitochondria. Peroxisomes are built via peroxins, the products of the Pex genes. So far, 3 of the 30 known peroxins, Pex3, Pex16, and Pex19, have been shown to be necessary for proper peroxisome assembly [139]. One of these peroxins, Pex19, was shown in a series of experiments to be involved in the transition to malignancy in prostate cancer through monocar- boxylate transporter 2 (MCT2). MCT2 is upregulated in prostate cancer and, like other MCTs, serves to facilitate the transport of lactic acid in glycolytic tumors. Immunoprecipitation experiments demonstrated that colocalization of MCT2 with peroxisomes was strongest at disease initiation and decreased as metastasis increased; furthermore, colocalization was absent in nonmalignant prostate cancer lines [140].

Other components of peroxisomes are the peroxisome proliferator-activated receptors (PPARs). Three PPARs (PPAR $\alpha$, PPAR $\gamma$, PPAR $\beta / \delta$ ) are known and have been described as ligand-activated transcription factors [141]. These three PPARs differ predominantly in tissue distribution, and their exact functions in cancer remain ambiguous. It has been shown that PPARs are key regulators that integrate lipid metabolism and inflammation [142]. Furthermore, the PPARs have been directly implicated in cancers as well as in cancer-related processes, including carcinogenesis and chemoresistance [143, 144].

The theme of heterogeneity persists within the various PPARs and across species. For example, long-term PPAR $\alpha$ agonism in rodents leads to the development of liver cancer. Interestingly, PPAR $\alpha$ is expressed at lower levels in human liver relative to rodent liver, and as such, PPAR $\alpha$ agonism does not lead to liver cancer in humans $[143,145]$. PPAR $\beta / \delta$ displays tissue-wide distribution. One of its functions is to reduce oxidative stress, such as in breast cancer [146]. However, it is expressed ubiquitously and has been shown to be involved in many cancer types, particularly in cancers under hypoxic environments, such as breast, colon, lung, and ovarian cancers, as well as chronic lymphocytic leukemia [143]. Its precise role remains controversial, but it appears that $\mathrm{PPAR} \beta / \delta$ may play a role as a lipid-activated mediator of an anti-inflammatory response. Like $\operatorname{PPAR} \beta / \delta$, mystery surrounds PPAR $\gamma$. Although it may be coded for by four mRNAs (PPARG1 through PPARG4), PPAR $\gamma 1$ and PPAR $\gamma 2$ are responsible for most PPAR $\gamma$ physiological actions [143]. PPAR $\gamma 1 \mathrm{mRNA}$ is found ubiquitously, whereas PPAR $\gamma 2$ mRNA is restricted to adipocytes [147]. Some, but not all, PPAR $\gamma$ agonists induce apoptosis in cancer cells and have 
also been reported to induce terminal differentiation. Targets of PPAR $\gamma$ include many genes involved in the cell cycle and apoptosis in tumors, such as p53 and PTEN. The increasing characterization of PPAR $\gamma$ as a biomarker in cancer led some investigators to speculate that it may be utilized in screens [148]. Together, the PPARs constitute an area of research that may prove critical in our understanding of tumor development and treatment.

The many aspects of peroxisomal signaling further convey the diversity of lipid signaling across many different types of cancers. Abnormalities within the peroxisomes themselves or within PPARs can alter the efficacy of the critical lipid signaling that cancer cells rely on. Further research, particularly in the form of genomic analyses, will be useful in harnessing this heterogeneity for personalized medicine approaches.

\section{Conclusion}

Therapeutic strategies targeting lipid metabolism are now in various stages of clinical development, and one approach worth highlighting is the "repurposing" of drugs from cardiology [149]. As emphasized, we urge caution based on the significance of heterogeneity in cancer lipid metabolism as we translate basic science into clinical applications. Drug combinations have become a cornerstone against refractory and heterogeneous tumors, so the question now is how to combine treatment options for maximum safety and efficacy [150-152]. Going forward, systems biology and bioinformatics will likely become essential tools for integrating various levels of -omic data $[153,154]$. Dissecting the spatial and temporal heterogeneity of lipid metabolism with these tools will likely accelerate the tailoring of clinical care according to patient-specific signatures, as envisioned by precision medicine (Fig. 1).

Acknowledgments We thank Dr. Resat Cinar, PhD, MBA, for his support and Mr. Daniel McCaskey, JD, for his review of the manuscript.

\section{References}

1. Ma, X., et al. (2016). Identification and quantitation of lipid $\mathrm{C}=\mathrm{C}$ location isomers: A shotgun lipidomics approach enabled by photochemical reaction. Proceedings of the National Academy of Sciences, 113(10), 2573-2578.

2. Shevchenko, A., \& Simons, K. (2010). Lipidomics: Coming to grips with lipid diversity. Nature Reviews Molecular Cell Biology, 11, 593.

3. Yang, K., \& Han, X. (2016). Lipidomics: Techniques, applications, and outcomes related to biomedical sciences. Trends in Biochemical Sciences, 41(11), 954-969.

4. DeBerardinis, R. J., \& Chandel, N. S. (2016). Fundamentals of cancer metabolism. Science Advances, 2(5), e1600200.

5. Beloribi-Djefaflia, S., Vasseur, S., \& Guillaumond, F. (2016). Lipid metabolic reprogramming in cancer cells. Oncogene, 5, e189.

6. Zalba, S., \& ten Hagen, T. L. M. (2017). Cell membrane modulation as adjuvant in cancer therapy. Cancer Treatment Reviews, 52, 48-57.

7. Rysman, E., et al. (2010). De novo lipogenesis protects cancer cells from free radicals and chemotherapeutics by promoting membrane lipid saturation. Cancer Research, 70(20), 8117-8126.

8. Jeon, S.-M., Chandel, N. S., \& Hay, N. (2012). AMPK regulates NADPH homeostasis to promote tumour cell survival during energy stress. Nature, $485,661$.

9. Ayala, A., et al. (2014). Lipid peroxidation: Production, metabolism, and signaling mechanisms of malondialdehyde and 4-hydroxy-2-nonenal. Oxidative Medicine and Cellular Longevity, 2014, 31 .

10. Keckesova, Z., et al. (2017). LACTB is a tumour suppressor that modulates lipid metabolism and cell state. Nature, 543, 681.

11. Wang, D., \& Dubois, R. N. (2010). Eicosanoids and cancer. Nature Reviews Cancer, 10(3), 181-193.

12. Pascual, G., et al. (2016). Targeting metastasisinitiating cells through the fatty acid receptor CD36. Nature, 541, 41.

13. Viswanathan, V. S., et al. (2017). Dependency of a therapy-resistant state of cancer cells on a lipid peroxidase pathway. Nature, 547, 453.

14. Luo, X., et al. (2017). Emerging roles of lipid metabolism in cancer metastasis. Molecular Cancer, 16, 76.

15. Hendrich, A. B., \& Michalak, K. (2003). Lipids as a target for drugs modulating multidrug resistance of cancer cells. Current Drug Targets, 4(1), 23-30.

16. Tadros, S., et al. (2017). De novo lipid synthesis facilitates gemcitabine resistance through endoplasmic reticulum stress in pancreatic cancer. Cancer Research, 77(20), 5503-5517.

17. Ellsworth, R. E., et al. (2017). Molecular heterogeneity in breast cancer: State of the science and 


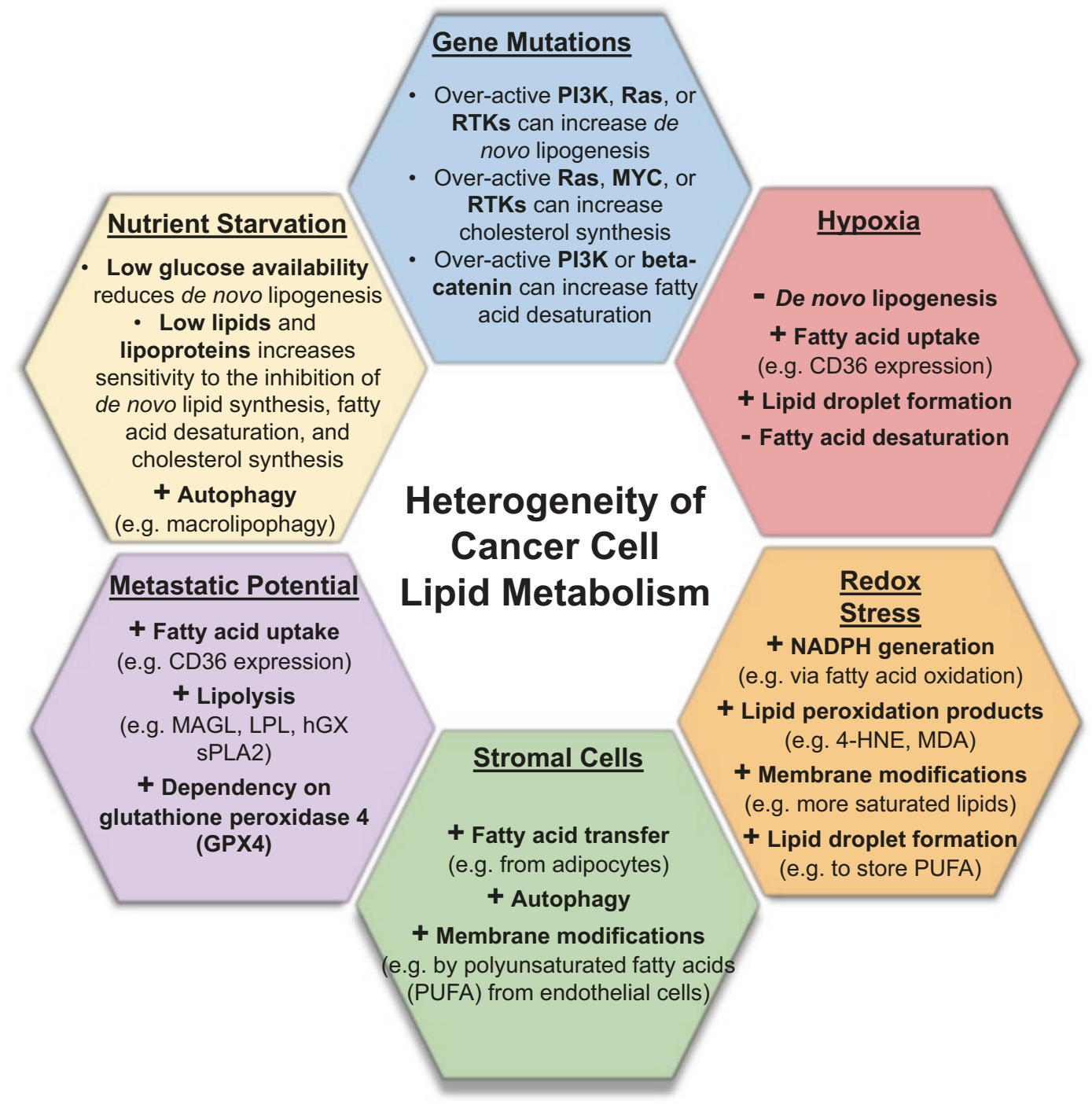

Fig. 1 Factors that can contribute to spatial and temporal heterogeneity in cancer cell lipid metabolism

implications for patient care. Seminars in Cell \& Developmental Biology, 64, 65-72.

18. Greaves, M. (2015). Evolutionary determinants of cancer. Cancer Discovery, 5(8), 806-820.

19. Dang, C. V., et al. (2011). Therapeutic targeting of cancer cell metabolism. Journal of Molecular Medicine (Berlin), 89(3), 205-212.

20. Hirschey, M. D., et al. (2015). Dysregulated metabolism contributes to oncogenesis. Seminars in Cancer Biology, 35(Suppl), S129-S150.

21. Strickaert, A., et al. (2016). Cancer heterogeneity is not compatible with one unique cancer cell metabolic map. Oncogene, 36, 2637.

22. Nabi, K., \& Le, A. (2021). The intratumoral heterogeneity of cancer metabolism. Advances in
Experimental Medicine and Biology, 1311, https:// doi.org/10.1007/978-3-030-65768-0_11

23. Antonio, M. J., Zhang, C., \& Le, A. (2021). Different tumor microenvironments lead to different metabolic phenotypes. Advances in Experimental Medicine and Biology, 1311, https:// doi.org/10.1007/978-3-030-65768-0_10

24. Catalina-Rodriguez, O., et al. (2012). The mitochondrial citrate transporter, CIC, is essential for mitochondrial homeostasis. Oncotarget, 3(10), 1220-1235.

25. Szutowicz, A., Kwiatkowski, J., \& Angielski, S. (1979). Lipogenetic and glycolytic enzyme activities in carcinoma and nonmalignant diseases of the human breast. British Journal of Cancer, 39(6), 681-687. 
26. Migita, T., et al. (2008). ATP citrate lyase: Activation and therapeutic implications in non-small cell lung cancer. Cancer Research, 68(20), 8547.

27. Yahagi, N., et al. (2005). Co-ordinate activation of lipogenic enzymes in hepatocellular carcinoma. European Journal of Cancer, 41(9), 1316-1322.

28. Turyn, J., et al. (2003). Increased activity of glycerol 3-phosphate dehydrogenase and other lipogenic enzymes in human bladder cancer. Hormone and Metabolic Research, 35(10), 565-569.

29. McGarry, J. D., Leatherman, G. F., \& Foster, D. W. (1978). Carnitine palmitoyltransferase. I. The site of inhibition of hepatic fatty acid oxidation by malonylCoA. Journal of Biological Chemistry, 253(12), 4128-4136.

30. Wang, C., et al. (2015). The acetyl-CoA carboxylase enzyme: A target for cancer therapy? Expert Review of Anticancer Therapy, 15(6), 667-676.

31. Savage, D. B., et al. (2006). Reversal of diet-induced hepatic steatosis and hepatic insulin resistance by antisense oligonucleotide inhibitors of acetylCoA carboxylases 1 and 2. Journal of Clinical Investigation, 116(3), 817-824.

32. Milgraum, L. Z., et al. (1997). Enzymes of the fatty acid synthesis pathway are highly expressed in in situ breast carcinoma. Clinical Cancer Research, 3(11), 2115-2120.

33. Swinnen, J. V., et al. (2000). Selective activation of the fatty acid synthesis pathway in human prostate cancer. International Journal of Cancer, 88(2), 176-179.

34. Nelson, M. E., et al. (2017). Inhibition of hepatic lipogenesis enhances liver tumorigenesis by increasing antioxidant defense and promoting cell survival. Nature Communications, 8, 14689.

35. The Cancer Genome Atlas Research Network. (2013). Comprehensive molecular characterization of clear cell renal cell carcinoma. Nature, 499(7456), 43-49.

36. Calvisi, D. F., et al. (2011). Increased lipogenesis, induced by AKT-mTORC1-RPS6 signaling promotes development of human hepatocellular carcinoma. Gastroenterology, 140(3), 1071-1083.e5.

37. Hilvo, M., et al. (2011). Novel theranostic opportunities offered by characterization of altered membrane lipid metabolism in breast Cancer progression. Cancer Research, 71(9), 3236-3245.

38. Beckers, A., et al. (2007). Chemical inhibition of acetyl-CoA carboxylase induces growth arrest and cytotoxicity selectively in cancer cells. Cancer Research, 67(17), 8180-8187.

39. Jones, J. E. C., et al. (2017). Inhibition of acetyl-CoA carboxylase 1 (ACC1) and 2 (ACC2) reduces proliferation and De novo lipogenesis of EGFRvIII human glioblastoma cells. PLoS One, 12(1), e0169566.

40. Petrova, E., et al. (2017). Acetyl-CoA carboxylase inhibitors attenuate WNT and hedgehog signaling and suppress pancreatic tumor growth. Oncotarget, 8(30), 48660-48670.

41. Rios Garcia, M., et al. (2017). Acetyl-CoA carboxylase 1-dependent protein acetylation controls breast cancer metastasis and recurrence. Cell Metabolism, 26(6), 842-855.e5.

42. Zakikhani, M., et al. (2006). Metformin is an AMP kinase-dependent growth inhibitor for breast cancer cells. Cancer Research, 66(21), 10269-10273.

43. Knowles, L. M., et al. (2008). Inhibition of fattyacid synthase induces caspase-8-mediated tumor cell apoptosis by up-regulating DDIT4. Journal of Biological Chemistry, 283(46), 31378-31384.

44. Moreau, K., et al. (2006). BRCA1 affects lipid synthesis through its interaction with acetyl- CoA carboxylase. Journal of Biological Chemistry, 281(6), 3172-3181.

45. Chajès, V., et al. (2006). Acetyl-CoA carboxylase $\alpha$ is essential to breast cancer cell survival. Cancer Research, 66(10), 5287-5294.

46. Swinnen, J. V., Brusselmans, K., \& Verhoeven, G. (2006). Increased lipogenesis in cancer cells: New players, novel targets. Current Opinion in Clinical Nutrition and Metabolic Care, 9(4), 358-365.

47. Alo, P. L., et al. (1996). Expression of fatty acid synthase (FAS) as a predictor of recurrence in stage I breast carcinoma patients. Cancer, 77(3), 474-482.

48. Swinnen, J. V., et al. (2002). Overexpression of fatty acid synthase is an early and common event in the development of prostate cancer. International Journal of Cancer, 98(1), 19-22.

49. Kridel, S. J., et al. (2004). Orlistat is a novel inhibitor of fatty acid synthase with antitumor activity. Cancer Research, 64(6), 2070-2075.

50. Zaytseva, Y. Y., et al. (2012). Inhibition of fatty acid synthase attenuates CD44-associated signaling and reduces metastasis in colorectal cancer. Cancer Research, 72(6), 1504-1517.

51. Heuer, T. S., et al. (2017). FASN inhibition and Taxane treatment combine to enhance anti-tumor efficacy in diverse Xenograft tumor models through disruption of tubulin palmitoylation and microtubule organization and FASN inhibition-mediated effects on oncogenic signaling and gene expression. eBioMedicine, 16, 51-62.

52. Jiang, L., et al. (2015). Metabolic reprogramming during TGF $\beta 1$-induced epithelial-to-mesenchymal transition. Oncogene, 34(30), 3908-3916.

53. Dean, E. J., et al. (2016). Preliminary activity in the first in human study of the first-in-class fatty acid synthase (FASN) inhibitor, TVB-2640. Journal of Clinical Oncology, 34(15_suppl), 2512-2512.

54. Falkenburger, B. H., et al. (2010). Phosphoinositides: Lipid regulators of membrane proteins. The Journal of Physiology, 588(Pt 17), 3179-3185.

55. Samuels, Y., et al. (2004). High frequency of mutations of the PIK3CA gene in human cancers. Science, 304(5670), 554. 
56. Samuels, Y., \& Velculescu, V. E. (2004). Oncogenic mutations of PIK3CA in human cancers. Cell Cycle, 3(10), 1221-1224.

57. Tennant, D. A., Duran, R. V., \& Gottlieb, E. (2010). Targeting metabolic transformation for cancer therapy. Nature Reviews Cancer, 10(4), 267-277.

58. Ricoult, S. J. H., et al. (2016). Oncogenic PI3K and K-Ras stimulate de novo lipid synthesis through mTORC1 and SREBP. Oncogene, 35(10), 1250-1260.

59. Gouw, A. M., et al. (2017). Oncogene KRAS activates fatty acid synthase, resulting in specific ERK and lipid signatures associated with lung adenocarcinoma. Proceedings of the National Academy of Sciences of the United States of America, 114(17), 4300-4305.

60. Polivka, J., \& Janku, F. (2014). Molecular targets for cancer therapy in the PI3K/AKT/mTOR pathway. Pharmacology \& Therapeutics, 142(2), 164-175.

61. Downward, J. (2003). Targeting RAS signaling pathways in cancer therapy. Nature Reviews Cancer, 3,11 .

62. Yang, Y.-A., et al. (2002). Activation of fatty acid synthesis during neoplastic transformation: Role of mitogen-activated protein kinase and phosphatidylinositol 3-kinase. Experimental Cell Research, 279(1), 80-90.

63. Che, L., et al. (2017). Oncogene dependent requirement of fatty acid synthase in hepatocellular carcinoma. Cell Cycle, 16(6), 499-507.

64. Ventura, R., et al. (2015). Inhibition of de novo palmitate synthesis by fatty acid synthase induces apoptosis in tumor cells by remodeling cell membranes, inhibiting signaling pathways, and reprogramming gene expression. eBioMedicine, 2(8), 808-824.

65. Hatzivassiliou, G., et al. (2005). ATP citrate lyase inhibition can suppress tumor cell growth. Cancer Cell, 8(4), 311-321.

66. Hanai, J.-I., et al. (2012). Inhibition of lung cancer growth: ATP citrate lyase knockdown and statin treatment leads to dual blockade of mitogen-activated protein kinase (MAPK) and phosphatidylinositol-3-kinase (PI3K)/AKT pathways. Journal of Cellular Physiology, 227(4), 1709-1720.

67. Svensson, R. U., et al. (2016). Inhibition of acetylCoA carboxylase suppresses fatty acid synthesis and tumor growth of non-small cell lung cancer in preclinical models. Nature Medicine, 22(10), 1108-1119.

68. Uddin, S., et al. (2010). Inhibition of fatty acid synthase suppresses c-Met receptor kinase and induces apoptosis in diffuse large B-cell lymphoma. Molecular Cancer Therapeutics, 9(5), 1244-1255.

69. Wieduwilt, M. J., \& Moasser, M. M. (2008). The epidermal growth factor receptor family: Biology driving targeted therapeutics. Cellular and Molecular Life Sciences: CMLS, 65(10), 1566-1584.
70. Sierra, J. R., \& Tsao, M.-S. (2011). c-MET as a potential therapeutic target and biomarker in cancer. Therapeutic Advances in Medical Oncology, 3(1 Suppl), S21-S35.

71. Hanai, J. I., et al. (2013). ATP citrate lyase knockdown impacts cancer stem cells in vitro. Cell Death \& Disease, 4(6), e696.

72. Chen, Y., et al. (2016). mTOR complex-2 stimulates acetyl-CoA and de novo lipogenesis through ATP citrate lyase in HER2/PIK3CA-hyperactive breast cancer. Oncotarget, 7(18), 25224-25240.

73. Corominas-Faja, B., et al. (2014). Chemical inhibition of acetyl-CoA carboxylase suppresses selfrenewal growth of cancer stem cells. Oncotarget, 5(18), 8306-8316.

74. Menendez, J. A., et al. (2004). Inhibition of fatty acid synthase (FAS) suppresses HER2/neu (erbB-2) oncogene overexpression in cancer cells. Proceedings of the National Academy of Sciences of the United States of America, 101(29), 10715-10720.

75. Giró-Perafita, A., et al. (2016). Preclinical evaluation of fatty acid synthase and EGFR inhibition in triplenegative breast cancer. Clinical Cancer Research, 22(18), 4687-4697.

76. Menendez, J. A., \& Lupu, R. (2017). Fatty acid synthase regulates estrogen receptor- $\alpha$ signaling in breast cancer cells. Oncogene, 6, e299.

77. Vellaichamy, A., et al. (2010). "Topological significance" analysis of gene expression and proteomic profiles from prostate cancer cells reveals key mechanisms of androgen response. PLoS One, 5(6), e10936.

78. Li, J.-N., et al. (2001). Pharmacological inhibition of fatty acid synthase activity produces both cytostatic and cytotoxic effects modulated by p53. Cancer Research, 61(4), 1493-1499.

79. Liu, D., et al. (2016). Wnt/ $\beta$-catenin signaling participates in the regulation of lipogenesis in the liver of juvenile turbot (Scophthalmus maximus L.). Comparative Biochemistry and Physiology Part B: Biochemistry and Molecular Biology, 191, 155-162.

80. Seo, M. H., et al. (2016). Exendin-4 inhibits hepatic lipogenesis by increasing $\beta$-catenin signaling. PLoS One, 11(12), e0166913.

81. Gelebart, P., et al. (2012). Blockade of fatty acid synthase triggers significant apoptosis in mantle cell lymphoma. PLoS One, 7(4), e33738.

82. Yoon, S., et al. (2007). Up-regulation of acetyl-CoA carboxylase $\alpha$ and fatty acid synthase by human epidermal growth factor receptor 2 at the translational level in breast cancer cells. Journal of Biological Chemistry, 282(36), 26122-26131.

83. Daemen, A., et al. (2015). Metabolite profiling stratifies pancreatic ductal adenocarcinomas into subtypes with distinct sensitivities to metabolic inhibitors. Proceedings of the National Academy of Sciences of the United States of America, 112(32), E4410-E4417.

84. Xie, H., \& Simon, M. C. (2017). Oxygen availability and metabolic reprogramming in cancer. The Journal of Biological Chemistry, 292(41), 16825-16832. 
85. Bensaad, K., et al. (2014). Fatty acid uptake and lipid storage induced by HIF- $1 \alpha$ contribute to cell growth and survival after hypoxia-reoxygenation. Cell Reports, 9(1), 349-365.

86. Kamphorst, J. J., et al. (2013). Hypoxic and Ras-transformed cells support growth by scavenging unsaturated fatty acids from lysophospholipids. Proceedings of the National Academy of Sciences of the United States of America, 110(22), 8882-8887.

87. Young, R. M., et al. (2013). Dysregulated mTORC1 renders cells critically dependent on desaturated lipids for survival under tumor-like stress. Genes \& Development, 27(10), 1115-1131.

88. Sounni, N. E., et al. (2014). Blocking lipid synthesis overcomes tumor regrowth and metastasis after antiangiogenic therapy withdrawal. Cell Metabolism, 20(2), 280-294.

89. Daniëls, V. W., et al. (2014). Cancer cells differentially activate and thrive on de novo lipid synthesis pathways in a low-lipid environment. PLoS One, 9(9), e106913.

90. Zaidi, N., et al. (2012). ATP citrate lyase knockdown induces growth arrest and apoptosis through different cell- and environment-dependent mechanisms. Molecular Cancer Therapeutics, 11(9), 1925-1935.

91. Lakhter, A. J., et al. (2016). Glucose-independent acetate metabolism promotes melanoma cell survival and tumor growth. The Journal of Biological Chemistry, 291(42), 21869-21879.

92. Tamura, K., et al. (2009). Novel lipogenic enzyme ELOVL7 is involved in prostate cancer growth through saturated long-chain fatty acid metabolism. Cancer Research, 69(20), 8133-8140.

93. Jump, D. B., Torres-Gonzalez, M., \& Olson, L. K. (2011). Soraphen A, an inhibitor of acetyl CoA carboxylase activity, interferes with fatty acid elongation. Biochemical Pharmacology, 81(5), 649-660.

94. Yang, W. S., et al. (2012). Proteomic approach reveals FKBP4 and S100A9 as potential prediction markers of therapeutic response to neoadjuvant chemotherapy in patients with breast cancer. Journal of Proteome Research, 11(2), 1078-1088.

95. Clendening, J. W., et al. (2010). Dysregulation of the mevalonate pathway promotes transformation. Proceedings of the National Academy of Sciences, 107(34), 15051-15056.

96. Platz, E. A., et al. (2006). Statin drugs and risk of advanced prostate Cancer. Journal of the National Cancer Institute, 98(24), 1819-1825.

97. Poynter, J. N., et al. (2005). Statins and the risk of colorectal cancer. New England Journal of Medicine, 352(21), 2184-2192.

98. Nielsen, S. F., Nordestgaard, B. G., \& Bojesen Statin, S. E. (2012). Use and reduced cancer-related mortality. New England Journal of Medicine, 367(19), 1792-1802.
99. Clendening, J. W., \& Penn, L. Z. (2012). Targeting tumor cell metabolism with statins. Oncogene, 31, 4967.

100. Campbell, M. J., et al. (2006). Breast cancer growth prevention by statins. Cancer Research, 66(17), 8707-8714.

101. Zhong, C., et al. (2014). HMGCR is necessary for the tumorigenicity of esophageal squamous cell carcinoma and is regulated by Myc. Tumor Biology, 35(5), 4123-4129.

102. Wang, X., et al. (2017). MYC-regulated mevalonate metabolism maintains brain tumor-initiating cells. Cancer Research, 77(18), 4947-4960.

103. Juneja, M., et al. (2017). Statin and rottlerin smallmolecule inhibitors restrict colon cancer progression and metastasis via MACC1. PLoS Biology, 15(6), e2000784.

104. Fujiwara, D., et al. (2017). Statins induce apoptosis through inhibition of Ras signaling pathways and enhancement of Bim and p27 expression in human hematopoietic tumor cells. Tumor Biology, 39(10), 1010428317734947.

105. Karagkounis, G., et al. (2017). Simvastatin enhances radiation sensitivity of colorectal cancer cells. Surgical Endoscopy, 32(3), 1533-1539.

106. Lipkin, S. M., et al. (2010). Genetic variation in 3-hydroxy-3-methylglutaryl CoA reductase modifies the chemopreventive activity of statins for colorectal cancer. Cancer Prevention Research, 3(5), 597-603.

107. Menter, D. G., et al. (2011). Differential effects of pravastatin and simvastatin on the growth of tumor cells from different organ sites. PLoS One, 6(12), e28813.

108. Lee, Y., et al. (2017). Randomized phase II study of afatinib plus simvastatin versus afatinib alone in previously treated patients with advanced nonadenocarcinomatous non-small cell lung cancer. Cancer Research and Treatment: Official Journal of Korean Cancer Association, 49(4), 1001-1011.

109. Baas, J. M., et al. (2015). Safety and efficacy of the addition of simvastatin to panitumumab in previously treated KRAS mutant metastatic colorectal cancer patients. Anti-Cancer Drugs, 26(8), 872-877.

110. Baas, J. M., et al. (2015). Safety and efficacy of the addition of simvastatin to cetuximab in previously treated KRAS mutant metastatic colorectal cancer patients. Investigational New Drugs, 33(6), 1242-1247.

111. Zaidi, N., et al. (2013). Lipogenesis and lipolysis: The pathways exploited by the cancer cells to acquire fatty acids. Progress in Lipid Research, 52(4), 585-589.

112. Pavlova, N. N., \& Thompson, C. B. (2016). The emerging hallmarks of cancer metabolism. Cell Metabolism, 23(1), 27-47.

113. Kuemmerle, N. B., et al. (2011). Lipoprotein lipase links dietary fat to solid tumor cell proliferation. Molecular Cancer Therapeutics, 10(3), 427-436. 
114. van't Veer, M. B., et al. (2006). The predictive value of lipoprotein lipase for survival in chronic lymphocytic leukemia. Haematologica, 91(1), 56-63.

115. Hale, J. S., et al. (2014). Cancer stem cell-specific scavenger receptor CD36 drives glioblastoma progression. Stem Cells, 32(7), 1746-1758.

116. Nath, A., et al. (2015). Elevated free fatty acid uptake via CD36 promotes epithelial-mesenchymal transition in hepatocellular carcinoma. Scientific Reports, 5,14752 .

117. Guillaumond, F., et al. (2015). Cholesterol uptake disruption, in association with chemotherapy, is a promising combined metabolic therapy for pancreatic adenocarcinoma. Proceedings of the National Academy of Sciences of the United States of America, 112(8), 2473-2478.

118. Chaffer, C. L., \& Weinberg, R. A. (2011). A perspective on cancer cell metastasis. Science, 331(6024), 1559-1564.

119. Hua, Y., et al. (2011). Dynamic metabolic transformation in tumor invasion and metastasis in mice with LM-8 osteosarcoma cell transplantation. Journal of Proteome Research, 10(8), 3513-3521.

120. Jung, Y. Y., Kim, H. M., \& Koo, J. S. (2015). Expression of lipid metabolism-related proteins in metastatic breast cancer. PLoS One, 10(9), e0137204.

121. Nath, A., \& Chan, C. (2016). Genetic alterations in fatty acid transport and metabolism genes are associated with metastatic progression and poor prognosis of human cancers. Scientific Reports, 6, 18669.

122. Uray, I. P., Liang, Y., \& Hyder, S. M. (2004). Estradiol down-regulates CD36 expression in human breast cancer cells. Cancer Letters, 207(1), 101-107.

123. Balaban, S., et al. (2015). Obesity and cancer progression: Is there a role of fatty acid metabolism? BioMed Research International, 2015, 274585.

124. Schoors, S., et al. (2015). Fatty acid carbon is essential for dNTP synthesis in endothelial cells. Nature, 520(7546), 192-197.

125. McDonnell, E., et al. (2016). Lipids reprogram metabolism to become a major carbon source for histone acetylation. Cell Reports, 17(6), 1463-1472.

126. Padanad, M. S., et al. (2016). Fatty acid oxidation mediated by acyl-CoA Synthetase long-chain 3 is required for mutant KRAS lung tumorigenesis. Cell Reports, 16(6), 1614-1628.

127. Liu, Y. (2006). Fatty acid oxidation is a dominant bioenergetic pathway in prostate cancer. Prostate Cancer and Prostatic Diseases, 9(3), 230-234.

128. Camarda, R., et al. (2016). Inhibition of fatty acid oxidation as a therapy for MYC-overexpressing triple-negative breast cancer. Nature Medicine, 22(4), 427-432.

129. Comerford, S. A., et al. (2014). Acetate dependence of tumors. Cell, 159(7), 1591-1602.

130. Mashimo, T., et al. (2014). Acetate is a bioenergetic substrate for human glioblastoma and brain metastases. Cell, 159(7), 1603-1614.
131. Qu, Q., et al. (2016). Fatty acid oxidation and carnitine palmitoyltransferase I: Emerging therapeutic targets in cancer. Cell Death \& Disease, 7(5), e2226.

132. Carrasco, P., et al. (2013). Carnitine palmitoyltransferase $1 \mathrm{C}$ deficiency causes motor impairment and hypoactivity. Behavioural Brain Research, 256, 291-297.

133. Zaugg, K., et al. (2011). Carnitine palmitoyltransferase $1 \mathrm{C}$ promotes cell survival and tumor growth under conditions of metabolic stress. Genes \& Development, 25(10), 1041-1051.

134. Wakil, S. J., \& Abu-Elheiga, L. A. (2009). Fatty acid metabolism: Target for metabolic syndrome. Journal of Lipid Research, 50(Suppl), S138-S143.

135. Du, W., et al. (2017). HIF drives lipid deposition and cancer in ccRCC via repression of fatty acid metabolism. Nature Communications, 8, 1769.

136. Huang, D., et al. (2014). HIF-1-mediated suppression of acyl-CoA dehydrogenases and fatty acid oxidation is critical for cancer progression. Cell Reports, 8(6), 1930-1942.

137. Fragasso, G., et al. (2009). Effects of metabolic approach in diabetic patients with coronary artery disease. Current Pharmaceutical Design, 15(8), 857-862.

138. Holubarsch, C. J., et al. (2007). A double-blind, randomized multicentre clinical trial to evaluate the efficacy and safety of two doses of etomoxir in comparison with placebo in patients with moderate congestive heart failure: The ERGO (etomoxir for the recovery of glucose oxidation) study. Clinical Science, 113(4), 205-212.

139. Lodhi, I. J., \& Semenkovich, C. F. (2014). Peroxisomes: A nexus for lipid metabolism and cellular signaling. Cell Metabolism, 19(3), 380-392.

140. Valença, I., et al. (2015). Localization of MCT2 at peroxisomes is associated with malignant transformation in prostate cancer. Journal of Cellular and Molecular Medicine, 19(4), 723-733.

141. Wang, Y.-X. (2010). PPARs: Diverse regulators in energy metabolism and metabolic diseases. Cell Research, 20(2), 124-137.

142. Bensinger, S. J., \& Tontonoz, P. (2008). Integration of metabolism and inflammation by lipid-activated nuclear receptors. Nature, 454, 470.

143. Peters, J. M., Shah, Y. M., \& Gonzalez, F. J. (2012). The role of peroxisome proliferator-activated receptors in carcinogenesis and chemoprevention. Nature Reviews Cancer, 12(3), 181-195.

144. Yousefi, B., et al. (2016). Peroxisome proliferatoractivated receptors and their ligands in cancer drug resistance: Opportunity or challenge. AntiCancer Agents in Medicinal Chemistry, 16(12), 1541-1548.

145. Holden, P. R., \& Tugwood, J. D. (1999). Peroxisome proliferator-activated receptor alpha: Role in rodent liver cancer and species differences. Journal of Molecular Endocrinology, 22(1), 1-8.

146. Wang, X., et al. (2016). PPAR-delta promotes survival of breast cancer cells in harsh metabolic conditions. Oncogene, 5(6), e232. 
147. Vidal-Puig, A. J., et al. (1997). Peroxisome proliferator-activated receptor gene expression in human tissues. Effects of obesity, weight loss, and regulation by insulin and glucocorticoids. Journal of Clinical Investigation, 99(10), 2416-2422.

148. Robbins, G. T., \& Nie, D. (2012). PPAR gamma, bioactive lipids, and cancer progression. Frontiers in Bioscience: A Journal and Virtual Library, 17, 1816-1834.

149. Corbet, C., \& Feron, O. (2017). Cancer cell metabolism and mitochondria: Nutrient plasticity for TCA cycle fueling. Biochimica et Biophysica Acta (BBA)Reviews on Cancer, 1868(1), 7-15.

150. Elgogary, A., et al. (2016). Combination therapy with BPTES nanoparticles and metformin targets the metabolic heterogeneity of pancreatic cancer. Proceedings of the National Academy of
Sciences of the United States of America, 113(36), E5328-E5336.

151. Bayat Mokhtari, R., et al. (2017). Combination therapy in combating cancer. Oncotarget, 8(23), 38022-38043.

152. Zhao, B., Hemann, M. T., \& Lauffenburger, D. A. (2014). Intratumor heterogeneity alters most effective drugs in designed combinations. Proceedings of the National Academy of Sciences of the United States of America, 111(29), 10773-10778.

153. Benfeitas, R., et al. (2017). New challenges to study heterogeneity in cancer redox metabolism. Frontiers in Cell and Development Biology, 5, 65.

154. Agren, R., et al. (2012). Reconstruction of genomescale active metabolic networks for 69 human cell types and 16 cancer types using INIT. PLoS Computational Biology, 8(5), e1002518.

Open Access This chapter is licensed under the terms of the Creative Commons Attribution 4.0 International License (http://creativecommons.org/licenses/by/4.0/), which permits use, sharing, adaptation, distribution and reproduction in any medium or format, as long as you give appropriate credit to the original author(s) and the source, provide a link to the Creative Commons license and indicate if changes were made.

The images or other third party material in this chapter are included in the chapter's Creative Commons license, unless indicated otherwise in a credit line to the material. If material is not included in the chapter's Creative Commons license and your intended use is not permitted by statutory regulation or exceeds the permitted use, you will need to obtain permission directly from the copyright holder. 


\section{Part II}

Heterogeneity of Cancer Metabolism 


\title{
The Multifaceted Glioblastoma: From Genomic Alterations to Metabolic Adaptations
}

\author{
Addison Quinones and Anne Le
}

\section{Keywords}

Glioblastoma $\cdot$ Metabolic profile $\cdot$ Glutamine metabolism · IDH1 mutation · mTOR signaling $\cdot$ Liquid biopsy $\cdot$ Glucose metabolism
CDKN2A Cyclin-dependent kinase inhibitor 2A

CDO Cysteine dioxygenase

CSA Cysteine sulfinic acid

EGFR Epidermal growth factor receptor

FABP7 Fatty acid-binding protein 7

FAS Fatty acid synthase

FUS Focused ultrasound

GABRA1 Gamma-aminobutyric acid type A receptor alpha-1

GBM Glioblastoma

Glutamate dehydrogenase

Glutaminase

Glioblastoma stem-like cell

Glutathione

Hexokinase

Isocitrate dehydrogenase

Macrophage 2

Myc-associated factor X

Mechanistic target of rapamycin or mammalian target of rapamycin

NAAG $\quad N$-acetyl-aspartyl-glutamate

NEFL Neurofilament light

NF1 Neurofibromatosis type 1

p53 Phosphoprotein 53

PDGFRA Platelet-derived growth factor receptor alpha

PDH Pyruvate dehydrogenase

PDK1 Pyruvate dehydrogenase kinase 1

PI3K Phosphoinositide 3-kinase

PTEN Phosphatase and tensin homolog

ROS Reactive oxygen species

Engineering, Johns Hopkins University Whiting

School of Engineering, Baltimore, MD, USA

e-mail: annele@jhmi.edu 


\section{RTK Receptor tyrosine kinase}

SLC12A5 Solute carrier family 12 member 5

SREBP-1 Sterol regulatory element-binding protein 1

SYT1 Synaptotagmin 1

TCA Tricarboxylic acid

TET Ten-eleven translocation

TME Tumor microenvironment

TMZ Temozolomide

TP73-AS1 Tumor protein 73 antisense RNA 1 VEGF Vascular endothelial growth factor

\section{Key Points}

- Glioblastoma (GBM) can be categorized into different subtypes based on diverse metabolic profiles.

- Characteristic genomic alterations lead to transformed metabolism.

- Synergistic therapies are beneficial to combat dynamic adaptations of glioblastoma metabolism.

- Advanced-grade brain tumors exhibit distinct metabolic profiles compared to lower grade tumors.

\section{Introduction}

Glioblastoma multiforme (GBM) develops on glial cells and is the most common as well as the deadliest form of brain cancer [1]. As in other cancers, distinct combinations of genetic alterations in GBM subtypes induce a diversity of metabolic phenotypes, which explains the variability of GBM sensitivity to current therapies targeting its reprogrammed metabolism. Therefore, it is becoming imperative for cancer researchers to account for the temporal and spatial heterogeneity within this cancer type before making generalized conclusions about a particular treatment's efficacy. Standard therapies for GBM have shown little success as the disease is almost always lethal; however, researchers are making progress and learning how to combine therapeutic strategies most effectively. GBMs can be classified initially into two subsets consisting of primary and secondary GBMs, and this categorization stems from cancer development. GBM is the highest grade of gliomas, which includes glioma I (low proliferative potential), glioma II (low proliferative potential with some capacity for infiltration and recurrence), glioma III (evidence of malignancy), and glioma IV (GBM) (malignant with features of necrosis and microvascular proliferation) [2]. Secondary GBM develops from a lowgrade glioma to an advanced-stage cancer, while primary GBM provides no signs of progression and is identified as an advanced-stage glioma from the onset. The differences in prognosis and histology correlated with each classification are generally negligible, but the demographics of individuals affected and the accompanying genetic/metabolic properties show distinct differentiation [3].

\section{GBM Classifications and Intratumoral Heterogeneity}

Previously, tumors had been classified based on histological and structural similarities without accounting for clinical disparities among them [4]. More recently, tumor classification has shifted toward a more molecular and genetic basis in combination with phenotypic information. This new-era classification allows practitioners to differentiate between biologically similar cases, allowing for more precise treatment and prognosis when encountering distinct mutant variants [4]. Phenotypic information must be used in accordance with genotypic data to determine tumor type and grade differentiation and to account for the rare occurrences when the phenotype differs from the usual criteria accompanying the defined genotype [5].

\subsection{GBM Subtype Classification}

A recent study identified four gene expression subtypes of GBM: mesenchymal, classical, proneural, and neural (Fig. 1).

- The mesenchymal subtype is characterized by high mutation rates of the tumor-suppressor 


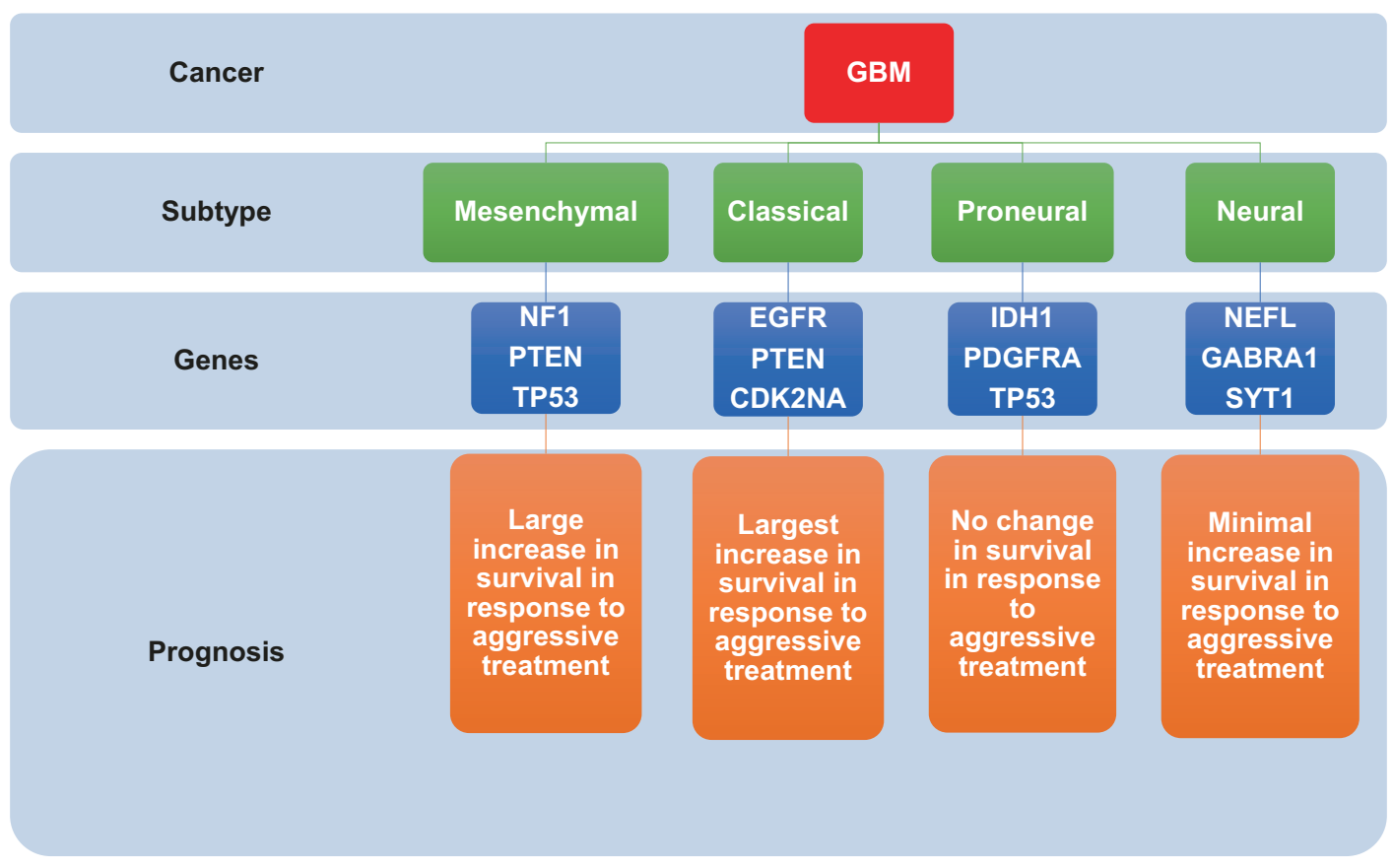

Fig. 1 Subtypes of glioblastoma, including the major genes altered and effect on prognosis following treatment. $N F 1$ neurofibromatosis type 1, PTEN phosphatase and tensin homolog, EGFR epidermal growth factor receptor, $C D K N 2 A$ cyclin-dependent kinase inhibitor 2A, IDH1 isocitrate dehydrogenase 1, PDGFRA platelet-derived growth factor receptor alpha, NEFL neurofilament light, GABRA1 gamma-aminobutyric acid type A receptor alpha-1, SYT1 synaptotagmin 1

genes: neurofibromatosis type 1 (NF1), phosphatase and tensin homolog (PTEN), and phosphoprotein 53 (p53). Following aggressive treatment, mesenchymal subtypes frequently display substantial increases in length of survival [6].

The mesenchymal subtype was discovered to have a large association with both the tumorpromoting M2 macrophage gene and the deactivation of NF1 [7]. This suggests a pathway linking the loss of function of NF1 to promoted macrophage/microglia recruitment and invasion of the tumor microenvironment (TME), leading to a poorer prognosis for patients afflicted with mesenchymal subtype expression factors [7]. High-grade gliomas containing altered NF1 frequently have an associated deactivation of cyclin-dependent kinase inhibitor 2A (CDKN2A) that inhibits Ras-mediated growth signaling, suggesting NF1 as another tumor-suppressor gene in the central nervous system. As a consequence of losing the NF1 function, Ras activity stimulates Ras effectors (PI3K, PAK, RAF, ERK1/2), increasing the proliferation of astrocytes, contributing to GBM growth [8].

- Classical GBM is defined by focal epidermal growth factor receptor (EGFR) mutation events in much larger frequencies than in the other three subtypes while containing zero mutations of the most altered gene in GBMs: p53. Similar to the mesenchymal subtype, classical subtypes tend to show the highest survival rates of all the subtypes when subjected to aggressive treatments [6].

- The proneural subtype carries mutations of p53, platelet-derived growth factor receptor alpha (PDGFRA), and isocitrate dehydrogenase-1 (IDH1) [6]. IDH1 and PDGFRA mutations can result in irregular cell/tumor growth. Proneural patients are characteristically younger than other subtype patients and have more prolonged survival, but their survival remains constant whether they are exposed to 
aggressive treatment or not. Furthermore, studies have demonstrated that classical and mesenchymal subtypes have a better response to therapy and better prognosis compared to the proneural subtype [6].

- The neural subtype is categorized based on the overexpression of neurofilament light (NEFL), gamma-aminobutyric acid type A receptor alpha-1 (GABRA1), synaptotagmin 1 (SYT1), and solute carrier family 12 member 5 (SLC12A5) neural markers. The gene expressions present within the neural subtype have been determined to be the most similar to normal brain tissue and are weakly characterized. Data suggests the average efficacy of treatment in the neural subtype, but it is not as effective as treatments of classical and mesenchymal subtypes [6]. These unique genetic alterations leading to subtype classifications result in different metabolic profiles of cancers depending on the specific genes altered.

There have been speculations that GBM subtypes do not remain stagnant during disease progression, nor while being bombarded with varying treatments [7]. A study by Wang et al. investigated phenotypic plasticity and genetic drivers behind the evolution of proneural, classical, and mesenchymal subtypes. Samples were collected from varying gliomas at the time of diagnosis as well as at the first onset of GBM recurrence, and genetic profiles were obtained to establish their molecular subtypes for comparison. After analysis, 50 of 91 (55\%) samples had their expression subtypes remain constant. After recurrence, the quantity of proneural and mesenchymal subtypes had increased, and the number of classical subtypes had decreased. There was no direct correlation observed between proneural and mesenchymal subtypes. The intratumoral heterogeneity of the initially collected samples was taken into account, and the samples with the lowest purity were typically the groups to undergo a transition of subtype.

GBMs are also divided into different groups based on the World Health Organization (WHO) classification system. Wild-type IDH accounts for $90 \%$ of diagnosed GBMs, which have a high correlation to primary GBM, especially in elderly patients. IDH-mutant GBM occurs in about 10\% of patients and has a higher correlation to secondary GBM, which is GBM progressing from previous lower grade gliomas. The final classification is reserved for cases in which a complete IDH evaluation cannot be completed. IDHwt versus IDHmu has significant implications on GBM cells' metabolism and needs to be investigated further. With all the heterogeneity that exists within glioblastomas, the classifications are continually changing, and the variants and patterns must frequently be updated to keep up with the evolving characteristics.

\subsection{Intratumoral Heterogeneity}

There are currently two proposed models that lead to tumor heterogeneity. The first model suggests that heterogeneity stems from clonal evolution, where there are changes in single cells that create survival advantages between clonal populations [9]. The second model relies on cancer stem cells to produce phenotypically diverse differentiated cells. These models are not mutually exclusive as the cancer stem cells have the opportunity to undergo clonal evolution for them to evolve into more aggressive, self-renewing stem cells [9].

The complexity of genotype and epigenetic states creates the intratumoral heterogeneity of metabolism [10-15]. In a study by Patel et al., single-cell RNA sequencing was used to create a profile for 430 cells harvested from 5 diverse glioblastomas. These cancer cells were categorized based on oncogenic signaling, proliferation, complement/immune response, and response to hypoxia. Variability between different tumors was evident, which led to different stages, gene and protein expression, and outcomes for therapeutic strategies [16].

\subsubsection{Liquid Biopsy as a Method for Detecting Heterogeneity and Longitudinal Tracking}

The existing spatial heterogeneity within individual gliomas has made therapy more difficult. Distinct cells contained within the same tumor 
can present with different mutations and phenotypic or epigenetic states, resulting in different subtypes being found in other compartments of the same tumor. These variances within the same tumor ultimately lead to the inefficiency of treatment and cancer recurrence. Thus, studies suggest that synergistic treatments will be the direction of new therapeutic strategies [16]. It is becoming more pressing to establish the spatial and temporal heterogeneity of any particular tumor in order to devise an effective treatment. As cancer develops, the longitudinal metabolic profile is not stagnant. Therefore, if we wish to inhibit metabolic targets, correct timing is essential. Surgical biopsies are the conventional strategy for gathering pathological information from GBM tumors. This fact is troublesome because a surgical biopsy will only provide a limited spatial and temporal snapshot of cancer, failing to reflect the intratumoral heterogeneity, not to mention there are significant risks associated with the procedure [17]. A newer approach is emerging that allows for assessment of the entire genetic landscape and longitudinal tracking, and is much less invasive: the liquid biopsy (Fig. 2).

Liquid biopsy has two potential sources, either the peripheral blood supply or the cerebrospinal fluid (CSF) via lumbar puncture. The liquid biopsy through the peripheral blood supply is best for measuring smaller metabolites that can cross the BBB easily and could be very important for longitudinal tracking of disease progression. Nguyen et al. demonstrated how concentrations of $\mathrm{N}$-acetyl-aspartyl-glutamate (NAAG) within tumor tissue were directly correlated with the advancement of GBM and lower grade gliomas with very minimal concentrations of NAAG. NAAG concentration in plasma was also directly correlated with NAAG concentration in the tumor and glioma advancement [18].

Additionally, it has been shown that focused ultrasound (FUS) enhances the release of biomarkers into the bloodstream [19]. This technique, in concordance with the liquid biopsy, could be pivotal in establishing precise spatial heterogeneity within a tumor. Liquid biopsy via lumbar puncture is also a viable, less invasive option for patients where surgical resection is too dangerous. It has been shown that there are greatly enhanced detectable levels of cf-DNA from malignant brain tumors. Liquid biopsy is highly beneficial as it could provide the entire genome of a cancer and allow for personalized oncology to target specific biomarkers [20]. Liquid biopsy could provide a broader overall look at the spatial heterogeneity present within a particular tumor. However, there are still obstacles to be overcome with liquid biopsy of the CSF because the amount of tumor cf-DNA leakage into the CSF appears to be relevant to the proximity of the tumor to the CSF reservoir, as well as the progression of the tumor.

\subsubsection{Glioblastoma Stem Cell Resistance and Recurrence Are Supported Through Mitochondrial Activity and Fatty Acid Oxidation}

A recent discovery that has a substantial impact on the spatial and temporal pathogenesis is the existence of glioblastoma cancer stem-like cells (GSCs). GSCs are not traditional stem cellsthey are tumor cells that develop an ultraaggressive phenotype that facilitates resistance and survival following standard therapies [21]. The effect that GSCs have on spatial pathogenesis can be explained with the "Go or Grow hypothesis," which postulates that infiltrative cells, such as GSCs, tend to diffuse and spread through tissues but have little proliferative potential. In contrast, tumor cells are proliferative and make up the bulk tumor mass (Fig. 3) [22]. The infiltration of these GSCs has the potential to seed different GBM cell subtype lineages, which contributes to the overall spatial heterogeneity.

The temporal pathogenesis of GBM is also affected by GSCs as they are the critical drivers for the recurrence of GBM, which is common following resection. Traits of GSCs that allow for recurrence are their diffuse infiltration, resistance, and initiation of growth following therapy. We have already discussed the diffuse infiltration of GSCs, but it is important to mention that slower proliferating GBM cells are better at initiating tumor growth and have increased resistance [23]. A 


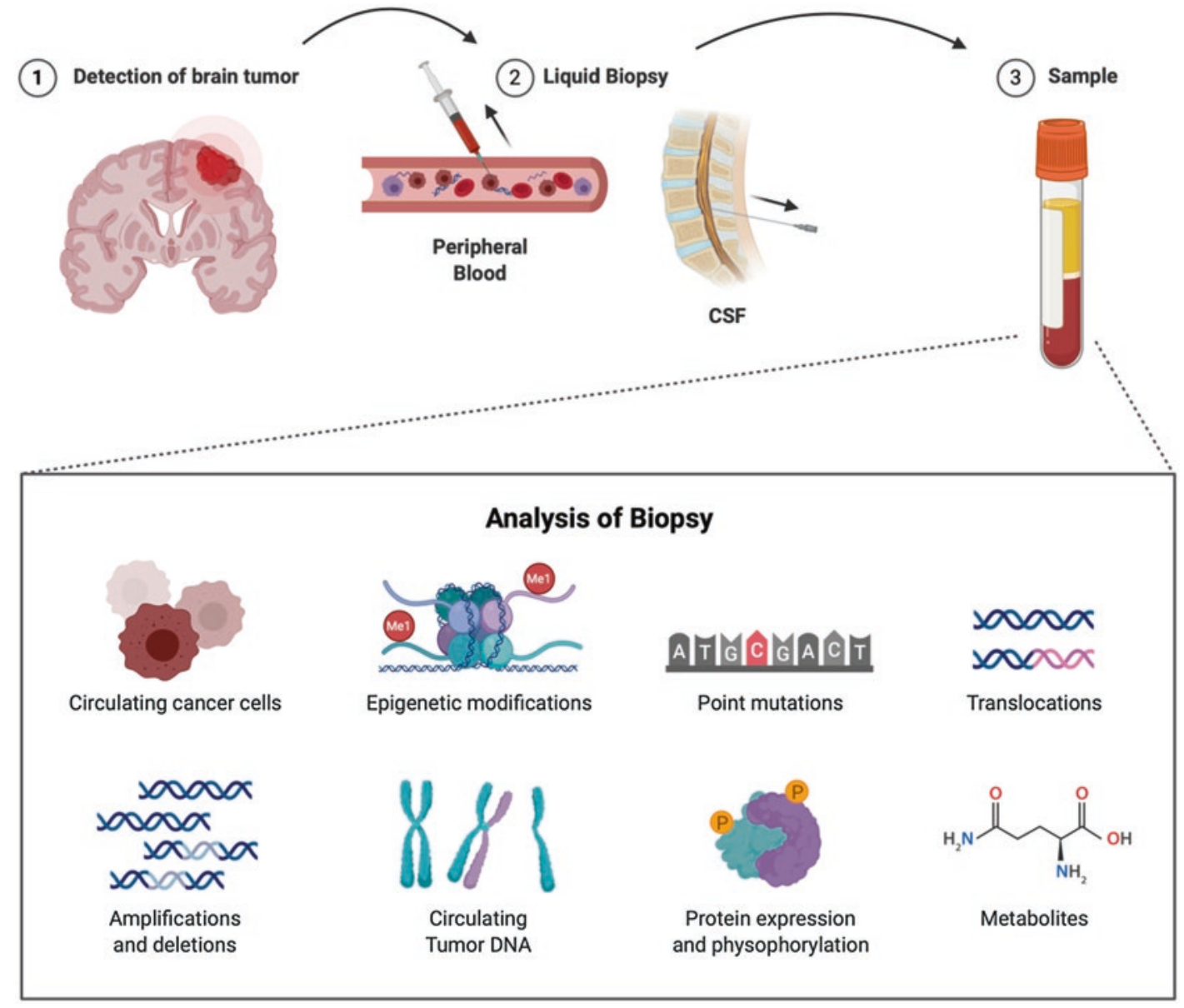

Fig. 2 Liquid biopsy and the analysis of biopsy: DNA, protein, and metabolites

recent discovery has determined that these infiltrating GSCs retain residual mitochondrial function, allowing them to perform oxidative phosphorylation along with fatty acid oxidation [23, 24]. This is the reason for resistance and recurrence: GSCs are able to withstand glucose deprivation and glycolytic disruption because they have other means to survive. These GSCs are incredibly adaptable to their environment as they are able to survive in four critical niches: hypoxic, perivascular, invasive, and acidic, allowing them to infiltrate various tissues without losing their stemness [21]. Recurrence typically occurs in resection-adjacent tissue, but even following a radical hemispherectomy, there is still contralateral recurrence. There is some evidence that injury, such as surgical resection, to the tumor may initiate proliferation and recurrence, but this process remains obscure [25]. Following the recurrence of GBM, when comparing the transcriptomes between primary and secondary tumors, the tumors have different metabolic signatures, with the recurrent tumor having similar metabolic activities to GSCs [23]. Experts are unsure as to the reason for the metabolic shifts, whether they are due to the changes in tumor microenvironment (TME) from first-line therapies or cell autonomous. 


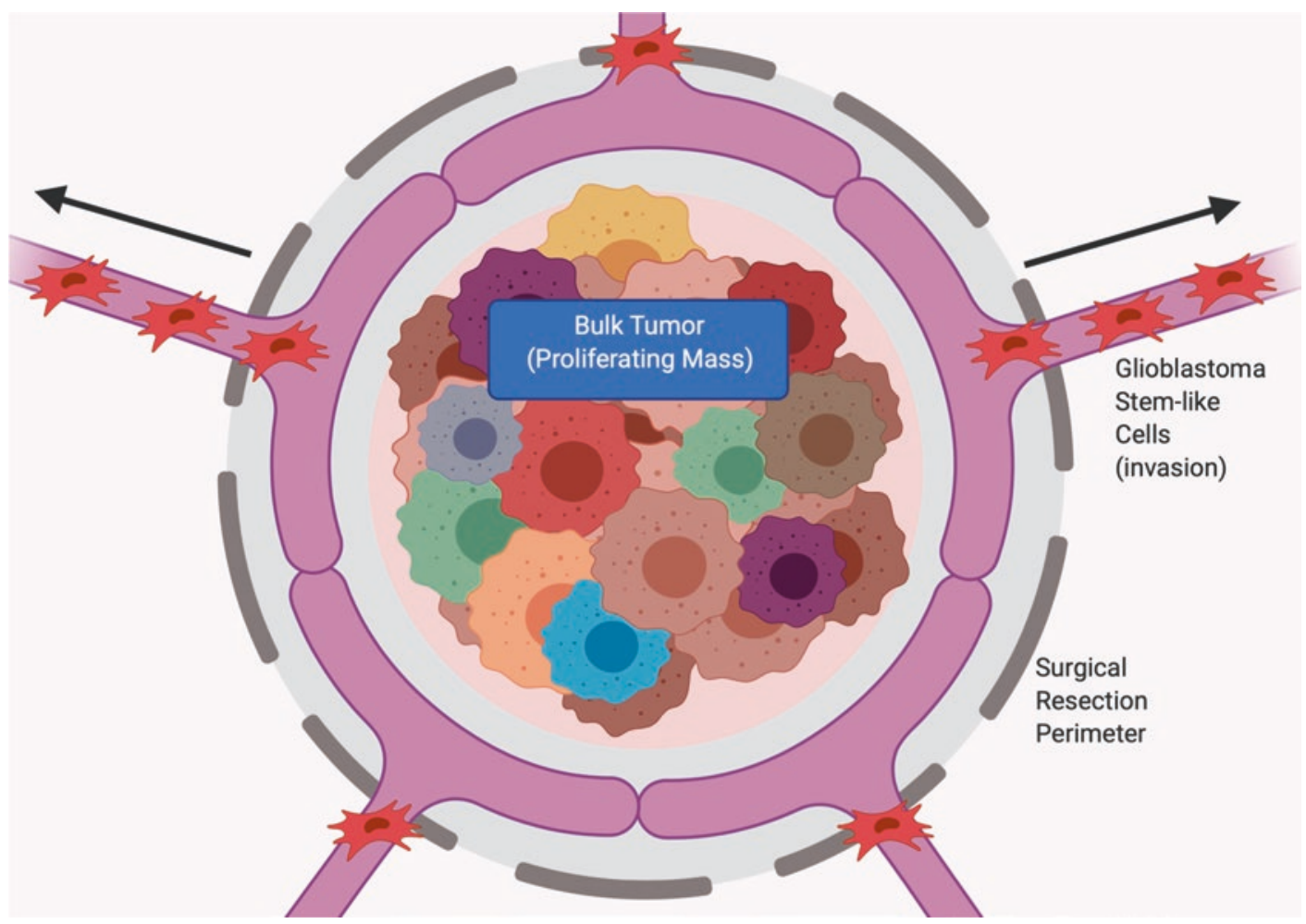

Fig. 3 Spatial representation of a GBM tumor exhibiting the proliferating mass of the bulk tumor compared with the diffuse infiltration of glioblastoma stem-like cells (GSCs) past the typical perimeter of surgical resection

\section{Genomic Alterations Lead to Distinct Metabolic Changes Allowing for Targeted Therapies}

As previously mentioned, GBMs can be classified according to their genetic and metabolic profiles. Genetic mutations are the cause of deviation of metabolism from the status quo. As genes are overexpressed, inactivated, or mutated, it leads to downstream effects. These downstream metabolic effects can be identified and investigated for therapeutic strategies, which is the current goal of cancer metabolism research.

\subsection{PTEN Mutations Lead to High Rates of Glycolysis, Facilitating Survival in Harsh Microenvironments}

In a study by Wolf et al., GBMs with loss of PTEN activity had high expression of the glycolytic enzyme hexokinase 2 (HK2), the first enzyme of glycolysis, enabling those GBM cells to survive and proliferate in a harsh TME [26]. HK2 is expressed in basal levels in adipose and skeletal tissues, but it is not expressed in normal brain tissue, which typically expresses HK1. Inhibition of HK2, without interfering with HK1 function, by siRNA led to a reverse of the 
Warburg effect to oxidative glucose metabolism, which ultimately led to impaired tumor growth. Also, HK2 inhibition sensitized GBM cells to multiple treatments, including the following: (1) temozolomide, the current chemotherapeutic GBM treatment; (2) radiation; and (3) hypoxiainduced apoptosis. Also, high HK2 expression predicted lower overall survival [26]. These findings support the genetically evolved metabolic heterogeneity in cancer cells.

\subsection{EGFR Mutations Shift Cancer Cells toward a Glycolytic Phenotype and Permit Survival under Glucose- Deprived Conditions}

Mutations in EGFR provide an additional example of genetic alterations that lead to changes in cancer cell metabolism [27]. In their study, Babic et al. revealed an activating EGFRvIII mutation, which causes an intracellular increase in heterogeneous nuclear ribonucleoprotein (hnRNP) A1 splicing factor. This upregulation, in turn, promotes the splicing of $M Y C$-associated factor $\mathrm{X}$ (MAX), a partner protein of MYC, which ultimately results in an upregulation of glycolysis, and shorter patient survival time [27]. There has been some success in utilizing the vulnerabilities created by the glycolytic phenotype of GBM as therapeutic strategies. A glucose analog, 2-deoxyD-glucose (2DG), has been shown to inhibit glycolysis and was well tolerated in clinical trials for other advanced cancers [28].

Growth factor signaling pathways are responsible for cellular metabolism, proliferation, and environmental adaptation [29, 30]. The growth factor signaling pathways are heavily dependent upon regulation from receptor tyrosine kinases (RTKs), showing that genetic mutations in RTKs such as EGFR lead to variable progression and growth of tumors stemming from the changes in the signaling pathways [31]. Furnari et al. used mouse models in correlation with clinical samples wherein the mouse growth factor signaling pathways were genetically modified to match the clinical samples [32]. The corresponding GBMs were determined to be histologically identical, indicating the importance of RTK alterations in the progression of GBMs. In a study of 251 patient-derived GBMs comprised mainly of de novo GBMs (95\%), there were alterations to RTKs in $66 \%$ of the samples, and the dominant alteration was to EGFR. This lesion was usually accompanied by activation of other PI3Ks, alteration, and deletion of CDKN2A. EGFRvIII ${ }^{+}$cells had a higher proliferation rate with less cell death in xenograft models when using EGFR-targeted therapies and showed increased glycolysis to fulfill the energy demand [30, 31, 33, 34]. Further studies are required to evaluate the mechanisms utilized by GBMs to sustain growth based on their environment.

\section{3 p53 Mutations Result in Activation of the Warburg Effect}

Tumor-suppressor gene p53 has been identified as a gene commonly mutated in many cancers, including GBM [35]. p53 serves to initiate cell cycle arrest and apoptosis when the cell is subjected to stressors, including hypoxia, hyperproliferative signals, nutrient deprivation, and DNA damage [36, 37]. Mutant p53 genes typically lead to complete inactivation of $\mathrm{p} 53$, which is characterized by a higher malignancy of cancer through greater rates of metastasis, genetic instability, and cellular differentiation [38-40]. Novel functions of p53 have emerged, showing its potential to regulate cellular metabolism. A signature feature of cancer is its reliance on the Warburg effect [41]. p53 has been shown to counter this oncogenic hallmark by activating the synthesis of cytochrome oxidase 2 to promote oxidative phosphorylation and inhibit glycolysis by repressing glucose transporters (SLC2A1, SLC2A4). Thus, the Warburg effect is more profound when p53 is inactivated [42-44]. Recently, there have been contradictory studies showing that the impact of p53 as a tumor suppressor is not as compelling as initially suggested. These studies found evidence to support the claim that cell cycle arrest and apoptosis, as a result of DNA 
damage, are not large contributors to tumor suppression depending on the cancer tissue examined [35]. These findings have yet to be replicated when evaluating GBM development, but further examination is necessary.

\subsection{GBM Exhibits Upregulated Glutamine Metabolism Allowing for Targeted Vulnerabilities Through GLS, GS, and mTOR}

As is true for many other cancers, GBM also exhibits "glutamine addiction" [45]. Glutamine serves as the major contributor to cell growth and energy production after it is converted into glutamate via glutaminase (GLS), and then into $\alpha$-ketoglutarate (aKG) via glutamate dehydrogenase $(\mathrm{GDH})$ or several aminotransferases. For this reason, GLS inhibition has become a popular therapeutic strategy to treat cancer patients and has reached clinical trials (NCT03528642). Oizel et al. showed how metabolic phenotype leading to subtype classification could help with targeted therapies. In their study, Oizel showed that mesenchymal GBM cells had significantly more uptake of glutamine and that glutamate derived from glutamine was converted to aKG to generate ATP. This altered phenotype was facilitated by upregulated SLC1A5 (glutamine transporter), GLS, and mitochondrial and cytosolic aminotransferase. Consequently, mesenchymal GBM was shown to be more susceptible to GLS inhibition [46]. Glutamine is essential in normal brain tissue, but it appears to have even higher concentrations in GBM tissue [47]. The primary source of glutamine in the brain comes from glutamine synthase (GS) as it combines glutamate and ammonia to form glutamine. GS levels in GBM have been shown to correlate with patient survival time, as those with lower GS levels could have up to a twofold increase in survival time [48]. The impact of GS expression levels on GBM progression, resistance, and patient survival time needs further investigation, but GS appears to be a reliable prognostic biomarker and could potentially lead to another therapeutic strategy.

The mechanistic target of rapamycin (mTOR) is a protein kinase that promotes oncogenic signaling through the phosphoinositide 3-kinase (PI3K) pathway, which in turn promotes cancer growth [49]. This has also made mTOR a popular target for cancers that use PI3K as a major pathway [50]. mTOR has been identified as a primary factor in downstream signaling for EGFR-mutant GBM, which is resistant to kinase inhibitors [51]. In a study by Tanaka et al., they found that mTOR-targeted treatments affected glutamine catabolism, increasing GLS expression, which is already highly expressed in GBM patients. mTOR-targeted therapies (by rapamycin or PP242) limited cell proliferation, glucose usage, and lactate production [50]. However, they were ineffective in promoting cell death. Following these results, Tanaka et al. performed an experiment in which the U87 and EGFRvIII GBM cells were subjected to glutamine deprivation through compound 968 (GLS inhibitor) and then treated with PP242, which was seen as more effective than mTOR-targeted treatment alone. Results showed that when used in combination, the GLSand mTOR-targeted therapies yielded a synergistic effect triggering enhanced tumor cell death compared to when either treatment was applied individually [50]. This combined treatment was then tested on normal human astroglial cells, and the results revealed that the treatment did not cause any normal cell death to occur. This synergistic treatment was then tested on GBM xenograft models of U87 and EGFRvIII GBM samples. The treatment resulted in $80 \%$ shrinkage of tumors and a sixfold increase in cell death from mTOR-targeted treatment alone. To determine the effects of the drug on the whole body and motor function, the same treatment was applied to normal mice. There were no changes in body weight, motor function, or cell morphology, indicating that the combination of GLS and mTOR inhibition has the potential to develop into an effective treatment for mTOR-targeted resistant GBM cancers [50]. 


\subsection{Lipid Metabolism Dysregulation Following BRAF Mutations and EGFR Signaling Provides Clues for New GBM Therapeutic Strategies}

Lipid synthesis is a limiting factor for cellular proliferation. GBMs must synthesize their own lipid components for proliferation leading to a potential vulnerability. Indeed, certain lipid levels-specifically free fatty acid levels-in malignant tissue are elevated when compared to normal brain tissue [52]. To synthesize fatty acids, cells must first generate cytosolic acetyl$\mathrm{CoA}$, and both tumor-suppressor genes and oncogenes regulate this crucial step in GBM. When there is a BRAF mutation concomitant with TP53 and PTEN deletion, it results in an increased expression of acetyl-CoA synthetase enzyme 2 (ACSS2), the enzyme responsible for converting acetate to acetyl-CoA in the cytosol [53, 54]. Another study discovered that EGFR-PI3K-Akt signaling, which results in sterol regulatory element-binding protein 1 (SREBP-1) cleavage and activation of acetyl coenzyme A (CoA) carboxylase (ACC) and fatty acid synthase (FAS), plays a role in the upregulation of lipogenesis in malignant gliomas. The reaction catalyzed by ACC has been determined as the rate-limiting step for de novo lipogenesis. For cells with upregulated EGFR signaling, targeting the downstream effectors of the pathway (SREBP-1, ACC, and FAS) results in GBM cell death but does not affect normal cells [55]. GSCs also contain elevated levels of lipid metabolites, which contribute to their resistance to glycolytic inhibition or glucose deprivation because when facing glycolytic inhibition or glucose deprivation, they have the ability to rely on fatty acid oxidation for energy. To combat this, Hoang-Minh et al. experimented with pharmacologic inhibition and CRISPR intervention of the fatty acid transporter fatty acid-binding protein 7 (FABP7) to prevent fatty acid uptake, which led to an increase in the overall survival of mice carrying GBM in vivo model [23].

\subsection{GBMs Rely on the TCA Cycle and Its Reductants}

Alterations of tumor-suppressor genes and oncogenes lead to dysregulations of the TCA cycle in GBM, which creates aberrant metabolism. IDH enzymes play a significant role in the TCA cycle for normal cells by catalyzing the oxidative decarboxylation of isocitrate to aKG using $\mathrm{NADP}^{+}$or $\mathrm{NAD}^{+}$as cofactors. IDHwt expression levels are elevated fourfold in GBM tumors when compared to normal brain tissue making IDH the most significant NADPHproducing enzyme and thus a promising therapeutic target. NADPH is necessary for GBM tumors as a reductant for antioxidant biomolecules that help to mitigate oxidative stress. The knockdown of IDH1 depletes stores of NADPH and sensitizes GBM cells to radiation, increasing cellular senescence [56].

In SF188 glioblastoma, high amplification of $M Y C[57,58]$ activates glutamate dehydrogenase (GDH), enabling cancer cell survival under glucose deprivation [59]. GDH, an enzyme necessary for the conversion of glutamate to aKG for incorporation into the TCA cycle, is upregulated in the absence of glucose. This upregulation allows glioblastoma cells to maximize the use of glutamine and thus contributes to the growth and proliferation of neoplastic cells in the absence of glucose [59].

As seen with the previous metabolic pathways, dysregulation of the TCA cycle results in aggressive GBM phenotypes resulting in greater proliferation and growth.

\subsection{IDH1 Mutations Lead to Oncometabolite Production and Glutamine Addiction and Act as a Prognostic Marker}

A study by Dang et al. demonstrated that mutations in IDH1 give rise to a novel function of this enzyme, which produces the oncometabolite (R)-2-hydroxyglutarate (2HG) from alphaketoglutarate (aKG) [60]. Struys et al. reported 
that "2HG," in fact, does not exist [61]. The compound has an asymmetric carbon atom that leads to $\mathrm{L}-2 \mathrm{HG}$ and $\mathrm{D}-2 \mathrm{HG}$, which are both stable endogenous metabolites in all bodily fluids. Routine analytical methods measure the sum of these two metabolites, which creates problems because IDH1 mutations solely result in increased levels of D-2HG. Therefore, an increase in L-2HG could yield false positives, and a small rise in D-2HG may be missed. This is why analytical methods able to distinguish L-2HG from D-2HG must be used [61, 62]. D-2HG not only is found in glioblastoma but has also been found to be sufficient in promoting several other types of cancers, such as leukemia, through mutations in a homolog to IDH1 and IDH2 [63, 64]. D-2HG retains a structure similar to that of $\mathrm{aKG}$, thus inhibiting enzymes from binding to aKG.

Both L- and D-2HG inhibit aKG-dependent histone demethylases and D-2HG occupies the active site of CeKDM7A, which is where aKG usually binds. Both L- and D-2HG also interferes with 10-11 translocation (TET) family interactions and regulates HIF-1a, which promotes hypermethylation, triggering cancer proliferation, and preservation $[65,66]$. The effect of IDH1mu on HIF-1a is currently being debated. HIF-1a-induced overexpression of target enzymes GLUT1, vascular endothelial growth factor (VEGF), and pyruvate dehydrogenase kinase 1 (PDK1) leads to increased tumor growth, invasion, and angiogenesis [67]. These mutations render cancer cells addicted to glutamine. Thus, glioblastoma cells with IDH1 mutations are more sensitive to GLS inhibition by bis-2-(5-phenylacetamido-1,3,4thiadiazol-2-yl)ethyl sulfide (BPTES), a smallmolecule selective inhibitor of GLS, as compared to their wild-type counterparts [68] (Fig. 4). Metabolic dependence on aKG was confirmed through rescue experiments showing that the supplement of aKG reduced the impact of BPTES on proliferation hindrance. However, wild-type IDH1 and IDH1 mutants were equally sensitive to glutamine deprivation, suggesting that there are different downstream effectors active when considering inhibition of glutamine metabolism versus inhibition of glutamine uptake. Metabolomics analysis [69] provided interesting findings that $2 \mathrm{HG}$ levels remained constant between the IDH1-mutant BPTES-treated group and the IDH1-mutant nontreated group. However, the glycolytic intermediate levels increased, and the TCA cycle intermediate levels decreased in the group treated with BPTES when compared to the non-treated group. The increase of glycolytic intermediates and the decrease of TCA cycle intermediates can be attributed to compensatory mechanisms attempting to maintain aKG levels by reallocating glutamine carbon from the TCA cycle to glycolysis [68]. The message from this specific example is that the mutations of IDH1 form a subset of glioblastomas that exhibit a shift toward glutaminedependent energy pathways. These pathways allow tumor cells to best utilize glutamine and its products in order to produce ATP as a fuel source for biosynthetic pathways. This ability is particularly advantageous when glucose is scarce. This signifies that these IDHmu tumors exhibit glutamine addiction, and therapies to target glutamine metabolism should prove to be particularly useful here. GLS inhibition could serve as a potential therapeutic target, and several BPTES analogs have been developed [70]. Still, they will most likely need to be used in conjunction with other treatment regiments to combat the dynamic properties of cellular metabolism [68].

In a study by Nobusawa et al., individuals with secondary glioblastoma who possessed IDH1 mutations had a more favorable prognosis than those with IDH1 wild-type [71]. Histologically, primary and secondary glioblastomas are identical; thus, clinical data is relied upon to determine their subdivision because they occur in patients of different ages and develop through different pathways [72, 73]. Through statistical analyses, it was determined that there was a positive correlation between IDH1 mutations accompanied by p53 mutations, diagnosis of secondary glioblastoma, more prolonged median survival, and younger patient age [74]. IDH1wt genes correlated with older patients, shorter median survival, and higher EGFR amplification. Secondary glioblastomas make up a smaller fraction of the disease compared to primary glioblastomas, but of the secondary cases sampled, an IDH1mu affected the majority, 


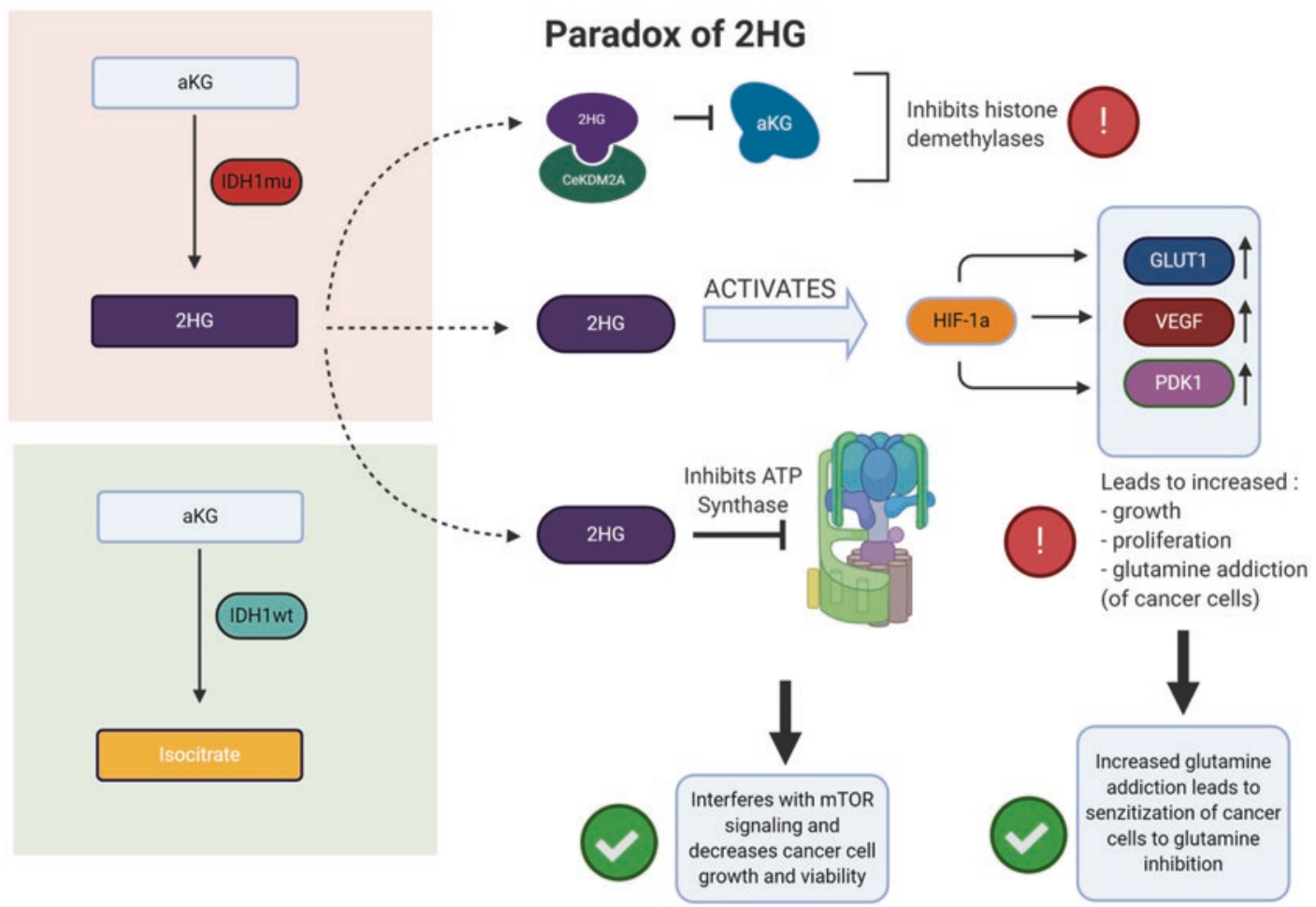

Fig. 4 The paradoxical nature of downstream effects of 2HG. 2HG inhibition of aKG-dependent histone demethylases, 2HG regulation of HIF-1a which induces overexpression of target enzymes GLUT1, VEGF, and PDK1 leading to increased tumor growth, proliferation, and glutamine addiction. However, that glutamine addiction creates a vulnerability within those GBM cells to targeted glutamine inhibition. 2HG also functions to inhibit ATP synthase and interferes with mTOR signaling causing decreased cancer cell growth and viability

while a minority of primary glioblastomas were affected by the IDH1 mutation. For the patients initially diagnosed as secondary GBM, which did not harbor an IDH1 mutation, and for the cases initially diagnosed as primary, which contained an IDH1 mutation, further analysis showed that these cases were likely misdiagnosed. The "primary" diagnosed patients with IDH1mu had every characteristic of secondary glioblastoma, while the opposite was true for "secondary" glioblastomas with IDH1wt. These discrepancies suggest incorrect diagnoses for gliomas that may have started at a low grade and then progressed quickly, making them look like a primary or higher grade glioma with some progression disguising it as secondary. Nobusawa et al. accurately identified IDH1 as a reliable signature marker for secondary glioblastoma with a more favorable outcome [71].
Furthermore, another study by Labussiere et al. showed that individuals with IDH1mu tumors lived longer than those who had IDH1wt tumors, despite all tumors being of the same grade [75]. Another classical function of IDH1 is to support oxidative decarboxylation of isocitrate to aKG coupled with the reduction of NADPH, allowing NADPH to promote the further reduction of glutathione (GSH), a crucial antioxidant $[76,77]$. When glioblastomas have IDH1mu, the loss of normal enzymatic function lowers the production of aKG and NADPH, subsequently lowering GSH, while increasing $2 \mathrm{HG}$ [60]. The surge of D-2HG increases oxidative stress present in cancer cells and the accumulation of reactive oxygen species (ROS), which encourages tumor cell growth [78]. These characteristics seemingly create a paradox wherein the accumulation of ROS could serve to further tumor development due to 
the increase of genetic instability, but on the other hand, the decrease of GSH levels leaves the tumor cells vulnerable for oxidative damage [75]. Both L- and D-2HG have also been shown to function as an ATP synthase inhibitor and interferes with mTOR signaling, which leads to a decrease in tumor growth and cell viability [79] (Fig. 4). There appears to be a greater response to radiation in patients with IDHmu tumors, which may be due to the effect D-2HG has as a radiosensitizing agent [80]. Both enantiomers of $2 \mathrm{HG}$ inhibit homologous recombination allowing for the greater effect of alkylating agents [81]. Controversy exists over the therapeutic potential of targeting $2 \mathrm{HG}$ because there appear to be both oncometabolite and tumor-suppressing capabilities of $2 \mathrm{HG}$. These conclusions suggest the need for further analyses on the mechanistic links between metabolic phenotype and clinical outcome. There appears to be a limited therapeutic window with IDHmu tumors where inhibition of IDH1/2 or $2 \mathrm{HG}$ could be beneficial earlier in pathogenesis to prevent invasion and progression, but further in development, these metabolic aberrations could hold the key to increased longevity due to the vulnerabilities created. This conflict illustrates the need for a deeper dive into the temporal pathogenesis of GBM with IDH mutations.

\section{$4 \quad$ Benefits of Combined Therapy}

Among the many struggles in treating cancer, tackling its inherent metabolic heterogeneity is a significant obstacle [69]. Metabolic pathways relevant to GBM have been established, but those pathways are dynamic, and cancer cells alter their metabolism as their environments change [82]. When a pathway is hit and deactivated, the ability of a cancer cell to work around it contributes to the complexity of treatments. After multiple pathways are inhibited, a cancer cell's metabolism will eventually be cornered with nowhere to turn (Fig. 5). Tanaka et al. successfully combined therapies inhibiting mTOR and GLS to limit cancer cell proliferation [50]. As mTOR-targeted treatments inactivate the PI3K,
GBM switches to higher expressions of GLS to rescue it from apoptosis [50]. Combining the mTOR-targeted treatment with GLS inhibition essentially traps specific GBM cells so that their metabolism cannot shift pathways to encourage cell survival. Heterogeneity creates problems when determining treatment because different metabolic profiles result in differences as to how cancer metabolism will change in response to treatment. These synergistic treatments are beneficial because they can work together when attempting to target different pathways. Still, the challenge remains to affect only the cancerous cells while not having a detrimental impact on benign, healthy cells.

The current treatment protocol for GBM is surgical resection, radiation, and chemotherapy, typically through temozolomide (TMZ), which is an alkylating agent that causes DNA damage to tumor cells to trigger apoptosis. Even with aggressive treatment, this disease is universally lethal. Interfering with GBM's metabolic pathways through metabolic targeting could serve as a way to enhance the effectiveness of standard therapies. Glycolytic phenotypes are typically accompanied by radiation resistance. Therefore if glycolytic inhibition is accompanied by radiation, it could improve the effectiveness of treatment. Indeed, these are the results obtained by Wolf et al. in their in vitro studies. As they depleted HK2 (a glycolytic enzyme) and combined it with radiation therapy, they observed reduced proliferation and increased apoptosis [26]. As discussed earlier, NADPH is a powerful reductant to help mediate oxidative stress created by radiation. Decreasing NADPH levels is also linked to high rates of cellular senescence in the presence of radiation [56]. As IDHwt is the driver for increased NADPH concentrations, IDHwt inhibition appears to be another potential synergistic strategy. Combination therapy with high pharmacological doses of ascorbate, which becomes a powerful prooxidant to inflict oxidative damage, and radiation is also a viable strategy to combat radiation resistance. Specifically, Herst et al. used in vitro studies to show that high doses of ascorbate combined with 1 Gy of radiation (which is lower than the typical amount of 


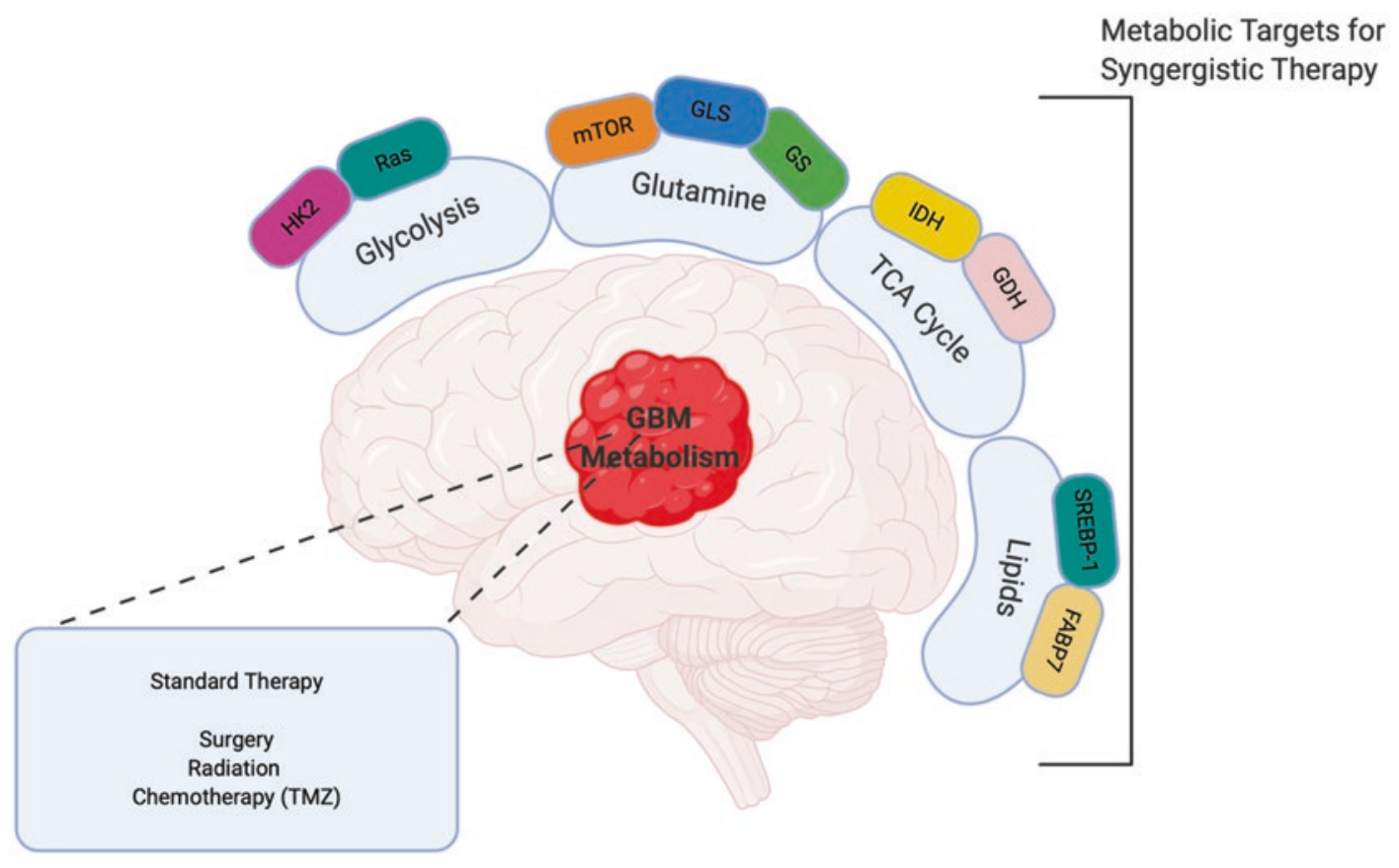

Fig. 5 Metabolic targets for GBM therapy (indicated by multicolored panels) accompanied by the specific metabolic pathways in which they participate. These targeted therapies can be used to supplement the standard therapy for treating GBM possibly resulting in synergistic effects. HK2 hexokinase 2, mTOR mechanistic target of rapamycin or mammalian target of rapamycin, $G L S$ glutaminase, $G S$ glutamine synthase, $I D H$ isocitrate dehydrogenase, $G D H$ glutamate dehydrogenase, $S R E B P-1$ sterol regulatory element-binding protein 1, FABP7 fatty acid-binding protein 7

2 Gy) resulted in greater cell death than either singular strategy and had less harmful effects on normal astrocytes [83]. Oxidative stress can be mediated by altering glutamine metabolism. Glutamine is converted to glutamate via GLS, and glutamate is an amino acid incorporated into GSH. Therefore, if GLS inhibition indirectly inhibits the formation of GSH, there are fewer antioxidants to provide radioprotection. CB-839, a potent GLS inhibitor, is currently undergoing a clinical trial (NCT03528642) in combination with TMZ and radiation.

Currently, it is not well understood how glycolytic metabolites contribute to chemotherapy resistance. However, as seen with radiation, the depletion of HK2 also appears to sensitize GBM to TMZ [26]. Tumor protein 73 antisense RNA 1 (TP73-AS1) has been found to have connections with GSCs that confers TMZ resistance. Mazor et al. demonstrated that silencing TP73-AS1 led to an increased sensitivity of GBM to TMZ therapy. The mechanism behind this effect is cur- rently being investigated. TP73-AS1 is known to encode aldehyde dehydrogenase 1 family member A1 (ALDH1A1), which is an established marker for cancer stem cells and promotes resistance. ALDH1A1 inhibition has a very similar sensitizing effect on GBM cells to TMZ [84].

\section{$5 \quad$ Advanced Brain Tumors (GBM) Display Distinct Metabolic Profiles Compared to Lower Grade Tumors}

While IDH1 mutations can distinguish between primary and secondary GBMs, another metabolic pathway involving cysteine catabolism is not highly activated in lower grade tumorigenesis [85]. The GSH synthesis pathway involving cysteine begins with a simultaneous efflux of glutamate and influx of cystine, which is then reduced to cysteine and converted to GSH when combined with glutamate and glycine. GSH functions 
as a central nervous system antioxidant increasing cancer cell survival when subjected to redox stress and hypoxia [86]. Prabhu et al. investigated another pathway involving cysteine, which resulted in the accumulation of cysteine sulfinic acid (CSA) through the regulatory enzyme cysteine dioxygenase-1 (CDO1). When comparing with grade 2 gliomas, there was a 23 -fold increase in the relative accumulation of CSA in GBM, ranking it as the highest change in concentration of any metabolite. This increase of metabolite concentration correlated with a higher expression of the CDO1 enzyme in GBM when evaluated using Western blot analysis. The buildup of CSA is associated with inhibited cellular respiration and decreased both oxidative phosphorylation and ATP production. CSA modulates mitochondrial function through inhibition of pyruvate dehydrogenase (PDH). PDH functions as a channel enzyme controlling the rate at which glycolysis occurs, and this enzyme was inhibited in a dose-dependent manner when treated with CSA using an established GBM cell line (U251). Further investigation is needed to determine how this alternative pathway of cysteine catabolism contributes to GBM tumorigenesis [85]. An analysis was conducted to uncover the impacts PDH modulation had on tumorigenesis in GBM and showed that PDH phosphatase expression regulated PDH activity as a result of Ras-mediated signaling. When the impairment of PDH was reversed, it inhibited tumor growth, making this pathway a possible therapeutic target to treat GBM in the future [87].

Nguyen et al. discovered a metabolite, NAAG, that serves as a glutamate reservoir for cancer cells in a glutamine-deprived environment, as mentioned earlier. Additionally, NAAG was found to be significantly elevated in GBM when compared to grade II-III gliomas and meningiomas [18]. As more of these biomarkers emerge, they could provide the key for tracking longitudinal pathogenesis and discovering new metabolic targets.

\section{Conclusion}

Glioblastoma is the most common and deadliest form of brain cancer in humans. Its poor prognosis and unreliable diagnosis are the results of its intricate heterogeneity and evolutionary characteristics. Experts have made substantial progress in characterizing this cancer with the use of improved technologies; most recently, there has been a spotlight on the use of metabolomics to discover its underlying molecular mechanisms. As more data and results are obtained to determine how different glioblastomas function and why they exhibit certain metabolic phenotypes, more therapeutic strategies can be developed to treat patients individually with respect to their genotypic and phenotypic profiles.

\section{References}

1. Purow, B. W., \& Schiff, D. (2010). Glioblastoma genetics: In rapid flux. Discovery Medicine, 9(45), 125-131.

2. Delgado-Martin, B., \& Medina, M. A. (2020). Advances in the knowledge of the molecular biology of glioblastoma and its impact in patient diagnosis, stratification, and treatment. Advanced Science (Weinh), 7(9), 1902971.

3. Kleihues, P., \& Ohgaki, H. (1999). Primary and secondary glioblastomas: From concept to clinical diagnosis. Neuro-Oncology, 1(1), 44-51.

4. Louis, D. N., et al. (2016). The 2016 World Health Organization classification of tumors of the central nervous system: A summary. Acta Neuropathologica, 131(6), 803-820.

5. Hainfellner, J., et al. (2014). Letter in response to David N. Louis et al, International Society Of Neuropathology-Haarlem Consensus Guidelines for Nervous System Tumor Classification and Grading, Brain Pathology, doi: 10.1111/bpa.12171. Brain Pathology, 24(6), 671-672.

6. Verhaak, R. G., et al. (2010). Integrated genomic analysis identifies clinically relevant subtypes of glioblastoma characterized by abnormalities in PDGFRA, IDH1, EGFR, and NF1. Cancer Cell, 17(1), 98-110.

7. Wang, Q., et al. (2017). Tumor evolution of gliomaintrinsic gene expression subtypes associates with immunological changes in the microenvironment. Cancer Cell, 32(1), 42-56. e6. 
8. Rubin, J. B., \& Gutmann, D. H. (2005). Neurofibromatosis type 1-a model for nervous system tumour formation? Nature Reviews. Cancer, 5(7), 557-564.

9. Visvader, J. E., \& Lindeman, G. J. (2008). Cancer stem cells in solid tumours: Accumulating evidence and unresolved questions. Nature Reviews. Cancer, 8(10), 755-768.

10. Navin, N., et al. (2011). Tumour evolution inferred by single-cell sequencing. Nature, 472(7341), 90-94.

11. Gerlinger, M., et al. (2012). Intratumor heterogeneity and branched evolution revealed by multiregion sequencing. The New England Journal of Medicine, 366(10), 883-892.

12. Driessens, G., et al. (2012). Defining the mode of tumour growth by clonal analysis. Nature, 488(7412), 527-530.

13. Schepers, A. G., et al. (2012). Lineage tracing reveals Lgr5+ stem cell activity in mouse intestinal adenomas. Science, 337(6095), 730-735.

14. Bedard, P. L., et al. (2013). Tumour heterogeneity in the clinic. Nature, 501(7467), 355-364.

15. Nabi, K., \& Le, A. (2021). The intratumoral heterogeneity of cancer metabolism. Advances in Experimental Medicine and Biology, 1311, https:// doi.org/10.1007/978-3-030-65768-0_11

16. Patel, A. P., et al. (2014). Single-cell RNA-seq highlights intratumoral heterogeneity in primary glioblastoma. Science, 344(6190), 1396-1401.

17. Simonelli, M., et al. (2020). Cerebrospinal fluid tumor DNA for liquid biopsy in glioma patients' management: Close to the clinic? Critical Reviews in Oncology/Hematology, 146, 102879.

18. Nguyen, T., et al. (2019). Uncovering the role of $\mathrm{N}$-acetyl-aspartyl-glutamate as a glutamate reservoir in cancer. Cell Reports, 27(2), 491-501. e6.

19. Zhu, L., et al. (2018). Focused ultrasound-enabled brain tumor liquid biopsy. Scientific Reports, 8(1), 6553.

20. Pan, W., et al. (2015). Brain tumor mutations detected in cerebral spinal fluid. Clinical Chemistry, 61(3), 514-522.

21. Badr, C. E., et al. (2020). Metabolic heterogeneity and adaptability in brain tumors. Cellular and Molecular Life Sciences, 77(24), 5101-5119.

22. Xie, Q., Mittal, S., \& Berens, M. E. (2014). Targeting adaptive glioblastoma: An overview of proliferation and invasion. Neuro-Oncology, 16(12), 1575-1584.

23. Hoang-Minh, L. B., et al. (2018). Infiltrative and drug-resistant slow-cycling cells support metabolic heterogeneity in glioblastoma. The EMBO Journal, 37, 23.

24. Lin, H., et al. (2017). Fatty acid oxidation is required for the respiration and proliferation of malignant glioma cells. Neuro-Oncology, 19(1), 43-54.

25. Osswald, M., et al. (2015). Brain tumour cells interconnect to a functional and resistant network. Nature, 528(7580), 93-98.

26. Wolf, A., et al. (2011). Hexokinase 2 is a key mediator of aerobic glycolysis and promotes tumor growth in human glioblastoma multiforme. The Journal of Experimental Medicine, 208(2), 313-326.

27. Babic, I., et al. (2013). EGFR mutation-induced alternative splicing of Max contributes to growth of glycolytic tumors in brain cancer. Cell Metabolism, 17(6), 1000-1008.

28. Dwarakanath, B. S., et al. (2009). Clinical studies for improving radiotherapy with 2-deoxy-D-glucose: Present status and future prospects. Journal of Cancer Research and Therapeutics, 5(Suppl 1), S21-S26.

29. Gschwind, A., Fischer, O. M., \& Ullrich, A. (2004). The discovery of receptor tyrosine kinases: Targets for cancer therapy. Nature Reviews. Cancer, 4(5), 361-370.

30. Lemmon, M. A., Schlessinger, J., \& Ferguson, K. M. (2014). The EGFR family: Not so prototypical receptor tyrosine kinases. Cold Spring Harbor Perspectives in Biology, 6(4), a020768.

31. Brennan, C. W., et al. (2013). The somatic genomic landscape of glioblastoma. Cell, 155(2), 462-477.

32. Furnari, F. B., et al. (2015). Heterogeneity of epidermal growth factor receptor signalling networks in glioblastoma. Nature Reviews. Cancer, 15(5), 302-310.

33. Nathanson, D. A., et al. (2014). Targeted therapy resistance mediated by dynamic regulation of extrachromosomal mutant EGFR DNA. Science, 343(6166), 72-76.

34. Dunn, G. P., et al. (2012). Emerging insights into the molecular and cellular basis of glioblastoma. Genes \& Development, 26(8), 756-784.

35. Bieging, K. T., Mello, S. S., \& Attardi, L. D. (2014). Unravelling mechanisms of p53-mediated tumour suppression. Nature Reviews. Cancer, 14(5), 359-370.

36. Giaccia, A. J., \& Kastan, M. B. (1998). The complexity of p53 modulation: Emerging patterns from divergent signals. Genes \& Development, 12(19), 2973-2983.

37. Hu, W., Feng, Z., \& Levine, A. J. (2012). The regulation of multiple p53 stress responses is mediated through MDM2. Genes \& Cancer, 3(3-4), 199-208.

38. Song, H., Hollstein, M., \& Xu, Y. (2007). p53 gainof-function cancer mutants induce genetic instability by inactivating ATM. Nature Cell Biology, 9(5), 573-580.

39. Olivier, M., \& Taniere, P. (2011). Somatic mutations in cancer prognosis and prediction: Lessons from TP53 and EGFR genes. Current Opinion in Oncology, 23(1), 88-92.

40. Rivlin, N., et al. (2011). Mutations in the p53 tumor suppressor gene: Important milestones at the various steps of tumorigenesis. Genes \& Cancer, 2(4), 466-474.

41. Bose, S., Zhang, C., \& Le, A. (2021). Glucose metabolism in cancer: The Warburg effect and beyond. Advances in Experimental Medicine and Biology, 1311, https://doi.org/10.1007/978-3-030-65768-0_1

42. Maddocks, O. D., \& Vousden, K. H. (2011). Metabolic regulation by $\mathrm{p} 53$. Journal of Molecular Medicine (Berlin), 89(3), 237-245. 
43. Kawauchi, K., et al. (2008). p53 regulates glucose metabolism through an IKK-NF-kappaB pathway and inhibits cell transformation. Nature Cell Biology, 10(5), 611-618.

44. Schwartzenberg-Bar-Yoseph, F., Armoni, M., \& Karnieli, E. (2004). The tumor suppressor p53 downregulates glucose transporters GLUT1 and GLUT4 gene expression. Cancer Research, 64(7), 2627-2633.

45. Li, T., Copeland, C., \& Le, A. (2021). Glutamine metabolism in cancer. Advances in Experimental Medicine and Biology, 1311, https://doi.org/10.1007/978-3-03065768-0_2

46. Oizel, K., et al. (2017). Efficient mitochondrial glutamine targeting prevails over glioblastoma metabolic plasticity. Clinical Cancer Research, 23(20), 6292-6304.

47. Marin-Valencia, I., et al. (2012). Analysis of tumor metabolism reveals mitochondrial glucose oxidation in genetically diverse human glioblastomas in the mouse brain in vivo. Cell Metabolism, 15(6), 827-837.

48. Rosati, A., et al. (2013). Glutamine synthetase expression as a valuable marker of epilepsy and longer survival in newly diagnosed glioblastoma multiforme. Neuro-Oncology, 15(5), 618-625.

49. Cancer Genome Atlas Research N. (2008). Comprehensive genomic characterization defines human glioblastoma genes and core pathways. Nature, 455(7216), 1061-1068.

50. Tanaka, K., et al. (2015). Compensatory glutamine metabolism promotes glioblastoma resistance to mTOR inhibitor treatment. The Journal of Clinical Investigation, 125(4), 1591-1602.

51. Duvel, K., et al. (2010). Activation of a metabolic gene regulatory network downstream of mTOR complex 1. Molecular Cell, 39(2), 171-183.

52. Guo, D., Bell, E. H., \& Chakravarti, A. (2013). Lipid metabolism emerges as a promising target for malignant glioma therapy. CNS Oncolology, 2(3), 289-299.

53. Mashimo, T., et al. (2014). Acetate is a bioenergetic substrate for human glioblastoma and brain metastases. Cell, 159(7), 1603-1614.

54. Park, J. K., et al. (2021). The heterogeneity of lipid metabolism in cancer. Advances in Experimental Medicine and Biology, 1311, https://doi. org/10.1007/978-3-030-65768-0_3

55. Guo, D., et al. (2009). EGFR signaling through an Akt-SREBP-1-dependent, rapamycin-resistant pathway sensitizes glioblastomas to antilipogenic therapy. Science Signaling, 2(101), ra82.

56. Wahl, D. R., et al. (2017). Glioblastoma therapy can be augmented by targeting IDH1-mediated NADPH biosynthesis. Cancer Research, 77(4), 960-970.

57. Dang, C. V., Le, A., \& Gao, P. (2009). MYC-induced cancer cell energy metabolism and therapeutic opportunities. Clinical Cancer Research, 15(21), 6479-6483.

58. Le, A., \& Dang, C. V. (2013). Studying Myc's role in metabolism regulation. Methods in Molecular Biology, 1012, 213-219.
59. Yang, C., et al. (2009). Glioblastoma cells require glutamate dehydrogenase to survive impairments of glucose metabolism or Akt signaling. Cancer Research, 69(20), 7986-7993.

60. Dang, L., et al. (2010). Cancer-associated IDH1 mutations produce 2-hydroxyglutarate. Nature, 465(7300), 966.

61. Struys, E. A. (2013). 2-Hydroxyglutarate is not a metabolite; D-2-hydroxyglutarate and L-2hydroxyglutarate are! Proceedings of the National Academy of Sciences of the United States of America, 110(51), E4939.

62. Karlstaedt, A., et al. (2016). Oncometabolite d-2hydroxyglutarate impairs alpha-ketoglutarate dehydrogenase and contractile function in rodent heart. Proceedings of the National Academy of Sciences of the United States of America, 113(37), 10436-10441.

63. Losman, J. A., et al. (2013). (R)-2-hydroxyglutarate is sufficient to promote leukemogenesis and its effects are reversible. Science, 339(6127), 1621-1625.

64. Gross, S., et al. (2010). Cancer-associated metabolite 2-hydroxyglutarate accumulates in acute myelogenous leukemia with isocitrate dehydrogenase 1 and 2 mutations. The Journal of Experimental Medicine, 207(2), 339-344.

65. Xu, W., et al. (2011). Oncometabolite 2-hydroxyglutarate is a competitive inhibitor of alpha-ketoglutarate-dependent dioxygenases. Cancer Cell, 19(1), 17-30.

66. Zhao, S., et al. (2009). Glioma-derived mutations in IDH1 dominantly inhibit IDH1 catalytic activity and induce HIF-1alpha. Science, 324(5924), 261-265.

67. Fu, Y., et al. (2012). Glioma derived isocitrate dehydrogenase-2 mutations induced up-regulation of HIF1alpha and beta-catenin signaling: Possible impact on glioma cell metastasis and chemo-resistance. The International Journal of Biochemistry \& Cell Biology, 44(5), 770-775.

68. Seltzer, M. J., et al. (2010). Inhibition of glutaminase preferentially slows growth of glioma cells with mutant IDH1. Cancer Research, 70(22), 8981-8987.

69. Hoang, G., Udupa, S., \& Le, A. (2019). Application of metabolomics technologies toward cancer prognosis and therapy. International Review of Cell and Molecular Biology, 347, 191-223.

70. Zimmermann, S. C., et al. (2016). Allosteric glutaminase inhibitors based on a 1,4-di(5-amino-1,3,4thiadiazol-2-yl)butane scaffold. ACS Medicinal Chemistry Letters, 7(5), 520-524.

71. Nobusawa, S., et al. (2009). IDH1 mutations as molecular signature and predictive factor of secondary glioblastomas. Clinical Cancer Research, 15(19), 6002-6007.

72. Ohgaki, H., et al. (2004). Genetic pathways to glioblastoma: A population-based study. Cancer Research, 64(19), 6892-6899.

73. Ohgaki, H., \& Kleihues, P. (2007). Genetic pathways to primary and secondary glioblastoma. The American Journal of Pathology, 170(5), 1445-1453. 
74. Parsons, D. W., et al. (2008). An integrated genomic analysis of human glioblastoma multiforme. Science, 321(5897), 1807-1812.

75. Labussiere, M., et al. (2010). IDH1 gene mutations: A new paradigm in glioma prognosis and therapy? The Oncologist, 15(2), 196-199.

76. Thompson, C. B. (2009). Metabolic enzymes as oncogenes or tumor suppressors. The New England Journal of Medicine, 360(8), 813-815.

77. Lee, S. M., et al. (2002). Cytosolic NADP(+)dependent isocitrate dehydrogenase status modulates oxidative damage to cells. Free Radical Biology \& Medicine, 32(11), 1185-1196.

78. Latini, A., et al. (2003). D-2-hydroxyglutaric acid induces oxidative stress in cerebral cortex of young rats. The European Journal of Neuroscience, 17(10), 2017-2022.

79. Fu, X., et al. (2015). 2-Hydroxyglutarate inhibits ATP synthase and mTOR signaling. Cell Metabolism, 22(3), 508-515.

80. Molenaar, R. J., et al. (2015). Radioprotection of IDH1-mutated cancer cells by the IDH1-mutant inhibitor AGI-5198. Cancer Research, 75(22), 4790-4802.

81. Sulkowski, P. L., et al. (2017). 2-Hydroxyglutarate produced by neomorphic IDH mutations suppresses homologous recombination and induces PARP inhibitor sensitivity. Science Translational Medicine, 9, 375 .

82. Antonio, M. J., Zhang, C., \& Le, A. (2021). Different tumor microenvironments lead to different metabolic phenotypes. Advances in Experimental Medicine and Biology, 1311, https:// doi.org/10.1007/978-3-030-65768-0_10.

83. Herst, P. M., et al. (2012). Pharmacological concentrations of ascorbate radiosensitize glioblastoma multiforme primary cells by increasing oxidative DNA damage and inhibiting G2/M arrest. Free Radical Biology \& Medicine, 52(8), 1486-1493.

84. Mazor, G., et al. (2019). The lncRNA TP73-AS1 is linked to aggressiveness in glioblastoma and promotes temozolomide resistance in glioblastoma cancer stem cells. Cell Death \& Disease, 10(3), 246.

85. Prabhu, A., et al. (2014). Cysteine catabolism: A novel metabolic pathway contributing to glioblastoma growth. Cancer Research, 74(3), 787-796.

86. Chung, W. J., et al. (2005). Inhibition of cystine uptake disrupts the growth of primary brain tumors. The Journal of Neuroscience, 25(31), 7101-7110.

87. Prabhu, A., et al. (2015). Ras-mediated modulation of pyruvate dehydrogenase activity regulates mitochondrial reserve capacity and contributes to glioblastoma tumorigenesis. Neuro-Oncology, 17(9), 1220-1230.

Open Access This chapter is licensed under the terms of the Creative Commons Attribution 4.0 International License (http://creativecommons.org/licenses/by/4.0/), which permits use, sharing, adaptation, distribution and reproduction in any medium or format, as long as you give appropriate credit to the original author(s) and the source, provide a link to the Creative Commons license and indicate if changes were made.

The images or other third party material in this chapter are included in the chapter's Creative Commons license, unless indicated otherwise in a credit line to the material. If material is not included in the chapter's Creative Commons license and your intended use is not permitted by statutory regulation or exceeds the permitted use, you will need to obtain permission directly from the copyright holder. 


\title{
The Intricate Metabolism of Pancreatic Cancers
}

\author{
Felipe Camelo and Anne Le
}

\section{Keywords \\ Pancreatic ductal adenocarcinoma $\cdot K R A S$ mutation · Glucose metabolism - Glutamine metabolism $\cdot$ Combined therapy}

\section{Abbreviations}

$\begin{array}{ll}\text { Asp } & \text { Aspartate } \\ \text { EGFR } & \text { Epidermal growth factor receptor } \\ \text { GLS } & \text { Glutaminase } \\ \text { GLUD1 } & \text { Glutamate dehydrogenase 1 } \\ \text { GLUT } & \text { Glucose transporter } \\ \text { GOT1 } & \text { Glutamic-oxaloacetic transaminase 1 } \\ \text { HCQ } & \text { Hydroxychloroquine } \\ \text { HIF-1 } \alpha & \text { Hypoxia-inducible factor 1-alpha } \\ \text { HK2 } & \text { Hexokinase 2 } \\ \text { KRAS } & \text { Kirsten rat sarcoma viral oncogene } \\ & \text { homolog }\end{array}$

F. Camelo

MD Program, Weill Cornell Medicine, New York, NY, USA

A. Le $(\bowtie)$

Department of Pathology and Oncology, Johns Hopkins University School of Medicine, Baltimore, MD, USA

Department of Chemical and Biomolecular Engineering, Johns Hopkins University Whiting School of Engineering, Baltimore, MD, USA e-mail: annele@jhmi.edu
LDH Lactate dehydrogenase

MAPK Mitogen-activated protein kinase

MCT Monocarboxylate transporter

MEK Mitogen-activated protein kinase kinase

MUC Mucin

NAA $\quad N$-acetyl-aspartate

NAAG $\quad N$-acetyl-aspartyl-glutamate

OAA Oxaloacetate

PDAC Pancreatic ductal adenocarcinoma

PDH Pyruvate dehydrogenase

PFK1 Phosphofructokinase 1

PPP Pentose phosphate pathway

ROS Reactive oxygen species

TCA Tricarboxylic acid

\section{Key Points}

- Oncogenic KRAS regulates glucose and glutamine metabolism in pancreatic cancer cells.

- MUC1 overexpression leads to increased glucose metabolism.

- p53 functions predict the sensitivity of pancreatic cancer tumors to glycolytic inhibition.

- Targeting alpha-ketoglutarate dehydrogenase function by CPI-613 slows mitochondrial metabolism.

- The antidiabetic drug, metformin, targets pancreatic cancer stem cells.

- Combined therapy is used to target pancreatic metabolism heterogeneity. 


\section{Introduction}

Currently, approximately $95 \%$ of pancreatic cancers are pancreatic ductal adenocarcinomas (PDAC), which are the most aggressive form and the fourth leading cause of cancer death with extremely poor prognosis [1]. Poor prognosis is primarily attributed to the late diagnosis of the disease when patients are no longer candidates for surgical resection [2]. Cancer cells are dependent on the oncogenes that allow them to proliferate limitlessly. Thus, targeting the expression of known oncogenes in pancreatic cancer has been shown to lead to more effective treatment [3]. This chapter discusses the complexity of metabolic features in pancreatic cancers. In order to comprehend the heterogeneous nature of cancer metabolism fully, we need to take into account the close relationship between cancer metabolism and genetics. Gene expression varies tremendously, not only among different types of cancers but also within the same type of cancer among different patients. Cancer metabolism heterogeneity is often prompted and perpetuated not only by mutations in oncogenes and tumorsuppressor genes but also by the innate diversity of the tumor microenvironment. Much effort has been focused on elucidating the genetic alterations that correlate with disease progression and treatment response [4, 5]. However, the precise mechanisms by which tumor metabolism contributes to cancer growth, survival, mobility, and aggressiveness represent a functional readout of tumor progression (Fig. 1).

\section{Oncogenic KRAS Regulates Metabolism in Pancreatic Cancer Cells (Fig. 2)}

\subsection{Oncogenic KRAS Regulates Glutamine Metabolism}

Cancer's specific metabolic adaptations in nutrient uptake and biosynthesis have been linked to a particular genetic mutation. The KRAS (Kirsten rat sarcoma) oncogene homolog is a known regulator of glutamine metabolism among other intermediary metabolic pathways that render cancer cells addicted to glutamine [6-9]. A range of mutations in the KRAS oncogene occur in over $90 \%$ of PDAC [10, 11]. Typically, glutamate feeds into the TCA cycle after being converted to alpha-ketoglutarate in the mitochondrion via glutamate dehydrogenase 1 (GLUD1) or aminotransferases. A study by Son et al. showed that KRAS regulated the reprogramming of glutamine metabolism through transcriptional regulation of key metabolic enzymes of transaminase reactions, which, in turn, determine PDAC tumor growth. Notably, they concluded that PDAC cells greatly depend on these reactions for redox homeostasis. Given that these pathways are nonessential in normal cells, the unique importance of these pathways in PDAC suggests novel approaches for therapy in treating PDAC [7]. $K R A S$ mutation led to the reprogramming of glutamine metabolism, which was partially due to increased cytosolic aspartate aminotransferase or glutamic-oxaloacetic transaminase 1 (GOT1) expression and decreased GLUD1 expression, which led to increased aspartate production via the mitochondrial isozyme GOT2. The change in the ratio of expression of GOT1 and GLUD1 thus shunts glutamine flux through the aspartate aminotransferase pathway. According to Lyssiotis et al., the observation that the glutamine metabolism pathway is downstream of mutant KRAS serves as an explanation for the distinct glutamine dependency of pancreatic cancer. Not only do their results yield novel targets for pancreatic cancer therapy, but they also suggest that inhibiting glutamine metabolism in pancreatic cancer therapies may synergize with therapies that increase reactive oxygen species (ROS) [8].

It has been recently found that oncogenic $K R A S$ also activates a nuclear factor erythroid 2-related factor 2 (Nrf2)-dependent antioxidant program to suppress ROS levels within the cells [12]. The upregulated Nrf2 pathway in cancer cells also increases the shunt of both glucose and glutamine towards anabolic metabolism, specifically towards the pentose phosphate pathway (PPP), when triggered by proliferative signaling [13]. Finally, Nrf2 also promotes pancreatic tumorigenesis and proliferation [14], further suggesting the multifaceted role of KRAS in driving pancreatic cancers. 
Fig. 1 Overview of pancreatic cancer metabolic heterogeneity. $P D A C$ pancreatic ductal adenocarcinoma, $M U C 1$ mucin 1

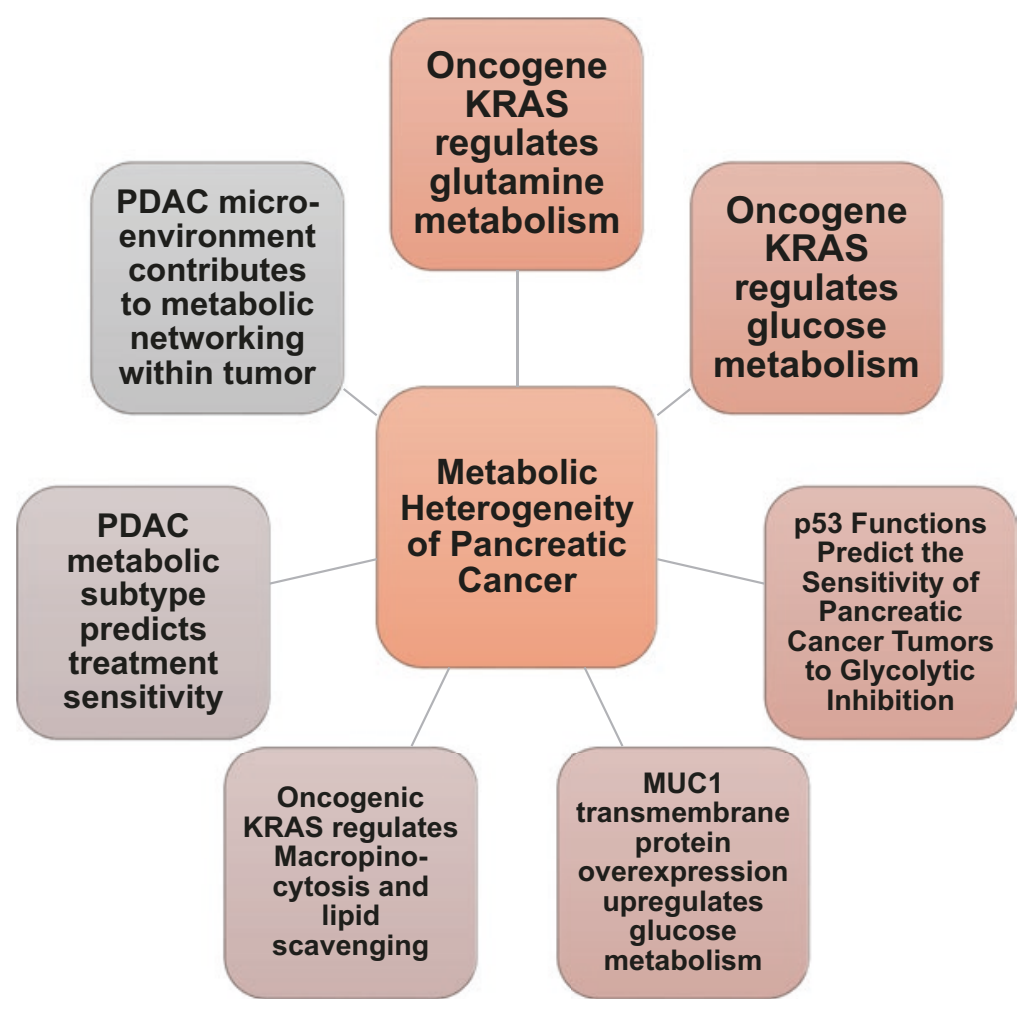

\subsection{Oncogenic KRAS Regulates Glucose Metabolism}

The KRAS oncogene is also known to contribute to the glucose metabolism in pancreatic cancer cells via the upregulation of glucose uptake and the diversion of glucose into the hexosamine biosynthesis pathways [15]. Oncogenic KRAS also controls the diversion of glycolytic intermediates into ribose biosynthesis pathways via the upregulation of the non-oxidative pentose phosphate pathway (PPP), a pathway that is fundamental to nucleic acid synthesis and thus cancer cell proliferation [15]. Expression of glucose transporter-1 (GLUT1), hexokinase-II (HK2), a kinase that phosphorylates glucose to drive intracellular glucose flux, and lactate dehydrogenase A (LDHA), an enzyme that catalyzes the reaction of pyruvate to lactate, are greatly enhanced by KRAS in pancreatic tumor cells [15]. Consequently, glycolytic flux, the production of lactate from glucose, was high in KRAS-mutant tumors. It is of note that these alterations are not nearly as pronounced in the stromal cells of these tumors, which can uptake the lactate generated by tumor cells and convert the lactate back to pyruvate in order to fuel the TCA cycle $[16,17]$. Yun et al. found that cells with mutated KRAS underwent the Warburg effect and survived better in low-glucose environments compared to cells with wild-type $K R A S$ due to the fact that KRAS upregulated GLUT1 [18, 19], suggesting that KRAS mutation is involved in the altering of a cancer cell's bioenergetics that is seen in most PDAC tumor cells, which take advantage of the altered metabolic pathways to proliferate and grow successfully.

\subsection{Oncogenic KRAS Upregulates Macropinocytosis and Lipid Scavenging}

Fatty acids are required for cancer cells to replicate their cell membranes and undergo essential lipid-dependent processes for proliferation. Besides shunting glutamine carbon to de novo 


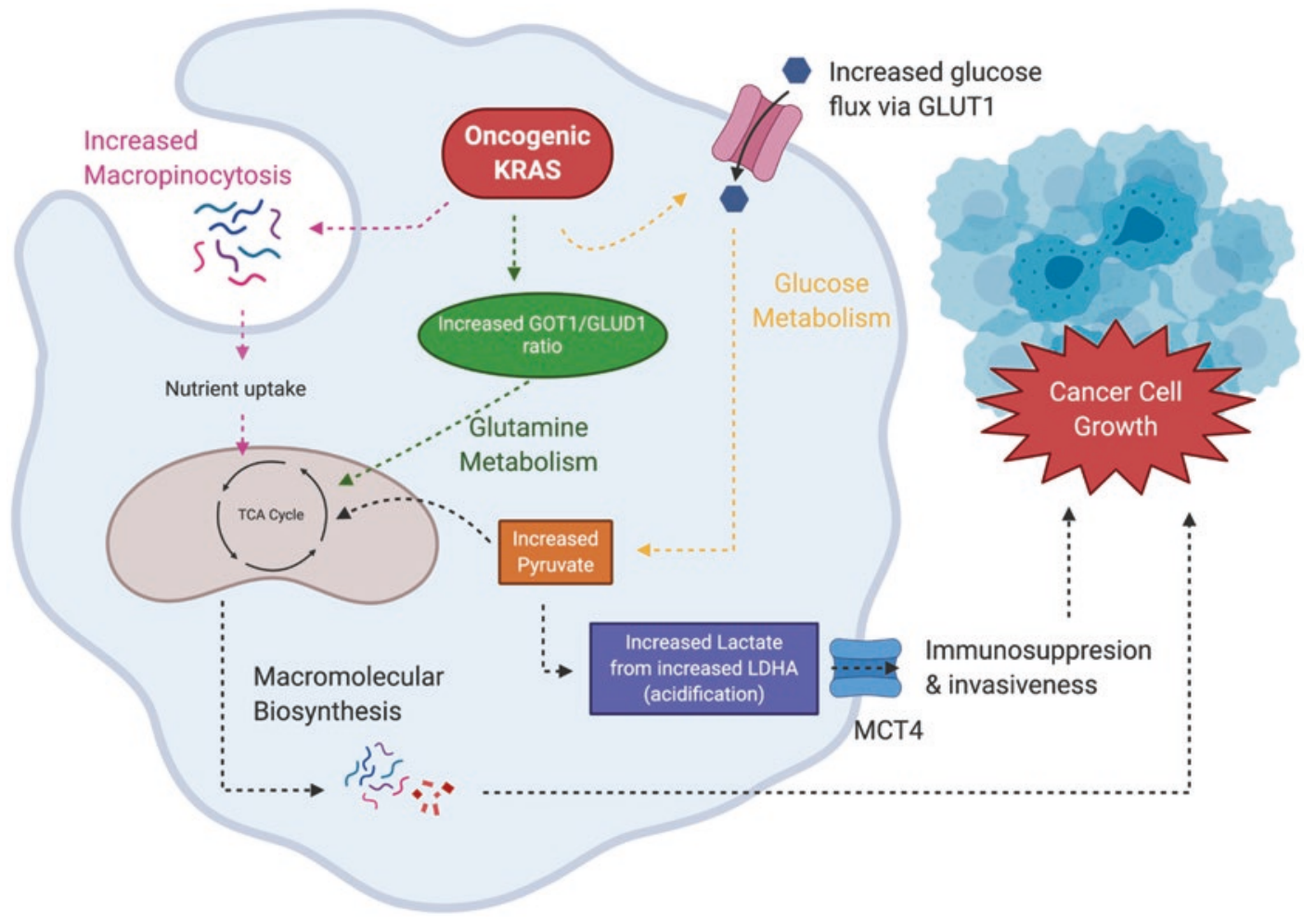

Fig. 2 Oncogenic KRAS affects multiple metabolic pathways that contribute to cancer cell growth. Of note are increased macropinocytosis, increased glutamine metabolism, and increased glucose metabolism. This leads to an increase in macromolecules that allow cancer cell growth. GOT1 glutamic-oxaloacetic transaminase s1, GLUD1 glutamate dehydrogenase 1, GLUT1 glucose transporter 1, MCT4 monocarboxylate transporter 4, LDHA lactate dehydrogenase A

fatty acid synthesis [20, 21], oncogenic KRAS also upregulates macropinocytosis, the endocytic process of cellular internalization of extracellular fluid, and its contents, further demonstrating the impact of oncogenic KRAS on PDAC. Recently, it was found that Ras-transformed cells utilize macropinocytosis as a way to uptake amino acids, including glutamine. In Ras-transformed pancreatic tumor xenografts, inhibiting macropinocytosis significantly inhibited tumor growth [22]. Ras-transformed cells, along with hypoxic cells, were also shown to support growth by increased scavenging for serum fatty acids [23]. Inhibiting low-density lipoprotein receptor (LDLR), which facilitates cholesterol intake and is associated with increased risk of PDAC recurrence, sensitized PDACs to chemotherapy drugs [24]. The metabolic importance of macropinocytosis in oncogenic KRAS-transformed cancer cells provides yet another metabolic target worth consid- ering for therapy. Contradictorily, multiple studies have demonstrated that lipids are decreased in both cancerous and noncancerous regions of PDACs [25, 26].

\section{Other Alternative Metabolisms in Pancreatic Cancer}

\subsection{MUC1 Overexpression Leads to Increased Glucose Metabolism}

A study by Chaika et al. revealed that the overexpression of transmembrane protein mucin 1 (MUC1) led to elevated glucose metabolism and related activities, such as increased glucose uptake and lactate production resulting from increases in GLUT1 expression and LDHA 
expression, respectively. These metabolic effects are particularly pronounced under hypoxic conditions, which are associated with the stabilization of hypoxia-inducible factor 1-alpha $(\mathrm{HIF}-1 \alpha)$, a transcription factor for many genes involved in regulating glucose uptake, through the overexpression of MUC1 [27]. Pancreatic cancer cells that do not overexpress MUC1 have a reduction in lactate and glycolytic intermediates. Overall, the overexpression of MUC1 is capable of influencing glucose metabolism, the elevation of amino acid metabolism, and the TCA cycle, all of which are important in the biosynthesis of cellular building blocks, and thus leading to tumorigenesis. The signaling pathway between MUC1 and HIF-1 $\alpha$ plays an important role in the facilitation of tumor growth and metastasis, serving as a potential target for manipulation in the treatment of diseases reliant upon these proteins [27].

\section{2 p53 Functions Predict the Sensitivity of Pancreatic Cancer Tumors to Glycolytic Inhibition}

The heterogeneity of metabolic alterations within the same cancer type is best illustrated in a study of Rajeshkumar et al. They showed that PDAC's sensitivity to the same metabolic inhibition could vary drastically from one tumor to another, depending on the specific tumor's genetic status and unique metabolic phenotype [28]. More specifically, they found that responses to LDHA inhibition by the small-molecule FX11 [29, 30] were determined by the status of a tumor's p53, a tumor-suppressor gene that is largely inactivated in many cancers $[28,31]$. Within the same PDAC type, tumors with wild-type TP53 demonstrated resistance to FX11, while those with mutant TP53 exhibited sensitivity in the form of increased apoptosis, reduced proliferation, and attenuated tumor growth. Their data show that FX11 specifically decreased pyruvate-to-lactate conversion by LDHA only in the TP53-mutant tumor, suggesting LDHA inhibition as a possible therapeutic target to reduce TP53-mutant tumor growth. Resistance in TP53-WT tumors is thought to result from reduced dependence on glucose, as corroborated by their data showing higher levels of TIGAR, a p53-inducible protein that lowers glycolytic flux, in these tumors [28, 32]. This study supports growing evidence for variable metabolic phenotypes not only across cancer types but also within cancers of the same type. From a clinical perspective, this insight emphasizes the importance of metabolic phenotypes in pancreatic cancer sub-characterization in order to pair drug therapies according to the phenotypic sensitivity for more selective and personalized treatment.

\subsection{Alternative Source of Glutamate in PDAC}

\subsubsection{Neurotransmitter $\mathbf{N}$-Acetyl- Aspartyl-Glutamate (NAAG) as a Glutamate Reservoir in Cancer}

In a recent study, Nguyen et al. found that in addition to the utilization of glutamate through traditional metabolic pathways, cancer cells actively convert glutamate into $\mathrm{N}$-acetyl-aspartylglutamate (NAAG), a metabolite commonly known as a neurotransmitter, which can be hydrolyzed back to glutamate when needed by the oncogenic cells via glutamate carboxypeptidase II (GCP II) [33]. They further demonstrated that knocking down GCPII expression or inhibiting GCPII led to a reduction in PDAC growth, suggesting that GCPII is a viable target for cancer therapy, either alone or in combination with glutaminase inhibition.

\subsubsection{Glutaminase II Pathway Is Another Source of Glutamate in Cancer}

Pancreatic cancer cells can utilize the conversion of glutamine to glutamate via glutaminase 1 (GLS1) to fuel their energetic needs [34]. Although inhibition of GLS1 led to decreased tumor growth and is being explored in clinical trials, there is still room for improvement. A recent study uncovered that pancreatic cancer cells utilized the glutaminase II 
pathway, an alternative pathway where glutamine is converted to alpha-ketoglutaramate, then eventually glutamate when GLS1 (glutaminase I pathway) is inhibited [35]. Knocking down glutamine transaminase K (GTK) expression, a key enzyme in the glutaminase II pathway, was found to inhibit the growth of pancreatic cancer cells in vitro. When translating to in vivo models, genetic suppression of GTK was found to inhibit tumorigenesis. The uncovering of the role of the glutaminase II pathway as a source of the carbon backbone of glutamate upon single-therapy GLS1 inhibition opens up new strategic approaches. Specifically, the study suggested a combination therapy of GLS1 and GTK inhibition for pancreatic cancer therapy.

\section{Pancreatic Tumor Microenvironment}

Pancreatic tumors were found to be hypovascular and constantly deprived of nutrients [36], leading to these tumors relying on alternative sources of nutrients to continue proliferating. The tumor microenvironment (TME) [37] was also found to be under such intense physical and oxidative stress that the interstitial pressures in pancreatic tumors induced vascular collapse [38]. This resulted in tumor hypoperfusion, limiting oxygen and nutrients, and drug delivery to cancer cells [39], creating an intratumoral heterogeneity of metabolism [40]. Due to this hindrance in nutrient acquisition, pancreatic cancer cells must support themselves using alternative sources. Part of sustaining tumor viability involved the dense stromal components that are largely populated by fibroblasts and immune cells [41]. Although this dense fibrotic stroma metabolically supported pancreatic cancer cells, studies have demonstrated that it could actually restrain cancer progression, likely due to the stroma's role in restraining angiogenesis $[42,43]$.

\subsection{PDACs are Dependent on Autophagy}

Yang et al. showed that pancreatic cancer cells are dependent on autophagy for tumor progression [44]. They found that suppression of autophagy via genetic depletion or pharmacological inhibition led to tumor regression in vivo and suppressed proliferation of multiple PDAC cell lines, along with an increase in ROS and a decrease in mitochondrial oxidative phosphorylation. However, in a recent study, Bryant and colleagues found that suppression of oncogenic $K R A S$ or mitogen-activated protein kinase (MAPK)/extracellular signal-regulated kinase (ERK) actually increased autophagic flux [45]. These results were surprising since it was previously believed that oncogenic KRAS increased autophagy in PDAC [46]. Bryant et al. also found that ERK inhibition sensitizes PDAC to chloroquine (CQ), an autophagy inhibitor, and decreased tumor cell proliferation and tumor growth in vivo. Similar results were obtained in a study by Kinsey et al., who found antitumor activity when combining hydroxychloroquine (HCQ) with MEK inhibitors [47]. These two studies point towards a combined therapy of HCQ with downstream KRAS inhibitors, with a current clinical trial of HCQ and MEK inhibitor combined treatment (NCT03825289) in progress. It is also worth noting that autophagy plays a paradoxical role in PDAC progression, in that genetic ablation of autophagy in the pancreas resulted in increased tumor initiation but impaired the tumor's ability to develop into invasive cancer [48]. Therefore, more studies into the role of autophagy in PDACs are required to better understand the clinical complexities of PDACs.

\subsection{Stromal Interactions Create Complex PDAC Metabolic Networks}

Metabolic networks within tumors can have profound effects on tumor progression and aggression. One example includes differences in regions of hypoxia and normoxia, where pancreatic cancer cells in hypoxic areas export lactate via monocarboxylate transporter 4 (MCT4) and PDAC cells in normoxic regions import this lactate via monocarboxylate 1 (MCT1) [49]. This increases the proliferation of PDAC cells in normoxic areas of the tumor. On the other hand, MCT4 expression has 
also been shown to promote an immunosuppressive environment and is associated with worse patient outcomes [50]. While PDAC cells can alter the tumor microenvironment to be immunosuppressive, PDAC cells can also stimulate stromal cells to release nutrients. Specifically, Sousa et al. found that stroma-associated pancreatic-stellate cells (PSC) secrete nonessential amino acids via autophagy and are critical for PDAC metabolism [51]. In fact, Sousa and colleagues found that alanine outperforms glucose and glutamine-derived carbon in PDAC to fuel TCA cycle intermediates by being converted to pyruvate. Other nutrients may also be taken up by PDAC cells via exosomes released by cancer-associated fibroblasts [52, 53]. Ultimately, there appears to be an incredibly complex metabolic network within PDAC tumors and the TME.

\section{$5 \quad$ Suggested Therapy (Fig. 3)}

Restricting fuel sources for homeostasis and proliferation in PDAC are the new therapeutic avenues for pancreatic cancer treatment [54]. KRAS appears to have a prominent role in the metabolic rewiring of PDAC tumors and PDAC pathogenesis [11]. Subsequently, it requires the cancer cell to become dependent on the oncogenic KRAS to continue proliferation [55]. This is known as oncogene addiction, in which the cancer cell becomes dependent on the activity of the oncogene for survival and proliferation [3]. Since KRAS mutations are found in a majority of PDAC cancer cells, and $K R A S$ regulates cancer cell's metabolism, targeting these regulations for cancer therapy is an approach that researchers are taking [55].

\subsection{Targeting Alpha- Ketoglutarate Dehydrogenase Complex Function by CPI-613 to Slow Mitochondrial Metabolism}

Drugs have been developed to target mitochondrial metabolism in cancers [55]. One of these drugs is CPI-613, an inhibitor of cancer-specific mitochondrial energy metabolism. The drug causes tumor cell apoptosis, necrosis, and autophagy by selectively targeting alterations in mitochondrial enzyme activities and redox status [56]. CPI-613 is a small molecule that attacks alpha-ketoglutarate dehydrogenase complex, an enzyme complex involved in the TCA cycle, in tumor cells through a redox process [57]. The drug is known to simultaneously attack multiple essential components of tumor cell regulation [57]. However, the exact mechanism is not well understood. Nevertheless, CPI-613 has been recognized to be effective against various types of cancers [58], including metastatic pancreatic cancer [56]. CPI-613 used in combination with modified FOLFIRINOX (mFOLFIRINOX) in patients with metastatic pancreatic cancer demonstrated better survival. However, since this phase I study was not designed to determine the efficacy of combining CPI-613 with mFOLFIRINOX, the results should be interpreted with caution. Nevertheless, Alistar et al. have obtained encouraging results from the phase I study and are currently performing a randomized phase III trial to compare FOLFIRINOX against mFOLFIRINOX with CPI-613 (NCT03504423). These results suggest that targeting mitochondrial metabolism holds enormous potential in combating pancreatic cancer.

\subsection{Antidiabetic Drug, Metformin, Targets Pancreatic Cancer Stem Cells}

Recent studies have shown that tumorigenic cancer stem cells (CSCs), a highly chemoresistant subclass of PDAC, are strongly dependent on oxidative metabolism [59, 60]. Retrospective analysis showed that oral administration of metformin in patients with type 2 diabetes was associated with a reduced risk of developing PDAC [61], along with a better outcome for patients that had established PDAC [62]. More recently, it has been discovered that metformin targets pancreatic CSCs but not the differentiated progenies (non-CSCs) [59]. KRAS targeting has resulted in tumor shrinkage but failed to kill all the CSCs [63]. Viale et al. established that dormant tumor 


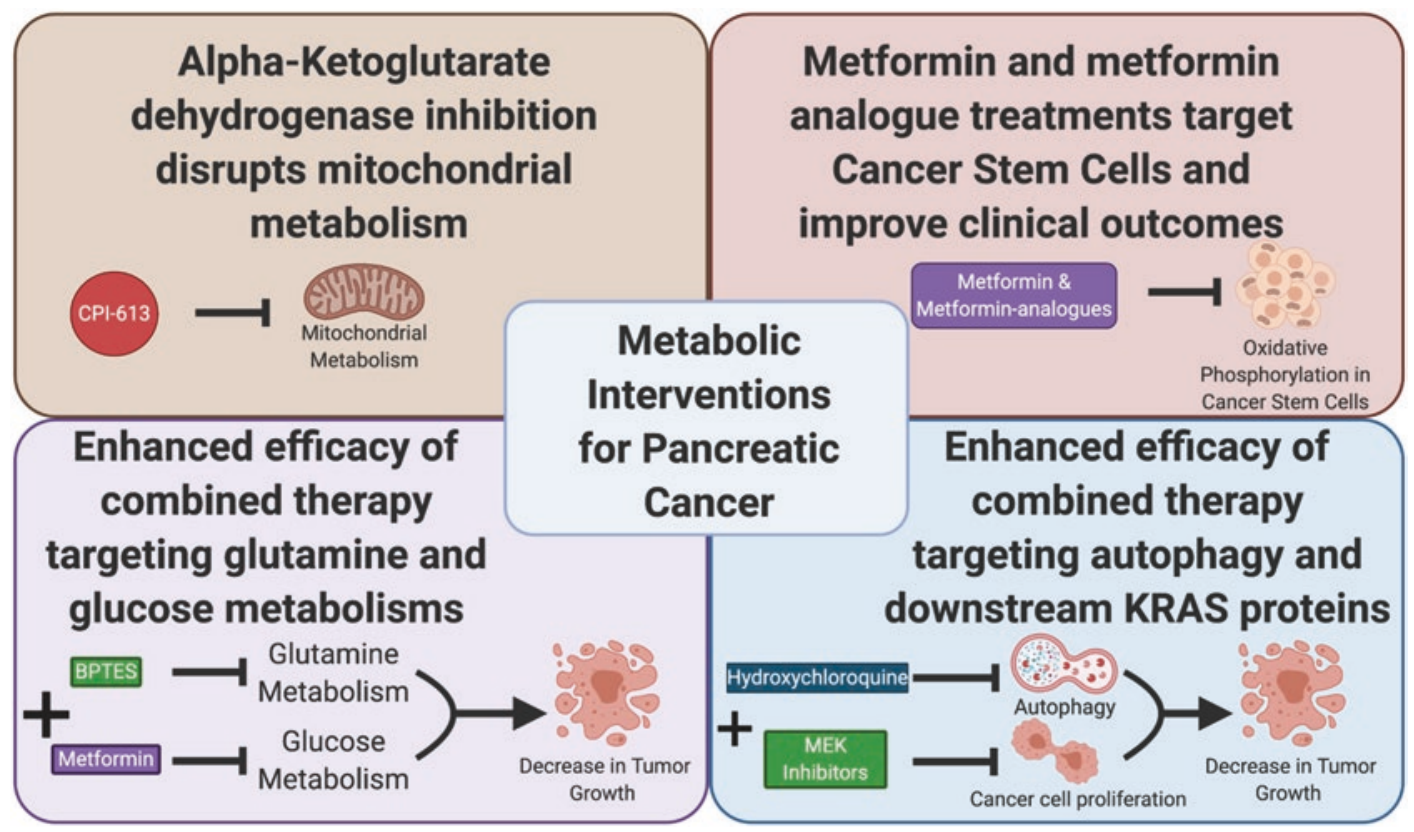

Fig. 3 Overview of therapeutic options targeting pancreatic cancer metabolism. Clinical trials in various stages are currently being conducted to determine the efficacy of these therapies. $M E K$ mitogen-activated protein kinase kinase

cells that survived oncogene ablation have high sensitivity to oxidative phosphorylation inhibitors [63]. Lonardo et al. showed that metformin uniformly reduced ATP levels in CSCs, but not in non-CSCs [60]. Although the mechanism of action for metformin in CSCs is largely unknown, what is known is that metformin slowly accumulates in the mitochondria and directly inhibits complex 1 (NADH dehydrogenase) in the electron transport chain, affecting oxidative phosphorylation [60]. However, phase II clinical trials found no benefit of metformin treatment when it is administered to patients with advanced or metastatic pancreatic cancers $[64,65]$. Although disappointing, there is still evidence to support the use of metformin as maintenance therapy in patients with stabilized metastatic PDAC [66], along with a recently finished clinical trial (NCT02048384). In addition, there may be more antitumor potential in metformin analogs with improved mitochondrial targeting ability [67]. Therefore, a potentially strong therapeutic strategy to manage pancreatic cancer is the combined targeting of the KRAS pathway and mitochondrial respiration [63].

\subsection{Combined Therapy to Target Pancreatic Metabolism Heterogeneity}

Combination therapy to target multiple metabolic pathways in pancreatic cancer has been demonstrated as a favorable therapeutic solution. Elgogary et al. found that targeting glutamine metabolism using the glutaminase inhibitor bis2-(5-phenylacetamido-1,2,4-thiadiazol-2-yl) ethyl sulfide (BPTES) encapsulated in nanoparticles effectively shrank pancreatic cancer tumor size and slowed proliferation [34]. They also found, using metabolomics technologies [68], that the tumor cells remaining after glutaminase inhibition were dependent on glycolysis and glycogen synthesis. Elgogary et al. continued the study by using both BPTES nanoparticles and metformin to target both glutamine and glucose metabolisms in pancreatic cancer cells. They discovered that the combined therapy provided enhanced efficacy that inhibited tumor growth significantly more compared to the single treatment of BPTES or metformin alone. This highlights the fact that there is great heterogeneity in 
pancreatic cell metabolism since targeting only glutamine metabolism did not kill all the pancreatic cancer cells, but targeting both glutamine and glucose metabolisms reduced tumor growth with considerably larger efficacy than targeting either glutamine or glucose metabolism alone. More clinical trials must be done in order to see whether combination therapy can assist in pancreatic cancer patient survival. BPTES analogs are being developed and investigated in glutamine-dependent tumors [34, 69-73].

\subsection{Targeting PDACs Based on Metabolic Subtype within the PDAC Tumor Microenvironment}

Increasing evidence within the past decade shows that PDAC heterogeneity can be characterized by the cell's molecular biology and tumor microenvironment. Daemen et al. investigated metabolic profiles of PDACs and defined two subtypes: glycolytic and lipogenic [74]. The glycolytic subtype have elevated gene expression associated with glycolysis and PPP, while the lipogenic subtype have increased gene expression associated with lipogenesis. Daemen et al. found strong associations between the glycolytic subtype with a mesenchymal phenotype and the lipogenic subtype with an epithelial phenotype. These results were consistent with the results of previous PDAC classification by Collison et al. [75]. Daemen et al. proposed a model where glycolytic (mesenchymal) PDACs favor utilizing glucose for glycolysis and lactate production and glutamine for the TCA cycle while lipogenic (epithelial) PDACs favor utilizing glucose for the TCA cycle and de novo lipogenesis. Using xenograft models, Daemen et al. found that patient-derived PDACs characterized as glycolytic were sensitive to LDHA knockdown, while those characterized as lipogenic were mildly affected, demonstrating functionally distinct PDAC subtypes with varying metabolic inhibition sensitivity [74]. It is worth noting that the PDAC subtype classification varies depending on what system each group utilized [76-78]. However, developing personal- ized therapies based on patients' PDAC subtypes appears to be a valid strategy as certain treatments appear to be better equipped in treating specific PDAC subtypes due to phenotypic differences.

\subsection{Autophagy Inhibition via Hydroxychloroquine}

As previously discussed, PDAC cells rely on autophagy. The Yang group furthered their study by taking the known autophagy inhibitor, hydroxychloroquine (HCQ), and demonstrating its antitumor effects in a mouse preclinical model using patient-derived xenografts [48]. Although HCQ has not demonstrated much success as a monotherapy [79], surgical outcomes have improved with combination therapy of HCQ with gemcitabine and nab-paclitaxel as a preoperative treatment in PDAC patients (NCT01978184) [80]. There may be more promise with the combination therapy of HCQ and MEK inhibitors (NCT03825289).

\section{Conclusion}

Pancreatic ductal adenocarcinoma (PDAC) is the fourth leading cause of cancer death in the United States and is expected to be the second largest by 2030 [81, 82]. The deadliness of this disease can be attributed to its metabolic heterogeneity, which develops through cancerous evolution. With that in mind, the investigation of PDAC within the past few years has been exponentially increasing with improved technologies and research methods that allow us to understand these intricate mechanisms better. It has also become clear that the heterogeneity in PDAC metabolism raises questions on how to approach new therapies that take into account a personalized approach to a patient's specific PDAC metabolic characteristics. Exploration of more aspects of pancreatic cells enables scientists and clinicians to better target multiple facets of pancreatic cancer cells, resulting in more effective therapeutic and diagnostic methods. 


\section{References}

1. Hariharan, D., Saied, A., \& Kocher, H. M. (2008). Analysis of mortality rates for gallbladder cancer across the world. HPB: The Official Journal of the International Hepato Pancreato Biliary Association, 10(5), 327-331.

2. Hidalgo, M. (2010). Pancreatic cancer. The New England Journal of Medicine, 362(17), 1605-1617.

3. Weinstein, I. B., \& Joe, A. (2008). Oncogene addiction. Cancer Research, 68(9), 3077-3080; discussion 3080.

4. Verhaak, R. G., et al. (2010). Integrated genomic analysis identifies clinically relevant subtypes of glioblastoma characterized by abnormalities in PDGFRA, IDH1, EGFR, and NF1. Cancer Cell, 17(1), 98-110.

5. Hirschey, M. D., et al. (2015). Dysregulated metabolism contributes to oncogenesis. Seminars in Cancer Biology, 35(Suppl), S129-S150.

6. Birnbaum, D. J., et al. (2011). Genome profiling of pancreatic adenocarcinoma. Genes, Chromosomes \& Cancer, 50(6), 456-465.

7. Son, J., et al. (2013). Glutamine supports pancreatic cancer growth through a KRAS-regulated metabolic pathway. Nature, 496(7443), 101-105.

8. Lyssiotis, C. A., et al. (2013). Pancreatic cancers rely on a novel glutamine metabolism pathway to maintain redox balance. Cell Cycle, 12(13), 1987-1988.

9. Li, T., Copeland, C., \& Le, A. (2021). Glutamine metabolism in cancer. Advances in Experimental Medicine and Biology, 1311, https://doi. org/10.1007/978-3-030-65768-0_2

10. di Magliano, M. P., \& Logsdon, C. D. (2013). Roles for KRAS in pancreatic tumor development and progression. Gastroenterology, 144(6), 1220-1229.

11. Sousa, C. M., \& Kimmelman, A. C. (2014). The complex landscape of pancreatic cancer metabolism. Carcinogenesis, 35(7), 1441-1450.

12. DeNicola, G. M., et al. (2011). Oncogene-induced Nrf2 transcription promotes ROS detoxification and tumorigenesis. Nature, 475(7354), 106-109.

13. Mitsuishi, Y., et al. (2012). Nrf2 redirects glucose and glutamine into anabolic pathways in metabolic reprogramming. Cancer Cell, 22(1), 66-79.

14. Chio, I. I. C., et al. (2016). NRF2 promotes tumor maintenance by modulating mRNA translation in pancreatic cancer. Cell, 166(4), 963-976.

15. Ying, H., et al. (2012). Oncogenic Kras maintains pancreatic tumors through regulation of anabolic glucose metabolism. Cell, 149(3), 656-670.

16. Chaika, N. V., et al. (2012). Differential expression of metabolic genes in tumor and stromal components of primary and metastatic loci in pancreatic adenocarcinoma. PLoS One, 7(3), e32996.

17. Maher, J. C., et al. (2005). Differential sensitivity to 2-deoxy-D-glucose between two pancreatic cell lines correlates with GLUT-1 expression. Pancreas, 30(2), e34-e39.
18. Yun, J., et al. (2009). Glucose deprivation contributes to the development of KRAS pathway mutations in tumor cells. Science, 325(5947), 1555-1559.

19. Bose, S., Zhang, C., \& Le, A. (2021). Glucose metabolism in cancer: The Warburg effect and beyond. Advances in Experimental Medicine and Biology, 1311, https://doi.org/10.1007/978-3-030-65768-0_1

20. Bar-Sagi, D., \& Feramisco, J. R. (1986). Induction of membrane ruffling and fluid-phase pinocytosis in quiescent fibroblasts by Ras proteins. Science, 233(4768), 1061-1068.

21. Park, J. K., et al. (2021). The heterogeneity of lipid metabolism in cancer. Advances in Experimental Medicine and Biology, 1311, https://doi. org/10.1007/978-3-030-65768-0_3

22. Commisso, C., et al. (2013). Macropinocytosis of protein is an amino acid supply route in Ras-transformed cells. Nature, 497(7451), 633-637.

23. Kamphorst, J. J., et al. (2013). Hypoxic and Rastransformed cells support growth by scavenging unsaturated fatty acids from lysophospholipids. Proceedings of the National Academy of Sciences of the United States of America, 110(22), 8882-8887.

24. Guillaumond, F., et al. (2015). Cholesterol uptake disruption, in association with chemotherapy, is a promising combined metabolic therapy for pancreatic adenocarcinoma. Proceedings of the National Academy of Sciences of the United States of America, 112(8), 2473-2478.

25. Ma, X., et al. (2011). The metabolic features of normal pancreas and pancreatic adenocarcinoma: Preliminary result of in vivo proton magnetic resonance spectroscopy at 3.0 T. Journal of Computer Assisted Tomography, 35(5), 539-543.

26. Yabushita, S., et al. (2013). Metabolomic and transcriptomic profiling of human K-ras oncogene transgenic rats with pancreatic ductal adenocarcinomas. Carcinogenesis, 34(6), 1251-1259.

27. Chaika, N. V., et al. (2012). MUC1 mucin stabilizes and activates hypoxia-inducible factor 1 alpha to regulate metabolism in pancreatic cancer. Proceedings of the National Academy of Sciences of the United States of America, 109(34), 13787-13792.

28. Rajeshkumar, N. V., et al. (2015). Therapeutic targeting of the Warburg effect in pancreatic cancer relies on an absence of p53 function. Cancer Research, 75(16), 3355-3364.

29. Dutta, P., et al. (2013). Evaluation of LDH-A and glutaminase inhibition in vivo by hyperpolarized 13C-pyruvate magnetic resonance spectroscopy of tumors. Cancer Research, 73(14), 4190-4195.

30. Le, A., et al. (2010). Inhibition of lactate dehydrogenase A induces oxidative stress and inhibits tumor progression. Proceedings of the National Academy of Sciences of the United States of America, 107(5), 2037-2042.

31. Surget, S., Khoury, M. P., \& Bourdon, J. C. (2013). Uncovering the role of p53 splice variants in human malignancy: A clinical perspective. OncoTargets and Therapy, 7, 57-68. 
32. Bensaad, K., et al. (2006). TIGAR, a p53-inducible regulator of glycolysis and apoptosis. Cell, 126(1), 107-120.

33. Nguyen, T., et al. (2019). Uncovering the role of $\mathrm{N}$-acetyl-aspartyl-glutamate as a glutamate reservoir in cancer. Cell Reports, 27(2), 491-501. e6.

34. Elgogary, A., et al. (2016). Combination therapy with BPTES nanoparticles and metformin targets the metabolic heterogeneity of pancreatic cancer. Proceedings of the National Academy of Sciences of the United States of America, 113(36), E5328-E5336.

35. Udupa, S., et al. (2019). Upregulation of the glutaminase II pathway contributes to glutamate production upon glutaminase 1 inhibition in pancreatic cancer. Proteomics, 19(21-22), e1800451.

36. Kamphorst, J. J., et al. (2015). Human pancreatic cancer tumors are nutrient poor and tumor cells actively scavenge extracellular protein. Cancer Research, 75(3), 544-553.

37. Antonio, M. J., Zhang, C., \& Le, A. (2021). Different tumor microenvironments lead to different metabolic phenotypes. Advances in Experimental Medicine and Biology, 1311, https:// doi.org/10.1007/978-3-030-65768-0_10

38. Provenzano, P. P., et al. (2012). Enzymatic targeting of the stroma ablates physical barriers to treatment of pancreatic ductal adenocarcinoma. Cancer Cell, 21(3), 418-429.

39. Olive, K. P., et al. (2009). Inhibition of Hedgehog signaling enhances delivery of chemotherapy in a mouse model of pancreatic cancer. Science, 324(5933), 1457-1461.

40. Nabi, K., \& Le, A. (2021). The intratumoral heterogeneity of cancer metabolism. Advances in Experimental Medicine and Biology, 1311, https:// doi.org/10.1007/978-3-030-65768-0_11

41. Chu, G. C., et al. (2007). Stromal biology of pancreatic cancer. Journal of Cellular Biochemistry, 101(4), 887-907.

42. Ozdemir, B. C., et al. (2014). Depletion of carcinomaassociated fibroblasts and fibrosis induces immunosuppression and accelerates pancreas cancer with reduced survival. Cancer Cell, 25(6), 719-734.

43. Rhim, A. D., et al. (2014). Stromal elements act to restrain, rather than support, pancreatic ductal adenocarcinoma. Cancer Cell, 25(6), 735-747.

44. Yang, S., et al. (2011). Pancreatic cancers require autophagy for tumor growth. Genes \& Development, 25(7), 717-729.

45. Bryant, K. L., et al. (2019). Combination of ERK and autophagy inhibition as a treatment approach for pancreatic cancer. Nature Medicine, 25(4), 628-640.

46. Guo, J. Y., et al. (2011). Activated Ras requires autophagy to maintain oxidative metabolism and tumorigenesis. Genes \& Development, 25(5), 460-470.

47. Kinsey, C. G., et al. (2019). Protective autophagy elicited by RAF-->MEK-->ERK inhibition suggests a treatment strategy for RAS-driven cancers. Nature Medicine, 25(4), 620-627.
48. Yang, A., et al. (2014). Autophagy is critical for pancreatic tumor growth and progression in tumors with p53 alterations. Cancer Discovery, 4(8), 905-913.

49. Guillaumond, F., et al. (2013). Strengthened glycolysis under hypoxia supports tumor symbiosis and hexosamine biosynthesis in pancreatic adenocarcinoma. Proceedings of the National Academy of Sciences of the United States of America, 110(10), 3919-3924.

50. Hutcheson, J., et al. (2016). Immunologic and metabolic features of pancreatic ductal adenocarcinoma define prognostic subtypes of disease. Clinical Cancer Research, 22(14), 3606-3617.

51. Sousa, C. M., et al. (2016). Pancreatic stellate cells support tumour metabolism through autophagic alanine secretion. Nature, 536(7617), 479-483.

52. Zhao, H., et al. (2016). Tumor microenvironment derived exosomes pleiotropically modulate cancer cell metabolism. eLife, 5, e10250.

53. Sazeides, C., \& Le, A. (2021). Metabolic relationship between cancerassociated fibroblasts and cancer cells. Advances in Experimental Medicine and Biology, 1311, https://doi.org/10.1007/978-3-030-65768-0_14

54. Le, A., et al. (2012). Conceptual framework for cutting the pancreatic cancer fuel supply. Clinical Cancer Research, 18(16), 4285-4290.

55. Weinberg, S. E., \& Chandel, N. S. (2015). Targeting mitochondria metabolism for cancer therapy. Nature Chemical Biology, 11(1), 9-15.

56. Alistar, A., et al. (2017). Safety and tolerability of the first-in-class agent CPI-613 in combination with modified FOLFIRINOX in patients with metastatic pancreatic cancer: A single-centre, open-label, doseescalation, phase 1 trial. The Lancet Oncology, 18(6), 770-778.

57. Stuart, S. D., et al. (2014). A strategically designed small molecule attacks alpha-ketoglutarate dehydrogenase in tumor cells through a redox process. Cancer \& Metabolism, 2(1), 4.

58. Pardee, T. S., et al. (2014). A phase I study of the first-in-class antimitochondrial metabolism agent, CPI-613, in patients with advanced hematologic malignancies. Clinical Cancer Research, 20(20), 5255-5264.

59. Sancho, P., et al. (2015). MYC/PGC-1alpha balance determines the metabolic phenotype and plasticity of pancreatic cancer stem cells. Cell Metabolism, 22(4), 590-605.

60. Lonardo, E., et al. (2013). Metformin targets the metabolic Achilles heel of human pancreatic cancer stem cells. PLoS One, 8(10), e76518.

61. Evans, J. M., et al. (2005). Metformin and reduced risk of cancer in diabetic patients. BMJ, 330(7503), 1304-1305.

62. Sadeghi, N., et al. (2012). Metformin use is associated with better survival of diabetic patients with pancreatic cancer. Clinical Cancer Research, 18(10), 2905-2912.

63. Viale, A., et al. (2014). Oncogene ablation-resistant pancreatic cancer cells depend on mitochondrial function. Nature, 514(7524), 628-632. 
64. Kordes, S., et al. (2015). Metformin in patients with advanced pancreatic cancer: A double-blind, randomised, placebo-controlled phase 2 trial. The Lancet Oncology, 16(7), 839-847.

65. Reni, M., et al. (2016). (Ir)relevance of metformin treatment in patients with metastatic pancreatic cancer: An open-label, randomized phase II trial. Clinical Cancer Research, 22(5), 1076-1085.

66. Yang, Y. X., \& Rustgi, A. K. (2016). Impact of metformin on advanced pancreatic cancer survival: Too little, too late? Clinical Cancer Research, 22(5), 1031-1033.

67. Cheng, G., et al. (2016). Mitochondria-targeted analogues of metformin exhibit enhanced antiproliferative and radiosensitizing effects in pancreatic cancer cells. Cancer Research, 76(13), 3904-3915.

68. Hoang, G., Udupa, S., \& Le, A. (2019). Application of metabolomics technologies toward cancer prognosis and therapy. International Review of Cell and Molecular Biology, 347, 191-223.

69. Gross, M. I., et al. (2014). Antitumor activity of the glutaminase inhibitor $\mathrm{CB}-839$ in triple-negative breast cancer. Molecular Cancer Therapeutics, 13(4), 890-901.

70. Robinson, M. M., et al. (2007). Novel mechanism of inhibition of rat kidney-type glutaminase by bis-2-(5phenylacetamido-1,2,4-thiadiazol-2-yl)ethyl sulfide (BPTES). The Biochemical Journal, 406(3), 407-414.

71. Wang, J. B., et al. (2010). Targeting mitochondrial glutaminase activity inhibits oncogenic transformation. Cancer Cell, 18(3), 207-219.

72. Willis, R. C., \& Seegmiller, J. E. (1977). The inhibition by 6-diazo-5-oxo-1-norleucine of glutamine catabolism of the cultured human lymphoblast. Journal of Cellular Physiology, 93(3), 375-382.

73. Zimmermann, S. C., et al. (2016). Allosteric glutaminase inhibitors based on a 1,4-di(5-amino-1,3,4- thiadiazol-2-yl)butane scaffold. ACS Medicinal Chemistry Letters, 7(5), 520-524.

74. Daemen, A., et al. (2015). Metabolite profiling stratifies pancreatic ductal adenocarcinomas into subtypes with distinct sensitivities to metabolic inhibitors. Proceedings of the National Academy of Sciences of the United States of America, 112(32), E4410-E4417.

75. Collisson, E. A., et al. (2011). Subtypes of pancreatic ductal adenocarcinoma and their differing responses to therapy. Nature Medicine, 17(4), 500-503.

76. Bailey, P., et al. (2016). Genomic analyses identify molecular subtypes of pancreatic cancer. Nature, 531(7592), 47-52.

77. Moffitt, R. A., et al. (2015). Virtual microdissection identifies distinct tumor- and stroma-specific subtypes of pancreatic ductal adenocarcinoma. Nature Genetics, 47(10), 1168-1178.

78. Puleo, F., et al. (2018). Stratification of pancreatic ductal adenocarcinomas based on tumor and microenvironment features. Gastroenterology, 155(6), 19992013. e3.

79. Wolpin, B. M., et al. (2014). Phase II and pharmacodynamic study of autophagy inhibition using hydroxychloroquine in patients with metastatic pancreatic adenocarcinoma. The Oncologist, 19(6), 637-638.

80. Bryant, K. L., \& Der, C. J. (2019). Blocking autophagy to starve pancreatic cancer. Nature Reviews. Molecular Cell Biology, 20(5), 265.

81. Rahib, L., et al. (2014). Projecting cancer incidence and deaths to 2030: The unexpected burden of thyroid, liver, and pancreas cancers in the United States. Cancer Research, 74(11), 2913-2921.

82. Rossi, M. L., Rehman, A. A., \& Gondi, C. S. (2014). Therapeutic options for the management of pancreatic cancer. World Journal of Gastroenterology, 20(32), 11142-11159.

Open Access This chapter is licensed under the terms of the Creative Commons Attribution 4.0 International License (http://creativecommons.org/licenses/by/4.0/), which permits use, sharing, adaptation, distribution and reproduction in any medium or format, as long as you give appropriate credit to the original author(s) and the source, provide a link to the Creative Commons license and indicate if changes were made.

The images or other third party material in this chapter are included in the chapter's Creative Commons license, unless indicated otherwise in a credit line to the material. If material is not included in the chapter's Creative Commons license and your intended use is not permitted by statutory regulation or exceeds the permitted use, you will need to obtain permission directly from the copyright holder.

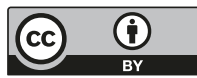




\title{
The Heterogeneity of Breast Cancer Metabolism
}

\author{
Jessica Tan and Anne Le
}

\section{Keywords}

Breast cancer - Estrogen receptor status ·

Metabolic fingerprint - Choline metabolism .

Estrogen metabolism - Serine biosynthesis .

Glycolytic upregulation · Intratumoral

heterogeneity $\cdot$ Metabolic adaptivity

\section{Abbreviations}

3HP 3-Phosphohydroxypyruvate

3PG 3-Phosphoglycerate

CK Choline kinase

COMT Catechol-O-methyltransferase

D-2HG D-2-Hydroxyglutarate

E2 17b-Estradiol

ECM Extracellular matrix

EMT Epithelial-to-mesenchymal transition

ER Estrogen receptor

GLUT Glucose transporter

GSTP Glutathione S-transferase P
HER2 Human epidermal growth factor receptor 2

LCMS Liquid chromatography mass spectrometry

MS Mass spectrometry

NADPH Nicotinamide adenine dinucleotide phosphate

NMR Nuclear magnetic resonance

PCho Phosphocholine

PGC-1a Peroxisome proliferation-activated receptor gamma coactivator-1a

PHGDH Phosphoglycerate dehydrogenase

PPP Pentose phosphate pathway

PR Progesterone receptor

PSAT1 Phosphoserine aminotransferase 1

PSPH Phosphoserine phosphatase

PtdCho Phosphatidylcholine

ROS Reactive oxygen species

TCA Tricarboxylic acid

TNBC Triple-negative breast cancer

$\alpha-\mathrm{KG} \quad$ Alpha-ketoglutarate

J. Tan

Wayne State University School of Medicine,

Detroit, MI, USA

\author{
A. Le $(\bowtie)$ \\ Department of Pathology and Oncology, Johns \\ Hopkins University School of Medicine, \\ Baltimore, MD, USA \\ Department of Chemical and Biomolecular \\ Engineering, Johns Hopkins University Whiting \\ School of Engineering, Baltimore, MD, USA \\ e-mail: annele@jhmi.edu
}




\section{Key Points}

- Aberrant metabolic pathways present in breast cancer contribute to breast cancer heterogeneity.

- Differences in glycolytic upregulation among breast cancer subtypes can be attributed to GLUT expression.

- Choline metabolism in breast cancer is strongly associated with tumor grades.

- Metabolic profiling of breast cancers can be used for clinical breast cancer diagnosis and prediction of recurrence or metastasis.

- Breast cancer metabolism has heterogeneous and adaptive characteristics from a spatial and temporal basis.

- Metabolic adaptability confers chemotherapyresistant phenotypes and promotes tumor evolution.

\section{Introduction}

Despite advances in screening, therapy, and surveillance that have improved patient survival rates, breast cancer is still the most commonly diagnosed cancer and the second leading cause of cancer mortality among women [1]. Breast cancer is a highly heterogeneous disease rooted in a genetic basis, influenced by extrinsic stimuli, and reflected in clinical behavior. The diversity of breast cancer hormone receptor status and the expression of surface molecules have guided therapy decisions for decades; however, subtype-specific treatment often yields diverse responses due to varying tumor evolution and malignant potential. Although the mechanisms behind breast cancer heterogeneity is not well understood, available evidence suggests that studying breast cancer metabolism has the potential to provide valuable insights into the causes of these variations as well as viable targets for intervention.

\section{Aberrant Metabolic Pathways Present in Breast Cancer Contribute to Breast Cancer Heterogeneity (Fig. 1)}

In order to sustain tumorigenic proliferation, cancer cells exploit diverse metabolic pathways. The diversity of hormone receptors present within breast cancer cells is classified into different subtypes. Breast cancers with hormone-positive receptors such as estrogen receptors (ER) and progesterone receptors (PR) rely on their respective hormones for growth. Patients with HER2+ breast cancer have overexpression of human epidermal growth factor receptor 2 (HER2). Patients negative for all three receptors are considered to have triple-negative breast cancer (TNBC) - the most heterogeneous molecular profile. This diversity, in turn, reflects the different metabolic phenotypes of breast cancer. Some of these core metabolic aberrations have fundamental effects on breast cancer tumorigenicity and offer rationale behind the aggressiveness of specific subtypes. Tumor evolution results in the reprogramming of cell metabolism in order to adapt to support cell proliferation. Specific mutations in oncogenes and tumor-suppressor genes are hypothesized to cause metabolic reprogramming within different breast cancer subtypes. Although several mutations are commonly seen in breast cancers, they appear in various combinations that are reflective of the diverse metabolic behaviors of breast cancers. For example, mutations in $B R A F, K R A S$, and HRAS were found to be metabolic regulators of TNBC [2]. These genetic alterations are known to regulate glutamine metabolism, which renders cancer cells dependent on glutamine for proliferation and survival $[3,4]$. The BRCA1 mutation is a good example of how genetic alterations lend to specific metabolic phenotypes that promote tumorigenesis. A study by Martinez-Outschoorn et al. showed that loss-of-function mutations in the 


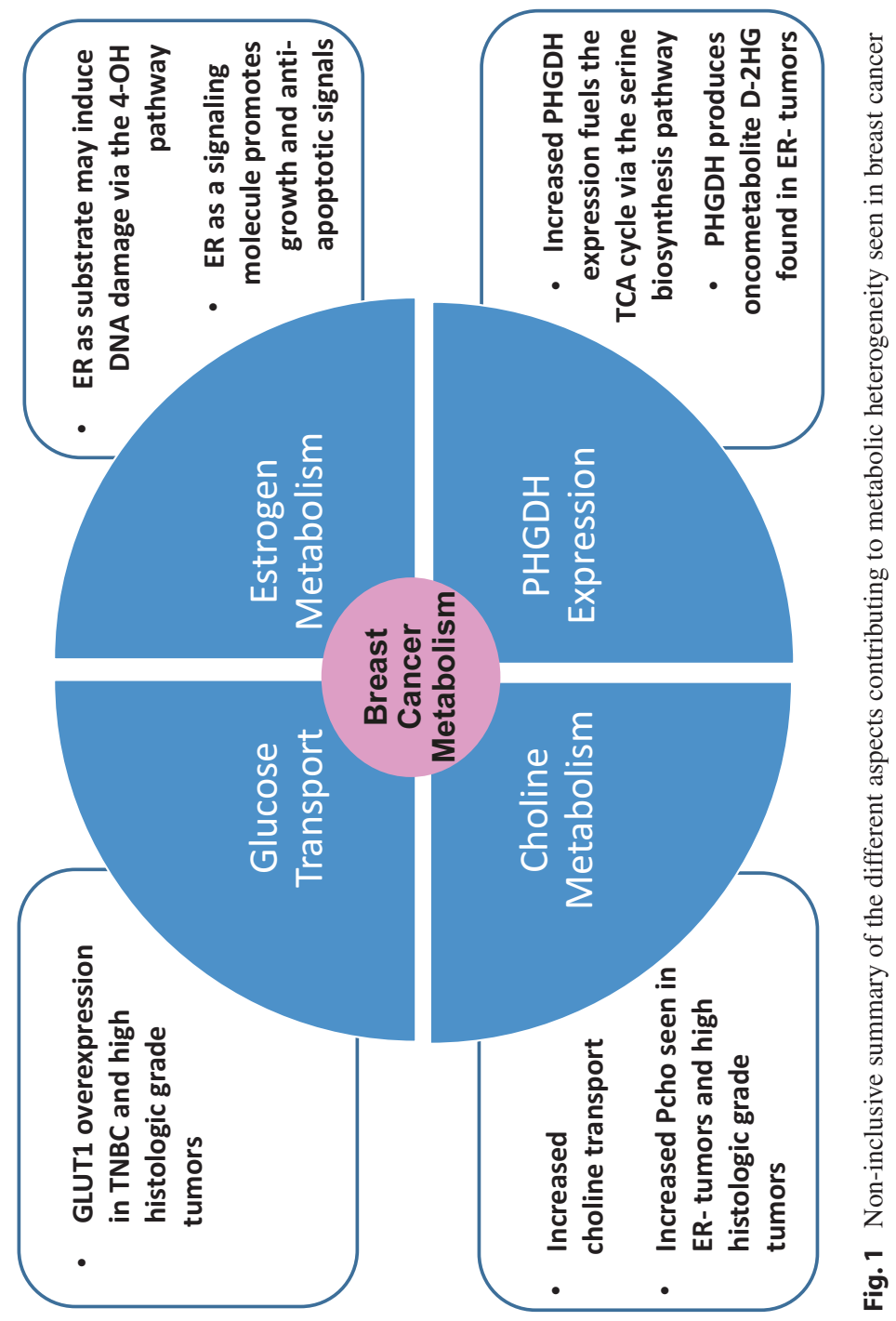


BRCA1 tumor-suppressor gene resulted in the production of hydrogen peroxide and oxidative stress in epithelial breast cancer cells and stromal fibroblasts [5]. This loss of function also causes elevated expression of monocarboxylate transporter 4 (a functional marker of oxidative stress and glycolytic activity) to shuttle L-lactate out of cells.

Furthermore, the loss of caveolin-1 in cancer-associated fibroblasts associated with elevated production of reactive oxygen species (ROS) and increased glycolysis in stromal cells, both of which play a fundamental role in tumorigenesis [6]. This encourages therapeutic targeting of cancer-associated fibroblasts that favor cancer progression [7]. Mutations in $B R C A 1$ are marked by high rates of proliferation and substantial cellular inflammation. This study suggests that antioxidant agents present promising therapies for BRCA1-mutated breast cancer.

\subsection{Differences in Glycolytic Upregulation Among Breast Cancer Subtypes Can Be Attributed to Glucose Transporter (GLUT) Expression}

First postulated by Otto Warburg in 1927 [8] and firmly established in the literature thereafter, a hallmark of cancer malignancy is an upregulation in aerobic glycolysis even in the presence of oxygen, known as the Warburg effect $[9,10]$. Lactate dehydrogenase A, a key enzyme of the Warburg effect that catalyzes the conversion of pyruvate to lactate, has been a studied target in several cancers [11-13]. Breast cancer tumors are no exception to the Warburg effect; however, there are variations in glycolytic rates and metaboliterelated protein expression among breast cancer subtypes that correlate with tumor aggressiveness. Previous in vitro studies have first observed that the glucose-dependent MCF-7 cell line is more sensitive to FX11, a lactate dehydrogenase A inhibitor, than the non-glucose-dependent
MDA-MB-453 cell line [11]. Another in vitro study found that noninvasive breast cancer cell lines showed a significantly lower rate of glucose intake compared to more aggressive, metastatic cells [14]. Higher rates of glucose uptake are accompanied by altered gene expression and translation of metabolism-related proteins as well. Glucose transporter (GLUT) expression has been studied extensively in breast cancer. GLUTs are integral transmembrane proteins that facilitate glucose delivery across the plasma membrane. They serve as a rate-limiting step that controls the amount of glucose accessible to the cell [15]. Studies have shown that different isoforms of GLUTs have been detected and/or overexpressed in breast cancer cells. Different GLUT expression patterns are found to be associated with various pathological grades and tumor aggressiveness in patient-derived samples. Choi et al. discovered that GLUT1, one of the isoforms of the GLUT family, had the highest expression in the TNBC subtype and tumors with high histologic grade [16]. As a result of increased glucose uptake, the increased rate of glycolysis subjects the cell to intracellular lactic acidosis-leading to cell death. Interestingly, the same group showed that TNBC had the highest expression of carbonic anhydrase IX, an enzyme that prevents acidosis and provides TNBC with an acid-resistant phenotype [16], suggesting that aggressive breast cancer subtypes adopt metabolic phenotypes able to suppress apoptosis. GLUT1 overexpression has also been linked to invasiveness in breast cancer [17].

The link between metabolic reprogramming and protein expression offers an adaptive advantage that contributes to a level of aggression specific to certain subtypes of breast cancer like TNBC, making them characteristically resilient and harder to treat.

\subsection{Choline Metabolism in Breast Cancer Is Strongly Associated with Tumor Grades}

The deregulation of choline metabolism and elevated levels of choline-containing compounds are 
frequently observed in breast cancer progression [18-21]. Choline plays an important role in supplying methyl groups through its metabolism and is essential for cellular structure as a precursor of phospholipids. Choline metabolism in breast tissue is distributed between two central pathways: (1) the biosynthesis of phosphatidylcholine (PtdCho) known as the Kennedy pathway and (2) the oxidation to betaine, a methyl group donor in many methylation reactions. A study by KatzBrull et al. revealed that breast cancer cells exhibited a higher choline transport rate compared to normal breast cells, and a majority of the choline was converted to phosphocholine (Pcho) through the Kennedy pathway while around approximately 25\% was oxidized to betaine [19]. Although levels of phospholipid-related metabolites are enhanced in most breast cancers [22], significantly higher levels of Pcho were found to be associated with ER tumors and the more aggressive histologic grade 3 tumors [23]. Because of this, choline-containing compounds have often been seen as biomarkers for breast tumor malignancy. Oncogenic expression of choline kinase (CK), the enzyme responsible for the conversion of choline to Pcho, is responsible for elevated levels of Pcho in breast cancer cells [19]. Furthermore, CK also showed a strong association with high histologic grade and ER- subtypes [24]. For this reason, $\mathrm{CK}$ is an attractive antitumor target for subsequent studies. Whether or not choline metabolism represents an agent of disease progression or merely a marker for transformation has still not been defined. CK inhibitors blocking choline metabolism have shown promising antitumor results. A study by Rodríguez-González et al. discovered that blocking the enzyme had no effect on normal cells but disrupted phospholipid production in tumor cells-resulting in apoptosis due to the accumulation of cytotoxic ceramide, the simplest class of sphingolipids [25].

\section{3 \\ Different Roles of Estrogen in Estrogen Metabolism and ER Binding Promote Breast Cancer Tumorigenicity}

Endogenous estrogens and their metabolism have been linked to breast carcinogenesis, especially in postmenopausal women [26]. 17b-Estradiol (E2), the main estrogen in breast tissue, acts as both a ligand for ER and a substrate in metabolism-roles which contribute to estrogen as a carcinogen. The mechanism of estrogen carcinogenesis is a combination of ER signaling and estrogen metabolism.

ERs, when activated, are responsible for the mediation of many downstream signaling pathways that function as transcription factors promoting cancer development [27]. In addition, ER signaling interacts with growth factor receptors and other signaling molecules to promote growth and anti-apoptotic signals [28]. ER activation has also been shown to promote downstream reprogramming in choline metabolism, an aberration in breast cancer [29].

As a substrate, the metabolism of estrogen through the 4-hydroxylation pathway produces specific catechol estrogens and estrogen quinones known to be carcinogenic. Estrogen is hydroxylated by cytochrome P450 enzymes and shuttled into three main pathways depending on the three different carbons hydroxylated: C2, C3, and $\mathrm{C} 16$. The catechol estrogens (2-OH E1, 2-OH E2, 4-OH E1, 4-OH E2) are either methylated by catechol-O-transferase (COMT), thereby reducing their mutational potential, or oxidized further to semiquinones or quinones. 4-OH catechol estrogen, when oxidized to a reactive estrogen quinone, leads to DNA damage by forming unstable DNA adducts between adenine and guanine nucleotides [30, 31]. 
Mutations caused by this mechanism have the potential to initiate breast cancer or increase cancer risk. In contrast, metabolites formed through the 2-OH pathway form stable DNA adducts and are anticarcinogenic-dubbing the 2-OH metabolites as "the good estrogen" in some cases [32]. Protective mechanisms such as estrogen quinone conjugation with glutathione via glutathione S-transferase P (GSTP) help lower the risk of cancerous mutations by detoxifying the estrogen quinones [30]. However, estrogenic imbalances lead to competition between the pathway forming the unstable DNA adducts and the detoxification of its cancer-promoting substrates [30]. Accordingly, hormone therapy for breast cancer has targeted ER+ subtypes with drugs such as tamoxifen, which acts as a competitive inhibitor that prevents estrogen from binding to the ER. Another important class of drugs inhibits aromatase, an important rate-limiting enzyme that converts androgens to estrogens, to lower estrogen levels in the body.

\subsection{PHGDH Overexpression in Serine Biosynthesis Fuels TCA Anaplerosis}

Serine biosynthesis is an essential pathway for breast cancer progression in specific subsets of breast tumors. Using RNAi-based loss-offunction screening, Possemato et al. identified phosphoglycerate dehydrogenase (PHGDH) in breast cancer with enhanced protein levels in $70 \%$ of aggressive ER- subtypes [33]. PHGDH catalyzes the committed-limiting step that oxidizes 3-phosphoglycerate (3PG) to 3-phosphohydroxypyruvate (3HP) substrates in the serine synthesis pathway. Enhanced PHGDH expression was associated with increased serine synthesis and glutamine uptake. Suppression of PHGDH expression led to a significant decrease in cell proliferation but did not affect intracellular serine levels; instead, researchers found a resulting drop in phosphoserine aminotransferase 1 (PSAT1)-dependent alpha-ketoglutarate $(\alpha-K G)$, an output of the serine pathway [33]. In cancer cells with overexpression of PHGDH, the serine synthesis pathway plays an important role in tricarboxylic acid (TCA) cycle anaplerosissupplying $\alpha-K G$ to support cell proliferation [33]. In addition, suppression of PSAT1 and phosphoserine phosphatase (PSPH) enzymes downstream in the serine pathway inhibits cell proliferation in PHGDH-enhanced cell lines as well [33]. Subsequent studies have revealed that in addition to 3PG oxidation, PHGDH also catalyzes the reduction of $\alpha-\mathrm{KG}$ to D-2-hydroxyglutarate (D-2HG) [34], an established oncometabolite [35, 36]. D-2HG acted as a competitive inhibitor of $\alpha$-KG-dependent dioxygenases, resulting in aberrations in histone methylation and DNA hypermethylation [37]. High levels of D-2HG and $\mathrm{N}$-acetyl-aspartate were found to accumulate preferentially in ERand basal-like tumors, which may contribute to their aggressive phenotypes [36] in contrast to the mixed effects on glioblastoma [38] and other cancers. In vitro experiments revealed that accumulation of D-2HG is associated with increased cell proliferation and inhibited apoptosis [36]; however, the oncogenic effects of D-2HG on breast cancer still need to be defined. Because of its deregulated expression and oncogenic effects, PHGDH is considered a promising target for therapy in breast cancers that exhibit PHGDH overexpression. Although a preliminary PHGDH inhibitor has been recently developed [39], PHGDH-targeted therapy is still in its infancy.

Using a novel computational method, Jerby et al. contributed further evidence that the metabolic profiles of ER+ and ER - subtypes are vastly different [40]. The stoichiometric analysis revealed serine metabolism to be coupled with glutamine uptake [40]. ER+ tumors exhibit a stronger preference for glutamine biosynthesis and secretion than ER- tumors [40]. In addition, their model identified ER+ phenotypes as having more capacity to convert glucose to lactate than ER - tumors. Due to higher rates of serine metabolism, ER- subtypes are rationalized to preferably divert 3PG toward serine metabolism via PHGDH to exploit alternative pathways for glutaminolysis [40]. In addition, a high $M Y C$ overexpression [41, 42], and low thioredoxin-interacting protein expression, an inhibitor of glucose utiliza- 

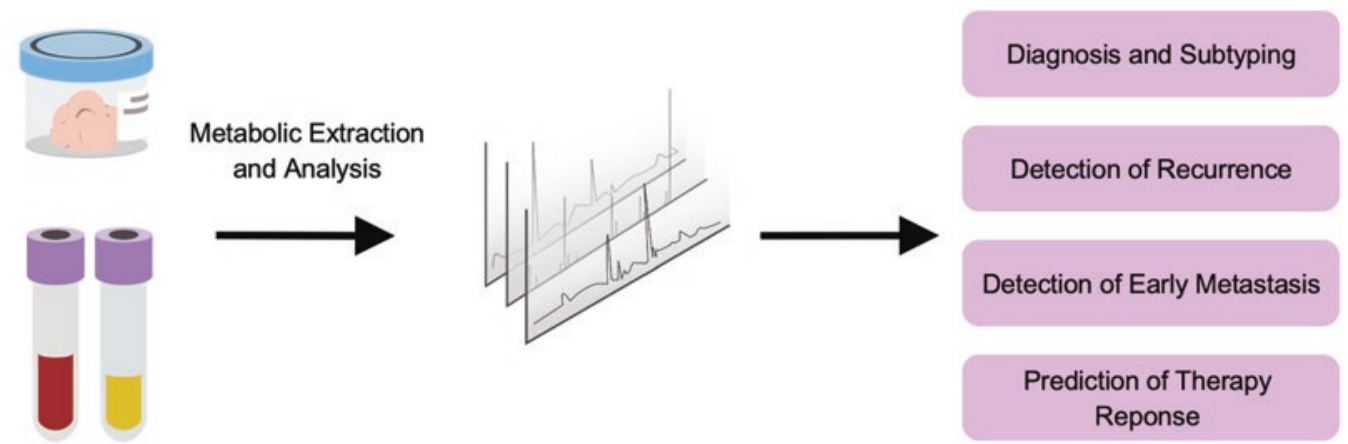

Fig. 2 The potential clinical applications of metabolic profiling for breast cancer using patient tumor, plasma, and serum samples

tion, was found to be a characteristic gene signature of TNBC and no other subtypes [43].

\section{The Clinical Applications of Metabolic Profiling}

Metabolic profiling has garnered much research interest within the past decade [44]. Although the mechanisms behind breast cancer transformation have not been firmly established, changes in tumor evolution have been investigated through metabolic variation. The exploitation of these metabolic signatures has the potential to improve clinical results through diagnosis confirmation, early detection, and prediction of disease progression [45, 46] (Fig. 2).

\subsection{Breast Cancer Diagnosis and Subtyping Using Metabolomics}

Studies have used metabolic profiling for the general diagnosis of breast cancer-using different techniques to build prediction models that distinguish specific metabolic fingerprints of breast cancer hormone receptor status, histologic grade, and axillary lymphatic spread [47-49]. Jove et al. used a combination of random forest classification and multivariate statistics to identify combinations of metabolites that were used to distinguish breast cancer plasma samples from healthy control samples [47]. On the other hand, Huang et al. sought out a model more tolerant of breast cancer heterogeneity by following metabolic pathways rather than metabolite-based biomarkers for early diagnosis of breast cancer [48]. Other studies have used metabolic profiling to build models to distinguish breast cancer stages [49] and levels of malignancy [50].

\subsection{Metabolic Profiling as a Strategy for Prediction of Recurrence in Breast Cancer}

Recurrence after initial therapy causes significant morbidity and mortality in breast cancer patients. Current methods for detecting recurrences such as medical imaging and serum tumor markers are not considered specific enough to be routinely recommended; therefore, there is still much room for improvement. A combination of nuclear magnetic resonance (NMR) and mass spectroscopy (MS) analysis and multivariate statistics on patient serum samples has been used to explore potential metabolic profiles sensitive to cancer recurrence [51]. Asiago et al. developed a prediction model built upon 11 biomarkers that correctly detected $55 \%$ of patients with breast cancer recurrence an average of 13 months prior to their clinical diagnosis using serum samples [51]. Although there is vast room for improvement on 
more specific and accurate models for early detection of recurrence, metabolic profiling of serum can be viewed as a promising noninvasive method for breast cancer surveillance.

\subsection{Metabolic Fingerprinting in Breast Cancer Metastasis}

Oakman et al. identified a preliminary metabolic fingerprint from patient serum samples that detected early and metastatic disease in breast cancer patients. In their study, higher levels of phenylalanine, glucose, proline, lysine, and $N$-acetyl cysteine and lower levels of lipids contributed to the metabolic profile of metastatic individuals [52]. Jobard et al. used similar serum NMR analysis to identify metabolic profiles between localized and metastatic breast cancer. They found eight statistically significant elevations of metabolite biomarkers in metastatic disease: histidine, acetoacetate, glycerol, pyruvate, $\mathrm{N}$-acetyl glycoproteins, mannose, glutamate, and phenylalanine [53]. Although there are differences in biomarkers between the two studies, it is notable that the same trends of variation in glucose concentration and lowered lipid levels were seen between early and metastatic breast cancer [53]. Defining an accurate metabolic fingerprint specific across all metastatic breast cancers is a challenge due to the variability and high mutational load of metastatic disease. Under changing tumor microenvironments [54], metastatic breast cancer cells readily switch between glycolysis and oxidative phosphorylation [55]. The metabolic plasticity of metastatic breast cancer may contribute to the inconsistencies of biomarkers across different tumors. However, studies attempting to identify these metabolic patterns provide great insights into the general characteristics of advanced diseases.

\subsection{Prediction of Response to Therapy Based on Metabolic Phenotypes}

Metabolic fingerprinting has also been used to predict responses to therapy and drug resistance. Using a combination of NMR and liquid chromatography-mass spectrometry (LC-MS), Wei et al. were able to identify four altered metabolites (threonine, glutamine, isoleucine, and linolenic acid) as indicators of adjuvant chemotherapy response within breast cancer [56]. A prediction model derived from these metabolic markers was able to distinguish between complete, partial, and no tumor response to chemotherapy in a neoadjuvant setting using patient samples [56]. The model was able to correctly identify $80 \%$ of patients whose tumors did not show a complete pathologic response to chemotherapy [56]. Collectively, these studies highlight the potential impact of metabolic profiling on the integration of metabolomics into clinical practice. Further advancements in profiling could improve diagnosis and early detection or at least offer confirmation in the treatment of breast cancer quickly and at low cost. Although most of the prediction models and metabolic phenotypes presented in these studies are in their preliminary stages, improvements could make way for more individualized treatments specific to each patient.

\section{$5 \quad$ Additional Perspectives on Breast Cancer Heterogeneity}

\subsection{Spatial Pathogenesis Observed in Breast Cancer Metabolism}

Metabolic heterogeneity within a single tumor adds another layer of complexity when trying to understand the dynamic processes of breast cancer metabolism [57]. Several studies have identified metabolic distinctions between breast tumor periphery and center. A study by Xu et al. analyzed the mitochondrial redox states of breast cancer xenografts of varying aggressiveness. In general, the researchers found more oxidized metabolic states in central regions and more reduced states in peripheral regions of the tumors [58]. The tumors also exhibited higher glucose uptake and NADH levels in tumor peripheries compared to the centers [58]. The authors presumed this was due to higher substrate availability at the peripheries 
from the tumor microenvironment. Furthermore, higher degrees of metabolic heterogeneity were consistently observed in larger and higher staged tumors $[58,59]$. When comparing metabolic profiles of clinical breast tumor samples, studies have observed higher levels of Pcho and phosphoethanolamine in the tumor core compared to tumor periphery [60]. Lactate and pyruvate were observed in higher levels in the tumor periphery compared to the tumor core [61]. Because the tumor periphery has direct interactions with the tumor microenvironment compared to the center, the differences in inputs translate into differences in metabolic phenotypes. In normal breast anatomy, the epithelia receive similar concentrations of oxygen, growth factors, and nutrients. The anatomic disorganization caused by breast cancer pathology alters the tumor microenvironment and intratumoral metabolism [57, 62]. It is unclear whether these observed differences are solely due to extrinsic inputs, genetic manifestations, or perhaps an interplay between both.

\subsection{Temporal Pathogenesis Observed in Breast Cancer Metabolism: Metabolic Differences Between Early Stage and Advanced Stage}

Temporal pathogenesis refers to tumor progression over time, starting from a single cancer cell to the formation of a primary tumor and then metastatic spread. The epithelial-to-mesenchymal transition (EMT) is one of the prerequisites of early metastasis. It describes the transition in which epithelial cells lose their polarity and cell adhesions to become mesenchymal cells with migratory properties. Cancer cells detach from their extracellular matrix (ECM) when they decide to metastasize. It has been shown in vivo that mammary epithelial cells with lost ECM attachment are unable to survive due to ATP deficiency from glucose deprivation [63]. ECM detachment is also accompanied by increased ROS [63, 64]. Overexpression of the HER2 oncogene rescued these cells by restoring glucose uptake and reducing ROS through the oxidative pentose phosphate pathway (PPP) [63]. Nicotinamide adenine dinucleotide phosphate (NADPH), a product of the PPP, serves as a reducing agent able to combat oxidative stress. The study by Schafer et al. also showed that the treatment of antioxidants alone was able to rescue matrix-detached cells-identifying oxidative resistance as an important property needed for metastatic migration [63]. Once cancer cells detach from the ECM, they will need to survive the journey in the oxidizing bloodstream. Many cells will undergo apoptosis in this environment, but the cells that acquire oxidative resistance have the adaptive advantage to metastasize. Although aerobic glycolysis is the most wellknown hallmark of cancer metabolism [10], research has also identified the importance of oxidative phosphorylation in cancer progression as well [65]. Increased mitochondrial biogenesis and respiration have been observed in cancer cell metastases through the modulation of peroxisome proliferation-activated receptor gamma coactivator-1a (PGC-1a) — a regulator of mitochondrial biogenesis and energy metabolism [66]. This is corroborated by the correlation between PGC-1a expression and formation of distant metastasis from patient breast tumors and breast cancer cell lines [66]. These changes in metabolic phenotype seen in migrating breast cancer cells are examples that highlight the importance of metabolic plasticity for cancer progression.

\subsection{Metabolic Heterogeneity Influences Effective Breast Cancer Drug Treatment}

In silico modeling of tumor progression by Robertson-Tessi et al. proposed that early stages of tumor growth have a stratified composition. Tumor centers have higher glycolytic activity and are, therefore, more aggressive compared to the periphery [67]. It is argued that cancer treatments should decrease or slow selective pressures in cells through the maintenance of less aggressive cancer cells within a tumor rather than aiming for eradication $[67,68]$. This concept is demonstrated in the antiangiogenic treatment of breast cancer. Antiangiogenic therapies 


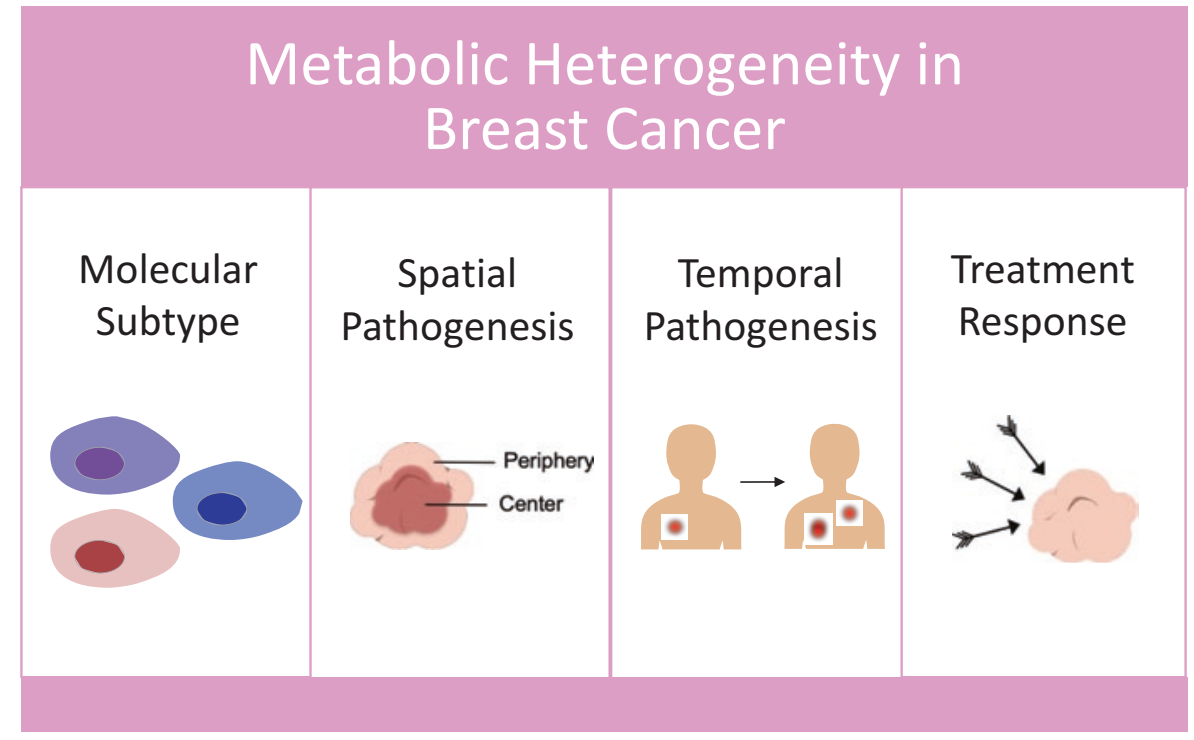

Fig. 3 Summary of the different levels of metabolic heterogeneity seen in breast cancer. Metabolic heterogeneity is demonstrated between molecular subtypes of breast cancer, between tumor core and periphery, in different stages of cancer progression, and in response to selective pressures from clinical treatment

aim to starve cancer cells of oxygen and nutrients by inhibiting tumor vascularization, creating pockets of intratumoral hypoxia. Cutting off nutrient supply in this way may be effective in stopping cancer cell growth, but it may also select for cells that are able to alter metabolism to adapt to hypoxic conditions [54], resulting in a drug-resistant phenotype. Although antiangiogenic therapies in breast cancer patients have been able to lengthen progression-free survival, data has shown that it does little to improve overall patient survival [69]. Aggressive relapse and enhanced metastasis in treated patients are not uncommon either [70]. Conley et al. were able to show that breast cancer xenografts, when treated with antiangiogenic drugs, developed hypoxiadriven cancer stem cell stimulation, which promoted tumorigenesis-opposite to the intended effect [70]. It would seem that the goal of chemotherapy is to halt tumorigenesis and shrink existing tumor populations as quickly and effectively as possible by delivering the drug at the highest dosage allowed. This objective, however, is a double-edged sword: if treatment is too aggressive, it puts selective pressure on the cells to enhance drug-resistant phenotypes that, in turn, escalate cancer progression. The adaptive nature of cancer metabolism is a significant obstacle for creating effective drug therapies. An effective treatment aims to find the delicate balance of delivering maximum cytotoxic effects while avoiding selective resistance.

\section{Conclusion}

Metabolomics serves as an essential utility in breast cancer research by offering a perspective that represents the net interactions between the tumor, the host, and the environment and within the tumor itself. The metabolic nuances across different breast cancer subtypes and treatment timelines can be taken advantage of when thinking about potential prognostic markers, prediction models, and mechanisms involved with breast cancer. Metabolic heterogeneity in breast cancer can be seen within a single tumor and in the different stages of the tumor's progression (Fig. 3). Understanding these dynamic processes and applying them to drug discovery and clinical practice have the potential to improve the lives of not only breast cancer patients but also all cancer patients. 


\section{References}

1. Gutierrez, T., et al. (2013). IL-21 promotes the production of anti-DNA IgG but is dispensable for kidney damage in lyn(-/-) mice. European Journal of Immunology, 43(2), 382-393.

2. Hu, X., et al. (2009). Genetic alterations and oncogenic pathways associated with breast cancer subtypes. Molecular Cancer Research, 7(4), 511-522.

3. Li, T., Copeland, C., \& Le, A. (2021). Glutamine metabolism in cancer. Advances in Experimental Medicine and Biology, 1311, https://doi. org/10.1007/978-3-030-65768-0_2.

4. Elgogary, A., et al. (2016). Combination therapy with BPTES nanoparticles and metformin targets the metabolic heterogeneity of pancreatic cancer. Proceedings of the National Academy of Sciences of the United States of America, 113(36), E5328-E5336.

5. Martinez-Outschoorn, U. E., et al. (2012). BRCA1 mutations drive oxidative stress and glycolysis in the tumor microenvironment: Implications for breast cancer prevention with antioxidant therapies. Cell Cycle, 11(23), 4402-4413.

6. Sazeides, C., \& Le, A. (2021). Metabolic relationship between cancer-associated fibroblasts and cancer cells. Advances in Experimental Medicine and Biology, 1311, https://doi.org/10.1007/978-3-030-65768-0_14.

7. Jung, J. G., \& Le, A. (2021). Targeting metabolic cross talk between cancer cells and cancerassociated fibroblasts. Advances in Experimental Medicine and Biology, 1311, https://doi. org/10.1007/978-3-030-65768-0_15.

8. Warburg, O., Wind, F., \& Negelein, E. (1927). The metabolism of tumors in the body. The Journal of General Physiology, 8(6), 519-530.

9. Vander Heiden, M. G., Cantley, L. C., \& Thompson, C. B. (2009). Understanding the Warburg effect: The metabolic requirements of cell proliferation. Science, 324(5930), 1029-1033.

10. Bose, S., Zhang, C., \& Le, A. (2021). Glucose metabolism in cancer: The Warburg effect and beyond. Advances in Experimental Medicine and Biology, 1311, https://doi.org/10.1007/978-3-030-65768-0_1.

11. Le, A., et al. (2010). Inhibition of lactate dehydrogenase A induces oxidative stress and inhibits tumor progression. Proceedings of the National Academy of Sciences of the United States of America, 107(5), 2037-2042.

12. Rajeshkumar, N. V., et al. (2015). Therapeutic targeting of the Warburg effect in pancreatic cancer relies on an absence of p53 function. Cancer Research, 75(16), 3355-3364.

13. Dutta, P., et al. (2013). Evaluation of LDH-A and glutaminase inhibition in vivo by hyperpolarized 13C-pyruvate magnetic resonance spectroscopy of tumors. Cancer Research, 73(14), 4190-4195.
14. Gatenby, R. A., \& Gillies, R. J. (2004). Why do cancers have high aerobic glycolysis? Nature Reviews. Cancer, 4(11), 891-899.

15. Waki, A., et al. (1998). The importance of glucose transport activity as the rate-limiting step of 2-deoxyglucose uptake in tumor cells in vitro. Nuclear Medicine and Biology, 25(7), 593-597.

16. Choi, J., Jung, W. H., \& Koo, J. S. (2013). Metabolismrelated proteins are differentially expressed according to the molecular subtype of invasive breast cancer defined by surrogate immunohistochemistry. Pathobiology, 80(1), 41-52.

17. Grover-McKay, M., et al. (1998). Role for glucose transporter 1 protein in human breast cancer. Pathology Oncology Research, 4(2), 115-120.

18. Lloyd, S. M., Arnold, J., \& Sreekumar, A. (2015). Metabolomic profiling of hormone-dependent cancers: A bird's eye view. Trends in Endocrinology and Metabolism, 26(9), 477-485.

19. Katz-Brull, R., et al. (2002). Metabolic markers of breast cancer: Enhanced choline metabolism and reduced choline-ether-phospholipid synthesis. Cancer Research, 62(7), 1966-1970.

20. Eliyahu, G., Kreizman, T., \& Degani, H. (2007). Phosphocholine as a biomarker of breast cancer: Molecular and biochemical studies. International Journal of Cancer, 120(8), 1721-1730.

21. Aboagye, E. O., \& Bhujwalla, Z. M. (1999). Malignant transformation alters membrane choline phospholipid metabolism of human mammary epithelial cells. Cancer Research, 59(1), 80-84.

22. Park, J. K., et al. (2021). The heterogeneity of lipid metabolism in cancer. Advances in Experimental Medicine and Biology, 1311, https://doi. org/10.1007/978-3-030-65768-0_3.

23. Hilvo, M., et al. (2011). Novel theranostic opportunities offered by characterization of altered membrane lipid metabolism in breast cancer progression. Cancer Research, 71(9), 3236-3245.

24. Ramirez de Molina, A., et al. (2002). Increased choline kinase activity in human breast carcinomas: Clinical evidence for a potential novel antitumor strategy. Oncogene, 21(27), 4317-4322.

25. Rodriguez-Gonzalez, A., et al. (2004). Choline kinase inhibition induces the increase in ceramides resulting in a highly specific and selective cytotoxic antitumoral strategy as a potential mechanism of action. Oncogene, 23(50), 8247-8259.

26. Fuhrman, B. J., et al. (2012). Estrogen metabolism and risk of breast cancer in postmenopausal women. Journal of the National Cancer Institute, 104(4), 326-339.

27. Cicatiello, L., et al. (2010). Estrogen receptor alpha controls a gene network in luminal-like breast cancer cells comprising multiple transcription factors and microRNAs. The American Journal of Pathology, 176(5), 2113-2130. 
28. Acconcia, F., \& Kumar, R. (2006). Signaling regulation of genomic and nongenomic functions of estrogen receptors. Cancer Letters, 238(1), 1-14.

29. Jia, M., et al. (2016). Estrogen receptor alpha promotes breast cancer by reprogramming choline metabolism. Cancer Research, 76(19), 5634-5646.

30. Devanesan, P., et al. (2001). Catechol estrogen conjugates and DNA adducts in the kidney of male Syrian golden hamsters treated with 4-hydroxyestradiol: Potential biomarkers for estrogen-initiated cancer. Carcinogenesis, 22(3), 489-497.

31. Cavalieri, E., et al. (2000). Estrogens as endogenous genotoxic agents-DNA adducts and mutations. Journal of the National Cancer Institute. Monographs, 27, 75-93.

32. Bradlow, H. L., et al. (1996). 2-Hydroxyestrone: The 'good' estrogen. The Journal of Endocrinology, 150(Suppl), S259-S265.

33. Possemato, R., et al. (2011). Functional genomics reveal that the serine synthesis pathway is essential in breast cancer. Nature, 476(7360), 346-350.

34. Fan, J., et al. (2015). Human phosphoglycerate dehydrogenase produces the oncometabolite D-2-hydroxyglutarate. ACS Chemical Biology, 10(2), 510-516.

35. Rakheja, D., et al. (2013). The emerging role of d-2-hydroxyglutarate as an oncometabolite in hematolymphoid and central nervous system neoplasms. Frontiers in Oncology, 3, 169.

36. Terunuma, A., et al. (2014). MYC-driven accumulation of 2-hydroxyglutarate is associated with breast cancer prognosis. The Journal of Clinical Investigation, 124(1), 398-412.

37. Yue, W., et al. (2003). Genotoxic metabolites of estradiol in breast: Potential mechanism of estradiol induced carcinogenesis. The Journal of Steroid Biochemistry and Molecular Biology, 86(3-5), 477-486.

38. Quinones, A., \& Le, A. (2021). The multifaceted glioblastoma: From genomic alterations to metabolic adaptations. Advances in Experimental Medicine and Biology, 1311, https://doi. org/10.1007/978-3-030-65768-0_4.

39. Mullarky, E., et al. (2016). Identification of a small molecule inhibitor of 3-phosphoglycerate dehydrogenase to target serine biosynthesis in cancers. Proceedings of the National Academy of Sciences of the United States of America, 113(7), 1778-1783.

40. Jerby, L., et al. (2012). Metabolic associations of reduced proliferation and oxidative stress in advanced breast cancer. Cancer Research, 72(22), 5712-5720.

41. Dang, C. V., Le, A., \& Gao, P. (2009). MYC-induced cancer cell energy metabolism and therapeutic opportunities. Clinical Cancer Research, 15(21), 6479-6483.

42. Le, A., \& Dang, C. V. (2013). Studying Myc's role in metabolism regulation. Methods in Molecular Biology, 1012, 213-219.
43. Shen, L., et al. (2015). Metabolic reprogramming in triple-negative breast cancer through Myc suppression of TXNIP. Proceedings of the National Academy of Sciences of the United States of America, 112(17), 5425-5430.

44. Hoang, G., Udupa, S., \& Le, A. (2019). Application of metabolomics technologies toward cancer prognosis and therapy. International Review of Cell and Molecular Biology, 347, 191-223.

45. Dang, C. V., et al. (2011). Therapeutic targeting of cancer cell metabolism. Journal of Molecular Medicine (Berlin), 89(3), 205-212.

46. Hirschey, M. D., et al. (2015). Dysregulated metabolism contributes to oncogenesis. Seminars in Cancer Biology, 35(Suppl), S129-S150.

47. Jove, M., et al. (2017). A plasma metabolomic signature discloses human breast cancer. Oncotarget, 8(12), 19522-19533.

48. Huang, S., et al. (2016). Novel personalized pathwaybased metabolomics models reveal key metabolic pathways for breast cancer diagnosis. Genome Medicine, 8(1), 34.

49. Giskeodegard, G. F., et al. (2010). Multivariate modeling and prediction of breast cancer prognostic factors using MR metabolomics. Journal of Proteome Research, 9(2), 972-979.

50. Mountford, C. E., et al. (2001). Diagnosis and prognosis of breast cancer by magnetic resonance spectroscopy of fine-needle aspirates analysed using a statistical classification strategy. The British Journal of Surgery, 88(9), 1234-1240.

51. Asiago, V. M., et al. (2010). Early detection of recurrent breast cancer using metabolite profiling. Cancer Research, 70(21), 8309-8318.

52. Oakman, C., et al. (2011). Identification of a serumdetectable metabolomic fingerprint potentially correlated with the presence of micrometastatic disease in early breast cancer patients at varying risks of disease relapse by traditional prognostic methods. Annals of Oncology, 22(6), 1295-1301.

53. Jobard, E., et al. (2014). A serum nuclear magnetic resonance-based metabolomic signature of advanced metastatic human breast cancer. Cancer Letters, 343(1), 33-41.

54. Antonio, M. J., Zhang, C., \& Le, A. (2021). Different tumor microenvironments lead to different metabolic phenotypes. Advances in Experimental Medicine and Biology, 1311, https:// doi.org/10.1007/978-3-030-65768-0_10.

55. Simoes, R. V., et al. (2015). Metabolic plasticity of metastatic breast cancer cells: Adaptation to changes in the microenvironment. Neoplasia, 17(8), 671-684.

56. Wei, S., et al. (2013). Metabolomics approach for predicting response to neoadjuvant chemotherapy for breast cancer. Molecular Oncology, 7(3), 297-307.

57. Nabi, K., \& Le, A. (2021). The intratumoral heterogeneity of cancer metabolism. Advances in Experimental Medicine and Biology, 1311, https:// doi.org/10.1007/978-3-030-65768-0_11. 
58. Xu, H. N., et al. (2013). Characterizing the metabolic heterogeneity in human breast cancer xenografts by $3 \mathrm{D}$ high resolution fluorescence imaging. Springerplus, 2(1), 73.

59. Son, S. H., et al. (2014). Prognostic implication of intratumoral metabolic heterogeneity in invasive ductal carcinoma of the breast. BMC Cancer, 14, 585.

60. Park, V. Y., et al. (2016). Intratumoral agreement of high-resolution magic angle spinning magnetic resonance spectroscopic profiles in the metabolic characterization of breast cancer. Medicine (Baltimore), 95(15), e3398.

61. Gallagher, F. A., et al. (2020). Imaging breast cancer using hyperpolarized carbon-13 MRI. Proceedings of the National Academy of Sciences of the United States of America, 117(4), 2092-2098.

62. Marusyk, A., Janiszewska, M., \& Polyak, K. (2020). Intratumor heterogeneity: The Rosetta Stone of therapy resistance. Cancer Cell, 37(4), 471-484.

63. Schafer, Z. T., et al. (2009). Antioxidant and oncogene rescue of metabolic defects caused by loss of matrix attachment. Nature, 461(7260), 109-113.

64. Lee, Y. J., et al. (1998). Glucose deprivation-induced cytotoxicity and alterations in mitogen-activated protein kinase activation are mediated by oxidative stress in multidrug-resistant human breast carcinoma cells. The Journal of Biological Chemistry, 273(9), 5294-5299.

65. Ahn, C. S., \& Metallo, C. M. (2015). Mitochondria as biosynthetic factories for cancer proliferation. Cancer \& Metabolism, 3(1), 1 .

66. LeBleu, V. S., et al. (2014). PGC-1alpha mediates mitochondrial biogenesis and oxidative phosphorylation in cancer cells to promote metastasis. Nature Cell Biology, 16(10), 992-1003. 1-15.

67. Robertson-Tessi, M., et al. (2015). Impact of metabolic heterogeneity on tumor growth, invasion, and treatment outcomes. Cancer Research, 75(8), 1567-1579.

68. Kerbel, R. S. (2009). Issues regarding improving the impact of antiangiogenic drugs for the treatment of breast cancer. Breast, 18(Suppl 3), S41-S47.

69. Ma, S., et al. (2018). The role of tumor microenvironment in resistance to anti-angiogenic therapy. F1000Res, 7, 326.

70. Conley, S. J., et al. (2012). Antiangiogenic agents increase breast cancer stem cells via the generation of tumor hypoxia. Proceedings of the National Academy of Sciences of the United States of America, 109(8), 2784-2789.

Open Access This chapter is licensed under the terms of the Creative Commons Attribution 4.0 International License (http://creativecommons.org/licenses/by/4.0/), which permits use, sharing, adaptation, distribution and reproduction in any medium or format, as long as you give appropriate credit to the original author(s) and the source, provide a link to the Creative Commons license and indicate if changes were made.

The images or other third party material in this chapter are included in the chapter's Creative Commons license, unless indicated otherwise in a credit line to the material. If material is not included in the chapter's Creative Commons license and your intended use is not permitted by statutory regulation or exceeds the permitted use, you will need to obtain permission directly from the copyright holder. 


\title{
Non-Hodgkin Lymphoma Metabolism
}

\author{
Brian James Kirsch, Shu-Jyuan Chang, \\ Michael James Betenbaugh, and Anne Le
}

\section{Keywords}

Heterogeneous malignant lymphomas .

Lactic acidosis - Aerobic glycolysis ·

Glutamine metabolism - Fatty acid metabo-

lism $\cdot$ Gene expression $\cdot$ PI3K/AKT/mTOR

pathway $\cdot\left[{ }^{18} \mathrm{~F}\right] \mathrm{FDG}$ PET/CT

\section{Abbreviations}

2-DG

2-Deoxyglucose

B. J. Kirsch

Department of Pathology, Johns Hopkins University

School of Medicine, Baltimore, MD, USA

Department of Chemical and Biomolecular Engineering, Johns Hopkins University Whiting

School of Engineering, Baltimore, MD, USA

\section{S.-J. Chang}

Graduate Institute of Medicine, College of Medicine,

Kaohsiung Medical University, Kaohsiung, Taiwan

M. J. Betenbaugh

Department of Chemical and Biomolecular

Engineering, Johns Hopkins University Whiting

School of Engineering, Baltimore, MD, USA

A. Le $(\bowtie)$

Department of Pathology and Oncology, Johns

Hopkins University School of Medicine,

Baltimore, MD, USA

Department of Chemical and Biomolecular Engineering, Johns Hopkins University Whiting School of Engineering, Baltimore, MD, USA

e-mail: annele@jhmi.edu
Acetyl-CoA

AKT

AMPK

ATP

AUC CSH

BCR

B-NHLs

CLL

CT

DLBCL

ETC

FAO

FAS

FASN

$\left[{ }^{18} \mathrm{~F}\right] \mathrm{FDG}$

FL

HIF-1

LDH

MCL

MDR

$\mathrm{MH}$

mTOR

mTORC1

MTV

NF- $\mathrm{BB}$
Acetyl coenzyme A

Protein kinase B

$5^{\prime}$-AMP-activated protein

kinase

Adenosine triphosphate

Area under the curve of the cumulative SUV histograms

B-cell receptor

B-cell non-Hodgkin

lymphomas

Chronic lymphocytic leukemia

Computed tomography

Diffuse large B-cell lymphoma

Electron transport chain

Fatty acid oxidation

Fatty acid synthesis

Fatty acid synthesizing enzyme

${ }^{18}$ F-Fluorodeoxyglucose

Follicular lymphoma

Hypoxia-inducible factor-1

Lactate dehydrogenase

Mantle cell lymphoma

Multidrug resistance

Metabolic heterogeneity

Mammalian target of rapamycin

Mammalian target of rapamycin complex 1

Metabolic tumor volume

Nuclear factor kappa-lightchain-enhancer of activated B cells 


$\begin{array}{ll}\text { NHLs } & \text { Non-Hodgkin lymphomas } \\ \text { OS } & \text { Overall survival } \\ \text { OXPHOS } & \text { Oxidative phosphorylation } \\ \text { PBL } & \text { Primary brain lymphoma } \\ \text { PDK1 } & \begin{array}{l}\text { Pyruvate dehydrogenase } \\ \text { kinase, isozyme 1 }\end{array} \\ \text { PEL } & \text { Primary effusion lymphoma } \\ \text { PET } & \text { Positron-emission tomography } \\ \text { PFS } & \text { Progression-free survival } \\ \text { PI3K } & \text { Phosphatidylinositol-3-kinase } \\ \text { POX/PRODH } & \text { Proline dehydrogenase } \\ \text { PRPS2 } & \text { Phosphoribosyl-pyrophosphate } \\ \text { RT } & \text { synthetase 2 } \\ \text { SREBP } & \text { Richter's transformation } \\ \text { SUV } & \text { Sterol regulatory element- } \\ \text { binding protein } \\ \text { tFL } & \text { Standardized uptake value } \\ \text { TLG } & \text { Tricarboxylic acid } \\ \text { TMTV } & \text { Transformed } \\ \text { TNFAIP3/A20 } & \text { lymphoma follicular } \\ \text { VEGF } & \text { Total lesion glycolysis } \\ & \text { induced protein 3 A20 } \\ & \text { Vascular endothelial growth } \\ \text { factor }\end{array}$

\section{Key Points}

- Aggressive lymphomas exhibit the Warburg effect.

- Lactic acidosis is a result of the overproduction of lactate and leads to a fatal prognosis.

- Mutation of p53 helps cancer cells survive glutamine deprivation.

- PI3K regulates fatty acid synthesis (FAS) in primary effusion lymphoma (PEL) and other B-NHLs.

- AMPK regulates NADPH balance for fatty acid oxidation (FAO) to supplement the TCA cycle.

- PRPS2 couples protein and nucleotide biosynthesis to drive lymphomagenesis.

- mTOR activation promotes fatty acid synthesis (FAS).

- $M Y C$ regulates cancer cell metabolism under glucose-deprived and hypoxic conditions.
- HIF-1 acts as a regulator in hypoxia adaption and the related metabolic changes.

- Knowledge of metabolic phenotypes in cancer can be used in tandem with genetic alterations to develop effective treatment strategies.

- $\left[{ }^{18} \mathrm{~F}\right] \mathrm{FDG}$ PET/CT is a valuable tool to visualize tumor glycolytic activity and characterize metabolic heterogeneity.

\section{Introduction}

Non-Hodgkin lymphomas (NHLs) are a heterogeneous group of lymphoid neoplasms with different biological characteristics. About $90 \%$ of all lymphomas in the United States originate from B lymphocytes, while the remaining originate from T cells [1]. The treatment of NHLs depends on the neoplastic histology and stage of the tumor, which will indicate whether radiotherapy, chemotherapy, or a combination is the best suitable treatment [2]. The American Cancer Society describes the staging of lymphoma as follows: Stage I is lymphoma in a single node or area. Stage II is when that lymphoma has spread to another node or organ tissue. Stage III is when it has spread to lymph nodes on two sides of the diaphragm. Stage IV is when cancer has significantly spread to organs outside the lymph system. Radiation therapy is the traditional therapeutic route for localized follicular and mucosa-associated lymphomas. Chemotherapy is utilized for the treatment of large-cell lymphomas and high-grade lymphomas [2]. However, the treatment of indolent lymphomas remains problematic as the patients often have metastasis, for which no standard approach exists [2].

Follicular lymphoma (FL), a form of nonHodgkin lymphoma, is the second most common form of B-cell lymphoma and remains incurable in the majority of cases, despite recent advances, including anti-CD20 antibodies (rituximab) and kinase inhibitors (ibrutinib) [3]. Following an indolent phase, $50 \%$ of patients suffer from disease transformation to an aggressive form of lymphoma (transformed FL; tFL) [4]. This dramatic switch in disease behavior typically culmi- 
nates in rapid deterioration and is usually fatal. Accordingly, much effort has been focused on understanding the genetics of transformation, which has resulted in the identification of key genetic lesions (e.g., MYC activation, loss of p53, activation of nuclear factor kappa-light-chainenhancer of activated B cells (NF-KB), loss of tumor necrosis factor-alpha-induced protein 3 A20 (TNFAIP3/A20)) [5-8]. However, exactly how tumor metabolism, which is altered by these genetic lesions, contributes to disease aggressiveness is not known. Therefore, the metabolic changes that occur during FL transformation are poorly understood. We need to understand the biological and metabolic changes upon disease transformation in order to develop effective treatment strategies.

Malignant cells have metabolic adaptations supporting bioenergetics, biosynthesis, and redox homeostasis in response to the development of the tumor microenvironment [9]. Metabolic heterogeneity is present in the tumor microenvironment, where concentrations of key resources can be spatially (localization) and temporally (stage of the diseases) varied [10], creating the heterogeneity of metabolism within the same tumor [11]. Cancer metabolism is influenced by tumor localization and vascularization status. Cancer cells can uptake nutrients and oxygen from the blood supply, which results in the production of ATP via aerobic oxidative phosphorylation (OXPHOS) as well as through anabolic pathways, supporting rapid cell proliferation.

In this chapter, we describe the intricacies of NHLs' metabolism resulting from alterations in gene expressions, which subsequently lead to poor prognosis. Furthermore, we explore how metabolomics technologies [12] and analysis can be applied to treatment strategies.

\section{Lymphoma Metabolism Exhibits Multifaceted Characteristic Features Which Are Correlated to Poor Prognosis}

\subsection{Aggressive Lymphomas Exhibit the Warburg Effect}

As described in the previous chapters, the drastic increase in glucose uptake of cancer cells is a feature of the distinctive metabolic rewiring known as the Warburg effect [13]. Recently, researchers have taken advantage of this metabolic shift to clinically detect localized glucose uptake of cancer cells using ${ }^{18} \mathrm{~F}$-fluorodeoxyglucose positronemission tomography $\left(\left[{ }^{18} \mathrm{~F}\right] \mathrm{FDG} P E T\right)$. High-grade NHL patients and intermediate-grade NHL patients with poor prognoses showed a high accumulation of $\left[{ }^{18} \mathrm{~F}\right] \mathrm{FDG}$ in their tumors $[14,15]$.

Primary effusion lymphoma (PEL) exhibits high glycolytic activity due to its hypoxic environment. This form of lymphoma requires aggressive treatment, but no standard therapy exists [16]. PEL is, however, highly sensitive to glucose withdrawal and glycolysis inhibitors, such as 2-deoxyglucose (2-DG) [16]. In this situation, the distinctive metabolic phenotype, glucose dependency, offers hope for effective treatments.

Cancer cells exhibiting the Warburg effect avidly take up glucose. After glucose uptake, cancer cells favor the conversion of glucosederived pyruvate to lactate. Recent reports showed that NHL patients had elevated plasma lactate and lactate dehydrogenase (LDH) levels, which were linked to poor survival rates [17-19]. Furthermore, inhibition of LDHA, the enzyme that catalyzes the conversion of pyruvate to 
lactate, has yielded positive results in tumor reduction in a $M Y C$-transformed Burkitt lymphoma model in vivo [20, 21]. Taken together, the characteristic metabolic features of the Warburg effect offer diagnostic tools as well as relevant therapeutic targets for NHL.

\subsection{Lactic Acidosis Is a Result of Overproduction of Lactate and Leads to a Fatal Prognosis}

Following the Warburg effect, lactic acidosis can occur when lactate homeostasis is disproportioned, due to overproduction and/or underutilization. Lactic acidosis is divided into two categories: type A and type B. Type A results from poor oxygenation in the tissue. Type B occurs in normoxic tissue as a result of a drug or toxin [22]. Type B lactic acidosis is the result of the alteration of glycolytic processes and their effects on redox [23, 24]. Type B lactic acidosis is present in many human malignancies, but notably in lymphomas and leukemias [22, 23, 25-27]. Once cancers exhibit type B lactic acidosis, these cases show poor prognoses and outcomes if not immediately treated [22].

Another notable cause of lactic acidosis is thiamine deficiency, an observable characteristic connected to type B lactic acidosis in some cancers. Thiamine is a cofactor that is necessary for the conversion of pyruvate into acetyl-CoA via pyruvate dehydrogenase. When malignant cells exhibit thiamine deficiency, pyruvate is heavily converted to lactate $[28,29]$. Subsequently, thiamine deficiency leads to lactic acidosis (Fig. 1).

\section{Genetic Alterations Lead to Different Metabolic Phenotypes in NHL (Fig. 2)}

NHLs often have abnormal activation of the mammalian target of rapamycin complex 1 (mTORC1) that reprograms multiple metabolic pathways, including nucleotide synthesis, amino acid synthesis, fatty acid synthesis, and glutaminolysis. Additionally, $M Y C$ is another important trigger for inducing many genes correlated with anabolic growth, including transporters and enzymes involved in glycolysis, mitochondrial biogenesis, fatty acid synthesis, and glutaminolysis [30-34]. MYC is a gene involved in cellular proliferation whose dysregulation was found in B-cell lymphomas [35]. Metabolic reprograming by transcription factors such as $M Y C$ and hypoxia-inducible factor 1 (HIF-1) in malignant tissues allows them to better survive the tumor microenvironmental alterations. These various genes can often influence each other; for instance, mTOR can also activate HIF-1 expression even under normoxic states [36] (Table 1).

\subsection{Mutation of p53 Helps Cancer Cells Survive Glutamine Deprivation}

As described in a previous chapter, many cancers depend on glutamine for bioenergy, redox homeostasis, and DNA synthesis, which are essential requirements for cancer cell survival. Therapeutic strategies often target cancer's glutamine dependency [37]. However, these treatments do not always have the intended impact, as many cancers are resistant to treatment. One such example is that of TP53, a protein responsible for tumor suppression, and its mutant form [38]. Specifically, in lymphoma cell lines, Tran et al. reported that mutp53 proteins could directly bind to the promoters of p53 target genes that regulate the cell cycle, which leads to cell cycle arrest and helps cancer cells survive in glutamine deprivation conditions [38]. Cancer cells expressing mutp53 proteins are able to survive the metabolic stress of glutamine deprivation in poorly vascularized tumor microenvironments, whereas p53-deficient cells and wtp53-expressing cells experience impaired proliferation and increased cell death [38]. The resistance to glutamine deprivation in mutp53-expressing malignant cells allows these cells to survive in metabolically restrictive environments. 

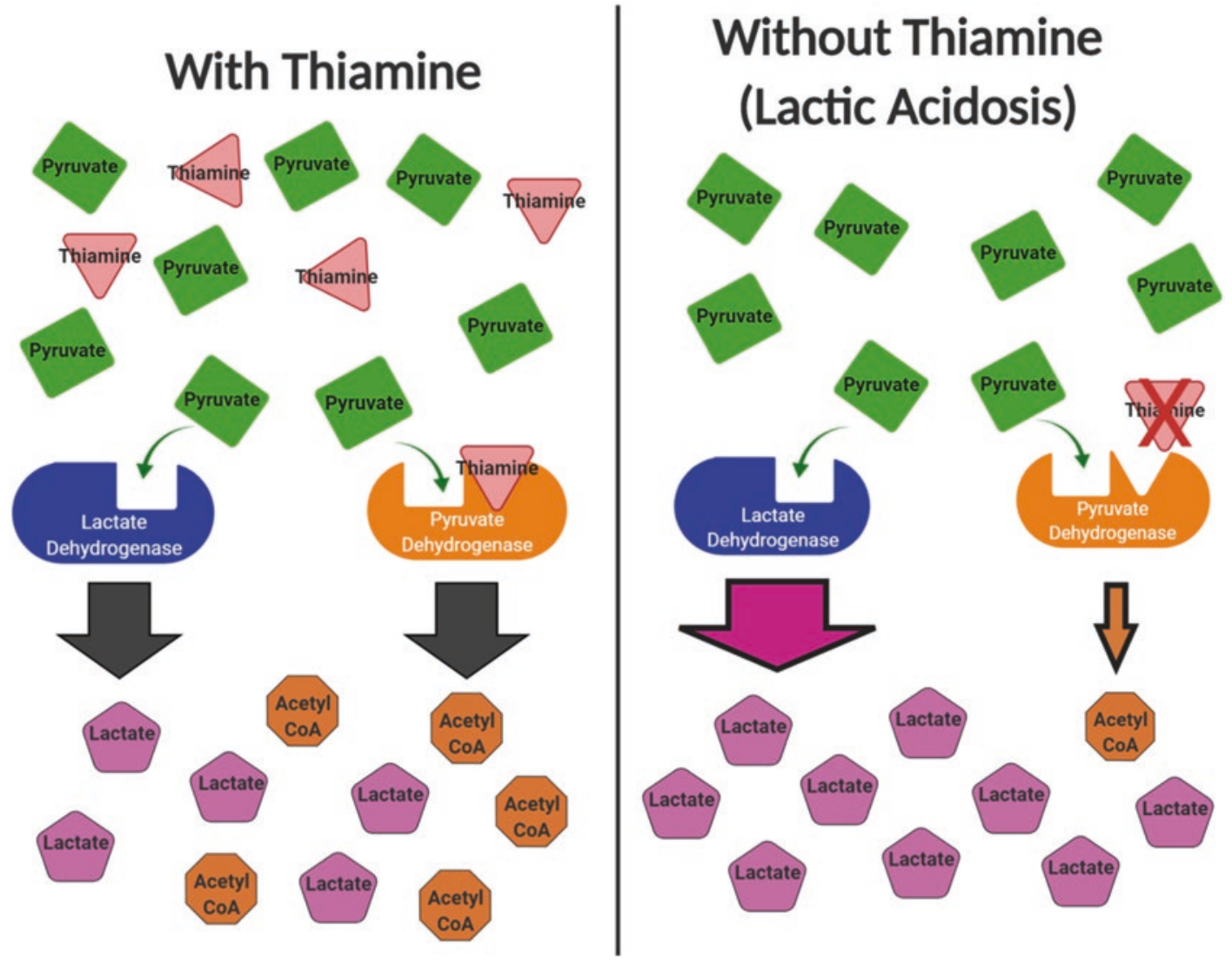

Fig. 1 The effect of thiamine on lactate and acetyl-CoA production. Some cancer cells produce lactate even in the presence of oxygen, an effect known as the Warburg effect. Lactic acidosis can result from unregulated lactic acid (lactate) buildup. When thiamine is present, there is a mixture of lactate and acetyl-CoA (left). When thiamine is deficient, acetyl-CoA production is impaired, and pyruvate is mainly converted to lactate resulting in lactate buildup (right)

\subsection{PI3K Regulates Fatty Acid Synthesis (FAS) in Primary Effusion Lymphoma (PEL) and Other B-NHLs}

While many lymphomas rely on glucose to produce lactate and energize their metabolism, this is not always the case. Dysregulation of cell metabolism in primary effusion lymphoma (PEL), an aggressive type of B-cell lymphoma, increased not only aerobic glycolysis but also fatty acid synthesis $[39,40]$. By using ${ }^{14} \mathrm{C}$-labeled glucose, Bhatt et al. showed that PEL created more lipids from glucose compared to primary B cells. Furthermore, these cells were sensitive to both an inhibitor of fatty acid synthase, C75, and an inhibitor of glycolysis, 2-DG. Each of these inhibitors affected both glycolysis and fatty acid synthesis (FAS) [39]. Bhatt et al. showed a significant difference in the metabolic profiles of primary B cells and those of human B-cell nonHodgkin lymphomas (B-NHL), including PEL. Poor-prognosis PEL and other B-NHLs exhibit high levels of aerobic glycolysis and FAS. This suggests that different types of malignant lymphomas can be distinguished by the rate of fatty acid biosynthesis, which may have the potential for targeted therapy against these aggressive lymphomas [39].

Previous work of Bhatt et al. and others showed that PEL cells exhibit high activities of phosphatidylinositol 3-kinase (PI3K), protein kinase B (AKT), and mTOR, genes related to proliferation and survival as well as glycolysis 
Fig. 2 Genetic alterations lead to phenotypes in nonHopkin lymphoma. Various lymphomas show these alterations in gene expression and the resulting changes in metabolism different metabolic

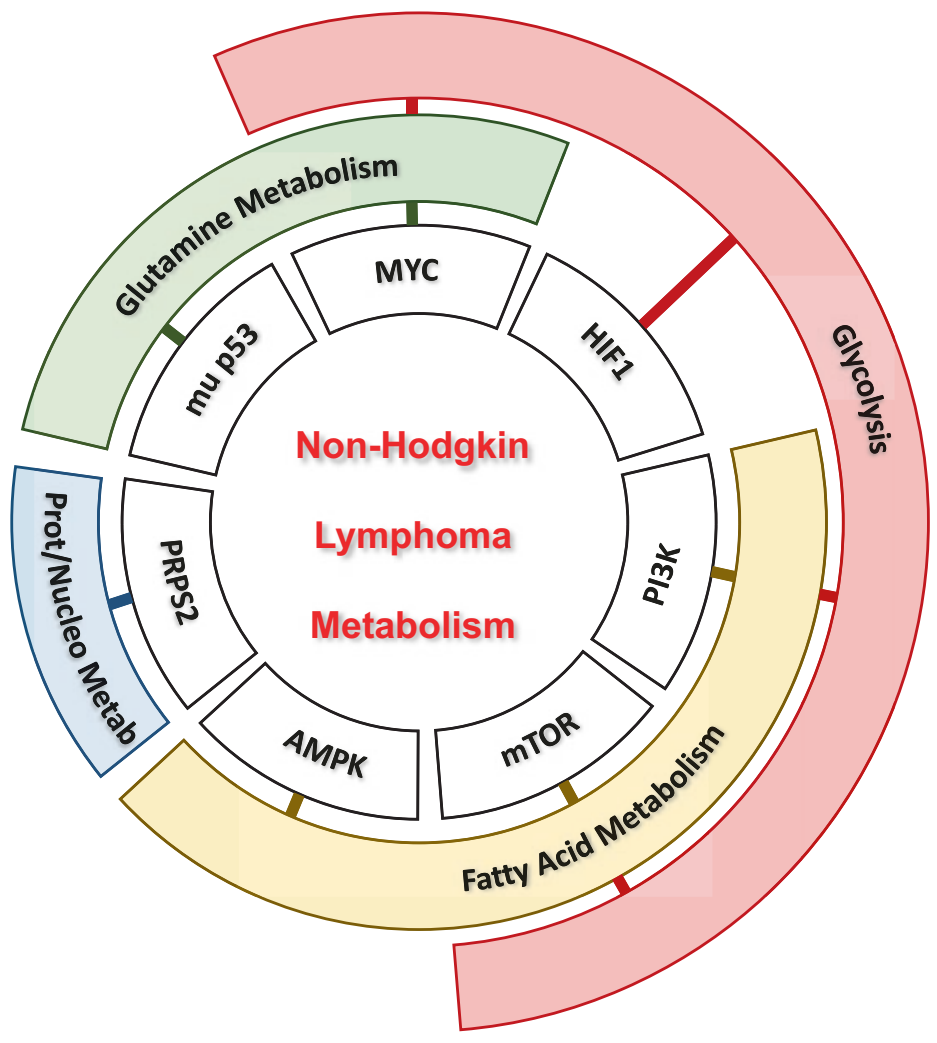

In summary, glucose was important to PEL

Table 1 Genetic alterations lead to different metabolic phenotypes in NHL

\begin{tabular}{l|l|l}
\hline Gene & Metabolic effect & Lymphoma type \\
\hline $\begin{array}{l}\text { Mutant } \\
T P 53\end{array}$ & $\begin{array}{l}\text { Allows cells to survive } \\
\text { glutamine deprivation }\end{array}$ & $\begin{array}{l}\text { B- and T-cell } \\
\text { lymphoma }\end{array}$ \\
\hline PI3K & $\begin{array}{l}\text { Regulates glycolysis and } \\
\text { FAS }\end{array}$ & $\begin{array}{l}\text { Primary } \\
\text { effusion } \\
\text { lymphoma }\end{array}$ \\
\hline AMPK & $\begin{array}{l}\text { Regulates FAO to } \\
\text { support TCA }\end{array}$ & $\begin{array}{l}\text { Diffuse large } \\
\text { B-cell } \\
\text { lymphoma }\end{array}$ \\
\hline PRPS2 & $\begin{array}{l}\text { Drives protein and } \\
\text { nucleotide synthesis }\end{array}$ & $\begin{array}{l}\text { B-cell } \\
\text { lymphoma }\end{array}$ \\
\hline mTOR & $\begin{array}{l}\text { Enhances glycolysis and } \\
\text { FAS }\end{array}$ & $\begin{array}{l}\text { B-cell } \\
\text { lymphoma }\end{array}$ \\
\hline MYC & $\begin{array}{l}\text { Regulates glycolysis, } \\
\text { TCA, glutamine, and } \\
\text { proline metabolism }\end{array}$ & $\begin{array}{l}\text { B-cell } \\
\text { lymphoma }\end{array}$ \\
\hline HIF-1 & $\begin{array}{l}\text { Promotes glycolysis, } \\
\text { particularly in hypoxia }\end{array}$ & $\begin{array}{l}\text { B-cell } \\
\text { lymphoma }\end{array}$ \\
\hline
\end{tabular}

[41-43]. In their more recent work, they showed that inhibiting PI3K by LY294002 decreased not only glycolytic flux but also the incorporation of ${ }^{14} \mathrm{C}$ glucose into lipids [39]. cells for providing not only energy but also acetyl-CoA for lipid synthesis. This illustrates that lymphoma's metabolism is complex and can exhibit multidimensional alterations.

\subsection{AMPK Regulates NADPH Balance for Fatty Acid Oxidation (FAO) as a Means of Supplementing the Tricarboxylic Acid (TCA) Cycle}

Jeon et al. showed that AMPK orchestrates NADPH consumption (by FAS) and production (from fatty acid oxidation (FAO)) in lymphoma to support ATP synthesis, redox-homeostasis, and biosynthesis under low-glucose environments [44]. By doing so, AMPK decreases pentose phosphate pathway activity and increases FAO [44]. 
Diffuse large B-cell lymphoma (DLBCL), a common lymphoma, utilizes FAO to support energy production and growth [45]. Fatty acids provide fuel for oxidative phosphorylation (OXPHOS), and to increase glutathione levels, and attenuate oxidative stress [45]. The DLBCL with OXPHOS is aggressive and resistant to ibrutinib, an inhibitor of B-cell receptor (BCR) survival signaling [46, 47]. More research into the combination of fatty acid oxidation-targeting drugs and the BCR inhibitor could provide potential therapeutic approaches for patients with DLBCL [45-48].

\subsection{PRPS2 Couples Protein and Nucleotide Biosynthesis to Drive Lymphomagenesis}

One of the current mainstay chemotherapeutic strategies involves targeting one-carbon metabolism in malignant cancers. This strategy reduces the production of nucleotides and ATP, as well as alters redox homeostasis. Individual drugs often inhibit the metabolism of folate, nucleotides, and, most notably, thymidine [48, 49]. Key enzyme targets of nucleic acid synthesis include dihydrofolate reductase, thymidylate synthase, adenine/adenosine deaminase, and DNA polymerase/ribonucleotide reductase [48-51]. As indicated by Cunningham et al., nucleotide biosynthesis is coupled to protein biosynthesis by a critical enzyme, phosphoribosyl-pyrophosphate synthetase 2 (PRPS2), which specifically promotes increased nucleotide biosynthesis in MYCdriven lymphoma. In these lymphomas, PRPS2 may be an effective anticancer target, and other enzymes in this pathway utilized by oncogenes may also exist as potential targets [52].

\section{5 mTOR Activation Promotes Fatty Acid Synthesis (FAS)}

mTOR activation during nutrient abundance enhances aerobic glycolysis and lipid synthesis, which is mediated by the sterol regulatory element-binding protein (SREBP) group by inducing the transcription of the fatty acidsynthesizing enzyme (FASN) [53]. FASN is present at elevated levels in the liver and at lower levels in other tissues, but cancerous tissues express excessive FASN, which has been identified as a metabolic oncogene [40, 54-56].

In PEL, rapamycin treatment improves survival time in the in vivo model by inhibiting autocrine signaling and vascular endothelial growth factor (VEGF) [43, 57]. On the other hand, Shestov et al. revealed that inhibition of mTOR has an impact on the flux of glycolysis, pentose phosphate pathway, and TCA cycle [58]. Thus, while mTOR activation is linked to FAS, inhibition of mTOR can impact other metabolic pathways critical to cancer growth.

\subsection{MYC Regulates Cancer Cell Metabolism under Glucose- Deprived and Hypoxic Conditions}

MYC is considered to be a regulator in glycolysis and mitochondrial respiration [33, 34, 59-63]. Using stable isotope-resolved metabolomics [12], Le et al. explored the metabolic alterations that occur in the oncogenic transcription factor c-MYC-inducible human Burkitt lymphoma model P493 cell line under aerobic and hypoxic conditions as well as glucose deprivation. They found the coexistence of oxidative and aerobic glycolysis. They also documented the prominent contribution of glutamine to the TCA cycle of proliferating cells and that hypoxic cancer cells continue to oxidize glutamine for cell growth and survival. Furthermore, this study showed that glutamine metabolism alone could sustain the TCA cycle for cell survival and growth in the absence of glucose [64]. This glucose-independent pathway reflects the dependence of cancer cells on metabolic reprogramming, allowing for the survival and proliferation of cancer cells under the harsh hypoxic and nutrient-deprived conditions of the tumor microenvironment. Their study demonstrated that inhibition of glutaminase, the enzyme that catalyzes the reaction of glutamine to glutamate, by BPTES, impaired MYC-transformed 
B lymphoma growth in vivo [64]. Other glutaminase inhibitors, such as BPTES analogs, have been developed to target glutamine metabolism in cancers [65].

$M Y C$ also regulates proline metabolism, as found by Liu et al. [66]. They found that proline dehydrogenase (POX/PRODH), the first enzyme in proline catabolism, was suppressed by $M Y C$ through upregulating miR-23b*. This study provided a deeper understanding of cancer metabolism while enabling the development of novel therapeutic strategies.

\subsection{HIF-1 Acts as a Regulator in Hypoxia Adaptation and the Related Metabolic Changes}

HIF-1 activity is enhanced by mTOR-altered metabolism and promotes glycolysis as a hypoxia-adaptive transcriptional program. The HIF-1 and HIF-2 heterodimers respond to and are stabilized by hypoxia, resulting in metabolic changes [67]. Of these two heterodimers, HIF-1 is a critical component involved in tumor metabolism that upregulates glucose transporters, glycolytic enzymes, and pyruvate dehydrogenase kinase, isozyme 1 (PDK1), an enzyme which prevents pyruvate from entering the TCA cycle [36]. Qiao et al. demonstrated that malignant lymphomas exhibit constitutive expression of HIF-1 $\alpha$. This expression is mediated by NF- $\kappa \mathrm{B}$, and ionizing radiation treatment of lymphoma showed increased NF- $\kappa$ B activation and elevated HIF- $1 \alpha$ levels. This indicates that additional treatment targeting HIF- $1 \alpha$ in combination with radiation therapy of lymphoma cells could potentially improve patient outcomes [68].

\subsection{Understanding the PI3K/AKT/ mTOR Pathway in Lymphoma Can Lead to a Variety of Treatments}

Thus far, we have discussed how metabolic analysis has-improved-our-understanding-of NHL metabolism. In some cases, the genetic altera- tions that lead to unique metabolic phenotypes, such as those discussed in Sect. 3, have been targeted for therapy and have resulted in clinical trials. Additionally, other tools such as $\left[{ }^{18} \mathrm{~F}\right] \mathrm{FDG}$ PET/computed tomography (CT). can help gauge the lymphoma metabolism to inform therapy and predict outcomes.

Sections 3.2 and 3.5 of this chapter have highlighted how PI3K and mTOR play key roles in controlling lymphoma metabolism. Due to the importance of the PI3K/AKT/mTOR pathway, targeting this pathway has already begun to show promising results in the treatment of different lymphoma types. For instance, in a 220-patient study of chronic lymphocytic leukemia (CLL), dual therapy with both idelalisib, a PI3K $\delta$ inhibitor, and rituximab, an established CD20 antibody commonly used against many lymphomas, improved the rate of progression-free survival (PFS) from $46 \%$ to $93 \%$ compared to monotherapy with rituximab alone [69].

However, in clinical studies, sometimes a less straightforward approach may be required for treatment due to unforeseen pathway changes. For instance, the PI3K/AKT/mTOR pathway is thought to play a role in refractory mantle cell lymphoma (MCL) resistance to ibrutinib [7072]. As such, one may logically reason that PI3K/ AKT/mTOR inhibitors would be a potential therapy for refractory MCL. However, the use of the PI3K inhibitor idelalisib and the rapamycin analog temsirolimus has provided underwhelming clinical outcomes [70, 73-75]. To explain this ineffective treatment, consider a study by Garcia et al. focusing on multidrug resistance (MDR), which coincided with an upregulated PI3K/AKT pathway. In this study, inhibition of PI3K via wortmannin and LY294002 induced cell death, but also coincided with the activation of NF- $\mathrm{BB}$, which can promote cell survival, thus mitigating the effect of PI3K-targeting treatment [76]. Zhang et al. postulate that understanding the metabolic reprogramming coincident with genetic alterations will be critical to a successful treatment approach. They identified a strong reliance on OXPHOS in refractory MCL, and subsequent treatment with IACS-010759, a clinically relevant inhibitor of complex 1 of the electron transport chain (ETC), yielded more promising 
results [70]. Additionally, glutamine uptake was also upregulated, and treatment with aminooxyacetate, which inhibits glutaminolysis, induced ROS formation and oxidative stress [70]. Thus, knowledge about both the genetic alterations and the metabolic shifts is key to developing effective treatment strategies.

\section{$4 \quad$ Metabolic Profiling for Monitoring Tumor Progression and Guiding Treatment}

\section{1 [ $\left[{ }^{18}\right.$ F]FDG PET/CT}

Regions of the body exhibiting elevated levels of glycolysis, such as many tumors, will show accumulation of the $\left[{ }^{18} \mathrm{~F}\right] \mathrm{FDG}$ as assessed by PET/ CT. Various metabolic parameters can be determined and used to diagnose a particular lymphoma case. The most frequently measured parameter is the standardized uptake value (SUV). The mean SUV and the max SUV are also useful parameters [77]. From the SUV, the metabolic tumor volume (MTV) and total lesion glycolysis (TLG) can be determined. MTV is the volume of the tumor that is metabolically active and is also sometimes referred to as total metabolic tumor volume (TMTV) when distinct regions are totaled. TLG is a dimensionless index rating the average glycolysis activity for the entire tumor and calculated by multiplying the mean SUV by the MTV [77, 78]. Additionally, metabolic heterogeneity can be approximated by the "area under the curve of the cumulative SUV histogram" (AUC CSH) method, where a lower AUC implies greater variability in metabolism [79]. Figure 3 shows a simplified visualization of the process for determining these key parameters and terms.

In DLBCL, patients with high TMTV and TLG exhibited a more advanced stage of lymphoma, and these parameters were strong inverse predictors of progression-free survival and overall survival (OS) outcomes [80, 81]. For instance, high TLG indicated a higher progression rate (41\% PFS) and worse overall survival (45\% OS) compared to low TLG (72\% PFS and $73 \%$ OS) [80]. Additionally, patients with overexpression of MYC were also at a greater risk for relapse and progression. In this study, Cottereau et al. combined molecular evaluation (MYC expression) and metabolic imaging (TMTV measurements) characteristics to more accurately diagnose and monitor patients and concluded that this approach could lead to patient-tailored therapies [80].

Interestingly, estimations of intertumoral metabolic heterogeneity ( $\mathrm{MH}$ ) were shown to be an accurate predictor of poor outcomes in patients with higher MTV, coinciding with shorter PFS and OS. Models taking into account both MTV and $\mathrm{MH}$ improved upon MTV alone as a predictor for PFS and OS [81, 82]. Similar results were found in primary mediastinal B-cell lymphoma (PMBCL), where a model combining both $\mathrm{MH}$ and TLG was found to be a more effective predictive tool than models using only one of these parameters [83]. Thus, $\left[{ }^{18} \mathrm{~F}\right] \mathrm{FDG}$ PET/CT can be used to determine key parameters relevant to the diagnosis and predicted outcomes of patients.

Evaluation of metabolism is not only helpful in evaluating DLBCL but also useful for other lymphomas as well. In FL, baseline TMTV was a strong predictor of outcome, where a TMTV of $510 \mathrm{~cm}^{3}$ cutoff is related to a less than 3-year PFS [84]. In primary brain lymphoma (PBL), MTV and TLG were the only $\left[{ }^{18} \mathrm{~F}\right]$ FDG-measured parameters shown to be correlated with PFS and OS [85]. Additionally, $\left[{ }^{18} \mathrm{~F}\right] \mathrm{FDG}$ PET/CT analysis is also a beneficial tool to monitor disease progression during treatment. In relapsed or refractory B-cell lymphoma treated with yttrium 90 (90Y) ibritumomab tiuxetan radioimmunotherapy, a drop in SUV of $49 \%$ or higher after either 2 or 6 months was shown to be an indicator of successful treatment, and lesser changes indicated a need for a different strategy [86]. $\left[{ }^{18} \mathrm{~F}\right] \mathrm{FDG}$ PET/CT is a flexible tool that can be applied to different forms of lymphoma and various therapeutic strategies.

$\left[{ }^{18} \mathrm{~F}\right]$ FDG PET/CT is also useful to predict disease transformation over time, such as Richter's transformation (RT), which is the conversion of CLL to DLBCL. In multiple studies, RT was shown to coincide with a max SUV greater than 5.0 with a relatively high predictive 


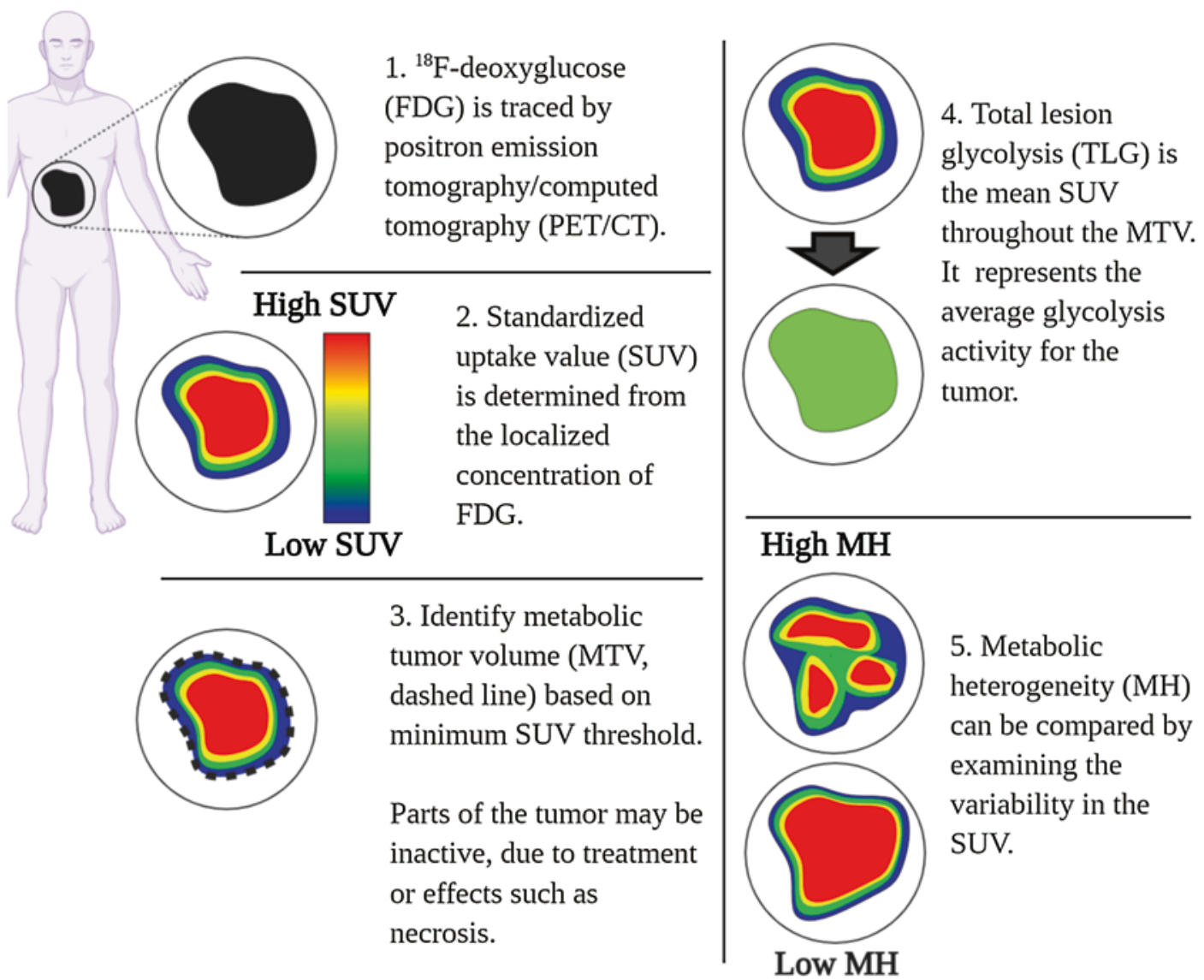

Fig. 3 Simplified $\left[{ }^{18} \mathrm{~F}\right] \mathrm{FDG}$ PET/CT process. Injection of the isotopic glucose analog $\left[{ }^{18} \mathrm{~F}\right] \mathrm{FDG}$ and subsequent analysis by PET/CT can determine key metabolic parameters (SUV, MTV, TLG, and MH) for research or clinical applications

potential [87-89]. Of particular note, a heterogeneous distribution of the $\left[{ }^{18} \mathrm{~F}\right] \mathrm{FDG}$ also coincided with lower survival, suggesting greater proliferation for these lymphomas [89].

$\left[{ }^{18} \mathrm{~F}\right] \mathrm{FDG}$ PET/CT has become a powerful metabolic tool for medical professionals for diagnosis and progression monitoring in various types of lymphoma. It can be used to visualize the glucose uptake and glycolytic activity of a tumor, as well as to characterize metabolic heterogeneity. Furthermore, it has been used as a methodology to direct patient treatment, where it can be used to confirm successful approaches or provide suggestions for alternative strategies for personalized medicine.

\subsection{Systemic NAAG Concentrations for Tumor Growth Monitoring}

One recent study found that concentrations of $\mathrm{N}$-acetyl-aspartyl-glutamate (NAAG) in brain tumors positively correlated with patient tumor grades [90]. The authors of this study then investigated the role of plasma NAAG concentrations in tumor growth monitoring using the $M Y C$ transformed human P493-6 model in vivo. They found that systemic NAAG concentrations in plasma of the mice bearing MYC-transformed P493-6 tumors strongly mirrored tumor growth where increases in NAAG concentrations pre- 
ceded the rise of tumor sizes when $M Y C$ was expressed and decreases in NAAG concentrations preceded the decrease of tumor sizes when $M Y C$ was suppressed. These findings suggest that plasma NAAG concentrations are linked to tumor growth rates and that changes in systemic NAAG concentrations are detectable before the corresponding changes in tumor sizes. Measurement of NAAG concentrations in peripheral blood is thus a promising non-invasive strategy for timely tumor growth monitoring during cancer treatment [90].

\section{Conclusion}

The therapeutic challenges in malignant lymphomas include chemoresistance, radiation tolerance, and multidrug resistance. Novel therapeutic strategies, which are based on the metabolic phenotypes of aggressive lymphomas are being pursued [51]. The malignant cells exhibit different pathways for altering catabolism and enhancing anabolism for rapid cell proliferation in order to adapt to the tumor microenvironment [9]. The metabolic differences can occur in many lymphomas; therefore, understanding and learning about these specific differences can lead to new targets for therapy, both individually and in combination with other treatments. Furthermore, metabolomics technologies can be critical to predict outcomes and to elucidate appropriate and novel treatment strategies going forward.

\section{References}

1. Shankland, K. R., Armitage, J. O., \& Hancock, B. W. (2012). Non-Hodgkin lymphoma. Lancet, 380(9844), 848-857.

2. Karen, M., Winkfield, R. W. T., \& Gospodarowicz, M. K. (2016). Chapter 77-Non-Hodgkin's lymphoma. Clinical Radiation Oncology (Fourth Edition), 2016, 1524-1546.e7.

3. Advani, R. H., et al. (2013). Bruton tyrosine kinase inhibitor ibrutinib (PCI-32765) has significant activity in patients with relapsed/refractory B-cell malignancies. Journal of Clinical Oncology, 31(1), 88-94.

4. Kridel, R., Sehn, L. H., \& Gascoyne, R. D. (2012). Pathogenesis of follicular lymphoma. The Journal of Clinical Investigation, 122(10), 3424-3431.

5. Wong, E., \& Dickinson, M. (2012). Transformation in follicular lymphoma: Biology, prognosis, and thera- peutic options. Current Oncology Reports, 14(5), 424-432.

6. Bouska, A., et al. (2014). Genome-wide copy-number analyses reveal genomic abnormalities involved in transformation of follicular lymphoma. Blood, 123(11), 1681-1690.

7. Okosun, J., et al. (2014). Integrated genomic analysis identifies recurrent mutations and evolution patterns driving the initiation and progression of follicular lymphoma. Nature Genetics, 46(2), 176-181.

8. Oricchio, E., et al. (2014). Frequent disruption of the RB pathway in indolent follicular lymphoma suggests a new combination therapy. The Journal of Experimental Medicine, 211(7), 1379-1391.

9. Antonio, M. J., Zhang, C., \& Le, A. (2021). Different tumor microenvironments lead to different metabolic phenotypes. Advances in Experimental Medicine and Biology, 1311, https:// doi.org/10.1007/978-3-030-65768-0_10

10. Biswas, S. K. (2015). Metabolic reprogramming of immune cells in cancer progression. Immunity, 43(3), 435-449.

11. Nabi, K., \& Le, A. (2021). The intratumoral heterogeneity of cancer metabolism. Advances in Experimental Medicine and Biology, 1311, https:// doi.org/10.1007/978-3-030-65768-0_11

12. Hoang, G., Udupa, S., \& Le, A. (2019). Application of metabolomics technologies toward cancer prognosis and therapy. International Review of Cell and Molecular Biology, 347, 191-223.

13. Bose, S., Zhang, C., \& Le, A. (2021). Glucose metabolism in cancer: The Warburg effect and beyond. Advances in Experimental Medicine and Biology, 1311, https://doi.org/10.1007/978-3-030-65768-0_1

14. Newman, J. S., et al. (1994). Imaging of lymphoma with PET with 2-[F-18]-fluoro-2-deoxy-D-glucose: Correlation with CT. Radiology, 190(1), 111-116.

15. Okada, J., et al. (1992). Positron emission tomography using fluorine-18-fluorodeoxyglucose in malignant lymphoma: A comparison with proliferative activity. Journal of Nuclear Medicine, 33(3), 325-329.

16. Mediani, L., et al. (2016). Reversal of the glycolytic phenotype of primary effusion lymphoma cells by combined targeting of cellular metabolism and PI3K/ Akt/mTOR signaling. Oncotarget, 7(5), 5521-5537.

17. Yadav, C., et al. (2016). Serum lactate dehydrogenase in non-Hodgkin's lymphoma: A prognostic indicator. Indian Journal of Clinical Biochemistry, 31(2), 240-242.

18. Fasola, G., et al. (1984). Serum LDH concentration in non-Hodgkin's lymphomas. Relationship to histologic type, tumor mass, and presentation features. Acta Haematologica, 72(4), 231-238.

19. Cowan, R. A., et al. (1989). Prognostic factors in high and intermediate grade non-Hodgkin's lymphoma. British Journal of Cancer, 59(2), 276-282.

20. Le, A., et al. (2010). Inhibition of lactate dehydrogenase A induces oxidative stress and inhibits tumor progression. Proceedings of the National Academy of Sciences of the United States of America, 107(5), 2037-2042. 
21. Dutta, P., et al. (2013). Evaluation of LDH-A and glutaminase inhibition in vivo by hyperpolarized 13C-pyruvate magnetic resonance spectroscopy of tumors. Cancer Research, 73(14), 4190-4195.

22. Claudino, W. M., et al. (2015). Type B lactic acidosis: A rare but life threatening hematologic emergency. A case illustration and brief review. American Journal of Blood Research, 5(1), 25-29.

23. de Groot, R., et al. (2011). Type B lactic acidosis in solid malignancies. The Netherlands Journal of Medicine, 69(3), 120-123.

24. Mizock, B. A. (1989). Lactic acidosis. Disease-aMonth, 35(4), 233-300.

25. Ruiz, J. P., Singh, A. K., \& Hart, P. (2011). Type B lactic acidosis secondary to malignancy: Case report, review of published cases, insights into pathogenesis, and prospects for therapy. ScientificWorldJournal, 11, 1316-1324.

26. Dogan, E., et al. (2005). Fatal lactic acidosis due to leukemic transformation in a patient with nonHodgkin's lymphoma: Case report. Advances in Therapy, 22(5), 443-446.

27. Chan, F. H., Carl, D., \& Lyckholm, L. J. (2009). Severe lactic acidosis in a patient with B-cell lymphoma: A case report and review of the literature. Case Reports in Medicine, 2009, 534561.

28. Andersen, L. W., et al. (2013). Etiology and therapeutic approach to elevated lactate levels. Mayo Clinic Proceedings, 88(10), 1127-1140.

29. Sia, P., Plumb, T. J., \& Fillaus, J. A. (2013). Type B lactic acidosis associated with multiple myeloma. American Journal of Kidney Diseases, 62(3), 633-637.

30. DeBerardinis, R. J., \& Chandel, N. S. (2016). Fundamentals of cancer metabolism. Science Advances, 2(5), e1600200.

31. Stine, Z. E., et al. (2015). MYC, metabolism, and cancer. Cancer Discovery, 5(10), 1024-1039.

32. Laplante, M., \& Sabatini, D. M. (2012). mTOR signaling in growth control and disease. Cell, 149(2), 274-293.

33. Dang, C. V., Le, A., \& Gao, P. (2009). MYC-induced cancer cell energy metabolism and therapeutic opportunities. Clinical Cancer Research, 15(21), 6479-6483.

34. Le, A., \& Dang, C. V. (2013). Studying Myc's role in metabolism regulation. Methods in Molecular Biology, 1012, 213-219.

35. Korac, P., et al. (2017). Role of MYC in B cell lymphomagenesis. Genes (Basel), 8, 4.

36. Cairns, R. A., Harris, I. S., \& Mak, T. W. (2011). Regulation of cancer cell metabolism. Nature Reviews. Cancer, 11(2), 85-95.

37. Li, T., Copeland, C., \& Le, A. (2021). Glutamine metabolism in cancer. Advances in Experimental Medicine and Biology, 1311, https://doi. org/10.1007/978-3-030-65768-0_2

38. Tran, T. Q., et al. (2017). Tumor-associated mutant p53 promotes cancer cell survival upon glutamine deprivation through p21 induction. Oncogene, 36(14), 1991-2001.

39. Bhatt, A. P., et al. (2012). Dysregulation of fatty acid synthesis and glycolysis in non-Hodgkin lymphoma. Proceedings of the National Academy of Sciences of the United States of America, 109(29), 11818-11823.

40. Park, J. K., et al. (2021). The heterogeneity of lipid metabolism in cancer. Advances in Experimental Medicine and Biology, 1311, https://doi. org/10.1007/978-3-030-65768-0_3.

41. Bhatt, A. P., et al. (2010). Dual inhibition of PI3K and mTOR inhibits autocrine and paracrine proliferative loops in PI3K/Akt/mTOR-addicted lymphomas. Blood, 115(22), 4455-4463.

42. Faber, A. C., et al. (2006). Inhibition of phosphatidylinositol 3-kinase-mediated glucose metabolism coincides with resveratrol-induced cell cycle arrest in human diffuse large B-cell lymphomas. Biochemical Pharmacology, 72(10), 1246-1256.

43. Sin, S. H., et al. (2007). Rapamycin is efficacious against primary effusion lymphoma (PEL) cell lines in vivo by inhibiting autocrine signaling. Blood, 109(5), 2165-2173.

44. Jeon, S. M., Chandel, N. S., \& Hay, N. (2012). AMPK regulates NADPH homeostasis to promote tumour cell survival during energy stress. Nature, 485(7400), 661-665.

45. Caro, P., et al. (2012). Metabolic signatures uncover distinct targets in molecular subsets of diffuse large B cell lymphoma. Cancer Cell, 22(4), 547-560.

46. Young, R. M., et al. (2015). B-cell receptor signaling in diffuse large B-cell lymphoma. Seminars in Hematology, 52(2), 77-85.

47. Havranek, O., et al. (2017). Tonic B-cell receptor signaling in diffuse large B-cell lymphoma. Blood, 130(8), 995-1006.

48. Martinez-Outschoorn, U. E., et al. (2017). Cancer metabolism: A therapeutic perspective. Nature Reviews. Clinical Oncology, 14(1), 11-31.

49. Wilson, P. M., et al. (2014). Standing the test of time: Targeting thymidylate biosynthesis in cancer therapy. Nature Reviews. Clinical Oncology, 11(5), 282-298.

50. Visentin, M., Zhao, R., \& Goldman, I. D. (2012). The antifolates. Hematology/Oncology Clinics of North America, 26(3), 629-648. ix.

51. Dang, C. V., et al. (2011). Therapeutic targeting of cancer cell metabolism. Journal of Molecular Medicine (Berlin), 89(3), 205-212.

52. Cunningham, J. T., et al. (2014). Protein and nucleotide biosynthesis are coupled by a single rate-limiting enzyme, PRPS2, to drive cancer. Cell, 157(5), 1088-1103.

53. Duvel, K., et al. (2010). Activation of a metabolic gene regulatory network downstream of mTOR complex 1. Molecular Cell, 39(2), 171-183.

54. Kuhajda, F. P. (2000). Fatty-acid synthase and human cancer: New perspectives on its role in tumor biology. Nutrition, 16(3), 202-208. 
55. Vazquez-Martin, A., et al. (2008). Overexpression of fatty acid synthase gene activates HER1/HER2 tyrosine kinase receptors in human breast epithelial cells. Cell Proliferation, 41(1), 59-85.

56. Flavin, R., et al. (2010). Fatty acid synthase as a potential therapeutic target in cancer. Future Oncology, 6(4), 551-562.

57. Gasperini, P., \& Tosato, G. (2009). Targeting the mammalian target of rapamycin to inhibit VEGF and cytokines for the treatment of primary effusion lymphoma. Leukemia, 23(10), 1867-1874.

58. Shestov, A. A., et al. (2016). (13)C MRS and LC-MS flux analysis of tumor intermediary metabolism. Frontiers in Oncology, 6, 135.

59. Dang, C. V. (2010). Rethinking the Warburg effect with Myc micromanaging glutamine metabolism. Cancer Research, 70(3), 859-862.

60. Kim, J., Lee, J. H., \& Iyer, V. R. (2008). Global identification of Myc target genes reveals its direct role in mitochondrial biogenesis and its E-box usage in vivo. PLoS One, 3(3), e1798.

61. Kim, J. W., et al. (2007). Hypoxia-inducible factor 1 and dysregulated c-Myc cooperatively induce vascular endothelial growth factor and metabolic switches hexokinase 2 and pyruvate dehydrogenase kinase 1 . Molecular and Cellular Biology, 27(21), 7381-7393.

62. Li, F., et al. (2005). Myc stimulates nuclearly encoded mitochondrial genes and mitochondrial biogenesis. Molecular and Cellular Biology, 25(14), 6225-6234.

63. Folmes, C. D., et al. (2011). Somatic oxidative bioenergetics transitions into pluripotency-dependent glycolysis to facilitate nuclear reprogramming. Cell Metabolism, 14(2), 264-271.

64. Le, A., et al. (2012). Glucose-independent glutamine metabolism via TCA cycling for proliferation and survival in B cells. Cell Metabolism, 15(1), 110-121.

65. Zimmermann, S. C., et al. (2016). Allosteric glutaminase inhibitors based on a 1,4-di(5-amino-1,3,4thiadiazol-2-yl)butane scaffold. ACS Medicinal Chemistry Letters, 7(5), 520-524.

66. Liu, W., et al. (2012). Reprogramming of proline and glutamine metabolism contributes to the proliferative and metabolic responses regulated by oncogenic transcription factor c-MYC. Proceedings of the National Academy of Sciences of the United States of America, 109(23), 8983-8988.

67. Bertout, J. A., Patel, S. A., \& Simon, M. C. (2008). The impact of $\mathrm{O} 2$ availability on human cancer. Nature Reviews. Cancer, 8(12), 967-975.

68. Qiao, Q., et al. (2010). NF-kappaB mediates aberrant activation of HIF-1 in malignant lymphoma. Experimental Hematology, 38(12), 1199-1208.

69. Furman, R. R., et al. (2014). Idelalisib and rituximab in relapsed chronic lymphocytic leukemia. The New England Journal of Medicine, 370(11), 997-1007.

70. Zhang, L., et al. (2019). Metabolic reprogramming toward oxidative phosphorylation identifies a therapeutic target for mantle cell lymphoma. Science Translational Medicine, 11, 491.
71. Zhao, X., et al. (2017). Unification of de novo and acquired ibrutinib resistance in mantle cell lymphoma. Nature Communications, 8, 14920.

72. Chiron, D., et al. (2014). Cell-cycle reprogramming for PI3K inhibition overrides a relapse-specific C481S BTK mutation revealed by longitudinal functional genomics in mantle cell lymphoma. Cancer Discovery, 4(9), 1022-1035.

73. Kahl, B. S., et al. (2014). A phase 1 study of the PI3Kdelta inhibitor idelalisib in patients with relapsed/refractory mantle cell lymphoma (MCL). Blood, 123(22), 3398-3405.

74. Hess, G., et al. (2009). Phase III study to evaluate temsirolimus compared with investigator's choice therapy for the treatment of relapsed or refractory mantle cell lymphoma. Journal of Clinical Oncology, 27(23), 3822-3829.

75. Witzig, T. E., et al. (2005). Phase II trial of singleagent temsirolimus (CCI-779) for relapsed mantle cell lymphoma. Journal of Clinical Oncology, 23(23), 5347-5356.

76. Garcia, M. G., et al. (2009). PI3K/Akt inhibition modulates multidrug resistance and activates NF-kappaB in murine lymphoma cell lines. Leukemia Research, 33(2), 288-296.

77. Kostakoglu, L., \& Chauvie, S. (2018). Metabolic tumor volume metrics in lymphoma. Seminars in Nuclear Medicine, 48(1), 50-66.

78. Schoder, H., \& Moskowitz, C. (2016). Metabolic tumor volume in lymphoma: Hype or hope? Journal of Clinical Oncology, 34(30), 3591-3594.

79. Watabe, T., et al. (2012). Intratumoral heterogeneity of F-18 FDG uptake differentiates between gastrointestinal stromal tumors and abdominal malignant lymphomas on PET/CT. Annals of Nuclear Medicine, 26(3), 222-227.

80. Cottereau, A. S., et al. (2016). Molecular profile and FDG-PET/CT total metabolic tumor volume improve risk classification at diagnosis for patients with diffuse large B-cell lymphoma. Clinical Cancer Research, 22(15), 3801-3809.

81. Ceriani, L., et al. (2020). SAKK38/07 study: Integration of baseline metabolic heterogeneity and metabolic tumor volume in DLBCL prognostic model. Blood Advances, 4(6), 1082-1092.

82. Senjo, H., et al. (2020). High metabolic heterogeneity on baseline 18FDG-PET/CT scan as a poor prognostic factor for newly diagnosed diffuse large B-cell lymphoma. Blood Advances, 4(10), 2286-2296.

83. Ceriani, L., et al. (2018). Metabolic heterogeneity on baseline 18FDG-PET/CT scan is a predictor of outcome in primary mediastinal B-cell lymphoma. Blood, 132(2), 179-186.

84. Meignan, M., et al. (2016). Baseline metabolic tumor volume predicts outcome in high-tumor-burden follicular lymphoma: A pooled analysis of three multicenter studies. Journal of Clinical Oncology, 34(30), 3618-3626. 
85. Albano, D., et al. (2018). Prognostic role of pretreatment 18F-FDG PET/CT in primary brain lymphoma. Annals of Nuclear Medicine, 32(8), 532-541.

86. Storto, G., et al. (2010). Assessment of metabolic response to radioimmunotherapy with 90Y-ibritumomab tiuxetan in patients with relapsed or refractory B-cell non-Hodgkin lymphoma. Radiology, 254(1), 245-252.

87. Bruzzi, J. F., et al. (2006). Detection of Richter's transformation of chronic lymphocytic leukemia by PET/ CT. Journal of Nuclear Medicine, 47(8), 1267-1273.
88. Falchi, L., et al. (2014). Correlation between FDG/ PET, histology, characteristics, and survival in 332 patients with chronic lymphoid leukemia. Blood, 123(18), 2783-2790.

89. Mauro, F. R., et al. (2015). Diagnostic and prognostic role of PET/CT in patients with chronic lymphocytic leukemia and progressive disease. Leukemia, 29(6), 1360-1365.

90. Nguyen, T., et al. (2019). Uncovering the role of $\mathrm{N}$-acetyl-aspartyl-glutamate as a glutamate reservoir in cancer. Cell Reports, 27(2), 491-501. e6.

Open Access This chapter is licensed under the terms of the Creative Commons Attribution 4.0 International License (http://creativecommons.org/licenses/by/4.0/), which permits use, sharing, adaptation, distribution and reproduction in any medium or format, as long as you give appropriate credit to the original author(s) and the source, provide a link to the Creative Commons license and indicate if changes were made.

The images or other third party material in this chapter are included in the chapter's Creative Commons license, unless indicated otherwise in a credit line to the material. If material is not included in the chapter's Creative Commons license and your intended use is not permitted by statutory regulation or exceeds the permitted use, you will need to obtain permission directly from the copyright holder. 


\title{
The Heterogeneity Metabolism of Renal Cell Carcinomas
}

\author{
Mohammadreza Zarisfi, Tu Nguyen, \\ Jessie R. Nedrow, and Anne Le
}

\section{Keywords}

Renal cell carcinoma $\cdot$ Metabolic phenotypes

- Glucose metabolism - Glutamine metabolism · Oncogenic heterogeneity_intratumoral heterogeneity

\section{Abbreviations}

ACADM

$$
\text { Medium-chain }
$$
dehydrogenase

ACC Acetyl-CoA carboxylase

AMPK AMP-activated protein kinase

ARID1A AT-rich interaction domain 1A

ASS1

ATP

BRG1
Arginosuccinate synthase

Adenosine triphosphate

Brahma-related gene 1
M. Zarisfi · J. R. Nedrow

Department of Radiology, Johns Hopkins University

School of Medicine, Baltimore, MD, USA

T. Nguyen

University of California, Los Angeles (UCLA) David

Geffen School of Medicine, Los Angeles, CA, USA

A. Le $(\bowtie)$

Department of Pathology and Oncology, Johns

Hopkins University School of Medicine,

Baltimore, MD, USA

Department of Chemical and Biomolecular Engineering, Johns Hopkins University Whiting

School of Engineering, Baltimore, MD, USA

e-mail: annele@jhmi.edu

$\begin{array}{ll}\text { BRM } & \text { Brahma gene } \\ \text { ccRCC } & \text { Clear-cell renal cell carcinoma } \\ \text { ePC } & \text { Ether-type PC } \\ \text { ePE } & \text { Ether-type PE } \\ \text { ERR } \alpha & \text { Estrogen-related receptor A } \\ \text { FASN } & \text { Fatty acid synthase } \\ \text { FDG } & \text { Fluorodeoxyglucose } \\ \text { FFAs } & \text { Free fatty acids } \\ \text { FH } & \text { Fumarate hydratase }\end{array}$

G6PH Glucose-6-phosphate dehydrogenase

GLS2 Glutaminase 2

Glu Glutamine

GLU-CYS Cysteine, $\gamma$-glutamyl cysteine

GLUT1 Glucose transporter 1

GLUT2 Glucose transporter 2

GSH Glutathione

GSSG Glutathione disulfide

HCC Hepatocellular carcinoma

HIF Hypoxia-inducible factor

HIF- $1 \alpha$ Hypoxia-inducible factor 1-alpha

HK2 Hexokinase 2

KDM5C Lysine-specific histone demethylase $5 \mathrm{C}$

LDHA Lactate dehydrogenase A

lncRNAs Long noncoding RNAs

MC1 Metabolic cluster 1

MC2 Metabolic cluster 2

MIR21 MicroRNA 21

MRI Magnetic resonance imaging

mTOR Mammalian target of rapamycin

mTORC1 Mechanistic target of rapamycin complex 1 


$\begin{array}{ll}\text { NADPH } & \begin{array}{l}\text { Nicotinamide adenine dinucleotide } \\ \text { phosphate }\end{array} \\ \text { NAE } & \text { N-acylethanolamine } \\ \text { PBRM1 } & \text { Polybromo-1 } \\ \text { PC } & \text { Phosphatidylcholine } \\ \text { PE } & \text { Phosphatidylethanolamine } \\ \text { PET } & \text { Positron-emission tomography } \\ \text { PGK1 } & \text { Phosphoglycerate kinase 1 } \\ \text { PGLS } & \text { 6-Phosphogluconolactonase } \\ \text { PHD } & \text { Prolyl hydroxylase } \\ \text { PI3K } & \text { Phosphatidylinositol-3 kinases } \\ \text { PKM2 } & \text { Pyruvate kinase isoform M2 } \\ \text { PPP } & \text { Pentose phosphate pathway } \\ \text { PTEN } & \text { Phosphatase and tensin homolog } \\ & \text { deleted in chromosome 10 } \\ \text { PS } & \text { Phosphatidylserine } \\ \text { RCC } & \text { Renal cell carcinoma } \\ \text { ROS } & \text { Reactive oxygen species } \\ \text { SETD2 } & \text { SET domain containing 2 } \\ \text { SLCs } & \text { Solute carriers } \\ \text { SM } & \text { Sphingomyelin } \\ \text { TALDO } & \text { Transaldolase } \\ \text { TCA } & \text { Tricarboxylic acid } \\ \text { TKT } & \text { Transketolase } \\ \text { TSC2 } & \text { Tuberous sclerosis 2 } \\ \text { VEGFR } & \text { Vascular endothelial growth factor } \\ & \text { receptor } \\ \text { VHL } & \text { Von Hippel-Lindau tumor-suppressor } \\ & \text { gene } \\ \alpha-K G & \alpha \text {-Ketoglutarate } \\ & \end{array}$

\section{Key Points}

- Different oncogenic mutations lead to various metabolic phenotypes in renal cell carcinomas (RCC).

- Loss of the von Hippel-Lindau (VHL) tumorsuppressor gene results in metabolic alterations, including shifts toward aerobic glycolysis in RCC.

- Fumarate hydratase mutations result in an increase in aerobic glycolysis in RCC.

- The upregulation of glycolysis enzymes is correlated with high-grade RCC.

- An increase in glutathione (GSH)/glutathione disulfide (GSSG) ratio protects RCC against oxidative stress in a grade-dependent manner.
- Metabolic spatial heterogeneity can be induced by both gene-dependent and independent processes.

- Metabolic intratumoral heterogeneity explains the failure of monotherapy and necessitates the need for personalized and/or combination therapies for RCC.

\section{Introduction}

According to data from the American Cancer Society, cancer is one of the deadliest health problems globally. Annually, renal cell carcinoma (RCC) causes more than 100,000 deaths worldwide [1-4], posing an urgent need to develop effective treatments to increase patient survival outcomes. New therapies are expected to address a major factor contributing to cancer's resistance to standard therapies: oncogenic heterogeneity. Gene expression can vary tremendously among different types of cancers, different patients of the same tumor type, and even within individual tumors; various metabolic phenotypes can emerge, making single-therapy approaches insufficient. Novel strategies targeting the diverse metabolism of cancers aim to overcome this obstacle. Though some have yielded positive results, it remains a challenge to uncover all of the distinct metabolic profiles of RCC. In the quest to overcome this obstacle, the metabolic oriented research focusing on these cancers has offered freshly new perspectives, which are expected to contribute heavily to the development of new treatments.

\section{Different Oncogenic Mutations Lead to Different Metabolic Phenotypes in RCC (Fig. 1)}

Renal cell carcinoma (RCC), or hypernephroma, is the most common type of kidney cancer in adults, responsible for approximately $90-95 \%$ of all cases. RCC originates from the network of convoluted tubules of the nephron [5] and consists of diverse histological subtypes, each with 

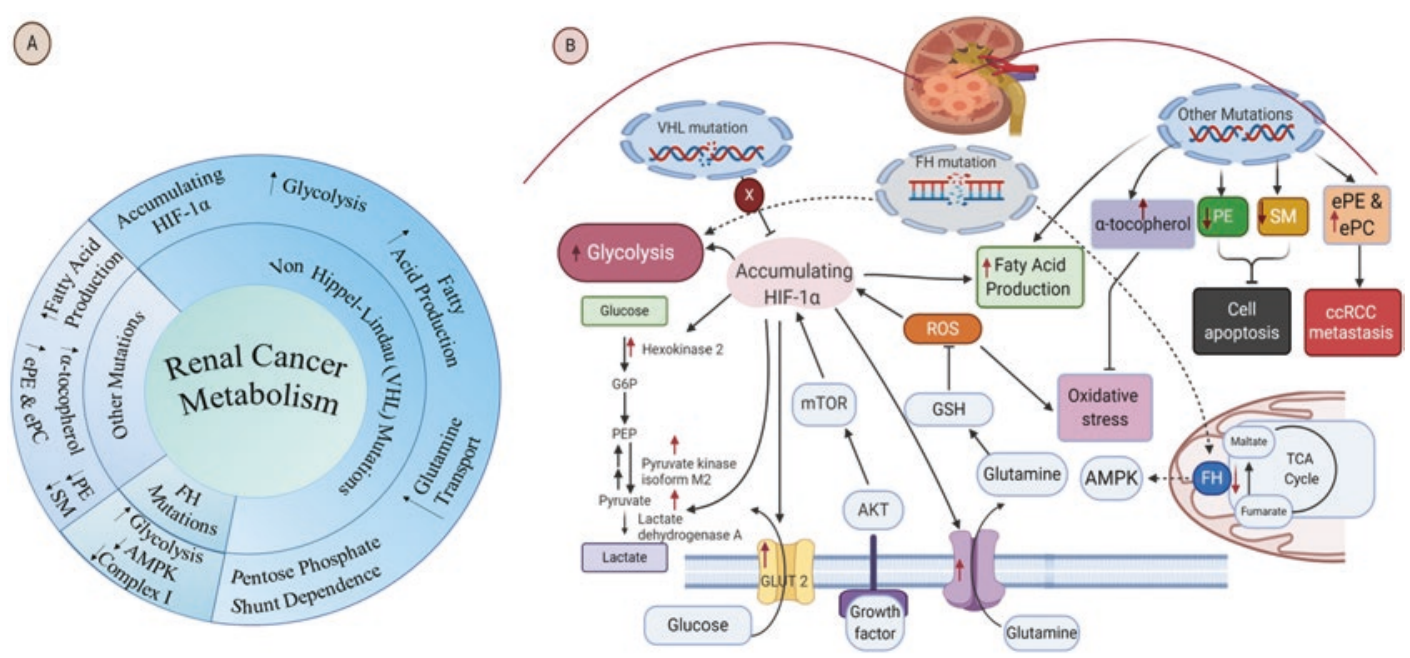

Fig. 1 Heterogeneous metabolic phenotypes in renal cell carcinoma as a result of different oncogenic mutations. $A K T$ protein kinase $\mathrm{B}, A M P K$ AMP-activated protein kinase, $e P C$ ether-type phosphatidylcholine, $e P E$ ether type, $F H$ fumarate hydratase, G6P glucose-6-phosphate, GLUT 2 glucose transporter 2, GSH glutathione, HIF-1 $\alpha$ hypoxia-inducible factor 1-alpha, $m T O R$ mammalian target of rapamycin, $P E P$ phosphoenolpyruvic acid, ROS reactive oxygen species, $S M$ sphingomyelin, TCA cycle tricarboxylic acid cycle

unique sets of metabolic rearrangements that can be traced back to gene alterations $[6,7]$. These genomic abnormalities provide cancer cells with the advantageous abilities to adapt to the limitations of their microenvironments and meet the demands of rapid and deleterious cell division.

\subsection{Loss of the von Hippel-Lindau Tumor-Suppressor Gene Results in Metabolic Alterations Including Shifts Toward Aerobic Glycolysis in RCC}

Loss of the von Hippel-Lindau (VHL) tumorsuppressor gene is the most prominent genetic alteration in RCC, commonly associated with over $80 \%$ of clear-cell renal cell carcinoma (ccRCC) tumors $[8,9]$. The protein product of VHL facilitates the degradation of hypoxiainducible factor (HIF) [10]. Thus, the loss of VHL leads to the accumulation of HIF- $1 \alpha$ and the constitutive activation of hypoxia-inducible genes, even in oxygenated conditions. This includes the enhanced expressions of glucose transporter 2 (GLUT2), hexokinase 2 (HK2), and lactate dehydrogenase A (LDHA), which are keys to the meta- bolic shift towards aerobic glycolysis in tumors with this genotype [11, 12]. In fact, enhanced HIF- $1 \alpha$ activity is thought to mediate the Warburg effect in RCC $[13,14]$. Moreover, a study by Gill et al. found that HIF upregulation led to increases in glucose and amino acid uptake, lipogenesis, and augmentation of glycolysis through upregulation of MET protein expression and subsequent phosphatidylinositol 3-kinase (PI3K)/Akt signaling pathway activation [14-16].

In addition, pentose phosphate shunt dependence, increases in glutamine transport, and fatty acid production all have been documented as VHL-associated metabolic alterations in ccRCC [17]. The increased activity of the pentose phosphate pathway (PPP) plays a significant role in protecting cancer cells from oxidative stress as this pathway generates NADPH and enables the maintenance of glutathione levels [13]. The Consortia of The Cancer Genome Atlas Research Network revealed that upregulation of PPP genes (G6PH, PGLS, TALDO, and TKT), fatty acid synthesis genes (ACC and FASN), and PI3K pathway-enhancing genes (MIR21) correlated with worse survival. In contrast, upregulation of AMP-activated protein kinase (AMPK) complex genes, multiple Krebs cycle genes, and PI3K pathway inhibitors (phosphatase and tensin 
homolog deleted in chromosome 10 (PTEN), tuberous sclerosis 2 (TSC2)) correlated with better survival [17].

\subsection{Fumarate Hydratase Mutations Result in an Increase in Aerobic Glycolysis in RCC}

Studies by Tong et al. indicated that RCC cells carrying inactivated fumarate hydratase $(\mathrm{FH})$, a tricarboxylic acid (TCA) cycle enzyme, demonstrated metabolic changes that were distinct from other genetically defined RCC, such as an increase in aerobic glycolysis and advanced tumorigenicity. Thus, fumarate hydratase-deficient kidney cancer has low oxygen consumption rates as well as low mitochondrial complex I activities [18, 19]. In addition to having a glycolytic shift, FH-deficient kidney tumors and cell lines from patients with hereditary leiomyomatosis and RCC also exhibited decreased levels of AMPK, a key metabolic regulator. Glycolytic upregulation allows the cells to adapt to growth demands by generating NADPH, acetyl-CoA, and precursors for ribose, protein, and fatty acid biosynthesis through reduced AMPK signaling. Furthermore, another study by Massari et al. demonstrated that intracellular augmentation of fumarate has an inhibitory effect on HIF prolyl hydroxylase (PHD) and consequently inhibited the activity of the VHL ubiquitination complex, which, in turn, led to the upregulation of HIF-target genes (VEGF and GLUT1) in a VHL-independent manner by stabilizing HIF1a [20]. On the other hand, intracellular reactive oxygen species (ROS) accumulation under glycolysis can also result in HIF1a stabilization [21].

LDHA inhibition has shown promise against FH-deficient RCC cells in vitro and in vivo [22] and other cancers [23-26]. Metformin, an antidiabetic medication, was reported to activate AMPK and inhibit RCC growth in vitro and in vivo [27].

\section{Metabolic Signatures of RCC}

\subsection{Metabolic Differences Between Normal Renal Cells and RCC}

To compensate for the high demands of energy and biosynthetic macromolecules for proliferation, RCC cells change their metabolic phenotypes from that of normal renal cells to satisfy the demands. This leads to the different metabolic signatures of RCC cells as compared to normal renal cells. One of the metabolic signatures presenting in RCC cells found by Saito et al. is the decrease in the level of phosphatidylethanolamine (PE) in RCC cells as compared to normal cells [28]. PE is one of the most abundant glycerophospholipids in eukaryotes and plays a crucial role in autophagy, cell division, and protein folding and acts as a precursor for synthesizing phosphatidylcholine (PC), phosphatidylserine (PS) N-acylethanolamine (NAE) [29-32]. Furthermore, the study by Saito et al. shows that increased PE, which is induced by ethanolamine, inhibits RCC cell proliferation [28]. Low levels of PE inhibit cell apoptosis, which explains why the downregulation of PE benefits RCC cells. The study also found that increases in ether-type PE (ePE) and ether-type phosphatidylcholine (ePC) are associated with ccRCC metastasis [28].

Interestingly, other studies showed that there is an upregulation of the fetal isoform of pyruvate kinase, isoform M2 (PKM2), across all grades of RCC, suggesting that tumor cells utilize different enzymes from those found in normal tissue to enhance their growth $[33,34]$.

Moreover, another metabolic feature of RCC is the decrease of sphingomyelin (SM), an essential component of the plasma membrane that regulates the formation of lipid microdomains through interacting with cholesterol and glycerophospholipids $[28,35]$. A high level of SM was reported to make the cells more vulnerable to apoptosis [36].

In addition to the metabolic signatures of RCC reported by Saito et al., the study by Catchpole et al. found that the upregulation of fatty acid levels was potentially linked to the metastatic 
stage of the malignancy [37]. The high fatty acid levels were the results of an increase in de novo fatty acid synthesis and/or a decrease in fatty acid oxidation, which often occurred during the invasive and metastatic stage of RCC [37, 38]. Another compound with high concentration, $\alpha$-tocopherol, was found in RCC and ovarian cancer to protect tumor cells against oxidative stress [37, 39].

Another interesting metabolic signature in RCC cells is related to amino acid metabolism. Specifically, while arginosuccinate synthase 1 (ASS1) is known to play an important role in the urea cycle via ammonia detoxification through the conversion of citrulline to arginine [40], the study by Yoon et al. revealed an absence or decrease of ASS1 in ccRCC; therefore, arginine was identified as an auxotrophic marker for ccRCC [41]. With the use of proteomics, another study by Perroud et al. similarly demonstrated the downregulation of ASS1 across all the grades of ccRCC [34]. Metabolic profiling using metabolomics technologies [42] has revealed different key metabolic phenotypes of RCC and identified potential targets for new treatments for RCC patients.

\subsection{Temporal Impact of RCC Metabolism (Fig. 2)}

Using the Fuhrman grading system, Wettersten et al. demonstrated that different grades of RCC were associated with different biochemical processes that have distinctive and prominent metabolic reprogramming, which is supported by the observation of clinically distinguishable features within various tumor grades [43]. In addition, Kang et al. confirmed the metabolic reprogramming in ccRCC by demonstrating the stagedependent alteration in the expression of solute carriers (SLCs) [44]. SLCs have an important role in transmembrane transportation of specific metabolic substrates, including inorganic ions, nucleotides, amino acids, fatty acids, and sugars [45].

Using a combination of proteomics and metabolomics analysis, Wettersten et al. correlated increasing amounts of metabolites in the aerobic glycolysis pathway, including glucose, glucose-6-phosphate, and fructose-6-phosphate, with higher tumor grade. Likewise, an indication that glucose metabolism is reprogrammed to the

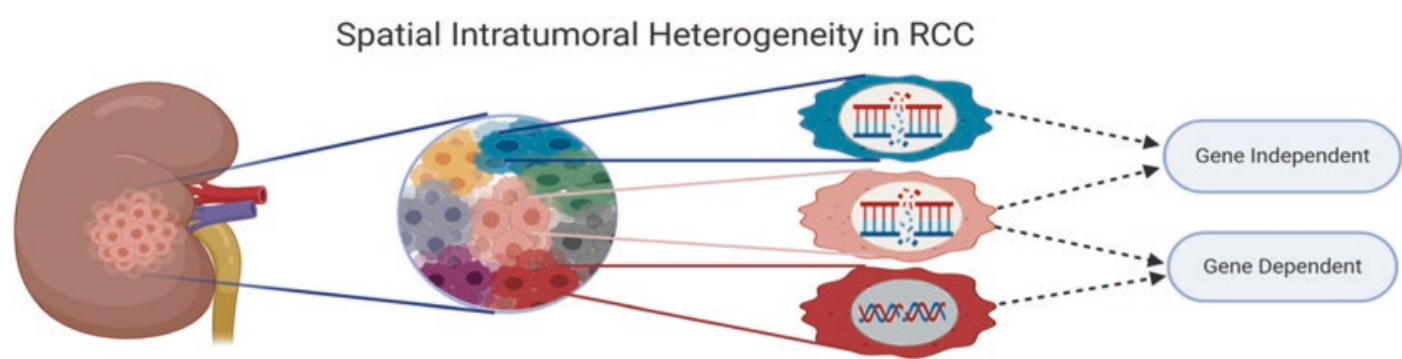

Temporal Intratumoral Heterogeneity in RCC
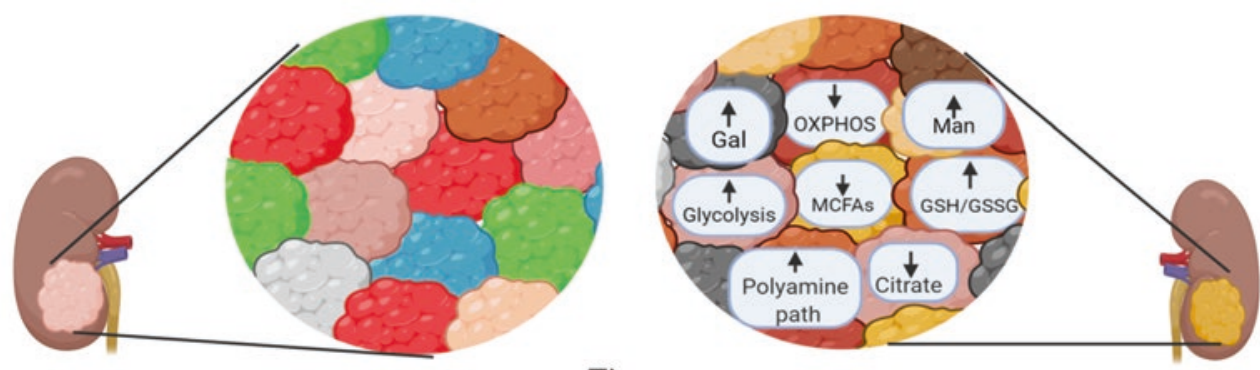

Time

Fig. 2 Spatial and temporal intratumoral heterogeneity in renal cell carcinoma. Gal galactose, GSH glutathione, GSSG glutathione disulfide, Man mannose, MCFAs medium-chain fatty acids 
lactate fermentation pathway, especially in advanced disease, is supported by the gradedependent upregulation of lactate [43]. Consistently, a study by Hakimi et al. reported a more than twofold downregulation of citrate concentration in high-stage tumors [46] that may contribute to a reduction in oxidative phosphorylation capacity. However, in a study conducted by Perroud et al., the correlation of the upregulation of phosphoglycerate kinase 1 (PGK1) and grade 1 and 2 tumors is debatable [34]. On the other hand, Hakimi et al. showed that high levels of galactose and mannose were associated with an advanced stage of tumor progression [46].

A study by Horiguchi et al. first revealed the correlation between fatty acid synthase expression and high grade and metastasis of RCC [47]. On the other hand, Wettersten et al. found a broad reduction in shorter chain free fatty acid (FFA) oxidation in high-grade tumors, whereas there was an increase in carnitine and acylcarnitine levels in all grades of RCC. In accordance with this finding, Hakimi et al. discovered in high-stage tumors a reduction in cis-aconitate and mediumchain fatty acids [43, 46]. Furthermore, Xiao et al. showed that genes related to fatty acid $\beta$-oxidation enzymes, EHHADH (3-hydroxyacyl CoA dehydrogenase and enoyl-CoA hydratase) and ACADM (medium-chain acyl-CoA dehydrogenase), were simultaneously downregulated at the mRNA and protein levels in opposite proportion to stages of ccRCC [48].

Glutathione (GSH)/glutathione disulfide (GSSG) ratio is an important indicator of oxidative stress. Wettersten et al. revealed that this ratio is grade dependent and consistent with the downregulation of the catabolic enzymes responsible for utilizing glutamine and glutamate in the TCA and urea cycles, indicating a protective role of glutamine reprogramming against oxidative stress in high-grade tumors [43, 49]. Similarly, upregulation of the majority of metabolites involved in the glutathione synthesis such as cysteine, $\boldsymbol{\gamma}$-glutamyl cysteine (GLU-CYS), and GSH was observed by Hakimi et al. in a stage-dependent manner [46]. Furthermore, they demonstrated that methionine augmentation at higher tumor stages could be related to the upregulation of glutathione metabolism. In fact, the glutathione regeneration pathway is carried out by homocysteine being converted to cysteine via cystathionine [50].

Interestingly, Hakimi et al. found a gradedependent increase in the level of methylthioadenosine (MTA). MTA is a downstream product of the polyamine biosynthetic pathway, implying that alterations in the polyamine pathway are correlated to tumor progression [46]. MTA is also part of the methionine and adenine salvage pathway, where phosphorylation of MTA via methylthioadenosine phosphorylase (MTAP) leads to the regeneration of adenine and methionine [51]. Using metabolomic approaches, $\mathrm{Xu}$ et al. recently identified a stage-dependent decrease in the expression of MTAP that consequently resulted in the accumulation of MTA in RCC cells [52].

\subsection{Intratumoral Heterogeneity of RCC (Fig. 2)}

\subsubsection{Gene Independence}

While intratumoral genetic heterogeneity has been extensively investigated in ccRCC [53, 54], only a small number of recent studies has documented the regional variations in metabolic patterns [55-57]. Specifically, mutations of SET domain containing 2 (SETD2), PTEN, and lysine-specific histone demethylase (KDM5C) were found within the same tumor, suggesting that convergent phenotypic evolution and mutations of VHL were ubiquitously detected by multi-region sequencing in all analyzed regions [54]. Using global metabolomics analyses, Okegawa et al. investigated the heterogeneity of intratumoral metabolic profile and gene mutations in multiple regions of a single primary RCC tumor. Two major metabolically different tumor clusters, metabolic cluster 1 (MC1) and metabolic cluster 2 (MC2), were identified among 32 spatially separated sections of the tumor tissue. They observed an upregulation of the glycolysisPPP, glutathione, and amino acids in the MC1 region as opposed to the downregulation of glycolysis-PPP metabolites and glutathione (reduced) in MC2 region. They also showed that the MC2 region had elevated levels of pyruvate, cystine, and 2-oxobutyric acid. This suggested 
that tumor cells are dependent on the presence of pyruvate in $\mathrm{MC} 2$ regions for growth stimulation, which may make pyruvate metabolism an ideal therapeutic target [55]. Importantly, they showed that the Warburg-like effect is present in all tumor sites without any correlation to the status of the VHL gene. Ultimately, the clear intratumoral metabolic heterogeneity identified within a single tumor may contribute to treatment resistance [56, 57]. In contrast, an almost uniform pattern of lipid profile was observed across the samples [55, 58]. Interestingly, the study also showed no correlation between the mutational status of genes and metabolic patterns, suggesting that other factors, such as noncanonical metabolic flux, tumor microenvironment, and epigenetics, may regulate the metabolic phenotype [55].

Accumulating evidence from various cancer studies suggests the important role of long noncoding RNAs (lncRNAs) in different cellular processes, including metabolism [59-61] through regulation of gene expression at the epigenetic level $[62,63]$. Specifically, a study by Li et al. found intertumoral heterogeneity of IncRNAs in ccRCC that can contribute to the regulation of the HIF-1 signaling pathway [64].

Using hyperpolarized carbon-13 $\left({ }^{13} \mathrm{C}\right)$ magnetic resonance imaging (HP-MRI) technique, Tran et al. noted the intertumoral heterogeneity of pyruvate delivery, pyruvate-to-lactate metabolic conversion, and ratio of lactate to pyruvate across the RCC but did not investigate whether this heterogeneity is gene dependent or not [65].

Using Dixon-based MRI technique, Zhang et al. demonstrated the correlation between fat fraction and intratumoral heterogeneity of metabolites; however, the heterogeneity was more prominent between different tumor types [66].

\subsubsection{Gene Dependence}

Using $\left[{ }^{18} \mathrm{~F}\right]$ fluorodeoxyglucose (FDG) and positron-emission tomography (PET)/MR imaging, Brooks et al. also demonstrated that the presence of glycolytic enzyme FBP1, higher levels of glucose transporter GLUT1, and lower expression of FBP1 and HIF- $1 \alpha$ transcription factor led to regional variation in metabolic heterogeneity. This study revealed an association between $\left[{ }^{18} \mathrm{~F}\right] \mathrm{FDG}$ avidity and distinct patterns of meta- bolic genes and protein expression. The $\left[{ }^{18} \mathrm{~F}\right]$ FDG-avid region had a lower expression of FBP1 and HIF1a and higher expression of glucose transporter GLUT1, which could be related to a higher metabolic activity supported by boosting glucose uptake [22].

Polybromo-1 (PBRM1) gene is one of the major subunits of PBAF (polybromo-associated BRG1 or BRM-associated factors). Loss of its expression is considered as another common mutation in tumor-suppressor gene in ccRCC, besides VHL $[67,68]$. Interestingly, Chowdhury et al. demonstrated that re-expression of PBAF in a PBAF-deficient ccRCC could lead to the reversion of several important metabolic signatures in RCC, including glucose and cholesterol metabolism [69]. Using tissue microarray (TMA)-based immunohistochemistry from each tumor, a study by Jiang et al. revealed different expressions of PBRM1, AT-rich interaction domain 1A (ARID1A), SETD2, brahma-related gene 1 (BRG1), and brahma gene (BRM) among 160 ccRCCs, indicating high intratumoral heterogeneity of PBRM1 expression and its associated proteins of the same tumor [70].

\section{$4 \quad$ RCC Therapy}

The current standard frontline therapies for metastatic RCC are largely VEGFR inhibitors, such as sunitinib and sorafenib. However, about 20-30\% of patients do not respond to these therapies, and among those, nearly all the patients become resistant to these drugs [71].

Activation of the mammalian target of rapamycin (mTOR), a member of PI3K-related kinases, through VEGF signaling pathways, can lead to protein synthesis and energy production in RCC [72]. Although a variety of agents have been investigated to target the mTOR pathway, only recently three mTOR inhibitors have been approved as second-line therapy in patients with RCC: temsirolimus (Torisel ${ }^{\circledR}-\mathrm{CCI}-779,4$ ), everolimus or rad001 (Afinitor ${ }^{\circledR}, 4 a$ ), and sirolimus (Rapamycin $\left.{ }^{\circledR}, 4 b\right)$ [73, 74]. Interestingly, a study by Li et al. revealed that mTOR inhibitor (everolimus) treatment could overcome the Warburg effect via mTOR pathway blocking and 
HIF1 $\alpha$ expression inhibition [75]. Recent work by Gameiro et al. found that loss of VHL rendered RCC cells sensitive to glutamine deprivation [76]. In line with this finding, they found that systematic treatment with glutaminase inhibitors suppressed ccRCC growth both in vitro and in vivo. Other metabolic targeting therapies for RCC include mitochondrial inhibition by auraptene [77] and GLUT1 inhibition by STF-3 [78].

\section{Conclusion}

Metabolic spatial and temporal heterogeneity represents one of the main mechanisms behind the high therapy resistance of RCC. Recent findings suggest that initial monotherapy failure can be associated with subclonal variation in metabolic heterogeneity. Targeting several metabolic pathways is the key strategy for effective therapy $[79,80]$. It is also important to mention that metabolic phenotypic heterogeneity is regulated through both gene-dependent and geneindependent manners. Taken together, RCC treatment has a variety of challenges posed by intratumoral metabolic heterogeneity, highlighting the need for the further development of novel approaches to identify clonally dominant metabolic targets for the future development of effective therapies for RCC.

\section{References}

1. Jemal, A., et al. (2011). Global cancer statistics. $C A$ : A Cancer Journal for Clinicians, 61(2), 69-90.

2. Global Burden of Disease Study. (2016). Global, regional, and national life expectancy, all-cause mortality, and cause-specific mortality for 249 causes of death, 1980-2015: A systematic analysis for the Global Burden of Disease Study 2015. Lancet, 388(10053), 1459-1544.

3. Siegel, R. L., Miller, K. D., \& Jemal, A. (2017). Cancer statistics, 2017. CA: A Cancer Journal for Clinicians, 67(1), 7-30.

4. Akinyemiju, T., et al. (2017). The burden of primary liver cancer and underlying etiologies from 1990 to 2015 at the global, regional, and national level: Results from the Global Burden of Disease Study 2015. JAMA Oncology, 3(12), 1683-1691.

5. Gu, F. L., et al. (1991). Cellular origin of renal cell carcinoma--an immunohistological study on mono- clonal antibodies. Scandinavian Journal of Urology and Nephrology. Supplementum, 138, 203-206.

6. Rini, B. I., Campbell, S. C., \& Escudier, B. (2009). Renal cell carcinoma. Lancet, 373(9669), 1119-1132.

7. Sudarshan, S., et al. (2013). Metabolism of kidney cancer: From the lab to clinical practice. European Urology, 63(2), 244-251.

8. Sato, Y., et al. (2013). Integrated molecular analysis of clear-cell renal cell carcinoma. Nature Genetics, 45(8), 860-867.

9. Nickerson, M. L., et al. (2008). Improved identification of von Hippel-Lindau gene alterations in clear cell renal tumors. Clinical Cancer Research, 14(15), 4726-4734.

10. Czyzyk-Krzeska, M. F., \& Meller, J. (2004). von Hippel-Lindau tumor suppressor: Not only HIF's executioner. Trends in Molecular Medicine, 10(4), 146-149.

11. Stubbs, M., \& Griffiths, J. R. (2010). The altered metabolism of tumors: HIF-1 and its role in the Warburg effect. Advances in Enzyme Regulation, 50(1), 44-55.

12. Gordan, J. D., Thompson, C. B., \& Simon, M. C. (2007). HIF and c-Myc: Sibling rivals for control of cancer cell metabolism and proliferation. Cancer Cell, 12(2), 108-113.

13. Pinthus, J. H., et al. (2011). Metabolic features of clear-cell renal cell carcinoma: Mechanisms and clinical implications. Canadian Urological Association Journal, 5(4), 274-282.

14. Semenza, G. L. (2007). HIF-1 mediates the Warburg effect in clear cell renal carcinoma. Journal of Bioenergetics and Biomembranes, 39(3), 231-234.

15. Gill, A. J., et al. (2014). Succinate dehydrogenase (SDH)-deficient renal carcinoma: A morphologically distinct entity: A clinicopathologic series of 36 tumors from 27 patients. The American Journal of Surgical Pathology, 38(12), 1588-1602.

16. Sulpice, E., et al. (2009). Cross-talk between the VEGF-A and HGF signalling pathways in endothelial cells. Biology of the Cell, 101(9), 525-539.

17. The Cancer Genome Atlas Research Network. (2013). Comprehensive molecular characterization of clear cell renal cell carcinoma. Nature, 499(7456), 43-49.

18. Yang, Y., et al. (2010). UOK 262 cell line, fumarate hydratase deficient (FH-/FH-) hereditary leiomyomatosis renal cell carcinoma: In vitro and in vivo model of an aberrant energy metabolic pathway in human cancer. Cancer Genetics and Cytogenetics, 196(1), $45-55$.

19. Tong, W. H., et al. (2011). The glycolytic shift in fumarate-hydratase-deficient kidney cancer lowers AMPK levels, increases anabolic propensities and lowers cellular iron levels. Cancer Cell, 20(3), 315-327.

20. Massari, F., et al. (2015). Metabolic alterations in renal cell carcinoma. Cancer Treatment Reviews, 41(9), 767-776.

21. Ishikawa, K., et al. (2008). ROS-generating mitochondrial DNA mutations can regulate tumor cell metastasis. Science, 320(5876), 661-664. 
22. Brooks, S. A., et al. (2016). Alternate metabolic programs define regional variation of relevant biological features in renal cell carcinoma progression. Clinical Cancer Research, 22(12), 2950-2959.

23. Le, A., et al. (2010). Inhibition of lactate dehydrogenase A induces oxidative stress and inhibits tumor progression. Proceedings of the National Academy of Sciences of the United States of America, 107(5), 2037-2042.

24. Rajeshkumar, N. V., et al. (2015). Therapeutic targeting of the Warburg effect in pancreatic cancer relies on an absence of p53 function. Cancer Research, 75(16), 3355-3364.

25. Dutta, P., et al. (2013). Evaluation of LDH-A and glutaminase inhibition in vivo by hyperpolarized 13C-pyruvate magnetic resonance spectroscopy of tumors. Cancer Research, 73(14), 4190-4195.

26. Bose, S., Zhang, C., \& Le, A. (2021). Glucose metabolism in cancer: The Warburg effect and beyond. Advances in Experimental Medicine and Biology, 1311, https://doi.org/10.1007/978-3-030-65768-0_1

27. Liu, J., et al. (2013). Metformin inhibits renal cell carcinoma in vitro and in vivo xenograft. Urologic Oncology, 31(2), 264-270.

28. Saito, K., et al. (2016). Lipidomic signatures and associated transcriptomic profiles of clear cell renal cell carcinoma. Scientific Reports, 6, 28932.

29. Fagone, P., \& Jackowski, S. (2013). Phosphatidylcholine and the CDP-choline cycle. Biochimica et Biophysica Acta, 1831(3), 523-532.

30. Vance, J. E., \& Tasseva, G. (2013). Formation and function of phosphatidylserine and phosphatidylethanolamine in mammalian cells. Biochimica et Biophysica Acta, 1831(3), 543-554.

31. Vance, J. E. (2008). Phosphatidylserine and phosphatidylethanolamine in mammalian cells: Two metabolically related aminophospholipids. Journal of Lipid Research, 49(7), 1377-1387.

32. Farine, L., \& Bütikofer, P. (2013). The ins and outs of phosphatidylethanolamine synthesis in Trypanosoma brucei. Biochimica et Biophysica Acta, 1831(3), 533-542.

33. Christofk, H. R., et al. (2008). Pyruvate kinase M2 is a phosphotyrosine-binding protein. Nature, 452(7184), 181-186.

34. Perroud, B., et al. (2009). Grade-dependent proteomics characterization of kidney cancer. Molecular \& Cellular Proteomics, 8(5), 971-985.

35. Barceló-Coblijn, G., et al. (2011). Sphingomyelin and sphingomyelin synthase (SMS) in the malignant transformation of glioma cells and in 2-hydroxyoleic acid therapy. Proceedings of the National Academy of Sciences of the United States of America, 108(49), 19569-19574.

36. Ding, T., et al. (2008). SMS overexpression and knockdown: Impact on cellular sphingomyelin and diacylglycerol metabolism, and cell apoptosis. Journal of Lipid Research, 49(2), 376-385.

37. Catchpole, G., et al. (2011). Metabolic profiling reveals key metabolic features of renal cell carcinoma. Journal of Cellular and Molecular Medicine, 15(1), 109-118.
38. Menendez, J. A., \& Lupu, R. (2007). Fatty acid synthase and the lipogenic phenotype in cancer pathogenesis. Nature Reviews. Cancer, 7(10), 763-777.

39. Ham, A. J., \& Liebler, D. C. (1997). Antioxidant reactions of vitamin $\mathrm{E}$ in the perfused rat liver: Product distribution and effect of dietary vitamin E supplementation. Archives of Biochemistry and Biophysics, 339(1), 157-164.

40. Haines, R. J., Pendleton, L. C., \& Eichler, D. C. (2011). Argininosuccinate synthase: At the center of arginine metabolism. International Journal of Biochemistry and Molecular Biology, 2(1), 8-23.

41. Yoon, C. Y., et al. (2007). Renal cell carcinoma does not express argininosuccinate synthetase and is highly sensitive to arginine deprivation via arginine deiminase. International Journal of Cancer, 120(4), 897-905.

42. Hoang, G., Udupa, S., \& Le, A. (2019). Application of metabolomics technologies toward cancer prognosis and therapy. International Review of Cell and Molecular Biology, 347, 191-223.

43. Wettersten, H. I., et al. (2015). Grade-dependent metabolic reprogramming in kidney cancer revealed by combined proteomics and metabolomics analysis. Cancer Research, 75(12), 2541-2552.

44. Kang, W., et al. (2020). The SLC family are candidate diagnostic and prognostic biomarkers in clear cell renal cell carcinoma. BioMed Research International, 2020, 1932948.

45. Hediger, M. A., et al. (2004). The ABCs of solute carriers: Physiological, pathological and therapeutic implications of human membrane transport proteins: Introduction. Pflügers Archiv, 447(5), 465-468.

46. Hakimi, A. A., et al. (2016). An integrated metabolic atlas of clear cell renal cell carcinoma. Cancer Cell, 29(1), 104-116.

47. Horiguchi, A., et al. (2008). Fatty acid synthase over expression is an indicator of tumor aggressiveness and poor prognosis in renal cell carcinoma. The Journal of Urology, 180(3), 1137-1140.

48. Xiao, H., et al. (2019). Three novel hub genes and their clinical significance in clear cell renal cell carcinoma. Journal of Cancer, 10(27), 6779-6791.

49. Li, T., Copeland, C., \& Le, A. (2021). Glutamine metabolism in cancer. Advances in Experimental Medicine and Biology, 1311, https://doi. org/10.1007/978-3-030-65768-0_2

50. Lu, S. C. (1999). Regulation of hepatic glutathione synthesis: current concepts and controversies. The FASEB Journal, 13(10), 1169-1183.

51. Kirovski, G., et al. (2011). Down-regulation of methylthioadenosine phosphorylase (MTAP) induces progression of hepatocellular carcinoma via accumulation of 5'-deoxy-5'-methylthioadenosine (MTA). The American Journal of Pathology, 178(3), 1145-1152.

52. Xu, J., et al. (2019). Targeting the insulin-like growth factor-1 receptor in MTAP-deficient renal cell carcinoma. Signal Transduction and Targeted Therapy, 4, 2.

53. Gerlinger, M., et al. (2014). Genomic architecture and evolution of clear cell renal cell carcinomas defined by multiregion sequencing. Nature Genetics, 46(3), 225-233. 
54. Gerlinger, M., et al. (2012). Intratumor heterogeneity and branched evolution revealed by multiregion sequencing. The New England Journal of Medicine, 366(10), 883-892.

55. Okegawa, T., et al. (2017). Intratumor heterogeneity in primary kidney cancer revealed by metabolic profiling of multiple spatially separated samples within tumors. eBioMedicine, 19, 31-38.

56. Nabi, K., \& Le, A. (2021). The intratumoral heterogeneity of cancer metabolism. Advances in Experimental Medicine and Biology, 1311, https:// doi.org/10.1007/978-3-030-65768-0_11

57. Antonio, M. J., Zhang, C., \& Le, A. (2021). Different tumor microenvironments lead to different metabolic phenotypes. Advances in Experimental Medicine and Biology, 1311, https:// doi.org/10.1007/978-3-030-65768-0_10

58. Park, J. K., et al. (2021). The heterogeneity of lipid metabolism in cancer. Advances in Experimental Medicine and Biology, 1311, https://doi. org/10.1007/978-3-030-65768-0_3

59. Xiong, H., et al. (2017). LncRNA HULC triggers autophagy via stabilizing Sirt1 and attenuates the chemosensitivity of HCC cells. Oncogene, 36(25), 3528-3540.

60. Rupaimoole, R., et al. (2015). Long noncoding RNA ceruloplasmin promotes cancer growth by altering glycolysis. Cell Reports, 13(11), 2395-2402.

61. Zheng, J., et al. (2016). Pancreatic cancer risk variant in LINC00673 creates a miR-1231 binding site and interferes with PTPN11 degradation. Nature Genetics, 48(7), 747-757.

62. Schmitt, A. M., \& Chang, H. Y. (2016). Long noncoding RNAs in cancer pathways. Cancer Cell, 29(4), 452-463.

63. Huarte, M. (2015). The emerging role of lncRNAs in cancer. Nature Medicine, 21(11), 1253-1261.

64. Li, X., et al. (2018). Dissecting LncRNA roles in renal cell carcinoma metastasis and characterizing genomic heterogeneity by single-cell RNA-seq. Molecular Cancer Research, 16(12), 1879-1888.

65. Tran, M., et al. (2019). First-in-human in vivo noninvasive assessment of intra-tumoral metabolic heterogeneity in renal cell carcinoma. BJR Case Report, 5, 3.

66. Zhang, Y., et al. (2017). Addressing metabolic heterogeneity in clear cell renal cell carcinoma with quantitative Dixon MRI. JCI Insight, 2, 15.

67. Peña-Llopis, S., et al. (2012). BAP1 loss defines a new class of renal cell carcinoma. Nature Genetics, 44(7), 751-759.
68. Varela, I., et al. (2011). Exome sequencing identifies frequent mutation of the SWI/SNF complex gene PBRM1 in renal carcinoma. Nature, 469(7331), 539-542.

69. Chowdhury, B., et al. (2016). PBRM1 regulates the expression of genes involved in metabolism and cell adhesion in renal clear cell carcinoma. PLoS One, 11(4), e0153718.

70. Jiang, W., et al. (2016). Immunohistochemistry successfully uncovers intratumoral heterogeneity and widespread co-losses of chromatin regulators in clear cell renal cell carcinoma. PLoS One, 11(10), e0164554.

71. Rini, B. I., \& Atkins, M. B. (2009). Resistance to targeted therapy in renal-cell carcinoma. The Lancet Oncology, 10(10), 992-1000.

72. Saxton, R. A., \& Sabatini, D. M. (2017). mTOR signaling in growth, metabolism, and disease. Cell, 169(2), 361-371.

73. Masoud, G. N., \& Li, W. (2015). HIF-1 $\alpha$ pathway: Role, regulation and intervention for cancer therapy. Acta Pharmaceutica Sinica B, 5(5), 378-389.

74. Kamli, H., Li, L., \& Gobe, G. C. (2019). Limitations to the therapeutic potential of tyrosine kinase inhibitors and alternative therapies for kidney cancer. The Ochsner Journal, 19(2), 138-151.

75. Li, X., et al. (2020). The tumor suppressor NDRG2 cooperates with an mTORC1 inhibitor to suppress the Warburg effect in renal cell carcinoma. Investigational New Drugs, 38(4), 956-966.

76. Gameiro, P. A., et al. (2013). In vivo HIF-mediated reductive carboxylation is regulated by citrate levels and sensitizes VHL-deficient cells to glutamine deprivation. Cell Metabolism, 17(3), 372-385.

77. Jang, Y., et al. (2015). Suppression of mitochondrial respiration with auraptene inhibits the progression of renal cell carcinoma: Involvement of HIF- $1 \alpha$ degradation. Oncotarget, 6(35), 38127-38138.

78. Chan, D. A., et al. (2011). Targeting GLUT1 and the Warburg effect in renal cell carcinoma by chemical synthetic lethality. Science Translational Medicine, 3(94), 94ra70.

79. Dang, C. V., et al. (2011). Therapeutic targeting of cancer cell metabolism. Journal of Molecular Medicine (Berlin), 89(3), 205-212.

80. Hirschey, M. D., et al. (2015). Dysregulated metabolism contributes to oncogenesis. Seminars in Cancer Biology, 35(Suppl), S129-S150.

Open Access This chapter is licensed under the terms of the Creative Commons Attribution 4.0 International License (http://creativecommons.org/licenses/by/4.0/), which permits use, sharing, adaptation, distribution and reproduction in any medium or format, as long as you give appropriate credit to the original author(s) and the source, provide a link to the Creative Commons license and indicate if changes were made.

The images or other third party material in this chapter are included in the chapter's Creative Commons license, unless indicated otherwise in a credit line to the material. If material is not included in the chapter's Creative Commons license and your intended use is not permitted by statutory regulation or exceeds the permitted use, you will need to obtain permission directly from the copyright holder.

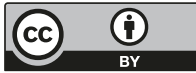




\section{The Heterogeneity of Liver Cancer Metabolism}

Javier Salazar and Anne Le

\section{Keywords}

Primary liver cancer - Metabolic phenotypes .

Glucose metabolism - Glutamine metabolism

- Oncogenic heterogeneity · Lipid metabolism

- Redox metabolism $\cdot$ Sorafenib

\section{Abbreviations}

ACC

ACSS 1

AFP

ATP

COX5B

CSA

DEN

$\mathrm{ERR} \alpha$

FAO

FASN

Acetyl-CoA carboxylase

Acyl-CoA synthetase short-chain family member 1

Alpha-fetoprotein

Adenosine triphosphate

Cytochrome $\mathrm{C}$ oxidase subunit 5B

Canavaninosuccinate

Diethylnitrosamine

Estrogen-related receptor A

Fatty acid oxidation
Fatty acid synthase

J. Salazar

Universidad Nacional Mayor de San Marcos, Lima, Peru

A. Le $(\bowtie)$

Department of Pathology and Oncology, Johns

Hopkins University School of Medicine,

Baltimore, MD, USA

Department of Chemical and Biomolecular Engineering, Johns Hopkins University Whiting

School of Engineering, Baltimore, MD, USA

e-mail: annele@jhmi.edu
FBP1 Fructose-1,6-bisphosphatase

G6P

G6PD

GCK

GLS2

Glu

GLUT1

GSH

GLUT2

$\mathrm{HCC}$

HK2

LCSCs

LDHA

LRH-1

MCT1

Me1

Glucose 6-phosphate

G 1 u c o s e - 6-phos phat e dehydrogenase

Glucokinase

Glutaminase 2

Glutamine

Glucose transporter 1

Glutathione

Glucose transporter 2

Hepatocellular carcinoma

Hexokinase 2

Liver cancer stem cells

Lactate dehydrogenase A

Liver receptor homolog 1

Protein monocarboxylate transporter isoform 1

mTORC1 Mechanistic target of rapamycin complex 1

NADPH Nicotinamide adenine dinucleotide phosphate

Non-LCSCs Non-liver cancer stem cells

PCK

Phosphoenolpyruvate

car-

boxykinase

PFK1 Phosphofructokinase-1

PGK1 Phosphoglycerate kinase 1

PKM Pyruvate kinase

ROS Reactive oxygen species

$\alpha$-KG $\quad \alpha$-Ketoglutarate 


\section{Key Points}

- Different oncogenic mutations lead to different metabolic phenotypes in primary liver cancer.

- $\quad M Y C$ and MET mutations regulate glucose and glutamine metabolism differently in primary liver cancer.

- Glucose metabolism increased by acetylated phosphoglycerate kinase 1 (PGK1) leads to the promotion of cancer cell proliferation and tumorigenesis in the liver.

- There exist metabolic differences between hepatocellular carcinoma (HCC) and normal liver tissue or other liver diseases.

1

\section{Introduction}

Primary liver cancer is the fourth leading cause of cancer death around the world. Histologically, it can be divided into two major groups, hepatocellular carcinoma ( $75 \%$ of all liver cancer) and intrahepatic cholangiocarcinoma (15\% of all liver cancer) $[1,2]$. Primary liver cancer usually happens in liver disease or cirrhosis patients [1], and the risk factors for developing HCC depend on the etiology [3] and the country of provenance [1]. There is an urgent need for an accurate diagnostic test given the high proportion of false positives and false negatives for alpha-fetoprotein (AFP), a common HCC biomarker [4]. Due to often being diagnosed in advanced stages, HCC-related deaths per year have doubled since 1999 [3]. With the use of metabolomics technologies [5], the aberrant metabolism characteristics of cancer tissues can be discovered and exploited for the new biomarkers and new therapies to treat $\mathrm{HCC}[6,7]$.

\section{Different Oncogenic Mutations Lead to Different Metabolic Phenotypes in Primary Liver Cancer}

Most patients with HCC are diagnosed at advanced stages, and the current effective treatments for these patients are limited. Nevertheless, if $\mathrm{HCC}$ patients are diagnosed at an early stage, the tumors can be resected or ablated. However, these patients often experience recurrence after resection/ablation [8]. Strategies to improve patient survival outcomes involve therapies exploiting the metabolic vulnerabilities of cancer cells. However, within the tumor microenvironments, the alterations in metabolic pathways [9, 10], resulting from the combinational effect of genetic, epigenetic, and transcriptomic variations $[11,12]$, occur frequently to accommodate the high energy demands of tumor growth. Consequently, the complexity of the heterogeneity of altered cancer metabolism leads to resistance in therapeutic cancer treatments [13, 14]. Additionally, different patients also exhibit different forms of liver cancer that correspond to genetic differences [11, 15-19]. Given the genetic and metabolic complexities of HCC, identifying core metabolic pathways utilized by the tumors to drive metabolic phenotypic plasticity of this neoplasm will substantially contribute to the development of effective metabolic therapies.

\subsection{MYC and MET Mutations Regulate Glucose and Glutamine Metabolism Differently in Primary Liver Cancer}

MYC is a well-known regulator of metabolism in cancer $[20,21]$. Yuneva et al. found that the reprogramming of glucose and glutamine metabolism was different depending on the activation of the MYC oncogene or the MET proto-oncogene even within a specific liver cancer type [22]. They found an increased uptake and catabolism of glucose in primary liver cancer as compared to the normal liver in both $M E T$ - and $M Y C$-induced liver tumors. However, $M Y C$-induced liver tumors exhibited the Warburg effect [23] in which these tumors produced significantly high levels of lactate, a phenotype not observed in METinduced tumors [22]. This study suggests that within the same cancer type, cells exhibit diverse genetic abnormalities that result in diverging and distinct metabolic manifestations. These numerous and remarkably pliant alterations appear to be essential for meeting the variety of demands 


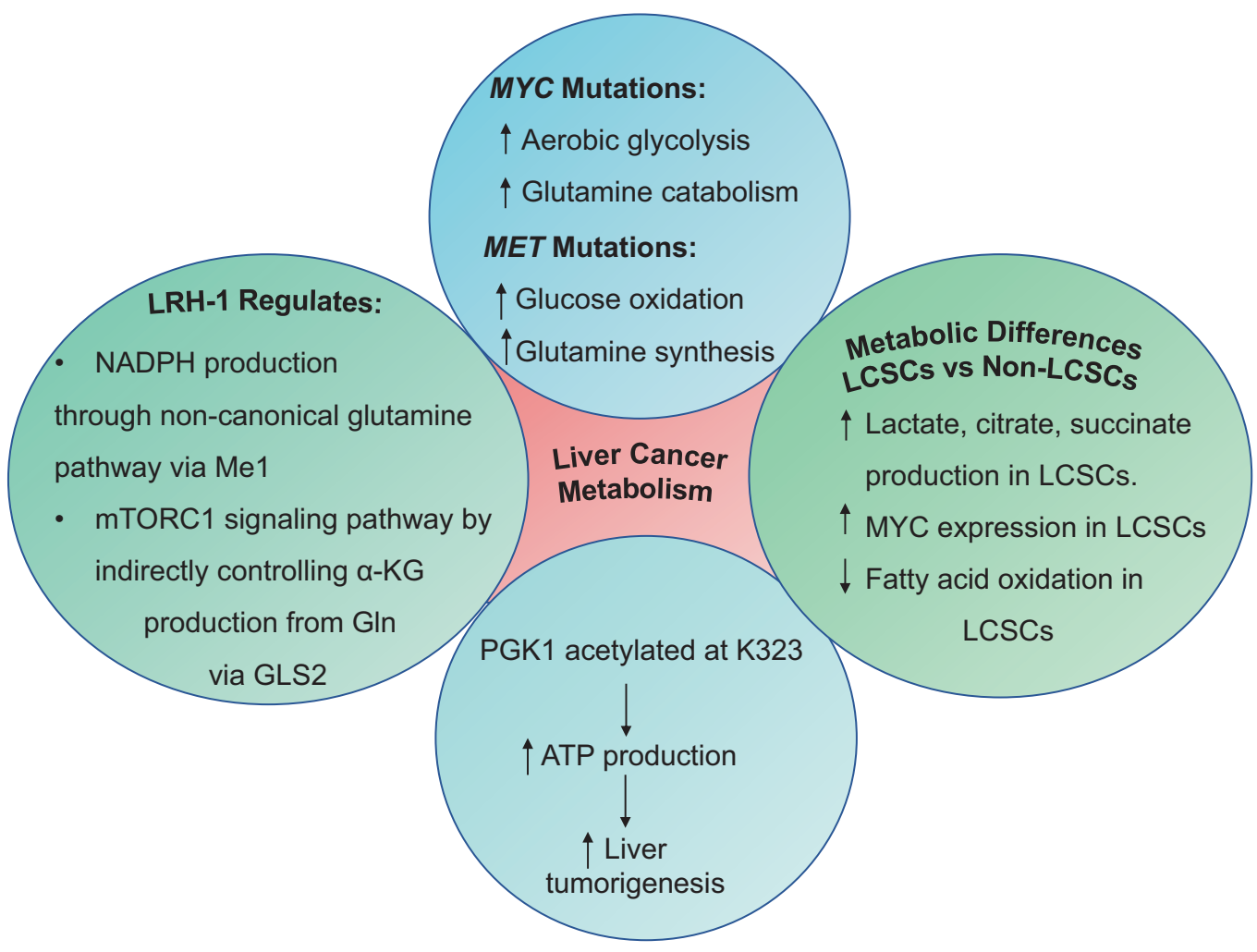

Fig. 1 The heterogeneity of liver cell carcinoma metabolism and its associated oncogenic mutations

of cell proliferation, which include ATP production, biosynthesis of cellular building blocks, reactive oxygen species (ROS) detoxification, and degradation of the extracellular matrix scaffolding to allow for angiogenesis and thus tumorigenesis.

Tumor cell metabolism of the same tissue type has been shown to depend on the identities of the genetic mutations. While $M Y C$-induced mouse liver tumors exhibit enhanced glutamine [24] and glucose [23] metabolism, accompanied by an increase in lactate production and Krebs cycle intermediates, MET-induced mouse liver tumors are found to consume glucose as a means of synthesizing glutamine [22] (Fig. 1). Thus, it is reasonable to conclude that these two genes dictate radically opposite roles for glutamine, a central player in cancer metabolism. This fact illustrates, once again, that cancer metabolism can be determined by the nature of the genetic alterations and that how the metabolism is altered across different tumors can be extremely substantial. This helps to explain how the same tissue of origin in different patients, in this case, can have different genetic alterations and metabolic phenotypes, thus substantiating the potential role of heterogeneity, even in a single tumor of the same patient.

\subsection{Liver Receptor Homolog 1 (LRH-1) Regulates Mitochondrial Glutamine Metabolism}

The study led by $\mathrm{Xu}$ et al. has revealed the crucial role of liver receptor homolog 1 (LRH-1) in regulating mitochondrial glutamine metabolism, which eventually leads to the production of NADPH through a noncanonical glutamine pathway. Specifically, the study found that the regulation of malic enzyme 1 (Me1), an enzyme that 
catalyzes the conversion of malate to pyruvate to produce NADPH through a noncanonical glutamine pathway [25], is dependent on LRH-1 [26].

Furthermore, using ${ }^{13} \mathrm{C}_{5}$-labeled glutamine, the study highlighted the essential role of LRH-1 in promoting the production of glutamate from glutamine via controlling mitochondrial glutaminase 2 (GLS2) [26]. Consequently, the production of $\alpha$-ketoglutarate $(\alpha-\mathrm{KG})$ from glutamate activates the mechanistic target of the rapamycin complex 1 (mTORC1) signaling pathway [26], a regulator of cell growth metabolism including proteins, lipids, and nucleotides [27]. Due to its pivotal role in the production of the reductive biosynthetic product NADPH and the activation of the mTORC1 signaling pathway through glutamine metabolism, LRH-1 promotes cell proliferation. Thus, loss of LRH-1 prevents diethylnitrosamine (DEN)-induced liver carcinogenesis [26]. Targeting glutamine metabolism as a strategy for the treatment has been studied in several cancers [24, 28-34] (Fig. 1).

\subsection{Glucose Metabolism Increased by Acetylated Phosphoglycerate Kinase 1 (PGK1) Leads to the Promotion of Cancer Cell Proliferation and Tumorigenesis in Liver}

Compared to normal liver cells, cancerous liver cells, and cancer cells, in general, need a much greater amount of energy to fuel their proliferation. One of the ways to satisfy these high demands of energy is to adjust the energyyielding pathways accordingly to produce energy in the most efficient manner. Deciphering different energy production-enhancing mechanisms in cancers has attracted a lot of attention because having a better understanding of these mechanisms provides strategies to advance treatments for cancers. Similarly, the study led by $\mathrm{Hu}$ et al. elucidated the role of phosphoglycerate kinase 1 (PGK1), an enzyme catalyzing the conversion of 1,3-bisphosphoglycerate to 3-phosphoglycerate yielding one ATP equivalent, in glycolysis, cell proliferation, and tumorigenesis. The formation of acetylated PGK1 at position K323 is required to activate PGK1 [35]. Activated PGK1, in turn, regulates cancer cell metabolism. Specifically, acetylated PGK1 enhances the production of energy in the form of ATP [35]. Ultimately, the acetylation of PGK1 at K323 promotes liver tumorigenesis. With this understanding of how PGK1 K323 acetylation functions in liver cancer, the emergence of new effective treatments using PGK1 as a therapeutic target for patients with liver cancer is promising (Fig. 1).

\section{$3 \quad$ Metabolic Differences Between Liver Cancer Stem Cells (LCSCs) and Non-liver Cancer Stem Cells (Non-LCSCs)}

Given their metabolic heterogeneity, LCSCs are able to adapt to many different environments, which causes therapeutic resistance to many available treatments for HCC. Understanding the metabolism of LCSCs is crucial not only for the improvement of currently available therapies but also for paving a new path for developing other therapies targeting the revealed metabolic pathways. In an effort to elaborate on the understanding of the metabolism of LCSCs, Hur et al. found the following differences in the metabolism of LCSCs as compared to nonLCSCs. The increased proliferation of LCSCs can be explained based on the metabolomics analysis, which reveals the higher presence of essential metabolites that are either resulting from highly activated catabolism or acting as substrates to promote other energy-yielding processes. Specifically, the study found a higher concentration of lactate, the final product from glycolysis, citrate, succinate, and several amino acids such as aspartate, glutamate, isoleucine, leucine, phenylalanine, tyrosine, and valine in LCSCs as compared to non-LCSCs. Moreover, they showed that $M Y C$, a known regulator of glycolytic metabolism [20, 36], is highly expressed in LCSCs as compared to non-LCSCs, and this resulted in an increased amount of energy available for the rapid proliferation of the cancer cells [37]. This study also found that fatty acid oxi- 
dation in LCSCs is less active than that in nonLCSCs [37]. Consequently, the production of NADPH from fatty acid oxidation, contributing to oxidative phosphorylation to generate ATP, is less for LCSCs. Nevertheless, the assessment of three genes - cytochrome Coxidase subunit 5B (COX5B), adenosine triphosphate-5-alpha (ATP5 $\alpha$ ), and estrogen-related receptor $\mathrm{A}(\mathrm{ERR} \alpha)$ - involved in oxidative phosphorylation showed no differences between LCSCs and non-LCSCs [37]. This means that LCSCs must have utilized more glycolysis to produce ATP to satisfy the demands of energy for their rapid proliferation (Fig. 1).

\section{$4 \quad$ Metabolic Signatures of Liver Cancer}

\subsection{Metabolism of HCC Is Different from that of Normal Liver Tissue}

This section presents evidence of changes in the concentrations of specific metabolites in liver cancer as compared to normal hepatocytes and how this knowledge allows the development of new therapeutic models.

From recent studies, it is known that glucose metabolism is reprogrammed in HCC with the aim of allowing its growth, proliferation, and metastasis. One of the most outstanding characteristics of cancer cell metabolism is the high demand for glucose [38]. Cassim et al. found that GLUT1 expression is increased in HCC tumorigenic cells as opposed to normal liver cells, which have high GLUT2 expression [39]. Furthermore, Kim et al. found that the expression of GLUT1 (SLC2A1) is increased in advanced stages of HCC [38] (Fig. 2). Moreover, there is a switch in the expression of hexokinase (HK) enzyme from low-affinity glucokinase (GCK) isoenzyme in normal hepatocytes to high-affinity hexokinase 2 (HK2) isoenzyme. DeWaal et al. showed that HCC cells predominantly use HK2, unlike noncancerous liver cells that use GCK (or HK4) [40]. In addition, they showed that there is an increased expression of HK2 in HCC cells and cells with evidence of dysplasia [40].
On the other hand, Fang et al. studied the influence of miR-383 on the expression of lactate dehydrogenase A (LDHA), which plays a key role in the progression of many types of cancer [41]. An overexpression of miR-383, an endogenous non-coding microRNA, has been reported to markedly inhibit HCC cells' glycolysis, proliferation, and invasion, through its binding to the LDHA-expressing gene [41] (Fig. 2). The LDHA inhibitor, FX11, has also shown promising results in several studies [42-44]. While it is known that there is an increase in the activity of aerobic glycolysis in HCC, Wang et al. showed that there is also a decrease in gluconeogenesis activity in HCC [45]. Björnson et al. discovered downregulation of phosphoenolpyruvate carboxykinase (PCK1 and PCK2), the first gluconeogenesis enzyme in HCC [46]. Hirata et al. also discovered that in HCC samples there is low expression of fructose-1,6-bisphosphatase (FBP1), a gene that encodes the gluconeogenic enzyme FBP1 [45].

Furthermore, they also discovered that FBP1 overexpression is associated with decreased expression of hexokinase-2 (HK2) and phosphofructokinase-1 (PFK1), key enzymes of aerobic glycolysis in cancer cells [45]. It was also shown that low FBP1 levels were associated with tumor progression in HCC samples [45] (Fig. 2).

Regarding the altered lipid metabolism [47], Björnson et al. found evidence of a significant increase in the expression of key genes related to fatty acid biosynthesis (FAB) and pentose phosphate pathway (PPP) in HCC samples compared to noncancerous liver samples [46] (Fig. 3). The upregulation of these genes and the corresponding enzymes thus provide the necessary supplies for the synthesis of fatty acids, nucleotides, and NADPH, essential for tumor growth and proliferation [46]. Acetyl-CoA is required for fatty acid biosynthesis, and it is commonly produced by the oxidation of fatty acids [46]. However, Björnson et al. found that fatty acid oxidation is decreased in $\mathrm{HCC}$ and the main supply for the generation of fatty acids is exogenously acquired acetate [46]. According to this study, fatty acid oxidation (FAO) enzymes such as carnitine palmitoyltransferase 2 (CPT2), acyl-CoA dehydrogenase family 


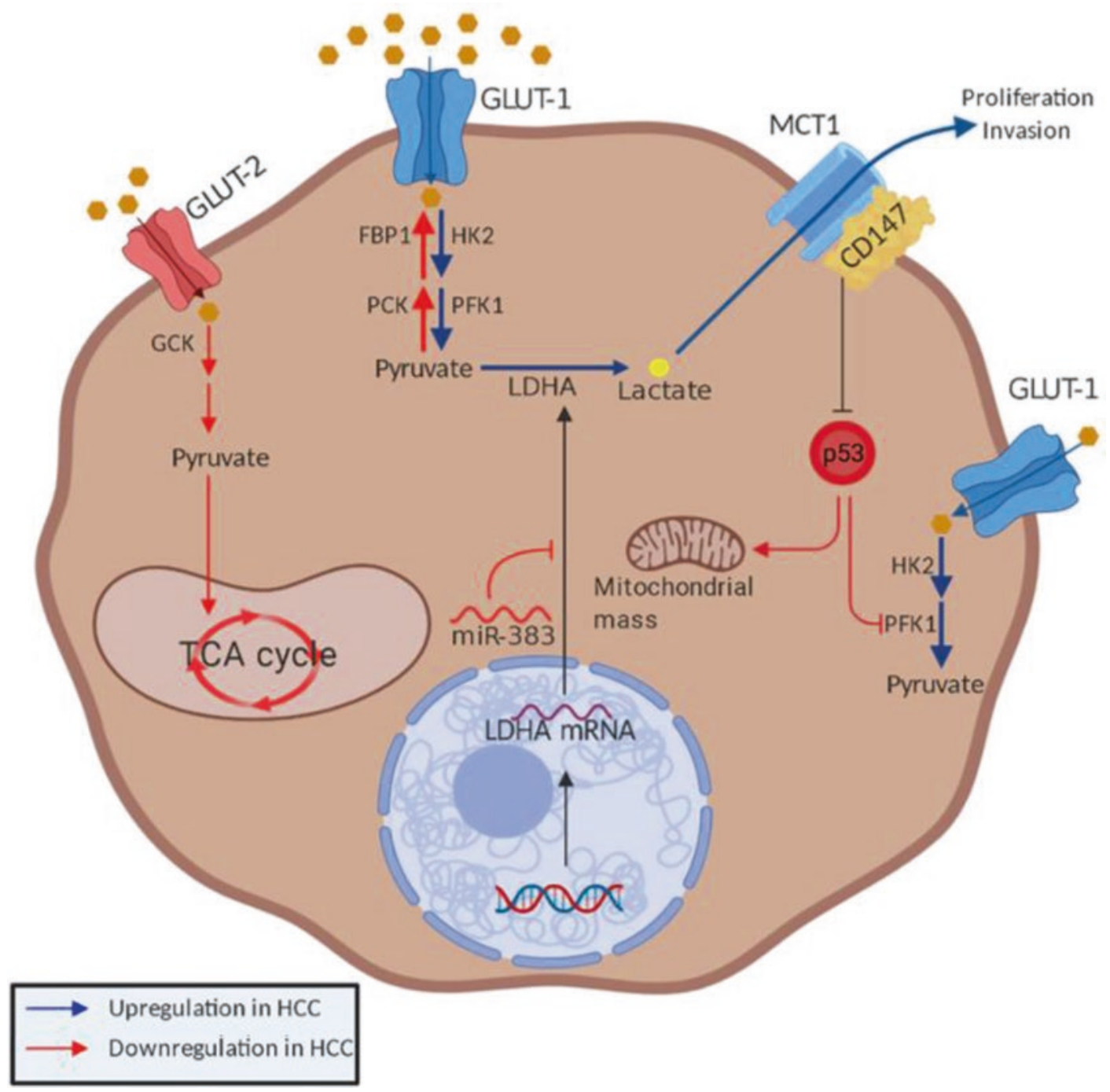

Fig. 2 The alteration of glucose metabolism involves upregulation of glycolysis enzymes and downregulation of gluconeogenic enzymes and mitochondrial aerobic activity. GLUT glucose transporter, $M C T 1$ protein monocarboxylate transporter isoform 1, HK2 hexokinase 2, PFK1 phosphofructokinase-1, FBP1 fructose-1,6-bisphosphatase, $P C K$ phosphoenolpyruvate carboxykinase, $G C K$ glucokinase, $T C A$ cycle tricarboxylic acid cycle, $m i R$-383 microRNA-383

(ACADSB), and hydroxyacyl-CoA dehydrogenases (HADH) are significantly downregulated in HCC tumors [46]. Acetate of exogenous origin enters the cell and, through acetyl-CoA synthases, is transformed into acetyl-CoA, a necessary substrate for the biosynthesis of fatty acids [46]. In addition, the expression of the acyl-CoA synthetase short-chain family member 1 (ACSS1) subtype, which resides in the mitochondria, ATP-citrate lyase (ACLY), acetyl-CoA carboxylase (ACC), and fatty acid synthase (FASN), are significantly increased in patients with HCC compared to noncancerous liver samples [46]. Furthermore, in the same study, two groups with highly differentiated ACSS1 expression levels were observed within the HCC patient samples [46] (Fig. 3). Further analysis revealed that the group with high levels of ACSS1 had a greater suppression of the enzymes related to FAO as compared to the group with low levels of ACSS1 [46]. Furthermore, it was also observed that the group with high ACSS1 expression had four times 


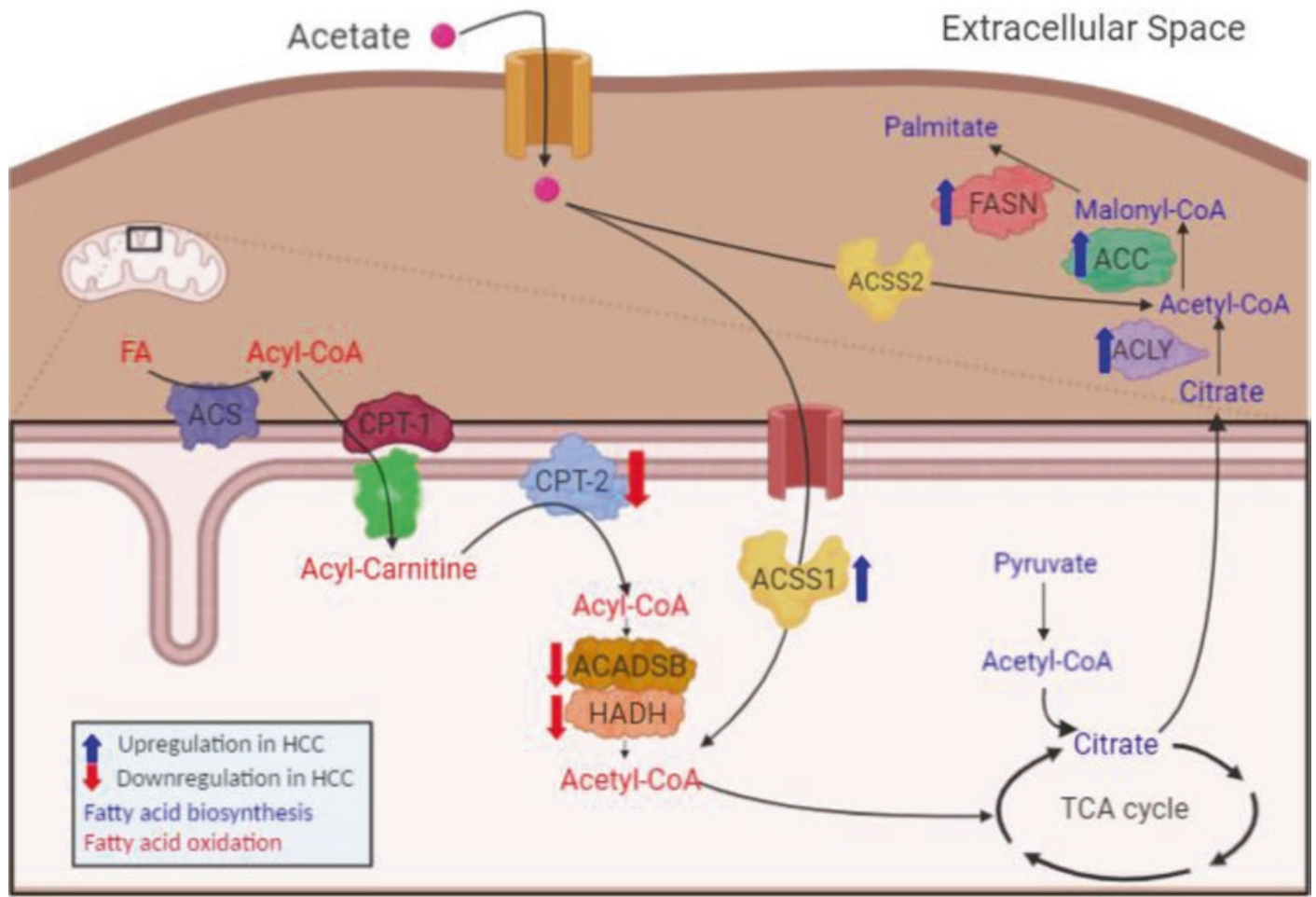

Fig. 3 The alteration of lipid metabolism involves upregulation of fatty acid biosynthesis enzymes and downregulation of fatty acid oxidation enzymes. FASN fatty acid synthase, ACC acetyl-CoA carboxylase, ACLY ATP citrate lyase, $A C S S 1$ acyl-CoA synthetase short-chain family member 1, ACADSB acyl-CoA dehydrogenase family, $H A D H$ hydroxyacyl-CoA dehydrogenases, $C P T$ carnitine palmitoyltransferase 2, ACS acetyl-coA synthase

the expression of pyruvate kinase (PKM) compared to the group with low levels of ACSS1 [46] (Fig. 3). Increased expression of PKM is related to a greater capacity for metabolic adaptation to different concentrations of nutrients and oxygen [46].

\subsection{Oxidative Stress Signature in HCC}

Oxidative stress, which is determined by the balance of oxidant and antioxidant species, also plays an important role in HCC. Among the antioxidant species, glutathione (GSH) is the major nonenzymatic regulator of intracellular redox homeostasis [48], and it is the most abundant antioxidant in hepatocytes [49]. In the serum of patients with HCC, a significant increase in amino acids related to the synthesis of GSH, the reduced form of glutathione, and glucose 6-phosphate (G6P), a compound necessary to form NADPH, has been evidenced
[50]. On the other hand, the quantification of oxidative damage is made possible through measuring oxidative products, and in HCC, 8-hydroxydeoxy guanosine and 4-hydroxynonenal (oxidative products of DNA and lipids, respectively) were found [49]. De Matteis et al. also found that there were elevated levels of 8-hydroxydeoxy-guanosine in the serum of patients with chronic hepatitis, which can lead to an increased risk of HCC [49].

It is important to note that Wang et al. found that a large elevation of canavaninosuccinate (CSA) in HCC patients from the first affiliated hospital of the medical school of Zhejiang University, as opposed to samples from cirrhosis patients, is a signature of oxidized stress [51]. CSA produces fumarate, which is a key metabolite of the tricarboxylic acid (TCA) cycle, and elevated CSA levels indicate that its conversion to fumarate is impaired and thus negatively impacting the subsequent TCA cycle intermediate formation while promoting Warburg's gly- 
colysis [52]. They also discovered the sensitivity and specificity of alpha-fetoprotein (AFP) and CSA to distinguish HCC patients from cirrhosis controls [51]. It is important to note that these authors do not discuss dietary factors due to the plant origin of canavanine.

\section{$5 \quad$ New Therapeutic Investigations Based on Metabolism Studies}

Currently, the multikinase inhibitor sorafenib is the main drug for the treatment of advanced HCC that delays tumur progression and improves overall survival. However, its ability to shrink the tumor in patients is very modest [53]. Therefore, other treatments that may potentiate or replace sorafenib are being studied. DeWaal et al. studied the synergism between HK2 silencing and sorafenib because the first one can induce cell death, and the second one can inhibit growth factor receptors. It was observed that HK2 silencing made HCC cells more sensitive to sorafenib. Therefore, the combination therapy using sorafenib and HK2 silencing decreased tumor growth substantially more than either treatment alone [40].

Another protein that has garnered attention because it is expressed in high amounts in many types of malignant cells is a transmembrane protein known as CD147 (Fig. 2). It is associated with the lactate transporter monocarboxylate transporter isoform 1 (MCT1), a key protein that exports lactate to the extracellular environment from HCC cells and is necessary for the proliferation of HCC [49]. Huang et al. found that CD147 overexpression significantly promoted glycolysis via p53/TIGAR/PFK and inhibited mitochondrial activity via p53 in HCC cells [54]. Furthermore, the silencing of CD147 was associated with a relative increase in mtDNA content in HCC cells compared to control cells [54]. Additionally, this silencing was associated with a high oxygen consumption rate, a decrease in the intracellular concentra- tion of glucose and pyruvate, a high intracellular lactate concentration, and a significant decrease in cell growth [54]. Inhibition of CD147 phosphorylation was found in HCC samples from patients with distant metastases and was associated with elevated levels of AFP. This phosphorylation requires further studies as a potential prognosis biomarker, as well as a potential strategy for the development of treatments against HCC metastasis [55].

\section{Conclusion}

Given the complex heterogeneity and intricate evolutionary characteristics of liver cancers, the increase in resistance rate to current therapies has emerged as the main obstacle that many studies focusing on HCC have been trying to overcome. Among the different methods available to tackle the problem, metabolomics-based approaches serve as powerful strategiesallowing researchers to uncover metabolic profiles of different cancers. In addition, using metabolomics technologies to track a variety of metabolites in cancers offers researchers a better picture of the interactions that occur within the tumor microenvironments. Understanding the heterogeneity of cancer metabolism will pave a new path for the development of metabolism-based therapies to improve the outcome of cancer therapy. As has been shown, genes such as MYC, MET, or LRH-1 activate various metabolic pathways for cell growth and survival. On the other hand, the combination of AFP with CSA has shown good results as a diagnostic test, which compels us to continue studying the metabolic pathway of CSA within HCC cells. However, more research is required to understand the progression of liver cancer and how to evaluate it at different stages for a more favorable prognosis, as well as less invasive and more effective treatments. The study of the metabolome is yielding good results, but it is still necessary to discover new relationships between the different metabolic pathways. 


\section{References}

1. Dasgupta, P., et al. (2020). Global trends in incidence rates of primary adult liver cancers: A systematic review and meta-analysis. Frontiers in Oncology, 10, 171.

2. Lin, H. S., et al. (2019). Identification of novel antiliver cancer small molecules with better therapeutic index than sorafenib via zebrafish drug screening platform. Cancers (Basel), 11, 6 .

3. Kim, H. S., \& El-Serag, H. B. (2019). The epidemiology of hepatocellular carcinoma in the USA. Current Gastroenterology Reports, 21(4), 17.

4. Gao, R., et al. (2015). Serum metabolomics to identify the liver disease-specific biomarkers for the progression of hepatitis to hepatocellular carcinoma. Scientific Reports, 5, 18175.

5. Hoang, G., Udupa, S., \& Le, A. (2019). Application of metabolomics technologies toward cancer prognosis and therapy. International Review of Cell and Molecular Biology, 347, 191-223.

6. Dang, C. V., et al. (2011). Therapeutic targeting of cancer cell metabolism. Journal of Molecular Medicine (Berlin), 89(3), 205-212.

7. Hirschey, M. D., et al. (2015). Dysregulated metabolism contributes to oncogenesis. Seminars in Cancer Biology, 35(Suppl), S129-S150.

8. Llovet, J. M., et al. (2016). Hepatocellular carcinoma. Nature Reviews. Disease Primers, 2, 16018.

9. Nabi, K., \& Le, A. (2021). The intratumoral heterogeneity of cancer metabolism. Advances in Experimental Medicine and Biology, 1311, https:// doi.org/10.1007/978-3-030-65768-0_11

10. Antonio, M. J., Zhang, C., \& Le, A. (2021). Different tumor microenvironments lead to different metabolic phenotypes. Advances in Experimental Medicine and Biology, 1311, https:// doi.org/10.1007/978-3-030-65768-0_10

11. Forner, A., Llovet, J. M., \& Bruix, J. (2012). Hepatocellular carcinoma. Lancet, 379(9822), 1245-1255.

12. Zucman-Rossi, J., et al. (2015). Genetic landscape and biomarkers of hepatocellular carcinoma. Gastroenterology, 149(5), 1226-1239. e4.

13. Schulze, K., Nault, J. C., \& Villanueva, A. (2016). Genetic profiling of hepatocellular carcinoma using next-generation sequencing. Journal of Hepatology, 65(5), 1031-1042.

14. Bobrovnikova-Marjon, E., \& Hurov, J. B. (2014). Targeting metabolic changes in cancer: Novel therapeutic approaches. Annual Review of Medicine, 65, 157-170.

15. Wolpaw, A. J., \& Dang, C. V. (2018). Exploiting metabolic vulnerabilities of cancer with precision and accuracy. Trends in Cell Biology, 28(3), 201-212.

16. Cancer Genome Atlas Research Network. (2017). Electronic address, w.b.e. and N. Cancer genome atlas research, comprehensive and integrative genomic characterization of hepatocellular carcinoma. Cell, 169(7), 1327-1341.e23.

17. Calderaro, J., et al. (2017). Histological subtypes of hepatocellular carcinoma are related to gene mutations and molecular tumour classification. Journal of Hepatology, 67(4), 727-738.

18. Zheng, H., et al. (2018). Single-cell analysis reveals cancer stem cell heterogeneity in hepatocellular carcinoma. Hepatology, 68(1), 127-140.

19. Xue, R., et al. (2016). Variable Intra-tumor genomic heterogeneity of multiple lesions in patients with hepatocellular carcinoma. Gastroenterology, 150(4), 998-1008.

20. Dang, C. V., Le, A., \& Gao, P. (2009). MYC-induced cancer cell energy metabolism and therapeutic opportunities. Clinical Cancer Research, 15(21), 6479-6483.

21. Le, A., \& Dang, C. V. (2013). Studying Myc's role in metabolism regulation. Methods in Molecular Biology, 1012, 213-219.

22. Yuneva, M. O., et al. (2012). The metabolic profile of tumors depends on both the responsible genetic lesion and tissue type. Cell Metabolism, 15(2), 157-170.

23. Bose, S., Zhang, C., \& Le, A. (2021). Glucose metabolism in cancer: The Warburg effect and beyond. Advances in Experimental Medicine and Biology, 1311, https://doi.org/10.1007/978-3-030-65768-0_1

24. Li, T., Copeland, C., \& Le, A. (2021). Glutamine metabolism in cancer. Advances in Experimental Medicine and Biology, 1311, https://doi. org/10.1007/978-3-030-65768-0_2

25. Wise, D. R., et al. (2008). Myc regulates a transcriptional program that stimulates mitochondrial glutaminolysis and leads to glutamine addiction. Proceedings of the National Academy of Sciences of the United States of America, 105(48), 18782-18787.

26. Xu, P., et al. (2016). LRH-1-dependent programming of mitochondrial glutamine processing drives liver cancer. Genes \& Development, 30(11), 1255-1260.

27. Laplante, M., \& Sabatini, D. M. (2012). mTOR signaling in growth control and disease. Cell, 149(2), 274-293.

28. Zimmermann, S. C., et al. (2016). Allosteric glutaminase inhibitors based on a 1,4-di(5-amino-1,3,4thiadiazol-2-yl)butane scaffold. ACS Medicinal Chemistry Letters, 7(5), 520-524.

29. Rais, R., et al. (2016). Discovery of 6-diazo-5-oxo1-norleucine (DON) prodrugs with enhanced CSF delivery in monkeys: A potential treatment for glioblastoma. Journal of Medicinal Chemistry, 59(18), 8621-8633.

30. Xiang, Y., et al. (2015). Targeted inhibition of tumorspecific glutaminase diminishes cell-autonomous tumorigenesis. The Journal of Clinical Investigation, 125(6), 2293-2306.

31. Le, A., et al. (2012). Glucose-independent glutamine metabolism via TCA cycling for proliferation and survival in B cells. Cell Metabolism, 15(1), 110-121. 
32. Elgogary, A., et al. (2016). Combination therapy with BPTES nanoparticles and metformin targets the metabolic heterogeneity of pancreatic cancer. Proceedings of the National Academy of Sciences of the United States of America, 113(36), E5328-E5336.

33. Udupa, S., et al. (2019). Upregulation of the glutaminase II pathway contributes to glutamate production upon glutaminase 1 inhibition in pancreatic cancer. Proteomics, 19(21-22), e1800451.

34. Nguyen, T., et al. (2019). Uncovering the role of $\mathrm{N}$-acetyl-aspartyl-glutamate as a glutamate reservoir in cancer. Cell Reports, 27(2), 491-501. e6.

35. Hu, H., et al. (2017). Acetylation of PGK1 promotes liver cancer cell proliferation and tumorigenesis. Hepatology, 65(2), 515-528.

36. Dang, C. V. (2010). Rethinking the Warburg effect with Myc micromanaging glutamine metabolism. Cancer Research, 70(3), 859-862.

37. Hur, W., et al. (2017). Systems approach to characterize the metabolism of liver cancer stem cells expressing CD133. Scientific Reports, 7, 45557.

38. Kim, Y. H., et al. (2017). SLC2A2 (GLUT2) as a novel prognostic factor for hepatocellular carcinoma. Oncotarget, 8(40), 68381-68392.

39. Cassim, S., et al. (2018). Metabolite profiling identifies a signature of tumorigenicity in hepatocellular carcinoma. Oncotarget, 9(42), 26868-26883.

40. DeWaal, D., et al. (2018). Hexokinase-2 depletion inhibits glycolysis and induces oxidative phosphorylation in hepatocellular carcinoma and sensitizes to metformin. Nature Communications, 9(1), 446.

41. Fang, Z., et al. (2017). The miR-383-LDHA axis regulates cell proliferation, invasion and glycolysis in hepatocellular cancer. Iranian Journal of Basic Medical Sciences, 20(2), 187-192.

42. Le, A., et al. (2010). Inhibition of lactate dehydrogenase A induces oxidative stress and inhibits tumor progression. Proceedings of the National Academy of Sciences of the United States of America, 107(5), 2037-2042.

43. Rajeshkumar, N. V., et al. (2015). Therapeutic targeting of the Warburg effect in pancreatic cancer relies on an absence of p53 function. Cancer Research, 75(16), 3355-3364.

44. Dutta, P., et al. (2013). Evaluation of LDH-A and glutaminase inhibition in vivo by hyperpolarized
13C-pyruvate magnetic resonance spectroscopy of tumors. Cancer Research, 73(14), 4190-4195.

45. Hirata, H., et al. (2016). Decreased expression of fructose-1,6-bisphosphatase associates with glucose metabolism and tumor progression in hepatocellular carcinoma. Cancer Research, 76(11), 3265-3276.

46. Bjornson, E., et al. (2015). Stratification of hepatocellular carcinoma patients based on acetate utilization. Cell Reports, 13(9), 2014-2026.

47. Park, J. K., et al. (2021). The heterogeneity of lipid metabolism in cancer. Advances in Experimental Medicine and Biology, 1311, https://doi. org/10.1007/978-3-030-65768-0_3

48. Arauz, J., Ramos-Tovar, E., \& Muriel, P. (2016). Redox state and methods to evaluate oxidative stress in liver damage: From bench to bedside. Annals of Hepatology, 15(2), 160-173.

49. De Matteis, S., et al. (2018). Aberrant metabolism in hepatocellular carcinoma provides diagnostic and therapeutic opportunities. Oxidative Medicine and Cellular Longevity, 2018, 7512159.

50. Andrisic, L., et al. (2018). Short overview on metabolomics approach to study pathophysiology of oxidative stress in cancer. Redox Biology, 14, 47-58.

51. Wang, B., et al. (2012). Metabonomic profiles discriminate hepatocellular carcinoma from liver cirrhosis by ultraperformance liquid chromatography-mass spectrometry. Journal of Proteome Research, 11(2), 1217-1227.

52. Fitian, A. I., \& Cabrera, R. (2017). Disease monitoring of hepatocellular carcinoma through metabolomics. World Journal of Hepatology, 9(1), 1-17.

53. Assenat, E., et al. (2019). Sorafenib alone vs. sorafenib plus GEMOX as 1(st)-line treatment for advanced HCC: The phase II randomised PRODIGE 10 trial. British Journal of Cancer, 120(9), 896-902.

54. Huang, Q., et al. (2014). CD147 promotes reprogramming of glucose metabolism and cell proliferation in HCC cells by inhibiting the p53-dependent signaling pathway. Journal of Hepatology, 61(4), 859-866.

55. Jin, J., et al. (2019). Hypo-phosphorylated CD147 promotes migration and invasion of hepatocellular carcinoma cells and predicts a poor prognosis. Cellular Oncology (Dordrecht), 42(4), 537-554.

Open Access This chapter is licensed under the terms of the Creative Commons Attribution 4.0 International License (http://creativecommons.org/licenses/by/4.0/), which permits use, sharing, adaptation, distribution and reproduction in any medium or format, as long as you give appropriate credit to the original author(s) and the source, provide a link to the Creative Commons license and indicate if changes were made.

The images or other third party material in this chapter are included in the chapter's Creative Commons license, unless indicated otherwise in a credit line to the material. If material is not included in the chapter's Creative Commons license and your intended use is not permitted by statutory regulation or exceeds the permitted use, you will need to obtain permission directly from the copyright holder.

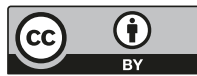




\title{
Different Tumor \\ Microenvironments Lead \\ to Different Metabolic Phenotypes
}

\author{
Marjorie Justine Antonio, Cissy Zhang, \\ and Anne Le
}

Keywords
Tumor microenvironments $\cdot$ Metabolic
phenotypes $\cdot$ Fatty acid oxidation $\cdot$ Metabolic
processes $\cdot$ Heterogeneity of cancer

Abbreviations

ACC

AMPK

ATP

CAF

Acetyl-CoA carboxylase

AMP-activated protein kinase

Adenosine triphosphate

Cancer-associated fibroblasts

\section{J. Antonio}

Department of Pathology, Johns Hopkins University

School of Medicine, Baltimore, MD, USA

C. Zhang

Department of Pathology, Johns Hopkins University

School of Medicine, Baltimore, MD, USA

Department of Biology, Johns Hopkins University

Krieger School of Arts and Sciences,

Baltimore, MD, USA

\section{A. Le $(\bowtie)$}

Department of Pathology and Oncology, Johns

Hopkins University School of Medicine,

Baltimore, MD, USA

Department of Chemical and Biomolecular Engineering, Johns Hopkins University Whiting

School of Engineering, Baltimore, MD, USA

e-mail: annele@jhmi.edu

$\begin{array}{ll}\text { Cav-1 } & \text { Caveolin-1 } \\ \text { CSC } & \text { Cancer stem cell } \\ \text { ETC } & \text { Electron transport chain } \\ \text { FABP4 } & \text { Fatty acid-binding protein } 4 \\ \text { FAO } & \text { Fatty acid oxidation } \\ \text { FASN } & \text { Fatty acid synthase } \\ \text { GLS } & \text { Glutaminase } \\ \text { GLUT1 } & \text { Glucose transporter 1 } \\ \text { HBx } & \text { Hepatitis B virus X protein } \\ \text { HCC } & \text { Hepatocellular carcinoma } \\ \text { hMSCs } & \text { Human mesenchymal stem cells } \\ \text { KRAS } & \text { Kirsten rat sarcoma viral oncogene } \\ & \text { homolog } \\ \text { MCT4 } & \text { Monocarboxylate transporter } 4 \\ \text { mTORC1 } & \text { Mammalian target of rapamycin } \\ & \text { complex 1 } \\ \text { NADPH } & \text { Nicotinamide adenine dinucleotide } \\ & \text { phosphate } \\ \text { NSCLC } & \text { Non-small cell lung cancer } \\ \text { OAA } & \text { Oxaloacetate } \\ \text { PanNET } & \text { Pancreatic neuroendocrine tumors } \\ \text { ROS } & \text { Reactive oxygen species } \\ \text { SCD1 } & \text { Stearoyl-CoA desaturase 1 } \\ \text { TCA } & \text { Tricarboxylic acid } \\ \text { TME } & \text { Tumor microenvironment } \\ \text { TSC1/2 } & \text { Tuberous sclerosis proteins } 1 / 2 \\ \text { VHL } & \text { Von Hippel-Lindau } \\ \alpha-\text { KG } & \alpha \text {-Ketoglutarate } \\ & \end{array}$

Cav-1

CSC

FABP4

FAO

GLS

GLUT

$\mathrm{HBx}$

hMSCs

KRAS

MCT4 


\section{Key Points}

- Cancer cells adapt to changes in nutrient and oxygen availability by adopting alternative metabolic pathways.

- Fatty acid oxidation in cancer cells is a survival mechanism under glucose deprivation.

- Lipid scavenging is utilized to enable cancer cells to survive periods of tumor regression.

- There is persistent glutamine oxidation under hypoxic and glucose deprivation conditions.

- Nutrient utilization can predict a tumor's metabolic dependencies in vivo.

- Distinct, and often complementary, metabolic processes operate concurrently within a single tumor.

\section{Introduction}

The beginning of the twenty-first century offered new advances in cancer research, including new knowledge about the tumor microenvironment (TME). Because TMEs provide the niches in which cancer cells, fibroblasts, lymphocytes, and immune cells reside, they play a crucial role in cancer cell development, differentiation, survival, and proliferation. Throughout cancer progression, the TME constantly evolves, causing cancer cells to adapt to the new conditions. The heterogeneity of cancer, evidenced by diverse proliferation rates, cellular structures, metabolisms, and gene expressions, presents challenges for cancer treatment despite the advances in research. This chapter discusses how different TMEs lead to specific metabolic adaptations that drive cancer progression.

\section{The Tumor Microenvironment}

The TME, the environment surrounding the cancer cells, is a heterogeneous mixture of immune cells, endothelial cells, materials secreted from cells and their organelles, and fibroblasts [1] (Fig. 1). Within this miniscule niche, the tumor survives in seemingly hostile conditions-hypoxia, nutrient deficiency, and necrosis - thanks to metabolic reprogramming [2]. The question is: How does a tumor microenvironment offer advantages for cancer cell survival under such conditions?

Hanahan and Weinberg suggest that there are six general characteristics of cancerous cells important for advancements toward malignant growth: (1) self-sufficiency in growth signals, (2) insensitivity to anti-growth signals, (3) evasion from apoptosis, (4) limitless replication potential, (5) sustained angiogenesis, and (6) tissue evasion and metastasis [3]. Despite the diversity of outcomes in tumor progression, these same capabilities are shared by most, if not all, tumor types. Moreover, these features develop differently in various tumor types through distinct mechanisms and at different time points during the multistep tumorigenesis enabled by genomic instability in cancer cells and tumor-promoting inflammation [4]. The hallmarks of cancer provide further insight into potential opportunities for early interventions for cancer treatment.

Among their basic needs, cancer cells require rapid ATP generation, biosynthesis of macromolecules, and maintenance of cellular redox status [5]. The insidious nature of cancer cells does not stop at their determination to live but also extends to the factors that sacrifice adjacent living tissue to propagate cancerous cells. Tumors create alternate pathways for nourishment and, most importantly, survival.

The differences in cancer origin and stage of progression ultimately lead to the heterogeneity of cancer and the corresponding components involved in cancer metabolism. 


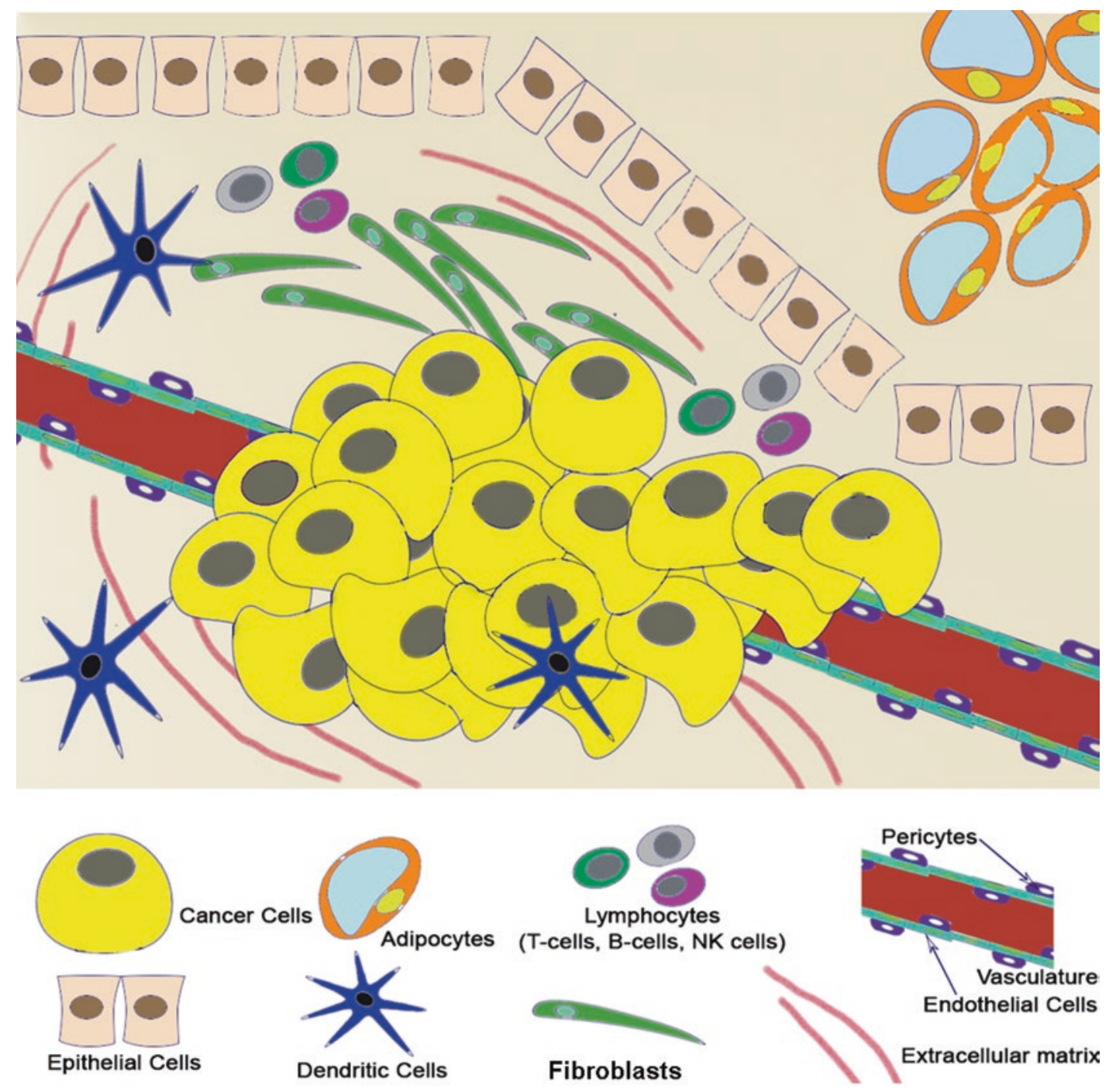

Fig. 1 The tumor microenvironment is composed of several components such as lymphocytes, adipocytes, fibroblasts, and dendritic cells

3

\section{Different Tumor \\ Microenvironments (TMEs) Lead to Different Metabolic Phenotypes}

3.1 Cancer Cells Adapt to Changes in Nutrient and Oxygen Availability by Adopting Alternative Metabolic Pathways (Fig. 2)
The harsh tumor microenvironment (TME), hypoxia, low $\mathrm{pH}$, and low nutrient concentrations are key characteristics in determining metabolic phenotypes. Various studies have demonstrated that cancer cells adapt to changes in nutrient and oxygen availability by adopting alternate metabolic pathways in order to continue providing the energy and macromolecules needed for cell proliferation. These pathways include fatty acid oxidation, lipid scavenging, and alternative cellular respiration pathways adopted by cancer cells under different TMEs [6-9]. 


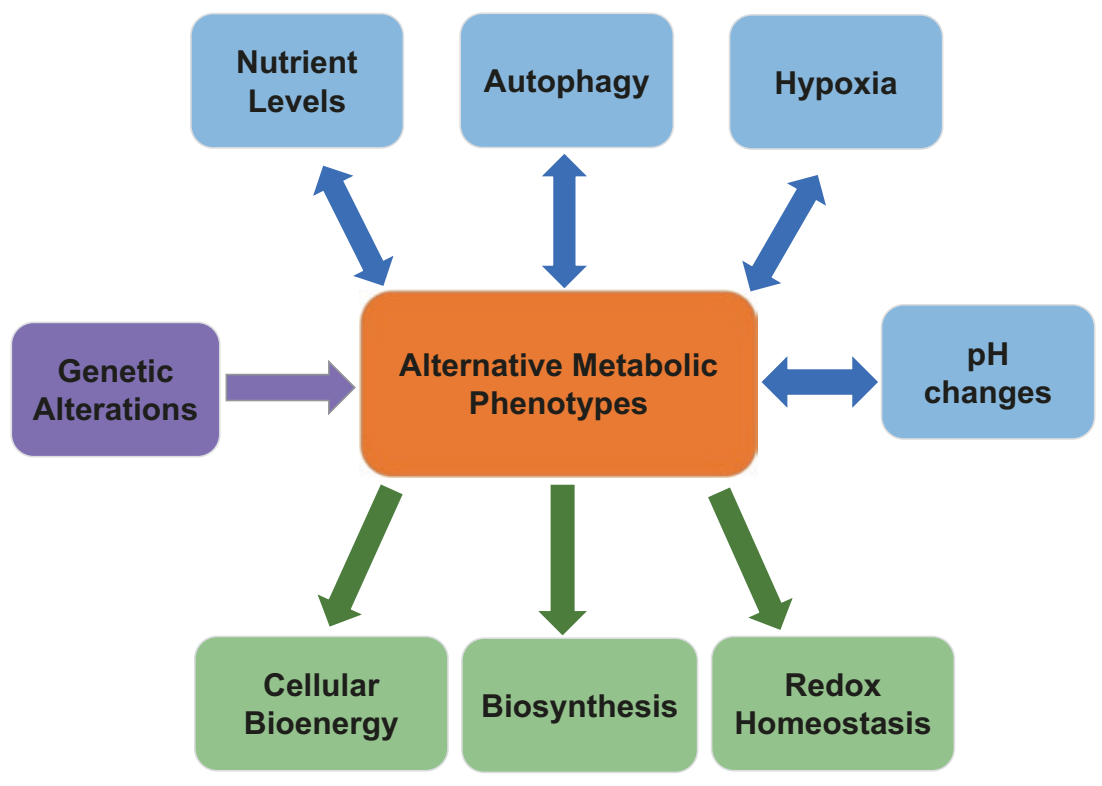

Fig. 2 The fundamental concept of how the tumor microenvironment (blue) leads to different metabolic phenotypes. Genetic alterations also contribute to the metabolic phenotype. The metabolic phenotype then propels bioenergetics, biosynthesis, and redox reactions in the tumor cells

The nutrient- and oxygen-poor internal conditions of TMEs incite cancer-friendly metabolic changes to help cancer cells survive in these harsh environments [10]. Under hypoxic conditions, oxidative phosphorylation or other aerobic reactions are limited. This state disrupts the redox balance and affects cell signaling. An increase in the levels of reactive oxygen species (ROS) is defined as oxidative stress [11]. Due to decreased oxygen tension, hypoxic cells depend mainly on anaerobic glycolysis for energy production, while their low oxygen supply allows limited ATP production via oxidative phosphorylation [12]. For example, breast cancer growth is attributed to the TME, which reacts to oxidative stress leading to the production of $\operatorname{ROS}[13,14]$. Similarly, a study by Le et al. found that there is an increase in ROS production in response to oxidative stress under hypoxia [15]. Thus, this study concluded that cancer cells become dependent on glutamine for bioenergetics and redox homeostasis as a way to survive in hypoxia [15].

Extracellular acidity is another crucial component of the TME [16]. When cancer cells undergo anaerobic glycolysis in hypoxia, lactic acid levels increase, causing the TME's extracellular $\mathrm{pH}$
$\left(\mathrm{pH}_{\mathrm{e}}\right)$ to diminish. This reaction generates an acidic TME [16]. Tumors that have an acidic TME have been shown to display more malignant phenotypes. Rofstad et al. treated melanoma cells with an acidic medium resulting in increased melanoma cells metastasizing to the lungs in mice [17]. The results seen in the study suggest that lower $\mathrm{pH}_{\mathrm{e}}$ can exacerbate malignant metastasis.

The heterogeneity of nutrient and oxygen supply and uptake within individual tumors, in conjunction with the evidence of the adaptive process of cancer cells in response to differing conditions, illustrates that cancers are composed of many different cells that are each capable of employing distinct metabolic pathways to supply energy and fuel biosynthesis as a means of maintaining tumorigenesis. Thus, the local TME holds the determining factors by which metabolic adaptation is acquired $[8,9,12,13]$.

The hypoxic conditions lead to pathways that would only be present due to the alterations made necessary by metabolic stress. Other cells respond to glucose deprivation by requiring less energy to survive or utilize alternative compounds to take glutamine's place in the tricarboxylic acid (TCA) cycle [15]. However, different 
cancer cells take varying initiatives in order to survive, further exemplifying the heterogeneity of cancer metabolism.

\subsection{Fatty Acid Oxidation (FAO) Is Used as a Survival Response to Glucose Deprivation}

Recently, with further study of the fatty acid oxidation pathway, there has been significant evidence presented to support a "lipolytic phenotype" of cancer. FAO is a part of various steps of tumorigenesis, including cancer cell growth and survival $[18,19]$. In addition, FAO also occurs in tumor-associated immune cells, endothelial cells, and adipocytes, which may lead to immune suppression in the tumor microenvironment [18]. As stated before, adipocytes are major elements of various tumor microenvironments. In a study of invasive melanoma by Lazar et al., adipocytes were found to secrete high numbers of exosomes, which are integrated by cancer cells, subsequently contributing to their migration and invasion [20]. Lazar et al. observed that the presence of adipocyte exosomes increased FAO in melanoma cells. Therefore, through the uptake of fatty acids from surrounding adipocytes, FAO was promoted in cancer cells [20]. Similarly, in a study by Wen et al., adipocytes promoted xenograft colon tumor growth in vivo [21]. In addition, they observed that adipocytes are crucial components for cancer stem cell (CSC) gene expression and downregulated intestinal epithelial cell differentiation gene expression in vitro [21]. Furthermore, Wen et al. also demonstrated how adipocytes within the tumor microenvironment lead to cancer cell proliferation and survival due to fatty acid uptake and FAO promotion [21]. Lazar et al. and Wen et al. both demonstrate how a lipolytic phenotype is promoted by the tumor microenvironment's associated components. Their findings suggest potential players to target within the FAO metabolic pathway to prevent tumorigenesis.

Cancer cells employ FAO as a means to survive in response to glucose deprivation $[6,7]$. FAO is utilized by tumor cells to produce ATP as an energy source [7, 22]. Over twice the amount of ATP can be made under mitochondrial oxidation of one mole of fatty acid as compared to oxidation of one mole of glucose [7]. Due to harsh TME conditions, for example, lack of nutrition, cancer cells adapt different metabolic phenotypes, such as transitioning from glycolytic to fatty acid oxidation phenotype $[6,22]$. The lack of nutrition also enhances both fatty acid synthesis and lipid droplet biogenesis to propel lipid oxidation for the maintenance of energy levels [6].

In a study conducted by Wang et al., the roles of the hepatitis B virus X protein (HBx) in hepatocellular carcinoma (HCC) adaption to metabolic stress were investigated. Wang et al. found that HBx activates FAO in glucose withdrawal [6], maintaining nicotinamide adenine dinucleotide phosphate (NADPH) and adenosine triphosphate (ATP) homeostasis. HBx promotes dynamic equilibrium, mobilizing, and oxidizing lipids to meet the demands for ATP [6]. These results suggest that $\mathrm{HBx}$ plays a key role in maintaining redox and energy levels by activating FAO, a necessary part of HCC cell survival under metabolic stress.

Most cancer cells synthesize de novo fatty acids during normoxia without nutrition deprivation [7, 22]. Fatty acid synthesis is a crucial step for tumor cell survival [22]. Cancer cells synthesize de novo fatty acids in order to sustain proliferation and energy production through FAO. Acetyl-CoA carboxylase (ACC) and fatty acid synthase (FASN) are essential enzymes in de novo fatty acid synthesis. Acidic and hypoxic environments induce FASN expression in cancer cells, which is an observable phenotype in a variety of human cancers [22].

According to Ackerman and Simon, adipocytes within TMEs play a key role in increasing lipolysis and secreting fatty acids for energy production, contributing to an aggressive growth phenotype [23]. Lipids produced from adipocytes were used by ovarian cancer cells in order to help tumor growth. These findings suggest that adipocytes are key players in tumor growth by supplying fatty acids [24]. Moreover, this study uncovered fatty acid-binding protein 4 (FABP4) as a potential target for cancer therapy. 


\subsection{Lipid Scavenging Is Utilized to Enable Cancer Cells to Survive Periods of Tumor Regression}

Under hypoxic conditions, oncogenic Kirsten rat sarcoma viral oncogene homolog $(K R A S)$ regulates lysophospholipids to replenish lipids for growth. The inhibition of stearoyl-CoA desaturase 1 (SCD1), which catalyzes the bypassing of saturated de novo fatty acids into lipids, was resisted by $K R A S$-derived tumor cells because of their adaption of lipid scavenging [7]. The increase in protein synthesis and a decrease in lipid desaturation ultimately resulted in cell death $[25,26]$. During tumor regression, cancer cell survival is made possible by FAO and other oxidative mitochondrial pathways. As demonstrated by $K R A S$-driven pancreatic cancer, tumor regression caused by kinase inhibitors or KRAS withdrawal resulted in inhibited oxidative respiration in tumor cells [27]. Lipid scavenging is an alternative pathway to gain fatty acids in hypoxia and fulfill the requirements for cell monounsaturated fatty acids by Ras-driven cancer cells [28]. The reduction of the need for de novo fatty acid synthesis is attributed to the increase in fatty acids being brought into the TME. Ras-driven cancer cells become immune to SCD1 inhibition, demonstrating the lipid scavenging phenotype [28].

\subsection{Persistence of Glutamine Oxidation Under Hypoxic and Glucose Deprivation Conditions}

As established in previous chapters, the tricarboxylic acid (TCA) cycle is crucial for producing energy and biosynthesis [29, 30]. However, how hypoxic TMEs influence the TCA cycle is still being investigated. Le et al. determined how hypoxic conditions could influence glutamine metabolism [15]. Their study showed that when deprived of glucose and oxygen, B-cell lymphoma exhibit an addiction to glutamine, where glutaminolysis is employed with a glucoseindependent TCA cycle to fuel cell proliferation
[15]. In this scenario, the glucose-independent TCA cycle is supported by glutamine. Similarly, hypoxic cells use glutamine to generate citrate from $\alpha$-ketoglutarate $(\alpha-K G)$ in response to a reduced supply of glucose-derived citrate [15]. Targeting glutamine metabolism was further investigated not only by their follow-up works [31-33] but also by other teams [34-36]. This dependence of cancer cells on glutamine metabolism has translated into clinical trials as a novel therapy for cancer patients. Collectively, these findings offer a cautionary note that therapeutic strategies targeting cancer metabolism should consider the metabolic heterogeneity in hypoxic cancer cells, particularly the non-Warburg cells that have so far been underrepresented in the cancer metabolism literature [37].

\section{$4 \quad$ Nutrient Utilization Can Predict a Tumor's Metabolic Dependencies In Vivo [38]}

As described by Sir Hans Kornberg, anaplerosis is the reloading of metabolic intermediates in the TCA cycle, which is a crucial part of energy production and biosynthetic pathways. Glutamine and glucose both contribute to TCA anaplerosis in non-small cell lung cancer (NSCLC) cells [38]. In this study by Davidson et al., the authors found that glucose is a carbon source of the metabolites in the TCA cycle, which is needed for tumorigenesis.

For continuous proliferation, cancer cells must maintain the necessary precursors of biosynthetic pathways, and glutamine serves as a major substrate for anaplerosis in many cancer cells [30]. For example, both hypoxic and normoxic renal cell carcinomas with a mutation in the von Hippel-Lindau (VHL) tumor-suppressor gene sustain lipogenesis by converting $\alpha-\mathrm{KG}$, derived from glutamine, to acetyl-CoA, which then allows them to utilize the glucoseindependent TCA cycle as a means of energy production $[8,9]$. On the other hand, when glutaminase is inhibited, the breakdown of glutamine is partially prevented and some cancer cells employ pyruvate carboxylase and use glucose- 
derived pyruvate as a substitute for glutamine to fuel anaplerosis [8].

Similarly, a study by Cheng et al. demonstrated that "glutamine-addicted" cells accomplished anaplerosis by utilizing pyruvate carboxylase [8, 39]. It was found that the glutamine-addicted cells utilized glucose-derived pyruvate for anaplerosis when glutaminase (GLS) was silenced. The data from this study supported the model of pyruvate carboxylase's role in cancer cell resistance against GLS inhibition or glutamine deprivation. Cells such as a hepatocellular carcinoma cell line, Huh7, use pyruvate carboxylase as a primary mechanism to resist the treatment of glutamine metabolism inhibition [8].

\subsection{Inhibition of mTORC1 Decreases Energy Consumption for Cancer Cell Survival}

The mammalian target of rapamycin complex 1 (mTORC1) is a protein that translates the TME into a growth phenotype through its control of autophagy and fatty acid oxidation (FAO). The inhibition of mTORC1 represses the AMPKdependent activation of tuberous sclerosis proteins $1 / 2\left(\mathrm{TSC}^{1} / 2\right)$ as a result of the withdrawal of glucose [9]. When energy consumption is reduced, oxaloacetate (OAA) or methyl pyruvate (MP) can be substituted for glutamine and still be able to maintain ATP levels and prevent cell death. The TSC-mTORC1 pathway balances energy supply and demand in a way that leads to a reduction of the energy needed to survive [9]. Choo et al. demonstrated that, under glucose deprivation, a decrease in anabolic reactions occurs in order to prevent cell death [9]. As shown with the decrease of energy consumption, the balance keeps the cancer cells alive through the dependence of $\mathrm{TSC}^{1} / 2$ cells on glutamate dehydrogenasedependent glutamine metabolism [9]. The results found in this study support the concept that tumor cells under stress create alternative pathways out of necessity. With glucose or glutamine metabolism inhibition, the potential treatment of TSCdeficient tumors may be possible.

\subsection{Cancer Cells with Functionally Defective Mitochondria Employ Glutamine- Dependent Reductive Carboxylation as an Alternative to Normal Oxidative Metabolism}

In normal cells, mitochondria play vital roles in regulating metabolic pathways and physiological states of the cell: they generate cellular energy, monitor cellular redox, and initiate cellular apoptosis. However, through investigation of mitochondria in cancer cells, it has become evident that mutations in mitochondrial genes correlate with tumorigenesis and metabolic adaptability [40]. Mitochondria in cancer cells subjected to hypoxia respond by releasing metabolites and proteins regulating metabolic pathways [40].

Cancer cells with functionally defective mitochondria employ glutamine-dependent reductive carboxylation, where glutamine is converted to citrate and then to acetyl-CoA and oxaloacetate, as an alternative to normal oxidative metabolism. Oxidative metabolism is favored in cells with normal mitochondria and provides the acetyl-CoA needed for lipogenesis and production of other metabolites of the TCA cycle, which serve as precursors of other biosynthetic pathways. Even in cells with altered mitochondrial function, the glutamine-dependent reductive metabolism still allows for the formation of these necessary metabolic precursors [41]. The glutamine-dependent reductive pathway permits glutamine to support cancer cell growth [41].

\section{Distinct, and Often Complementary, Metabolic Processes Operate Concurrently Within a Single Tumor}

The particular alternative metabolic pathways adopted by cancer cells are associated with specific genetic alterations that allow the cancer cells to express certain enzymes in higher than 
usual amounts. The production of these enzymes allows cancer cells to use the available nutrients in their microenvironment to fuel cell survival and proliferation. For example, genetic alterations that result in the deactivation of caveolin-1 (Cav-1) expression lead to autophagy and aerobic glycolysis in cancer-associated fibroblasts [42]. Subsequently, lactate, glutamine, and other metabolites that fuel biosynthesis are synthesized and exported to initiate oxidative metabolism in neighboring cancer cells [42].

Other studies have revealed that distinct, and often complementary, metabolic processes operate concurrently within a single tumor. Hypoxic breast cancer cells and stromal cells in the TME exhibit a mutualistic relationship employing complementary metabolic processes [43]. When subjected to hypoxia, breast cancer cells demonstrate an increase in lactate secretion. The elevation in lactate concentration in the TME results in the migration of specific stromal cells called human mesenchymal stem cells (hMSCs) toward hypoxic tumor cells. These hMSCs, along with stromal cancer-associated fibroblasts (CAFs), consume the newly produced lactate and convert it to pyruvate to be used in the TCA cycle. Lactate consumption by stromal cells serves two purposes: the breakdown of lactate serves as an energy source for the proliferating cancer cells, and the conversion of lactate to pyruvate, and ultimately to $\alpha-\mathrm{KG}$ in the TCA cycle, prevents acidification of the TME [43].

Another example of this phenomenon of cells in the TME pairing metabolic processes is evident in ovarian cancers. Adipocytes in breast cancer microenvironments employ lipolysis to release fatty acids which provide energy to fuel rapidly proliferating ovarian cancer cells [24]. Within one region of the TME, two different types of cells undergo vastly different, yet complementary, metabolic processes in order to fuel tumorigenesis, thus demonstrating the heterogeneity of cancer metabolism.

\subsection{Metabolic Symbiosis as a Result of Tumor Angiogenesis Inhibition Can Be Stopped by mTOR Signaling Inhibition [44]}

Coordinated metabolic pathways with respect to glucose and lactate metabolism between cells within the TME have been observed in various cancers [45]. Allen et al. observed metabolic symbiosis with their work in tumor angiogenesis inhibition [44]. When angiogenesis is targeted using VEGF inhibitors in mice bearing pancreatic neuroendocrine tumors (PanNET), cancer cells formed next to the remaining blood vessels [44]. The cancer cells compartmentalized in response to insufficient vascularization-creating distal hypoxic cells and proximal normoxic cells [44]. Upon further observation, increased glucose transporter 1 (GLUT1) and monocarboxylate transporter 4 (MCT4) were found in tumor cells far from blood vessels indicating glycolysis [44]. Moreover, signs of mTOR signaling, in the form of ribosomal protein s6 ( $\mathrm{p}-\mathrm{S} 6$ ) expression, were found in tumor cells near blood vessels [44]. In these metabolic pathways known as metabolic symbiosis, the hypoxic cells take up glucose and secrete lactate, which is then taken up and catabolized by normoxic cells [44]. As a result of increased lactate catabolism, the normoxic cells' mTOR signaling through glutamine metabolism is increased [44].

The metabolic symbiosis that Allen et al. found is due to the compartmental expression of GLUT1/MCT4 [44]. For metabolic symbiosis to occur, the lactate that is secreted must be taken up and used for energy metabolism by the normoxic cancer cells. Within their study, Allen et al. also demonstrated that normoxic cancer cells in vitro and in vivo take up and catabolize lactate - which reinforces the notion that lactate is used for energy metabolism [44]. 
Allen et al.'s experiments demonstrate how PanNET tumor cells adapt to evade targeted antiangiogenesis therapy. While Allen et al. were able to target the metabolic symbiosis by inhibiting mTOR signaling [44], the initial adoption of metabolic symbiosis demonstrates how the tumor cells circumnavigate the initial treatment by creating new pathways for energy production. Furthermore, metabolic symbiosis as a result of tumor angiogenesis inhibition reflects how distinct and complementary metabolic processes occur within the same tumor.

\section{Conclusion}

As cancer cells seek to survive, alternate metabolic pathways adapt to different TME stresses. These adaptations, often through genetic alterations or coordination with other metabolic processes, illustrate how precisely the TME can alter metabolic characteristics. With the advancements in research into TMEs and the use of metabolomics technologies [46], there is a tremendous opportunity for uncovering new therapeutic targets and creating treatments that target TMEs $[47,48]$. The heterogeneity of cancer metabolism is evident in genetic mutations in oncogenes and tumor-suppressor genes, as well as the diversity of the TME.

\section{References}

1. Ward, P. S., \& Thompson, C. B. (2012). Metabolic reprogramming: A cancer hallmark even Warburg did not anticipate. Cancer Cell, 21(3), 297-308.

2. Nabi, K., \& Le, A. (2021). The intratumoral heterogeneity of cancer metabolism. Advances in Experimental Medicine and Biology, 1311, https:// doi.org/10.1007/978-3-030-65768-0_11

3. Hanahan, D., \& Weinberg, R. A. (2000). The hallmarks of cancer. Cell, 100(1), 57-70.

4. Hanahan, D., \& Weinberg, R. A. (2011). Hallmarks of cancer: The next generation. Cell, 144(5), 646-674.

5. Cairns, R. A., Harris, I. S., \& Mak, T. W. (2011). Regulation of cancer cell metabolism. Nature Reviews. Cancer, 11(2), 85-95.
6. Wang, M. D., et al. (2016). HBx regulates fatty acid oxidation to promote hepatocellular carcinoma survival during metabolic stress. Oncotarget, 7(6), 6711-6726.

7. Boroughs, L. K., \& DeBerardinis, R. J. (2015). Metabolic pathways promoting cancer cell survival and growth. Nature Cell Biology, 17(4), 351-359.

8. Cheng, T. L., et al. (2011). Pyruvate carboxylase is required for glutamine-independent growth of tumor cells. Proceedings of the National Academy of Sciences of the United States of America, 108(21), 8674-8679.

9. Choo, A. Y., et al. (2010). Glucose addiction of TSC null cells is caused by failed mTORC1-dependent balancing of metabolic demand with supply. Molecular Cell, 38(4), 487-499.

10. Pavlova, N. N., \& Thompson, C. B. (2016). The emerging hallmarks of cancer metabolism. Cell Metabolism, 23(1), 27-47.

11. Schieber, M., \& Chandel, N. S. (2014). ROS function in redox signaling and oxidative stress. Current Biology, 24(10), R453-R462.

12. Miyata, T., Takizawa, S., \& de Strihou, C. V. (2011). Hypoxia. 1. Intracellular sensors for oxygen and oxidative stress: Novel therapeutic targets. American Journal of Physiology-Cell Physiology, 300(2), C226-C231.

13. Jezierska-Drutel, A., Rosenzweig, S. A., \& Neumann, C. A. (2013). Role of oxidative stress and the microenvironment in breast cancer development and progression. Advances in Cancer Research, 119(119), 107-125.

14. Stolarek, R. A., et al. (2010). Increased H2O2 level in exhaled breath condensate in primary breast cancer patients. Journal of Cancer Research and Clinical Oncology, 136(6), 923-930.

15. Le, A., et al. (2012). Glucose-independent glutamine metabolism via TCA cycling for proliferation and survival in B cells. Cell Metabolism, 15(1), 110-121.

16. Kato, Y., et al. (2013). Acidic extracellular microenvironment and cancer. Cancer Cell International, 13(1), 89.

17. Rofstad, E. K., et al. (2006). Acidic extracellular pH promotes experimental metastasis of human melanoma cells in athymic nude mice. Cancer Research, 66(13), 6699-6707.

18. Ma, Y., et al. (2018). Fatty acid oxidation: An emerging facet of metabolic transformation in cancer. Cancer Letters, 435, 92-100.

19. Park, J. K., et al. (2021). The heterogeneity of lipid metabolism in cancer. Advances in Experimental Medicine and Biology, 1311, https://doi. org/10.1007/978-3-030-65768-0_3

20. Lazar, I., et al. (2016). Adipocyte exosomes promote melanoma aggressiveness through fatty acid oxidation: A novel mechanism linking obesity and cancer. Cancer Research, 76(14), 4051-4057. 
21. Wen, Y. A., et al. (2017). Adipocytes activate mitochondrial fatty acid oxidation and autophagy to promote tumor growth in colon cancer. Cell Death \& Disease, 8(2), e2593.

22. Mashima, T., Seimiya, H., \& Tsuruo, T. (2009). De novo fatty-acid synthesis and related pathways as molecular targets for cancer therapy. British Journal of Cancer, 100(9), 1369-1372.

23. Ackerman, D., \& Simon, M. C. (2014). Hypoxia, lipids, and cancer: Surviving the harsh tumor microenvironment. Trends in Cell Biology, 24(8), 472-478.

24. Nieman, K. M., et al. (2011). Adipocytes promote ovarian cancer metastasis and provide energy for rapid tumor growth. Nature Medicine, 17(11), 1498-1503.

25. DeBerardinis, R. J., et al. (2007). Beyond aerobic glycolysis: Transformed cells can engage in glutamine metabolism that exceeds the requirement for protein and nucleotide synthesis. Proceedings of the National Academy of Sciences of the United States of America, 104(49), 19345-19350.

26. Young, R. M., et al. (2013). Dysregulated mTORC1 renders cells critically dependent on desaturated lipids for survival under tumor-like stress. Genes \& Development, 27(10), 1115-1131.

27. Viale, A., et al. (2014). Oncogene ablation-resistant pancreatic cancer cells depend on mitochondrial function. Nature, 514(7524), 628-632.

28. Kamphorst, J. J., et al. (2013). Hypoxic and Ras-transformed cells support growth by scavenging unsaturated fatty acids from lysophospholipids. Proceedings of the National Academy of Sciences of the United States of America, 110(22), 8882-8887.

29. Bose, S., Zhang, C., \& Le, A. (2021). Glucose metabolism in cancer: The Warburg effect and beyond. Advances in Experimental Medicine and Biology, 1311, https://doi.org/10.1007/978-3-030-65768-0_1

30. Li, T., Copeland, C., \& Le, A. (2021). Glutamine metabolism in cancer. Advances in Experimental Medicine and Biology, 1311, https://doi. org/10.1007/978-3-030-65768-0_2

31. Elgogary, A., et al. (2016). Combination therapy with BPTES nanoparticles and metformin targets the metabolic heterogeneity of pancreatic cancer. Proceedings of the National Academy of Sciences of the United States of America, 113(36), E5328-E5336.

32. Nguyen, T., et al. (2019). Uncovering the role of $\mathrm{N}$-acetyl-aspartyl-glutamate as a glutamate reservoir in cancer. Cell Reports, 27(2), 491-501. e6.

33. Udupa, S., et al. (2019). Upregulation of the glutaminase II pathway contributes to glutamate production upon glutaminase 1 inhibition in pancreatic cancer. Proteomics, 19(21-22), e1800451.

34. Xiang, Y., et al. (2015). Targeted inhibition of tumorspecific glutaminase diminishes cell-autonomous tumorigenesis. The Journal of Clinical Investigation, 125(6), 2293-2306.
35. Zimmermann, S. C., et al. (2016). Allosteric glutaminase inhibitors based on a 1,4-di(5-amino-1,3,4thiadiazol-2-yl)butane scaffold. ACS Medicinal Chemistry Letters, 7(5), 520-524.

36. Rais, R., et al. (2016). Discovery of 6-diazo-5-oxo1-norleucine (DON) prodrugs with enhanced CSF delivery in monkeys: A potential treatment for glioblastoma. Journal of Medicinal Chemistry, 59(18), 8621-8633.

37. Le, A., et al. (2014). Tumorigenicity of hypoxic respiring cancer cells revealed by a hypoxia-cell cycle dual reporter. Proceedings of the National Academy of Sciences of the United States of America, 111(34), 12486-12491.

38. Davidson, S. M., et al. (2016). Environment impacts the metabolic dependencies of ras-driven non-small cell lung cancer. Cell Metabolism, 23(3), 517-528.

39. Wise, D. R., et al. (2008). Myc regulates a transcriptional program that stimulates mitochondrial glutaminolysis and leads to glutamine addiction. Proceedings of the National Academy of Sciences of the United States of America, 105(48), 18782-18787.

40. Solaini, G., et al. (2010). Hypoxia and mitochondrial oxidative metabolism. Biochimica et Biophysica Acta-Bioenergetics, 1797(6-7), 1171-1177.

41. Mullen, A. R., et al. (2012). Reductive carboxylation supports growth in tumour cells with defective mitochondria. Nature, 481(7381), 385-U171.

42. Sotgia, F., et al. (2012). Caveolin-1 and cancer metabolism in the tumor microenvironment: Markers, models, and mechanisms. Annual Review of Pathology: Mechanisms of Disease, 7(7), 423-467.

43. Rattigan, Y. L., et al. (2012). Lactate is a mediator of metabolic cooperation between stromal carcinoma associated fibroblasts and glycolytic tumor cells in the tumor microenvironment. Experimental Cell Research, 318(4), 326-335.

44. Allen, E., et al. (2016). Metabolic symbiosis enables adaptive resistance to anti-angiogenic therapy that is dependent on mTOR signaling. Cell Reports, 15(6), 1144-1160.

45. Lyssiotis, C. A., \& Kimmelman, A. C. (2017). Metabolic interactions in the tumor microenvironment. Trends in Cell Biology, 27(11), 863-875.

46. Hoang, G., Udupa, S., \& Le, A. (2019). Application of metabolomics technologies toward cancer prognosis and therapy. International Review of Cell and Molecular Biology, 347, 191-223.

47. Jung, J. G., \& Le, A. (2021). Targeting metabolic cross talk between cancer cells and cancerassociated fibroblasts. Advances in Experimental Medicine and Biology, 1311, https://doi. org/10.1007/978-3-030-65768-0_15

48. Hirschey, M. D., et al. (2015). Dysregulated metabolism contributes to oncogenesis. Seminars in Cancer Biology, 35(Suppl), S129-S150. 
Open Access This chapter is licensed under the terms of the Creative Commons Attribution 4.0 International License (http://creativecommons.org/licenses/by/4.0/), which permits use, sharing, adaptation, distribution and reproduction in any medium or format, as long as you give appropriate credit to the original author(s) and the source, provide a link to the Creative Commons license and indicate if changes were made.

The images or other third party material in this chapter are included in the chapter's Creative Commons license, unless indicated otherwise in a credit line to the material. If material is not included in the chapter's Creative Commons license and your intended use is not permitted by statutory regulation or exceeds the permitted use, you will need to obtain permission directly from the copyright holder.

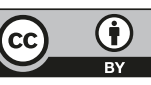




\title{
The Intratumoral Heterogeneity of Cancer Metabolism
}

\author{
Karim Nabi and Anne Le
}

Keywords
Intratumoral heterogeneity $\cdot$ Metabolism
Genetic and metabolic adaptation
Angiogenesis $\cdot$ Hypoxia

\section{Abbreviations}

\begin{tabular}{|c|c|}
\hline$\alpha-K G$ & $\alpha$-Ketoglutarate \\
\hline BPTES & $\begin{array}{l}\text { Bis-2-(5-phenylacetamido-1,3,4- } \\
\text { thiadiazol-2-yl)ethyl sulfide }\end{array}$ \\
\hline $\mathrm{CAF}$ & Cancer-associated fibroblasts \\
\hline $\mathrm{CSC}$ & Cancer stem cell \\
\hline DTC & Disseminated tumor cells \\
\hline $\mathrm{EC}$ & Endothelial cells \\
\hline FBP1 & Fructose-1,6-bisphosphatase 1 \\
\hline FBP2 & Fructose-1,6-bisphosphatase 2 \\
\hline FDG & {$\left[{ }^{18} \mathrm{~F}\right]$-Fluorodeoxyglucose } \\
\hline FH & Fumarate hydratase \\
\hline FMISO & {$\left[{ }^{18} \mathrm{~F}\right]$-Fluoromisonidazole } \\
\hline GLUT-1 & Glucose transporter type 1 \\
\hline
\end{tabular}

K. Nabi

Department of Pathology, Johns Hopkins University School of Medicine, Baltimore, MD, USA
A. Le $(\bowtie)$
Department of Pathology and Oncology, Johns Hopkins University School of Medicine,
Baltimore, MD, USA
Department of Chemical and Biomolecular Engineering, Johns Hopkins University Whiting School of Engineering, Baltimore, MD, USA e-mail: annele@jhmi.edu

HIF- $1 \alpha \quad$ Hypoxia-inducible factor- $1 \alpha$

HK-2 Hexokinase 2

LDHA Lactate dehydrogenase A

MC Metabolic cluster

MTV Metabolic tumor volume

NR2F1 Nuclear receptor subfamily 2 group F member 1

OXPHOS Oxidative phosphorylation

PET Positron-emission tomography

PKM2 Pyruvate kinase muscle isoform 2

SDH Succinate dehydrogenase

SUV Standardized uptake values of glucose

TCA Tricarboxylic acid

VEGF Vascular endothelial growth factor

\section{Key Points}

- Heterogeneity is a hallmark of cancer.

- Clonal evolution theory and cancer stem cell theory explain tumor subpopulation growth.

- Intratumoral metabolic heterogeneity follows intratumoral genetic alterations.

- Epigenetics alterations lead to intratumoral metabolic heterogeneity.

- Intratumoral metabolic adaptation and heterogeneity are due to the extreme conditions of the tumor microenvironment.

- Spatial and temporal heterogeneity provides survival advantages to tumors.

- Metabolic profile-targeted therapeutics can result in successful clinical outcomes. 
Cancer is one of the deadliest diseases in the world, causing over half a million deaths a year in the USA alone. Despite recent advances made in the field of cancer biology and the therapies that have been developed [1,2], it is clear that more advances are necessary for us to classify cancer as curable. The logical question that arises is simple: Why, despite all the technologies and medical innovations of our time, has a complete cure eluded us? This chapter sheds light on one of cancer's most impactful attributes: its heterogeneity and, more specifically, the intratumoral heterogeneity of cancer metabolism. Simply put, what makes cancer one of the deadliest diseases is its ability to change and adapt. Cancer cells' rapid evolution, coupled with their irrepressible ability to divide, gives most of them the advantage over our immune systems. In this chapter, we delve into the complexities of this adaptability and the vital role that metabolism plays in the rise and progression of this heterogeneity.

\section{Multiple Theories Explain Cancer's Heterogeneous Nature}

In any observable tumor, there is much more than what meets the eye. In the carcinogenic environment, we can observe a microcosm of the theory of evolution at play. While Darwin's theory was proposed to explain the evolution of species due to slowly cumulative changes that arise from natural selection, cancer cells, driven by their genetic instability and high reproductive rates, develop and evolve in a fraction of our lifetime, leading to dangerous and unpredictable outcomes. The genetic instability associated with cancerous cells gives rise to a plethora of downstream metabolic phenotypes. These phenotypes offer cancerous cells one of the most valued assets in their battle for survival: their metabolic diversity, which can explain why it is so difficult to find effective therapies for most cancers.
The explanation of intratumoral heterogeneity using the theory of evolution provides a solid basis for understanding why and how tumors possess this medley of metabolic phenotypes. Tumors have different genetic and metabolic phenotypes due to different environmental pressures such as vascularization, oxygen supply, and other factors such as drug treatments. While certain subpopulations with defined metabolic phenotypes may be sensitive to a suitable metabolic inhibitor, other subclones with different metabolic phenotypes may well be resistant to that drug. This explains why patients may become unresponsive to secondround treatment after an initial successful first round in which most of the tumor was targeted and eliminated by the treatment, but small subpopulations were not [3]. These selective pressures promote the survival and propagation of genetically and even epigenetically diverse subclones that lead to the downstream array of distinct metabolic phenotypes in each subclone (Fig. 1). It is important to mention another heterogeneity-emerging theory, namely the cancer stem cell (CSC) theory, which challenges the previously mentioned clonal evolution theory. The clonal evolution theory claims that genetically and metabolically distinct subpopulations arise from a previously larger population of cancer cells due to the expansion of the population, genetic diversification, and selection of certain subclones over others. On the other hand, the CSC theory states that a significant source of heterogeneity in cancer cells is due to CSCs, which are undifferentiated and have high rates of division. These cancer stem cells possess largely variable metabolic phenotypes through their differentiation into different types of cells [4]. They are also capable of differentiating into metabolically and functionally diverse subclones within a single tumor. Moreover, they are usually resistant to many therapeutic methods due to their undifferentiated state. This fact is supported by findings suggesting that more differentiated cancer stem cells tend to lead to better prognoses due to their decreased tumorigenic potential [5]. In fact, the mechanism behind many therapies for cancer patients induces differentiation of CSCs. The origin of these CSCs ranges from tumor cells that acquired stem cell properties to differentiated stem 
Fig. 1 Bottleneck effect in tumors. Metabolically different subclones of a tumor, each represented by a different color. Survival and growth rates of subclones depend on the various selective environmental pressures applied such as blood supply shortage, low oxygen levels, and drug treatment

\section{Bottleneck Effect}

Selective Pressure

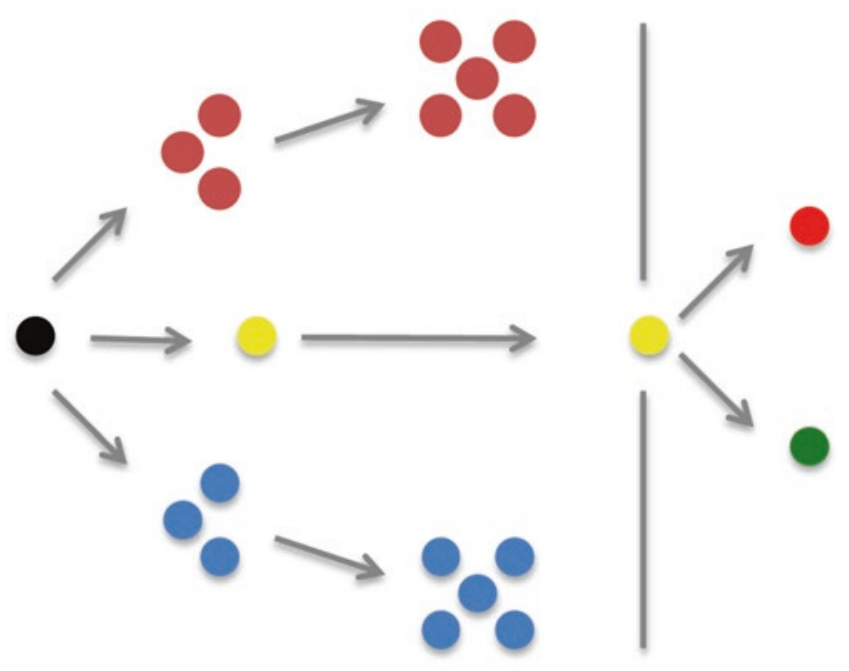

Metabolic Heterogeneity

cells that simply accumulated mutations that turn them into CSCs [6].

Cancer is further complicated by the fact that the different sources of heterogeneity, namely CSC-derived heterogeneity, evolution-derived heterogeneity, and heterogeneity related to environmental factors, can all coexist at once [7]. This makes it a much more arduous feat to eradicate all subclones within any given tumor which then leads to the following question: Why do cancer cells employ various biological processes, even within a single tumor from a single patient? The ultimate advantage of intratumoral heterogeneity of cancer cell metabolism is that it confers on the cancer cells an ability to survive and proliferate within the tremendously variable, and often harsh, tumor microenvironment. The diversity of the tumor microenvironment - characterized by areas of poor oxygenation, increased acidity, sparse nutrients, or growth factors-is the challenge that cancer cells must overcome in order to achieve the goals of survival and continued rapid cell proliferation.

How do these diverse metabolic phenotypes arise? We know that the different microenvironments in any given tumor provide different selective pressures that lead to the propagation of specific advantageous mutations in each respective cancer subclone. We also know that CSCs can contribute to the heterogeneous aspect of a tumor by providing differentiated subpopulations with varied genetic expressions and metabolic pathways. These changes result in a variety of proteins and, most importantly among them, enzymes necessary to effectively convert locally available nutrients into energy and useful products suited for each microenvironment to obtain what is required for the production of a specific metabolic phenotype for each subclone.

\section{$3 \quad$ Intratumoral Metabolic Heterogeneity Follows Intratumoral Genetic Alterations}

The intricate relationship between genetics and metabolism in cancer is arguably the main reason why the diverse metabolic phenotypes within a given tumor can arise. All of the genetic changes, if occurring in different regions of a tumor, can 
lead to a diverse array of differently regulated metabolic processes in a tumor.

Genetic alterations, which are often the result of a response to the tumor microenvironment, are the means by which cancer cells are able to produce the enzymes necessary to effectively convert locally available nutrients into energy and useful products to achieve their goals. Oxygen and nutrient supply varies across individual tumors. Thus, intratumoral gene expression is diverse, and it is this genetic heterogeneity that allows cancer cells to adapt to the diverse and taxing conditions of the tumor microenvironment. These adaptations in nutrient uptake and biosynthesis, which have been linked to particular genetic mutations, must follow from altered gene expression in cancer cells. As such, the enzymes produced are the proximate causes of the adoption of alternative metabolic pathways, which contribute to the cancer cells' successful survival and growth.

In light of the evidence of intratumoral genetic heterogeneity, along with the fact that changes in cancer cell metabolism are the consequences of alterations in gene expression, cancer metabolism must be vastly diverse across a single tumor. A recent study noted the coexistence of various genetically different subclones in advanced tumors, challenging the previously held notion that a dominant subclone usually appears in a given tumor [8]. Furthermore, based on the expression of 110 genes, another study showed that different subpopulations in one clear cell carcinoma could be classified as either clear cell A (associated with good prognosis) or B (associated with poor prognosis) [8, 9]. These results emphasize not only how varied gene expression within a single tumor can be, but also the need to accurately use prognostic markers due to the different genotypes within each subclone, as not doing so could potentially lead to erroneous prognoses (Fig. 2).

Another study has shown that intratumoral genetic diversity is also widely prevalent within tumor cell populations in breast carcinomas [10, 11]. These tumor cell populations are composed of stem cell-like or more differentiated cell populations expressing different clusters of differenti- ation and antigens on their surface [10]. These subpopulations were further found to exhibit highly heterogeneous genetic compositions, implying different biological and metabolic functions and, most likely, different responses to the same treatments [10].

\section{$4 \quad$ Epigenetics Alterations Lead to Intratumoral Metabolic Heterogeneity}

It is important to note that not all heterogeneity arises from genetic alterations. New studies point to the important role of epigenetics in tumor heterogeneity. Epigenetics studies have recently uncovered increased methylation in promoters of a variety of important genes in tumor progression, such as tumor-suppressor genes [12]. Other findings also showed similar roles of epigenetics in cancer evolution. We can observe an example of the effect of intratumoral epigenetic heterogeneity in a study which revealed that in a given glioblastoma [14] tumor, 40\% transcriptional heterogeneity was observed in a gene encoding a DNA repair enzyme: O6-methylguanine DNA methyltransferase (MGMT) [13]. Furthermore, $14 \%$ of the heterogeneity was attributed to the methylation levels of the promoter of that gene, whose methylation status has been used for clinical purposes as a marker that correlates with therapeutic response [13]. However, these variations in expression across a single tumor pose a threat to the effectiveness of this enzyme as a clinical marker. In addition to the genetic heterogeneity observed, researchers and clinicians need to keep in mind the variability displayed on an epigenetic level across a single tumor. Therefore, it is fair to keep in mind the potential effect epigenetics could have on metabolism.

In a study by Okegawa et al., the characterization of kidney tumors revealed distinct metabolic profiles in different regions of the same tumor [15]. The study identified two distinct tumor metabolic clusters, MC1 and MC2, where MC2 displayed upregulated pyruvate metabolism, which was confirmed using isotope tracing in tumor slices. This suggests that pyruvate metabolism 
Fig. 2 The intratumoral heterogeneity of cancer metabolism. Clear cell carcinoma tumor (shown in blue) with subclones (shown in orange and gray). The orange subclone consists of clear cell A cells (associated with good prognoses), and the gray subpopulation consists of clear cell B cells (associated with poor prognoses). Single biopsies taken from one population may indicate misleading prognoses

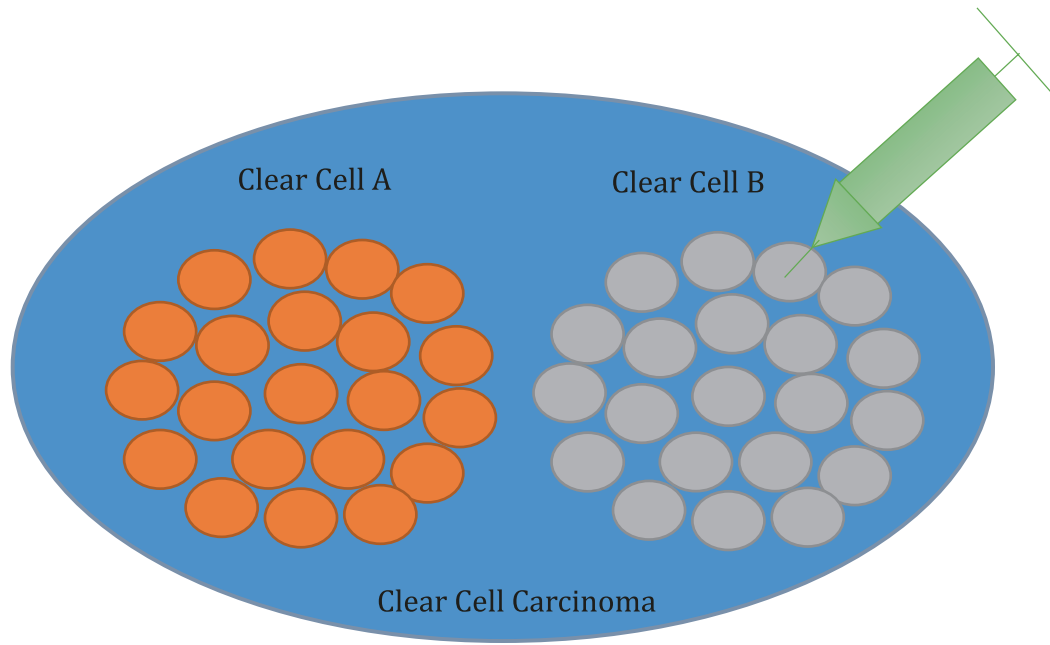

may be a potential therapeutic target due to some clones' reliance on it. However, genetic differences between subpopulations did not match the metabolic profiles of such subclones, suggesting that factors other than genetics, such as epigenetics, may play a role in developing distinct metabolic phenotypes.

\section{Intratumoral Metabolic Adaptation and Heterogeneity Are Due to the Intemperate Conditions of the Tumor Microenvironment}

Now we will take a closer look at how a tumor can metabolically adapt to its ever-changing environment. These adaptations also reflect an evolutionary advantage in cancer cells and give rise to the heterogeneity found in cancer. As a tumor grows in size, it develops hypoxic regions that are beyond the diffusion limits of oxygen in existing vasculature. Tumor hypoxia, in addition to its role in the mutation of oncogenes and tumor-suppressor genes, plays a major role in the overexpression of hypoxia-inducible factor- $1 \alpha$ (HIF- $1 \alpha)$ in cancer. HIF- $1 \alpha$ is part of a heterodimeric protein that acts as a transcriptional regulator for many genes involved in angiogenesis, erythropoietin production, and cell survival. While HIF- $1 \alpha$ usually degrades quickly under normal conditions, degradation is suppressed in hypoxic environments. Therefore, increased HIF- $1 \alpha$ level upregulates the expression of genes that code for adaptive metabolic changes, switching cancer cell metabolism from oxidative phosphorylation (OXPHOS) to glycolysis, increasing the conversion of glucose to glycogen as a glucose reservoir, and using glutamine as the major substrate for fatty acid synthesis [16, 17]. Furthermore, HIF-1 directly transactivates lactate dehydrogenase A (LDHA) expression under hypoxia [17], which explains how hypoxia further promotes glycolysis [18-20].

In order for the tumor to metastasize and grow beyond a few millimeters, angiogenesis is necessary [21]. HIF-1 $\alpha$ also upregulates the expression of genes that code for angiogenesis. One of the most notable of such genes is the gene encoding proangiogenic vascular endothelial growth factor (VEGF), which induces the proliferation of endothelial cells (ECs), a key process in angiogenesis [22-24]. Surprisingly, several studies found that ECs mainly rely on glycolysis rather than oxidative phosphorylation (OXPHOS) despite their ideal location that promotes their function as endothelium and in maintaining vascular barrier homeostasis and bioenergetics [25-30]. Similar to cancer cells, ECs choose aerobic glycolysis over OXPHOS due to their rapid growth, which is necessary to fulfill the demands of forming new blood vessels [25]. Reducing glycolysis by 
silencing its stimulator phosphofructokinase-2/ fructose-2,6-bisphosphatase 3 (PFKFB3) decreased angiogenesis [25]. Moreover, PFKFB3-deficient ECs display poor vessel growth in several in vivo models of angiogenesis. VEGF, in turn, also promotes glycolysis through the upregulation of glucose transporter type 1 (GLUT-1), which facilitates glucose uptake [31].

The tumor microenvironment is tremendously dynamic and diverse regarding nutrient and oxygen supply, both spatially and temporally within a single tumor. Temporal variations of the partial pressure of oxygen within a specified region of the tumor referred to as intermittent or cyclic hypoxia occur in different regions throughout the tumor [32]. The occurrence of cyclic hypoxia is attributed to variations in red blood cell flux, which is thought to be a result of changes in blood flow resistance that arise from angiogenesis and other structural changes to the vasculature $[32,33]$. Regions of the tumor with adequate vasculature are much more resistant to intermittent hypoxia than regions with insufficient vasculature [34]. Although reduced oxygenation to select either regions or the entire tumor can induce hypoxia, an increase of equal magnitude in oxygen consumption is disproportionately more effective in inducing hypoxia [35-39]. These variations in oxygen and nutrient delivery, as well as in oxygen consumption within a tumor, are fundamental to the pervasive metabolic heterogeneity exhibited by different types of cancers, patients with the same cancer type, and, most notably, a single tumor from any given patient.

\section{$6 \quad$ Metabolic Heterogeneity Leads to Unpredictable Outcomes}

Now that we have a basic understanding of how the various metabolic phenotypes in a given tumor arise and the different processes driving it, we can take a look at some specific examples and cases of intratumoral metabolic heterogeneity.

\subsection{Spatial Heterogeneity Provides a Survival Advantage to Tumors}

For a long time, it was believed that cancer cells' major metabolic footprint was the Warburg effect, which dictates that cancer cells undergo glycolysis to produce lactic acid even in the presence of oxygen, a process termed aerobic glycolysis. Although the Warburg effect is still relevant, it has recently become clear that the metabolic phenotypes of cancer cells are far more varied and intricate. In a recent study published by Le et al. [40], the identification of genetic variability within the same tumor also revealed distinct metabolic profiles of each cell subpopulation within a given tumor. In addition to the hypoxiainducible factor (HIF) positive and/or cycling cells (Warburg effect-displaying cells), they found that the subpopulation that was HIF negative and non-cycling expressed a distinct set of genes with increased expression of mitochondrial genes as compared to other subpopulations. This subpopulation respires under hypoxia, supported by the fact that it has the highest oxygen consumption rate and mitochondrial capacity. The non-cycling and HIF-negative subclone is able to produce a tumor when purified and injected as a xenograft in vivo. This points to the importance of understanding how cancer metabolism allows for tremendous metabolic variegation.

Hypoxic cells can also coexist with aerobic cells, those that undergo oxidative phosphorylation, in a commensal manner. Hypoxic cells provide lactate that can be converted to pyruvate in the aerobic cells, which use it to run the TCA cycle and undergo oxidative phosphorylation [41]. These aerobic cells are oxygenated due to their proximity to a nearby blood supply. Therefore, they can survive in this manner and are more suited to doing so than hypoxic cells. However, in addition to these two types of cancerous cells, Le et al. recently uncovered the existence of a non-Warburg metabolic phenotype in B lymphoma cells that undergo hypoxic respiration by activating the TCA cycle through glutamine oxidation [19]. Oxidation of glutamine allows it to be used as a source for running the 
TCA cycle and enables the decrease of reliance on glucose as a primary fuel source for cancer cells [42]. This revelation once again supports the existence of diverse metabolic phenotypes in any given tumor.

The metabolic nature of cancer is muddled. Not only do some cancer subclones form commensal relationships with each other, but cancer cells can also form similar relationships with cancer-associated fibroblasts (CAFs) [43, 44]. CAFs are a subpopulation of cells that reside within the tumor microenvironment and support the proliferation and growth of tumor cells. By providing lactate and ketone bodies, acidic compounds that can form acetyl-CoA in a reversible manner, and by taking up reactive oxygen species that promote glycolytic metabolic pathways, CAFs establish a fundamental relationship with adjacent cancer cells [45]. CAFs are also involved in the maintenance of an acidic extracellular environment, providing suitable conditions for optimal cancer cell growth [45].

Elgogary et al. present another case of spatial metabolic heterogeneity. Pancreatic tumors were targeted by bis-2-(5-phenylacetamido-1,3,4thiadiazol-2-yl)ethyl sulfide (BPTES), a glutaminase 1 (GLS1) inhibitor, which was encapsulated in a nanoparticle to enhance drug delivery [46]. The drug decreased tumor sizes, but metabolomics analysis [47] revealed that surviving tumors relied on glycolysis and glycogen synthesis instead. Thus, further combination therapy of BPTES and metformin, a drug frequently used to treat diabetes by blocking glucose synthesis, further reduced tumor size. These results support the prevailing consensus that different metabolic phenotypes in any given tumor require specific therapeutic actions based on each subclonal phenotype (Fig. 3).

As previously noted, hypoxia has been found to play an important role in the development of heterogeneous phenotypes in cancer cells. In a recent study, Fluegen et al. investigated the fate of disseminated tumor cells (DTCs). They revealed that these post-hypoxia DTCs carry an array of upregulated genes, such as dormancy (nuclear receptor subfamily 2 group F member 1 (NR2F1), DEC2, p27) and hypoxia (HIF-1 $\alpha$ ) genes, in addi- tion to the GLUT-1 gene [48]. This dormant subpopulation, which evades many chemotherapies, as the authors of the paper noted, could explain relapse and the poor survival rates. As a result, heterogeneity in cancer metabolism comes in a variety of forms, and the same factor, in this case, hypoxia, can come into play through different approaches depending on each scenario.

While the different aspects of cancer metabolism may seem to intertwine neatly, the relationship between these parts is far more complex. For instance, while glutamine utilization in the TCA cycle is heavily linked to low oxygen consumption and hypoxia, the latter can sometimes occur independently of the former. Thus, these pathways may overlap when intracellular lactate causes an increase in glutamine uptake and metabolism. However, anaplerosis (pyruvate conversion into oxaloacetate), an alternative use of pyruvate in hypoxic conditions, can sometimes lead to the conversion of glucose to glutamate, taking away glutamine's role as the glutamate and $\alpha$-ketoglutarate $(\alpha-K G)$ provider needed to run the TCA cycle [49].

Despite our tendency to separate different metabolic pathways and to assign rigid pathways to cancer metabolism, it must be noted that different pathways often cross-talk and that the correlative nature of many metabolic pathways does not necessarily point to a causative relationship.

\subsection{Temporal Heterogeneity Provides Cancer with Short- Term Adaptive Capabilities}

As discussed earlier, tumors tend to evolve rapidly and produce dissimilar subclones through their interaction with the microenvironment. There exists a similar sort of evolution in single cancer cells: a form of temporal heterogeneity. Cancer cells are also astoundingly plastic regarding their metabolism. For example, they can switch their mitochondrial energy source between glutamine and glucose through the utilization of different transcriptional factors that encode enzymes required for each respective metabolic pathway. Cancer cells achieve this kind of plasticity through a variety of mecha- 

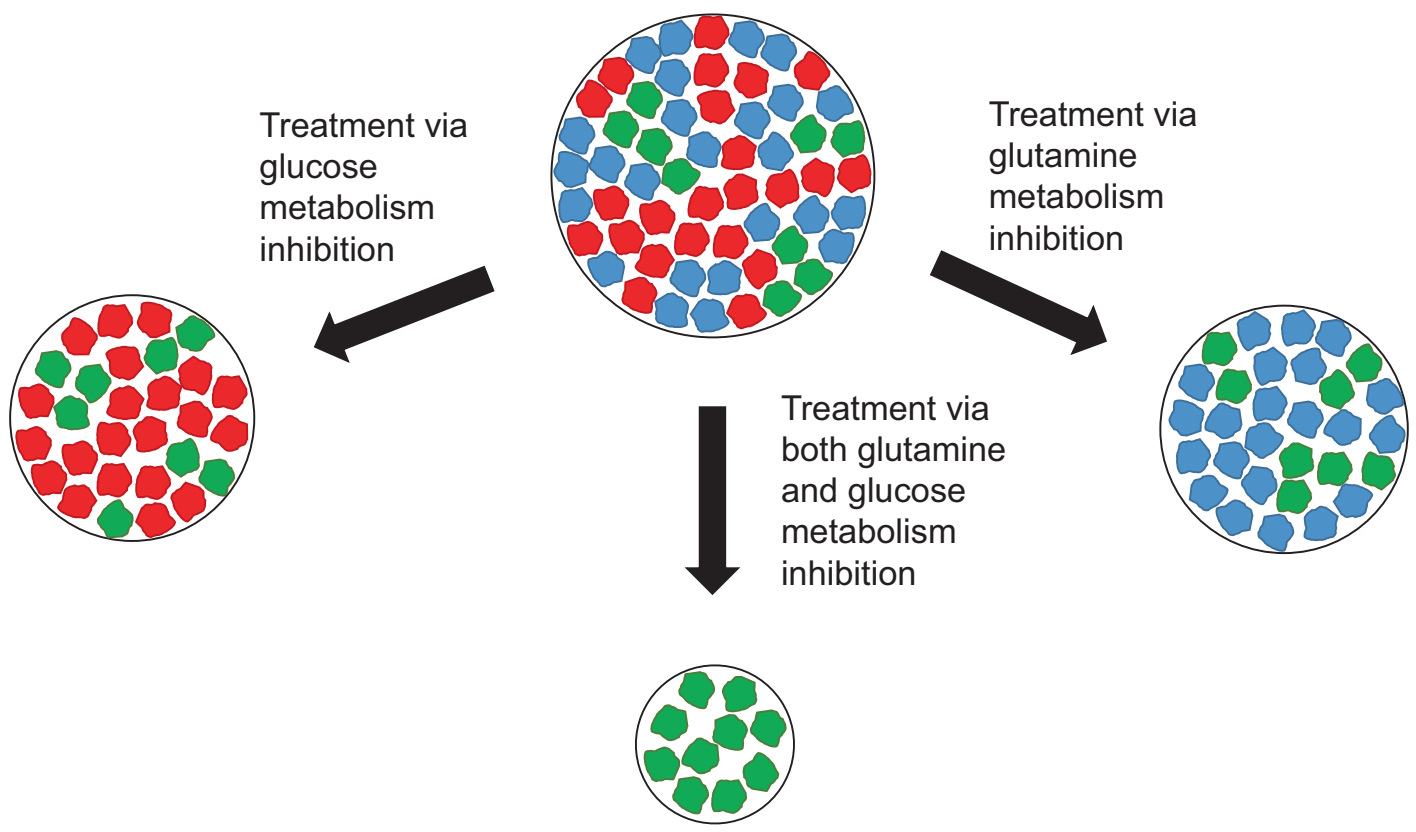

Fig. 3 Depiction of effects of combined therapy based on cancer metabolism. Depicted is an in vivo tumor containing different subpopulations of cancer cells (glycolytic cells presented as blue, glutamine-dependent cells presented as red, and other metabolic pathway-dependent cells presented as green)

nisms. Posttranslational modifications allow a quick and immediate response to changes in the environment, which could be useful in the sense that blood supply changes can be very rapid. Slower modifications do also exist, such as changes in gene expression and epigenetic modifications.

An example of such posttranslational modifications and an illustration of cancer's remarkable plasticity are seen once again in hypoxic cancer cells. Hypoxic cancer cells increase the transcription of pyruvate kinase muscle isoform 2 (PKM2), an enzyme responsible for the final nonreversible step in glycolysis, the conversion of phosphoenolpyruvate to pyruvate. This is achieved as the first intron of the PKM2 gene contains a hypoxia response element that is a target for HIF- $1 \alpha$. PKM2 is produced through the alternative splicing of the precursor mRNA PKM and is controlled by $c-M Y C[50,51]$. As such, high PKM2 levels are correlated with poor survival rates in gastric cancers as this upregulation of PKM2 helps cancer cells dedicate most of their glucose towards lactate production quickly and efficiently under hypoxic conditions [52]. Therefore, this posttranslational modification allows cancer cells to switch their metabolic profiles quickly and efficiently when faced with varying environmental conditions.

\section{$7 \quad$ Tailored Clinical Applications and Therapies Targeting Metabolic Pathways Can Lead to Better Clinical Outcomes}

Given the different tumor microenvironments, the diversity of cancer cell metabolism, and their genetic and epigenetic composition, various techniques have been developed to visualize the different tumor microenvironments in a given tumor. These imaging techniques have further propelled us in the search for effective cancer therapies targeting different cancer cell metabolism that can be specialized and tailor-made for every different microenvironment.

The most successful methods currently used to identify different tumor microenvironments include positron-emission tomography (PET) and 
computed tomography (PET-CT) scans. ${ }^{18} \mathrm{~F}$-Fluorodeoxyglucose-positron-emission tomography (FDG-PET) images of individual cervical tumors have revealed varying levels of glucose consumption across different regions of a single tumor [53]. The variation of glucose consumption within a tumor has been associated with increased expression of glucose transporters GLUT-1, GLUT-3, and hexokinase 2 (HK-2), the first key enzyme of glucose metabolism [54]. PET scans can also be used to identify hypoxic microenvironments through the use of isotopically labeled ${ }^{18} \mathrm{~F}$-fluoromisonidazole (FMISO), which is injected and taken up by cells through passive diffusion. In the absence of oxygen, FMISO accumulates in cells to generate an image of the hypoxic regions within a tumor [55]. PET scans can also be used to measure various tumor microenvironments based on other variables such as the partial pressure of oxygen [56].

Intratumoral metabolic heterogeneity in cancer can also serve as a useful tool for prognosis. In a study done by Mena et al., intratumoral metabolic heterogeneity was measured across 105 patients with oropharyngeal squamous cell carcinoma, along with either standardized uptake values of glucose (SUV) or metabolic tumor volume (MTV). These measurements were shown to have effective capacities as prognostic markers ( $\mathrm{p}=0.026$ and 0.022 , respectively), with higher levels indicating poorer prognoses, supporting the notion that the more diverse metabolic phenotypes exist within a tumor, the more arduous a task it becomes to eradicate all different subclones in the tumor [57].

Besides prognostication, increased knowledge of cancer's heterogeneous metabolic nature, and specifically its intratumoral heterogeneity, can enable specific targeting of subclones in a single tumor and has resulted in a surge in specific tailormade cancer therapies. One of the earliest hallmarks of cancer is its ability to take up increased amounts of glucose, through the utilization of many GLUTs. Cancer cells are also capable of metabolizing glucose at much quicker rates than normal cells. Consequently, this has resulted in increased research addressing the production of commercial GLUT inhibitors and the transporter isoform specificity of inhibition [58]. Other drugs have been developed to target the hypoxic pathways of cancer cells, such as topotecan, which inhibits hypoxia-inducible factor 1 (HIF-1) transcriptional activity and HIF- $1 \alpha$ protein accumulation in hypoxia-treated U251 human glioma cells [59]. This has generated increased interest in mRNA-regulating agents that target HIF- $1 \alpha$. Many drugs have followed with variable success that act by blocking mRNA transcription of the HIF- $1 \alpha$ gene. Recent research has also provided many other pathways in cancer metabolism that can be targeted with effective results. For example, lactate dehydrogenase A (LDHA), an enzyme involved in the generation of lactate from glucose in Warburg effect-displaying cells, has also been found to be a suitable target for effective tumor reduction through small-molecule inhibition. Decreased expression of LDHA through small-molecule FX11 inhibition elevated oxidative stress levels and ultimately resulted in cell death and tumor volume reduction [60-62]. Other methods targeting HIF-1 $\alpha$ and hypoxia have been formulated through the integration of different therapies to each specific tumor microenvironment [63], making complete cancer recession very promising. Again, it is vital to realize the complex nature of cancer metabolism and the need for specific therapies to be directed at the individual metabolic phenotypes in order to see effective responses in patients (Fig. 4).

\section{Conclusion}

Despite the challenges, there is much hope in the field of cancer therapies. The recently discovered and understood aspects of cancer's metabolic heterogeneity, including its intricate interactions with CAFs, the exchanges between its distinct subclones, and its impressive plasticity, promise to greatly advance this field. The importance of accounting for intratumoral heterogeneity in any given tumor has never been as widely understood as it is now. The latest findings we have discussed in this chapter give us a more solid understanding of cancer complexities, which we can seek to translate into effective and strategic therapies in the near future. 
Fig. 4 Various factors associated with intratumoral metabolic heterogeneity

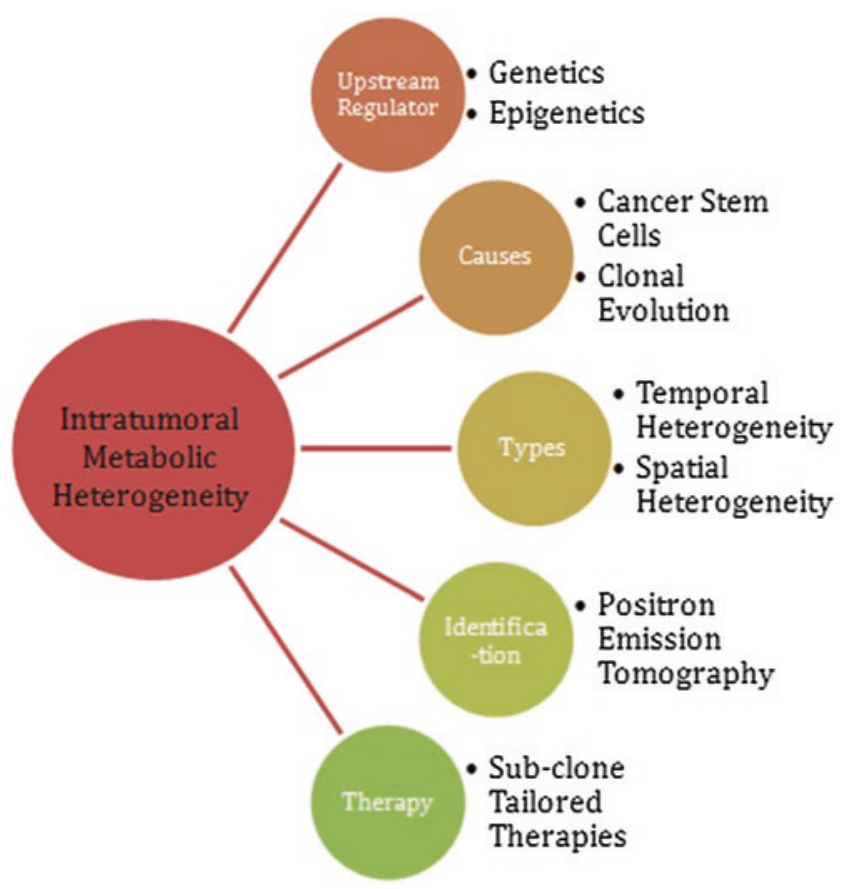

9. Gerlinger, M., et al. (2012). Intratumor heterogeneity and branched evolution revealed by multiregion sequencing. The New England Journal of Medicine, 366(10), 883-892.

1. Dang, C. V., et al. (2011). Therapeutic targeting of cancer cell metabolism. Journal of Molecular Medicine (Berlin), 89(3), 205-212.

2. Hirschey, M. D., et al. (2015). Dysregulated metabolism contributes to oncogenesis. Seminars in Cancer Biology, 35(Suppl), S129-S150.

3. Gonzalez-Angulo, A. M., Morales-Vasquez, F., \& Hortobagyi, G. N. (2007). Overview of resistance to systemic therapy in patients with breast cancer. Advances in Experimental Medicine and Biology, 608, 1-22.

4. Prasetyanti, P. R., \& Medema, J. P. (2017). Intratumor heterogeneity from a cancer stem cell perspective. Molecular Cancer, 16(1), 41.

5. Jogi, A., et al. (2012). Cancer cell differentiation heterogeneity and aggressive behavior in solid tumors. Upsala Journal of Medical Sciences, 117(2), 217-224.

6. Bu, Y., \& Cao, D. (2012). The origin of cancer stem cells. Frontiers in Bioscience (Scholar Edition), 4, 819-830.

7. Yang, T., et al. (2014). Cancer stem cells: Constantly evolving and functionally heterogeneous therapeutic targets. Cancer Research, 74(11), 2922-2927.

8. Gonzalez-Garcia, I., Sole, R. V., \& Costa, J. (2002). Metapopulation dynamics and spatial heterogeneity in cancer. Proceedings of the National Academy of Sciences of the United States of America, 99(20), 13085-13089.
10. Park, S. Y., et al. (2010). Cellular and genetic diversity in the progression of in situ human breast carcinomas to an invasive phenotype. The Journal of Clinical Investigation, 120(2), 636-644.

11. Tan, J., \& Le, A. (2021). The heterogeneity of breast cancer metabolism. Advances in Experimental Medicine and Biology, 1311, https://doi. org/10.1007/978-3-030-65768-0_6

12. Greger, V., et al. (1994). Frequency and parental origin of hypermethylated RB1 alleles in retinoblastoma. Human Genetics, 94(5), 491-496.

13. Parker, N. R., et al. (2016). Intratumoral heterogeneity identified at the epigenetic, genetic and transcriptional level in glioblastoma. Scientific Reports, 6, 22477.

14. Quinones, A., \& Le, A. (2021). The multifaceted glioblastoma: From genomic alterations to metabolic adaptations. Advances in Experimental Medicine and Biology, 1311, https://doi. org/10.1007/978-3-030-65768-0_4

15. Okegawa, T., et al. (2017). Intratumor heterogeneity in primary kidney cancer revealed by metabolic profiling of multiple spatially separated samples within tumors. eBioMedicine, 19, 31-38.

16. Masson, N., \& Ratcliffe, P. J. (2014). Hypoxia signaling pathways in cancer metabolism: The importance of co-selecting interconnected physiological pathways. Cancer \& Metabolism, 2(1), 3.

17. Semenza, G. L. (2013). HIF-1 mediates metabolic responses to intratumoral hypoxia and oncogenic 
mutations. The Journal of Clinical Investigation, 123(9), 3664-3671.

18. Bose, S., Zhang, C., \& Le, A. (2021). Glucose metabolism in cancer: The Warburg effect and beyond. Advances in Experimental Medicine and Biology, 1311, https://doi.org/10.1007/978-3-030-65768-0_1

19. Le, A., et al. (2012). Glucose-independent glutamine metabolism via TCA cycling for proliferation and survival in B cells. Cell Metabolism, 15(1), 110-121.

20. Lee, S. L., \& Fanburg, B. L. (1987). Glycolytic activity and enhancement of serotonin uptake by endothelial cells exposed to hypoxia/anoxia. Circulation Research, 60(5), 653-658.

21. Cox, T. R., \& Erler, J. T. (2011). Remodeling and homeostasis of the extracellular matrix: Implications for fibrotic diseases and cancer. Disease Models \& Mechanisms, 4(2), 165-178.

22. Forsythe, J. A., et al. (1996). Activation of vascular endothelial growth factor gene transcription by hypoxia-inducible factor 1. Molecular and Cellular Biology, 16(9), 4604-4613.

23. Mazure, N. M., et al. (1996). Oncogenic transformation and hypoxia synergistically act to modulate vascular endothelial growth factor expression. Cancer Research, 56(15), 3436-3440.

24. Brychtova, S., et al. (2008). The role of vascular endothelial growth factors and their receptors in malignant melanomas. Neoplasma, 55(4), 273-279.

25. De Bock, K., et al. (2013). Role of PFKFB3-driven glycolysis in vessel sprouting. Cell, 154(3), 651-663.

26. Parra-Bonilla, G., et al. (2010). Critical role for lactate dehydrogenase $\mathrm{A}$ in aerobic glycolysis that sustains pulmonary microvascular endothelial cell proliferation. American Journal of Physiology. Lung Cellular and Molecular Physiology, 299(4), L513-L522.

27. Peters, K., et al. (2009). Changes in human endothelial cell energy metabolic capacities during in vitro cultivation. The role of "aerobic glycolysis" and proliferation. Cellular Physiology and Biochemistry, 24(5-6), 483-492.

28. Polet, F., \& Feron, O. (2013). Endothelial cell metabolism and tumour angiogenesis: Glucose and glutamine as essential fuels and lactate as the driving force. Journal of Internal Medicine, 273(2), 156-165.

29. Merchan, J. R., et al. (2010). Antiangiogenic activity of 2-deoxy-D-glucose. PLoS One, 5(10), e13699.

30. Wang. (2011). Q., et al., 2-Deoxy-D-glucose treatment of endothelial cells induces autophagy by reactive oxygen species-mediated activation of the AMP-activated protein kinase. PLoS One, 6(2), e17234.

31. Yeh, W. L., Lin, C. J., \& Fu, W. M. (2008). Enhancement of glucose transporter expression of brain endothelial cells by vascular endothelial growth factor derived from glioma exposed to hypoxia. Molecular Pharmacology, 73(1), 170-177.

32. Kimura, H., et al. (1996). Fluctuations in red cell flux in tumor microvessels can lead to transient hypoxia and reoxygenation in tumor parenchyma. Cancer Research, 56(23), 5522-5528.
33. Lanzen, J., et al. (2006). Direct demonstration of instabilities in oxygen concentrations within the extravascular compartment of an experimental tumor. Cancer Research, 66(4), 2219-2223.

34. Bennewith, K. L., \& Durand, R. E. (2004). Quantifying transient hypoxia in human tumor xenografts by flow cytometry. Cancer Research, 64(17), 6183-6189.

35. Secomb, T. W., et al. (1993). Analysis of oxygen transport to tumor tissue by microvascular networks. International Journal of Radiation Oncology, Biology, Physics, 25(3), 481-489.

36. Barger, J. F., \& Plas, D. R. (2010). Balancing biosynthesis and bioenergetics: Metabolic programs in oncogenesis. Endocrine-Related Cancer, 17(4), R287-R304.

37. Schafer, Z. T., et al. (2009). Antioxidant and oncogene rescue of metabolic defects caused by loss of matrix attachment. Nature, 461(7260), 109-113.

38. Samudio, I., et al. (2010). Pharmacologic inhibition of fatty acid oxidation sensitizes human leukemia cells to apoptosis induction. The Journal of Clinical Investigation, 120(1), 142-156.

39. Buzzai, M., et al. (2005). The glucose dependence of Akt-transformed cells can be reversed by pharmacologic activation of fatty acid beta-oxidation. Oncogene, 24(26), 4165-4173.

40. Le, A., et al. (2014). Tumorigenicity of hypoxic respiring cancer cells revealed by a hypoxia-cell cycle dual reporter. Proceedings of the National Academy of Sciences of the United States of America, 111(34), 12486-12491.

41. Zheng, J. (2012). Energy metabolism of cancer: Glycolysis versus oxidative phosphorylation (Review). Oncology Letters, 4(6), 1151-1157.

42. Li, T., Copeland, C., \& Le, A. (2021). Glutamine metabolism in cancer. Advances in Experimental Medicine and Biology, 1311, https://doi. org/10.1007/978-3-030-65768-0_2

43. Sazeides, C., \& Le, A. (2021). Metabolic relationship between cancer-associated fibroblasts and cancer cells. Advances in Experimental Medicine and Biology, 1311, https://doi.org/10.1007/978-3-030-65768-0_14

44. Jung, J. G., \& Le, A. (2021). Targeting metabolic cross talk between cancer cells and cancerassociated fibroblasts. Advances in Experimental Medicine and Biology, 1311, https://doi. org/10.1007/978-3-030-65768-0_15

45. Martinez-Outschoorn, U. E., Lisanti, M. P., \& Sotgia, F. (2014). Catabolic cancer-associated fibroblasts transfer energy and biomass to anabolic cancer cells, fueling tumor growth. Seminars in Cancer Biology, $25,47-60$.

46. Elgogary, A., et al. (2016). Combination therapy with BPTES nanoparticles and metformin targets the metabolic heterogeneity of pancreatic cancer. Proceedings of the National Academy of Sciences of the United States of America, 113(36), E5328-E5336.

47. Hoang, G., Udupa, S., \& Le, A. (2019). Application of metabolomics technologies toward cancer prog- 
nosis and therapy. International Review of Cell and Molecular Biology, 347, 191-223.

48. Fluegen, G., et al. (2017). Phenotypic heterogeneity of disseminated tumour cells is preset by primary tumour hypoxic microenvironments. Nature Cell Biology, 19(2), 120-132.

49. Eales, K. L., Hollinshead, K. E., \& Tennant, D. A. (2016). Hypoxia and metabolic adaptation of cancer cells. Oncogene, 5, e190.

50. Dang, C. V., Le, A., \& Gao, P. (2009). MYC-induced cancer cell energy metabolism and therapeutic opportunities. Clinical Cancer Research, 15(21), 6479-6483.

51. Le, A., \& Dang, C. V. (2013). Studying Myc's role in metabolism regulation. Methods in Molecular Biology, 1012, 213-219.

52. Wong, N., De Melo, J., \& Tang, D. (2013). PKM2, a central point of regulation in cancer metabolism. International Journal of Cell Biology, 2013, 242513.

53. Kidd, E. A., \& Grigsby, P. W. (2008). Intratumoral metabolic heterogeneity of cervical cancer. Clinical Cancer Research, 14(16), 5236-5241.

54. Zhao, S., et al. (2005). Biologic correlates of intratumoral heterogeneity in 18F-FDG distribution with regional expression of glucose transporters and hexokinase-II in experimental tumor. Journal of Nuclear Medicine, 46(4), 675-682.

55. Farwell, M. D., Pryma, D. A., \& Mankoff, D. A. (2014). PET/CT imaging in cancer: Current applications and future directions. Cancer, 120(22), 3433-3445.

56. Plathow, C., \& Weber, W. A. (2008). Tumor cell metabolism imaging. Journal of Nuclear Medicine, 49(Suppl 2), 43S-63S.
57. Mena, E., et al. (2017). Value of intratumoral metabolic heterogeneity and quantitative 18F-FDG PET/ CT parameters to predict prognosis in patients with HPV-positive primary oropharyngeal squamous cell carcinoma. Clinical Nuclear Medicine, 42(5), e227-e234.

58. Ojelabi, O. A., et al. (2016). WZB117 (2-fluoro-6-(mhydroxybenzoyloxy) phenyl m-hydroxybenzoate) inhibits GLUT1-mediated sugar transport by binding reversibly at the exofacial sugar binding site. The Journal of Biological Chemistry, 291(52), 26762-26772.

59. Rapisarda, A., et al. (2004). Topoisomerase I-mediated inhibition of hypoxia-inducible factor 1: Mechanism and therapeutic implications. Cancer Research, 64(4), 1475-1482.

60. Le, A., et al. (2010). Inhibition of lactate dehydrogenase A induces oxidative stress and inhibits tumor progression. Proceedings of the National Academy of Sciences of the United States of America, 107(5), 2037-2042.

61. Rajeshkumar, N. V., et al. (2015). Therapeutic targeting of the warburg effect in pancreatic cancer relies on an absence of p53 function. Cancer Research, 75(16), 3355-3364.

62. Dutta, P., et al. (2013). Evaluation of LDH-A and glutaminase inhibition in vivo by hyperpolarized 13C-pyruvate magnetic resonance spectroscopy of tumors. Cancer Research, 73(14), 4190-4195.

63. Antonio, M. J., Zhang, C., \& Le, A. (2021). Different tumor microenvironments lead to different metabolic phenotypes. Advances in Experimental Medicine and Biology, 1311, https:// doi.org/10.1007/978-3-030-65768-0_10.

Open Access This chapter is licensed under the terms of the Creative Commons Attribution 4.0 International License (http://creativecommons.org/licenses/by/4.0/), which permits use, sharing, adaptation, distribution and reproduction in any medium or format, as long as you give appropriate credit to the original author(s) and the source, provide a link to the Creative Commons license and indicate if changes were made.

The images or other third party material in this chapter are included in the chapter's Creative Commons license, unless indicated otherwise in a credit line to the material. If material is not included in the chapter's Creative Commons license and your intended use is not permitted by statutory regulation or exceeds the permitted use, you will need to obtain permission directly from the copyright holder. 


\section{Cancer Stem Cell Metabolism}

Fidelia B. Alvina, Arvin M. Gouw, and Anne Le

\section{Keywords}

Cancer stem cell · Metabolic plasticity .

Glucose metabolism - Glutamine metabolism ·

Mitochondrial metabolism · Lipid metabolism

\section{Abbreviations}

$\begin{array}{ll}\text { 2-DG } & \text { 2-Deoxy-D-glucose } \\ \text { ACC1 } & \text { Acetyl carboxylase A } \\ \text { ACLY } & \text { ATP citrate lyase } \\ \text { AML } & \text { Acute myeloid leukemia } \\ \text { AMPK } & \text { AMP-activated protein kinase } \\ \text { Ape1/Ref-1 } & \begin{array}{l}\text { Apurinic/apyrimidinic endonucle- } \\ \text { ase/redox effector factor }\end{array} \\ \text { ASCT2 } & \begin{array}{l}\text { Alanine, serine, cysteine trans- } \\ \text { porter 2 }\end{array} \\ \text { ATP } & \text { Adenosine triphosphate } \\ & \end{array}$

F. B. Alvina

University of Wisconsin-Madison,

Madison, WI, USA

\begin{abstract}
A. M. Gouw
Division of Oncology, Departments of Medicine and

Pathology, Stanford University School of Medicine,

Stanford, CA, USA
\end{abstract}

\section{A. Le $(\bowtie)$}

Department of Pathology and Oncology, Johns

Hopkins University School of Medicine,

Baltimore, MD, USA

Department of Chemical and Biomolecular Engineering, Johns Hopkins University Whiting School of Engineering, Baltimore, MD, USA

e-mail: annele@jhmi.edu
CPT1 Carnitine palmitoyltransferase 1

CS

CSCs

ECAR

EMT

FAO

FAS

FASN

FP

G6Pase

GLS1

GLUT1

GOT1

GPI

HK2

HMGCR

HMGCS

IR

Citrate synthase

Cancer stem cells

Extracellular acidification rate

Epithelial-mesenchymal transition

Fatty acid oxidation

Fatty acid synthesis

Fatty acid synthase

Fat pad

Glucose-6-phosphatase

Glutaminase 1

Glucose transporter 1

Glutamic-oxaloacetic transaminase 1

LDH-A Lactate dehydrogenase A

mtDNA Mitochondrial deoxyribonucleic acid

MVK Mevalonate kinase

NSCLC Non-small cell lung carcinoma

OAA Oxaloacetate

OCR Oxygen consumption rate

OXPHOS Oxidative phosphorylation

PDHC Pyruvate dehydrogenase complex

PDK4 Pyruvate dehydrogenase kinase 4

Pepck Phosphoenolpyruvate carboxykinase

PGAM1 Phosphoglycerate mutase 1

PMVK Phosphomevalonate kinase 


$\begin{array}{ll}\text { PPPs } & \text { Pyrophosphates } \\ \text { ROS } & \text { Reactive oxygen species } \\ \text { SCD } & \text { Stearoyl CoA desaturase } \\ \text { SREBPs } & \text { Sterol regulatory element-binding } \\ & \text { proteins } \\ \text { TCA } & \text { Tricarboxylic acid } \\ \text { TGs } & \text { Triglycerides } \\ \text { TICs } & \text { Tumor-initiating cells }\end{array}$

\section{Key Points}

- CSCs are different from stem cells and nonCSC tumor cells metabolically, but CSCs share some similarities with both stem cells and cancer cells in glucose, glutamine, oxidative phosphorylation (OXPHOS), and lipid metabolism.

- The commonly shared metabolic pathways indicate that CSCs are a subtype of cancer cells that genetically evolved from normal stem/progenitor cells.

- The commonly shared metabolic pathways between CSCs, stem cells, and non-CSC tumor cells indicate that these metabolic pathways are selected due to their ability to sustain the catabolic and anabolic needs of highly proliferating cells.

- The fact that CSCs share metabolic pathways with stem cells and cancer cells poses a challenge for developing cancer metabolic drugs that do not target normal stem cells.

- Further studies are needed to shed light on how to develop specific metabolic drugs targeting CSCs in specific cancer types.

\section{Introduction}

Cancer stem cells (CSCs), also known as tumorinitiating cells (TICs), are a group of cells found within cancer cells. Like normal stem cells, CSCs can proliferate, engage in self-renewal, and are often implicated in the recurrence of tumors after therapy $[1,2]$. The existence of CSCs in various types of cancer has been proven, such as in acute myeloid leukemia (AML) [3], breast [4], pancreatic [5], and lung cancers [6], to name a few. There are two theories regarding the origin of
CSCs. First, CSCs may have arisen from normal stem/progenitor cells that experienced changes in their environment or genetic mutations. On the other hand, CSCs may also have originated from differentiated cells that underwent genetic and/or heterotypic modifications [7]. Either way, CSCs reprogram their metabolism in order to support tumorigenesis.

Metabolism plays a crucial role in cellular function and survival, and it is no different for CSCs. Metabolic rewiring is necessary for CSCs as it enables them to adapt to different environments and survive. For example, metabolic rewiring to oxidative phosphorylation (OXPHOS) from glycolysis makes CSCs more efficient in generating adenosine triphosphate (ATP) and more resistant to microenvironmental pressures such as lack of nutrients [8]. Understanding the metabolism of CSCs would enhance targeting CSCs, and in turn improve cancer therapy. In this chapter, we will be looking at four key metabolism of CSCs-glucose metabolism, glutamine metabolism, mitochondrial metabolism, and lipid metabolism-and how they affect the state of CSCs (Fig. 1).

\section{$2 \quad$ High Levels of Glycolytic Enzymes and Activities in CSCs (Fig. 2)}

In Chap. 1, glucose metabolism and the Warburg effect have been described as key processes in cancer cells [9]. Not only are these processes observed in cancer cells, but they are also relevant to CSCs. Stemness features of CSCs, such as proliferation, are shown to be reliant on the Warburg effect [10].

CSCs across different types of cancers are found to express high levels of glycolytic genes. Song et al. found that CD133(+) liver CSCs exhibit increased expressions of glycolytic genes: glucose transporter 1 (GLUT1), hexokinase II (HK2), pyruvate dehydrogenase kinase 4 (PDK4), and phosphoglycerate mutase 1 (PGAM1). Furthermore, they observed a high extracellular acidification rate (ECAR) and decreased expressions of gluconeogenetic genes: 


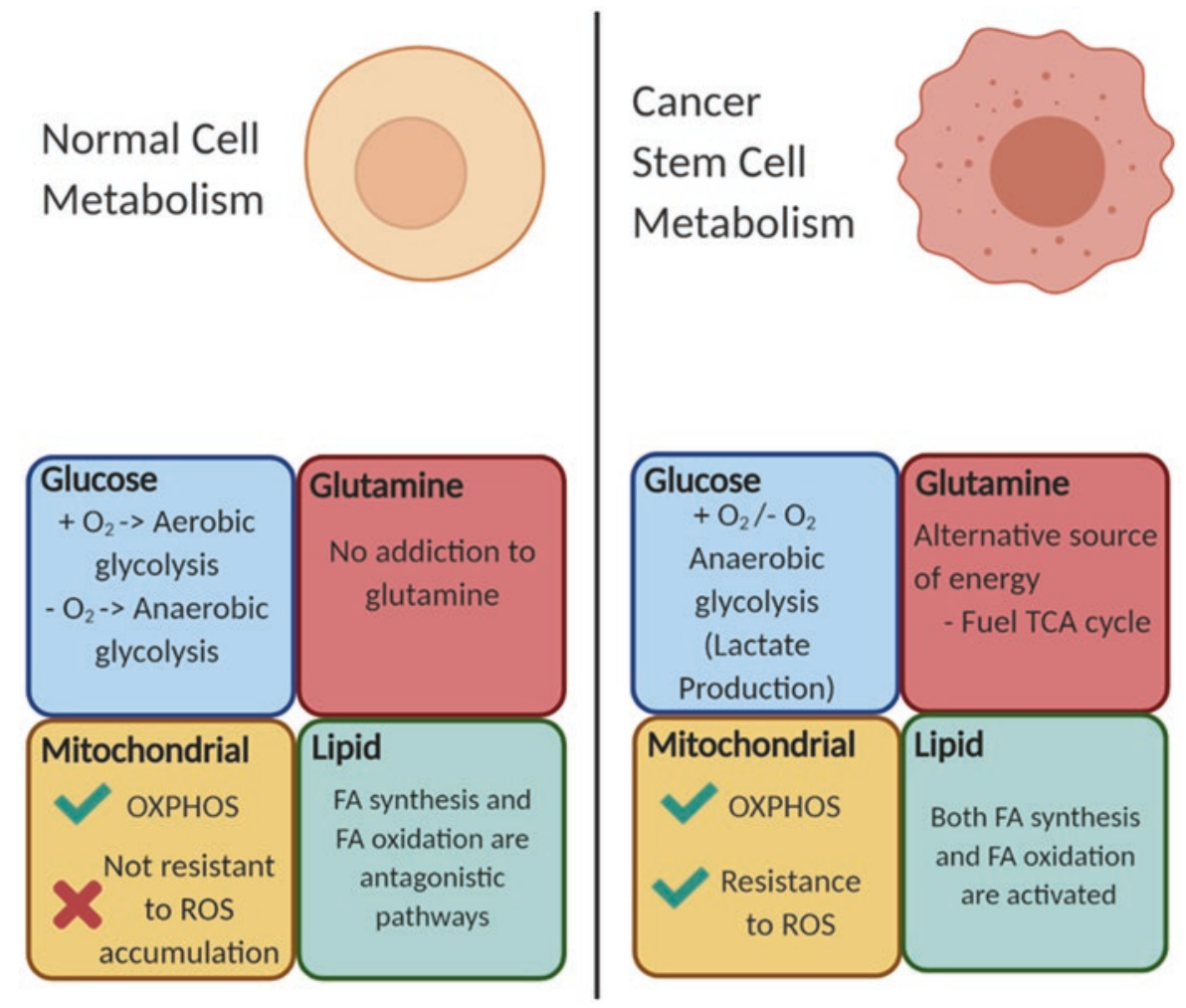

Fig. 1 Cancer stem cell metabolism compared to normal cell metabolism

glucose-6-phosphatase (G6Pase) and phosphoenolpyruvate carboxykinase (Pepck) [11]. These findings align with observations made in nasopharyngeal carcinoma (NPC) CSCs. In comparison to their parental cells, NPC CSCs express higher levels of GLUT1, HK2, and glucose-6phosphate isomerase (GPI) [12]. The high levels of glycolytic genes found in CSCs suggest that CSCs depend on glucose metabolism as a source of energy.

Studies have demonstrated the importance of glycolytic enzymes in CSCs, such as regulating the self-renewal and spheroid formation ability of CSCs. Shibuya et al. studied the role of GLUT1 in the CSCs of glioblastoma, pancreatic, and ovarian cancers. They found that the inhibition of GLUT1, through either siRNA-mediated knockdown or pharmacological inhibition by WZB117, a small-molecule inhibitor, resulted in a decrease in the uptake of glucose, a reduction in the expressions of stem cell markers (Sox2, Bmi1, Nanog), and an increase in the expressions of dif- ferentiation markers (GFAP in glioblastoma and B-actin in glioblastoma, pancreatic, and ovarian CSCs). Furthermore, these inhibitions impaired the spheroid-formation ability of CSCs, and inhibition by WZB117 did so without affecting their proliferative potential [13]. These findings suggest that GLUT1 plays a crucial role in maintaining the glucose metabolism and stemness characteristics of CSCs.

PDK4, one of the highly expressed genes in liver CSCs mentioned earlier, is an inhibitor of pyruvate dehydrogenase complex (PDHC). PDHC regulates the entrance of glucose-derived pyruvate into the mitochondria, which will subsequently form acetyl-CoA for the tricarboxylic acid (TCA) cycle. Song et al. found that low levels of liver-specific miR-122, which targets PDK4, are expressed in CD133+ cells. This allows for the upregulation of PDK4 and the subsequent inhibition of $\mathrm{PDH}$, which in turn results in the failure of pyruvate to enter the mitochondrial matrix. Instead of going through subsequent 


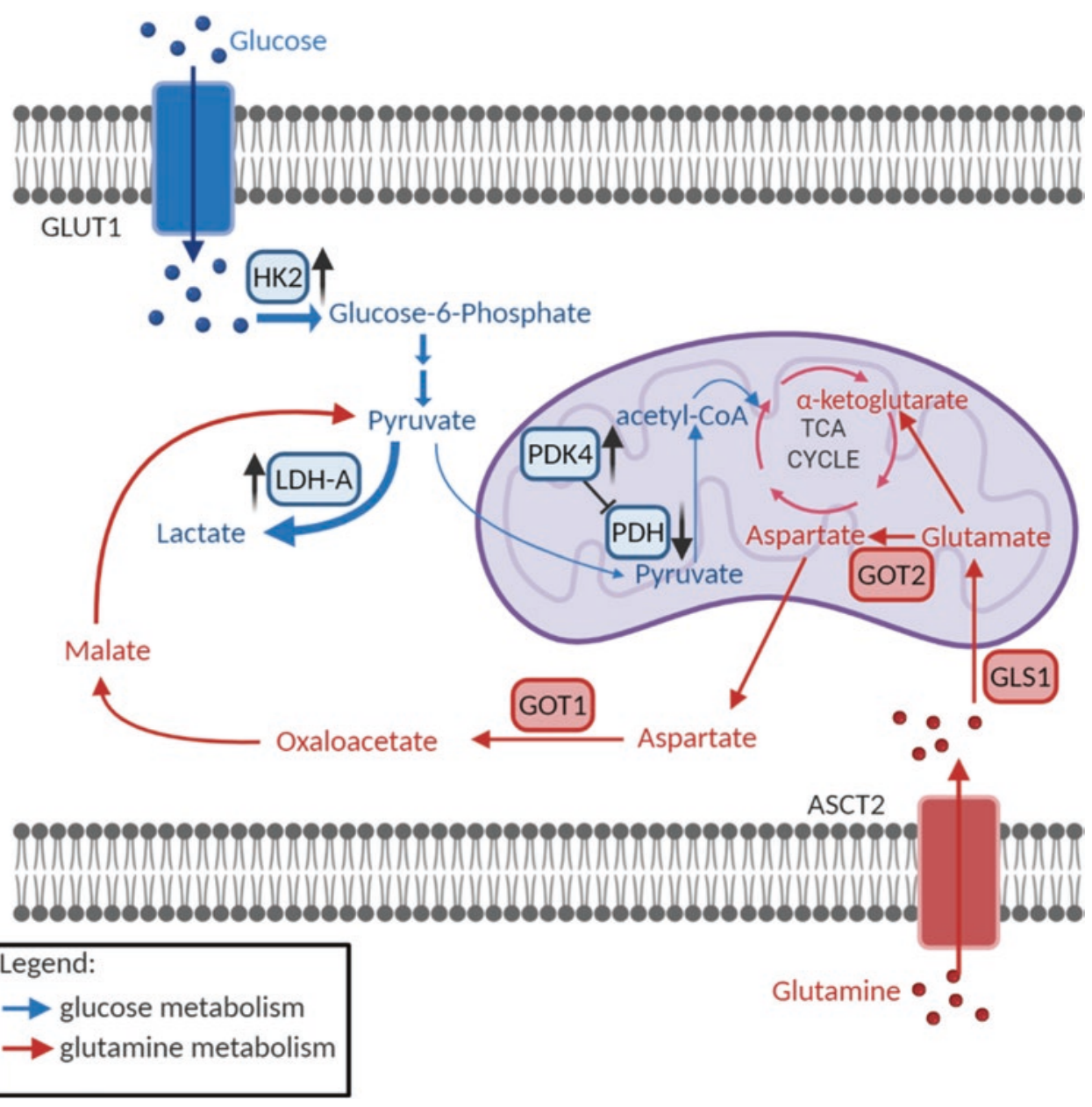

Fig. 2 Glucose and glutamine metabolism in cancer stem cells. GLUT1 glucose transporter 1, HK2 hexokinase II, $L D H-A$ lactate dehydrogenase A, $P D H C$ pyruvate dehydrogenase complex, $P D K 4$ pyruvate dehydrogenase kinase 4, GOT glutamic-oxaloacetic transaminase, GLS1 glutaminase 1, ASCT2 alanine, serine, cysteine transporter 2

oxidative phosphorylation (OXPHOS), lactate production in the cytosol occurs [11]. Therefore, this supports the idea that the Warburg effect is of relevance to CSCs. In fact, high levels of miR-122 impair the spheroid-formation ability of CSCs [11]. Hence, suppression of miR-122 in liver CSCs allows the induction of glucose metabolism and preference for fermentation instead of OXPHOS.

The conversion of pyruvate to lactate in the cytosol is regulated by lactate dehydrogenase A (LDH-A), which was found to be upregulated in breast CSCs compared to spheroid-derived adherent cells (SDACs) [14]. The importance of LDH-A can be concluded from the deleterious effect of
LDH-A suppression on CSCs. The knockdown of LDH-A caused liver CD133+ CSCs to produce fewer transcriptional factors (Sox2, Nanog, Oct4) that give rise to stemness characteristics, and their spheroid-formation ability was impaired [11]. Furthermore, in non-small cell lung carcinoma (NSCLC), the suppression of LDH-A by shRNA resulted in a decrease of CSCs in the A549 cell line and a decrease in their ability to form tumorspheres [15]. These findings reinforce the idea that glucose metabolism, which results in lactate production, is central to the survival of CSCs and the maintenance of their stemness characteristics. LDH-A inhibitor such as FX-11 has been tested in 
several types of cancers $[9,16-18]$ and could be a potential strategy in targeting CSCs.

\section{Effects of Deregulation of Glutamine Metabolism on CSCs (Fig. 2)}

Glutamine metabolism is also a source of energy for cancer cells that is an alternative to glucose metabolism [19, 20]. For CSCs, a lack of glucosederived pyruvate for the TCA cycle would mean that reliance on other metabolites to drive the TCA cycle is needed. Compared to non-CSCs, metabolites from oxidative glutamine metabolism contribute to the TCA cycle in CSCs [10]. Additionally, both glucose deprivation and treatment using the glycolytic inhibitor 2-deoxyglucose (2-DG) resulted in an increase in the uptake of glutamine into CSCs but not into nonCSCs, further suggesting the metabolic plasticity of CSCs [10]. Colorectal CSCs of the SW620 cell line were shown to be resistant to the effects of inhibition of mitochondrial metabolism by metformin because they compensated by deriving energy from glutamine metabolism [21]. These findings suggest the importance of glutamine metabolism in CSC survival. Targeting of glutamine metabolism has been extensively studied by many researchers [22-29]. These strategies could have the potential of eliminating CSCs.

Glutaminolysis starts with the intake of glutamine into the cell via transporters such as alanine, serine, and cysteine transporter 2 (ASCT2). Kim et al. found that the CSCs of colorectal cancer, both HT29 and SW260 cell lines, have a higher amount of ASCT2 than non-CSCs, which means that a higher glutamine uptake occurs in CSCs. After ASCT2 knockdown, the number of CD133+/CD44+ CSCs drastically decreased. In metformin-resistant cell line SW260, the siRNAmediated knockdown of glutaminase 1 (GLS1) and ASCT2 decreased the number of CSCs, with a more significant decrease observed during ASCT2 knockdown only [21].
Another study on pancreatic CSCs has demonstrated the importance of glutamine metabolism to CSCs. When there is a lack of glutamine, pancreatic CSCs have a decreased ability to form both primary and secondary spheroids. Furthermore, the lack of glutamine also resulted in reduced expression of stemness-related genes and accumulation of intracellular reactive oxygen species (ROS). Oxaloacetate (OAA), an intermediate of the TCA cycle, was found to alleviate the high ROS levels. Furthermore, the knockdown of cytosolic glutamic-oxaloacetic transaminase 1 (GOT1), which converts aspartate to OAA, together with the administration of lowdose ionizing radiation (IR) resulted in impairment of spheroid-formation ability, and the spheroids formed experienced apoptosis within 10 days of their formation [30]. These findings revealed the important role that OAA plays in ensuring a balanced ROS level in CSCs. Therefore, the noncanonical pathway of glutamine metabolism helps CSCs in their ability to resist ROS accumulation and its effects.

\section{Mitochondrial Metabolism (Fig. 3)}

The term "mitostemness" describes the crucial role that mitochondria play in the ability of CSCs to self-renew and resist differentiating and losing their stemness properties [31]. The ability of CSCs to switch across different types of metabolism for better survival in different environmental conditions has resulted in contradictory findings regarding their metabolism. Some studies have shown that CSCs depend on glycolysis [32, 33], while others have described CSCs to be mostly dependent on OXPHOS [33-35].

In addition to their energetics contributions, mitochondrial metabolic pathways also epigenetically regulate CSC stemness. Mitochondrial metabolism is important because the TCA cycle and OXPHOS are involved in the production of metabolites such as acetyl-CoA, alphaketoglutarate, NAD+, and S-adenosyl methio- 


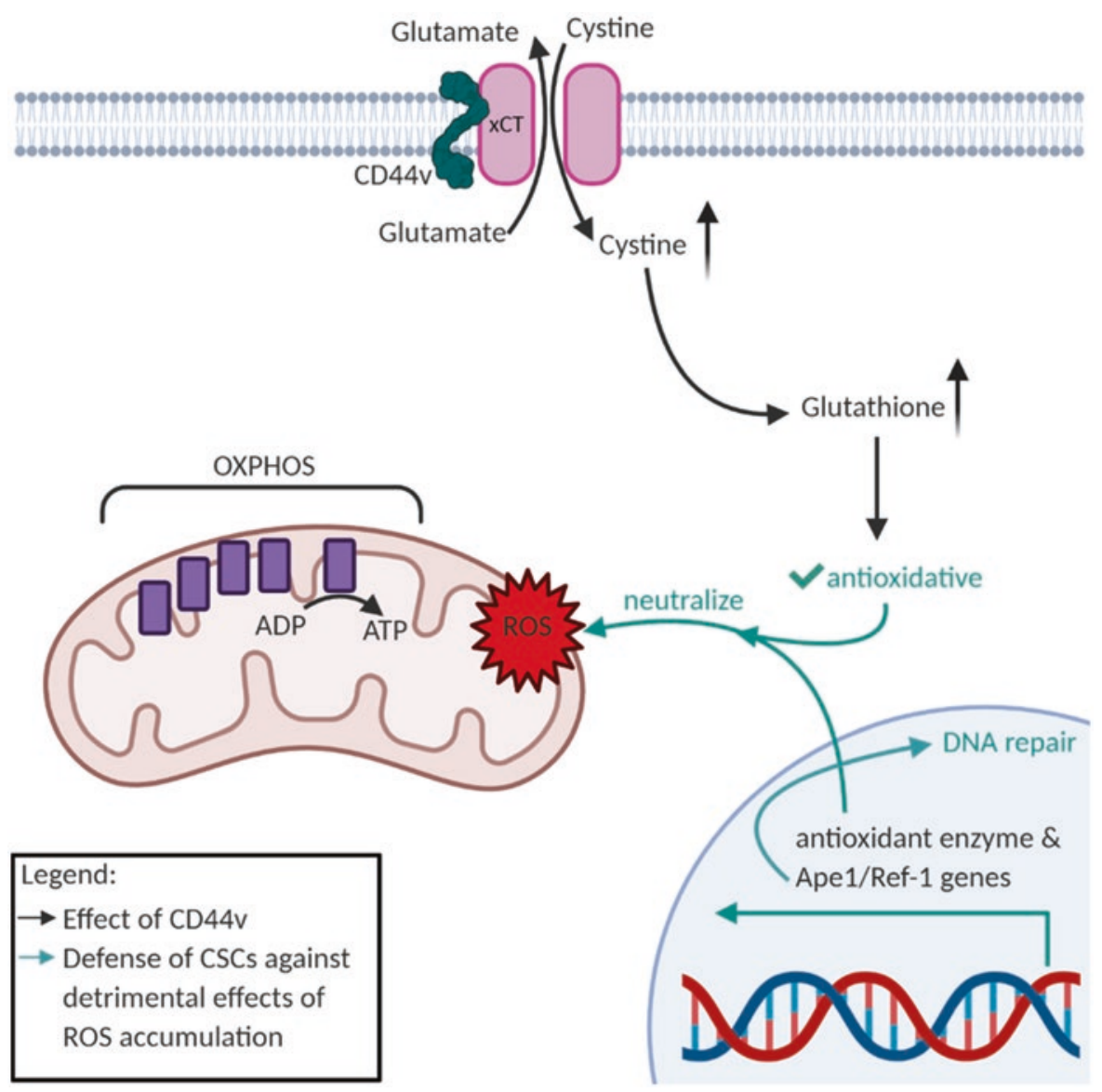

Fig. 3 Mitochondrial metabolism in cancer stem cells. OXPHOS oxidative phosphorylation, ROS reactive oxygen species, CSCs cancer stem cells

nine, which are substrates for chromatin-state modifications, whether acetylation or methylation [36]. In turn, these epigenetic modifications have been found to alter the stemness of CSCs [37].

\subsection{OXPHOS}

OXPHOS, which involves the mitochondrial respiratory chain, is the last step of aerobic glucose metabolism where energy is generated as ATP, which is used for cell survival and growth. In a study done on pancreatic CSCs, it was found that in comparison to normal adherent cells, CSCs have a higher number of mitochondria and form more components that are required for OXPHOS. Furthermore, compared to non-CSCs (CD133- differentiated cells), CD133+ CSCs have a higher mitochondrial oxygen consumption rate (OCR) [35], suggesting the reliance of CSCs on OXPHOS for their growth and survival. In fact, the inhibition of OXPHOS resulted in CSCs experiencing an energy crisis. However, there is a subset of CSCs that are resistant to inhibition of mitochondrial metabolism by metformin, and they are found to have enhanced glycolytic capacity [35], suggesting the metabolic plasticity of CSCs.

Studies have shown that the inhibition of OXPHOS can have detrimental effects on CSCs. Salinomycin, a K+ ionophore, which changes the mitochondrial membrane potential of cells, inhibits OXPHOS [38] and decreases the number of CSCs in breast [39], gastric [40], and pancreatic cancers [41]. The change in mitochondrial 


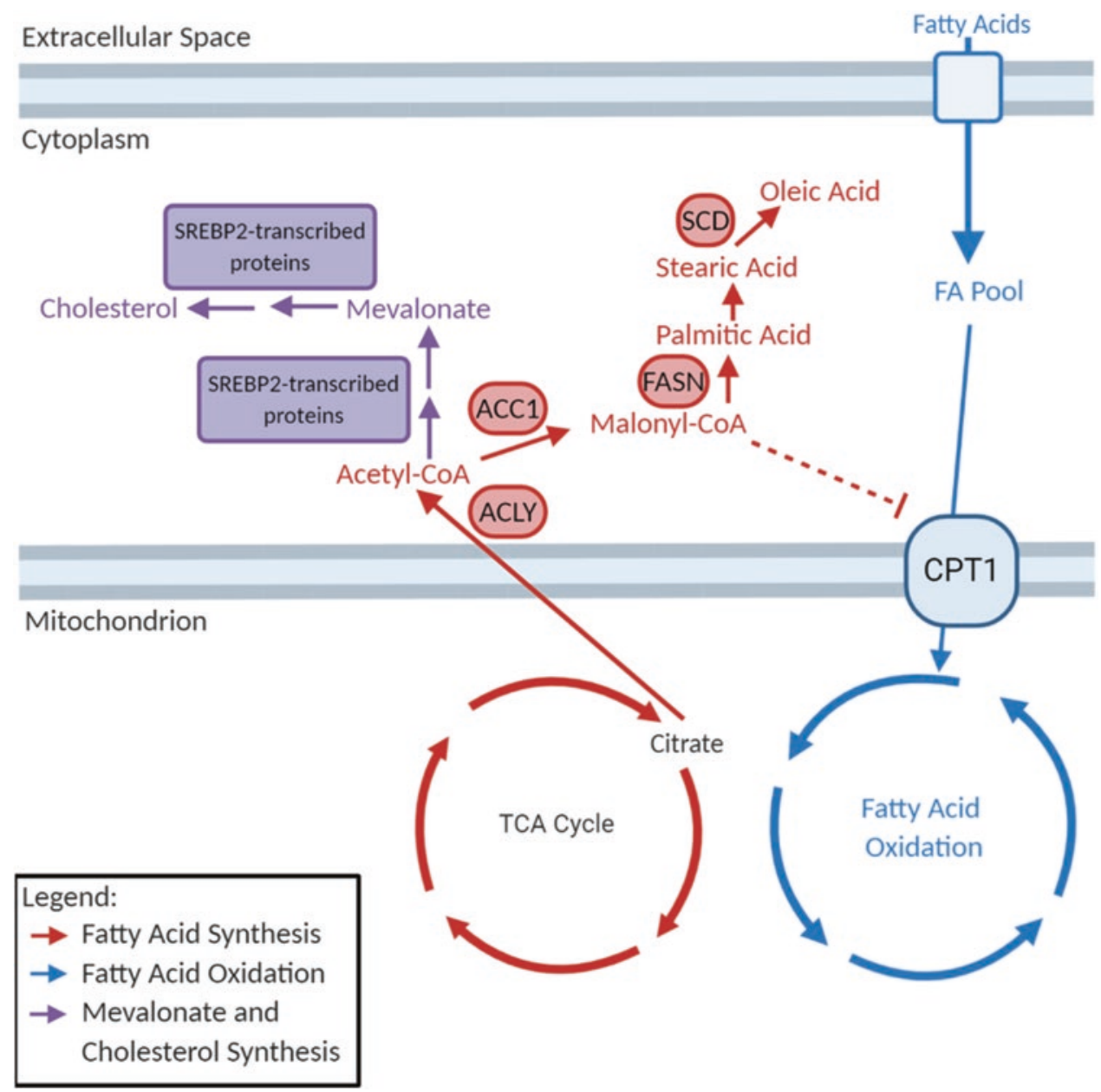

Fig. 4 Lipid metabolism in cancer stem cells. SREBP sterol regulatory element-binding protein, $A C C 1$ acetyl carboxylase A, ACLY ATP citrate lyase, FA fatty acid, FASN fatty acid synthase, $S C D$ stearoyl CoA desaturase, $C P T 1$ carnitine palmitoyltransferase 1

membrane potential caused by salinomycin causes mitochondrial imbalance. In turn, ATP depletion activates AMP-activated protein kinase (AMPK) and results in apoptosis of CSCs [42]. A balanced and well-regulated mitochondrion is, therefore, important to CSCs.

The mitochondrial respiratory chain is comprised of four enzymatic complexes for the transfer of electrons to $\mathrm{O}_{2}$ and generate ATP, which is used for cell survival. Only complex II is completely encoded by the nuclear genome, while for complexes I, III, and IV, mitochondrial DNA (mtDNA) plays a role in encoding them [43]. The inhibition of these complexes is detrimental to the survival and growth of CSCs and, therefore, they serve as possible targets for eliminating CSCs. The inhibition of complex I in pancreatic cancer by metformin resulted in a cell cycle arrest in non-CSCs, while in CSCs, it led to apoptosis [34]. This observation aligns with the finding that metformin results in the selective killing of CD44+/CD24- CSCs of MCF10-A and MCF-7 cell lines, and works well with doxorubicin in reducing both CSCs and non-CSCs [44]. Another study showed that the inhibition of complexes I and II by pyrvinium pamoate caused dysregulated mitochondrial metabolism in the fat pad (FP) CSCs, but this specific mechanism was not 
toxic to CSCs [45]. Treatment using atovaquone, a complex III inhibitor, decreased proliferation of CD44+/CD24- MCF7 breast CSCs, and induced apoptosis in dose-independent conditions, but had no effect on mitochondrial respiration in normal fibroblasts [46]. Furthermore, complex V inhibitor bedaquiline, which causes ATP depletion, was found to target MCF7 breast CSCs preferentially and did not affect the viability of cancer cells and normal human fibroblasts [47]. These studies indicated the importance of OXPHOS across different CSCs as the inhibition of the complexes involved in OXPHOS negatively impacted the survival and growth of CSCs. However, it is important to keep in mind the different responses to mitochondrial respiratory chain complexes' inhibition seen in different CSCs. For instance, complex I inhibition by metformin in pancreatic CSCs led to their apoptosis, but no cytotoxic effect was brought about in FP-CSCs when pyrvinium pamoate inhibited complexes I and II. Further studies regarding the differences in the mechanism and regulation of OXPHOS across different CSCs are required. Nevertheless, it is certain that OXPHOS is necessary and important to the survival of CSCs and its enzymatic complexes serve as potential targets in the elimination of CSCs.

\subsection{Resistance of CSCs Against ROS}

ROS are reactive products generated from mitochondrial metabolism. Mitochondria are the major source of intracellular ROS, such as free radicals, superoxide, and peroxides [42, 48]. Excess ROS causes apoptosis via oxidative stress [49]. Radiotherapy and most of the chemotherapies increase ROS levels and thereby affect cancer cell viability and growth $[50,51]$. Despite such therapy, tumor recurrence does occur due to the failure to eliminate CSCs during such treatment $[52,53]$. One of the mechanisms of CSC resistance is through increased $\mathrm{Bcl}-2$ expression and radioresistance.

Studies have shown the resistance of CSCs against the effects of ROS in CD44+ CSCs of prostate, breast, and colorectal cancers [54-56].
An isoform of CD44, CD44v, was found to protect CSCs of gastric cancer from the detrimental effects of ROS accumulation. CD44v stabilizes the $\mathrm{xCT}$ subunit of cystine-glutamate exchange transporter, allowing for the uptake of cystine needed for glutathione (GSH) synthesis. The upregulation of GSH synthesis makes CSCs more antioxidative [57]. Other than containing CD44, CSCs increase the expression of antioxidant enzyme genes that neutralize ROS and the multifunctional protein apurinic/apyrimidinic endonuclease/redox effector factor (Ape1/Ref1). In turn, they neutralize the intracellular ROS and reduce DNA damage via DNA repair [58]. Therefore, through their antioxidative capability, CSCs are resistant to the effects of ROS produced during therapy and can, therefore, cause recurrence of tumors $[58,59]$. The defense mechanism against ROS of CSCs needs to be targeted for improved cancer treatment.

\section{$5 \quad$ Lipid Metabolism (Fig. 4)}

Lipid anabolic metabolism consists of both ex novo incorporation of lipids and de novo syntheses of lipids [60, 61]. De novo lipogenesis consists of three major pathways: fatty acid synthesis (FAS), mevalonate biosynthesis, and cholesterol biosynthesis [62]. CSCs exhibit both ex novo and de novo lipid anabolic pathways. Interestingly, despite the activation of anabolic pathways, CSCs also activate catabolic lipid pathways, such as fatty acid oxidation (FAO). Classically, FAS and FAO are antagonistic pathways due to the role of FAS intermediate malonyl CoA, which suppresses carnitine palmitoyltransferase 1 (CPT1), inactivating FAO in normal cellular metabolism [63]. However, it seems that in CSCs, both pathways are activated to allow them to adapt best to their environmental pressures and cellular demands for proliferation.

Lipogenesis via uptake from the environment has been reported in CSCs [64]. Given the complexity of certain lipids such as phospholipids and cholesterol, which rely on the stoichiometric balancing of fatty acid with glycerol in various combinations, it makes sense that CSCs have been found to incorporate these complex 
lipids. Mass spectrometry studies reveal that CSCs have abundant unsaturated lipids, cholesterol, and triglycerides (TGs) which are important for their stemness [61]. The combination of external lipid uptake and de novo lipogenesis seems to be the most efficient route for fulfilling the increasing demands of membrane production for the proliferating CSCs.

De novo lipogenesis, however, still plays a major role despite CSC's ex novo sources of lipids. The FAS pathway, being the initial step of de novo lipogenesis, is activated in CSCs, as it is in some cancer cells, such as those driven by MYC $[62,65,66]$. The key enzymes in the FAS pathway are ATP citrate lyase (ACLY), acetyl carboxylase A (ACC1), fatty acid synthase (FASN), and stearoyl-CoA desaturase (SCD). ACLY is connected directly to the TCA cycle via citrate synthase (CS). Thus, increased TCA mitochondrial metabolism in CSCs would also drive the flux into FAS via CS into ACLY and the rest of the FAS pathway [62]. Though de novo lipogenesis may appear to be a secondary or passenger effect of increased glucose and glutamine metabolism in CSCs, inhibition of virtually any enzyme of the FAS pathway in CSCs leads to apoptosis. ACLY knockdown reduced the proliferation of lung cancer [67] and breast cancer CSCs [68]. ACC1 inhibition suppressed the stemness of CSCs [69]. Inhibition of FASN reduced the stemness of glioma stem cells [70]. SCD inhibition has been correlated with the suppression of various CSCs [71].

Like the FAS pathway, the cholesterol synthesis pathways via mevalonate are regulated by sterol regulatory element-binding proteins (SREBPs). SREBP2 actively transcribes HMGCoA synthase (HMGCS), HMG-CoA reductase (HMGCR), mevalonate kinase (MVK), and phosphomevalonate kinase (PMVK) for the production of pyrophosphate-containing intermediates, which leads to the production of cholesterol. The mevalonate and cholesterol synthesis pathways are connected to the acetyl-CoA pool from ACLY in FAS via acetoacetyl CoA transferase (ACAT), which feeds into HMGCS. During epithelial-mesenchymal transition (EMT), the mevalonate and cholesterol pathways are activated, such that inhibition of any of the aforemen- tioned enzymes disrupts CSCs' stemness and the overall EMT [72].

In addition to the induction of the anabolic lipid metabolic pathways mentioned above (ex novo incorporation of lipids and the three de novo lipogenesis pathways: FAS, mevalonate, and cholesterol), CSCs also upregulate the catabolic lipid metabolic pathway: FAO [73]. FAO takes place in mitochondria following the uptake of fatty acids via CPT1. CPT1 can be inhibited by malonyl CoA, which is a product of ACC 1 of the FAS pathway, ensuring the inhibition of running a futile cycle between FAS and FAO. However, due to the highly proliferative state of FAO and the ex vivo uptake of lipids, FAO is also upregulated in CSCs. This FAO pathway may be necessary for CSCs to replenish NADH and $\mathrm{FADH}_{2}$, which will help facilitate the electron transport chain (ETC) in OXPHOS [63]. Indeed, inhibition of FAO has been reported to lower the number of CSCs [74].

\section{Conclusion}

From our brief overview, it is clear that CSCs have diverse metabolic profiles that are quite specific to each cancer type and tissue type, which is a break from convention with normal cell metabolism. For example, the activation of OXPHOS alongside the Warburg effect presents a complexity that demands further studies. The shunting of glucose-derived pyruvate to lactate instead of the TCA cycle by the Warburg effect should preclude the flow of glucose carbons to OXPHOS unless somehow the glutaminolysis carbons can compensate for all the TCA cycle to push forward to OXPHOS independent of glucose [20]. Regardless, CSCs seem to exhibit both the Warburg effect and OXPHOS simultaneously. Another example is the activation of mitochondrial metabolism alongside lipogenesis, which also challenges conventional wisdom concerning cellular metabolism. Typically, the activation of lipogenesis would block fatty acid oxidation in the mitochondria due to the inhibition of CPT1 by malonyl-CoA. However, CSCs seem to have no problem activating both pathways. 
The metabolic assessment of CSCs using metabolomics technologies [75] reflects variation within the CSC population. Single-cell metabolic analyses would enable us to identify such diversity in the CSC population. Moreover, there is tissue and microenvironmental specificity to CSC metabolic profiles. For example, breast CSCs that are surrounded by lipids may "prefer" to activate fatty acid oxidation instead of inducing de novo lipogenesis. This may not be the case for lymphomas that travel within the blood vessels, which may prefer to use glucose as a primary energy source instead of lipids. Furthermore, CSCs seem to run futile cycles metabolically (i.e., activating lipogenesis and fatty acid oxidation and OXPHOS simultaneously), because these processes are not absolutely efficient. Thus CSCs activate both pathways to make sure that all residual metabolites are processed efficiently catabolically and anabolically to support their high demand for energy and biomass.

Despite the differences in CSC metabolic profiles, CSCs do share some similarities with both stem cells and cancer cells in glucose, glutamine, OXPHOS, and lipid metabolism. This makes it difficult to distinguish CSCs from stem cells and non-CSC tumor cells metabolically, and conventional CSC markers are still needed (i.e., CD44 and CD133). However, the fact that there are commonly activated metabolic pathways between CSCs, stem cells, and non-CSC tumor cells is consistent with three ideas pertaining to the nature of CSCs. First, the commonly shared metabolic pathways indicate that CSCs are indeed a subtype of cancer cells that genetically evolved from normal stem/progenitor cells. Second, the commonly shared metabolic pathways between the three indicate that these metabolic pathways are selected due to their ability to sustain the catabolic and anabolic needs of highly proliferating cells. Third, the fact that CSCs share metabolic pathways with stem cells and cancer cells presents a challenge for developing cancer metabolic drugs that do not target normal stem cells.

Overall, this is a fascinating field in metabolism, where researchers need to further delineate metabolic similarities and differences within the CSC population itself as well as with other stem cell and cancer cell types. These intra- and interdifferences will shed light on how to best develop specific metabolic drugs targeting CSCs in specific cancer types that would not be toxic to normal stem cells.

\section{References}

1. Dick, J. E. (2008). Stem cell concepts renew cancer research. Blood, 112(13), 4793-4807.

2. Reya, T., et al. (2001). Stem cells, cancer, and cancer stem cells. Nature, 414(6859), 105-111.

3. Bonnet, D., \& Dick, J. E. (1997). Human acute myeloid leukemia is organized as a hierarchy that originates from a primitive hematopoietic cell. Nature Medicine, 3(7), 730-737.

4. Crabtree, J. S., \& Miele, L. (2018). Breast cancer stem cells. Biomedicine, 6, 3 .

5. Hermann, P. C., et al. (2007). Distinct populations of cancer stem cells determine tumor growth and metastatic activity in human pancreatic cancer. Cell Stem Cell, 1(3), 313-323.

6. Ho, M. M., et al. (2007). Side population in human lung cancer cell lines and tumors is enriched with stemlike cancer cells. Cancer Research, 67(10), 4827-4833.

7. Yu, Z., et al. (2012). Cancer stem cells. The International Journal of Biochemistry \& Cell Biology, 44(12), 2144-2151.

8. Sancho, P., Barneda, D., \& Heeschen, C. (2016). Hallmarks of cancer stem cell metabolism. British Journal of Cancer, 114(12), 1305-1312.

9. Bose, S., Zhang, C., \& Le, A. (2021). Glucose metabolism in cancer: The Warburg effect and beyond. Advances in Experimental Medicine and Biology, 1311, https://doi.org/10.1007/978-3-030-65768-0_1

10. Aguilar, E., et al. (2016). Metabolic reprogramming and dependencies associated with epithelial cancer stem cells independent of the epithelial-mesenchymal transition program. Stem Cells, 34(5), 1163-1176.

11. Song, K., et al. (2015). Active glycolytic metabolism in CD133(+) hepatocellular cancer stem cells: Regulation by MIR-122. Oncotarget, 6(38), 40822-40835

12. Shen, Y. A., et al. (2015). Metabolic reprogramming orchestrates cancer stem cell properties in nasopharyngeal carcinoma. Cell Cycle, 14(1), 86-98.

13. Shibuya, K., et al. (2015). Targeting the facilitative glucose transporter GLUT1 inhibits the self-renewal and tumor-initiating capacity of cancer stem cells. Oncotarget, 6(2), 651-661.

14. Ciavardelli, D., et al. (2014). Breast cancer stem cells rely on fermentative glycolysis and are sensitive to 2-deoxyglucose treatment. Cell Death \& Disease, 5, e1336.

15. Xie, H., et al. (2014). Targeting lactate dehydrogenase--a inhibits tumorigenesis and tumor progression in mouse models of lung cancer and impacts tumorinitiating cells. Cell Metabolism, 19(5), 795-809.

16. Le, A., et al. (2010). Inhibition of lactate dehydrogenase A induces oxidative stress and inhibits tumor progres- 
sion. Proceedings of the National Academy of Sciences of the United States of America, 107(5), 2037-2042.

17. Rajeshkumar, N. V., et al. (2015). Therapeutic targeting of the Warburg effect in pancreatic cancer relies on an absence of p53 function. Cancer Research, 75(16), 3355-3364.

18. Dutta, P., et al. (2013). Evaluation of LDH-A and glutaminase inhibition in vivo by hyperpolarized 13C-pyruvate magnetic resonance spectroscopy of tumors. Cancer Research, 73(14), 4190-4195.

19. Li, T., Copeland, C., \& Le, A. (2021). Glutamine metabolism in cancer. Advances in Experimental Medicine and Biology, 1311, https://doi. org/10.1007/978-3-030-65768-0_2

20. Le, A., et al. (2012). Glucose-independent glutamine metabolism via TCA cycling for proliferation and survival in B cells. Cell Metabolism, 15(1), 110-121.

21. Kim, J. H., et al. (2018). Effects of metformin on colorectal cancer stem cells depend on alterations in glutamine metabolism. Scientific Reports, 8(1), 409.

22. Zimmermann, S. C., et al. (2016). Allosteric glutaminase inhibitors based on a 1,4-di(5-amino-1,3,4thiadiazol-2-yl)butane scaffold. ACS Medicinal Chemistry Letters, 7(5), 520-524.

23. Rais, R., et al. (2016). Discovery of 6-diazo-5-oxol-norleucine (DON) prodrugs with enhanced CSF delivery in monkeys: a potential treatment for glioblastoma. Journal of Medicinal Chemistry, 59(18), 8621-8633.

24. Xiang, Y., et al. (2015). Targeted inhibition of tumorspecific glutaminase diminishes cell-autonomous tumorigenesis. The Journal of Clinical Investigation, 125(6), 2293-2306.

25. Dang, C. V., et al. (2011). Therapeutic targeting of cancer cell metabolism. Journal of Molecular Medicine (Berlin), 89(3), 205-212.

26. Hirschey, M. D., et al. (2015). Dysregulated metabolism contributes to oncogenesis. Seminars in Cancer Biology, 35(Suppl), S129-S150.

27. Elgogary, A., et al. (2016). Combination therapy with BPTES nanoparticles and metformin targets the metabolic heterogeneity of pancreatic cancer. Proceedings of the National Academy of Sciences of the United States of America, 113(36), E5328-E5336.

28. Udupa, S., et al. (2019). Upregulation of the glutaminase II pathway contributes to glutamate production upon glutaminase 1 inhibition in pancreatic cancer. Proteomics, 19(21-22), e1800451.

29. Nguyen, T., et al. (2019). Uncovering the role of $\mathrm{N}$-acetyl-aspartyl-glutamate as a glutamate reservoir in cancer. Cell Reports, 27(2), 491-501. e6.

30. Li, D., et al. (2015). Inhibition of glutamine metabolism counteracts pancreatic cancer stem cell features and sensitizes cells to radiotherapy. Oncotarget, 6(31), 31151-31163.

31. Cuyas, E., et al. (2018). Mitostemness. Cell Cycle, 17(8), 918-926.

32. Liu, P. P., et al. (2014). Metabolic regulation of cancer cell side population by glucose through activation of the Akt pathway. Cell Death and Differentiation, 21(1), 124-135.
33. Peiris-Pages, M., et al. (2016). Cancer stem cell metabolism. Breast Cancer Research, 18(1), 55.

34. Lonardo, E., et al. (2013). Metformin targets the metabolic Achilles heel of human pancreatic cancer stem cells. PLoS One, 8(10), e76518.

35. Sancho, P., et al. (2015). MYC/PGC-1alpha balance determines the metabolic phenotype and plasticity of pancreatic cancer stem cells. Cell Metabolism, 22(4), 590-605.

36. Reid, M. A., Dai, Z., \& Locasale, J. W. (2017). The impact of cellular metabolism on chromatin dynamics and epigenetics. Nature Cell Biology, 19(11), 1298-1306.

37. Wainwright, E. N., \& Scaffidi, P. (2017). Epigenetics and cancer stem cells: Unleashing, hijacking, and restricting cellular plasticity. Trends Cancer, 3(5), 372-386.

38. Mitani, M., et al. (1976). Salinomycin effects on mitochondrial ion translocation and respiration. Antimicrobial Agents and Chemotherapy, 9(4), 655-660.

39. Gupta, P. B., et al. (2009). Identification of selective inhibitors of cancer stem cells by high-throughput screening. Cell, 138(4), 645-659.

40. Zhi, Q. M., et al. (2011). Salinomycin can effectively kill ALDH (high) stem-like cells on gastric cancer. Biomedicine \& Pharmacotherapy, 65(7), 509-515.

41. Zhang, G. N., et al. (2011). Combination of salinomycin and gemcitabine eliminates pancreatic cancer cells. Cancer Letters, 313(2), 137-144.

42. Lyakhovich, A., \& Lleonart, M. E. (2016). Bypassing mechanisms of mitochondria-mediated cancer stem cells resistance to chemo- and radiotherapy. Oxidative Medicine and Cellular Longevity, 2016, 1716341.

43. Garcia-Heredia, J. M., \& Carnero, A. (2015). Decoding Warburg's hypothesis: Tumor-related mutations in the mitochondrial respiratory chain. Oncotarget, 6(39), 41582-41599.

44. Hirsch, H. A., et al. (2009). Metformin selectively targets cancer stem cells, and acts together with chemotherapy to block tumor growth and prolong remission. Cancer Research, 69(19), 7507-7511.

45. Dattilo, R., et al. (2020). Pyrvinium pamoate induces death of triple-negative breast cancer stem-like cells and reduces metastases through effects on lipid anabolism. Cancer Research, 80(19), 4087-4102.

46. Fiorillo, M., et al. (2016). Repurposing atovaquone: Targeting mitochondrial complex III and OXPHOS to eradicate cancer stem cells. Oncotarget, 7(23), 34084-34099.

47. Fiorillo, M., et al. (2016). Bedaquiline, an FDA-approved antibiotic, inhibits mitochondrial function and potently blocks the proliferative expansion of stem-like cancer cells (CSCs). Aging (Albany NY), 8(8), 1593-1607.

48. Sosa, V., et al. (2013). Oxidative stress and cancer: An overview. Ageing Research Reviews, 12(1), 376-390.

49. Redza-Dutordoir, M., \& Averill-Bates, D. A. (2016). Activation of apoptosis signalling pathways by reactive oxygen species. Biochimica et Biophysica Acta, 1863(12), 2977-2992. 
50. Fan, P. C., et al. (2019). Quantitative proteomics reveals mitochondrial respiratory chain as a dominant target for carbon ion radiation: Delayed reactive oxygen species generation caused DNA damage. Free Radical Biology \& Medicine, 130, 436-445.

51. Yang, H., et al. (2018). The role of cellular reactive oxygen species in cancer chemotherapy. Journal of Experimental \& Clinical Cancer Research, 37(1), 266.

52. Piao, L. S., et al. (2012). CD133+ liver cancer stem cells modulate radioresistance in human hepatocellular carcinoma. Cancer Letters, 315(2), 129-137.

53. Gomez-Casal, R., et al. (2013). Non-small cell lung cancer cells survived ionizing radiation treatment display cancer stem cell and epithelial-mesenchymal transition phenotypes. Molecular Cancer, 12(1), 94.

54. Collins, A. T., et al. (2005). Prospective identification of tumorigenic prostate cancer stem cells. Cancer Research, 65(23), 10946-10951.

55. Al-Hajj, M., et al. (2003). Prospective identification of tumorigenic breast cancer cells. Proceedings of the National Academy of Sciences of the United States of America, 100(7), 3983-3988.

56. Dalerba, P., et al. (2007). Phenotypic characterization of human colorectal cancer stem cells. Proceedings of the National Academy of Sciences of the United States of America, 104(24), 10158-10163.

57. Ishimoto, T., et al. (2011). CD44 variant regulates redox status in cancer cells by stabilizing the $\mathrm{xCT}$ subunit of system xc(-) and thereby promotes tumor growth. Cancer Cell, 19(3), 387-400.

58. Diehn, M., et al. (2009). Association of reactive oxygen species levels and radioresistance in cancer stem cells. Nature, 458(7239), 780-783.

59. Schulz, A., et al. (2019). Cancer stem cells and radioresistance: DNA repair and beyond. Cancers (Basel), 11, 6.

60. Park, J. K., et al. (2021). The heterogeneity of lipid metabolism in cancer. Advances in Experimental Medicine and Biology, 1311, https://doi. org/10.1007/978-3-030-65768-0_3

61. Sun, M., \& Yang, Z. (2019). Metabolomic studies of live single cancer stem cells using mass spectrometry. Analytical Chemistry, 91(3), 2384-2391.

62. Gouw, A. M., et al. (2019). The MYC oncogene cooperates with sterol-regulated element-binding protein to regulate lipogenesis essential for neoplastic growth. Cell Metabolism, 30(3), 556-572. e5.
63. Foster, D. W. (2012). Malonyl-CoA: The regulator of fatty acid synthesis and oxidation. The Journal of Clinical Investigation, 122(6), 1958-1959.

64. Begicevic, R. R., Arfuso, F., \& Falasca, M. (2019). Bioactive lipids in cancer stem cells. World Journal of Stem Cells, 11(9), 693-704.

65. Dang, C. V., Le, A., \& Gao, P. (2009). MYC-induced cancer cell energy metabolism and therapeutic opportunities. Clinical Cancer Research, 15(21), 6479-6483.

66. Le, A., \& Dang, C. V. (2013). Studying Myc's role in metabolism regulation. Methods in Molecular Biology, 1012, 213-219.

67. Hanai, J. I., et al. (2013). ATP citrate lyase knockdown impacts cancer stem cells in vitro. Cell Death \& Disease, 4, e696.

68. Rios Garcia, M., et al. (2017). Acetyl-CoA carboxylase 1-dependent protein acetylation controls breast cancer metastasis and recurrence. Cell Metabolism, 26(6), 842-855. e5.

69. Corominas-Faja, B., et al. (2014). Chemical inhibition of acetyl-CoA carboxylase suppresses self-renewal growth of cancer stem cells. Oncotarget, 5(18), 8306-8316.

70. Yasumoto, Y., et al. (2016). Inhibition of fatty acid synthase decreases expression of stemness markers in glioma stem cells. PLoS One, 11(1), e0147717.

71. Tracz-Gaszewska, Z., \& Dobrzyn, P. (2019). StearoylCoA desaturase 1 as a therapeutic target for the treatment of cancer. Cancers (Basel), 11, 7.

72. Gruenbacher, G., \& Thurnher, M. (2018). Mevalonate metabolism in cancer stemness and trained immunity. Frontiers in Oncology, 8, 394.

73. Chen, C. L., et al. (2016). NANOG metabolically reprograms tumor-initiating stem-like cells through tumorigenic changes in oxidative phosphorylation and fatty acid metabolism. Cell Metabolism, 23(1), 206-219.

74. Kuo, C. Y., \& Ann, D. K. (2018). When fats commit crimes: Fatty acid metabolism, cancer stemness and therapeutic resistance. Cancer Communications (Lond), 38(1), 47.

75. Hoang, G., Udupa, S., \& Le, A. (2019). Application of metabolomics technologies toward cancer prognosis and therapy. International Review of Cell and Molecular Biology, 347, 191-223.

Open Access This chapter is licensed under the terms of the Creative Commons Attribution 4.0 International License (http://creativecommons.org/licenses/by/4.0/), which permits use, sharing, adaptation, distribution and reproduction in any medium or format, as long as you give appropriate credit to the original author(s) and the source, provide a link to the Creative Commons license and indicate if changes were made.

The images or other third party material in this chapter are included in the chapter's Creative Commons license, unless indicated otherwise in a credit line to the material. If material is not included in the chapter's Creative Commons license and your intended use is not permitted by statutory regulation or exceeds the permitted use, you will need to obtain permission directly from the copyright holder. 


\title{
Metabolism of Immune Cells in the Tumor Microenvironment
}

\author{
Jin G. Jung and Anne Le
}

Keywords
Immunometabolism · Tumor immunity ·
Metabolic competition · Metabolic barrier ·
CAR T lymphocytes

\section{Abbreviations}

ASCT2 Alanine, serine, cysteine trans-

$\begin{array}{ll} & \text { porter } 2 \\ \text { CAFs } & \text { Cancer-associated fibroblasts } \\ \text { CAR } & \text { Chimeric antigen receptor } \\ \text { CTL } & \text { Cytotoxic T lymphocytes } \\ \text { ECM } & \text { Extracellular matrix } \\ \text { ERR } \alpha & \text { Estrogen-related receptor alpha } \\ \text { FAO } & \text { Fatty acid oxidation } \\ \text { GLUT } & \text { Glucose transporter }\end{array}$

J. G. Jung

Department of Pathology, Johns Hopkins University

School of Medicine, Baltimore, MD, USA

\section{A. Le $(\bowtie)$}

Department of Pathology and Oncology, Johns

Hopkins University School of Medicine,

Baltimore, MD, USA

Department of Chemical and Biomolecular Engineering, Johns Hopkins University Whiting School of Engineering, Baltimore, MD, USA e-mail: annele@jhmi.edu
GPCRs $\quad G$ protein-coupled receptors

HIF1 $\alpha \quad$ Hypoxia-inducible factor $1 \alpha$

HPSE Heparanase

HSPG Heparan sulfate proteoglycans

IFN $\gamma \quad$ Interferon-gamma

IL

LDHA

MCT

MDSC

mTOR

NK cells

OXPHOS

PCK1

PDK1

PEP

PGC1 $\alpha$

PHD

PI3K

SNAT

TAM

$\mathrm{T}_{\mathrm{cm}}$

TCR

$\mathrm{T}_{\text {eff }}$

TILs

TLR

$\mathrm{TNF} \alpha$

$\mathrm{T}_{\text {reg }}$
Interleukin

Lactate dehydrogenase A

Monocarboxylate lactate transporters

Myeloid-derived suppressor cells Mammalian target of rapamycin

Natural killer cells

Oxidative phosphorylation

Phosphoenolpyruvate carboxykinase 1

Pyruvate dehydrogenase kinase

Phosphoenolpyruvate

PPAR-gamma coactivator $1 \alpha$

Prolyl-hydroxylase

Phosphatidylinositol-4,5-bisphos phate 3-kinase

Sodium-coupled neutral amino acid transporter

Tumor-associated macrophages

Central memory T cells

T-cell receptor

Effector T cells

T-cell infiltrating lymphocytes

Toll-like receptor

Tumor necrosis factor alpha

Regulatory T cells 
TRUCKs $\mathrm{T}$ cells redirected for antigenunrestricted cytokine-initiated killing

Tscm Stem memory T cells

\section{Key Points}

- Tumor cells produce numerous substances to create an immunosuppressive tumor microenvironment.

- The tumor microenvironment physically constitutes a barrier against T-cell infiltration.

- Activated T cells reprogram OXPHOS and FAO to glycolysis and glutaminolysis.

- Tumors escape immunity via T-cell dysfunction or hyporesponsiveness by upregulation of several inhibitory receptors.

- Increased glucose uptake by cancer cells restricts T-cell function by decreasing mTOR activity.

- Treatments using immune checkpoint inhibitors increase extracellular glucose levels to improve T-cell infiltrating lymphocytes' function.

\section{Introduction}

The tumor microenvironment (TME) is a complex biological structure surrounding tumor cells and includes blood vessels, immune cells, fibroblasts, adipocytes, and extracellular matrix (ECM) $[1,2]$. These heterogeneous surrounding structures provide nutrients, metabolites, and signaling molecules to provide a cancer-friendly environment. The metabolic interplay between immune cells and cancer cells in the TME is a key feature not only for understanding tumor biology but also for discovering cancer cells' vulnerability. As cancer immunotherapy to treat cancer patients and the use of metabolomics technologies become more and more common [3], the importance of the interplay between cancer cells and immune cells in the TME is emerging with respect to not only cell-to-cell interactions but also metabolic pathways. This interaction between immune cells and cancer cells is a complex and dynamic process in which immune cells act as a determinant factor of can- cer cells' fate and vice versa. In this chapter, we provide an overview of the metabolic interplay between immune cells and cancer cells and discuss the therapeutic opportunities as a result of this interplay in order to define targets for cancer treatment. It is important to understand and identify therapeutic targets that interrupt this cancerpromoting relationship between cancer cells and the surrounding immune cells, allowing for maximum efficacy of immune checkpoint inhibitors as well as other genetic and cellular therapies.

\section{Tumor Immunity and the Various Roles of Immune Cells}

The immune system's antitumor activity is mainly carried out by tumor antigen-specific cytotoxic T lymphocytes (CTL), T effector $\left(\mathrm{T}_{\text {eff }}\right)$ cells, antibody-producing $\mathrm{B}$ cells, as well as antigen-presenting dendritic cells (DC), which lead to adaptive immunity by directly recognizing and eliminating cancer cells. Natural killer (NK) cells, macrophages, and NK-T cells also play crucial roles in suppressing tumor progression via a nonspecific immune response. Even though this defense system is well developed, the tumor often has the ability to develop an immunosuppressive microenvironment favorable to its progression. Specifically, myeloid-derived suppressor cells (MDSC), regulatory $\mathrm{T}\left(\mathrm{T}_{\text {reg }}\right)$ cells, and tumor-associated macrophages (TAMs) are well-known players. These tumor-friendly immune cells suppress the settlement of tumorinfiltrating lymphocytes (TILs) by expressing essential amino acid (EAA)-degrading enzymes including arginase 1 (Arg1) and indoleamine2,3-dioxygenase (IDO) [4-7], and inhibitors targeting Arg1 and IDO are being investigated in ongoing clinical trials $[8,9]$. The TME is an environment composed of multifaceted components with tumor-friendly or antitumoral characteristics where there is strong competition for metabolites and nutrients. Studies have shown that T-cell-mediated adaptive response is a promising therapeutic strategy to strengthen the antitumor activity of the immune system [10-12]. 


\subsection{Metabolic Competition and Tumor Immunity}

With strong evidence showing how $\mathrm{T}$ lymphocytes infiltrate into the tumor niche and how checkpoint inhibitors or chimeric antigen receptor (CAR) $\mathrm{T}$ cells inhibit the growth of cancer cells, a new era of immunotherapy has just begun with successful clinical development [13]. However, cancer cells can escape immune recognition via "immunoediting," allowing cancer cell clones without detectable cancer antigens to dominate and escape from the pressure of immune checkpoint inhibitors [14].

Rapidly growing tumor cells require nutrients, oxygen, and essential metabolites to proliferate and, at the same time, create an immunosuppressive microenvironment. How immune cells and cancer cells share or compete in these harsh environmental conditions and how the TME alters immunometabolism are important questions to address. Specifically, it needs to be addressed how cancer cells and neighboring immune cells compete to take up nutrients and metabolites, which consequently influences signaling cascades, metabolic activities, and tumor progression.

Cancer-associated adipocytes: It is well known that adipocytes play an important role in communicating with cancer cells by excreting inflammatory factors, adipokines, and free fatty acids, which help cancer growth. In addition, immune cell functions are heavily regulated by lipids, lipoproteins, and cholesterol within the TME. For example, elevated levels of oxidized lipoproteins as a result of incorporation via scavenger receptors and formation of lipid droplets can compromise the ability of dendritic cells (DCs) to activate $\mathrm{T}$ cells by presenting tumor antigens $[15,16]$. Also, it is well known that cancer cells instruct neighboring adipocytes to increase lipolysis [17].

Cancer-associated fibroblasts [18, 19]: It is reported that tryptophan catabolism by CAFs causes the starvation of immune cells and results in the production of kynurenine, an immunosuppressive metabolite [20]. Cancer cells also produce hydrogen peroxide $\left(\mathrm{H}_{2} \mathrm{O}_{2}\right)$, which can lead to oxidative stress in CAFs. Oxidative stress is associated with impaired mitochondrial function, which results in upregulated glucose uptake and elevated reactive oxygen species (ROS) levels [21]. In addition, glucose is also a key metabolite for the antitumor activities of effector $\mathrm{T}$ (Teff) cells and M1 macrophages because aerobic glycolysis is necessary for their activation [22, 23].

Altered amino acid levels: Amino acids in the TME are not only a resource competed for by cancer and immune cells but also another metabolic checkpoint regulating antitumor immunity. For example, glutamine is a precursor for the tricarboxylic acid (TCA) cycle [24] and lipid synthesis in hypoxic cancer cells [25] and Teff cells [26]. As such, glutaminolysis, a series of biochemical reactions that start with the conversion of glutamine carbon to glutamate and aspartate, is essential for cancer cells by providing nutrients and metabolites through anaplerotic reactions, and leads to tumor cells' and TILs' competition for glutamine, the pathway's starting material [27-29]. Moreover, it is known that glutamine activates the mammalian target of rapamycin (mTOR) signaling cascades in T cells and macrophages and is important for protein O-GlcNAcylation and synthesis of S-2hydroxyglutarate (S-2GH), a regulator of effector $\mathrm{T}$ ( $\left.\mathrm{T}_{\text {eff }}\right)$ cell function [30, 31]. Consequently, it was found that there is an upregulation of the major glutamine transporter alanine, serine, cysteine transporter 2 (ASCT2), also known as SLC1A5, for several types of cancer [32].

The proliferation of immune cells relies on growth factors for efficient nutrient utilization. For example, interleukin-2 (IL-2) promotes increased expression of glucose transporters (GLUT) and thus enhances glycolysis in activated T cells [33-36]. There is a question about how metabolites activate signaling pathways to induce changes in immune cell functions. A classic example is the binding of metabolites and energy substrates to $G$ protein-coupled receptors (GPCRs) [37]. For example, succinate leads to increased chemotaxis and activation of dendritic cells after toll-like receptor (TLR) agonist treatment by binding to the succinate receptor GPR91 [38]. On the other hand, adenosine, by binding to $\mathrm{A} 2 \mathrm{~B}$ and $\mathrm{A} 2 \mathrm{~A}$ adenosine receptors, leads to 
increased interleukin 4 (IL-4)-induced M2 macrophage activation [39]. Moreover, it is recently reported that there is a significantly reduced arginine level in the TME as a result of inducible nitric oxide synthase (iNOS) and arginase expressed by myeloid-derived suppressor cells, indicating that rapid dynamic changes of amino acids can happen in the TME [40].

\subsection{Antitumor T-Cell Metabolisms in the TME}

As $T$ cells play a critical role in antigen-specific adaptive immunity against the tumor, it is fundamentally important to understand T-cell biology. $\mathrm{T}$ lymphocytes respond to the presence of antigens and evolve rapidly. This response first requires T-cell growth; then their drastic expansion, differentiation, and death; and lastly, the formation and preservation of the memory of the immune response.

$\mathrm{T}$ lymphocyte proliferation in the TME requires a switch in its metabolism. While naïve $\mathrm{T}$ cells utilize fatty acid $\beta$-oxidation, activated $\mathrm{T}$ cells mainly use glycolysis, pentose phosphate pathway, and glutaminolysis [41, 42]. Additionally, it is reported that distinct transcriptional programs and signaling pathways are involved in this metabolic shift, including the transcription factor c-Myc [43, 44], estrogenrelated receptor alpha $(\mathrm{ERR} \alpha)[41,45,46]$, phosphatidylinositol-3-OH kinase $(\mathrm{PI}(3) \mathrm{K})$, and GLUT1-dependent Akt pathways [45]. This significant metabolic reprogramming of activated $\mathrm{T}$ cells is required for their proliferation and expansion. Consistently, it is also reported that activated $\mathrm{T}$ cells switch from oxidative phosphorylation (OXPHOS) and fatty acid oxidation (FAO) to glycolysis and glutaminolysis, which are characteristics of Teff cells, induced by antigenic stimulation through the T-cell receptor (TCR) and engagement of CD28 with a ligand on antigen-presenting cells (APC) [26]. Although glycolysis produces less ATP than OXPHOS, it is very efficient at producing biosynthetic precursors [47], which can further support the rapid proliferation and pro-inflammatory functions of Teff. Moreover, it is consistent with the findings that depletion of GLUT1 impaired T-cell proliferation and functions [36], while elevated expression of GLUT1 increased Teff cell functions [48]. In addition, demands for nutrients, such as glucose, glutamine, and other amino acids, lead to upregulations of transporters in T cells, including GLUT1 [36, 45, 49], glutamine transporters and sodium-coupled neutral amino acid transporters 1 and 2 (SNAT1 and SNAT2) [50], and monocarboxylate lactate transporters MCT1 and MCT4 to export lactate produced via aerobic glycolysis [51].

This metabolic shift from OXPHOS and FAO to glycolysis and glutaminolysis during T-cell activation is mediated by several crucial regulators. It is reported that TCR directly induces $\mathrm{PI} 3 \mathrm{~K} / \mathrm{Akt} / \mathrm{mTORC} 1$ and MYC pathways, which not only activate effector $\mathrm{T}$ cells but are also crucial for their proliferation and biological functions [26]. Indeed, an activated mTOR pathway promotes glycolysis by upregulating c-MYC and hypoxia-inducible factor $1 \alpha(\mathrm{HIF} 1 \alpha)$ [41, 45, 48, $49,52,53] . M Y C$ then induces the transcriptional factor AP4, which further upregulates glycolytic enzyme gene expressions [54]. Moreover, increased HIF1 $\alpha$ expression and activity upregulate pyruvate dehydrogenase kinase (PDK1) and lactate dehydrogenase A (LDHA), leading to increased aerobic glycolysis and decreased OXPHOS $[55,56]$, thus switching pyruvate away from the TCA cycle to lactate production. HIF1 $\alpha$ also promotes glycolysis by upregulating GLUT1 and MCT4 expression, in addition to glycolytic enzymes and regulators [57].

After fulfilling their duties, activated $\mathrm{T}$ cells undergo apoptosis during a time period called the contraction phase [58], while $\mathrm{T}_{\text {reg }}$ cells and memory $\mathrm{T}\left(\mathrm{T}_{\mathrm{mem}}\right)$ cells, by using lipid oxidation for energy production, remain in peripheral tissues or secondary lymphoid organs without undergoing apoptosis $[48,57,59,60]$. 


\subsection{Cancer Cells' Impacts on T-Cell Metabolism in the TME}

It is well known that T-cell dysfunction, or hyporesponsiveness, can result in tumors escaping immunity. This dysfunction or hyporesponsiveness is due to exhaustion and senescence of $\mathrm{T}$ cells [61]. For instance, tumor cells are shown to express indoleamine 2,3-dioxygenase (IDO), an enzyme that results in decreased tryptophan levels and inhibition of T-cell proliferation [62, 63]. Lactate produced by tumor cells can also lead to reduced T-cell function by blocking their lactate export [64]. Intracellular lactate accumulation impairs their aerobic glycolysis and thus limits their function [65].

Moreover, increased glucose uptake and consumption by cancer cells [66] impair T-cell function by decreasing their mTOR activity, glycolysis, and INF- $\gamma$ production. These negative consequences on $\mathrm{T}$ cells help promote tumor progression, which is also facilitated by decreased cytokine production due to the lack of glucose in the microenvironment. It is also supported by the fact that many types of tumors have high glycolysis rates [67, 68]. Moreover, lack of glucose impairs IFN- $\gamma$ production of $\mathrm{T}$ cells and proinflammatory signals in macrophages [36, 65, 69]. In addition, increased glycolysis rate in tumor cells as a result of the overexpression of the glycolytic enzyme hexokinase 2 (HK2) reduced glucose uptake and IFN- $\gamma$ production in TILs, which led to a more tumor-friendly microenvironment $[69,70]$.

\subsection{Cancer Cell-Induced Metabolically Harsh Environment Impairs T-Cell Function}

As the tumor grows larger, (1) oxygen supply becomes limited, thus creating a hypoxic condition; (2) nutrients become deficient; and (3) the microenvironment becomes acidic. Recent findings suggest that these harsh metabolic states significantly disrupt T-cell function. Therefore, the influence of cancer cell metabolism on the TME may directly control the metabolic pathways in surrounding $\mathrm{T}$ cells [71]. The tumor microenvi- ronment physically constitutes a barrier against T-cell infiltration, as it is a compact structure with tight interactions among cancer cells, fibroblasts, immune cells, and ECM. Indeed, tumor cells generate numerous substances to create an immunosuppressive microenvironment. For example, hypoxic cancer cells release prostaglandin E2 (PGE2) and adenosine, which can result in $\mathrm{T}$ lymphocyte proliferation inhibition by activating G protein-coupled receptors (GPCR) and protein kinase A [72]. Among the GPCRs, chemokine (C-X-C motif) receptor 3 (CXCR3) and chemokine (C-C motif) receptor 5 (CCR5) are often expressed in active lymphocytes that have infiltrated the tumors in melanoma, breast, and colorectal cancers [73] (Fig. 1).

In order to reach tumor cells and to enhance the efficacy of immunotherapy, $\mathrm{T}$ lymphocytes have to degrade the ECM and heparan sulfate proteoglycans (HSPGs) [74]. It is reported that chimeric antigen receptor (CAR) $\mathrm{T}$ cells need to release heparanase (HPSE) to successfully degrade HSPGs, which then allows T cells to gain access to the solid tumor [75] (Table 1 and Fig. 1).

\section{Targeting the Metabolism of Immune Cells for Cancer Treatment}

Accumulating evidence from the past decade indicates that metabolic reprogramming greatly affects $\mathrm{T}$ cells. Indeed, when $\mathrm{T}$ cells recognize antigens, they are activated to proliferate and produce effector molecules to eliminate the foreign antigens. During this course of the immune response, immune cells respond to changes in the metabolic microenvironment, which serves as a "metabolic checkpoint" responsible for connecting the metabolic states with signaling pathways in immune cells, which further determines their immune functions [47]. Accordingly, metabolic reprogramming of cells, such as a switch from OXPHOS and FAO to glycolysis and glutaminolysis in naïve and memory $\mathrm{T}$ cells, helps provide energy and other building block materials to generate new biomass. The manipulation of metabolic enzyme expressions helps T cells adapt in the tumor-suppressive microenvironment and 


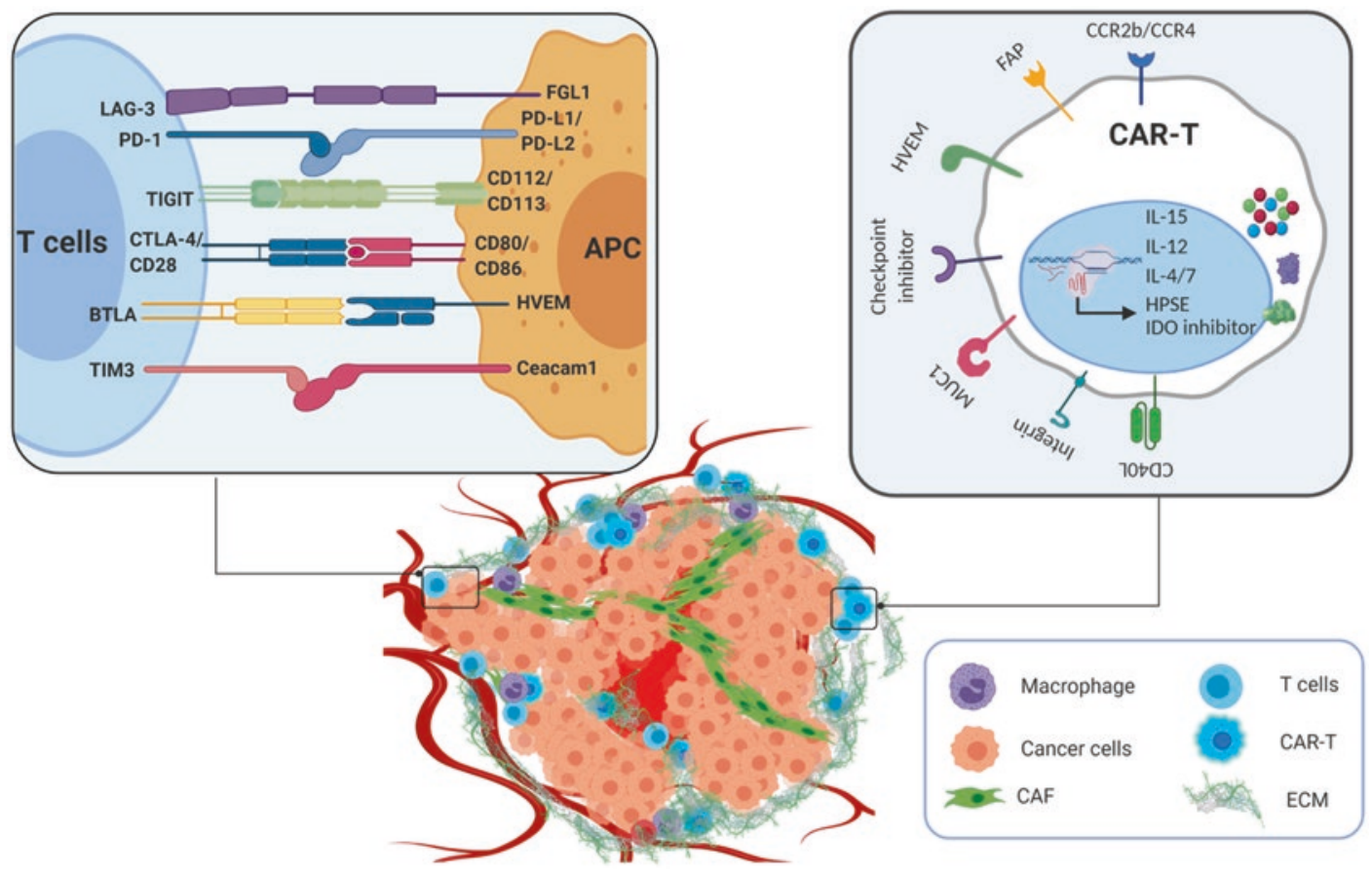

Fig. 1 Potential immunometabolism-targeting strategy in the TME. APC antigen-presenting cells, CAR T chimeric antigen receptor T cell, $C A F$ cancer-associated fibroblast, ECM extracellular matrix

restore their functions. Specifically, overexpression of phosphoenolpyruvate carboxykinase 1 (PCK1) results in a high level of the glycolytic metabolite phosphoenolpyruvate (PEP). High PEP level then enhances T-cell effector functions through T-cell receptor-mediated $\mathrm{Ca}^{2+}$-dependent nuclear factor of activated T-cell (NFAT) signaling. PCK1-overexpressing $\mathrm{T}$ cells inhibited melanoma tumor growth in vivo [70]. Another example is the oxygen-sensing prolyl-hydroxylase (PHD) proteins, which, as oxygen sensors in $\mathrm{T}$ cells, support cancer metastasis to the lung. Indeed, targeting T-cell-intrinsic PHD proteins resulted in increased antitumor immunity [112]. Also, as TILs usually have impaired mitochondrial function after infiltrating the tumors, reactivation of PPAR-gamma coactivator $1 \alpha(\mathrm{PGC} 1 \alpha)$ by suppressing Akt signaling in these T cells can induce mitochondrial biogenesis. Thus, increasing the expression of PGC1 $\alpha$ in these T cells also activates their functions [113]. These approaches may improve antitumor immunity for adoptive T-cell therapy, which is a personalized therapy for cancer via T-cell manipulation [70, 112, 113].

\subsection{The Metabolism of the Immune Checkpoint Blockades}

When $\mathrm{T}$ cells infiltrate the TME, they gradually lose several abilities, including responsiveness to T-cell receptor (TCR) stimuli and production of antitumor cytokines, in a phenomenon referred to as T-cell exhaustion or hyporesponsiveness. This is the result of the upregulation of several inhibitory receptors such as PD-1, LAG3, TIGIT, and CTLA-4 that make T cells less sensitive to tumor antigens [114]. In particular, the PD-1:PD-L1 axis and CTLA-4 are critical immune checkpoints for $\mathrm{T}$ cells, and targeting these receptors breaks down the cross talk between cancer cells and exhausted $\mathrm{T}$ cells. This result is supported by numerous clinical successes of immune checkpoint inhibitors, including ipilimumab, nivolumab, pembrolizumab, atezolizumab, avelumab, durvalumab, and cemiplimab-rwlc [115].

Interestingly, glucose deprivation caused by rapid uptake by actively growing cancer cells and glucose competition between cancer cells and 
Table 1 Tumor microenvironment-related obstacles and possible solutions (modified from [76])

\begin{tabular}{|c|c|c|c|}
\hline Obstacle & Factor & Solution & References \\
\hline \multirow[t]{3}{*}{ Tumor stroma } & Cancer-associated fibroblast & Anti-FAP CAR T cells & [77] \\
\hline & Heparan sulfate proteoglycan & Heparanase-secreting CAR T cells & [75] \\
\hline & ECM-targeting CAR T & Oncolytic virus & [78] \\
\hline \multirow[t]{2}{*}{ Tumor antigen } & integrin & $\begin{array}{l}\text { Integrin } \alpha v \beta 6 \text {, integrin } \beta \text {-targeting CAR } \\
\mathrm{T}\end{array}$ & {$[79,80]$} \\
\hline & MUC1 & $\begin{array}{l}\text { Tumor-expressing MUC1-targeting } \\
\text { CAR T }\end{array}$ & {$[81,82]$} \\
\hline \multirow[t]{6}{*}{ Cytokines and enzymes } & $\begin{array}{l}\text { Immunosuppression of the } \\
\text { TME }\end{array}$ & $\begin{array}{l}\text { TRUCK T cells expressing IL-12, } \\
\text { IL-15, IL-18 }\end{array}$ & {$[83]$} \\
\hline & TGF- $\beta$ & TGF- $\beta$ dominant-negative CAR T cells & [84] \\
\hline & IL-4 & 4/7 ICR CAR T cells (IL-4 exodomain) & {$[85]$} \\
\hline & IL-7 & CCL19 CAR T cells & [86] \\
\hline & Adenosine & A2AR receptor antagonist & {$[87,88]$} \\
\hline & CCR & CCR2/4-targeting CAR T & [89] \\
\hline \multirow[t]{5}{*}{ Metabolism and hypoxia } & CD73 & CD73 inhibitor & [90] \\
\hline & ROS and $\mathrm{H} 2 \mathrm{O} 2$ & Catalase CAR T & [91] \\
\hline & $\begin{array}{l}\text { Indolamine- } 2,3 \text { dioxygenase } \\
\text { (IDO) }\end{array}$ & IDO inhibitor & {$[92]$} \\
\hline & Protein kinase A & RIAD-CAR T & [93] \\
\hline & High levels of antioxidants & $\mathrm{N}$-acetyl cysteine & [94] \\
\hline \multirow{15}{*}{$\begin{array}{l}\text { Immune inhibitory } \\
\text { checkpoints }\end{array}$} & CTLA-1 & CTLA-4 inhibitor & {$[95]$} \\
\hline & & CTLA-4 knockout in CAR T & [96] \\
\hline & & $\begin{array}{l}\text { Anti-CTLA-1 antibody-producing CAR } \\
\mathrm{T}\end{array}$ & [95] \\
\hline & PD-1 & PD-1 inhibitor & [97] \\
\hline & & PD-1 KO in CAR T & [98] \\
\hline & & PD1-CD28 CAR T & [99] \\
\hline & & Anti-PD-1 antibody-producing CAR T & [99] \\
\hline & LAG-3 & Blockade of LAG-3 and PD-1 & {$[100]$} \\
\hline & TIM3 & TIM3 KO in CAR T & {$[100]$} \\
\hline & BTLA-4 & BTLA-4 inhibitor & {$[100]$} \\
\hline & & Blockade of BTLA-4 and PD-1 & [101] \\
\hline & & HVEM-targeting CAR T & [102] \\
\hline & $\mathrm{A} 2 \mathrm{AR}$ & A2AR antagonist & [88] \\
\hline & TIGIT & Blockade of TIGIT and PD-1 & [103] \\
\hline & CD40L & CD40L-CAR T & [104] \\
\hline \multirow[t]{6}{*}{ Immunosuppressive cells } & MDSC & CXC15-CXCR2 inhibitor & [105] \\
\hline & $\mathrm{T}_{\mathrm{reg}}$ & ALTRA-CAR T & [106] \\
\hline & & $\begin{array}{l}\text { Genetic depletion and anti-PD-L1 } \\
\text { blocking } \mathrm{Ab} \text { of } \mathrm{T}_{\mathrm{reg}}\end{array}$ & [107] \\
\hline & & $\begin{array}{l}\text { Use of IL-2, IL-7, and IL-21 with CAR } \\
\mathrm{T}\end{array}$ & $\begin{array}{l}{[108,} \\
109]\end{array}$ \\
\hline & TAM & $\begin{array}{l}\text { Induction of TAM to produce nitric } \\
\text { oxide }\end{array}$ & {$[110]$} \\
\hline & iDC & Expression of IL-18 by CAR T & $\begin{array}{l}{[110,} \\
111]\end{array}$ \\
\hline
\end{tabular}

other cells in the TME further upregulates PD-1 expression [114, 116]. In fact, PD-1 activation leads to suppressed T-cell receptor (TCR), PI3K, and mTOR signaling in T cells and reduced glycolysis, which may lead to increased accumulation of regulatory CD4 $\left(\mathrm{T}_{\text {reg }}\right)$ cells in the TME 
[117-119]. If PD-L1 on the surface of tumor cells binds to PD-1 on $\mathrm{T}$ cells, referred to as the engagement of PD-1, T-cell proliferation, cytokine production, and cytolytic function are inhibited, which promotes cancer cell proliferation [120]. It has also been shown that the degree of PD-L1 expression correlates with glycolysis rates, as well as the expression levels of glycolytic enzymes [116]. Moreover, $\alpha$-PD-L1 antibody treatment increases extracellular glucose levels in vivo, which results in improved TIL function and subsequently reduced tumor growth. Indeed, intrinsic PD-1 expression promotes mTOR signaling and tumor growth [121], while blockade of PD-1 signaling activates glycolysis and anabolic pathways in exhausted $\mathrm{T}$ cells via mTORC-1 [69, 122]. Thus, this metabolic shift provides the rationale for the clinical development of combination therapy with immune checkpoint blockade and mTOR inhibitors. Indeed, multiple clinical trials are under investigation with those drug combinations in patients with TNBC and renal cell carcinoma (NCT03805399, NCT04203901). Collectively, these results imply that the most promising therapy should target the co-inhibitory receptor-toligand interactions and re-sensitize exhausted $\mathrm{T}$ cells in the TME.

\subsection{The Metabolism of Chimeric Antigen Receptor (CAR) T Cells}

Recent clinical progress with genetically engineered chimeric antigen receptor (CAR) $\mathrm{T}$ cells for cancer therapy opens up a new era of cell/ gene therapy. However, its success is limited thus far to acute lymphoblastic leukemia (ALL) and lymphoma, whereas it shows less promising results for solid tumor treatment [72]. It is widely accepted that the major cause of the limited efficacy of CAR T cells is the poor accessibility of $T$ cells to the TME and the low-nutrient, hypoxic environment that provides suboptimal conditions for T-cell proliferation and cytokine production [123]. Thus, CAR T-cell infiltration into the tumor is a critical step to enhance their antitumor efficacy in solid tumors (Fig. 1).
The lack of therapeutic effects of CAR T cells in solid tumors is due, in part, to the immunosuppressive TME, which acts as a critical barrier. As such, new strategies to increase CAR T cells' accessibility to TME in solid tumors have been proposed. For example, stabilization of HIF1 $\alpha$ under hypoxic conditions regulates cellular metabolism, which is a critical feature in the hypoxic TME. A recent study found that targeting an oxygen-sensitive subdomain of HIF1 $\alpha$ enhances the CAR-T activity in solid tumors [124]. Another example of new strategies for CAR T therapy is the targeting of heparanase (HPSE). Stroma and tumor cells in the TME are linked together through the ECM which contains a considerable amount of heparan sulfate proteoglycan (HSPG) [75]. To explore whether HSPG can be targeted in solid tumors, Caruana et al. generated HPSE-expressing CAR $\mathrm{T}$ cells that showed ECM degradation ability in solid tumors, which resulted in increased infiltration and antitumor activity [75]. This approach may imply the therapeutic benefits of the use of CAR T immunotherapy coupled with HPSE degradation to access tumor niches.

Another approach of engineering CAR T cells to target solid tumors is the development of the nuclear factor of activated $\mathrm{T}$ cells, which is referred to as $\mathrm{T}$ cells redirected for antigen-unrestricted cytokine-initiated killing (TRUCKs). For instance, engineered CAR T cells with several cytokines, including interleukin-7, $-12,-15$, and -18 , are being explored for TRUCKs [125, 126]. The underlying physiological functions of these interleukins in CAR T-cell therapies are summarized in the reference [126]:

- Interleukin-2: proliferation of T-cell differentiation of $\mathrm{Te}_{\mathrm{ff}}$, development of $\mathrm{T}_{\text {reg }}$ in thymus

- Interleukin-4: differentiation of Th2 and Th9 cells, survial of B-cells and T-cells

- Interleukin-7: development of T-cell in thymus, survival of and homeostasis in memory and naïve T cells

- Interleukin-9: mast cell proliferation, increased antitumor immunity

- Interleukin-15: development of $\mathrm{CD}^{+} \mathrm{T}$-cell memory, survival of and homeostasis in $\mathrm{CD} 8^{+}$ T-cells 
- Interleukin-21: suppression of $\mathrm{T}_{\text {reg }}$, survial and proliferation of $\mathrm{CD} 4^{+}$Th17 cells

Among them, engineered CAR T cells with IL-2, IL-7, IL-15, and IL-21 NFATs are being investigated in clinical trials [126] (Table 1 and Fig. 1).

In addition, it is also known that cytokines can be manipulated to control the metabolism of stem memory $\mathrm{T}$ cells $\left(\mathrm{T}_{\mathrm{SCM}}\right)$ and central memory $\left(\mathrm{T}_{\mathrm{cm}}\right)$ $\mathrm{T}$ cells. Of note, T-cell activation by interleukin families, including IL-15 and IL-17, leads to an increased $\mathrm{T}_{\mathrm{SCM}}$-like phenotype as well as increased interferon-gamma (IFN $\gamma$ ), tumor necrosis factor alpha (TNF $\alpha)$, and IL-2 production [127]. Moreover, it has been reported that IL-15 activates fatty acid oxidation (FAO) and mitochondrial spare respiratory capacity (SRC) as an alternate way for energy production in $\mathrm{T}$ cells [60]. Taken together, IL-15 may provide therapeutic benefits in the form of $\mathrm{T}$ memory cell differentiation and mitochondrial metabolism [60]. In addition to mitochondrial metabolism, manipulation of ion and $\mathrm{pH}$ levels in the tumor microenvironment, such as decreasing the concentration of potassium, can also enhance T-cell antitumor activity [128]. As such, these metabolism-targeting approaches will provide the rationales for future clinical developments and therapeutic use of CAR T-cell immunotherapy for cancer patients.

\section{Conclusion}

The immunosuppressive microenvironments in solid tumors are physically and functionally hostile for immune cells, including immune checkpoint inhibitors and CAR T cells. The reasons for less promising efficacy of immunotherapies vary and include the immune cells' poor accessibility to tumor cells in the TME due to physical and metabolic barriers, including a lack of nutrients and acidosis. In order to improve the therapeutic efficacy of immunotherapies, the tightly controlled microenvironment has to be modified by targeting the metabolic vulnerability of cancer cells. This includes either targeting metabolic enzymes to regulate the metabolism of cancer cells or disrupting the tumor-friendly microenvironment. As metabolism is fundamental for biological and cellular functions, targeting the tumor microenvironment itself or modifying T-cell metabolism is a promising strategy to improve current treatment efficacy.

\section{References}

1. Antonio, M. J., Zhang, C., \& Le, A. (2021). Different tumor microenvironments lead to different metabolic phenotypes. Advances in Experimental Medicine and Biology, 1311, https:// doi.org/10.1007/978-3-030-65768-0_10

2. Nabi, K., \& Le, A. (2021). The intratumoral heterogeneity of cancer metabolism. Advances in Experimental Medicine and Biology, 1311, https:// doi.org/10.1007/978-3-030-65768-0_11

3. Hoang, G., Udupa, S., \& Le, A. (2019). Application of metabolomics technologies toward cancer prognosis and therapy. International Review of Cell and Molecular Biology, 347, 191-223.

4. Munn, D. H., et al. (2002). Potential regulatory function of human dendritic cells expressing indoleamine 2,3-dioxygenase. Science, 297(5588), 1867-1870.

5. Lee, G. K., et al. (2002). Tryptophan deprivation sensitizes activated $\mathrm{T}$ cells to apoptosis prior to cell division. Immunology, 107(4), 452-460.

6. Rodriguez, P. C., et al. (2004). Arginase I production in the tumor microenvironment by mature myeloid cells inhibits T-cell receptor expression and antigenspecific T-cell responses. Cancer Research, 64(16), 5839-5849.

7. Uyttenhove, C., et al. (2003). Evidence for a tumoral immune resistance mechanism based on tryptophan degradation by indoleamine 2,3-dioxygenase. Nature Medicine, 9(10), 1269-1274.

8. Adams, J. L., et al. (2015). Big opportunities for small molecules in immuno-oncology. Nature Reviews. Drug Discovery, 14(9), 603-622.

9. Mondanelli, G., et al. (2019). Immunoregulatory interplay between arginine and tryptophan metabolism in health and disease. Frontiers in Immunology, $10,1565$.

10. June, C. H. (2007). Adoptive T cell therapy for cancer in the clinic. The Journal of Clinical Investigation, 117(6), 1466-1476.

11. Leen, A. M., Rooney, C. M., \& Foster, A. E. (2007). Improving $\mathrm{T}$ cell therapy for cancer. Annual Review of Immunology, 25, 243-265.

12. Kershaw, M. H., Westwood, J. A., \& Darcy, P. K. (2013). Gene-engineered T cells for cancer therapy. Nature Reviews. Cancer, 13(8), 525-541. 
13. Ribas, A. (2015). Adaptive immune resistance: How cancer protects from immune attack. Cancer Discovery, 5(9), 915-919.

14. Vesely, S., et al. (2013). Parameters derived from the postoperative decline in ultrasensitive PSA improve the prediction of radical prostatectomy outcome. World Journal of Urology, 31(2), 299-304.

15. Cubillos-Ruiz, J. R., et al. (2015). ER stress sensor XBP1 controls anti-tumor immunity by disrupting dendritic cell homeostasis. Cell, 161(7), 1527-1538.

16. Ramakrishnan, R., et al. (2014). Oxidized lipids block antigen cross-presentation by dendritic cells in cancer. Journal of Immunology, 192(6), 2920-2931.

17. Nieman, K. M., et al. (2011). Adipocytes promote ovarian cancer metastasis and provide energy for rapid tumor growth. Nature Medicine, 17(11), 1498-1503.

18. Sazeides, C., \& Le, A. (2021). Metabolic relationship between cancer-associated fibroblasts and cancer cells. Advances in Experimental Medicine and Biology, 1311, https://doi. org/10.1007/978-3-030-65768-0_14

19. Jung, J. G., \& Le, A. (2021). Targeting metabolic cross talk between cancer cells and cancer associated fibroblasts. Advances in Experimental Medicine and Biology, 1311, https://doi. org/10.1007/978-3-030-65768-0_15

20. Hsu, Y. L., et al. (2016). Lung cancer-derived galectin-1 contributes to cancer associated fibroblastmediated cancer progression and immune suppression through TDO2/kynurenine axis. Oncotarget, 7(19), 27584-27598.

21. Arcucci, A., et al. (2016). Cancer: An oxidative crosstalk between solid tumor cells and cancer associated fibroblasts. BioMed Research International, 2016, 4502846.

22. Buck, M. D., O’Sullivan, D., \& Pearce, E. L. (2015). $\mathrm{T}$ cell metabolism drives immunity. The Journal of Experimental Medicine, 212(9), 1345-1360.

23. O’Neill, L. A., \& Pearce, E. J. (2016). Immunometabolism governs dendritic cell and macrophage function. The Journal of Experimental Medicine, 213(1), 15-23.

24. Le, A., et al. (2012). Glucose-independent glutamine metabolism via TCA cycling for proliferation and survival in B cells. Cell Metabolism, 15(1), 110-121.

25. Pavlova, N. N., \& Thompson, C. B. (2016). The emerging hallmarks of cancer metabolism. Cell Metabolism, 23(1), 27-47.

26. Andrejeva, G., \& Rathmell, J. C. (2017). Similarities and distinctions of cancer and immune metabolism in inflammation and tumors. Cell Metabolism, 26(1), 49-70.

27. Li, T., Copeland, C., \& Le, A. (2021). Glutamine metabolism in cancer. Advances in Experimental Medicine and Biology, 1311, https://doi. org/10.1007/978-3-030-65768-0_2

28. Jin, L., Alesi, G. N., \& Kang, S. (2016). Glutaminolysis as a target for cancer therapy. Oncogene, 35(28), 3619-3625.
29. Perez-Escuredo, J., et al. (2016). Lactate promotes glutamine uptake and metabolism in oxidative cancer cells. Cell Cycle, 15(1), 72-83.

30. Swamy, M., et al. (2016). Glucose and glutamine fuel protein O-GlcNAcylation to control T cell selfrenewal and malignancy. Nature Immunology, 17(6), 712-720.

31. Tyrakis, P. A., et al. (2016). S-2-hydroxyglutarate regulates $\mathrm{CD} 8(+)$ T-lymphocyte fate. Nature, 540(7632), 236-241.

32. Wang, Q., et al. (2015). Targeting ASCT2-mediated glutamine uptake blocks prostate cancer growth and tumour development. The Journal of Pathology, 236(3), 278-289.

33. Fox, C. J., Hammerman, P. S., \& Thompson, C. B. (2005). Fuel feeds function: Energy metabolism and the T-cell response. Nature Reviews. Immunology, 5(11), 844-852.

34. Rathmell, J. C., et al. (2001). IL-7 enhances the survival and maintains the size of naive T cells. Journal of Immunology, 167(12), 6869-6876.

35. Wofford, J. A., et al. (2008). IL-7 promotes Glut1 trafficking and glucose uptake via STAT5-mediated activation of Akt to support T-cell survival. Blood, 111(4), 2101-2111.

36. Macintyre, A. N., et al. (2014). The glucose transporter Glut1 is selectively essential for CD4 T cell activation and effector function. Cell Metabolism, 20(1), 61-72.

37. Blad, C. C., Tang, C., \& Offermanns, S. (2012). G protein-coupled receptors for energy metabolites as new therapeutic targets. Nature Reviews. Drug Discovery, 11(8), 603-619.

38. Rubic, T., et al. (2008). Triggering the succinate receptor GPR91 on dendritic cells enhances immunity. Nature Immunology, 9(11), 1261-1269.

39. Csoka, B., et al. (2012). Adenosine promotes alternative macrophage activation via $\mathrm{A} 2 \mathrm{~A}$ and $\mathrm{A} 2 \mathrm{~B}$ receptors. The FASEB Journal, 26(1), 376-386.

40. Kidani, Y., \& Bensinger, S. J. (2012). Liver X receptor and peroxisome proliferator-activated receptor as integrators of lipid homeostasis and immunity. Immunological Reviews, 249(1), 72-83.

41. Wang, R., et al. (2011). The transcription factor Myc controls metabolic reprogramming upon T lymphocyte activation. Immunity, 35(6), 871-882.

42. Gerriets, V. A., \& Rathmell, J. C. (2012). Metabolic pathways in $\mathrm{T}$ cell fate and function. Trends in Immunology, 33(4), 168-173.

43. Dang, C. V., Le, A., \& Gao, P. (2009). MYC-induced cancer cell energy metabolism and therapeutic opportunities. Clinical Cancer Research, 15(21), 6479-6483.

44. Le, A., \& Dang, C. V. (2013). Studying Myc's role in metabolism regulation. Methods in Molecular Biology, 1012, 213-219.

45. Frauwirth, K. A., et al. (2002). The CD28 signaling pathway regulates glucose metabolism. Immunity, 16(6), 769-777. 
46. Michalek, R. D., et al. (2011). Estrogen-related receptor-alpha is a metabolic regulator of effector T-cell activation and differentiation. Proceedings of the National Academy of Sciences of the United States of America, 108(45), 18348-18353.

47. Wang, R., \& Green, D. R. (2012). Metabolic checkpoints in activated $\mathrm{T}$ cells. Nature Immunology, 13(10), 907-915.

48. Michalek, R. D., et al. (2011). Cutting edge: Distinct glycolytic and lipid oxidative metabolic programs are essential for effector and regulatory CD4+ T cell subsets. Journal of Immunology, 186(6), 3299-3303.

49. Jacobs, S. R., et al. (2008). Glucose uptake is limiting in $\mathrm{T}$ cell activation and requires CD28-mediated Akt-dependent and independent pathways. Journal of Immunology, 180(7), 4476-4486.

50. Carr, E. L., et al. (2010). Glutamine uptake and metabolism are coordinately regulated by ERK/ MAPK during $\mathrm{T}$ lymphocyte activation. Journal of Immunology, 185(2), 1037-1044.

51. Murray, C. M., et al. (2005). Monocarboxylate transporter MCT1 is a target for immunosuppression. Nature Chemical Biology, 1(7), 371-376.

52. Doedens, A. L., et al. (2013). Hypoxia-inducible factors enhance the effector responses of CD8(+) $\mathrm{T}$ cells to persistent antigen. Nature Immunology, 14(11), 1173-1182.

53. Finlay, D. K., et al. (2012). PDK1 regulation of mTOR and hypoxia-inducible factor 1 integrate metabolism and migration of $\mathrm{CD} 8+\mathrm{T}$ cells. The Journal of Experimental Medicine, 209(13), 2441-2453.

54. Chou, C., et al. (2014). c-Myc-induced transcription factor AP4 is required for host protection mediated by CD8+ T cells. Nature Immunology, 15(9), 884-893.

55. Kim, J. W., et al. (2006). HIF-1-mediated expression of pyruvate dehydrogenase kinase: A metabolic switch required for cellular adaptation to hypoxia. Cell Metabolism, 3(3), 177-185.

56. Papandreou, I., et al. (2006). HIF-1 mediates adaptation to hypoxia by actively downregulating mitochondrial oxygen consumption. Cell Metabolism, 3(3), 187-197.

57. Shi, L. Z., et al. (2011). HIF1alpha-dependent glycolytic pathway orchestrates a metabolic checkpoint for the differentiation of TH17 and Treg cells. The Journal of Experimental Medicine, 208(7), 1367-1376.

58. Harty, J. T., \& Badovinac, V. P. (2008). Shaping and reshaping CD8+ T-cell memory. Nature Reviews. Immunology, 8(2), 107-119.

59. Rosenblum, M. D., Way, S. S., \& Abbas, A. K. (2016). Regulatory T cell memory. Nature Reviews. Immunology, 16(2), 90-101.

60. van der Windt, G. J., \& Pearce, E. L. (2012). Metabolic switching and fuel choice during T-cell differentiation and memory development. Immunological Reviews, 249(1), 27-42.
61. Crespo, J., et al. (2013). T cell anergy, exhaustion, senescence, and stemness in the tumor microenvironment. Current Opinion in Immunology, 25(2), 214-221.

62. Munn, D. H., \& Mellor, A. L. (2013). Indoleamine 2,3 dioxygenase and metabolic control of immune responses. Trends in Immunology, 34(3), 137-143.

63. Munn, D. H., et al. (1999). Inhibition of T cell proliferation by macrophage tryptophan catabolism. The Journal of Experimental Medicine, 189(9), 1363-1372.

64. Fischer, K., et al. (2007). Inhibitory effect of tumor cell-derived lactic acid on human T cells. Blood, 109(9), 3812-3819.

65. Cham, C. M., et al. (2008). Glucose deprivation inhibits multiple key gene expression events and effector functions in CD8+ T cells. European Journal of Immunology, 38(9), 2438-2450.

66. Bose, S., Zhang, C., \& Le, A. (2021). Glucose metabolism in cancer: The Warburg effect and beyond. Advances in Experimental Medicine and Biology, 1311, https://doi. org/10.1007/978-3-030-65768-0_1

67. Gatenby, R. A., \& Gillies, R. J. (2004). Why do cancers have high aerobic glycolysis? Nature Reviews. Cancer, 4(11), 891-899.

68. Warburg, O. (1956). On the origin of cancer cells. Science, 123(3191), 309-314.

69. Chang, C. H., et al. (2015). Metabolic competition in the tumor microenvironment is a driver of cancer progression. Cell, 162(6), 1229-1241.

70. Ho, P. C., et al. (2015). Phosphoenolpyruvate is a metabolic checkpoint of anti-tumor T cell responses. Cell, 162(6), 1217-1228.

71. Sugiura, A., \& Rathmell, J. C. (2018). Metabolic barriers to $\mathrm{T}$ cell function in tumors. Journal of Immunology, 200(2), 400-407.

72. D'Aloia, M. M., et al. (2018). CAR-T cells: The long and winding road to solid tumors. Cell Death \& Disease, 9(3), 282.

73. Mikucki, M. E., et al. (2015). Non-redundant requirement for $\mathrm{CXCR} 3$ signalling during tumoricidal T-cell trafficking across tumour vascular checkpoints. Nature Communications, 6, 7458.

74. Stewart, M. D., \& Sanderson, R. D. (2014). Heparan sulfate in the nucleus and its control of cellular functions. Matrix Biology, 35, 56-59.

75. Caruana, I., et al. (2015). Heparanase promotes tumor infiltration and antitumor activity of CAR-redirected T lymphocytes. Nature Medicine, 21(5), 524-529.

76. Tahmasebi, S., Elahi, R., \& Esmaeilzadeh, A. (2019). Solid tumors challenges and new insights of CAR $\mathrm{T}$ cell engineering. Stem Cell Reviews and Reports, 15(5), 619-636.

77. Wang, L. C., et al. (2014). Targeting fibroblast activation protein in tumor stroma with chimeric antigen receptor $\mathrm{T}$ cells can inhibit tumor growth and augment host immunity without severe toxicity. Cancer Immunology Research, 2(2), 154-166. 
78. Nishio, N., \& Dotti, G. (2015). Oncolytic virus expressing RANTES and IL-15 enhances function of CAR-modified $\mathrm{T}$ cells in solid tumors. Oncoimmunology, 4(2), e988098.

79. Whilding, L. M., et al. (2019). CAR T-cells targeting the integrin alphavbeta6 and co-expressing the chemokine receptor CXCR2 demonstrate enhanced homing and efficacy against several solid malignancies. Cancers (Basel), 11, 5.

80. Hosen, N., et al. (2017). The activated conformation of integrin beta7 is a novel multiple myelomaspecific target for CAR $\mathrm{T}$ cell therapy. Nature Medicine, 23(12), 1436-1443.

81. Posey, A. D., Jr., et al. (2016). Engineered CAR T cells targeting the cancer-associated Tn-glycoform of the membrane mucin MUC1 control adenocarcinoma. Immunity, 44(6), 1444-1454.

82. Zhou, R., et al. (2019). CAR T cells targeting the tumor MUC1 glycoprotein reduce triple-negative breast cancer growth. Frontiers in Immunology, 10, 1149.

83. Koneru, M., et al. (2015). IL-12 secreting tumortargeted chimeric antigen receptor $\mathrm{T}$ cells eradicate ovarian tumors in vivo. Oncoimmunology, 4(3), e994446.

84. Zhang, L., et al. (2013). Inhibition of TGF-beta signaling in genetically engineered tumor antigenreactive $\mathrm{T}$ cells significantly enhances tumor treatment efficacy. Gene Therapy, 20(5), 575-580.

85. Mohammed, S., et al. (2017). Improving chimeric antigen receptor-modified $\mathrm{T}$ cell function by reversing the immunosuppressive tumor microenvironment of pancreatic cancer. Molecular Therapy, 25(1), 249-258.

86. Adachi, K., et al. (2018). IL-7 and CCL19 expression in CAR-T cells improves immune cell infiltration and CAR-T cell survival in the tumor. Nature Biotechnology, 36(4), 346-351.

87. Arab, S., \& Hadjati, J. (2019). Adenosine blockage in tumor microenvironment and improvement of cancer immunotherapy. Immune Network, 19(4), e23.

88. Beavis, P. A., et al. (2017). Targeting the adenosine $2 \mathrm{~A}$ receptor enhances chimeric antigen receptor $\mathrm{T}$ cell efficacy. The Journal of Clinical Investigation, 127(3), 929-941.

89. Srivastava, S., \& Riddell, S. R. (2018). Chimeric antigen receptor T cell therapy: Challenges to benchto-bedside efficacy. Journal of Immunology, 200(2), 459-468.

90. Arab, S., et al. (2017). Increased efficacy of a dendritic cell-based therapeutic cancer vaccine with adenosine receptor antagonist and CD73 inhibitor. Tumour Biology, 39(3), 1010428317695021.

91. Ligtenberg, M. A., et al. (2016). Coexpressed catalase protects chimeric antigen receptor-redirected $\mathrm{T}$ cells as well as bystander cells from oxidative stress-induced loss of antitumor activity. Journal of Immunology, 196(2), 759-766.

92. Ninomiya, S., et al. (2015). Tumor indoleamine 2,3-dioxygenase (IDO) inhibits CD19-CAR T cells and is downregulated by lymphodepleting drugs. Blood, 125(25), 3905-3916.

93. Newick, K., Moon, E., \& Albelda, S. M. (2016). Chimeric antigen receptor T-cell therapy for solid tumors. Molecular Therapy Oncolytics, 3, 16006.

94. Scheffel, M. J., et al. (2016). Efficacy of adoptive T-cell therapy is improved by treatment with the antioxidant $\mathrm{N}$-acetyl cysteine, which limits activation-induced T-cell death. Cancer Research, 76(20), 6006-6016.

95. Peggs, K. S., et al. (2009). Blockade of CTLA-4 on both effector and regulatory $\mathrm{T}$ cell compartments contributes to the antitumor activity of anti-CTLA-4 antibodies. The Journal of Experimental Medicine, 206(8), 1717-1725.

96. Ren, J., et al. (2017). A versatile system for rapid multiplex genome-edited CAR $\mathrm{T}$ cell generation. Oncotarget, 8(10), 17002-17011.

97. John, L. B., et al. (2013). Anti-PD-1 antibody therapy potently enhances the eradication of established tumors by gene-modified T cells. Clinical Cancer Research, 19(20), 5636-5646.

98. Rupp, L. J., et al. (2017). CRISPR/Cas9-mediated PD-1 disruption enhances anti-tumor efficacy of human chimeric antigen receptor T cells. Scientific Reports, 7(1), 737.

99. Liu, X., et al. (2016). A chimeric switch-receptor targeting PD1 augments the efficacy of secondgeneration CAR T cells in advanced solid tumors. Cancer Research, 76(6), 1578-1590.

100. Yoon, D. H., et al. (2018). Incorporation of immune checkpoint blockade into chimeric antigen receptor T cells (CAR-Ts): Combination or built-In CAR-T. International Journal of Molecular Sciences, 19, 2.

101. Fourcade, J., et al. (2012). CD8(+) T cells specific for tumor antigens can be rendered dysfunctional by the tumor microenvironment through upregulation of the inhibitory receptors BTLA and PD-1. Cancer Research, 72(4), 887-896.

102. Boice, M., et al. (2016). Loss of the HVEM tumor suppressor in lymphoma and restoration by modified CAR-T cells. Cell, 167(2), 405-418. e13.

103. Johnston, R. J., Yu, X., \& Grogan, J. L. (2015). The checkpoint inhibitor TIGIT limits antitumor and antiviral CD8(+) T cell responses. Oncoimmunology, 4(9), e1036214.

104. Kuhn, N. F., et al. (2019). CD40 ligand-modified chimeric antigen receptor $\mathrm{T}$ cells enhance antitumor function by eliciting an endogenous antitumor response. Cancer Cell, 35(3), 473-488. e6.

105. Kershaw, M. H., et al. (2002). Redirecting migration of $\mathrm{T}$ cells to chemokine secreted from tumors by genetic modification with CXCR2. Human Gene Therapy, 13(16), 1971-1980.

106. Long, A. H., et al. (2016). Reduction of MDSCs with all-trans retinoic acid improves CAR therapy efficacy for sarcomas. Cancer Immunology Research, 4(10), 869-880.

107. Zhou, Q., et al. (2010). Program death-1 signaling and regulatory $\mathrm{T}$ cells collaborate to resist the function of adoptively transferred cytotoxic $\mathrm{T}$ lympho- 
cytes in advanced acute myeloid leukemia. Blood, 116(14), 2484-2493.

108. Markley, J. C., \& Sadelain, M. (2010). IL-7 and IL-21 are superior to IL-2 and IL-15 in promoting human $\mathrm{T}$ cell-mediated rejection of systemic lymphoma in immunodeficient mice. Blood, 115(17), 3508-3519.

109. Yao, X., et al. (2012). Levels of peripheral CD4(+) FoxP3(+) regulatory $\mathrm{T}$ cells are negatively associated with clinical response to adoptive immunotherapy of human cancer. Blood, 119(24), 5688-5696.

110. Spear, P., et al. (2012). Chimeric antigen receptor $\mathrm{T}$ cells shape myeloid cell function within the tumor microenvironment through IFN-gamma and GM-CSF. Journal of Immunology, 188(12), 6389-6398.

111. Chmielewski, M., \& Abken, H. (2017). CAR T cells releasing IL-18 convert to T-Bet(high) FoxO1(low) effectors that exhibit augmented activity against advanced solid tumors. Cell Reports, 21(11), 3205-3219.

112. Clever, D., et al. (2016). Oxygen sensing by T cells establishes an immunologically tolerant metastatic niche. Cell, 166(5), 1117-1131. e14.

113. Scharping, N. E., et al. (2016). The tumor microenvironment represses $\mathrm{T}$ cell mitochondrial biogenesis to drive intratumoral $\mathrm{T}$ cell metabolic insufficiency and dysfunction. Immunity, 45(2), 374-388.

114. Wherry, E. J., \& Kurachi, M. (2015). Molecular and cellular insights into $\mathrm{T}$ cell exhaustion. Nature Reviews. Immunology, 15(8), 486-499.

115. Vaddepally, R. K., et al. (2020). Review of indications of FDA-approved immune checkpoint inhibitors per NCCN guidelines with the level of evidence. Cancers (Basel), 12, 3.

116. Chang, K. C., et al. (2013). Blockade of the negative co-stimulatory molecules PD-1 and CTLA-4 improves survival in primary and secondary fungal sepsis. Critical Care, 17(3), R85.
117. Bengsch, B., et al. (2016). Bioenergetic insufficiencies due to metabolic alterations regulated by the inhibitory receptor PD-1 are an early driver of CD8(+) $\mathrm{T}$ cell exhaustion. Immunity, 45(2), 358-373.

118. Parry, R. V., et al. (2005). CTLA-4 and PD-1 receptors inhibit T-cell activation by distinct mechanisms. Molecular and Cellular Biology, 25(21), 9543-9553.

119. Patsoukis, N., et al. (2015). PD-1 alters T-cell metabolic reprogramming by inhibiting glycolysis and promoting lipolysis and fatty acid oxidation. Nature Communications, 6, 6692.

120. Riley, J. L. (2009). PD-1 signaling in primary T cells. Immunological Reviews, 229(1), 114-125.

121. Kleffel, S., et al. (2015). Melanoma cell-intrinsic PD-1 receptor functions promote tumor growth. Cell, 162(6), 1242-1256.

122. Staron, M. M., et al. (2014). The transcription factor FoxO1 sustains expression of the inhibitory receptor PD-1 and survival of antiviral CD8(+) T cells during chronic infection. Immunity, 41(5), 802-814.

123. Ma, S., et al. (2019). Current progress in CAR-T cell therapy for solid tumors. International Journal of Biological Sciences, 15(12), 2548-2560.

124. Juillerat, A., et al. (2017). An oxygen sensitive selfdecision making engineered CAR T-cell. Scientific Reports, 7, 39833.

125. Petersen, C. T., \& Krenciute, G. (2019). Next generation CAR T cells for the immunotherapy of highgrade glioma. Frontiers in Oncology, 9, 69.

126. Dwyer, C. J., et al. (2019). Fueling cancer immunotherapy with common gamma chain cytokines. Frontiers in Immunology, 10, 263.

127. Gomez-Eerland, R., et al. (2014). Manufacture of gene-modified human T-cells with a memory stem/ central memory phenotype. Human Gene Therapy Methods, 25(5), 277-287.

128. Eil, R., et al. (2016). Ionic immune suppression within the tumour microenvironment limits $\mathrm{T}$ cell effector function. Nature, 537(7621), 539-543.

Open Access This chapter is licensed under the terms of the Creative Commons Attribution 4.0 International License (http://creativecommons.org/licenses/by/4.0/), which permits use, sharing, adaptation, distribution and reproduction in any medium or format, as long as you give appropriate credit to the original author(s) and the source, provide a link to the Creative Commons license and indicate if changes were made.

The images or other third party material in this chapter are included in the chapter's Creative Commons license, unless indicated otherwise in a credit line to the material. If material is not included in the chapter's Creative Commons license and your intended use is not permitted by statutory regulation or exceeds the permitted use, you will need to obtain permission directly from the copyright holder.

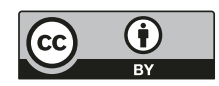




\section{Part III}

Relationship Between Cancer Cells and Cancer-Associated Fibroblasts 


\title{
Metabolic Relationship Between Cancer-Associated Fibroblasts and Cancer Cells
}

\author{
Christos Sazeides and Anne Le
}

Keywords
Cancer-associated fibroblasts $\cdot$ CAF-derived
exosomes $\cdot$ Glutamine metabolism $\cdot$ Hypoxia-
inducible factor- $1 \cdot$ Reverse Warburg effect $\cdot$
miRNA $\cdot$ TGF- $\beta \cdot$ Alanine $\cdot$ Cav-1

Abbreviations

$\begin{array}{ll}\text { ATG16L1 } & \begin{array}{l}\text { Autophagy-related 16 like 1 } \\ \text { BNIP3 }\end{array} \\ & \begin{array}{l}\text { BCL2/adenovirus E1B 19 } \mathrm{kDa} \\ \text { protein-interacting protein 3 }\end{array} \\ \text { BNIP3L } & \begin{array}{l}\text { BCL2/adenovirus E1B 19 kDa } \\ \text { protein-interacting protein 3-like }\end{array} \\ \text { CAF } & \begin{array}{l}\text { Cancer-associated fibroblast } \\ \text { Cav-1 }\end{array} \\ \text { CDE } & \text { CAveolin-1 } \\ \text { CTSB } & \text { Cathepsin B }\end{array}$

C. Sazeides

Icahn School of Medicine at Mount Sinai,

New York, NY, USA

\section{A. Le $(\bowtie)$}

Department of Pathology and Oncology, Johns

Hopkins University School of Medicine,

Baltimore, MD, USA

Department of Chemical and Biomolecular Engineering, Johns Hopkins University Whiting School of Engineering, Baltimore, MD, USA e-mail: annele@jhmi.edu
EMT Epithelial-mesenchymal transition

FASN Fatty acid synthase

FH Fumarase

HIF-1 Hypoxia-inducible factor-1

I $\mathrm{B} \quad$ Inhibitor of NF- $\mathrm{KB}$

IкBK IкB kinase

LDHA

LDHB

MCT

miRNA

Lactate dehydrogenase A

Lactate dehydrogenase B

Monocarboxylate transporter

mtROS Mitochondrial ROS

$\mathrm{NF}$

$\mathrm{NF \kappa B}$

NHE1

PDAC

PGC- $1 \alpha$

PKFM

PKM2

PSC

ROS

SDH

SIRT

TCA

TGF- $\beta$

TME

TP53INP1

Normal fibroblasts

Nuclear factor kappa-light-chainenhancer of activated B cells

Sodium-hydrogen exchanger 1

Pancreatic ductal adenocarcinoma

PPARG coactivator 1 alpha

6-Phosphofructokinase, muscle type

Pyruvate kinase isozymes M1/M2

Pancreatic stellate cells

Reactive oxygen species

Succinate dehydrogenase

Sirtuin

Tricarboxylic acid

Transforming growth factor-beta

Tumor microenvironment

Tumor protein p53-inducible nuclear protein 1

$\alpha$-KG $\quad \alpha$-Ketoglutarate

$\alpha$-SMA $\quad \alpha$-Smooth muscle actin 


\section{Key Points}

- Cancer-associated fibroblasts undergo the reverse Warburg effect and provide cancer cells with glycolytic metabolites.

- The interaction between cancer cells and CAFs helps cancer cells manage the Warburg effect.

- Loss of stromal Cav-1 is a biomarker of poor prognosis in breast cancers.

- Exogenous and endogenous miRNAs are crucial in the metabolic reprogramming of CAFs.

- CAF-derived exosomes (CDEs) can reprogram the metabolic pathway of cancer cells.

- CAF-derived lactate is crucial in prostate cancer metabolic transformation towards OXPHOS.

- CAFs can transfer functional mitochondria to prostate cancer cells.

- CAFs augment cancer's addiction to glutamine and its metabolically relevant consequences.

- Alanine secreted by pancreatic stellate cells supports tumor metabolism.

- CAFs act as lipid synthesis factories for colorectal cancer cells.

\section{Introduction}

Cancer-associated fibroblasts (CAFs), a major component of the tumor microenvironment (TME), play an important role in cancer initiation, progression, and metastasis. Recent findings have demonstrated that the TME not only provides physical support for cancer cells but also directs cell-to-cell interactions (in this case, the interaction between cancer cells and CAFs). As cancer progresses, the CAFs also coevolve, transitioning from an inactivated state to an activated state. The elucidation and understanding of the interaction between cancer cells and CAFs will pave the way for new cancer therapies [1-3].

The TME is a heterogeneous environment consisting of fibroblasts, tumor-associated macrophages, adipocytes, an extracellular matrix, and mesenchymal stem cells [4]. The exact composition of each stroma varies depending on cancer and tissue type. To add to this variation, there is heterogeneity even within the CAF population itself. Different CAFs express different markers and influence stromal pro-tumorigenic capacity and cancer progression in diverse ways $[5,6]$.

CAFs, unlike normal fibroblasts (NF), are not passive bystanders. They possess similar characteristics to myofibroblasts, the fibroblasts responsible for wound healing and chronic inflammation, such as the expression of $\alpha$-smooth muscle actin ( $\alpha$-SMA) $[7,8]$. Regarded in a similar light, cancer might be considered a wound that cannot be healed. CAFs can originate from the activation and differentiation of quiescent fibroblasts, bone marrow-derived mesenchymal stem cells, and epithelial and endothelial cells [9].

The interaction of the TME, specifically among CAFs with cancer cells, is incontrovertible. The effect of CAFs on cancer is dependent on cancer type and stage. The production and secretion of growth factors, chemokines, cytokines, metabolites, and extracellular matrix components aid in the recruitment of various cell types, such as pericytes and endothelial cells, facilitating angiogenesis and bestowing chemoresistant properties to the cancer cells. In this chapter, we discuss the properties and characteristics of CAFs, and their importance in cancer progression.

As mentioned in the chapter "Different Tumor Microenvironments Lead to Different Metabolic Phenotypes" [10], Hanahan and Weinberg [11] have identified six hallmark capabilities of cancer cells: (1) self-sufficiency in growth signals, (2) insensitivity to anti-growth signals, (3) evasion of apoptosis, (4) limitless replicative potential, (5) sustained angiogenesis, and (6) tissue invasion and metastasis. The exact mechanisms by which the TME can influence cancer and lead to the acquisition of those hallmark capabilities are not yet fully understood. However, there is growing evidence suggesting that the manipulation of signal transduction pathways in cancer cells, CAFs, and altered metabolic pathways may play a role in the transformation process [12-17]. 


\section{CAFs Undergo the Reverse Warburg Effect and Provide Cancer Cells with Glycolytic Metabolites}

As mentioned in previous chapters, cancer cells undergo a phenomenon known as the Warburg effect, an increase in aerobic glycolysis to produce ATP even in normoxic conditions (normoxia or normal oxygen levels) [18]. Warburg initially attributed this phenomenon to malfunctioning mitochondria forcing the cancer cells to rely on glycolysis for energy production. Pyruvate and lactate, the two end products of glycolysis, were believed to be secreted by the hypoxic core of the tumor through monocarboxylate transporters (MCT4) for the adjacent oxygenated cancer cells to take up (via MCT1) and utilize as substrates for the tricarboxylic acid (TCA) cycle [19-21].

Recent studies, however, have revolutionized the way scientists view the TME, especially the cross talk between CAFs and cancer cells and the effect of this cross talk on metabolism. The Warburg effect, a phenomenon initially believed to be limited to cancer cells, has also been observed in the fibroblasts surrounding the cancer cells. To distinguish this CAF-related phenomenon from its cancer cell-related counterpart, Pavlides et al. named it the reverse Warburg effect [17]. Caveolin-1 (Cav-1) is a transforming growth factor-beta (TGF- $\beta$ ) type I receptor kinase inhibitor, and the loss of Cav-1 expression causes a myofibroblastic phenotype. By using Cav-1(-/-) fibroblasts, Pavlides et al. induced myofibroblastic differentiation to mimic CAFs. With the use of proteomics, they identified 25 proteins that were overexpressed when Cav-1 was suppressed. Eight of those proteins were glycolytic enzymes (Table 1), including M2-type pyruvate kinase (PKM2) and lactate dehydrogenase A (LDHA) [17]. These two enzymes are known to play crucial roles in the Warburg effect [22, 23]. Additionally, two enzymes involved in oxidative stress, peroxiredoxin 1 and catalase, were overexpressed under normoxic conditions, which indicates an increase of reactive oxygen species (ROS) in Cav-1(-/-) fibroblasts. Hypoxiainducible factor-1 (HIF-1) is a transcription factor that responds to low oxygen concentrations. Under high levels of ROS, HIF-1 is stabilized. Subsequently, HIF-1, a regulator of all glycolytic enzymes, as well as glucose transporters, GLUT1 and GLUT3, induces aerobic glycolysis [17, 23].

A similar study performed by Shan et al. provided further evidence to support the reverse Warburg effect hypothesis. In this study, pancreatic associated fibroblasts expressed elevated levels of the glycolytic enzymes LDHA and PKM2, as well as the MCT4 transporter responsible for lactate secretion. Additionally, they observed that when pancreatic cancer cells were exposed to CAF-conditioned media, they underwent enhanced aerobic activity, causing an observable enlargement of the mitochondria. Furthermore, pancreatic cancer cells significantly increased the expression of MCT1, fumarate hydratase $(\mathrm{FH})$, and succinate dehydrogenase (SDH). The overexpression of these enzymes further indicated the existence of metabolic coupling between CAFs and cancer cells [24].

\section{The Interaction Between Cancer Cells and CAFs Helps Cancer Cells Manage the Warburg Effect}

Even though the extratumoral high lactate concentration produced by CAFs is crucial for the progression of cancer, high intracellular lactate concentration causes a dramatic drop in the $\mathrm{pH}$, which, if left untreated, results in the death of the cell. Interestingly, experimental research revealed a few mechanisms by which cancer cells manage the elevated lactate level as a result of the Warburg effect [25-28]. Cancer cells overexpress a $\mathrm{Na}^{+} / \mathrm{H}^{+}$ transporter, NHE1 (sodium-hydrogen exchanger 1), that pumps $\mathrm{H}^{+}$out of the cell and $\mathrm{Na}^{+}$into it, therefore neutralizing this decrease in $\mathrm{pH}$ caused by lactate [26]. Under hypoxic conditions, cancer cells overexpress carbonic anhydrase 9 (CA9), which is responsible for the conversion of carbon dioxide to bicarbonate to neutralize increased acidity [27]. Certain cancer cells also overexpress MCT4, the transporter involved in secreting lactate out of the cell. By doing this, if intracel- 
Table 1 Glycolytic enzymes upregulated in Cav-1(-/-) mammary stromal fibroblasts

\begin{tabular}{l|l}
\hline Glycolytic and metabolic enzymes & Metabolic reaction involved \\
\hline M2-type pyruvate kinase & Phosphoenolpyruvate $\rightarrow$ pyruvate \\
\hline Phosphoglycerate kinase I & Glycerate-1,3P2 $\leftrightarrow$ glycerate-3P \\
\hline Lactate dehydrogenase A & Lactate $\leftrightarrow$ pyruvate \\
\hline Fructose-bisphosphate aldolase A & Fructose-1,6P2 $\leftrightarrow$ glyceraldehyde-3P + dihydroxyacetone-P \\
\hline Glycerol 3-phosphate dehydrogenase 2 & Dihydroxyacetone-P $\leftrightarrow$ glycerol-3P \\
\hline Enolase I & Glycerate-2P $\leftrightarrow$ phosphoenolpyruvate \\
\hline Triosephosphate isomerase I & Glyceraldehyde-3P $\leftrightarrow$ dihydroxyacetone-P \\
\hline Phosphoglycerate mutase & Glycerate-3P $\leftrightarrow$ glycerate-2P \\
\hline
\end{tabular}

All eight enzymes lead to the overproduction of pyruvate and lactate, which are then secreted in the medium for adjacent cancer cells to take up and utilize as an energy source

lular lactate concentration goes too high, some of it can be secreted to prevent the $\mathrm{pH}$ from dropping too low [28]. Cancer cells adjacent to autophagic CAFs upregulate TP53-induced glycolysis and the apoptosis regulator (TIGAR). TIGAR is capable of protecting cancer cells against oxidative stress by inhibiting autophagy and apoptosis while simultaneously shifting cells towards oxidative phosphorylation (OXPHOS) and away from aerobic glycolysis [29]. Finally, several antioxidant enzymes, such as peroxiredoxin-1, have been observed to be upregulated in certain cancer cells [2]. It is likely that as more experiments are performed involving the TME and cancer cells, more evasion mechanisms will be elucidated.

\section{$4 \quad$ Loss of Stromal Cav-1 Is an Indicator of Poor Prognosis in Breast Cancers}

The importance of Cav-1 in transdifferentiating normal fibroblasts into myofibroblasts is well established. Recent experiments have shed light on the complex mechanisms by which cancer cells modulate their environment and manage to downregulate Cav-1 expression in fibroblasts. Cav-1 inhibits TGF- $\beta$ type I receptor kinase. The lack of Cav-1 expression in the Cav-1(-/-) null skin fibroblasts can induce a myofibroblastic phenotype. One of the most widely known tumorderived factors involved in the activation of CAFs is TGF- $\beta 1[1,30,31]$. Interestingly, in the absence of CAFs, TGF- $\beta$ itself in cancer cells has no direct effect on cancer proliferation and survival [31]. It is believed that cancer-derived TGF- $\beta$ acts in a paracrine manner and causes the downregulation of Cav- $1 \alpha$ in CAFs. This event results in the overexpression of ROS by CAFs that can act both in an autocrine and a paracrine fashion, stimulating themselves and nearby fibroblasts to acquire a myofibroblastic phenotype. ROS inhibit prolyl hydroxylase (PHD) from targeting the transcription factor HIF- $1 \alpha$ for degradation [32-34]. As a result, HIF-1 $\alpha$ gets stabilized and translocated into the nucleus causing the overexpression of autophagy genes, BNIP3 (BCL2/adenovirus E1B $19 \mathrm{kDa}$ proteininteracting protein 3) and BNIP3L (BCL2/adenovirus $\mathrm{E} 1 \mathrm{~B} 19 \mathrm{kDa}$ protein-interacting protein 3-like), which compete with Beclin-1. Beclin-1 then acts as a mitophagy/autophagy factor causing the dysfunction of mitochondria and, thus, the increase of ROS, acting on a positive feedback loop [35, 36]. HIF-1 $\alpha$ also upregulates CTSB (cathepsin B) and ATG16L1 (autophagyrelated 16 like 1), which are markers for autophagy and mitophagy, respectively [29]. Additionally, TGF- $\beta$ causes the upregulation of BNIP3, BNIP3L, and CTSB (cathepsin B), all of which can induce mitophagy/autophagy and therefore shift the cell away from OXPHOS and towards aerobic glycolysis [31]. BNIP3, BNIP3L, and CTSB increase lactate production, whereas ATG16L1 increases ketone production [37]. Lactate and ketone bodies can then be utilized by cancer cells to enhance tumor growth [37]. TGF$\beta$, therefore, promotes tumorigenesis via CAF metabolism, and specifically TGF- $\beta$ in fibroblasts 


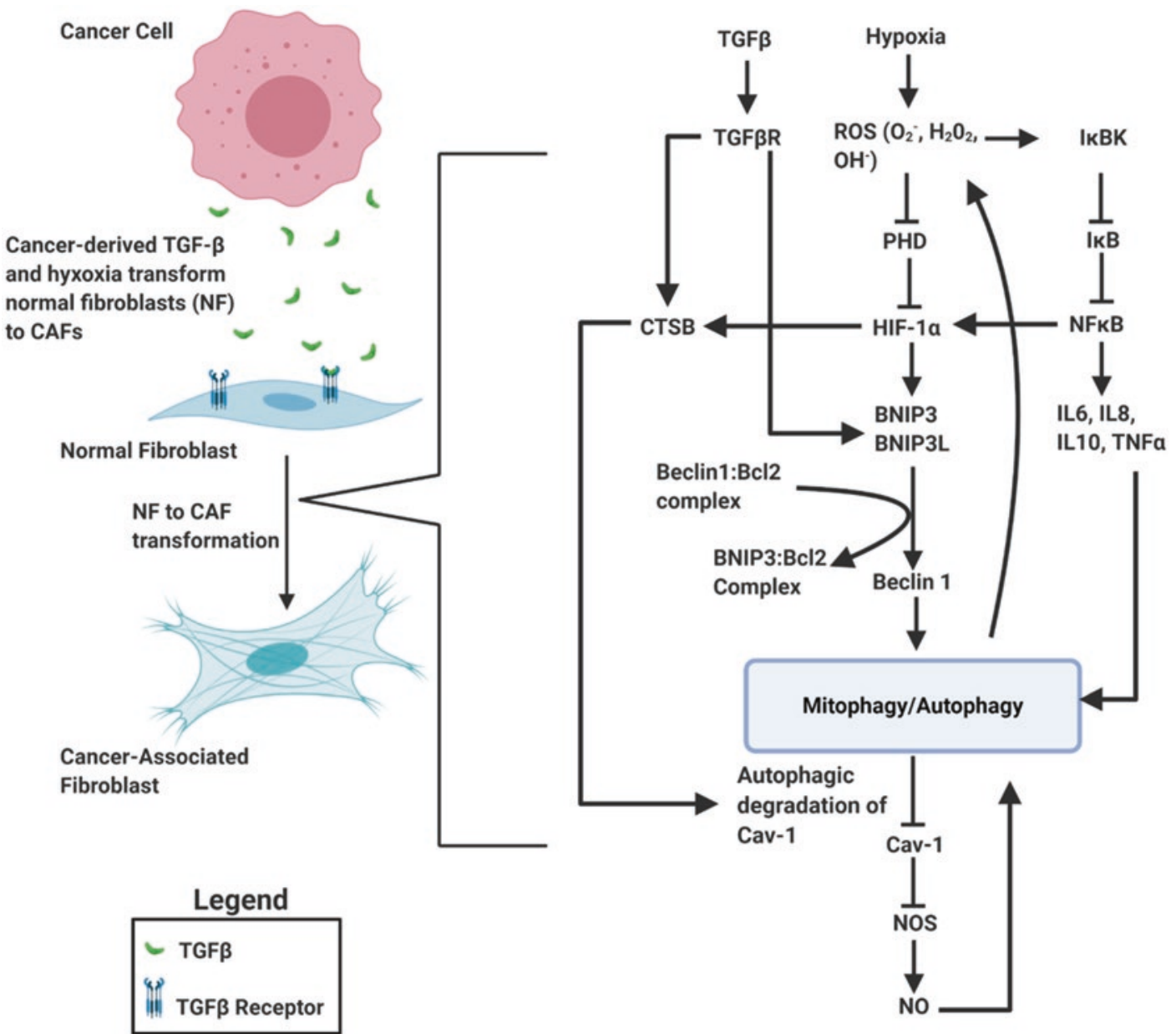

Fig. 1 Conversion of normal fibroblasts (NF) to cancer-associated fibroblasts (CAF) through cancer-induced hypoxia and cancer-derived TGF $\beta$

leads to the upregulated mitochondrial activity of cancer cells and tumor growth [31] (Fig. 1).

The rapid proliferation of cancer, without a significant increase in vascularization, limits oxygen availability for normal fibroblasts, thus creating a hypoxic environment that forces the fibroblasts to undergo metabolic changes [1]. Hypoxia results in the stabilization of HIF-1 $\alpha$, which, as described previously, is a very important transcription factor for genes involved in autophagy, mitochondrial biogenesis, and general energy homeostasis [29]. Furthermore, under normoxia, NFKB (nuclear factor kappa-light-chain-enhancer of activated $\mathrm{B}$ cells), a key inducer of autophagy, is inhibited by

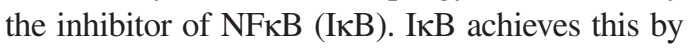

sequestering the nuclear localization signal of $\mathrm{NF \kappa B}$, therefore rendering it inactive in the cytoplasm [38]. However, hypoxic conditions activate

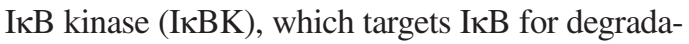
tion by phosphorylation and thus promotes the activation of NFkB [39]. Even though the exact mechanism by which $\mathrm{NF \kappa B}$ is able to direct autophagy is unclear, it is believed that this transcription factor upregulates the expression of certain inflammatory cytokines, such as IL-6, IL-8, IL-10, and TNFo [40, 41]. These inflammatory mediators are able to induce autophagy independent from each other [40, 41]. Finally, NFkB also binds to the HIF-1 $\alpha$ promoter and results in its upregulation [42] (Fig. 1). Hypoxia- and TGF- $\beta$ - 
induced autophagy cause the lysosomal degradation of Cav-1 as well as mitochondrial dysfunction and degradation, leading to a highly glycolytic state in CAFs. Cav- $1 \alpha$ normally inhibits nitric oxide synthase and prevents the accumulation of nitric oxide (NO). In the absence of Cav-1 $\alpha$, NO accumulates and inhibits cytochrome c oxidase, causing mitochondrial uncoupling and thus rendering mitochondria susceptible to mitophagy [43]. This results in high amounts of lactate, pyruvate, ketone bodies, glutamine, and free fatty acids [2, $24,44]$ that can be utilized by adjacent cancer cells.

The aforementioned oxidative stress and hypoxia derived from Cav-1 loss lead to mitochondrial dysfunction and degradation. Mitochondrial dysfunction causes the premature reaction of electrons with oxygen, leading to the generation of ROS, such as $\mathrm{O}^{2-}, \mathrm{H}_{2} \mathrm{O}_{2}$, and $\mathrm{OH}$. [2]. ROS induce oxidative stress, stabilize HIF-1 $\alpha$, and inhibit NFKB in a positive feedback manner. The fact that TGF was not able to stimulate a significant increase in angiogenesis and vascularization suggests that the growth stimulated by CAFs depends on the paracrine supply of high-energy molecules such as lactate, pyruvate, ketone bodies, amino acids, and fatty acids [45, 46].

\section{5 miRNAs Play a Crucial Role in CAF Metabolic Reprogramming}

MicroRNAs (miRNAs) are short noncoding RNAs that target mRNA and, therefore, can regulate gene transcription at the posttranscriptional level [47]. miRNAs have been shown to be upregulated in CAFs, as well as secreted by various tumors into the TME in microvesicles (MV) [47-51].

\subsection{The Role of Endogenous miRNAs in the Metabolic Reprogramming of CAFs}

Isocitrate dehydrogenase $3 \alpha$ (IDH $3 \alpha)$, the enzyme responsible for the conversion of isocitrate to $\alpha$-ketoglutarate, is downregulated in colon cancer CAFs and melanoma CAFs following TGF $\beta$ exposure [48]. This downregulation of
IDH $3 \alpha$ is attributed to the increased levels of miR-424 which leads to the accumulation of succinate and fumarate, which in turn inhibits the activation of PHD2 (the predominant enzyme that degrades HIF-1 $\alpha$ by hydroxylation), thus leading to stabilization of HIF- $1 \alpha[48,52]$. It is this HIF- $1 \alpha$ stabilization and activity that leads to the upregulation of transporters and various glycolytic enzymes, such as glucose transporter 1 (GLUT1), hexokinase 2 (HK2), and 6-phosphofructokinase, muscle type (PKFM) [48], and causes an increase in glucose uptake and lactate production, as well as a decrease in oxygen consumption by these fibroblasts [48].

Additionally, HIF- $1 \alpha$ in colon cancer CAFs and melanoma CAFs is able to inhibit OXPHOS by downregulating mitochondria complex I through the overexpression of a complex I inhibitor, known as NADH dehydrogenase 1 alpha subcomplex 4-like 2 (NDUFA4L2) [48]. It is noteworthy that the expression of NDUFA4L2 is TGF $\beta$ dose and time dependent [48].

In another set of experiments, miR-21 was shown to play a critical role in pancreatic cancer CAF development [49]. Metabolic coupling was evident between CAFs that had higher expression of miR-21 and pancreatic cancer cells. miR-21 upregulation increases glucose uptake and lactate production in CAFs, while at the same time upregulating SDH and FH in pancreatic cancers [49].

\subsection{The Role of Exogenous miRNA in the Metabolic Reprogramming of CAFs}

Yao et al. studied the interaction between pancreatic cancer cells and fibroblasts in 2015 [47]. They identified that miR-155 was packaged in MVs and secreted by pancreatic cancer cells [47]. Once in NFs, miR-155 exerted its transformative role by targeting the TP53INP1 gene and transforming NFs into CAFs $[47,50]$. The transformative ability of miR-155 could be explained by the fact that TP53INP1 has pro-apoptotic properties and its downregulation by miR-155 leads to decreased mitophagy and accumulation of dysfunctional mitochondria that produce high levels of ROS [47, 51]. 
Table 2 Amino acids present in various CDEs

\begin{tabular}{l|l|l}
\hline Amino acid & $\begin{array}{l}\text { CDE from } \\
\text { prostate CAF }\end{array}$ & $\begin{array}{l}\text { CDE from } \\
\text { pancreatic CAF }\end{array}$ \\
\hline Alanine & Yes & Yes \\
\hline Anserine & & Yes \\
\hline Arginine & Yes & \\
\hline Asparagine & Yes & \\
\hline Citrulline & Yes & Yes \\
\hline Cysteine & Yes & Yes \\
\hline Glutamic acid & Yes & Yes \\
\hline Glutamine & Yes & Yes \\
\hline Glycine & Yes & Yes \\
\hline Histidine & Yes & Yes \\
\hline Isoleucine & & Yes \\
\hline Leucine & Yes & Yes \\
\hline Lysine & Yes & Yes \\
\hline Methionine & Yes & Yes \\
\hline Ornithine & Yes & Yes \\
\hline Phenylalanine & Yes & \\
\hline Phosphoserine & Yes & Yes \\
\hline Proline & Yes & Yes \\
\hline Serine & Yes & Yes \\
\hline Threonine & & Yes \\
\hline Tryptophan & & Yes \\
\hline Valine & & \\
\hline
\end{tabular}

Table 3 The ten most abundant miRNAs present in CDE and their respective target genes

\begin{tabular}{l|l}
\hline $\begin{array}{l}\text { Exosomal } \\
\text { miRNA }\end{array}$ & OXPHOS gene silenced \\
\hline miR-302d-3p & UQCRFS1 \\
\hline miR-29b-3p & $\begin{array}{l}\text { NDUFA10, ATP5G1, ATP6V1A, } \\
\text { ATP5G3 }\end{array}$ \\
\hline miR-22-3p & ATP6V1A \\
\hline miR-155-5p & ATP5G3 \\
\hline miR-25-3p & NDUFS4 \\
\hline miR-29a-3p & ATP5G1, ATP6V1A, ATP5G3 \\
\hline miR-23a-3p & UQCRFS1, NDUFS4, ATP6V0E2, \\
\hline miR-21-5p & ATP5L, ATP5G2 \\
\hline miR-16-5p & ATP5G3 \\
\hline miR-222-3p & ATP6V1A \\
\hline
\end{tabular}

6

CAF-Derived Exosomes
(CDEs) Can Reprogram
the Metabolic Pathway
of Cancer Cells

Much research has been focused on exosomes secreted by cancer cells, while little is known about exosomes secreted by CAFs. Zhao et al., with the use of isotopologue tracing [53], showed that CAF-derived exosomes (CDEs) are taken up by cancer cells in a KRAS-independent mechanism and are, indeed, capable of reprogramming the metabolic activity of pancreatic and prostate cancer cells [54]. They demonstrated how CDE can sustain the rapidly dividing cancer cells under hypoxic conditions or when the normal oxidative mitochondrial function has been disabled. Additionally, the presence of CDEs can rescue prostate and pancreatic cancer cells from starvation by providing de novo-synthesized metabolites, such as amino acids (Table 2). This suggests that there is constant communication between the cancer cells and the adjacent fibroblasts, where both constantly coevolve [54].

\subsection{CDEs Contain miRNAs that Downregulate Oxidative Phosphorylation of Cancer Cells}

CAF-derived exosomes (CDEs) contain amino acids, fatty acids, pyruvate, lactate, miRNA, and many other compounds. miRNAs are essential in regulating gene expression [54]. Zhao et al. showed that miRNAs present in CDEs are capable of downregulating all 109 OXPHOS-related genes in cancer cells. As shown in Table 3, the ten most abundant miRNAs present in these CDEs target one or more OXPHOS genes, leading to decreased OXPHOS. Therefore, cancer cells must rely on alternative metabolic pathways to maintain their rapid proliferation [54].

\subsection{Effect of CDEs on Glycolysis and TCA of Cancer Cells}

With the use of GC-MS and ${ }^{13} \mathrm{C}_{6}$-glucose, Zhao et al. showed that glucose from CDEs was the main glycolytic substrate for cancer cells. This was evident due to the increase in labeled glycolytic metabolites, lactate and pyruvate, in prostate cancer cells cultured with CDEs, and the reduced amount of non-labeled pyruvate and lactate (Fig. 2). Additionally, they further 
showed that the labeled metabolites involved in the TCA cycle (citrate, $\alpha$-ketoglutarate, fumarate, and malate) are found in significantly lower concentrations when cultured with CDEs. Therefore, the increase of labeled glycolytic metabolites and the decrease of labeled TCA metabolites suggest that glucose provided by CDEs is primarily used in glycolysis and not in mitochondrial oxidative phosphorylation [54].

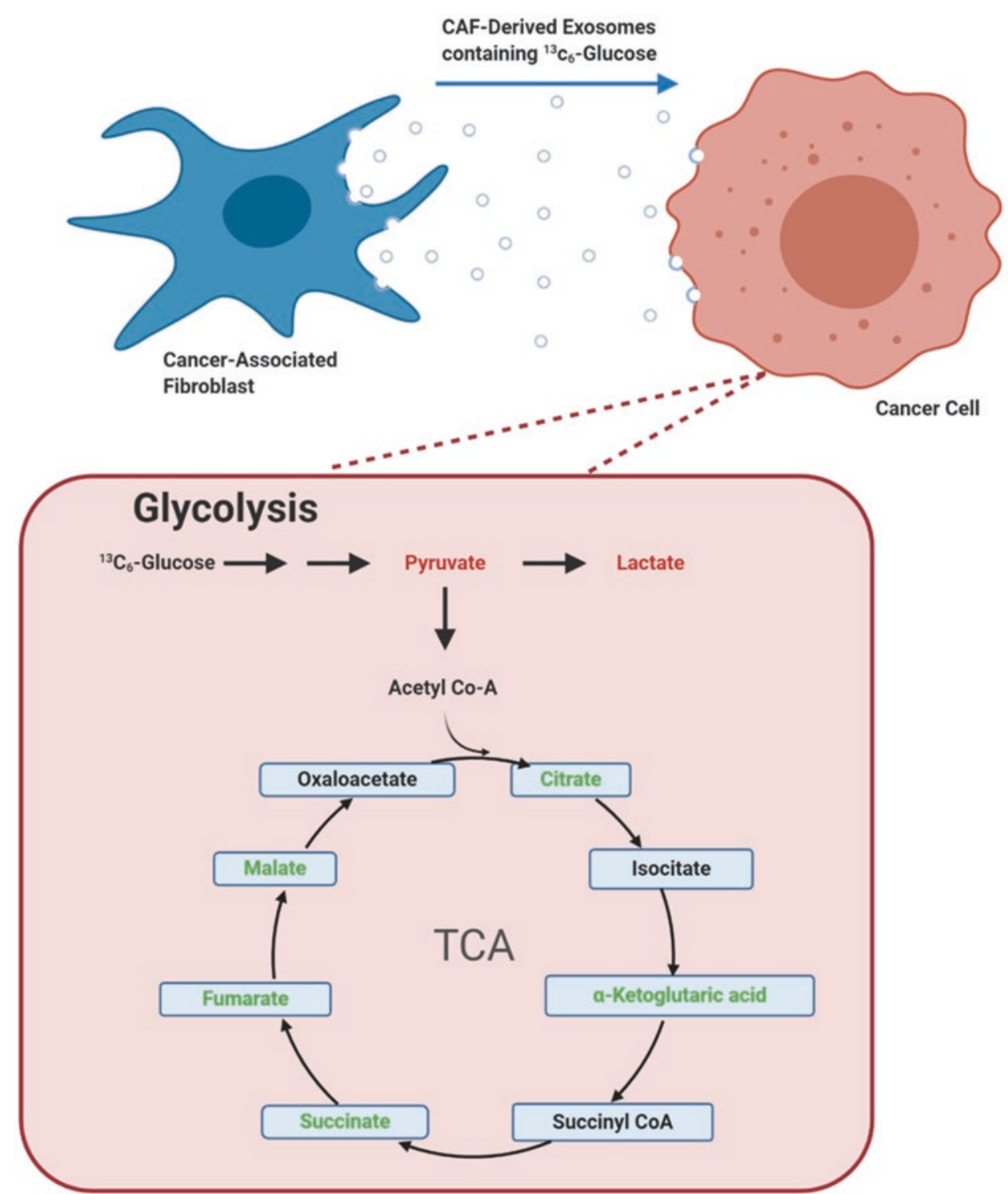

Fig. 2 CDE-derived glucose is mainly used in cancer cell glycolysis and, to a lesser extent, the TCA cycle. Metabolites in red represent the metabolites found in cancer cells present in high concentrations resulting from CDE-derived glucose. Metabolites in green represent the metabolites found in cancer cells present in low concentrations from CDEderived glucose 


\subsection{Glutamine from CDEs Undergoes Mainly Reductive Metabolism that Also Results in Aberrant Lipogenesis in Adjacent Cancer Cells}

Glutamine is another major carbon source for the TCA cycle and a nitrogen source for protein synthesis [55-58]. Zhao et al. identified the contribution of CDE-derived glutamine to the TCA cycle in the cancer cells using $\mathrm{U}_{-}{ }^{13} \mathrm{C}_{5}$-glutamine isotopologue tracing [54]. Under both normoxic and hypoxic conditions, glutamine can enter the oxidative metabolic pathway and produce oxaloacetate, which then combines with acetyl-CoA to form citrate, a fatty acid precursor. Additionally, under hypoxic conditions, glutamine enters the reductive metabolic pathway generating $\alpha$-ketoglutarate and then citrate [59]. As shown in Fig. 3, citrate is eventually converted to fumarate and then malate. The presence of $\mathrm{M}+5$ citrate, $\mathrm{M}+3$ fumarate, and $\mathrm{M}+3$ malate in high concentrations suggests that cancer cells mainly rely on the reductive glutamine metabolism when the normal mitochondrial function of the cell is disrupted. Additional evidence to support this is the decreased $M+4 / M+5$ citrate ratio. $M+4$ citrate is derived from the oxidative pathway of glutamine, whereas $M+5$ citrate is from the reductive pathway, and therefore this reduced $M+4 / M+5$ citrate ratio confirms the predominance of the glutamine reductive pathway [54]. Furthermore, a major component and requirement for cell proliferation is lipogenesis, the generation of fatty acids for cell membranes [60]. Zhao et al. also showed that exposure to CDEs resulted in increased acetate contribution and simultaneously decreased pyruvate contribution to lipogenesis. This event suggests that the main source of carbon for acetyl-CoA upon exposure to CDEs is the glutamine reductive carboxylation pathway and not the oxidative glucose pathway. Finally, metabolic analysis of CDEs revealed significant amounts of stearate and palmitate that can be directly utilized by the cancer cells for lipid synthesis [54]. It is worth mentioning that fatty acid synthase (FASN) expression has been found to be elevated in numerous types of cancer [61]. Even though there is still no direct link between CAFs and overexpression of FASN, this could be the result of the coevolution of stroma and cancer. However, more research is required before conclusions can be drawn.

\section{CAF-Derived Lactate Is more Than Just a Metabolite}

Sirtuins (SIRTs) are deacetylases that are activated when NADH/NAD+ ratios are unbalanced and act as sensors of nutrient deprivation [62-64]. SIRT1, specifically, targets and activates peroxisome proliferator-activated receptor gamma coactivator 1-alpha (PGC-1 $\alpha$ ), a transcription factor that promotes mitochondrial respiration and OXPHOS [65-68]. CAF-derived lactate is converted to pyruvate by the upregulated enzyme lactate dehydrogenase B (LDHB), leading to the accumulation of NADH. High levels of NADH result in the increased expression of SIRT1. Currently, lactate is the only metabolite capable of inducing SIRT1 activation and PGC- $1 \alpha$ deacetylation and therefore proves the importance of lactate as more than just a metabolite. NADH is then oxidized back to NAD+, and the unbalanced high levels of NAD+ cause the activation of SIRT1, thus help maintain the increased mitochondrial activity and OXPHOS in cancer cells [69].

As mentioned earlier, increased mitochondrial activity and oxygen consumption have been recorded in many tumors when co-cultured with CAFs. Prostate cancers are an example [69]. CAF-derived lactate enters the TCA cycle, leading to the accumulation of citrate, succinate, fumarate, and malate, but not to a significant increase in $\alpha-K G$ [69]. Additionally, in prostate cancer cells, mitochondrial complexes II-III are downregulated, and complex I is upregulated, leading to the accumulation of mitochondrial reactive oxygen species (mtROS) [69]. The increased complex II dysfunction leads to the accumulation of succinate [69].

mtROS oxidize and activate Scr, a crucial and mandatory step for CAF-derived lactate addiction of prostate cancer cells [69]. Scr is able to sustain the activation of SIRT1/PGC- $1 \alpha$. 


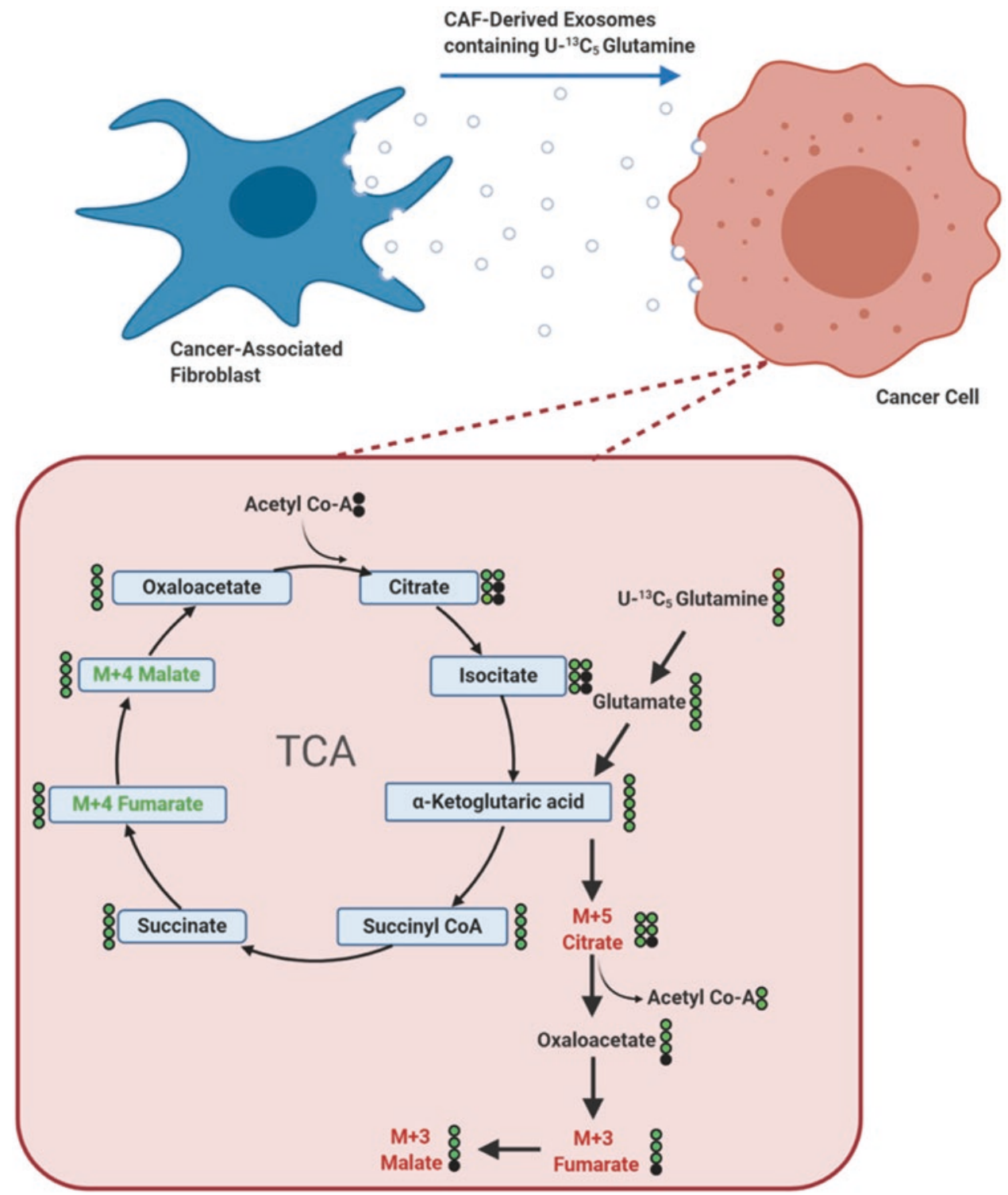

Fig. 3 Oxidative and reductive glutaminolysis. Green circles represent labeled carbons $\left({ }^{13} \mathrm{C}\right)$, and black circles represent non-labeled carbons $\left({ }^{12} \mathrm{C}\right)$. Glutamine derived from CDEs is fully labeled, and its subsequent pathways (oxidative or reductive) in cancer cells are analyzed. Metabolites in red represent the metabolites of the reductive metabolic pathway of glutamine found. Metabolites in green represent the metabolites of the oxidative metabolic pathway of glutamine found

In the absence of Scr activity, prostate cancer cells are unable to utilize CAF-derived lactate in this manner and show no SIRT1/PGC- $1 \alpha$ activity [69].

Even though the increase in both mtROS and PGC- $1 \alpha$ might seem controversial since PGC- $1 \alpha$ is a regulator of the antioxidant response, it is believed that there is a fine balance between the two that helps aid prostate cancer survival and promotes OXPHOS [69]. PGC- $1 \alpha$ could be acting in a negative feedback loop, ensuring that mtROS remain under control [69]. Taken together, CAF-derived lactate is a crucial metabolite in prostate cancer metabolic transformation 
towards OXPHOS through mtROS production, Src activation, and SIRT1/PGC-1 $\alpha$ activity.

\section{CAFs"Surrender" Their Functional Mitochondria to Prostate Cancer Cells}

In vitro experiments showed that CAFs are able to surrender their functional mitochondria to prostate cancer cells [69]. This effect is more prominent in higher grade prostate tumors [69]. Interestingly, after exposure to CAF-conditioned media, significantly more prostate tumor cells were able to receive CAF-derived mitochondria. This suggests that CAFs act in a paracrine manner to prime prostate cancer cells and prepare them to receive functional mitochondria [69]. Additionally, confocal microscopy and imaging techniques were able to show the transfer of mitochondria from CAFs to cancer cells through nanotubule formation. NFs were unable to donate their mitochondria, suggesting that this is a property limited to CAFs and not to all fibroblasts [69].

Prostate cancer cells that received CAFderived mitochondria express higher levels of MCT1, the transporter that transports lactate into the cell. Those prostate cancer cells also upregulate SIRT- 1 and PGC- $1 \alpha$. Interestingly, inhibition of MCT1 prevents the receipt of CAF-derived mitochondria. The exact mechanism and relation of MCT1 with the mitochondrial transfer are still unknown, but its importance in enhancing OXPHOS metabolism in mitochondrial recipient prostate cancer cells is evident [69]. An increased number of mitochondria and OXPHOS can aid higher grade tumors to meet the high energy demands [69].

\section{CAFs Augment Cancer Addiction to Glutamine and Its Metabolically Relevant Consequences}

As mentioned earlier, there exists constant coevolution between cancer cells and the TME. Cancer cells stop directing glucose into the TCA cycle and, instead, use glucose for the production of nucleotides [70]. Consequently, cancer cells start relying on other carbon sources for oxidative phosphorylation, particularly on glutamine derived from CAFs [54]. However, during glutaminolysis, specifically during the conversion of glutamine to glutamate and then to $\alpha$-ketoglutarate (which enters the TCA cycle), ammonia is released as a by-product [57, 71, 72]. Ammonia is a diffusible compound and an inducer of autophagy. This has detrimental effects on the surrounding stroma, as it causes autophagy in the adjacent CAFs. CAFs, subsequently, undergo autophagy and further release glutamine to be metabolized by the cancer cells. Therefore, a positive feedback loop exists between cancer's addiction to glutamine and CAF's conversion/ autophagy [71, 72].

\section{Alanine Secreted by Pancreatic Stellate Cells Supports Pancreatic Cancer Metabolism}

In one study, Sousa et al. discovered that myofibroblast-like pancreatic stellate cells (PSCs) secreted alanine and were able to support pancreatic cancer metabolism [70]. Among the 200 metabolites analyzed, only alanine and aspartate met the following criteria: (1) increased amounts of metabolite in PSC medium, (2) decreased amounts of metabolite in PSC medium when exposed to pancreatic cancer (PDAC) cells, and (3) increased amounts of metabolite in PDAC after exposure to PSC medium. Furthermore, kinetic studies showed that alanine is secreted even more rapidly than lactate. In fact, PSCderived alanine does not contribute to the production of glycolytic intermediates or alter the NAD+/ $\mathrm{NADH}$ ratio, but rather, it is transaminated to pyruvate, providing additional substrates for the TCA cycle. This intermediate contribution to the TCA cycle subsequently increases oxygen consumption [70]. Alanine-derived pyruvate enters the TCA cycle in the mitochondria and contributes predominantly to the generation of citrate (23-46\% among different PDAC cell lines) and 
isocitrate. To a lesser extent, it also contributes to the generation of malate, fumarate, aspartate, and glutamate. Alanine, therefore, fuels mitochondrial metabolism without affecting glycolysis. Alaninederived citrate is then transported from the mitochondria to the cytosol for lipogenesis. Metabolite tracing showed that alanine significantly contributed to the generation of palmitate and stearate, more than $20 \%$ and $10 \%$ of the total concentrations, respectively [70]. In the presence of alanine, glucose enters the serine biosynthetic pathway in PDAC cells and produces serine and glycine. Serine and glycine can then be used in the biosynthesis of nucleic acids. Under nutrient-deprived conditions, the entry of glucose into the serine biosynthetic pathway is more evident. This suggests that in cases of glucose-deprived conditions, alanine can take over aerobic respiration by providing TCA cycle intermediate metabolites, and subsequently, glucose can then enter different metabolic pathways, such as the serine biosynthetic pathway [70].

The induction of alanine secretion by PSCs for PDAC cells to take up is a two-way intratumoral cross talk. PDAC cells initially stimulate PSCs to undergo autophagy and thus lead to the release of alanine. PSC-derived alanine is then taken up by PDAC cells to contribute to metabolic pathways. In nutrient-rich conditions, the PSC autophagic alanine secretion has a minimum effect on PDAC proliferation. However, in nutrient-deprived conditions, PSC autophagic alanine secretion can significantly rescue and promote the growth of PDAC cells. This effect mainly occurs during the early stages of cancer development. Interestingly, autophagy does not influence the proliferation rate of PSCs themselves [70].

\section{CAFs Act as Lipid Synthesis Factories for Colorectal Cancer Cells}

New research performed by Zhao et al. in 2020 has shown that CAFs overexpress FASN and undergo lipidomic reprogramming to help colorectal cancer cells meet their high energy demands by providing them with newly synthe- sized lipids [73]. Nineteen lipids were specifically identified that were produced and secreted by CAFs and taken up by colorectal cancer cells [73]. However, more research is needed to identify the exact mechanisms that stimulate this lipidomic reprogramming in CAFs and how the de novo lipids are utilized by cancer cells [74].

\section{Reciprocal Communication Is Essential for Cancer Progression}

The importance of KRAS in supporting heterocellular communication was demonstrated by Tape et al. [25]. When PDAC cells were exposed to homocellular conditions, mitochondrial function decreased, and superoxide concentrations increased. However, when PDAC cells were exposed to heterocellular conditions (i.e., cocultured together with CAFs), mitochondrial function was restored, and superoxide concentrations were well regulated. These results suggest that heterocellular and reciprocal communication between CAFs and cancer cells is essential for the progression of cancer. In this experiment, PDAC cells with KRAS mutation initially stimulated the surrounding CAFs to undergo metabolic and cellular changes. Reciprocal stimulation between CAFs and PDAC cells prevents cancer cell mitochondrial dysfunction and superoxide production. The exact signals involved in this dialogue between CAFs and PDAC cells are still unclear, and further research is required to unravel this mechanism [25].

\section{Conclusion}

As cancer research progresses, the significance of the TME in cancer progression is better elucidated [75]. Tumor cell-derived TGF- $\beta$ causes the lysosomal targeting of fibroblastic Cav-1, inducing a myofibroblastic phenotype (activated form). Additionally, the increased oxygen consumption of cancer cells with no significant increase in vascularization induces hypoxia and oxidative stress, causing the stabilization of HIF- $1 \alpha$ and inhibition of I $\mathrm{\kappa B}$ in CAFs. Stabilized HIF-1 $\alpha$ induces 
autophagy and mitophagy. Subsequently, CAFs rely on glycolysis for energy, producing a high amount of lactate, ketone bodies, glutamine, and fatty acids, which are then secreted and taken up by the surrounding cancer cells.

In the case of PDAC, it is evident that the secretion of alanine by PSCs (myofibroblast-like pancreatic stellate cells) is sufficient to rescue cancer cells in low-nutrient environments. It was noted that alanine, not glucose, is used in the TCA cycle. This allows glucose to enter the serine biosynthetic pathway to generate nucleic acids, further contributing to the rapid cancer cell proliferation.

Furthermore, miRNAs play a significant role in metabolically coupling CAFs and various cancers, such as pancreatic cancer. miRNAs can lead to increased glucose uptake and lactate production in CAFs, while at the same time SDH and $\mathrm{FH}$ are upregulated in pancreatic cancers. CAFs have also been demonstrated to "surrender" their functional mitochondria to prostate cancer cells to help cancers meet their high energy demands. In other cases, such as with colorectal cancer, CAFs act as lipid synthesis factories.

CAFs' contribution to cancer progression does not end here. Exosomes derived from CAFs (CDEs) contain a variety of miRNAs responsible for downregulating genes involved in OXPHOS and therefore contribute to the reprogramming of the metabolic activity of cancer cells. CDEs also contain de novo metabolites that enable the rapidly dividing cancer cells to survive in low-nutrient conditions. For example, CDE-derived glutamine undergoes reductive metabolism and generates acetyl-CoA for lipogenesis. However, during glutamine metabolism, ammonia, a diffusible autophagy factor, is produced as a by-product. CAFs that are stimulated by ammonia undergo autophagy and, in turn, further release more glutamine to be metabolized by cancer cells in a positive feedback loop. It is clear that cancer should not be regarded as an individual entity anymore, but rather it should be viewed within the context of its microenvironment. The understanding of the extent of the stromal impact on cancer metabolism and progression can provide new targets for cancer therapy [76].

\section{References}

1. Zhao, X., He, Y., \& Chen, H. (2013). Autophagic tumor stroma: Mechanisms and roles in tumor growth and progression. International Journal of Cancer, 132(1), 1-8.

2. Martinez-Outschoorn, U. E., et al. (2010). Oxidative stress in cancer associated fibroblasts drives tumorstroma co-evolution: A new paradigm for understanding tumor metabolism, the field effect and genomic instability in cancer cells. Cell Cycle, 9(16), 3256-3276.

3. Gascard, P., \& Tlsty, T. D. (2016). Carcinomaassociated fibroblasts: Orchestrating the composition of malignancy. Genes \& Development, 30(9), 1002-1019.

4. Spill, F., et al. (2016). Impact of the physical microenvironment on tumor progression and metastasis. Current Opinion in Biotechnology, 40, 41-48.

5. Chen, Z., et al. (1999). Expression of proinflammatory and proangiogenic cytokines in patients with head and neck cancer. Clinical Cancer Research, 5(6), 1369-1379.

6. Sriram, G., Bigliardi, P. L., \& Bigliardi-Qi, M. (2015). Fibroblast heterogeneity and its implications for engineering organotypic skin models in vitro. European Journal of Cell Biology, 94(11), 483-512.

7. Shimoda, M., Mellody, K. T., \& Orimo, A. (2010). Carcinoma-associated fibroblasts are a rate-limiting determinant for tumour progression. Seminars in Cell \& Developmental Biology, 21(1), 19-25.

8. Rasanen, K., \& Vaheri, A. (2010). Activation of fibroblasts in cancer stroma. Experimental Cell Research, 316(17), 2713-2722.

9. Xouri, G., \& Christian, S. (2010). Origin and function of tumor stroma fibroblasts. Seminars in Cell \& Developmental Biology, 21(1), 40-46.

10. Antonio, M. J., Zhang, C., \& Le, A. (2021). Different tumor microenvironments lead to different metabolic phenotypes. Advances in Experimental Medicine and Biology, 1311, https:// doi.org/10.1007/978-3-030-65768-0_10.

11. Hanahan, D., \& Weinberg, R. A. (2000). The hallmarks of cancer. Cell, 100(1), 57-70.

12. Pavlides, S., et al. (2010). Loss of stromal caveolin-1 leads to oxidative stress, mimics hypoxia and drives inflammation in the tumor microenvironment, conferring the "reverse Warburg effect": A transcriptional informatics analysis with validation. Cell Cycle, 9(11), 2201-2219.

13. Hu, Y., et al. (2015). Fibroblast-derived exosomes contribute to chemoresistance through priming cancer stem cells in colorectal cancer. PLoS One, 10(5), e0125625.

14. DeFilippis, R. A., et al. (2014). Stress signaling from human mammary epithelial cells contributes to phenotypes of mammographic density. Cancer Research, 74(18), 5032-5044.

15. Pietras, K., \& Ostman, A. (2010). Hallmarks of cancer: Interactions with the tumor stroma. Experimental Cell Research, 316(8), 1324-1331. 
16. Semenza, G. L. (2008). Tumor metabolism: Cancer cells give and take lactate. The Journal of Clinical Investigation, 118(12), 3835-3837.

17. Pavlides, S., et al. (2009). The reverse Warburg effect: Aerobic glycolysis in cancer associated fibroblasts and the tumor stroma. Cell Cycle, 8(23), 3984-4001.

18. Bose, S., Zhang, C., \& Le, A. (2021). Glucose metabolism in cancer: The Warburg effect and beyond. Advances in Experimental Medicine and Biology, 1311, https://doi. org/10.1007/978-3-030-65768-0_1.

19. Warburg, O., Wind, F., \& Negelein, E. (1927). The metabolism of tumors in the body. The Journal of General Physiology, 8(6), 519-530.

20. Crabtree, H. G. (1929). Observations on the carbohydrate metabolism of tumours. The Biochemical Journal, 23(3), 536-545.

21. Feron, O. (2009). Pyruvate into lactate and back: From the Warburg effect to symbiotic energy fuel exchange in cancer cells. Radiotherapy and Oncology, 92(3), 329-333.

22. Christofk, H. R., et al. (2008). The M2 splice isoform of pyruvate kinase is important for cancer metabolism and tumour growth. Nature, 452(7184), 230-233.

23. Vander Heiden, M. G., Cantley, L. C., \& Thompson, C. B. (2009). Understanding the Warburg effect: The metabolic requirements of cell proliferation. Science, 324(5930), 1029-1033.

24. Shan, T., et al. (2017). Cancer-associated fibroblasts enhance pancreatic cancer cell invasion by remodeling the metabolic conversion mechanism. Oncology Reports, 37(4), 1971-1979.

25. Tape, C. J., et al. (2016). Oncogenic KRAS regulates tumor cell signaling via stromal reciprocation. Cell, 165(4), 910-920.

26. Chiche, J., Brahimi-Horn, M. C., \& Pouyssegur, J. (2010). Tumour hypoxia induces a metabolic shift causing acidosis: A common feature in cancer. Journal of Cellular and Molecular Medicine, 14(4), 771-794.

27. Swietach, P., et al. (2010). New insights into the physiological role of carbonic anhydrase IX in tumour $\mathrm{pH}$ regulation. Oncogene, 29(50), 6509-6521.

28. Gerlinger, M., et al. (2012). Genome-wide RNA interference analysis of renal carcinoma survival regulators identifies MCT4 as a Warburg effect metabolic target. The Journal of Pathology, 227(2), 146-156.

29. Martinez-Outschoorn, U. E., et al. (2010). Autophagy in cancer associated fibroblasts promotes tumor cell survival: Role of hypoxia, HIF1 induction and NFkappaB activation in the tumor stromal microenvironment. Cell Cycle, 9(17), 3515-3533.

30. Lohr, M., et al. (2001). Transforming growth factorbeta1 induces desmoplasia in an experimental model of human pancreatic carcinoma. Cancer Research, 61(2), 550-555.

31. Guido, C., et al. (2012). Metabolic reprogramming of cancer-associated fibroblasts by TGF-beta drives tumor growth: Connecting TGF-beta signaling with "Warburg-like" cancer metabolism and L-lactate production. Cell Cycle, 11(16), 3019-3035.
32. Pan, Y., et al. (2007). Multiple factors affecting cellular redox status and energy metabolism modulate hypoxia-inducible factor prolyl hydroxylase activity in vivo and in vitro. Molecular and Cellular Biology, 27(3), 912-925.

33. Chandel, N. S., et al. (2000). Reactive oxygen species generated at mitochondrial complex III stabilize hypoxia-inducible factor-1alpha during hypoxia: A mechanism of $\mathrm{O} 2$ sensing. The Journal of Biological Chemistry, 275(33), 25130-25138.

34. Salceda, S., \& Caro, J. (1997). Hypoxia-inducible factor 1alpha (HIF-1alpha) protein is rapidly degraded by the ubiquitin-proteasome system under normoxic conditions. Its stabilization by hypoxia depends on redox-induced changes. The Journal of Biological Chemistry, 272(36), 22642-22647.

35. Bellot, G., et al. (2009). Hypoxia-induced autophagy is mediated through hypoxia-inducible factor induction of BNIP3 and BNIP3L via their BH3 domains. Molecular and Cellular Biology, 29(10), 2570-2581.

36. Klimova, T., \& Chandel, N. S. (2008). Mitochondrial complex III regulates hypoxic activation of HIF. Cell Death and Differentiation, 15(4), 660-666.

37. Capparelli, C., et al. (2012). Autophagy and senescence in cancer-associated fibroblasts metabolically supports tumor growth and metastasis via glycolysis and ketone production. Cell Cycle, 11(12), 2285-2302.

38. Mercurio, F., et al. (1997). IKK-1 and IKK-2: Cytokine-activated IkappaB kinases essential for NF-kappaB activation. Science, 278(5339), 860-866.

39. Cummins, E. P., et al. (2006). Prolyl hydroxylase-1 negatively regulates IkappaB kinase-beta, giving insight into hypoxia-induced NFkappaB activity. Proceedings of the National Academy of Sciences of the United States of America, 103(48), 18154-18159.

40. Martinez-Outschoorn, U. E., et al. (2011). Cytokine production and inflammation drive autophagy in the tumor microenvironment: Role of stromal caveolin-1 as a key regulator. Cell Cycle, 10(11), 1784-1793.

41. Wu, S., \& Sun, J. (2011). Vitamin D, vitamin D receptor, and macroautophagy in inflammation and infection. Discovery Medicine, 11(59), 325-335.

42. Bonello, S., et al. (2007). Reactive oxygen species activate the HIF-1alpha promoter via a functional NFkappaB site. Arteriosclerosis, Thrombosis, and Vascular Biology, 27(4), 755-761.

43. Garcia-Cardena, G., et al. (1997). Dissecting the interaction between nitric oxide synthase (NOS) and caveolin. Functional significance of the nos caveolin binding domain in vivo. The Journal of Biological Chemistry, 272(41), 25437-25440.

44. Martinez-Outschoorn, U. E., et al. (2011). Energy transfer in "parasitic" cancer metabolism: Mitochondria are the powerhouse and Achilles' heel of tumor cells. Cell Cycle, 10(24), 4208-4216.

45. Sotgia, F., et al. (2012). Caveolin-1 and cancer metabolism in the tumor microenvironment: Markers, models, and mechanisms. Annual Review of Pathology, 7 , 423-467. 
46. Martinez-Outschoorn, U. E., Sotgia, F., \& Lisanti, M. P. (2012). Power surge: Supporting cells "fuel" cancer cell mitochondria. Cell Metabolism, 15(1), 4-5.

47. Pang, W., et al. (2015). Pancreatic cancer-secreted miR-155 implicates in the conversion from normal fibroblasts to cancer-associated fibroblasts. Cancer Science, 106(10), 1362-1369.

48. Zhang, D., et al. (2015). Metabolic reprogramming of cancer-associated fibroblasts by IDH3alpha downregulation. Cell Reports, 10(8), 1335-1348.

49. Chen, S., et al. (2018). MiR-21-mediated metabolic alteration of cancer-associated fibroblasts and its effect on pancreatic cancer cell behavior. International Journal of Biological Sciences, 14(1), 100-110.

50. Gironella, M., et al. (2007). Tumor protein 53-induced nuclear protein 1 expression is repressed by miR-155, and its restoration inhibits pancreatic tumor development. Proceedings of the National Academy of Sciences of the United States of America, 104(41), 16170-16175.

51. Saadi, H., Seillier, M., \& Carrier, A. (2015). The stress protein TP53INP1 plays a tumor suppressive role by regulating metabolic homeostasis. Biochimie, 118, 44-50.

52. Selak, M. A., et al. (2005). Succinate links TCA cycle dysfunction to oncogenesis by inhibiting HIF-alpha prolyl hydroxylase. Cancer Cell, 7(1), 77-85.

53. Hoang, G., Udupa, S., \& Le, A. (2019). Application of metabolomics technologies toward cancer prognosis and therapy. International Review of Cell and Molecular Biology, 347, 191-223.

54. Zhao, H., et al. (2016). Tumor microenvironment derived exosomes pleiotropically modulate cancer cell metabolism. eLife, 5, e10250.

55. Dang, C. V. (2010). Glutaminolysis: Supplying carbon or nitrogen or both for cancer cells? Cell Cycle, 9(19), 3884-3886.

56. Daye, D., \& Wellen, K. E. (2012). Metabolic reprogramming in cancer: Unraveling the role of glutamine in tumorigenesis. Seminars in Cell \& Developmental Biology, 23(4), 362-369.

57. Li, T., Copeland, C., \& Le, A. (2021). Glutamine metabolism in cancer. Advances in Experimental Medicine and Biology, 1311, https://doi. org/10.1007/978-3-030-65768-0_2.

58. Le, A., et al. (2012). Glucose-independent glutamine metabolism via TCA cycling for proliferation and survival in B cells. Cell Metabolism, 15(1), 110-121

59. Metallo, C. M., et al. (2011). Reductive glutamine metabolism by IDH1 mediates lipogenesis under hypoxia. Nature, 481(7381), 380-384.

60. Kamphorst, J. J., et al. (2014). Quantitative analysis of acetyl-CoA production in hypoxic cancer cells reveals substantial contribution from acetate. Cancer \& Metabolism, 2, 23.
61. Kumar-Sinha, C., et al. (2003). Transcriptome analysis of HER2 reveals a molecular connection to fatty acid synthesis. Cancer Research, 63(1), 132-139.

62. Guarente, L. (2013). Calorie restriction and sirtuins revisited. Genes \& Development, 27(19), 2072-2085.

63. Houtkooper, R. H., Pirinen, E., \& Auwerx, J. (2012). Sirtuins as regulators of metabolism and healthspan. Nature Reviews. Molecular Cell Biology, 13(4), 225-238.

64. Gambini, J., et al. (2011). Free [NADH]/[NAD(+)] regulates sirtuin expression. Archives of Biochemistry and Biophysics, 512(1), 24-29.

65. Nemoto, S., Fergusson, M. M., \& Finkel, T. (2005). SIRT1 functionally interacts with the metabolic regulator and transcriptional coactivator PGC-1\{alpha\}. The Journal of Biological Chemistry, 280(16), 16456-16460.

66. Benton, C. R., et al. (2008). PGC-1alpha increases skeletal muscle lactate uptake by increasing the expression of MCT1 but not MCT2 or MCT4. Physiological Genomics, 35(1), 45-54.

67. Rodgers, J. T., et al. (2005). Nutrient control of glucose homeostasis through a complex of PGC-1alpha and SIRT1. Nature, 434(7029), 113-118.

68. Tan, Z., et al. (2016). The role of PGC1alpha in cancer metabolism and its therapeutic implications. Molecular Cancer Therapeutics, 15(5), 774-782.

69. Ippolito, L., et al. (2019). Cancer-associated fibroblasts promote prostate cancer malignancy via metabolic rewiring and mitochondrial transfer. Oncogene, 38(27), 5339-5355.

70. Sousa, C. M., et al. (2016). Pancreatic stellate cells support tumour metabolism through autophagic alanine secretion. Nature, 536(7617), 479-483.

71. Marino, G., \& Kroemer, G. (2010). Ammonia: A diffusible factor released by proliferating cells that induces autophagy. Science Signaling, 3(124), pe19.

72. Eng, C. H., \& Abraham, R. T. (2010). Glutaminolysis yields a metabolic by-product that stimulates autophagy. Autophagy, 6(7), 968-970.

73. Gong, J., et al. (2020). Reprogramming of lipid metabolism in cancer-associated fibroblasts potentiates migration of colorectal cancer cells. Cell Death \& Disease, 11(4), 267.

74. Park, J. K., et al. (2021). The heterogeneity of lipid metabolism in cancer. Advances in Experimental Medicine and Biology, 1311, https://doi. org/10.1007/978-3-030-65768-0_3.

75. Nabi, K., \& Le, A. (2021). The intratumoral heterogeneity of cancer metabolism. Advances in Experimental Medicine and Biology, 1311, https:// doi.org/10.1007/978-3-030-65768-0_11.

76. Jung, J. G., \& Le, A. (2021). Targeting metabolic cross talk between cancer cells and cancerassociated fibroblasts. Advances in Experimental Medicine and Biology, 1311, https://doi. org/10.1007/978-3-030-65768-0_15 
Open Access This chapter is licensed under the terms of the Creative Commons Attribution 4.0 International License (http://creativecommons.org/licenses/by/4.0/), which permits use, sharing, adaptation, distribution and reproduction in any medium or format, as long as you give appropriate credit to the original author(s) and the source, provide a link to the Creative Commons license and indicate if changes were made.

The images or other third party material in this chapter are included in the chapter's Creative Commons license, unless indicated otherwise in a credit line to the material. If material is not included in the chapter's Creative Commons license and your intended use is not permitted by statutory regulation or exceeds the permitted use, you will need to obtain permission directly from the copyright holder.

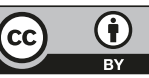




\title{
Targeting Metabolic Cross Talk Between Cancer Cells and Cancer- Associated Fibroblasts
}

\author{
Jin G. Jung and Anne Le
}

Keywords
Cancer-associated fibroblasts $\cdot$ Cancer-
associated adipocytes $\cdot$ Tumor microenviron-
ment $\cdot$ Metabolism $\cdot$ Metabolites $\cdot$ Cancer
therapy

Abbreviations

$\begin{array}{ll}\text { ACAT1 } & \text { Acetyl-CoA acetyltransferase } \\ \text { ACC } & \text { Acetyl-CoA carboxylase } \\ \text { ACCA } & \text { Alpha-cyano-4-hydroxycinnamic } \\ & \text { acid }\end{array}$

ASCT2 Alanine, serine, cysteinepreferring transporter 2

BDH1 3-Hydroxybutyrate dehydrogenase 1

CAAs Cancer-associated adipocytes

CAFs Cancer-associated fibroblasts

ECM Extracellular matrix

J. G. Jung

Department of Pathology, Johns Hopkins University School of Medicine, Baltimore, MD, USA

\footnotetext{
A. Le $(\bowtie)$

Department of Pathology and Oncology, Johns Hopkins University School of Medicine, Baltimore, MD, USA

Department of Chemical and Biomolecular Engineering, Johns Hopkins University Whiting School of Engineering, Baltimore, MD, USA e-mail: annele@jhmi.edu
}

$\begin{array}{ll}\text { EGF } & \text { Epidermal growth factor } \\ \text { HGH } & \text { Human growth hormone } \\ \text { HMG-CoA } & \text { 3-Hydroxy-3-methylglutaryl-CoA } \\ \text { HMGCS2 } & \begin{array}{l}\text { 3-Hydroxy-3-methylglutaryl-CoA } \\ \text { synthase 2 }\end{array} \\ \text { HSP60 } & \text { Heat-shock protein 60 } \\ \text { IL-1 } & \text { Interleukin-1 } \\ \text { MCT1 } & \text { Monocarboxylate transporter 1 } \\ \text { MCT4 } & \text { Monocarboxylate transporter 4 } \\ \text { MMP } & \text { Matrix metalloprotease } \\ \text { NF-кB } & \text { Nuclear factor kappa-light chain- } \\ & \text { enhancer of activated B cells } \\ \text { OXPHOS } & \text { Oxidative phosphorylation } \\ \text { SLC38A5 } & \text { Solute carrier family 38 member 5 } \\ \text { TCA } & \text { Tricarboxylic acid } \\ \text { TME } & \text { Tumor microenvironment } \\ \text { VEGF } & \text { Vascular endothelial growth factor } \\ \beta O H B & \beta \text {-Hydroxybutyrate }\end{array}$

\section{Key Points}

- Metabolic cross talk between CAFs and cancer can be a metabolic target for cancer therapy.

- The reverse Warburg effects can be targeted via disruption of the "lactate shuttle" by MCT1/MCT 4 inhibitors.

- Metformin can be used to inhibit glycolysis and block the function of CAFs, which promote cancer cell growth. 
- Blocking the glutamine uptake of cancer cells from CAFs is a strategy in targeting glutaminolysis.

- Ketone bodies and ketosis in CAFs can be targeted for cancer treatment.

- Fatty acid metabolism from cancer-associated adipocytes (CAAs) serves as a nutrient reservoir for cancer cell growth and is another target for cancer therapy.

\section{Introduction}

Although cancer has classically been regarded as a genetic disease of uncontrolled cell growth, the importance of the tumor microenvironment (TME) $[1,2]$ is continuously emphasized by the accumulating evidence that cancer growth is not simply dependent on the cancer cells themselves $[3,4]$ but also dependent on angiogenesis [5-8], inflammation $[9,10]$, and the supporting roles of cancer-associated fibroblasts (CAFs) [11-13]. After the discovery that CAFs are able to remodel the tumor matrix within the TME and provide the nutrients and chemicals to promote cancer cell growth [14], many studies have aimed to uncover the cross talk between cancer cells and CAFs. Moreover, a new paradigm in cancer metabolism shows how cancer cells act like "metabolic parasites" to take up the high-energy metabolites, such as lactate, ketone bodies, free fatty acids, and glutamine from supporting cells, including CAFs and cancer-associated adipocytes (CAAs) $[15,16]$. This chapter provides an overview of the metabolic coupling between CAFs and cancer cells to further define the therapeutic options to disrupt the CAF-cancer cell interactions.

\section{Overview of the Metabolism of CAFs in Solid Tumors}

Pathological analysis shows that CAFs either are located in the tumor margin or infiltrate the tumor mass, indicating that CAFs and cancer cells are physically and functionally connected to each other $[17,18]$. Of note, other than their locations, the physiological roles of CAFs depend on the existence of neighboring cancer cells [19], lead- ing Madar et al. to propose a new concept: a "CAF state" instead of "cell type" [20].

Studying cancer metabolism is difficult due to the dynamic and rapid metabolic influx/efflux of heterogeneous cancer cells [2]. However, it is clear that the reprogramming of energy metabolism is one of the hallmarks of cancer [21]. Thus, researchers have sought to identify the metabolic vulnerabilities of cancer cells and exploit them for therapy [22, 23]. Cancerfriendly fibroblasts are the most abundant noncancerous cells in solid tumors, and they promote cancer cell growth and induce chemotherapy resistance [24]. Unfortunately, the underlying mechanism of how CAFs help in tumor cell growth remains unclear. However, recent progress in metabolic technologies, including stable isotope-resolved metabolomics, NMR-based metabolomics, and fluorescence-activated cell sorting [25-29], is deepening our insight into the metabolic cross talk between CAFs and cancer cells in the context of metabolic alterations. Using these advanced technologies, CAF-cancer cell interactions have been investigated in various types of cancers, including breast cancer [30], prostate cancer, ovarian cancer, lymphomas [31], non-small cell lung cancer, and head and neck cancers [24]. For instance, CAF produces and releases lactate to the TME, while cancer cells simultaneously utilize this lactate for mitochondrial oxidative phosphorylation (OXPHOS) in order to produce ATP efficiently and rapidly (Fig. 1). Interestingly, not only do CAFs produce these metabolites to help cancer cells grow, but cancer cells also release epidermal growth factor (EGF) to enhance the production and secretion of leptin by CAFs, which eventually leads to tumor progression [32]. Additionally, tumor cells express pro-inflammatory genes, including nuclear factor kappa-light-chainenhancer of activated $\mathrm{B}$ cells $(\mathrm{NF}-\mathrm{\kappa B})$ and interleukin-1 (IL-1), so that normal fibroblasts can be guided by cancer cells to become pro-inflammatory CAFs [33] (Fig. 1).

Moreover, several studies have identified CAF gene expression profiles, including certain extracellular matrix (ECM) components and several matrix metalloproteases (MMP2, MMP11, 


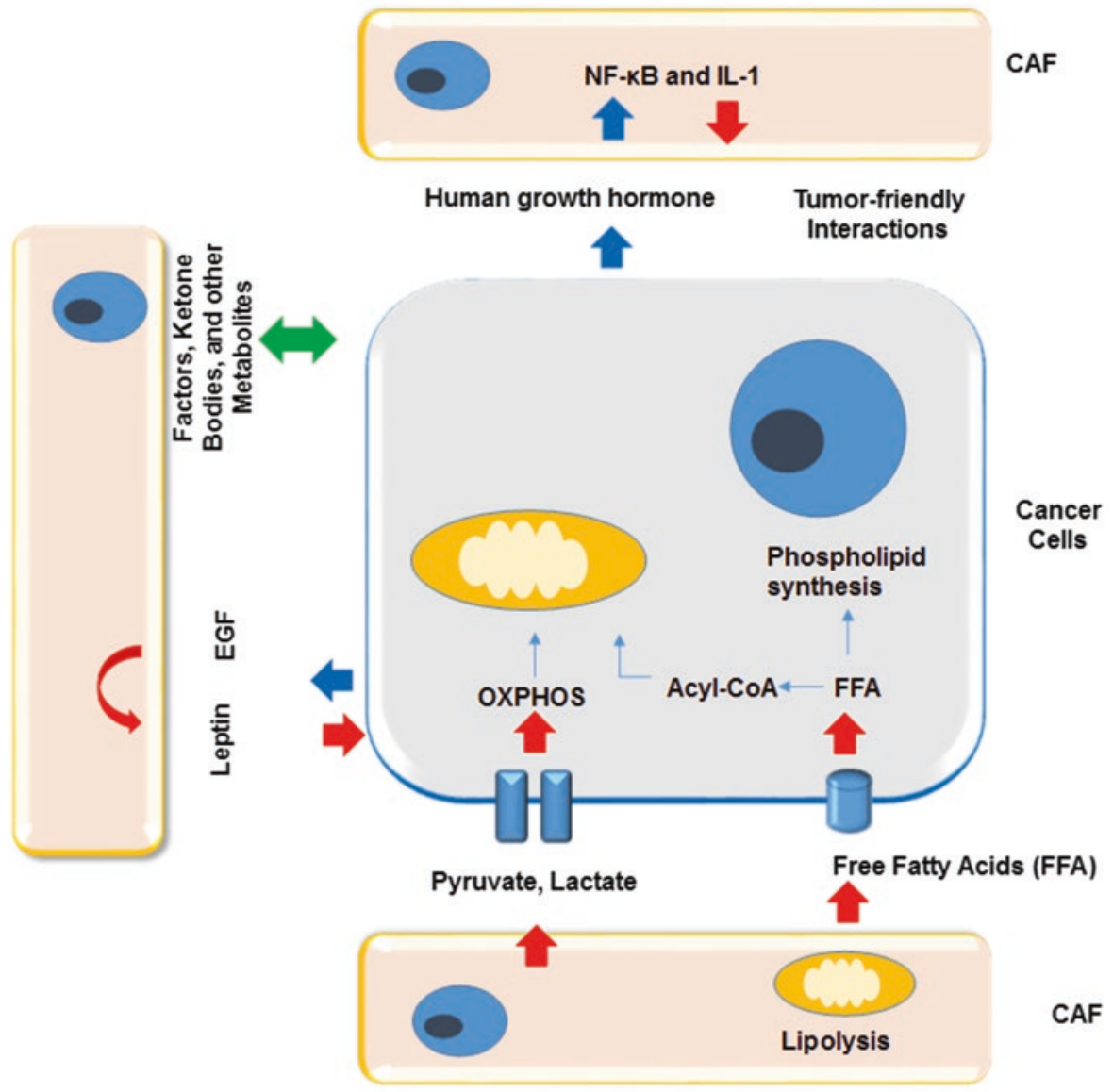

Fig. 1 Overview of CAF metabolism in solid tumors

and MMP14). These results suggest that ECM biosynthesis and remodeling is one of the critical features of the interplay between CAFs and cancer [34-36]. For instance, in ovarian and small cell lung cancers, many ECM genes remarkably elevate their expression levels in chemotherapytreated cancer cells to induce chemoresistance [37, 38]. Additionally, CAFs bypass and help tumor cells resist anti-vascular endothelial growth factor (VEGF) treatment caused by angiogenesis inhibition by activating the plateletderived growth factor C (PDGF-C) pathway [39], and stroma cells mediate RAF inhibitor resistance in BRAF-mutant melanoma through human growth hormone (HGH) secretion [40]. Even though gene expression profiles provide a method to predict the risks of metabolic coupling between cancer cells and CAFs, gene expression levels do not always correlate with metabolic changes. Therefore, measuring the metabolite levels in a sample could be a more accurate method to predict the risk of the aforementioned metabolic interplay.

\section{$3 \quad$ Targeting the Metabolic Exchanges Between CAFs and Cancer Cells}

\subsection{Targeting the Reverse Warburg Effect via Disruption of the "Lactate Shuttle" by MCT1/MCT4 Inhibitors}

Glycolysis, the process of converting glucose to pyruvate, is an essential metabolic pathway to produce energy in the form of ATP in cells. In the 1920s, Otto Warburg found that cancer cells pref- 
erably produce energy by converting glucose to lactic acid, even in aerobic conditions, to generate ATP rapidly. This is known as the Warburg effect $[41,42]$. Interestingly, it was suggested that the reverse Warburg effect is the result of fibroblasts secreting lactate/pyruvate and epithelial cancer cells simultaneously taking up the energy-rich metabolites to utilize in the tricarboxylic acid (TCA) cycle and promote energy production for their growth [43]. In this hypothesis, cancer cells first guide the normal stromal cells to become CAFs, providing a tumor-friendly microenvironment to activate tumor growth. Next, lactate from CAFs is directly absorbed by the cancer cells as fuel for OXPHOS after the conversion of lactate to pyruvate [44]. Accordingly, the expression levels of glycolytic enzymes, such as lactate dehydrogenase and pyruvate kinase isozymes M2, and the lactate transporter, monocarboxylate transporter 4 (MCT4), are elevated in CAFs within breast and lung cancer tumors $[24,45]$. Of note, lactate plays an important role in generating energy for the brain and the heart [46-49] and serves as an energy interplay shuttle between stromal cells and various types of cancer cells $[45,49,50]$. In this scenario, surrounding CAFs can communicate with cancer cells through direct cell-to-cell contact by releasing an exosome packaged with CAF-produced metabolites [51]. This coincides with neovascularization, inflammatory cell infiltration, and extensive remodeling of extracellular matrix in the TME [52]. Evidence supporting the "lactate shuttle" in human cancers $[16,53,54]$ further shows that lactate can be directly transferred from CAFs to adjacent tumor cells under the premise that (1) CAFs overexpress the transmembrane monocarboxylate transporter 4 (MCT4) for lactate efflux from CAFs to cancer cells [53], (2) cancer cells overexpress monocarboxylate transporter 1 (MCT1) for lactate influx into cancer cells [45, 55], and (3) cancer cells finally utilize lactate as fuel for producing ATP via the TCA cycle [56-58]. Of note, MCT1 and MCT4, the main transporters of lactate, are key modulators of lactate homeostasis [59]. The elevated expression levels of genes involved in the lactate shuttle system, including high expression levels of MCT4, are associated with poor prognosis in the prostate, pancreas, and triple-negative breast cancers [59-61]. Consequently, accumulating evidence suggests that MCT1 and MCT4 transporters could be promising targets for cancer therapy.

Over the last decade, there has been significant progress in understanding the roles of the TME in tumorigenesis and the development of effective strategies for cancer therapy. In order to disrupt the metabolic bridge in CAF-cancer cell interactions through glycolysis and lactate metabolism inhibition, three potential strategies have been proposed. First, elevated expression of the lactate transporter MCT1 in cancer cells is a potential target for blocking cellular uptake of two types of mitochondrial fuels, ketone bodies and lactate [62]. MCT1 and MCT2 inhibitors can block the influx and efflux of lactate produced by either CAFs or cancer cells. Thus, due to the rapid accumulation of lactate and protons within cancer cells by inhibiting lactate influx/efflux, rapid acidification can occur in cancer cells and the TME, resulting in lactic acidosis [63-65]. For instance, alpha-cyano-4-hydroxy-cinnamic acid (ACCA), an MCT inhibitor, not only inhibits lactic acid efflux in glycolytic gliomas but also disrupts redox hemostasis and enhances radiosensitivity [66, 67]. AZD3965, an MCT1 inhibitor, is currently being tested in phase I clinical trial in solid tumors, including lymphoma, prostate cancer, and gastric cancer (NCT01791595) (Fig. 2). However, there are concerns over the alternative effects of MCT1 inhibitors, which include the blockage of lactate transport in muscles, gastrointestinal tract, and liver [68, 69].

\subsection{Blocking the Function of CAFs by Metformin (Fig. 2)}

Metformin, a drug that has been widely used for type 2 diabetes treatment, has found new applications as an anticancer drug for its glucose-targeting effects. Metformin activates the AMPK pathway and simultaneously inhibits cancer cell growth through the inhibition of glycolysis by facilitating the trafficking of glucose transporters 1 and 4 [70, 71]. Recent studies have also shown the therapeutic potential of met- 


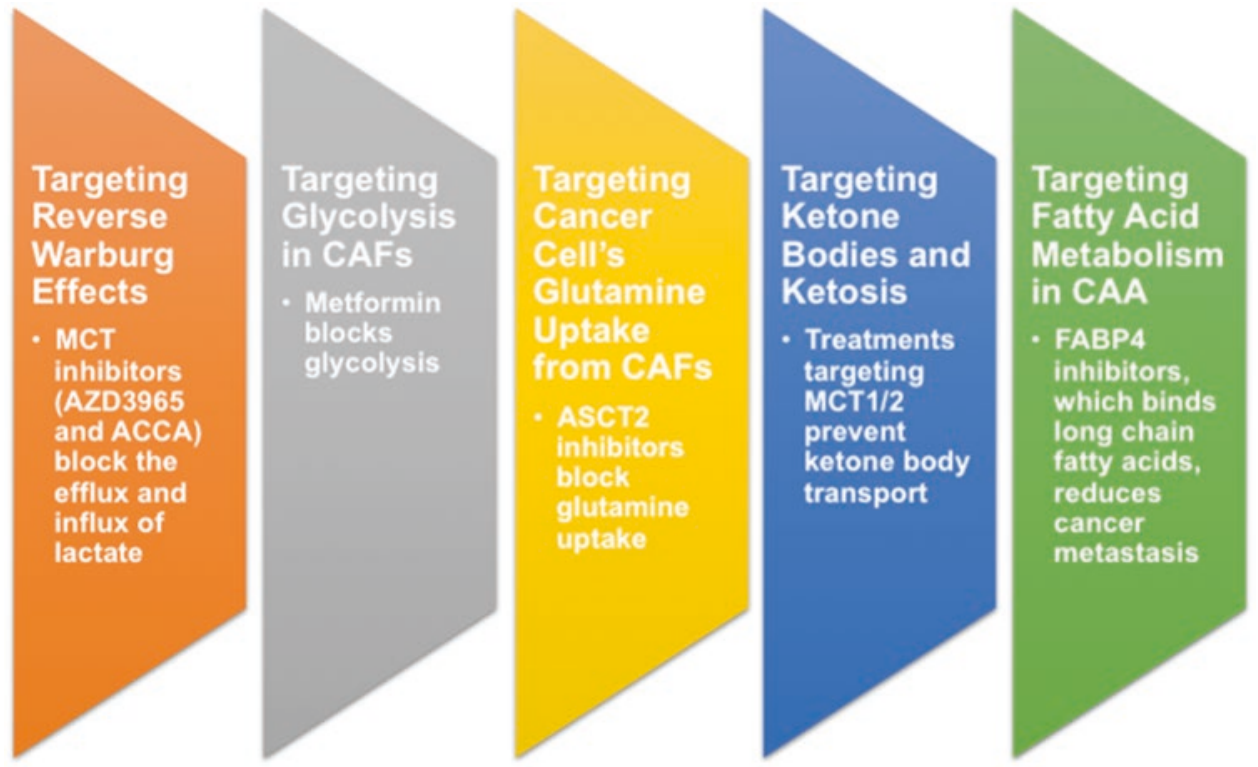

Fig. 2 Therapies targeting metabolic cross talk between cancer cells and cancer-associated fibroblasts

formin in blocking the function of CAFs [72]. In other words, metformin is sufficient to reverse the effects of CAFs on cancer cell growth [72], providing a rationale for why metformin is actively being tested in multiple clinical trials in solid tumors and lymphoma (NCTNCT00659568, NCT00881725, NCT00984490, and NCT00909506).

\section{$4 \quad$ Targeting the Glutamine Uptake of Cancer Cells from CAFs}

Glutamine addiction is a physiological phenomenon where cancer cells rely on the presence of exogenous glutamine to be used as a fuel for the TCA cycle and as a nitrogen donor for nucleotide and amino acid synthesis [73-76]. It was recently revealed that CAFs produce and release glutamine, while cancer cells take up and utilize this glutamine from the TME as an alternative carbon and nitrogen source $[51,77]$. This explains why glutamine transporters, alanine, serine, cysteine preferring transporter 2 (ASCT2), and solute carrier family 38 member 5 (SLC38A5) are usually overexpressed in breast and prostate cancers [7880]. Of note, ASCT2 mediates the uptake of glutamine, an essential amino acid in triple-negative basal-like breast cancer [80]. And ASCT2 inhibitors, such as benzylserine and L- $\gamma$-glutamyl- $p$ nitroanilide, have been shown to inhibit glutamine uptake and cell growth in melanoma and endometrial carcinoma [81-83] (Fig. 2). Additionally, FDA-approved tamoxifen and raloxifene also suppress estrogen receptor-negative breast cancer growth by inhibiting glutamine uptake [84].

\section{$5 \quad$ Targeting Ketone Bodies and Ketosis in CAFs}

Ketone bodies such as acetoacetate, $\beta$-hydroxybutyrate $(\beta \mathrm{OHB})$, and acetone are produced through fatty acid metabolism in the liver [85]. Liver hepatocytes convert fatty acids into ketone bodies and release them into the bloodstream, especially under starvation conditions or after excessive exercises. Consequently, through ketolysis, a process of the conversion of ketone bodies into acetylCoA, they feed into the TCA cycle or OXPHOS to generate ATP [86]. Recent studies have shown that CAFs secrete ketone bodies and cancer cells utilize them as energy sources [77, $85,87]$. Furthermore, Bonuccelli et al. observed that ketone bodies, especially $\beta \mathrm{OHB}$, serve as a 
more powerful energy source for cancer cell growth in comparison to lactate [88]. Genes associated with ketolysis and ketogenesis in CAFs, including 3-hydroxybutyrate dehydrogenase 1 (BDH1) and 3-hydroxy-3-methylglutaryl-CoA synthase 2 (HMGCS2), were overexpressed [89, 90]. Specifically, BDH1 catalyzes the conversion of acetoacetate to $\beta O H B$. HMGCS2, a member of the 3-hydroxy3-methylglutaryl-CoA (HMG-CoA) synthase family, generates HMG-CoA [86, 91]. In contrast to the gene expression profile of the surrounding epithelial cells, cancer cells themselves have upregulated gene expressions associated with ketone reutilization (acetylCoA acetyltransferase, ACAT1) and mitochondrial biogenesis (heat-shock protein 60, HSP60) [89]. Moreover, ketone bodies can be a source of lactate and pyruvate because acetone-an end product of ketosis-can be metabolized to lactate and pyruvate [92, 93]. Taken together, this suggests that the ketone bodies produced by CAFs can serve as an energy fuel for tumor growth and have further implications as a potential therapeutic target for cancer therapy. Furthermore, ketone bodies, including $\beta \mathrm{OHB}$, are transported by the monocarboxylate transporters (MCT1 and MCT2), which also transport them across the blood-brain barrier [91, 94]. Accordingly, treatments targeting MCT1 and MCT2 are currently being tested in phase I clinical trials in solid tumor cancers (NCT01791595) [95]. Thus, the MCT1 and MCT2 inhibitors may effectively block the transport of lactate and ketone bodies, both generated by CAFs (Fig. 2).

\section{Targeting Fatty Acid, a Nutrient Reservoir for Cancer Cell Growth, from Cancer-Associated Adipocytes (CAAs)}

Cancer-associated adipocytes (CAAs) play an important tumorigenic role in fatty acid metabolism in the TME. For instance, omental adipo- cytes promote the migration and invasion of ovarian cancer cells to the omentum [96, 97]. It is known that omental adipocytes generate free fatty acids (FFAs) that are further transferred to cancer cells to generate ATP via $\beta$-oxidation [98] (Fig. 1). Therefore, in order to utilize FFAs, a subset of cancer cells overexpress the fatty acidbinding protein 4 (FABP4), which plays a key role in fatty acid transport for ovarian and breast cancer metastasis [15, 96, 99] (Fig. 1). It has been shown that a FABP4 inhibitor, which binds longchain fatty acids, reduces metastasis of prostate cancer and regulates fatty acid production in ovarian and prostate cancers [96, 100] (Fig. 2). Because adipocytes are a major component of the TME in breast and ovarian cancers, it may be a rationale for FABP4's effectiveness in those cancers $[96,101]$. Accumulation of fatty acids in the TME could serve as a nutrient reservoir for cancer cell growth during nutrient deprivation. Taken together, stromal catabolites, such as free fatty acids, promote tumor growth, and act as chemoattractants to metastasizing cancer cells in the omentum.

\section{Conclusion}

The TME is comprised of cancer cells, CAFs, immune cells, adipocytes, and other supporting cells. Of these, CAFs are one of the key regulators of tumorigenesis. Within the TME of solid tumors, heterogeneous cancer cells and CAFs interact by transferring their metabolites, including amino acids, fatty acids, ketone bodies, cytokines, and growth factors, which reciprocally facilitate the growth of cancer cells. Moreover, CAFs provide not only a structural matrix for providing a tumor-friendly microenvironment to cancer cells but also nutrients for cancer cells. As such, the metabolic interplay between CAFs and cancer cells is considered as an area of vulnerability among cancer cells given that (1) cancer cells release $\mathrm{HGH}$ and induce pro-inflammatory gene expression in CAFs, (2) CAFs produce and release highenergy metabolites to the TME, and (3) cancer cells take up those metabolites to produce 
energy for cancer growth. Therefore, it is widely accepted that CAF-mediated metabolism plays a key role in tumorigenesis and that targeting the metabolic cross talk between cancer cells and CAFs can serve as a potential target for cancer therapy. Consequently, researchers have made continuous efforts to exploit areas of metabolic vulnerability by targeting (1) glycolysis and lactate metabolism, (2) glutaminolysis, (3) ketone bodies and ketosis, and (4) fatty acid metabolism.

\section{References}

1. Antonio, M. J., Zhang, C., \& Le, A. (2021). Different tumor microenvironments lead to different metabolic phenotypes. Advances in Experimental Medicine and Biology, 1311, https:// doi.org/10.1007/978-3-030-65768-0_10.

2. Nabi, K., \& Le, A. (2021). The intratumoral heterogeneity of cancer metabolism. Advances in Experimental Medicine and Biology, 1311, https:// doi.org/10.1007/978-3-030-65768-0_11.

3. Bissell, M. J., \& Radisky, D. (2001). Putting tumours in context. Nature Reviews. Cancer, 1(1), 46-54.

4. Tlsty, T. D., \& Coussens, L. M. (2006). Tumor stroma and regulation of cancer development. Annual Review of Pathology, 1, 119-150.

5. Folkman, J. (1971). Tumor angiogenesis: Therapeutic implications. The New England Journal of Medicine, 285(21), 1182-1186.

6. O'Reilly, M. S., et al. (1997). Endostatin: An endogenous inhibitor of angiogenesis and tumor growth. Cell, 88(2), 277-285.

7. Kalluri, R. (2003). Basement membranes: Structure, assembly and role in tumour angiogenesis. Nature Reviews. Cancer, 3(6), 422-433.

8. Kim, K. J., et al. (1993). Inhibition of vascular endothelial growth factor-induced angiogenesis suppresses tumour growth in vivo. Nature, 362(6423), 841-844.

9. Coussens, L. M., \& Werb, Z. (2002). Inflammation and cancer. Nature, 420(6917), 860-867.

10. de Visser, K. E., Korets, L. V., \& Coussens, L. M. (2005). De novo carcinogenesis promoted by chronic inflammation is B lymphocyte dependent. Cancer Cell, 7(5), 411-423.

11. Tlsty, T. D., \& Hein, P. W. (2001). Know thy neighbor: Stromal cells can contribute oncogenic signals. Current Opinion in Genetics \& Development, 11(1), 54-59.

12. Elenbaas, B., \& Weinberg, R. A. (2001). Heterotypic signaling between epithelial tumor cells and fibroblasts in carcinoma formation. Experimental Cell Research, 264(1), 169-184.
13. Sazeides, C., \& Le, A. (2021). Metabolic relationship between cancer-associated fibroblasts and cancer cells. Advances in Experimental Medicine and Biology, 1311, https://doi.org/10.1007/ 978-3-030-65768-0_14.

14. Calvo, F., et al. (2015). Cdc42EP3/BORG2 and septin network enables mechano-transduction and the emergence of cancer-associated fibroblasts. Cell Reports, 13(12), 2699-2714.

15. Martinez-Outschoorn, U. E., Sotgia, F., \& Lisanti, M. P. (2012). Power surge: Supporting cells "fuel" cancer cell mitochondria. Cell Metabolism, 15(1), 4-5.

16. Martinez-Outschoorn, U. E., et al. (2011). Energy transfer in "parasitic" cancer metabolism: Mitochondria are the powerhouse and Achilles' heel of tumor cells. Cell Cycle, 10(24), 4208-4216.

17. Kilvaer, T. K., et al. (2015). Cancer associated fibroblasts in stage I-IIIA NSCLC: Prognostic impact and their correlations with tumor molecular markers. PLoS One, 10(8), e0134965.

18. Ha, S. Y., et al. (2014). The prognostic significance of cancer-associated fibroblasts in esophageal squamous cell carcinoma. PLoS One, 9(6), e99955.

19. Cheteh, E. H., et al. (2017). Human cancerassociated fibroblasts enhance glutathione levels and antagonize drug-induced prostate cancer cell death. Cell Death \& Disease, 8(6), e2848.

20. Madar, S., Goldstein, I., \& Rotter, V. (2013). 'Cancer associated fibroblasts'-more than meets the eye. Trends in Molecular Medicine, 19(8), 447-453.

21. Hanahan, D., \& Weinberg, R. A. (2011). Hallmarks of cancer: The next generation. Cell, 144(5), 646-674.

22. Dang, C. V., et al. (2011). Therapeutic targeting of cancer cell metabolism. Journal of Molecular Medicine (Berlin), 89(3), 205-212.

23. Hirschey, M. D., et al. (2015). Dysregulated metabolism contributes to oncogenesis. Seminars in Cancer Biology, 35(Suppl), S129-S150.

24. Martinez-Outschoorn, U. E., Lisanti, M. P., \& Sotgia, F. (2014). Catabolic cancer-associated fibroblasts transfer energy and biomass to anabolic cancer cells, fueling tumor growth. Seminars in Cancer Biology, 25, 47-60.

25. Hoang, G., Udupa, S., \& Le, A. (2019). Application of metabolomics technologies toward cancer prognosis and therapy. International Review of Cell and Molecular Biology, 347, 191-223.

26. Bruntz, R. C., et al. (2017). Exploring cancer metabolism using stable isotope-resolved metabolomics (SIRM). The Journal of Biological Chemistry, 292(28), 11601-11609.

27. Fan, T. W., et al. (2009). Altered regulation of metabolic pathways in human lung cancer discerned by (13)C stable isotope-resolved metabolomics (SIRM). Molecular Cancer, 8, 41.

28. Elgogary, A., et al. (2016). Combination therapy with BPTES nanoparticles and metformin targets the metabolic heterogeneity of pancreatic 
cancer. Proceedings of the National Academy of Sciences of the United States of America, 113(36), E5328-E5336.

29. Lane, A. N., \& Fan, T. W. (2017). NMR-based stable isotope resolved metabolomics in systems biochemistry. Archives of Biochemistry and Biophysics, 628, 123-131.

30. Tan, J., \& Le, A. (2021). The heterogeneity of breast cancer metabolism. Advances in Experimental Medicine and Biology, 1311, https:// doi.org/10.1007/978-3-030-65768-0_6.

31. Kirsch, B. J., et al. (2021). Non-Hodgkin lymphoma metabolism. Advances in Experimental Medicine and Biology, 1311, https://doi. org/10.1007/978-3-030-65768-0_7.

32. Barone, I., et al. (2012). Leptin mediates tumorstromal interactions that promote the invasive growth of breast cancer cells. Cancer Research, 72(6), 1416-1427.

33. Erez, N., et al. (2010). Cancer-associated fibroblasts are activated in incipient neoplasia to orchestrate tumor-promoting inflammation in an NF-kappaB-dependent manner. Cancer Cell, 17(2), 135-147.

34. Casey, T., et al. (2009). Molecular signatures suggest a major role for stromal cells in development of invasive breast cancer. Breast Cancer Research and Treatment, 114(1), 47-62.

35. Ma, X. J., et al. (2009). Gene expression profiling of the tumor microenvironment during breast cancer progression. Breast Cancer Research, 11(1), R7.

36. Finak, G., et al. (2008). Stromal gene expression predicts clinical outcome in breast cancer. Nature Medicine, 14(5), 518-527.

37. Sherman-Baust, C. A., et al. (2003). Remodeling of the extracellular matrix through overexpression of collagen VI contributes to cisplatin resistance in ovarian cancer cells. Cancer Cell, 3(4), 377-386.

38. Hodkinson, P. S., Mackinnon, A. C., \& Sethi, T. (2007). Extracellular matrix regulation of drug resistance in small-cell lung cancer. International Journal of Radiation Biology, 83(11-12), 733-741.

39. Crawford, Y., et al. (2009). PDGF-C mediates the angiogenic and tumorigenic properties of fibroblasts associated with tumors refractory to anti-VEGF treatment. Cancer Cell, 15(1), 21-34.

40. Straussman, R., et al. (2012). Tumour microenvironment elicits innate resistance to RAF inhibitors through HGF secretion. Nature, 487(7408), 500-504.

41. Bose, S., Zhang, C., \& Le, A. (2021). Glucose metabolism in cancer: The Warburg effect and beyond. Advances in Experimental Medicine and Biology, 1311, https://doi.org/10.1007/978-3-030-65768-0_1.

42. Warburg, O. (1956). On the origin of cancer cells. Science, 123(3191), 309-314.

43. Pavlides, S., et al. (2009). The reverse Warburg effect: Aerobic glycolysis in cancer associated fibroblasts and the tumor stroma. Cell Cycle, 8(23), 3984-4001.
44. Lee, M., \& Yoon, J. H. (2015). Metabolic interplay between glycolysis and mitochondrial oxidation: The reverse Warburg effect and its therapeutic implication. World Journal of Biological Chemistry, 6(3), 148-161.

45. Faubert, B., et al. (2017). Lactate metabolism in human lung tumors. Cell, 171(2), 358-371. e9.

46. van Hall, G., et al. (2009). Blood lactate is an important energy source for the human brain. Journal of Cerebral Blood Flow and Metabolism, 29(6), 1121-1129.

47. Medina, J. M., \& Tabernero, A. (2005). Lactate utilization by brain cells and its role in CNS development. Journal of Neuroscience Research, 79(1-2), $2-10$.

48. Bartelds, B., et al. (1999). Myocardial lactate metabolism in fetal and newborn lambs. Circulation, 99(14), 1892-1897.

49. Fujiwara, S., et al. (2015). Lactate, a putative survival factor for myeloma cells, is incorporated by myeloma cells through monocarboxylate transporters 1. Experimental Hematology \& Oncology, 4, 12.

50. Hirschhaeuser, F., Sattler, U. G., \& Mueller-Klieser, W. (2011). Lactate: A metabolic key player in cancer. Cancer Research, 71(22), 6921-6925.

51. Zhao, H., et al. (2016). Tumor microenvironment derived exosomes pleiotropically modulate cancer cell metabolism. eLife, 5, e10250.

52. Ronnov-Jessen, L., Petersen, O. W., \& Bissell, M. J. (1996). Cellular changes involved in conversion of normal to malignant breast: Importance of the stromal reaction. Physiological Reviews, 76(1), 69-125.

53. Whitaker-Menezes, D., et al. (2011). Evidence for a stromal-epithelial "lactate shuttle" in human tumors: MCT4 is a marker of oxidative stress in cancerassociated fibroblasts. Cell Cycle, 10(11), 1772-1783.

54. Rattigan, Y. I., et al. (2012). Lactate is a mediator of metabolic cooperation between stromal carcinoma associated fibroblasts and glycolytic tumor cells in the tumor microenvironment. Experimental Cell Research, 318(4), 326-335.

55. Hong, C. S., et al. (2016). MCT1 modulates cancer cell pyruvate export and growth of tumors that co-express MCT1 and MCT4. Cell Reports, 14(7), 1590-1601.

56. Gladden, L. B. (2004). Lactate metabolism: A new paradigm for the third millennium. The Journal of Physiology, 558(Pt 1), 5-30.

57. Draoui, N., \& Feron, O. (2011). Lactate shuttles at a glance: From physiological paradigms to anti-cancer treatments. Disease Models \& Mechanisms, 4(6), 727-732.

58. Sonveaux, P., et al. (2008). Targeting lactate-fueled respiration selectively kills hypoxic tumor cells in mice. The Journal of Clinical Investigation, 118(12), 3930-3942.

59. Baek, G., et al. (2014). MCT4 defines a glycolytic subtype of pancreatic cancer with poor prognosis and unique metabolic dependencies. Cell Reports, 9(6), 2233-2249. 
60. Pertega-Gomes, N., et al. (2014). A lactate shuttle system between tumour and stromal cells is associated with poor prognosis in prostate cancer. $B M C$ Cancer, 14, 352.

61. Witkiewicz, A. K., et al. (2012). Using the "reverse Warburg effect" to identify high-risk breast cancer patients: Stromal MCT4 predicts poor clinical outcome in triple-negative breast cancers. Cell Cycle, 11(6), 1108-1117.

62. Lamb, R., et al. (2014). Mitochondria as new therapeutic targets for eradicating cancer stem cells: Quantitative proteomics and functional validation via MCT1/2 inhibition. Oncotarget, 5(22), 11029-11037.

63. Wu, H., et al. (2012). Central role of lactic acidosis in cancer cell resistance to glucose deprivationinduced cell death. The Journal of Pathology, 227(2), 189-199.

64. Xie, J., et al. (2014). Beyond Warburg effect--dual metabolic nature of cancer cells. Scientific Reports, 4, 4927.

65. Romero-Garcia, S., et al. (2016). Lactate contribution to the tumor microenvironment: Mechanisms, effects on immune cells and therapeutic relevance. Frontiers in Immunology, 7, 52.

66. Colen, C. B., et al. (2011). Metabolic targeting of lactate efflux by malignant glioma inhibits invasiveness and induces necrosis: An in vivo study. Neoplasia, 13(7), 620-632.

67. Colen, C. B., et al. (2006). Metabolic remodeling of malignant gliomas for enhanced sensitization during radiotherapy: An in vitro study. Neurosurgery, 59(6), 1313-1323. discussion 1323-4.

68. Ovens, M. J., et al. (2010). AR-C155858 is a potent inhibitor of monocarboxylate transporters MCT1 and MCT2 that binds to an intracellular site involving transmembrane helices 7-10. The Biochemical Journal, 425(3), 523-530.

69. Perez-Escuredo, J., et al. (2016). Monocarboxylate transporters in the brain and in cancer. Biochimica et Biophysica Acta, 1863(10), 2481-2497.

70. Ben Sahra, I., et al. (2010). Metformin in cancer therapy: A new perspective for an old antidiabetic drug? Molecular Cancer Therapeutics, 9(5), 1092-1099.

71. Kozka, I. J., et al. (1995). The effects of insulin on the level and activity of the GLUT4 present in human adipose cells. Diabetologia, 38(6), 661-666.

72. Martinez-Outschoorn, U. E., et al. (2010). Oxidative stress in cancer associated fibroblasts drives tumorstroma co-evolution: A new paradigm for understanding tumor metabolism, the field effect and genomic instability in cancer cells. Cell Cycle, 9(16), 3256-3276.

73. Li, T., Copeland, C., \& Le, A. (2021). Glutamine metabolism in cancer. Advances in Experimental Medicine and Biology, 1311, https://doi.org/10.1007/ 978-3-030-65768-0_2.

74. Le, A., et al. (2012). Glucose-independent glutamine metabolism via TCA cycling for proliferation and survival in B cells. Cell Metabolism, 15(1), 110-121.
75. Wise, D. R., \& Thompson, C. B. (2010). Glutamine addiction: A new therapeutic target in cancer. Trends in Biochemical Sciences, 35(8), 427-433.

76. Romero, I. L., et al. (2015). Molecular pathways: Trafficking of metabolic resources in the tumor microenvironment. Clinical Cancer Research, 21(4), 680-686.

77. Pavlides, S., et al. (2010). The autophagic tumor stroma model of cancer: Role of oxidative stress and ketone production in fueling tumor cell metabolism. Cell Cycle, 9(17), 3485-3505.

78. Wang, Q., et al. (2015). Targeting ASCT2-mediated glutamine uptake blocks prostate cancer growth and tumour development. The Journal of Pathology, 236(3), 278-289.

79. Gupta, S., Roy, A., \& Dwarakanath, B. S. (2017). Metabolic cooperation and competition in the tumor microenvironment: Implications for therapy. Frontiers in Oncology, 7, 68.

80. van Geldermalsen, M., et al. (2016). ASCT2/ SLC1A5 controls glutamine uptake and tumour growth in triple-negative basal-like breast cancer. Oncogene, 35(24), 3201-3208.

81. Wang, Q., et al. (2014). Targeting glutamine transport to suppress melanoma cell growth. International Journal of Cancer, 135(5), 1060-1071.

82. Esslinger, C. S., Cybulski, K. A., \& Rhoderick, J. F. (2005). Ngamma-aryl glutamine analogues as probes of the ASCT2 neutral amino acid transporter binding site. Bioorganic \& Medicinal Chemistry, 13(4), 1111-1118.

83. Marshall, A. D., et al. (2017). ASCT2 regulates glutamine uptake and cell growth in endometrial carcinoma. Oncogene, 6(7), e367.

84. Todorova, V. K., et al. (2011). Tamoxifen and raloxifene suppress the proliferation of estrogen receptornegative cells through inhibition of glutamine uptake. Cancer Chemotherapy and Pharmacology, 67(2), 285-291.

85. Wu, D., Zhuo, L., \& Wang, X. (2017). Metabolic reprogramming of carcinoma-associated fibroblasts and its impact on metabolic heterogeneity of tumors. Seminars in Cell \& Developmental Biology, 64, $125-131$.

86. Grabacka, M., et al. (2016). Regulation of ketone body metabolism and the role of PPARalpha. International Journal of Molecular Sciences, 17, 12.

87. Fiaschi, T., et al. (2012). Reciprocal metabolic reprogramming through lactate shuttle coordinately influences tumor-stroma interplay. Cancer Research, 72(19), 5130-5140.

88. Bonuccelli, G., et al. (2010). Ketones and lactate "fuel" tumor growth and metastasis: Evidence that epithelial cancer cells use oxidative mitochondrial metabolism. Cell Cycle, 9(17), 3506-3514.

89. Martinez-Outschoorn, U. E., et al. (2012). Ketone bodies and two-compartment tumor metabolism: Stromal ketone production fuels mitochondrial biogenesis in epithelial cancer cells. Cell Cycle, 11(21), 3956-3963. 
90. Saraon, P., et al. (2013). Quantitative proteomics reveals that enzymes of the ketogenic pathway are associated with prostate cancer progression. Molecular \& Cellular Proteomics, 12(6), 1589-1601.

91. Newman, J. C., \& Verdin, E. (2014). Ketone bodies as signaling metabolites. Trends in Endocrinology and Metabolism, 25(1), 42-52.

92. Glew, R. H. (2010). You can get there from here: Acetone, anionic ketones and even-carbon fatty acids can provide substrates for gluconeogenesis. Nigerian Journal of Physiological Sciences, 25(1), $2-4$.

93. Miller, O. N., \& Bazzano, G. (1965). Propanediol metabolism and its relation to lactic acid metabolism. Annals of the New York Academy of Sciences, 119(3), 957-973.

94. Pellerin, L., et al. (2005). Cellular and subcellular distribution of monocarboxylate transporters in cultured brain cells and in the adult brain. Journal of Neuroscience Research, 79(1-2), 55-64.

95. Halford, S., et al. (2017). A first-in-human firstinclass (FIC) trial of the monocarboxylate transporter 1 (MCT1) inhibitor AZD3965 in patients with advanced solid tumours. Journal of Clinical Oncology, 35(15_Suppl), 2516.

96. Nieman, K. M., et al. (2011). Adipocytes promote ovarian cancer metastasis and provide energy for rapid tumor growth. Nature Medicine, 17(11), 1498-1503.

97. Vinokurov, V. L., \& Kolosov, A. E. (1980). Ovarian cancer metastasis to the greater omentum. Voprosy Onkologii, 26(2), 30-34.

98. Park, J. K., et al. (2021). The heterogeneity of lipid metabolism in cancer. Advances in Experimental Medicine and Biology, 1311, https://doi. org/10.1007/978-3-030-65768-0_3.

99. Guaita-Esteruelas, S., et al. (2017). Exogenous FABP4 increases breast cancer cell proliferation and activates the expression of fatty acid transport proteins. Molecular Carcinogenesis, 56(1), 208-217.

100. Uehara, H., et al. (2014). Exogenous fatty acid binding protein 4 promotes human prostate cancer cell progression. International Journal of Cancer, 135(11), 2558-2568.

101. Dirat, B., et al. (2011). Cancer-associated adipocytes exhibit an activated phenotype and contribute to breast cancer invasion. Cancer Research, 71(7), 2455-2465.

Open Access This chapter is licensed under the terms of the Creative Commons Attribution 4.0 International License (http://creativecommons.org/licenses/by/4.0/), which permits use, sharing, adaptation, distribution and reproduction in any medium or format, as long as you give appropriate credit to the original author(s) and the source, provide a link to the Creative Commons license and indicate if changes were made.

The images or other third party material in this chapter are included in the chapter's Creative Commons license, unless indicated otherwise in a credit line to the material. If material is not included in the chapter's Creative Commons license and your intended use is not permitted by statutory regulation or exceeds the permitted use, you will need to obtain permission directly from the copyright holder. 


\section{Part IV}

The Metabolic Interplay Between Cancer and Other Diseases 


\title{
Diabetes and Cancer: \\ The Epidemiological \\ and Metabolic Associations
}

\author{
Cissy Zhang and Anne Le
}

\section{Keywords}

Cancer - Type 2 diabetes - Glucose metabolism $\cdot$ Hexosamine biosynthetic pathway . $K R A S$ mutation $\cdot$ Hyperinsulinemia $\cdot$ mTOR signaling $\cdot$ Metformin

\section{Abbreviations}

4E-BP1

AMPK

BPTES

CDC
eIF4E-binding protein 1

Adenosine monophosphateactivated protein kinase Bis-2-(5-phenylacetamido1,2,4-thiadiazol-2-yl)ethyl sulfide

Centers for Disease Control and Prevention

C. Zhang

Department of Pathology, Johns Hopkins University

School of Medicine, Baltimore, MD, USA

Department of Biology, Johns Hopkins University

Krieger School of Arts \& Sciences,

Baltimore, MD, USA

A. Le $(\bowtie)$

Department of Pathology and Oncology, Johns

Hopkins University School of Medicine,

Baltimore, MD, USA

Department of Chemical and Biomolecular Engineering, Johns Hopkins University Whiting School of Engineering, Baltimore, MD, USA e-mail: annele@jhmi.edu

eIF4E

GDH

GFA

GLS

GSH

HBP

IARC

IR

IRS-1

KRAS

MAPK

mTOR

mTORC1

$O$-GlcNAcylation

OGT

PDAC

PI3K

PPAR $\gamma$

PPRE

PTM
Eukaryotic translation initiation factor $4 \mathrm{E}$

Glutamate dehydrogenase Glutamine:fructose-6phosphate amidotransferase Glutaminase Glutathione Hexosamine biosynthetic pathway

International Agency for Research on Cancer Insulin receptor Insulin receptor substrate 1 Kirsten rat sarcoma viral oncogene homolog

Mitogen-activated protein kinase

Mammalian target of rapamycin

Mammalian target of rapamycin complex 1

$O$-linked $\mathrm{N}$-acetylglucosami nylation

$O$-GlcNAc transferase

Pancreatic ductal adenocarcinoma

Phosphoinositide 3-kinase

Peroxisome proliferatoractivated receptor $\gamma$

Peroxisome proliferatorresponsive element

Posttranslational modification 


$\begin{array}{ll}\text { PTP } & \text { Protein tyrosine phosphatase } \\ \text { RNR } & \text { Ribonucleotide reductase } \\ \text { ROS } & \text { Reactive oxygen species } \\ \text { S6K } & \text { S6 kinase } \\ \text { SAM } & \text { S-adenosylmethionine } \\ \text { T1D } & \text { Type 1 diabetes } \\ \text { T2D } & \text { Type 2 diabetes } \\ \text { TCA } & \text { Tricarboxylic acid } \\ \text { UDP-GlcNAc } & \text { Uridine diphosphate } N \text {-acetyl } \\ & \text { glucosamine } \\ \alpha-\text { KG } & \text { Alpha-ketoglutarate }\end{array}$

\section{Key Points}

- Diabetes is correlated with increased risk for several types of cancer.

- Diabetes and cancer both exhibit abnormal glucose metabolism: hyperglycemia and the Warburg effect, respectively.

- High glucose levels can lead to increased $O$-GlcNAcylation through upregulation of the hexosamine biosynthetic pathway (HBP), which contributes to insulin resistance in diabetes and de novo KRAS mutations which can result in pancreatic cancer.

- Hyperinsulinemia, a condition commonly associated with diabetes, can promote cancer proliferation.

- Obesity can result in insulin resistance and increased oxidative stress contributing to diabetes and cancer.

- Dysregulation of leucine metabolism, which regulates insulin secretion and mTOR signaling, can promote type 2 diabetes (T2D) pathogenesis, insulin resistance, and cancer growth.

- Glutamine regulates insulin secretion contributes to gluconeogenesis in T2D, and can promote cancer growth.

- Different antidiabetic drugs have varied effects on cancer.

\section{Introduction}

Diabetes mellitus, commonly known as diabetes, and cancer are two of the most common diseases plaguing the world today. According to the Centers for Disease Control and Prevention
(CDC), there are currently more than 20 million people with diabetes in the United States [1]. According to the International Agency for Research on Cancer (IARC), there were around 18 million people diagnosed with cancer, with approximately ten million deaths globally in 2018 [2]. Given the prevalence and deadliness of diabetes and cancer, these two diseases have long been the focus of many researchers with the goal of improving treatment outcomes. While diabetes and cancer may seem to be two very different diseases at first glance, they share several similarities, especially regarding their metabolic characteristics. This chapter discusses the similarities and relationships between the metabolism of diabetes, especially type 2 diabetes (T2D), and cancer, including their abnormal glucose and amino acid metabolism, the contribution of hyperglycemia to oncogenic mutations, and the contribution of hyperinsulinemia to cancer progression. Investigating the metabolic interplay between diabetes and cancer in an effort to exploit this connection for cancer treatment has the potential to significantly improve clinical efficacy.

\section{The Epidemiological Association: Diabetes Correlates with Increased Risk for Many Types of Cancer}

As two of the most common diseases in the world, diabetes and cancer have attracted the attention of a great number of investigators since the 1950s with the intention of discovering their epidemiological connections [3]. It was found through these studies that patients with diabetes have a higher risk of developing many different types of cancer, including liver, pancreatic, endometrium, breast, colon, and rectum cancers [3]. Specifically, it was found that the risk for colorectal cancer is $30 \%$ greater for patients with T2D, which is caused by insulin resistance, as compared to people who do not have diabetes $[4,5]$. Among all types of cancers, pancreatic cancer has the most drastic associa- 
tion with diabetes. As Pannala et al. discovered, around $50 \%$ of the patients with pancreatic cancer have T2D [5, 6]. However, while the epidemiological connections between diabetes and cancer have long been known, the exact mechanisms behind the relationships are not well understood, and further studies are needed.

While it seems reasonable to speculate that high glucose levels in diabetes can promote cancer proliferation, some clinical data contradict this speculation. For example, it was found that there is a significantly decreased risk for prostate cancer in patients with T2D [7] and that the chances for developing lung [3] and ovarian cancers $[7,8]$ are not associated with T2D. These exceptions call for further investigations to fully understand the association between diabetes, especially T2D, and cancer, as well as the mechanisms behind it.

\section{Abnormal Glucose Metabolism Serves as a Link Between Diabetes and Cancer}

\begin{abstract}
Abnormally high blood glucose levels, or hyperglycemia, is one of the defining characteristics of diabetes caused by either insulin secretion defects (T1D) or insulin resistance (T2D). Persistent hyperglycemia over a long period of time in diabetic patients eventually causes damage to important organs, including the eyes, heart, and kidneys, thus leading to the observed symptoms and detrimental effects of diabetes [9]. For many cancers, glucose metabolism is indispensable due to their high need for energy and materials to sustain growth and proliferation. The Warburg effect is one of the most well-known metabolic adaptations of cancers, where the cancer cells readily convert glucose to pyruvate and lactate even under normoxic conditions in order to satisfy their increased need for energy [10, 11]. Diabetes and cancer both have abnormal glucose metabolism, but with different mechanisms: one leads to deterioration of tissues while the other fuels uncontrolled cell growth.
\end{abstract}

\subsection{High Glucose Levels Lead to Hexosamine Biosynthetic Pathway (HBP) Upregulation, Contributing to Insulin Resistance in T2D and Oncogenic Mutations} (Fig. 1)

As mentioned previously, it has been well established that patients with diabetes have a higher risk of developing many types of cancers. However, the mechanisms behind this association are less well known. Only recently was one possible mechanism discovered, where high glucose is linked to de novo mutation of the Kirsten rat sarcoma viral oncogene homolog $(K R A S)$ in pancreatic cells, thus leading to a greater chance of tumorigenesis and an increased risk for cancer $[12,13]$.

From the glycolysis pathway, glucose can be converted to glucose-6-phosphate, which then alternatively enters the hexosamine biosynthetic pathway (HBP). The HBP utilizes around 2-3\% of the total glucose taken up by normal cells [14]. The final product of the HBP is uridine diphosphate $\quad N$-acetylglucosamine (UDP-GlcNAc). UDP-GlcNAc is essential for the production of glycans, such as $O$-glycans and $\mathrm{N}$-glycans, which are important for various cellular functions that are involved in cancer, such as signaling pathways and cell proliferation regulation [15]. UDP-GlcNAc is also the substrate for $O$-GlcNAc transferase (OGT), an enzyme responsible for the $O$-linked $\mathrm{N}$-acetylglucosaminylation (O-GlcNAcylation) of many proteins in the cytoplasm, the nucleus, and the mitochondria [16]. $O$-GlcNAcylation is a posttranslational modification (PTM) where the $O$-linked GlcNAc moieties are attached to the serine or threonine residues of the proteins.

In diabetes, increased HBP activity is caused by high glucose levels and overexpression of glutamine: fructose-6-phosphate amidotransferase (GFA), the enzyme in HBP that converts fructose 6-phosphate to glucosamine 6-phosphate, which can lead to increased insulin resistance [17, 18]. This results in a feedback loop where hyperglycemia leads to an upregulation in HBP, which in turn leads to insulin resistance and worsens hyper- 


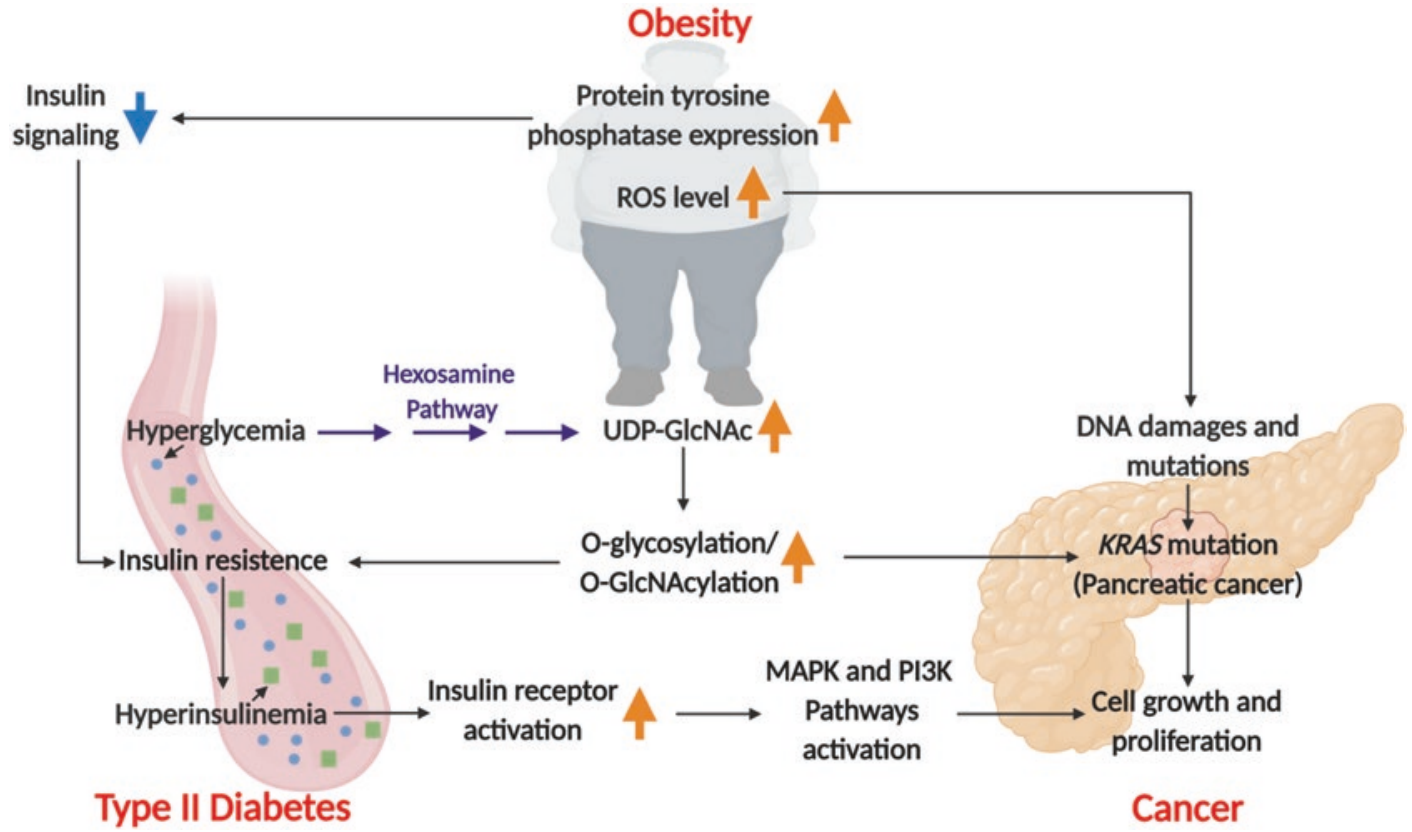

Fig. 1 Associations between type 2 diabetes, cancer, and obesity. High blood glucose levels in T2D lead to upregulation of the hexosamine biosynthetic pathway (HBP). The upregulation of HBP, in turn, results in high UDP-GlcNAc levels and increased protein $O$-glycosylation/O-GlcNAcylation. This can lead to insulin resistance in T2D and $K R A S$ mutation in pancreatic cancer. Insulin resistance can contribute to hyperinsulinemia. Hyperinsulinemia can result in increased insulin receptor activation, which can further activate the MAPK and PI3K pathways that promote cell growth and proliferation. Obesity can result in increased protein tyrosine phosphatase expression, which can impair insulin signaling and contribute to insulin resistance. Obesity can also increase ROS levels which lead to DNA damages and mutations and contribute to cancer. Blue circles represent glucose molecules. Green squares represent insulin molecules

glycemia. In the study by $\mathrm{Hu}$ et al., the upregulation of HBP and increased UDP-GlcNAc leads to increased $O$-GlcNAcylation of ribonucleotide reductase (RNR) [13]. Subsequently, this results in reduced activity of RNR, which coverts NDPs to the corresponding dNDPs, thus depleting the dNTP pool in the cells. Since dNTPs are indispensable for DNA synthesis and repair, the decreased dNTP pool impairs the DNA repair system leading to an increased mutation rate, including mutations in important oncogenes such as $K R A S$. High glucose levels thus led to upregulated HBP and increased $O$-GlcNAcylation, which eventually led to de novo KRAS mutation in pancreatic cells. Mutant KRAS is present in more than $95 \%$ of pancreatic ductal adenocarcinoma (PDAC) [19, 20]. It is responsible for regulating several key metabolic pathways of the cells, including glucose and glutamine metabolism, leading to PDAC's aggressive growth and prolif- eration. KRAS mutations caused by increased $O$-GlcNAcylation thus lead to increased risk for developing pancreatic cancer. Besides contributing to KRAS mutation, $O$-GlcNAcylation is also involved in regulating key oncoproteins such as MYC and tumor suppressors such as p53 and is found to be upregulated in many cancers, such as colon and breast cancers, along with an increase in OGT level [21]. Furthermore, the inhibition of OGT has been shown to lead to breast cancer cell apoptosis [22].

Taken together, the HBP plays an important role in both diabetes and cancer. Given the role of $O$-GlcNAcylation in pancreatic cancer tumorigenesis, it is worth investigating its role in other cancer types. Downregulating the HBP by inhibiting enzymes such as GFA and/or inhibiting $O$-GlcNAcylation can be a potential target for cancer treatment. Indeed, several studies have shown that inhibiting GFA has tumor reduction 
effects in both in vitro and in vivo models and cancer patients [23]. In addition, with evidence supporting the association between high glucose levels and pancreatic cancer, more attention should be paid to mediating these high glucose levels early in diabetic patients not only to prevent symptoms associated with diabetes but also to prevent cancer development.

\subsection{Hyperinsulinemia in Diabetes Promotes Cancer Growth Through Insulin Receptors and the Subsequent Signaling Pathways (Fig. 1)}

Hyperinsulinemia is another common characteristic of T2D, where there are frequently high levels of insulin circulating in patients. It is usually caused by insulin resistance and subsequent treatments, such as the use of insulin secretagogues, which can lead to increased insulin secretion. Many studies have found that high levels of insulin can lead to cancer progression through increased activation of insulin receptors (IRs) and the subsequent cell signaling pathways, thus offering another possible explanation for the observed association between diabetes and cancer [3]. IRs are tyrosine kinases that are found in two isoforms: IR-A and IR-B. IR-A was found to activate the mitogen-activated protein kinase (MAPK) pathways [7]. These MAPK pathways are responsible for regulating many important cellular aspects, such as gene expression, metabolism, mitosis, and apoptosis through a series of phosphorylations [24]. IR-B, on the other hand, activates the phosphoinositide 3-kinase (PI3K) pathway [7], which promotes the synthesis of glycogens, lipids, and proteins [25]. Therefore, hyperinsulinemia can result in increased insulin binding to IRs, which can lead to increased activation of the MAPK pathways and the PI3K pathway. The activation of these pathways then promotes cell proliferation. Hence, diabetic treatments, which have the potential to promote hyperinsulinemia, should be considered more thoroughly before being administered to patients, especially those with cancer, in order to prevent cancer growth. Inhibiting IRs or decreasing insulin levels can also have potential as a cancer therapy.

\section{$4 \quad$ Obesity Leads to Insulin Resistance in Diabetes and Oxidative Stress, Which Can Lead to Cancer (Fig. 1)}

Obesity, another common condition associated with dysregulated metabolism, serves as another link between type 2 diabetes (T2D) and cancer. Obesity has been shown to increase the expression and activity of protein tyrosine phosphatases (PTPs), enzymes that remove the phosphate group from protein tyrosine residues, which can lead to impaired insulin signaling and thus result in insulin resistance [26]. Insulin resistance can then result in T2D and hyperinsulinemia. Hyperinsulinemia, as discussed in Sect. 3.2, has the potential to promote cancer growth. Obesity can also increase reactive oxygen species (ROS) levels in the cells through adipokine production by adipose tissue leading to increased oxidative stress [27]. Elevated ROS levels can result in DNA damage and mutations. The accumulation of mutations in cells that escape apoptosis can ultimately lead to cancer [28]. It has been found that several types of cancer, including breast cancer [29], pancreatic cancer [20], and liver cancer, are associated with obesity [3]. Therefore, more effort should be devoted to raising awareness of the connections between obesity, diabetes, and cancer. Inhibiting PTPs and reducing adipokine production as well as ROS levels can potentially help prevent cancer in patients with obesity.

\section{$5 \quad$ Amino Acid Metabolism Plays Important Roles in Both T2D and Cancer}

Aside from glucose metabolism, amino acid metabolism is also deeply involved in both cancer and T2D. Amino acids are the building blocks for protein synthesis and are required for almost all cellular functions. Dysregulation of amino 
acid metabolism can thus contribute to the pathogenesis and progression of many diseases, including diabetes and cancer. Several key amino acids involved in diabetes and cancer include leucine, glutamine, methionine, and cysteine. Therefore, targeting dysregulated amino acid metabolism may serve as a promising strategy for cancer treatments [30, 31].

\subsection{Leucine's Regulation of Insulin Secretion and mTOR Signaling Promotes T2D Pathogenesis, Insulin Resistance, and Cancer Growth}

While it is well known that glucose uptake stimulates the secretion of insulin, the amino acid leucine can also lead to insulin secretion. Leucine allosterically activates glutamate dehydrogenase $(\mathrm{GDH})$, an enzyme that converts glutamate to alpha-ketoglutarate $(\alpha-K G)$ to enter the tricarboxylic acid (TCA) cycle, which leads to the subsequent production of metabolic coupling factors which are needed for insulin secretion [32, 33]. Dysregulation of leucine metabolism can thus result in dysregulated insulin secretion contributing to diabetes. Specifically, it was found that constantly increased leucine levels for a long period of time can be detrimental to the function of $\beta$-cells [34], which are responsible for insulin synthesis and secretion. Leucine is also crucial for the activation of the mammalian target of rapamycin (mTOR) signaling pathway, specifically the mammalian target of rapamycin complex 1 (mTORC1) [35]. The activation of mTOCR1 can lead to the activation of S6 kinases (S6Ks), which results in the downregulation of insulin signaling by phosphorylating insulin receptor substrate 1 (IRS-1). This downregulation of insulin signaling then leads to insulin resistance and T2D [35]. Leucine and mTOR signaling pathway also play important roles in cancer. Studies have shown that mTORC1 is responsible for signaling cell cycle progression and cell survival through eIF4E-binding protein 1 (4E-BP1) and eukaryotic translation initiation factor $4 \mathrm{E}$ (eIF4E), thus promoting cancer growth
[36]. In fact, various mTOR inhibitors are being tested in both preclinical and clinical studies for many cancer types with promising results [37]. Therefore, targeting leucine metabolism and inhibiting the mTOR signaling pathway are promising strategies for the treatment of cancer.

\subsection{Glutamate Regulates Insulin Secretion, Contributes to Gluconeogenesis in T2D, and Promotes Cancer Growth}

As mentioned above, GDH is regulated by leucine, which can lead to insulin secretion. Thus, glutamate, the substrate for GDH, is indirectly involved in regulating insulin secretion even though it cannot stimulate insulin secretion on its own [33]. Therefore, dysregulation in glutamine metabolism may also lead to dysregulated insulin secretion and diabetes. It has also been shown that mutations in GDH can result in hyperinsulinemia [32], which has a growth-promoting effect on cancer cells. Aside from its influence on insulin secretion, a study by Miller et al. has shown that glutamine's contribution to gluconeogenesis is important in T2D with dysregulation of the glycogen signaling pathway, which can lead to hyperglycemia during the fasting state [38]. The role of glutamine in promoting cancer survival and proliferation as an important source of energy and building block materials is well known and also well described in the previous glutamine metabolism chapter [39, 40]. The important roles that glutamine and glutamate play in both diabetes and cancer thus serve as another link between the two diseases.

\subsection{Increased Methionine and Cysteine Levels in T2D Can Promote Cancer Growth}

Other amino acids are also implicated in T2D. Specifically, studies have found that the levels of methionine and cysteine are elevated in the blood of patients with T2D [41]. Methionine is involved in polyamine biosynthesis and one- 
carbon metabolism, which is important for redox homeostasis and nucleotide synthesis [42, 43]. Increased methionine levels can lead to increased nucleotide synthesis and contribute to cancer growth. Methionine metabolism also leads to $S$-adenosylmethionine (SAM) production, the substrate for methyltransferases, which are responsible for the methylation of secondary metabolites, lipids, nucleic acids, and proteins [44]. SAM is involved in histone methylation, which can control gene expression, the dysregulation of which can lead to cancer [43, 45]. In fact, for some cancers, methionine is indispensable, suggesting a condition called "methionine dependence" [46]. On the other hand, cysteine is an important component of glutathione (GSH), an antioxidant used by cancer cells to mediate oxidative stress [47]. Therefore, elevated cysteine levels can result in increased GSH production protecting cancer cells against oxidative stress. In short, increased methionine and cysteine levels in T2D can promote cancer growth, and inhibiting methionine and cysteine metabolism or decreasing their concentrations should be investigated as a potential therapy for cancer.

\section{Exploiting the Similarities and Relationships Between Diabetes and Cancer Metabolism for Cancer Treatment (Fig. 2)}

\subsection{Metformin, a Drug Developed for Diabetes, Can Inhibit Cancer Growth and Proliferation}

As new research provides more insights into these similarities and relationships, it opens a new avenue of repurposing therapeutic strategies used for one disease for the treatment of another. One prominent example is the use of metformin, which was developed decades ago and is a commonly used drug for the treatment of diabetes [48]. It has the ability to reduce the production of glucose in the liver, activate adenosine monophosphate-activated protein kinase
(AMPK), and improve insulin sensitivity [48]. Metformin administration leads to reduced pancreatic and breast cancer risk and prolonged survival for cancer patients even though the specific mechanisms behind its effects are not yet well understood [49]. Metformin has yielded positive results in in vivo studies, including in lung cancer xenografts [50], gastric cancer xenografts [51], and prostate cancer xenografts [52]. It is also currently under clinical trials (e.g., NCT01101438, NCT02122185, NCT01750567) for cancer treatment [53]. A study by Elgogary et al. demonstrated that combination therapy using metformin with the glutaminase inhibitor bis-2-(5-phenylacetamido-1,2,4-thiadiazol2-yl)ethyl sulfide (BPTES) encapsulated in nanoparticles resulted in a more pronounced reduction in pancreatic tumor growth in vivo than either treatment alone since both glucose metabolism and glutamine metabolism of the cancer cells were inhibited [54]. Metformin thus serves as a positive example for which a drug developed for one disease, in this case, diabetes, can be repurposed for the treatment of cancer because of the metabolic similarities between the two diseases. Therefore, the strategy of using drugs developed for diabetes for cancer treatment seems promising and is worth investigating further.

\subsection{Polyphenols Prevent Diabetes and Reduce Cancer Growth}

Polyphenols, such as flavonoids, are naturally occurring compounds found in plants that are being investigated for the treatment of several diseases, including diabetes and cancer [55]. A study by Rienks et al. found that polyphenols, especially flavonoids, can help reduce the risk for T2D [56]. Polyphenols' antihyperglycemic effects are associated with their ability to regulate glucose metabolism and enhance $\beta$-cell function as well as their inhibition of mTOR signaling [35, 57]. In cancers, flavonoids have been shown to modulate several signaling pathways leading to reduced cell proliferation and increased apoptosis [58]. Specifically, Zhang 


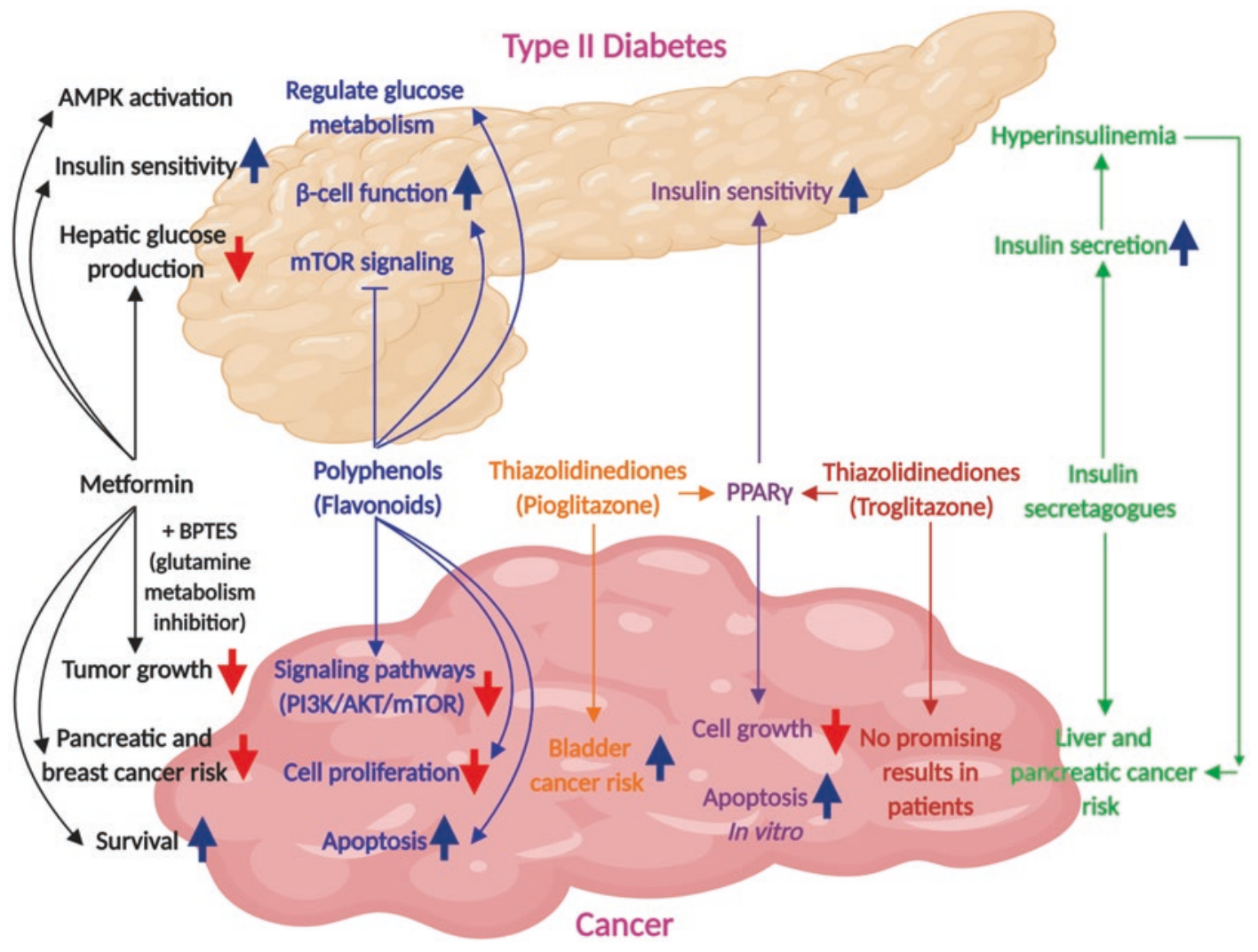

Fig. 2 Antidiabetic drugs and their effects on cancer. Metformin can activate AMPK, increase insulin sensitivity, and decrease hepatic glucose production in T2D while it also leads to reduced pancreatic and breast cancer risk, increased survival, and reduced tumor growth together with glutamine metabolism inhibition by BPTES. Polyphenols such as flavonoids regulate glucose metabolism, increase $\beta$-cell function, and inhibit mTOR signaling in T2D while they impair signaling pathways such as PI3K/AKT/mTOR pathways, decrease cell proliferation, and increase apoptosis in cancer. Thiazolidinediones such as pioglitazone and troglitazone can activate PPAR $\gamma$, leading to increased insulin sensitivity in T2D. However, in cancer, while they can lead to decreased cell growth and increased apoptosis in vitro, pioglitazone is associated with increased bladder cancer risk, and troglitazone showed no promising results in patients. Insulin secretagogues can promote insulin secretion in T2D which can potentially help to explain their observed association with increased liver and pancreatic cancer risk through the effects of hyperinsulinemia

et al. showed that treatment with flavonoids resulted in reduced levels of phospho-PI3K, phospho-AKT, phospho-mTOR, phosphop70S6K, and phospho-ULK, thereby impairing the PI3K/AKT/mTOR/p70S6K/ULK signaling pathways, contributing to their observed effects [59]. Polyphenols and flavonoids thus should be investigated further as potential drugs for cancer treatment.

\subsection{Thiazolidinediones and Their Varied Effects on Cancer}

Thiazolidinediones are antidiabetic drugs that can help improve insulin sensitivity by activating peroxisome proliferator-activated receptor $\gamma$ $(\operatorname{PPAR} \gamma)$ [3, 49]. While PPAR $\gamma$ agonists have been shown to inhibit cell growth and increase apoptosis in vitro, in vivo studies have shown that 
they can actually lead to tumorigenesis, which is potentially caused by varied conditions within the model used that can lead to varied peroxisome proliferator-responsive element (PPRE) activation by PPAR $\gamma$ agonists $[3,60]$. In fact, several cohort studies with patients have indicated an increased risk for bladder cancer associated with the long-term and high-dose exposure to pioglitazone, a thiazolidinedione [49]. Troglitazone, another thiazolidinedione, has been tested in phase II clinical trial for the treatment of refractory metastatic breast cancer but did not exhibit promising results [61]. Therefore, although thiazolidinediones should theoretically have anticancer effects as PPAR $\gamma$ agonists, more research is required before applying them to cancer treatment in patients because of their observed association with increased cancer risk.

\subsection{Insulin Secretagogues Can Lead to Increased Cancer Risks}

Despite the positive effects of certain diabetes drugs on cancer, there are some drugs that can actually promote cancer, especially the drugs that induce insulin secretion in diabetic patients. For example, insulin secretagogues, such as sulfonylureas and meglitinides, which stimulate insulin secretion, have been found to be associated with increased risks for liver and pancreatic cancers [49]. This is likely the result of increased insulin binding to insulin receptors, which, as discussed in Sect. 4, can promote cancer growth through subsequent signaling pathways. Therefore, caution must be exercised, and the drug's mechanism of action and effects must be thoroughly investigated in preclinical models before repurposing it for the treatment of another disease.

\section{Conclusion}

Diabetes and cancer are two of the most common diseases around the world that have many similarities and associations regarding their metabolic characteristics. Diabetes is correlated with an increased risk for many types of cancer. Certain features of diabetes metabolism, such as upregulated HBP and hyperinsulinemia, can actually contribute to cancer pathogenesis and growth. Understanding these associations with the use of metabolomics technologies [62] and exploiting them for cancer treatment, as demonstrated by the repurposing of metformin for cancer treatment, can potentially improve current clinical outcomes. There should also be heightened awareness about the connections between diabetes and cancer so that more efforts can be directed to prevent cancer in diabetic patients, given the metabolic similarities and associations between these two diseases.

\section{References}

1. Prevention, C.f.D.C.a. (2020). In U.S.D.o.H.a.H. Services (Ed.), National diabetes statistics report, 2020. GA: Atlanta.

2. Bray, F., et al. (2018). Global cancer statistics 2018: GLOBOCAN estimates of incidence and mortality worldwide for 36 cancers in 185 countries. CA: A Cancer Journal for Clinicians, 68(6), 394-424.

3. Giovannucci, E., et al. (2010). Diabetes and cancer: A consensus report. Diabetes Care, 33(7), 1674-1685.

4. Sacerdote, C., \& Ricceri, F. (2018). Epidemiological dimensions of the association between type 2 diabetes and cancer: A review of observational studies. Diabetes Research and Clinical Practice, 143, 369-377.

5. Le, A., Udupa, S., \& Zhang, C. (2019). The Metabolic Interplay between Cancer and Other Diseases. Trends Cancer, 5(12), 809-821.

6. Pannala, R., et al. (2008). Prevalence and clinical profile of pancreatic cancer-associated diabetes mellitus. Gastroenterology, 134(4), 981-987.

7. Orgel, E., \& Mittelman, S. D. (2013). The links between insulin resistance, diabetes, and cancer. Current Diabetes Reports, 13(2), 213-222.

8. Gapstur, S. M., et al. (2012). Type II diabetes mellitus and the incidence of epithelial ovarian cancer in the cancer prevention study-II nutrition cohort. Cancer Epidemiology, Biomarkers \& Prevention, 21(11), 2000-2005.

9. American Diabetes, A. (2010). Diagnosis and classification of diabetes mellitus. Diabetes Care, 33(Suppl 1), S62-S69.

10. Warburg, O. (1924). Über den stoffwechsel der carcinomzelle. Naturwissenschaften, 1924, 1131-1137.

11. Bose, S., Zhang, C., \& Le, A. (2021). Glucose metabolism in cancer: The Warburg effect and beyond. Advances in Experimental Medicine and Biology, 1311, https://doi.org/10.1007/978-3-030-65768-0_1. 
12. Waters, A. M., \& Der, C. J. (2018). KRAS: The critical driver and therapeutic target for pancreatic cancer. Cold Spring Harbor Perspectives in Medicine, 8, 9.

13. Hu, C. M., et al. (2019). High glucose triggers nucleotide imbalance through O-GlcNAcylation of key enzymes and induces KRAS mutation in pancreatic cells. Cell Metabolism, 29(6), 1334-1349. e10.

14. Marshall, S., Bacote, V., \& Traxinger, R. R. (1991). Discovery of a metabolic pathway mediating glucoseinduced desensitization of the glucose transport system. Role of hexosamine biosynthesis in the induction of insulin resistance. The Journal of Biological Chemistry, 266(8), 4706-4712.

15. Stowell, S. R., Ju, T., \& Cummings, R. D. (2015). Protein glycosylation in cancer. Annual Review of Pathology, 10, 473-510.

16. Yang, X., \& Qian, K. (2017). Protein O-GlcNAcylation: Emerging mechanisms and functions. Nature Reviews. Molecular Cell Biology, 18(7), 452-465.

17. Cooksey, R. C., \& McClain, D. A. (2011). Increased hexosamine pathway flux and high fat feeding are not additive in inducing insulin resistance: Evidence for a shared pathway. Amino Acids, 40(3), 841-846.

18. Buse, M. G. (2006). Hexosamines, insulin resistance, and the complications of diabetes: Current status. American Journal of Physiology. Endocrinology and Metabolism, 290(1), E1-E8.

19. Bryant, K. L., et al. (2014). KRAS: Feeding pancreatic cancer proliferation. Trends in Biochemical Sciences, 39(2), 91-100.

20. Camelo, F., \& Le, A. (2021). The intricate metabolism of pancreatic cancers. Advances in Experimental Medicine and Biology, 1311, https:// doi.org/10.1007/978-3-030-65768-0_5.

21. Fardini, Y., et al. (2013). O-GlcNAcylation: A new cancer hallmark? Frontiers in Endocrinology (Lausanne), 4, 99.

22. Barkovskaya, A., et al. (2019). O-GlcNAc transferase inhibition differentially affects breast cancer subtypes. Scientific Reports, 9(1), 5670.

23. Yang, C., et al. (2016). High expression of GFAT1 predicts poor prognosis in patients with pancreatic cancer. Scientific Reports, 6, 39044.

24. Cargnello, M., \& Roux, P. P. (2011). Activation and function of the MAPKs and their substrates, the MAPK-activated protein kinases. Microbiology and Molecular Biology Reviews, 75(1), 50-83.

25. Wilcox, G. (2005). Insulin and insulin resistance. Clinical Biochemist Reviews, 26(2), 19-39.

26. Kahn, B. B., \& Flier, J. S. (2000). Obesity and insulin resistance. The Journal of Clinical Investigation, 106(4), 473-481.

27. Fernandez-Sanchez, A., et al. (2011). Inflammation, oxidative stress, and obesity. International Journal of Molecular Sciences, 12(5), 3117-3132.

28. Liou, G. Y., \& Storz, P. (2010). Reactive oxygen species in cancer. Free Radical Research, 44(5), 479-496.

29. Tan, J., \& Le, A. (2021). The heterogeneity of breast cancer metabolism. Advances in Experimental
Medicine and Biology, 1311, https://doi. org/10.1007/978-3-030-65768-0_6.

30. Dang, C. V., et al. (2011). Therapeutic targeting of cancer cell metabolism. Journal of Molecular Medicine (Berlin), 89(3), 205-212.

31. Hirschey, M. D., et al. (2015). Dysregulated metabolism contributes to oncogenesis. Seminars in Cancer Biology, 35(Suppl), S129-S150.

32. Li, C., et al. (2003). Regulation of leucine-stimulated insulin secretion and glutamine metabolism in isolated rat islets. The Journal of Biological Chemistry, 278(5), 2853-2858.

33. Newsholme, P., et al. (2005). New insights into amino acid metabolism, beta-cell function and diabetes. Clinical Science (London, England), 108(3), 185-194.

34. Liu, Z., et al. (2012). Chronic exposure to leucine in vitro induces beta-cell dysfunction in INS-1E cells and mouse islets. The Journal of Endocrinology, 215(1), 79-88.

35. Melnik, B. C. (2012). Leucine signaling in the pathogenesis of type 2 diabetes and obesity. World Journal of Diabetes, 3(3), 38-53.

36. Zoncu, R., Efeyan, A., \& Sabatini, D. M. (2011). mTOR: From growth signal integration to cancer, diabetes and ageing. Nature Reviews. Molecular Cell Biology, 12(1), 21-35.

37. Tian, T., Li, X., \& Zhang, J. (2019). mTOR signaling in cancer and mTOR inhibitors in solid tumor targeting therapy. International Journal of Molecular Sciences, 20, 3.

38. Miller, R. A., et al. (2018). Targeting hepatic glutaminase activity to ameliorate hyperglycemia. Nature Medicine, 24(4), 518-524.

39. Le, A., et al. (2012). Glucose-independent glutamine metabolism via TCA cycling for proliferation and survival in B cells. Cell Metabolism, 15(1), 110-121.

40. Li, T., Copeland, C., \& Le, A. (2021). Glutamine metabolism in cancer. Advances in Experimental Medicine and Biology, 1311, https://doi. org/10.1007/978-3-030-65768-0_2.

41. Adams, S. H. (2011). Emerging perspectives on essential amino acid metabolism in obesity and the insulin-resistant state. Advances in Nutrition, 2(6), 445-456.

42. Gao, X., et al. (2019). Dietary methionine influences therapy in mouse cancer models and alters human metabolism. Nature, 572(7769), 397-401.

43. Gao, X., et al. (2017). Metabolic interactions with cancer epigenetics. Molecular Aspects of Medicine, $54,50-57$.

44. Yang, M., \& Vousden, K. H. (2016). Serine and one-carbon metabolism in cancer. Nature Reviews. Cancer, 16(10), 650-662.

45. Song, Y., Wu, F., \& Wu, J. (2016). Targeting histone methylation for cancer therapy: Enzymes, inhibitors, biological activity and perspectives. Journal of Hematology \& Oncology, 9(1), 49.

46. Cavuoto, P., \& Fenech, M. F. (2012). A review of methionine dependency and the role of methio- 
nine restriction in cancer growth control and lifespan extension. Cancer Treatment Reviews, 38(6), 726-736.

47. Traverso, N., et al. (2013). Role of glutathione in cancer progression and chemoresistance. Oxidative Medicine and Cellular Longevity, 2013, 972913.

48. Rena, G., Hardie, D. G., \& Pearson, E. R. (2017). The mechanisms of action of metformin. Diabetologia, 60(9), 1577-1585.

49. Nguyen, Q. T., et al. (2012). Diabetes medications and cancer risk: Review of the literature. American Health \& Drug Benefits, 5(4), 221-229.

50. Yousef, M., \& Tsiani, E. (2017). Metformin in lung cancer: Review of in vitro and in vivo animal studies. Cancers (Basel), 9, 5.

51. Kato, K., et al. (2012). The antidiabetic drug metformin inhibits gastric cancer cell proliferation in vitro and in vivo. Molecular Cancer Therapeutics, 11(3), 549-560.

52. Ben Sahra, I., et al. (2008). The antidiabetic drug metformin exerts an antitumoral effect in vitro and in vivo through a decrease of cyclin D1 level. Oncogene, 27(25), 3576-3586.

53. Chae, Y. K., et al. (2016). Repurposing metformin for cancer treatment: Current clinical studies. Oncotarget, 7(26), 40767-40780.

54. Elgogary, A., et al. (2016). Combination therapy with BPTES nanoparticles and metformin targets the metabolic heterogeneity of pancreatic cancer. Proceedings of the National Academy of Sciences of the United States of America, 113(36), E5328-E5336.

55. Pandey, K. B., \& Rizvi, S. I. (2009). Plant polyphenols as dietary antioxidants in human health and disease. Oxidative Medicine and Cellular Longevity, 2(5), 270-278.

56. Rienks, J., et al. (2018). Polyphenol exposure and risk of type 2 diabetes: Dose-response meta-analyses and systematic review of prospective cohort studies. The American Journal of Clinical Nutrition, 108(1), 49-61.

57. Bahadoran, Z., Mirmiran, P., \& Azizi, F. (2013). Dietary polyphenols as potential nutraceuticals in management of diabetes: A review. Journal of Diabetes and Metabolic Disorders, 12(1), 43.

58. Abotaleb, M., et al. (2018). Flavonoids in cancer and apoptosis. Cancers (Basel), 11, 1.

59. Zhang, H. W., et al. (2018). Flavonoids inhibit cell proliferation and induce apoptosis and autophagy through downregulation of PI3Kgamma mediated $\mathrm{PI} 3 \mathrm{~K} / \mathrm{AKT} / \mathrm{mTOR} / \mathrm{p} 70 \mathrm{~S} 6 \mathrm{~K} / \mathrm{ULK}$ signaling pathway in human breast cancer cells. Scientific Reports, 8(1), 11255 .

60. Clay, C. E., et al. (2001). Magnitude of peroxisome proliferator-activated receptor-gamma activation is associated with important and seemingly opposite biological responses in breast cancer cells. Journal of Investigative Medicine, 49(5), 413-420.

61. Burstein, H. J., et al. (2003). Use of the peroxisome proliferator-activated receptor (PPAR) gamma ligand troglitazone as treatment for refractory breast cancer: A phase II study. Breast Cancer Research and Treatment, 79(3), 391-397.

62. Hoang, G., Udupa, S., \& Le, A. (2019). Application of metabolomics technologies toward cancer prognosis and therapy. International Review of Cell and Molecular Biology, 347, 191-223.

Open Access This chapter is licensed under the terms of the Creative Commons Attribution 4.0 International License (http://creativecommons.org/licenses/by/4.0/), which permits use, sharing, adaptation, distribution and reproduction in any medium or format, as long as you give appropriate credit to the original author(s) and the source, provide a link to the Creative Commons license and indicate if changes were made.

The images or other third party material in this chapter are included in the chapter's Creative Commons license, unless indicated otherwise in a credit line to the material. If material is not included in the chapter's Creative Commons license and your intended use is not permitted by statutory regulation or exceeds the permitted use, you will need to obtain permission directly from the copyright holder.

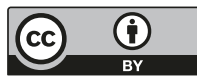




\title{
Bridging the Metabolic Parallels Between Neurological Diseases and Cancer
}

\author{
Shenghao Guo, Yanni Gu, Jiayin Qu, and Anne Le
}

Keywords

Glutamine $\cdot$ Glutamate $\cdot$ NAAG $\cdot$ GABA .

GCP II · Cancer · Neurological diseases

\section{Abbreviations}

2-MPPA

2-(3-Mercaptopropyl) pentanedioic acid

\section{S. Guo}

Department of Pathology, Johns Hopkins University

School of Medicine, Baltimore, MD, USA

Department of Biomedical Engineering, Johns

Hopkins University Whiting School of Engineering,

Baltimore, MD, USA

Y. Gu

Program of Neuroscience, Johns Hopkins University

Krieger School of Arts and Sciences,

Baltimore, MD, USA

J. Qu

Program of Neuroscience, Johns Hopkins University

Krieger School of Arts and Sciences,

Baltimore, MD, USA

Department of Applied Mathematics and Statistics, Johns Hopkins University Whiting School of

Engineering, Baltimore, MD, USA

\section{A. Le $(\bowtie)$}

Department of Pathology and Oncology,

Johns Hopkins University School of Medicine,

Baltimore, MD, USA

Department of Chemical and Biomolecular Engineering, Johns Hopkins University Whiting

School of Engineering, Baltimore, MD, USA

e-mail: annele@jhmi.edu
2-PMPA

$\alpha-\mathrm{KG}$

$\mathrm{AD}$

ADHD

ALS

AMPAR

$\mathrm{A} \beta$

cAMP

CNS

CREB

GABA

GABAAR

GABABR-ab

GABARAP

GAD

GAD-A

GCP II

GLS

GS

HD

KGM

LE

mGluR1

mGluR3

MMP
2-(Phosphonomethyl) pentanedioic acid

Alpha-ketoglutarate

Alzheimer's disease

Attention-deficit/hyperactivity disorder

Amyotrophic lateral sclerosis

$\alpha$-Amino-3-hydroxy-5-methyl-

4-isoxazolepropionic acid receptor

Amyloid-beta

Cyclic adenosine monophosphate Central nervous system

cAMP response element-binding protein

Gamma-aminobutyric acid

GABAA receptor

GABAB receptor antibodies

GABAAR-related protein

Glutamate decarboxylase

GAD autoantibodies

Glutamate carboxypeptidase II

Glutaminase

Glutamine synthetase

Huntington's disease

Alpha-ketoglutaramate

Limbic encephalitis

Metabotropic glutamate receptor I

Group II type 3 metabotropic glutamate receptor

Matrix metalloproteinase 


$\begin{array}{ll}\text { NAA } & N \text {-Acetyl-aspartate } \\ \text { NAAG } & N \text {-Acetyl-aspartyl-glutamate } \\ \text { NMDAR } & N \text {-methyl-D-aspartate receptor } \\ \text { NNK } & \text { 4-(Methylnitrosamino)-1- } \\ & \text { (3-pyridyl)-1-butanone } \\ \text { NO } & \text { Nitric oxide } \\ \text { NOS } & \text { Nitric oxide synthase } \\ \text { OSCC } & \text { Oral squamous cell carcinoma } \\ \text { PAC } & \text { Pulmonary adenocarcinoma } \\ \text { PCP } & \text { Phencyclidine } \\ \text { PD } & \text { Parkinson's disease } \\ \text { PLP } & \text { Pyridoxal 5'-phosphate } \\ \text { PP-2A } & \text { Protein phosphatase 2A } \\ \text { PSMA } & \text { Prostate-specific } \\ & \text { antigen } \\ \text { SCLC } & \text { Small cell lung cancer } \\ \text { SHR } & \text { Spontaneous hypertensive rat } \\ \text { SPS } & \text { Stiff-person syndrome }\end{array}$

\section{Key Points}

- The elevated presence of glutamine in cancer and neurological diseases leads to two distinctive disease progressions and compromises patient survival.

- Glutamate has a prognostic role in cancer, schizophrenia, and hyperammonemia.

- Downregulation of glutamate transporters causes excessive extracellular glutamate in cancer, hyperammonemia diseases, and neurodegenerative diseases.

- NMDA receptor activation protects cancer cells as well as neurons in psychiatric disorders but results in cell deaths for neurodegenerative diseases and hyperammonemia diseases.

- AMPAR expression plays a contrasting role in disease progression of cancer and Alzheimer's disease.

- The complex relationship between glutamine metabolism and $M Y C$ contributes significantly to the pathophysiology of both cancer and neurodegenerative diseases.

- Elevated GAD expression is found in acute stress and contributes to oral squamous cell carcinoma invasiveness.

- GABA level is characteristic of various cancers and contributes to Alzheimer's disease and attention-deficit/hyperactivity disorder (ADHD).
- GABA receptors contribute to Alzheimer's disease pathogenesis, Parkinson's disease severity, and cancer invasiveness.

- Autoimmune disorder's attack on the GABAergic system correlates with neurological diseases and cancers.

- NAAG affects both cancer and neurological disease progression via glutamate.

- Targeting GCP II is a very promising strategy for cancer treatment.

- NAAG inhibits GABA release and indirectly affects both cancer and neurological diseases via GABA-ergic system.

\section{Introduction}

Despite the many recent breakthroughs in cancer research, oncology has traditionally been seen as a distinct field from other diseases. Recently, more attention has been paid to repurposing established therapeutic strategies and targets of other diseases towards cancer treatment, with some of these attempts generating promising outcomes [1,2]. Recent studies using advanced metabolomics technologies [3] have shown evidence of close metabolic similarities between cancer and neurological diseases. These studies have unveiled several metabolic characteristics shared by these two categories of diseases, including metabolisms of glutamine, gammaaminobutyric acid (GABA), and $\mathrm{N}$-acetylaspartyl-glutamate (NAAG) [4-6]. The striking metabolic overlap between cancer and neurological diseases sheds light on novel therapeutic strategies for cancer treatment. For example, 2-(phosphonomethyl) pentanedioic acid (2-PMPA), one of the glutamate carboxypeptidase II (GCP II) inhibitors that prevent the conversion of NAAG to glutamate, has been shown to suppress cancer growth $[6,7]$. These promising results have led to an increased interest in integrating this metabolic overlap between cancer and neurological diseases into the study of cancer metabolism. The advantages of studying this metabolic overlap include not only drug repurposing but also translating existing knowledge from neurological diseases to the field of cancer research. This chapter discusses the spe- 
cific overlapping metabolic features between cancer and neurological diseases, focusing on glutamine, GABA, and NAAG metabolisms. Understanding the interconnections between cancer and neurological diseases will guide researchers and clinicians to find more effective cancer treatments.

\section{Glutamine Plays a Vital Role in Both Cancer Growth and Neurological Diseases}

Glutamine metabolism is a vital contributor to cancer growth and is discussed thoroughly in Chap. 2 [8]. The survival of some tumors depends entirely on the presence of exogenous glutamine, a condition known as glutamine addiction [9]. In addition, the severity of glutamine addiction observed in cancer is positively associated with the degree of tumor malignancies [10]. Besides its role in cancer growth, glutamine is also a well-established precursor of neurotransmitters, glutamate, and GABA [11], and glutamate is involved in learning and memory, especially long-term potentiation [12]. A growing body of evidence has shown that abnormalities in the glutamatergic neurotransmission, including excessive glutamate release and dysfunction of glutamate receptors, play a significant role in a number of neurological diseases, such as Alzheimer's disease (AD), Huntington's disease (HD), hyperammonemic disease, schizophrenia, and other psychotic disorders [13-16]. In this section, examining the glutamine metabolism in neurological diseases may provide further understanding of glutamine's role in cancer and provide insights into glutamine-targeting cancer therapies.

\subsection{The Elevated Presence of Glutamine in Cancer and Neurological Diseases Leads to Two Distinctive Disease Progressions and Compromised Patient Survival}

Glutamine addiction and an elevated level of glutamine are observed in a variety of cancers and promote tumor growth by serving as a major energy source. Similarly, the high presence of glutamine was also observed in hyperammonemia diseases, where ammonia level increases abnormally in blood. Hyperammonemic diseases are associated with many brain injuries, including cerebral edema, and is believed to be the primary cause of hepatic encephalopathy [17]. Glutamine acts as an astroglial intracellular idiogenic osmole, disturbing the delicate balance between water and glutamine concentrations inside and outside of astrocytes. The result is an influx of water into the cells, causing cerebral edema. The suppression of glutamine accumulation results in reduced swelling of glial cells [13]. Thus, although the role of elevated levels of glutamine in cancer progression and aggressiveness is very different from its negative effects in edema under hyperammonemia, both impair patient survival.

Glutaminase (GLS) catalyzes the conversion of glutamine to glutamate. Because it plays a central role in the glutamine/glutamate metabolic cycle, GLS is extremely important in cancer and neurological disorders. As previously discussed in Chap. 2, inhibition of GLS suppresses the growth of various tumors [8, 18-21]. Moreover, the upregulated conversion from glutamine to glutamate as a source of cellular bioenergy is a common characteristic observed in many cancers [22]. Therefore, the activity of GLS is crucial to cancer development.

An increase in GLS enzymatic activity, as well as transcription, has also been observed in schizophrenia, though without a detailed mechanism as to how the enzyme contributes to the disease pathophysiology [16]. Nevertheless, it is fair to connect the increase of GLS to glutamate accumulation and thus to the disease symptomology. In both cancer and schizophrenia, GLS upregulation is thought to be a major factor in disease progression.

On the other hand, glutamine-fueled tricarboxylic acid cycle (TCA cycle activity) via the GLS pathway [23] has differing impacts on cancer and Alzheimer's disease. In contrast to the increased GLS activation in cancer, a downregulation in GLS has been observed in AD pathophysiology [14]. Reduced GLS activation, resulting in hampered oxidative glutamine metabolism, is a poten- 
tial early marker of $\mathrm{AD}$ pathogenesis, preceding amyloid plaque formation in mice [14]. A significant decrease in glutamine-fueled TCA cycle activity, which requires GLS activity, has been observed in early AD [14], suggesting the importance of glutamine/glutamate-derived energy against disease development. It was found that a compromise in both ATP synthesis rate and cellular energy homeostasis caused by decreased TCA cycle activity likely results in amyloid plaque deposition [14], and thus is responsible for the core progression of $\mathrm{AD}$.

Opposing mechanisms involving GLS, alphaketoglutarate $(\alpha-\mathrm{KG})$, and TCA cycle have been observed in both cancer and AD. While a decrease in glutamine-fueled TCA cycle activity is beneficial in terms of tumor suppression, it actually promotes $\mathrm{AD}$ progression, causing plaque formation and leading to disease development, which may appear to be a new complication for glutamine-targeting cancer therapies.

\subsection{The Prognostic Role of Glutamate in Cancer, Schizophrenia, and Hyperammonemia}

In addition to the elevated presence of glutamine, excessive glutamate, a direct product of glutamine via the glutaminase I pathway, also significantly impacts cancer. Glutamate acts as an energy source for cancer cells and can be directly converted to $\alpha-\mathrm{KG}$, a TCA cycle intermediate. Glutamate concentration positively correlates with the severity of cancer, as measured by the Gleason score in prostate cancer [24]. Glutamate deprivation or blockage with antagonists of metabotropic glutamate receptor I (mGluR1) results in decreased cancer cell growth, migration, and invasion and eventually contributes to apoptotic cancer cell death [24]. With the direct correlation of glutamate concentration in serum and cancer severity, glutamate can thus serve as a prominent prognostic indicator of cancer development.

Excessive glutamate levels have also been observed in a wide range of neurological diseases. This accumulation results in neuronal cell deaths due to neuro-excitotoxicity, underlying the pathophysiology of neuronal loss in multiple neurological diseases $[15,25]$. Excess glutamate amplifies and exacerbates excitotoxicity through the positive feedback mechanism involving $\mathrm{Ca}^{2+}$ influx $[25,26]$. Moreover, recent research in schizophrenia has shown that a higher glutamate level results in more severe disease symptoms and lower remission rates after treatment [26]. Interestingly, as schizophrenia moves from onset to the chronic stage, glutamate level, especially the ratio of glutamate/glutamine, increases significantly [27, 28]. Dysfunction of glutamate neurotransmission with lower glutamate levels during the disorder's onset and with higher glutamate levels for the chronic stage suggests the facilitating role of glutamate in the course of schizophrenia progression. The relationship between glutamate elevation and disease severity is also seen in hyperammonemia diseases [17]. The increasing extracellular glutamate concentration is also observed to be in a positive feedback loop with nitric oxide (NO) [17], thus contributing to the ever-worsening progression of hyperammonemia and neuronal death from both glutamate and NO toxicity.

A higher level of glutamate contributes to increased disease severity in cancer, schizophrenia, and hyperammonemia and thus is responsible for the longitudinal development of all three diseases. It is clear that the negative impact of glutamate accumulation exacerbates a variety of diseases ranging from cancer to a multitude of neurological disorders, indicating the prognostic role of excessive glutamate in these diseases.

Glutamine synthetase (GS) transforms glutamate into glutamine, preventing excitotoxicity due to glutamate accumulation. Therefore, dysfunction in GS results in an imbalance of glutamate and glutamine, causing pathophysiology in many diseases. Due to the importance of glutamate and glutamine in cancer, GS dysfunction and its implications are widely studied. Bode et al. showed that GS contributed to cancer cell proliferation, resistance, and aggression [4]. As cancer consumed glutamine to support the TCA cycle, GS provided an alternative influx of glutamine under glutamine-depleted conditions [4]. An increase in GS/GLS ratio was observed at both mRNA and enzymatic activity levels start- 
ing at $24 \mathrm{~h}$ after tumor implantation in liver and kidney tumor models [10], suggesting an elevated need for glutamine production in cancer cells.

GS dysregulation is also seen in Huntington's disease (HD). An increase in GS activity has been observed to predominate in severely affected areas of the brain [29], indicating the relation between GS upregulation and symptomology and severity in HD. Therefore, GS may be responsible for disease aggression through glutamatergic energy production in cancer as well as upregulated expression in HD. In hyperammonemia, a decrease in GS activity in non-glutamatergic areas leads to ammonia and glutamate accumulation, since GS converts glutamate and ammonia into glutamine. This inability to keep the glutamate concentration from exceeding the normal physiological limit may be the root of neuronal toxicity observed in hyperammonemia [17] (Fig. 1).

The dysregulation of GS thus exerts divergent influences on different diseases. GS activity promotes aggression in both cancer and HD. On the other hand, in hyperammonemia diseases, GS activity prevents excitotoxicity from elevated glutamate levels. As glutamine and glutamate have opposite implications in diseases under certain conditions, the proper balance of these two, regulated by GS and GLS, is a fine line to navigate.

\subsection{Downregulation of Glutamate Transporters Causes Excessive Extracellular Glutamate in Cancer, Hyperammonemic Diseases, and Neurodegenerative Diseases}

Given the effect of excessive glutamate, targeting the cause behind high glutamate concentration is a promising therapeutic strategy. One of the reasons for the elevated concentration of glutamate is the downregulation of glutamate transporters, which are proteins embedded in the astroglial membrane. Impaired glutamate transporters often result in glutamate accumulation in the extracellular space, which underlies a wide range of diseases discussed above. As a result, the dysfunction of glutamate transporters is a contributing factor to the pathophysiology of many diseases, including cancers and neurological diseases [30]. For example, glutamate transporters were found to support cancer cells' survival, growth, and invasion [31]. In cancer, and especially in gliomas, the downregulation of glutamate transporters resulting in a glutamate excess is associated with the extreme aggressiveness of malignant gliomas [31]. This suggests that the hypofunction of glutamate transporters plays a role in energy provision in cancer, which eventu-

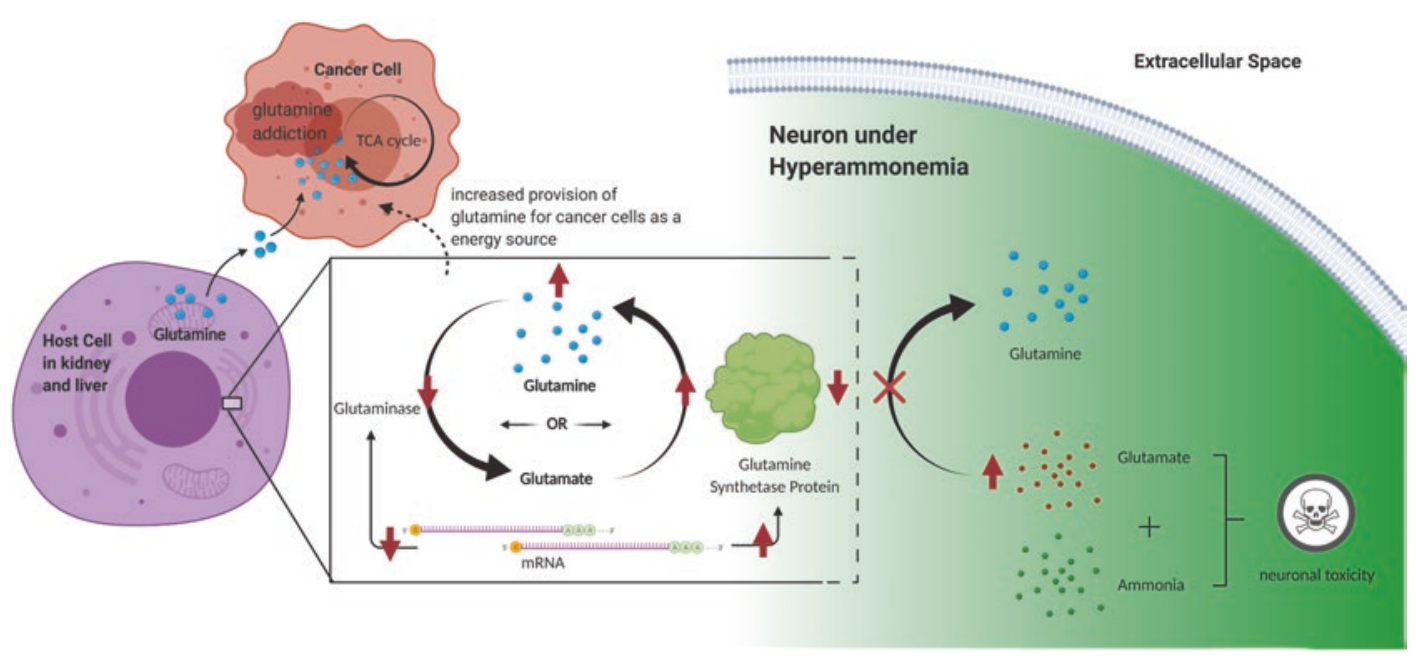

Fig. 1 The effects of glutamine synthetase dysregulation on both cancer and hyperammonemic diseases 
ally results in the development of devastating tumors.

Downregulation of glutamate transporters is also observed in multiple neurological diseases [17]. Specifically, in hyperammonemia diseases, the reduced expression of astrocytic glutamate transporters possibly contributes to the delayed degeneration of a certain number of neurons as well as the increased extracellular concentrations of glutamate [15, 17], which is thought to contribute to hyperammonemia severity and other underlying abnormalities in acute liver failure [17]. In addition, neurodegenerative diseases, such as HD and AD, are also associated with excitotoxic impacts from elevated glutamate due to the downregulation of glial transporters [15]. Previous studies have shown that elicitation of glutamate transporters has neuroprotective implications, especially in $\mathrm{AD}$ and depression [25, 32], suggesting a direct correlation of reduced expression of glutamate transporters to disease pathophysiology.

The downregulation of glutamate transporters correlates with tumor malignancy and aggression, possibly via increasing extracellular glutamate levels. Downregulated glutamate transporters also cause neuronal deaths in hyperammonemia and neurodegenerative diseases. Despite the different effects of glutamate transporter hypofunction in cancer versus neurological diseases, they all contribute to the progression of these diseases.

\subsection{NMDA Receptor Leads to Opposite Cell Fates in Cancer and Psychiatric Disorders Versus Neurodegenerative Diseases and Hyperammonemic Diseases}

Although the exact mechanisms involving glutamate receptors have not yet been discovered completely in cancer, it is widely known that antagonists of the $N$-methyl-D-aspartate receptor (NMDAR), an ionotropic glutamate receptor, can decrease cancer cell viability and prevent tumor growth [33].
In schizophrenia and bipolar disorder, NMDAR activity has a neuroprotective advantage. Hypofunction of NMDAR was observed in both diseases [28, 34]. In addition, the downregulation of NMDAR induced and exacerbated schizophrenia symptoms [28]. Possible explanations for downregulated NMDAR-induced abnormalities have been suggested for schizophrenia. The receptor is likely responsible for cognitive development, axon pruning, and neuron preservation [35], which are all significantly impaired in schizophrenia symptomology [35].

Although NMDAR is essential for tumor survival and development as well as possible neuron preservation in schizophrenia, NMDAR activation is, in fact, related to cell death in neurodegenerative diseases. In neurodegenerative diseases, high $\mathrm{Ca}^{2+}$ permeability in NMDAR contributed to chronic neurodegeneration due to excitotoxicity [26]. Research has confirmed that neuronal survival can be maintained via NMDAR blockage [25, 26], indicating the direct causal role of NMDAR in cellular necrosis. Specifically, NMDAR activation, which reduces protein phosphatase 2A (PP-2A) activity, is a major contributor to the hyperphosphorylation of microtubule-associated protein Tau $(\tau)$ in AD [25]. In hyperammonemia diseases, NMDAR activation can result in ammonia-induced death of animals [17]. Moreover, excessive stimulation of NMDAR is also responsible for NO formation through the activation of nitric oxide synthase (NOS) [17]. NO accumulation through acute and chronic exposure is toxic to neuronal cells, which is observed in several neurological diseases, including Huntington's disease, Alzheimer's disease, and hyperammonemia [17].

It is safe to say that NMDAR is closely associated with the viability and growth of cancer cells and normal neurons in psychiatric patients' brains as well as with the toxic cellular deaths observed in neurodegenerative diseases and hyperammonemia diseases. The abundant evidence suggests that NMDAR activation leads to vastly different responses in cancer and neurological diseases. Thus, NMDAR has the potential as a drug target in cancer treatment. 


\subsection{AMPAR Expression Played a Contrasting Role in Disease Progression of Cancer and Alzheimer's Disease}

$\alpha$ - A m in o- 3 - h y d rox y - 5 - m e t h y $1-4-$ isoxazolepropionic acid receptor (AMPAR), an ionotropic glutamate receptor in the central nervous system, is also a major contributor to disease development in both cancer and neurological diseases. A study by Herner et al. has shown that the inhibition of GluR1 and GluR2 subunits of AMPAR significantly decreases tumor invasion [36]. Specifically, AMPAR activation could be a switch to activate the Kras/MAPK cascade, which induces oncogenic signaling and eventually leads to tumor invasion, migration, and malignant transformation [36]. Interestingly, though both AMPAR and NMDAR are involved in neuroexcitotoxicity, the mechanism of AMPAR works opposite to that of NMDAR. In AD, a loss in GluR2 of AMPAR results in $\mathrm{Ca}^{2+}$-mediated neurotoxicity and severe neuropathology [25]. Excitotoxicity could be induced by a loss of GluR2 subunit alone, and upregulation of GluR2 subunit can confer a protective advantage under increased $\mathrm{Ca}^{2+}$ levels [25]. Although both NMDAR and AMPAR are activated by the same neurotransmitter, glutamate, their effects on pathophysiology in cancer and $\mathrm{AD}$ are widely different. AMPAR expression plays a contrasting role in the two diseases' progression. While AMPAR activity in cancer is to promote tumor invasion and malignancy transformation, in $\mathrm{AD}$, its role is neuroprotective against $\mathrm{Ca}^{2+}$-mediated neurotoxicity and disease severity [25].

\subsection{The Complex Relationship Between Glutamine Metabolism and MYC Contributes Significantly to the Pathophysiology of Both Cancer and Neurodegenerative Diseases}

MYC is a family of regulator genes and protooncogenes that codes for transcription factors and plays a significant role in cancer progression [3739]. In addition, $M Y C$ is also closely associated with the glutamine metabolism in both cancers and neurological diseases. MYC upregulates GLS expression and is primarily responsible for glutamine addiction in cancer [40-42]. The upregulation of GLS, as discussed in Sects. 2.1 and 2.2, leads to enhanced glutaminolysis and glutamate accumulation, and in turn promotes cancer growth [24]. Therefore, it is not surprising that an overexpression of $M Y C$ is associated with tumorigenesis [43]. Across various cancer types, MYC suppression results in reduced cancer cell growth, impaired colony formation, decreased tumor progression, and even induced cancer cell apoptosis [44-46]. In a recent study, new light has been shone on what was originally considered to be a one-way effect on glutamine metabolism by $M Y C$. There actually exists a reciprocal regulation between $M Y C$ and glutamine metabolism, meaning that suppression of glutamine metabolism can prevent MYC transcription [47]. This mutual balance and regulation between the oncogene and the amino acid cause significant pathophysiological impacts when they affect each other, which can be a new research target for cancer treatment.

Interestingly, $M Y C$, a proto-oncogene, is also closely involved in neurodegenerative diseases, especially $\mathrm{HD}$ and $\mathrm{AD}$, via glutamine metabolism. The excitotoxic pathway through NMDAR activation in HD involves the induction of MYC and other pro-apoptotic proteins that eventually result in neuronal deaths, significant underlying pathophysiology in neurodegenerative diseases [48]. More specifically, MYC induces significant cognitive deficits and cell cycle reentry, which is believed to be responsible for neuronal cell death through loss of trophic support during development, thus leading to neurodegeneration in $\mathrm{AD}$ [43] (Fig. 2).

$M Y C$ and glutamine pathways are therefore clearly associated with each other and also with the pathophysiology of both cancer and neurodegenerative diseases. This is another indication of the close interplay between the metabolisms of cancer and neurological diseases, which also confirms glutamine as being a very promising drug target for cancer treatment. 


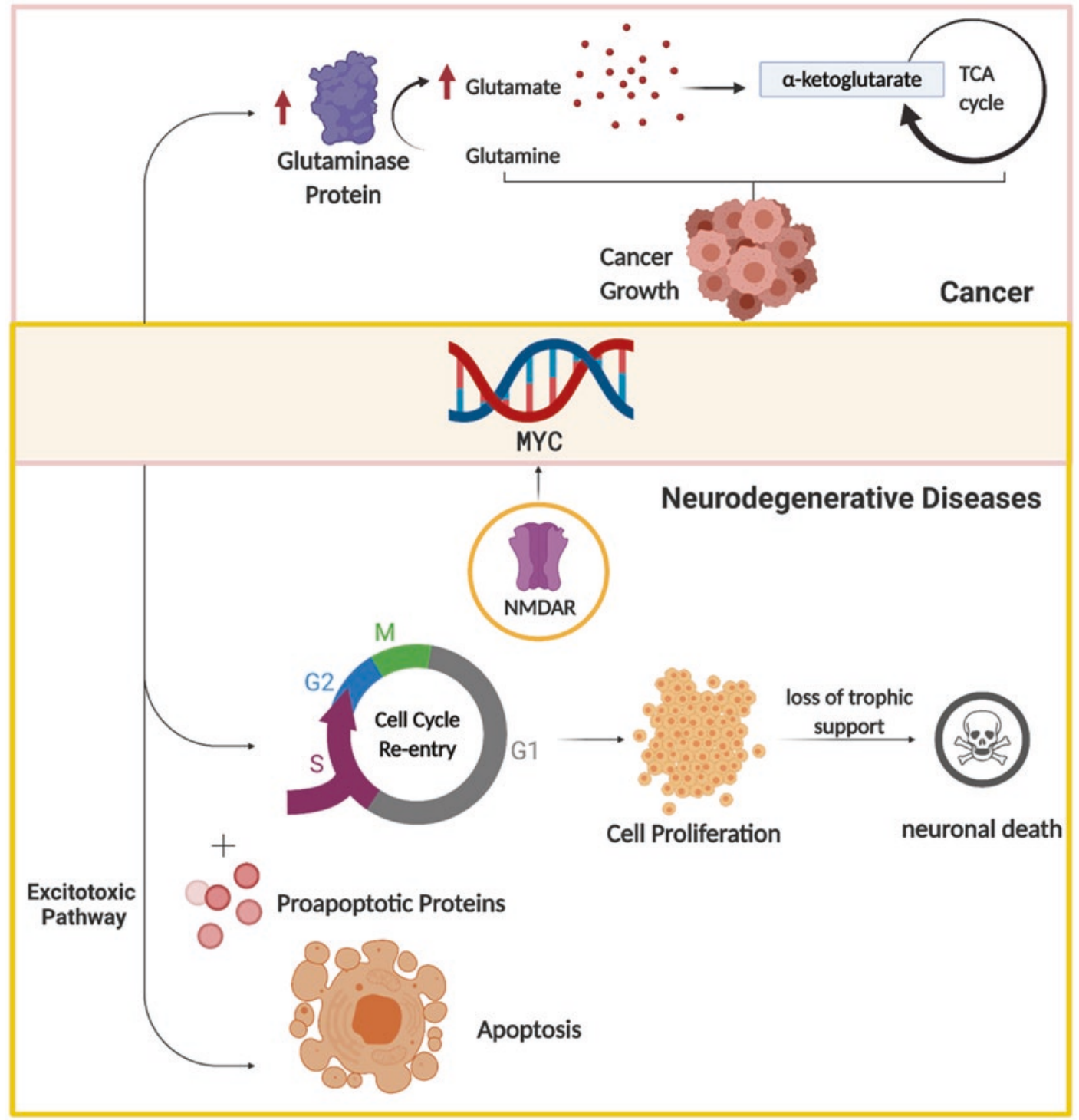

Fig. $2 \mathrm{MYC}$ and its contributions in cancer and neurodegenerative diseases. NMDAR N-methyl-D-aspartate receptor

\subsection{Alpha-ketoglutaramate and Its Shared Pathway in Cancer and Hyperammonemic Diseases}

Recent study revealed that alpha-ketoglutaramate $(\mathrm{KGM})$ is a significant component in glutamine addiction, connecting glutamine to the TCA cycle via the glutaminase II pathway, in which glutamine is converted to KGM by transamination with a suitable $\alpha$-keto acid. KGM is then hydro- lyzed to $\alpha-\mathrm{KG}$, an intermediate metabolite within the TCA cycle, by an enzyme known as $\omega$-amidase [49]. The presence of glutamine, KGM, and $\alpha-\mathrm{KG}$, therefore, promotes tumor growth. In addition, the blockage of glutamine's conversion to KGM in the glutaminase II pathway results in the complete inhibition of pancreatic tumorigenesis in vivo, suggesting the important role KGM plays in tumor progression [2, 22]. A recently publicized study correlates increasing prostate cancer cell aggressiveness with an upregulation of the glutaminase II pathway [50]. 
$\mathrm{KGM}$ is a major contributor not only in cancer but also in hyperammonemia diseases. It is a biomarker for both primary and secondary hyperammonemia diseases, including hepatic encephalopathy [49]. KGM concentration was shown to be correlated with the degree of encephalopathy. Although the specific mechanisms are still unknown, the study indicated a direct relationship between KGM and hyperammonemia disease progression [51].

This association of hyperammonemia and cancer involving KGM is a significant discovery in the metabolic interplay between cancer and neurological diseases. KGM not only promotes tumor growth through the glutaminase II pathway [50], but also serves as an excellent biomarker for hyperammonemia diseases and an indicator for encephalopathy severity.

\section{GABA and Its Multiple Functions in Neurological Diseases and Cancer}

Gamma-aminobutyric acid (GABA) serves as an inhibitory neurotransmitter in the central nervous system, and can also be detected in peripheral tissues. Dysregulation of the GABAergic system and related metabolisms are observed in various cancers and neurological diseases. In neurological diseases, GABA works through the neural networks and mainly serves as an inhibitory neurotransmitter [52], whereas in cancer, GABA is found in peripheral tissues, serving as an onco-metabolite [53] by affecting multiple cell functions, including cell proliferation and mobility (Fig. 3).

\subsection{Elevated GAD Expression Is Found in Acute Stress and Contributes to Oral Squamous Cell Carcinoma Invasiveness}

Glutamate decarboxylase (GAD) is a ratelimiting enzyme that catalyzes the production of GABA from glutamate [54]. GAD has two enzymes: GAD65 and GAD67. The two enzymes have different subcellular locations and interact differently with the cofactor pyridoxal 5 '-phos- phate (PLP) [55]. GAD65 and GAD67 are encoded by genes GAD2 and GAD1, respectively. In both acute stress and oral squamous cell carcinoma (OSCC), the upregulation of GAD67 is observed [56].

Acute stress primarily affects GAD67: increased GAD67 mRNA level is observed in various brain regions, including the arcuate nucleus, dorsomedial hypothalamic nucleus, and hippocampus [57]. Increased GAD67 mRNA is preferentially induced in acute stress, compared to chronic stress, possibly due to GAD67's potentiality of rapid activation as almost all GAD67 is bounded to its cofactor in the CNS [57].

In the case of OSCC, overexpression of GAD1 is found to be a characteristic event. The knockout of GAD1 in OSCC-derived cell lines interfered with the invasive ability. It is hypothesized that the process is completed through GAD1 regulation of $\beta$-catenin, which leads to the activation of MMP7, a gene found to be expressed in many cancers, including breast cancer, lung cancer, and prostate cancer, that contributes to tumor invasiveness and metastasis [58].

Although the underlying mechanisms are different, the upregulation of GAD67 is observed in both acute stress and OSCC. Hence, GAD1 and GAD67 mRNA might be possible targets in the treatment of OSCC.

\subsection{GABA Levels Are Characteristic of Various Cancers and Contribute to Alzheimer's Disease and Attention-Deficit/ Hyperactivity Disorder (ADHD)}

Dysregulation of GABA metabolism is observed in many CNS disorders and various types of cancers. For instance, elevated GABA levels are characteristic of both $\mathrm{AD}$ and gastric cancer [5]. In $\mathrm{AD}$ patients, astrocytes in the dentate gyrus exhibit increased GAD activation, leading to an elevated level of GABA [59]. One direct result of the increased GABA level is enhanced tonic neural inhibition, one of the major symptoms in $\mathrm{AD}$ [59]. In gastric cancer, evaluation of gastric neoplastic tissue revealed that GAD activity is sig- 


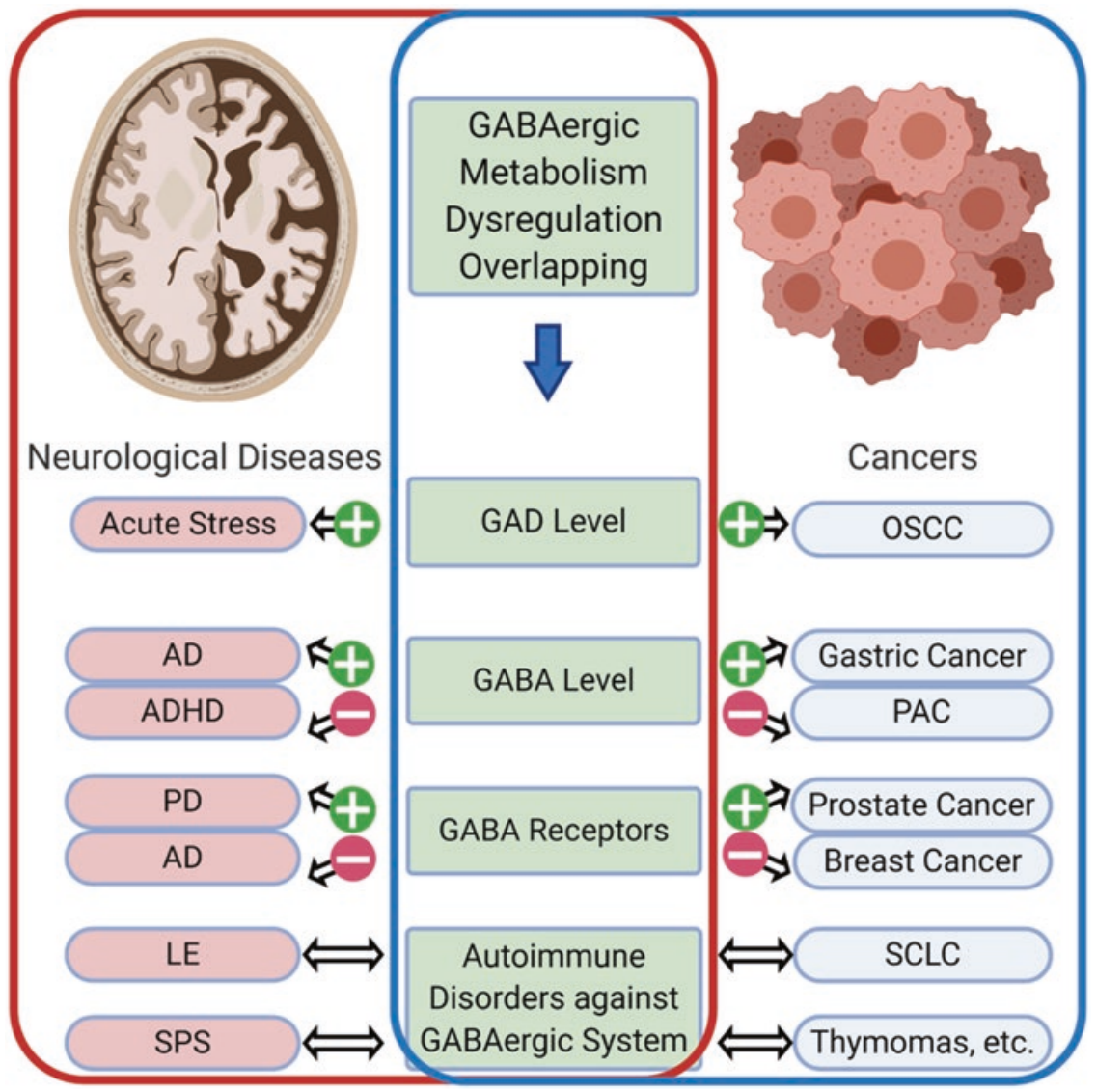

Fig. 3 A general view of GABA-ergic system-related metabolism with corresponding cancers and neurological diseases. $G A D$ glutamate decarboxylase, $A D$ Alzheimer's disease, $A D H D$ attention-deficit/hyperactivity disorder, $P D$ Parkinson's disease, $L E$ limbic encephalitis, SPS stiff-person syndrome, $O S C C$ oral squamous cell carcinoma, PAC pulmonary adenocarcinoma, $S C L C$ small cell lung cancer

nificantly higher than in surrounding normal tissue and that GABA content increases above normal levels [5]. Similar results have also been obtained with other cancers, including breast cancer, colon cancer, and prostate cancer [5]. The exact underlying mechanism of the abnormal GABA metabolism in cancer remains unclear. It is still practical, however, to deem an elevated GABA level as a good indicator of both $\mathrm{AD}$ and various cancers.

Not all the neurological diseases and cancers have upregulated levels of GABA. For example, GABA downregulation is observed in ADHD and pulmonary adenocarcinoma (PAC). ADHD is the most diagnosed psychiatric disorder in children and adolescents. Recent research based on the spontaneous hypertensive rat
(SHR) models suggested that a decrease in the extracellular concentration of GABA in the SHR hippocampus may be the underlying reason for the ADHD-like behaviors [60]. A decreased GABA level was also observed in PAC. It was found that tobacco-specific nitrosamine 4-(methylnitrosamino)-1-(3-pyridyl)1-butanone (NNK) can significantly reduce GABA in the lungs and that NNK-induced PAC expresses particularly low levels of GABA. Data showed that GABA could limit PAC cell proliferation and migration through the inhibition of cAMP signaling, suggesting that PAC cells downregulate GABA to promote tumor growth and invasion [61]. The underlying mechanism for how NNK suppresses GABA level remains unclear, however. 


\subsection{GABA Receptors Contribute to Alzheimer's Disease Pathogenesis, Parkinson's Disease Severity, and Cancer Invasiveness}

GABA receptors can be divided into two classes: GABAA receptors and GABAB receptors. Downregulation of ionotropic GABAA receptor (GABAAR) is observed in both $\mathrm{AD}$ [62] and breast cancer [63]. A recent study of AD explored amyloid beta's $(A \beta)$ ability to weaken synaptic inhibition through the downregulation of GABAAR, indicating that such regulation is achieved through induced endocytosis of GABAAR [62]. Impaired inhibitory circuits, coupled with neuronal hyperexcitability [62], might contribute to the characteristic formation of amyloid plaque in $\mathrm{AD}$ [64].

In breast cancer, downregulation of GABAAR is achieved through decreased expression of GABAAR-related protein (GABARAP). In invasive ductal and invasive lobular carcinomas, GABARAP mRNA and GABARAP expression were all found to be significantly downregulated [63]. GABARAP was suggested to be a tumor suppressor, but how GABARAP is suppressed and overcome by the tumor cells is yet to be determined. Although the underlying mechanisms are different, downregulation of the GABAAR was observed in both breast cancer and AD [63]. Moreover, understanding the mechanism behind GABARAP suppression in cancer and restoring its function could be a possible strategy for cancer treatment. In the case of prostate cancer, the GABAAR plays an important role in the proliferation of prostate cancer cells. Non-cancer prostate epithelial tissues showed no GABAAR expression, whereas $15 \%$ of prostate cancer epithelial tissue samples showed GABAAR expression to various extents [1]. Applying antagonists of various receptors, including GABAAR antagonist, picrotoxin, inhibited the proliferation of prostate cancer cells. Moreover, applying a GABAAR agonist stimulates the proliferation of several prostate cancer cell lines [1]. Therefore, GABAAR is shown to significantly promote prostate cancer growth, and inhibiting GABAAR becomes a potential strategy in prostate cancer treatment.

Just like the opposite trends of GABA concentration between different neurological diseases and cancers, besides its downregulation, GABAAR upregulation is also observed in Parkinson's disease (PD). PD is the second most common neurodegenerative disease. In patients with PD, the concentration of GABAAR, especially those containing the $\alpha 4$ subunit, is increased approximately 22 -fold in the caudate nucleus in basal ganglia [65]. Increased GABAAR levels also strengthened the tonic inhibition by astrocytes, indicating a direct relationship between increased GABAAR and PD severity [65].

Given the malignant effect that GABAAR has on both PD and prostate cancer, it is worth exploring whether reducing GABAAR expression or suppressing GABAAR function could help mitigate prostate cancer. This is another excellent example of how the overlap between neurological diseases and cancer sheds light on cancer treatment.

Besides its role in a variety of cancers, GABABR is also a possible target for the treatment of various types of cancers and neurological diseases. Specifically, baclofen, an agonist of GABABR, has been shown to be effective in inhibiting tumor growth in rat models, which further exemplifies the suppressive role of GABABR activation in tumor development [66]. Of particular note, clinical data also suggest that baclofen can be used to reduce certain types of drug addiction, including cocaine, alcohol, nicotine, and heroin [52]. Positive modulation of GABABR was also shown to be effective in treating anxiety in rat models. Rats with no GABABR were more anxious, whereas acute and chronic treatments with positive GABABR modulator CG39783 decreased anxiety levels generated in rats [67].

Research on the influence of GABA on human prostate cancer cells in vitro suggests that matrix metalloproteinase (MMP) induced by GABA greatly promotes the invasive ability of the cells [68]. Applying GABABR antagonists significantly reduced MMP production as well as prostate cancer cell invasiveness, whereas applying 
GABABR agonists induced the opposite effect [68]. The overall result suggests that positive modulation of GABABR is implicated in the invasive ability of prostate cancer cells.

A blockade of GABABR using GABABR antagonist or GABABR knockout was found to produce an antidepressant-like phenotype in rat models. One hypothesis suggests that the antidepressant function is based on the interaction between the GABABR and the serotonergic system and neurotrophic factors [69].

Although GABABR function is distinctly different in cancers and neurological diseases, significant effects in cancers are observed with both the upregulation and downregulation of the receptors. Therefore, it is still very promising to develop treatments based on GABAB receptor modulators after taking cancer type-specific effects into consideration.

\subsection{Autoimmune Disorders' Attack on the GABA-ergic System Correlates with Neurological Diseases and Cancers}

Autoimmune disorders are observed to attack the GABA-ergic system, and two of these disorders are closely intertwined with specific types of neurological diseases and cancers.

The first disease is limbic encephalitis (LE), which is frequently related to small cell lung cancer (SCLC). LE mainly affects the medial temporal lobes and patients exhibit symptoms including seizures, short-term memory impairment, and anxiety [70]. In a study of 20 patients who have GABAB receptor antibodies (GABABR-ab), 19 (95\%) patients eventually developed LE. 10 of these 19 patients developed SCLC [71]. The specific relationship between LE and SCLC is not yet clear, but the close correlation between the two could still provide valuable insights toward treatment, with GABABR-ab serving as a practical diagnostic factor.

The second disease is stiff-person syndrome (SPS), often seen accompanied by thymomas and breast cancer. SPS is a rare neurological disease with 1-2 cases/million [72, 73], and is characterized by rigidity and spasm of skeletal muscles in limbs and trunks. Patients showed increased sensitivity to stimuli, such as noise and emotional perturbation [74]. The majority of SPS patients exhibited an increased concentration of autoantibodies against both forms of GADs in cerebral fluids [75]. Additionally, $83 \%$ of sera from SPS patients with positive GAD autoantibodies (GAD-A) decreased GABA production in brain extracts of rats [75]. As a direct consequence of GAD malfunctioning, GABA synthesis is interrupted, and magnetic resonance spectroscopy revealed a reduced level of GABA in the motor cortices of people with SPS [76]. In the case of paraneoplastic SPS, research showed that $53 \%$ of the tumors in patients with SPS are thymomas and breast cancer [72]. Given that GADs are expressed in breast cancer, and the overall GABA metabolism mediates breast cancer metastasis, the association may be caused by the cancerinduced autoimmune disorder [53]. Thus, an increased GAD level might trigger an autoimmune response, leading to the production of GAD-A [72].

Although the underlying mechanisms of the interplay between autoimmune system disorders, neurological diseases, and cancers have yet to be identified, the altered metabolic signatures could serve as a prognostic indicator of cancers and neurological disease progression.

\section{$4 \quad$ NAAG and Its Versatile Role in Neurological Diseases and Cancer}

As one of the most prevalent and widely distributed neuropeptides found in the mammalian nervous system, $\mathrm{N}$-acetyl-aspartyl-glutamate (NAAG) was not initially considered a neurotransmitter when it was first discovered in 1965 , due to its abundance and identity as a peptide. It was not tested against neurotransmitter criteria until the 1980s, and only then was it formally established as a new neurotransmitter. Its 
role in the neurological system was later found to be more versatile than simply being the precursor of glutamate, since it also functions as an agonist of group II metabotropic glutamate receptor (mGluR3), synaptic GABA release inhibitor, and cyclic AMP inhibitor [77].

The recognition of NAAG's role in cancer growth was even more delayed. Only recently a study by Nguyen et al. revealed that NAAG plays an important role as a glutamate reservoir for cancer growth via GCP II [6]. Moreover, NAAG can also serve as an indicator of cancer progression as increases in NAAG concentrations were observed in higher grade lymphoma, ovarian cancers, and gliomas compared to their lower grade counterparts $[6,78,79]$. Therefore, the direct conversion of NAAG to glutamate established NAAG as a juncture between the fields of cancer and neurological disease and became a shared characteristic pathway and a drug target for the two disease groups. Despite its positive correlation with cancer progression, the correlation between NAAG concentration and neurological disease progression varies depending on the type of neurological disease. In general, NAAG is established as a neuroprotective neurotransmitter in the neurological system, where a decreased level of NAAG is correlated with neuronal loss [80]. Besides serving as a neurotransmitter in neurological diseases, NAAG can also reduce other synaptic neurotransmitter levels, namely GABA [81] and glutamate, by activating a group II G protein-coupled metabotropic glutamate (mGlu) receptor, mGluR3, to achieve neuroprotective effects and reduce neurotoxicity caused by glutamate accumulation [82]. Meanwhile, mGluR3 is also known to promote cancer growth [8390]. Previous studies have shown that mGluR3 expression is associated with glioma growth and poor GBM survival rates [85]. In GCP II-negative glioblastoma, it is suspected that NAAG could be taking on another role to promote cell growth through an alternative pathway by acting as an agonist to mGluR3, thereby precluding the role of NAAG as a glutamate provider.

\subsection{NAAG Affects Both Cancer and Neurological Disease Progression via Glutamate}

As a cancer metabolite and a neurotransmitter, NAAG has an effect on cancer metabolism and neurological diseases, which largely relies on its ability to regulate and be directly converted to another cancer metabolite/neurotransmitter, glutamate. Via hydrolysis by GCP II, NAAG can be catabolized into $N$-Acetyl-aspartate (NAA) and glutamate. This process has proven to be a crucial glutamate production pathway in cancer [6], and the produced glutamate can serve as a source of carbon and nitrogen to support the elevated need of energy, nucleotides, and protein of the cancer cells [91]. Specifically, the resulting glutamate can be further converted to $\alpha-\mathrm{KG}$ and it participates in the TCA cycle for energy production in cancer cells. Therefore, NAAG promotes cancer growth by playing the role of a glutamate reservoir [6].

The conversion of NAAG to glutamate also plays an important role in neurological diseases. Glutamate has long been recognized as a neurodegenerative neurotransmitter, and its excessive release leads to neuronal loss and neural excitotoxicity. The excitotoxicity caused by glutamate is very complicated, and is promoted by extracellular $\mathrm{Ca}^{+}$, mediated by $\mathrm{NO}$, and involves synaptic free radicals and zinc accumulation [92]. In the neurodegenerative disorder, amyotrophic lateral sclerosis (ALS), increased GCP II activity is observed, which further supports NAAG's role as a major source of extracellular glutamate [93]. Thus, this conversion of NAAG to glutamate contributes not only to cancer growth but also to a wide range of neurological diseases. This points to the significance of the NAAG-glutamate pathway as a very promising potential target in cancer therapy. Inhibition of the conversion of NAAG to NAA and glutamate has been the focus of researchers working with neurological diseases for years [94]. As we begin to uncover the valuable interplay between cancer and neurological disease, previous and ongoing research may serve to catalyze potential developments for cancer therapies. 
Due to the diverse nature of different neurological diseases, the direct relationships between NAAG and these diseases are not consistent. As an established neuroprotective neurotransmitter, NAAG typically reduces neurotoxicity caused by excitatory neuroactivity, and suppresses and reverses the effect of neurodegenerative neurotransmitters like glutamate [77]. The decrease of NAAG levels in most cases is related to neuronal loss and consequent neurodegenerative disorders. In a study of $\mathrm{HD}$ and $\mathrm{AD}$, researchers observed a decrease in NAAG level and GCP II activity and its correlation with the neuronal loss [80]. A similar trend was also observed in another neurodegenerative disease, ALS, where decreased NAAG levels in all regions of the spinal cord, except for the posterior column, were observed in ALS patients. However, in this case, GCP II activity was found to be increased, which led to excessive hydrolysis of GCP II and to a subsequent decrease in the NAAG levels [93]. Therefore, decreased NAAG level reflects the pathogenesis and progression of neurodegenerative diseases.

A reverse relationship between NAAG and disease progression, however, was observed in cancer. A recent study showed that NAAG has great potential as a noninvasive cancermonitoring biomarker. Moreover, the NAAG concentration in plasma reflects its concentration in tumors, which indicates a higher translational value of NAAG as a cancer biomarker [6]. Another translational aspect of NAAG concentration is in monitoring cancer growth as a NAAG concentration spike could be detected prior to tumor growth surge and thus allow for the monitoring of tumor growth in a timely fashion [6, 95]. Therefore, considering the ability of NAAG concentration to reflect cancer grade, the mirror relationship between plasma NAAG concentration and tumor NAAG concentration, and its predictive time point of occurrence, NAAG shows great potential as a real-time tumor growth monitor.

Few studies have been conducted on the role of NAAG in another major neurological disease category, psychiatric disorders, and the results are more controversial. In one study of the effect of NAAG on schizophrenia, an increase in brain NAAG level and decreases in GCP II and glutamate levels were observed, which indicates a completely reverse relationship between NAAG and psychiatric disorders compared with its relationship with neurodegenerative disorders [96]. However, in another study, brain NAAG was found to be higher in younger schizophrenia subjects compared to control counterparts, while a reverse relationship was observed in the older groups [97]. Therefore, possibly due to the complex nature and disease dynamics of psychiatric disorders and their reduced reliance on neuron loss, NAAG does not completely reflect the disease progression in this category of neurological disorders compared to cancer and neurodegenerative disorders.

\subsection{Targeting GCP II Is a Promising Strategy for Cancer Treatment}

After recognizing the crucial role of NAAG in both cancer growth and neurological disease progression via its catabolism to NAA and glutamate, we can shift our attention from NAAG itself to its conversion process. As mentioned in Sect. 4.1, a very promising strategy for both neurological disease and cancer treatment would be shutting down the hydrolysis of NAAG to glutamate. GCP II, also known as $N$-acetyl-L-aspartyl-L-glutamate peptidase (NAALADase), is the key enzyme in NAAG's hydrolysis to glutamate and NAA [98]. Due to its dominant role in this process, GCP II has long been in consideration as a drug target. Generations of GCP II inhibitors have been developed to treat cancer and neurological diseases, including 2-(phosphonomethyl) pentanedioic acid (2-PMPA), thiol and indole thiol derivatives, and NAAG analogs. Among these, 2-(3-mercaptopropyl) pentanedioic acid (2-MPPA) showed great tolerance in a Phase I clinical trial, and a Phase II clinical trial has been initiated [94].

The use of GCP II inhibitors on a variety of neurological diseases has been proven to be effective. The GCP II inhibitors (2-PMPA, thiolbased 2-MPPA, and urea-based ZJ43) reduce 
inflammatory pain and neuronal loss in rat models, and another GCP II inhibitor, GPI5232, greatly reduces the number of seizures in a rat stroke model compared to vehicle control [94]. Besides these acute neurological diseases, the effect of GCP II inhibitors was also shown in chronic neurological disorders. ZJ43 was shown to reduce dissociative anesthetic phencyclidine (PCP)-induced motor activation, falling, stereotypic circling behavior, and head movements in schizophrenia patients. In addition, GPI5332 reduces diabetic neuropathy, and 2-PMPA and 2-MPPA delay mortality and pathological abnormalities of ALS in vitro and in vivo, respectively [94]. All the above positive effects of GCP II inhibitors over neurological diseases are driven by a two-faceted mechanism. Firstly, GCP II inhibition cuts off the supply of glutamate from NAAG hydrolysis and results in a direct decrease of glutamate level. Secondly, although GCP II inhibition reduces extracellular glutamate directly, its therapeutic effects seem to primarily rely on inhibition of the synaptic release of glutamate [94]. An observation in a rat stroke model showed that after GCP II inhibition, the decrease in extracellular glutamate level exceeded extracellular NAAG levels. Via this two-faceted mechanism, GCP II exhibits great potential and effectiveness as a treatment for neurological diseases (Fig. 4). The success of the use of GCP II inhibitors in neurological diseases and the development of reliable GCP II inhibitors provides further evidence for this strategy's potential in cancer treatment and it exhibits great translational value due to the shared metabolic pathway. Likewise, GCP II inhibitors have gained interest in cancer treatment. A study by Nguyen et al. showed that the use of 2-PMPA reduces both tumor growth and glutamate concentration in vivo [6]. This was further supported by the finding that the combination use of 2-PMPA and glutaminase inhibition accentuated the effects by further cutting off glutamate supply [95]. Therefore, inhibiting GCP II and in turn shutting down the hydrolysis of NAAG to glutamate have proven to be effective in cancer treatment, and thus GCP II has become a very promising drug target.
Besides its function in hydrolyzing NAAG, GCP II is also connected with cancer from other aspects. In prostate cancer, GCP II is also known as prostate-specific membrane antigen (PSMA), which serves as a biomarker that serves to cleave terminal carboxyl glutamate from NAAG [99]. In prostate cancer, its concentration greatly increases. A study by Evans et al. has shown that reducing GCP II expression led to cell cycle arrest, decreased cell proliferation, and diminished invasiveness in prostate cancer. This trait makes GCP II a tool for prostate cancer imaging and treatment [100]. The strategy of targeting GCP II in cancer treatment, therefore, becomes more promising by the addition of this connection between GCP II and prostate cancer.

\subsection{NAAG Inhibits GABA Release and Indirectly Affects Both Cancer and Neurological Diseases via the GABA-ergic System}

Besides its direct effect on cancer and neurological diseases, NAAG also interacts with other molecules to indirectly influence the two disease groups. The activation of mGluR3 and the decrease of the cAMP level induced by NAAG inhibit the calcium-dependent, KCl-induced GABA release [81]. As discussed in Sect. 3, GABA is found to contribute to the TCA cycle and cancer energy supply by its conversion to succinate. The inhibition of GABA release is observed when either NAAG or DCG-IV, another mGluR3 agonist, is applied. The addition of forskolin, which leads to forskolin-induced cAMP synthesis, reverses the inhibition of GABA release caused by NAAG [81]. Therefore, NAAG potentially inhibits GABA release by mGluR3 activation and consequent cAMP decrease, and in turn downregulates the GABA-ergic system. As discussed in Sect. 3 of this chapter, this downregulation could have multifaceted effects on cancer and neurological diseases, including relieving $\mathrm{AD}$ and deducing the invasiveness of prostate cancer. 
Fig. 4 Regulation of NAAG metabolism and its contribution to cancer. $A D$ Alzheimer's disease, $H D$

Huntington's disease, $A L S$ amyotrophic lateral sclerosis, GCP II glutamate carboxypeptidase II

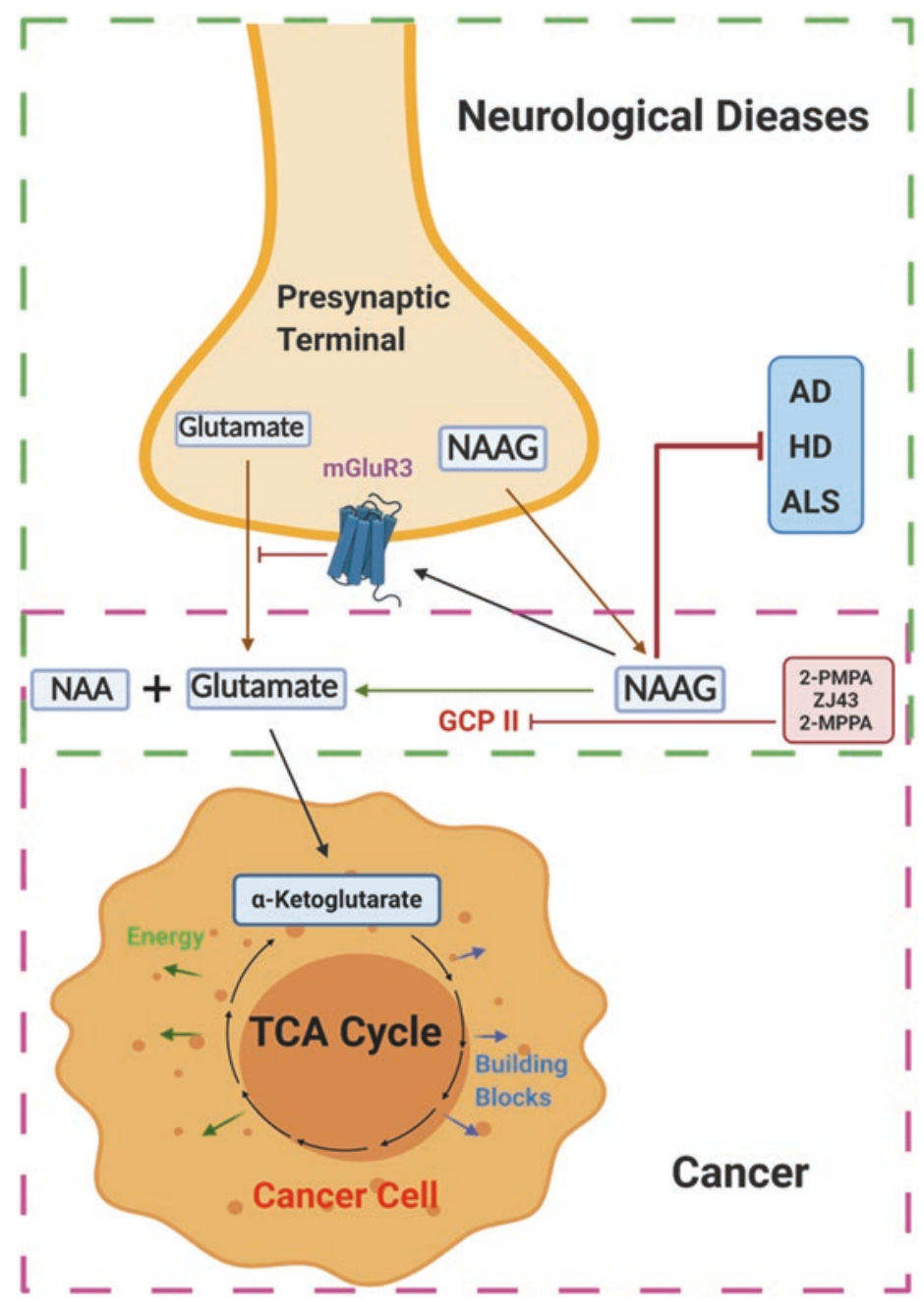

\section{Conclusion}

Glutamine/glutamate, GABA, and NAAG metabolism are closely involved in both cancer and neurological diseases. As important factors in neurotransmission in neurological diseases and as significant components in cancer metabolism and energy supply, glutamine/glutamate, GABA, and NAAG introduce considerable overlaps between the two categories of diseases. Possibly due to the complex nature of the neurological system and the divergent causes of different neurological disorders, the effect and correlation of one neurotransmitter are very specific to that disease type, sometimes even opposite to its effect on other diseases. However, these neurotransmitters, as cancer metabolites and signaling molecules, affect cancer development in unique ways. Glutamine and glutamate, in general, serve as cancer cell energy sources, while GABA and NAAG both have more versatile roles serving as signaling factors, metabolic reservoirs, and prognostic indicators for cancer. Therefore, taking insights gleaned from the metabolic interplay between neurological diseases and cancer and applying them to the field of oncology may not only provide further understanding of the mechanisms by which these metabolites affect cancer growth but also allow established therapeutic strategies in neurological diseases to be repur- 
posed for cancer treatment and even shed light on novel drug targets. As discussed in this chapter, the regulation of these four metabolites/neurotransmitters and their related enzymes and receptors greatly impacts diagnosis, monitoring, and treatments for cancer, with some already in the process of being translated into cancer clinical trials. The future direction includes investigating potential therapeutic methods involving intertwining glutamine metabolism, GABA regulation, and GCP II/GLS/GS inhibition, which can eventually be utilized for cancer and neurological disease treatment.

\section{References}

1. Young, S. Z., \& Bordey, A. (2009). GABA's control of stem and cancer cell proliferation in adult neural and peripheral niches. Physiology (Bethesda), 24, 171-185.

2. Udupa, S., et al. (2019). Upregulation of the glutaminase II pathway contributes to glutamate production upon glutaminase 1 inhibition in pancreatic cancer. Proteomics, 19(21-22), e1800451.

3. Hoang, G., Udupa, S., \& Le, A. (2019). Application of metabolomics technologies toward cancer prognosis and therapy. International Review of Cell and Molecular Biology, 347, 191-223.

4. Mates, J. M., et al. (2019). Dysregulation of glutaminase and glutamine synthetase in cancer. Cancer Letters, 467, 29-39.

5. Matuszek, M., Jesipowicz, M., \& Kleinrok, Z. (2001). GABA content and GAD activity in gastric cancer. Medical Science Monitor, 7(3), 377-381.

6. Nguyen, T., et al. (2019). Uncovering the role of $\mathrm{N}$-acetyl-aspartyl-glutamate as a glutamate reservoir in cancer. Cell Reports, 27(2), 491-501. e6.

7. Yao, V., et al. (2010). Expression of prostate-specific membrane antigen (PSMA), increases cell folate uptake and proliferation and suggests a novel role for PSMA in the uptake of the non-polyglutamated folate, folic acid. Prostate, 70(3), 305-316.

8. Li, T., Copeland, C., \& Le, A. (2021). Glutamine metabolism in cancer. Advances in Experimental Medicine and Biology, 1311, https://doi. org/10.1007/978-3-030-65768-0_2.

9. Still, E. R., \& Yuneva, M. O. (2017). Hopefully devoted to Q: Targeting glutamine addiction in cancer. British Journal of Cancer, 116(11), 1375-1381.

10. Medina, M. A. (2001). Glutamine and cancer. The Journal of Nutrition, 131(9 Suppl), 2539S-2542S; discussion 2550S-1S.

11. Struzynska, L., \& Sulkowski, G. (2004). Relationships between glutamine, glutamate, and GABA in nerve endings under Pb-toxicity conditions. Journal of Inorganic Biochemistry, 98(6), 951-958.
12. Meldrum, B. S. (2000). Glutamate as a neurotransmitter in the brain: Review of physiology and pathology. The Journal of Nutrition, 130(4S Suppl), 1007S-1015S.

13. Takahashi, H., et al. (1991). Inhibition of brain glutamine accumulation prevents cerebral edema in hyperammonemic rats. The American Journal of Physiology, 261(3 Pt 2), H825-H829.

14. Andersen, J. V., et al. (2017). Alterations in cerebral cortical glucose and glutamine metabolism precedes amyloid plaques in the APPswe/PSEN1dE9 mouse model of Alzheimer's disease. Neurochemical Research, 42(6), 1589-1598.

15. Leegwater-Kim, J., \& Cha, J. H. (2004). The paradigm of Huntington's disease: Therapeutic opportunities in neurodegeneration. NeuroRx, 1(1), $128-138$.

16. Burbaeva, G., et al. (2003). Glutamine synthetase and glutamate dehydrogenase in the prefrontal cortex of patients with schizophrenia. Progress in NeuroPsychopharmacology \& Biological Psychiatry, 27(4), 675-680.

17. Suarez, I., Bodega, G., \& Fernandez, B. (2002). Glutamine synthetase in brain: Effect of ammonia. Neurochemistry International, 41(2-3), 123-142.

18. Elgogary, A., et al. (2016). Combination therapy with BPTES nanoparticles and metformin targets the metabolic heterogeneity of pancreatic cancer. Proceedings of the National Academy of Sciences of the United States of America, 113(36), E5328-E5336.

19. Xiang, Y., et al. (2015). Targeted inhibition of tumorspecific glutaminase diminishes cell-autonomous tumorigenesis. The Journal of Clinical Investigation, 125(6), 2293-2306.

20. Zimmermann, S. C., et al. (2016). Allosteric glutaminase inhibitors based on a 1,4-di(5-amino-1,3,4thiadiazol-2-yl)butane scaffold. ACS Medicinal Chemistry Letters, 7(5), 520-524.

21. Rais, R., et al. (2016). Discovery of 6-diazo-5-oxol-norleucine (DON) prodrugs with enhanced CSF delivery in monkeys: A potential treatment for glioblastoma. Journal of Medicinal Chemistry, 59(18), 8621-8633.

22. Le, A., Udupa, S., \& Zhang, C. (2019). The metabolic interplay between cancer and other diseases. Trends Cancer, 5(12), 809-821.

23. Le, A., et al. (2012). Glucose-independent glutamine metabolism via TCA cycling for proliferation and survival in B cells. Cell Metabolism, 15(1), 110-121.

24. Koochekpour, S., et al. (2012). Serum glutamate levels correlate with Gleason score and glutamate blockade decreases proliferation, migration, and invasion and induces apoptosis in prostate cancer cells. Clinical Cancer Research, 18(21), 5888-5901.

25. Walton, H. S., \& Dodd, P. R. (2007). Glutamateglutamine cycling in Alzheimer's disease. Neurochemistry International, 50(7-8), 1052-1066.

26. Hynd, M. R., Scott, H. L., \& Dodd, P. R. (2004). Glutamate-mediated excitotoxicity and neurodegeneration in Alzheimer's disease. Neurochemistry International, 45(5), 583-595. 
27. Zheng, P., et al. (2019). The gut microbiome from patients with schizophrenia modulates the glutamate-glutamine-GABA cycle and schizophrenia-relevant behaviors in mice. Science Advances, 5(2), eaau8317.

28. Madeira, C., et al. (2018). Blood levels of glutamate and glutamine in recent onset and chronic schizophrenia. Frontiers in Psychiatry, 9, 713.

29. Behrens, P. F., et al. (2002). Impaired glutamate transport and glutamate-glutamine cycling: Downstream effects of the Huntington mutation. Brain, 125(Pt 8), 1908-1922.

30. O’Donovan, S. M., Sullivan, C. R., \& McCullumsmith, R. E. (2017). The role of glutamate transporters in the pathophysiology of neuropsychiatric disorders. NPJ Schizophrenia, 3(1), 32.

31. Robert, S. M., \& Sontheimer, H. (2014). Glutamate transporters in the biology of malignant gliomas. Cellular and Molecular Life Sciences, 71(10), 1839-1854.

32. Arnone, D., et al. (2015). Indirect evidence of selective glial involvement in glutamate-based mechanisms of mood regulation in depression: Metaanalysis of absolute prefrontal neuro-metabolic concentrations. European Neuropsychopharmacology, 25(8), 1109-1117.

33. North, W. G., et al. (2017). NMDA receptors are important regulators of pancreatic cancer and are potential targets for treatment. Clinical Pharmacology, 9, 79-86.

34. Li, C. T., Yang, K. C., \& Lin, W. C. (2018). Glutamatergic dysfunction and glutamatergic compounds for major psychiatric disorders: Evidence from clinical neuroimaging studies. Frontiers in Psychiatry, 9, 767.

35. Meador-Woodruff, J. H., \& Healy, D. J. (2000). Glutamate receptor expression in schizophrenic brain. Brain Research. Brain Research Reviews, 31(2-3), 288-294.

36. Herner, A., et al. (2011). Glutamate increases pancreatic cancer cell invasion and migration via AMPA receptor activation and Kras-MAPK signaling. International Journal of Cancer, 129(10), 2349-2359.

37. Dang, C. V., Le, A., \& Gao, P. (2009). MYC-induced cancer cell energy metabolism and therapeutic opportunities. Clinical Cancer Research, 15(21), 6479-6483.

38. Le, A., \& Dang, C. V. (2013). Studying Myc's role in metabolism regulation. Methods in Molecular Biology, 1012, 213-219.

39. Gabay, M., Li, Y., \& Felsher, D. W. (2014). MYC activation is a hallmark of cancer initiation and maintenance. Cold Spring Harbor Perspectives in Medicine, 4, 6 .

40. Wise, D. R., et al. (2008). Myc regulates a transcriptional program that stimulates mitochondrial glutaminolysis and leads to glutamine addiction. Proceedings of the National Academy of Sciences of the United States of America, 105(48), 18782-18787.
41. Hu, W., et al. (2010). Glutaminase 2, a novel p53 target gene regulating energy metabolism and antioxidant function. Proceedings of the National Academy of Sciences of the United States of America, 107(16), 7455-7460.

42. Gao, P., et al. (2009). c-Myc suppression of miR$23 \mathrm{a} / \mathrm{b}$ enhances mitochondrial glutaminase expression and glutamine metabolism. Nature, 458(7239), 762-765.

43. Lee, H. G., et al. (2009). The neuronal expression of MYC causes a neurodegenerative phenotype in a novel transgenic mouse. The American Journal of Pathology, 174(3), 891-897.

44. Niu, Z., et al. (2015). Knockdown of c-Myc inhibits cell proliferation by negatively regulating the $\mathrm{Cdk} /$ $\mathrm{Rb} / \mathrm{E} 2 \mathrm{~F}$ pathway in nasopharyngeal carcinoma cells. Acta Biochimica et Biophysica Sinica Shanghai, 47(3), 183-191.

45. Zhang, X., Ge, Y. L., \& Tian, R. H. (2009). The knockdown of c-myc expression by RNAi inhibits cell proliferation in human colon cancer HT-29 cells in vitro and in vivo. Cellular \& Molecular Biology Letters, 14(2), 305-318.

46. Lukey, M. J., Katt, W. P., \& Cerione, R. A. (2017). Targeting amino acid metabolism for cancer therapy. Drug Discovery Today, 22(5), 796-804.

47. Jiang, J., Srivastava, S., \& Zhang, J. (2019). Starve cancer cells of glutamine: Break the spell or make a hungry monster? Cancers (Basel), 11, 6 .

48. Dong, X. X., Wang, Y., \& Qin, Z. H. (2009). Molecular mechanisms of excitotoxicity and their relevance to pathogenesis of neurodegenerative diseases. Acta Pharmacologica Sinica, 30(4), 379-387.

49. Cooper, A. J., \& Kuhara, T. (2014). alphaKetoglutaramate: An overlooked metabolite of glutamine and a biomarker for hepatic encephalopathy and inborn errors of the urea cycle. Metabolic Brain Disease, 29(4), 991-1006.

50. Dorai, T., et al., High Levels of Glutaminase II Pathway Enzymes in Normal and Cancerous Prostate Suggest a Role in 'Glutamine Addiction'. Biomolecules, 2019. 10(1).

51. Vergara, F., F. Plum, and T.E. Duffy, Alphaketoglutaramate: increased concentrations in the cerebrospinal fluid of patients in hepatic coma. Science, 1974. 183(4120): p. 81-3.

52. Wong, C. G., Bottiglieri, T., \& Snead, O. C., 3rd. (2003). GABA, gamma-hydroxybutyric acid, and neurological disease. Annals of Neurology, 54(Suppl 6), S3-S12.

53. Zhang, D., et al. (2014). GABAergic signaling facilitates breast cancer metastasis by promoting ERK1/2-dependent phosphorylation. Cancer Letters, 348(1-2), 100-108.

54. Watanabe, M., et al. (2002). GABA and GABA receptors in the central nervous system and other organs. International Review of Cytology, 213, 1-47.

55. Feldblum, S., Erlander, M. G., \& Tobin, A. J. (1993). Different distributions of GAD65 and GAD67 mRNAs suggest that the two glutamate decarbox- 
ylases play distinctive functional roles. Journal of Neuroscience Research, 34(6), 689-706.

56. Hettema, J. M., et al. (2006). Association between glutamic acid decarboxylase genes and anxiety disorders, major depression, and neuroticism. Molecular Psychiatry, 11(8), 752-762.

57. Bowers, G., Cullinan, W. E., \& Herman, J. P. (1998). Region-specific regulation of glutamic acid decarboxylase (GAD) mRNA expression in central stress circuits. The Journal of Neuroscience, 18(15), 5938-5947.

58. Kimura, R., et al. (2013). Glutamate acid decarboxylase 1 promotes metastasis of human oral cancer by beta-catenin translocation and MMP7 activation. BMC Cancer, 13, 555.

59. Wu, Z., et al. (2014). Tonic inhibition in dentate gyrus impairs long-term potentiation and memory in an Alzheimer's [corrected] disease model. Nature Communications, 5, 4159.

60. Sterley, T. L., Howells, F. M., \& Russell, V. A. (2013). Evidence for reduced tonic levels of GABA in the hippocampus of an animal model of ADHD, the spontaneously hypertensive rat. Brain Research, 1541, 52-60.

61. Schuller, H. M., Al-Wadei, H. A., \& Majidi, M. (2008). Gamma-aminobutyric acid, a potential tumor suppressor for small airway-derived lung adenocarcinoma. Carcinogenesis, 29(10), 1979-1985.

62. Ulrich, D. (2015). Amyloid-beta impairs synaptic inhibition via GABA(A) receptor endocytosis. The Journal of Neuroscience, 35(24), 9205-9210.

63. Klebig, C., et al. (2005). Characterization of \{gamma\}-aminobutyric acid type A receptorassociated protein, a novel tumor suppressor, showing reduced expression in breast cancer. Cancer Research, 65(2), 394-400.

64. Bero, A. W., et al. (2011). Neuronal activity regulates the regional vulnerability to amyloid-beta deposition. Nature Neuroscience, 14(6), 750-756.

65. Kim, Y. S., \& Yoon, B. E. (2017). Altered GABAergic signaling in brain disease at various stages of life. Experimental Neurobiology, 26(3), 122-131.

66. Jiang, X., et al. (2012). GABAB receptor complex as a potential target for tumor therapy. The Journal of Histochemistry and Cytochemistry, 60(4), 269-279.

67. Mombereau, C., et al. (2004). Genetic and pharmacological evidence of a role for $\mathrm{GABA}(\mathrm{B})$ receptors in the modulation of anxiety- and antidepressantlike behavior. Neuropsychopharmacology, 29(6), 1050-1062.

68. Azuma, H., et al. (2003). Gamma-aminobutyric acid as a promoting factor of cancer metastasis; induction of matrix metalloproteinase production is potentially its underlying mechanism. Cancer Research, 63(23), 8090-8096.

69. Cryan, J. F., \& Slattery, D. A. (2010). GABAB receptors and depression. Current status. Advances in Pharmacology, 58, 427-451.

70. Budhram, A., et al. (2019). Diagnosing autoimmune limbic encephalitis. CMAJ, 191(19), E529-E534.
71. Lin, J., et al. (2019). Encephalitis with antibodies against the GABAB receptor: High mortality and risk factors. Frontiers in Neurology, 10, 1030.

72. Arino, H., et al. (2015). Paraneoplastic neurological syndromes and glutamic acid decarboxylase antibodies. JAMA Neurology, 72(8), 874-881.

73. Nene, Y., et al. (2019). A case of anti-glutamic acid decarboxylase-65 antibody positive stiff person syndrome presenting initially as acute peripheral vestibulopathy, leading to delayed diagnosis after multiple hospitalizations. Cureus, 11(11), e6083.

74. Lee, Y. Y., et al. (2019). Association of stiff-person syndrome with autoimmune endocrine diseases. World Journal of Clinical Cases, 7(19), 2942-2952.

75. Dinkel, K., et al. (1998). Inhibition of gammaaminobutyric acid synthesis by glutamic acid decarboxylase autoantibodies in stiff-man syndrome. Annals of Neurology, 44(2), 194-201.

76. Rakocevic, G., \& Floeter, M. K. (2012). Autoimmune stiff person syndrome and related myelopathies: Understanding of electrophysiological and immunological processes. Muscle \& Nerve, 45(5), 623-634.

77. Neale, J. H., Bzdega, T., \& Wroblewska, B. (2000). N-Acetylaspartylglutamate: The most abundant peptide neurotransmitter in the mammalian central nervous system. Journal of Neurochemistry, 75(2), 443-452.

78. Kirsch, B. J., et al. (2021). Non-Hodgkin lymphoma metabolism. Advances in Experimental Medicine and Biology, 1311, https://doi. org/10.1007/978-3-030-65768-0_7.

79. Quinones, A., \& Le, A. (2021). The multifaceted glioblastoma: From genomic alterations to metabolic adaptations. Advances in Experimental Medicine and Biology, 1311, https://doi. org/10.1007/978-3-030-65768-0_4.

80. Passani, L. A., et al. (1997). $\mathrm{N}$-acetylaspartylglutamate, $\mathrm{N}$-acetylaspartate, and $\mathrm{N}$-acetylated alpha-linked acidic dipeptidase in human brain and their alterations in Huntington and Alzheimer's diseases. Molecular and Chemical Neuropathology, 31(2), 97-118.

81. Zhao, J., et al. (2001). NAAG inhibits KCl-induced [(3)H]-GABA release via mGluR3, cAMP, PKA and L-type calcium conductance. The European Journal of Neuroscience, 13(2), 340-346.

82. Cartmell, J., \& Schoepp, D. D. (2000). Regulation of neurotransmitter release by metabotropic glutamate receptors. Journal of Neurochemistry, 75(3), 889-907.

83. D’Onofrio, M., et al. (2003). Pharmacological blockade of mGlu2/3 metabotropic glutamate receptors reduces cell proliferation in cultured human glioma cells. Journal of Neurochemistry, 84(6), 1288-1295.

84. Brocke, K. S., et al. (2010). Glutamate receptors in pediatric tumors of the central nervous system. Cancer Biology \& Therapy, 9(6), 455-468.

85. Ciceroni, C., et al. (2013). Type-3 metabotropic glutamate receptors regulate chemoresistance in glioma stem cells, and their levels are inversely related to survival in patients with malignant 
gliomas. Cell Death and Differentiation, 20(3), 396-407.

86. Arcella, A., et al. (2005). Pharmacological blockade of group II metabotropic glutamate receptors reduces the growth of glioma cells in vivo. NeuroOncology, 7(3), 236-245.

87. Stepulak, A., et al. (2009). Expression of glutamate receptor subunits in human cancers. Histochemistry and Cell Biology, 132(4), 435-445.

88. Aronica, E., et al. (2003). Expression and functional role of mGluR3 and mGluR5 in human astrocytes and glioma cells: Opposite regulation of glutamate transporter proteins. The European Journal of Neuroscience, 17(10), 2106-2118.

89. Zhou, K., et al. (2014). mGlu3 receptor blockade inhibits proliferation and promotes astrocytic phenotype in glioma stem cells. Cell Biology International, 38(4), 426-434.

90. Ciceroni, C., et al. (2008). Type-3 metabotropic glutamate receptors negatively modulate bone morphogenetic protein receptor signaling and support the tumourigenic potential of glioma-initiating cells. Neuropharmacology, 55(4), 568-576.

91. Wise, D. R., \& Thompson, C. B. (2010). Glutamine addiction: A new therapeutic target in cancer. Trends in Biochemical Sciences, 35(8), 427-433.

92. Lau, A., \& Tymianski, M. (2010). Glutamate receptors, neurotoxicity and neurodegeneration. Pflügers Archiv, 460(2), 525-542.
93. Tsai, G. C., et al. (1991). Reductions in acidic amino acids and $\mathrm{N}$-acetylaspartylglutamate in amyotrophic lateral sclerosis CNS. Brain Research, 556(1), 151-156.

94. Zhou, J., et al. (2005). NAAG peptidase inhibitors and their potential for diagnosis and therapy. Nature Reviews. Drug Discovery, 4(12), 1015-1026.

95. Asaka, R., \& Le, A. (2019). Dual role of N-acetylaspartyl-glutamate metabolism in cancer monitor and therapy. Molecular \& Cellular Oncology, 6(5), e1627273.

96. Tsai, G., et al. (1995). Abnormal excitatory neurotransmitter metabolism in schizophrenic brains. Archives of General Psychiatry, 52(10), 829-836.

97. Rowland, L. M., et al. (2013). In vivo measurements of glutamate, GABA, and NAAG in schizophrenia. Schizophrenia Bulletin, 39(5), 1096-1104.

98. Mesters, J. R., et al. (2006). Structure of glutamate carboxypeptidase II, a drug target in neuronal damage and prostate cancer. The EMBO Journal, 25(6), 1375-1384.

99. Ghosh, A., \& Heston, W. D. (2004). Tumor target prostate specific membrane antigen (PSMA) and its regulation in prostate cancer. Journal of Cellular Biochemistry, 91(3), 528-539.

100. Evans, J. C., et al. (2016). The therapeutic and diagnostic potential of the prostate specific membrane antigen/glutamate carboxypeptidase II (PSMA/ GCPII) in cancer and neurological disease. British Journal of Pharmacology, 173(21), 3041-3079.

Open Access This chapter is licensed under the terms of the Creative Commons Attribution 4.0 International License (http://creativecommons.org/licenses/by/4.0/), which permits use, sharing, adaptation, distribution and reproduction in any medium or format, as long as you give appropriate credit to the original author(s) and the source, provide a link to the Creative Commons license and indicate if changes were made.

The images or other third party material in this chapter are included in the chapter's Creative Commons license, unless indicated otherwise in a credit line to the material. If material is not included in the chapter's Creative Commons license and your intended use is not permitted by statutory regulation or exceeds the permitted use, you will need to obtain permission directly from the copyright holder. 


\title{
Metabolic Intersection of Cancer and Cardiovascular Diseases: Opportunities for Cancer Therapy
}

\author{
Giang Hoang, Kiet Nguyen, and Anne Le
}

\section{Keywords}

Glutamine metabolism · Fatty acid oxidation

- Tryptophan catabolism - Pyruvate dehydrogenase complex $\cdot$ Cancer $\cdot$ Cardiovascular diseases

\section{Abbreviations}

3-KAT 3-Ketoacyl-CoA thiolase

AHR Aryl hydrocarbon receptor

ATP Adenosine triphosphate

BMI Body mass index

G. Hoang

Department of Pathology, Johns Hopkins University

School of Medicine, Baltimore, MD, USA

Department of Biomedical Engineering, Johns

Hopkins University Whiting School of Engineering, Baltimore, MD, USA

K. Nguyen

Department of Chemistry and Biology, Emory

University, Atlanta, GA, USA

\section{A. Le $(\square)$}

Department of Pathology and Oncology, Johns Hopkins University School of Medicine,

Baltimore, MD, USA

Department of Chemical and Biomolecular Engineering, Johns Hopkins University Whiting School of Engineering, Baltimore, MD, USA e-mail: annele@jhmi.edu
BPTES Bis-2-(5-phenylacetamido-1,2,4thiadiazol-2-yl)ethyl sulfide

CD36 Cluster of differentiation 36

CLL Chronic lymphocytic leukemia

CPT Carnitine palmitoyltransferase

DCA Dichloroacetate

ECM Extracellular matrix

FA Fatty acid

FAO Fatty acid oxidation

FASN Fatty acid synthase

GLS Glutaminase

GS Glutamine synthetase

GSH Glutathione

HDL High-density lipoproteins

IDO Indoleamine 2,3-dioxygenase

KTR Kynurenine/tryptophan ratio

LDL Low-density lipoproteins

PAH Pulmonary arterial hypertension

PDHC Pyruvate dehydrogenase complex

PDK Pyruvate dehydrogenase kinase

siRNA Small interfering RNA

TCA Tricarboxylic acid

TDO Tryptophan 2,3-dioxygenase

TFP Trifunctional protein

\section{Key Points}

- Similarities in metabolic dysregulations between cardiovascular diseases and cancer lead to the identification of common treatment targets. 
- Alteration in circulating glutamine and glutamate levels is indicative of both cancer proliferation and cardiometabolic diseases.

- Glutaminolysis is upregulated in cancer and pulmonary arterial hypertension.

- Glutaminase is a treatment target for cancer, hypertension, and hyperglycemia.

- Glutamine supplementation is implemented in the treatment of cancer to support immunoregulation and the treatment of many cardiovascular diseases.

- Pharmacological inhibition of fatty acid oxidation is proven effective in slowing cancer progression and treating some cardiovascular diseases.

- Inhibition of fatty acid synthesis is proven both effective in cancer treatment and protective against pulmonary hypertension.

- Upregulated tryptophan catabolism has been linked to the progression of cardiovascular diseases and enhanced immune system evasion in cancer.

- Pyruvate dehydrogenase complex downregulation is associated with many cardiovascular diseases and chemoresistance in cancer.

\section{Introduction}

According to data from the World Health Organization, cardiovascular diseases and cancer are the two leading causes of mortality in the world [1]. Despite the immense effort to study these diseases and the constant innovation in treatment modalities, the number of deaths associated with cardiovascular diseases and cancer is predicted to increase in the coming decades [1]. From 2008 to 2030, due to population growth and population aging in many parts of the world, the number of deaths caused by cancer globally is projected to increase by $45 \%$, corresponding to an annual increase of around four million people [1]. For cardiovascular diseases, this number is six million people [1]. In the United States, treatments for these two diseases are among the most costly and result in a disproportionate impact on low- and middle-income people. As the fight against these fatal diseases continues, it is crucial that we continue our investigation and broaden our understanding of cancer and cardiovascular diseases to innovate our prognostic and treatment approaches. Even though cardiovascular diseases and cancer are usually studied independently [2-12], there are some striking overlaps between their metabolic behaviors and therapeutic targets, suggesting the potential application of cardiovascular disease treatments for cancer therapy. More specifically, both cancer and many cardiovascular diseases have an upregulated glutaminolysis pathway, resulting in low glutamine and high glutamate circulating levels. Similar treatment modalities, such as glutaminase (GLS) inhibition and glutamine supplementation, have been identified to target glutamine metabolism in both cancer and some cardiovascular diseases. Studies have also found similarities in lipid metabolism, specifically fatty acid oxidation (FAO) and synthesis. Pharmacological inhibition of FAO and fatty acid synthesis have proven effective against many cancer types as well as specific cardiovascular conditions. Many of these treatments have been tested in clinical trials, and some have been medically prescribed to patients to treat certain diseases, such as angina pectoris [13, 14]. Other metabolic pathways, such as tryptophan catabolism and pyruvate metabolism, were also dysregulated in both diseases, making them promising treatment targets. Understanding the overlapping traits exhibited by both cancer metabolism and cardiovascular disease metabolism can give us a more holistic view of how important metabolic dysregulation is in the progression of diseases. Using established links between these illnesses, researchers can take advantage of the discoveries from one field and potentially apply them to the other. In this chapter, we highlight some promising therapeutic discoveries that can support our fight against cancer, based on common metabolic traits displayed in both cancer and cardiovascular diseases. 
2

\section{Glutamine Metabolism as a Prognostic and Therapeutic Target in Cancer and Cardiovascular Diseases (Fig. 1)}

\subsection{Alterations in Circulating Glutamine and Glutamate Levels Are Indicative of Both Cancer Proliferation and Cardiometabolic Diseases}

Glutamine metabolism in cancer has been extensively studied [15, 16], in which glutamine addiction has been highlighted as a signature cancer behavior. This rapid consumption of glutamine by tumors eventually results in glutamine depletion in the patient's body and can serve as an indicator of cancer proliferation [17]. However, glutamine depletion is not always an immediate result of cancer onset. The presence of a tumor alters the host's glutamine metabolism and creates a net flux of glutamine from other tissues and organs towards the tumor [12]. Specifically, as described in a review paper by Medina et al., glutamine synthesis is upregulated in the liver and skeletal muscles of carcinomabearing animals, reflected by an increase in the glutamine synthetase (GS)/glutaminase (GLS) ratio [18]. The synthesized glutamine is then secreted into the circulatory system and results in an initial elevated glutamine plasma level within the first 24 hours of tumor transplantation [18]. However, this initial increase is later overshadowed by the rapid glutamine consumption by the tumor which eventually leads to glutamine depletion in the body. The active absorption of glutamine by cancer is shown by the upregulated glutamine transportation through the plasma membrane in malignant cells $[19$, 20]. For cardiovascular diseases, many clinical and experimental studies have shown that the low presence of circulating glutamine is indicative of cardiometabolic diseases and hemolytic disorders [11, 21, 22]. Specifically, the low glutamine/glutamate ratio in the plasma is asso-

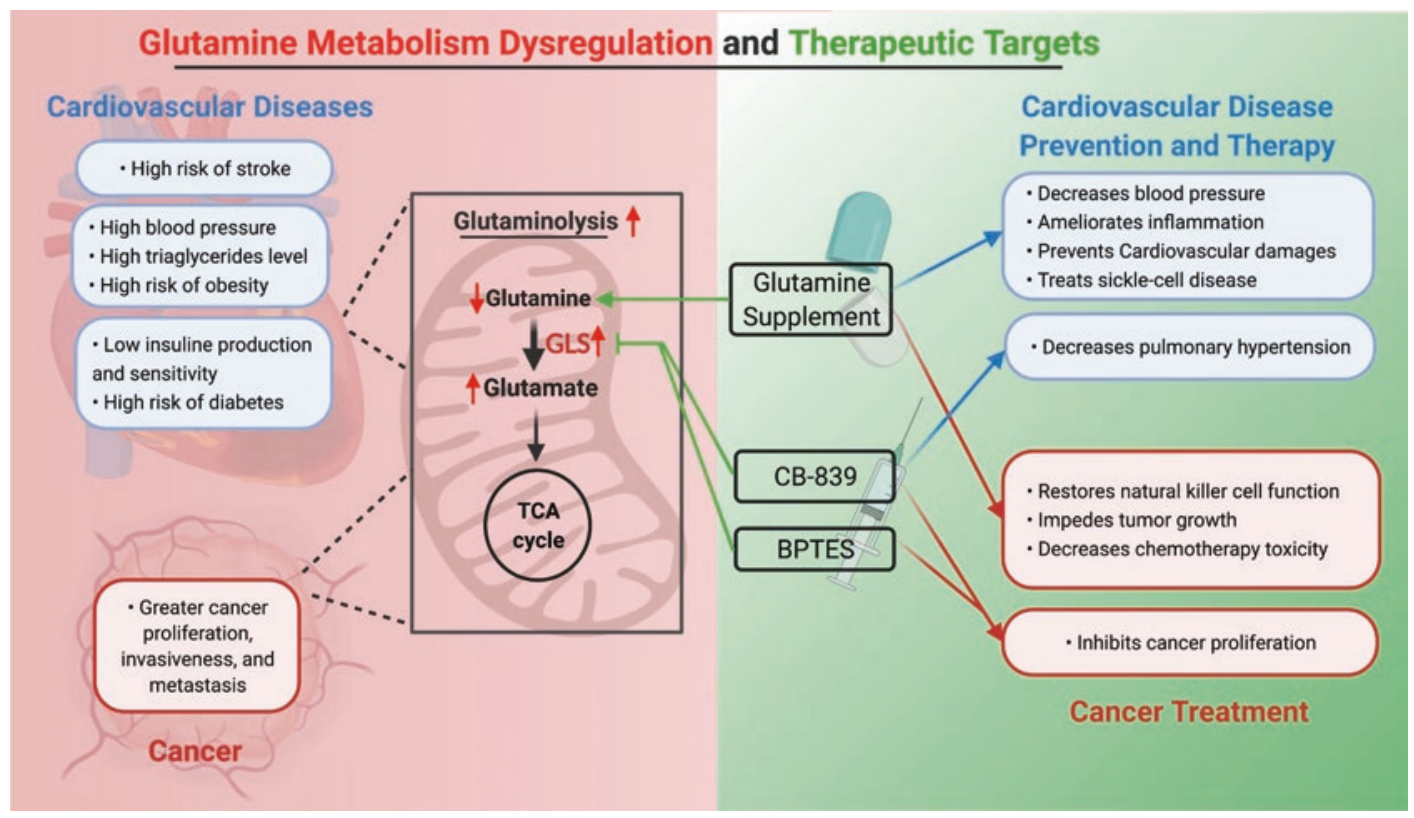

Fig. 1 Glutamine metabolism dysregulation and corresponding therapeutic targets in cancer and cardiovascular diseases. The upregulation of glutaminolysis pathway, resulting in low glutamine and high glutamate concentrations, is observed in many cancers and cardiovascular diseases. Pharmacological inhibition of glutaminase (GLS) with BPTES and CB-839, as well as dietary glutamine supplementation, has been incorporated into the treatment of both conditions and has shown promising results 
ciated with higher blood pressure, higher circulating triglycerides, obesity, and insulin-resistant traits [11]. In contrast, a high glutamine/glutamate ratio is connected to lower risks of stroke and diabetes mellitus and is generally related to better cardiovascular health [23]. In patients with sickle cell diseases, glutamine concentration in plasma and endothelial cells has been reported to decrease significantly [24].

On the other hand, circulating glutamate levels have been shown to increase in breast and prostate cancer patients and reflect tumor progression to some extent [25-27]. The increase in glutamate levels is also associated with increased risks of cardiovascular events and decreased insulin production and sensitivity, and is positively correlated with diabetes $[11,28]$. In other words, a decrease in glutamine levels and an increase in glutamate levels are generally associated with adverse health outcomes in both cancer and cardiovascular diseases. There are, however, exceptions to this trend: elevated serum glutamine levels are observed in acute myocardial ischemia patients [29].

Even though there has not been clear evidence suggesting that circulating glutamine and glutamate levels could be used for prognosis, their abnormalities have been consistently demonstrated as consequences of cancer and a variety of cardiovascular disorders. This imbalance in glutamine and glutamate levels further highlights the upregulated conversion from glutamine to glutamate, and more generally, the glutaminolysis pathway in both diseases.

\subsection{Upregulation of Glutaminolysis in Cancer and Pulmonary Arterial Hypertension}

The role of glutaminolysis in cancer proliferation has been well established as it contributes to tumor growth by both promoting cell proliferation and inhibiting cell death. Glutaminolysis fuels energy metabolism via the tricarboxylic acid (TCA) cycle and serves as a source of materials for biosynthesis, especially for purine and pyrimidine metabolism $[15,16]$. Glutaminolysis upregulation is also associated with cancer aggressiveness, invasiveness, and metastasis [25, 30]. The upregulation of glutaminolysis has been extensively studied in many cancer types and has proven crucial for cancer development, making it a well-known target for cancer therapy. However, it is important to note that the upregulation of glutaminolysis is a trait exhibited by cancer cells, localized to the tumor, and not a signature trait exhibited by normal cells in the patient body $[15,18]$.

For cardiovascular diseases, glutaminolysis has been shown to increase in pulmonary endothelial cells of patients with pulmonary arterial hypertension (PAH) [11]. PAH is a potentially fatal condition caused by the abnormal proliferation of vascular cells, forming lesions that obstruct blood flow [31]. Vascular transformation, coupled with fibrosis and vasoconstriction, can lead to an increase in blood pressure and, if left untreated, can cause right ventricular failure and death [11]. Studies have shown an increased GLS1 expression and decreased glutamine levels in pulmonary endothelial cells and in myocardiocytes of the right ventricle in $\mathrm{PAH}$ in vivo rat model [32]. An upregulation in glutaminolysis is found in PAH patients' right ventricular samples, suggesting that upregulated glutaminolysis is associated with PAH [32]. Furthermore, increasing glutaminolysis can result in an increased alpha-ketoglutarate level, a direct product of glutamate that helps promote the translation and stabilization of collagens, which stimulates fibrosis and worsens PAH [33]. As a result, glutaminolysis has also become a promising treatment target for PAH.

Thus, as an important energetic pathway frequently dysregulated in both cancer and cardiovascular disease metabolism, glutaminolysis has been widely investigated as a treatment target, resulting in the development of several pharmacological innovations and dietary implementations, discussed in detail in the next sections. 


\subsection{Glutaminase Is a Treatment Target for Cancer, Hypertension, and Hyperglycemia}

GLS is an aminohydrolase enzyme that catalyzes the hydrolysis of glutamine to glutamate, which marks the initiation of glutaminolysis. There are two main types of GLS expressed in humans: GLS1, the kidney-type glutaminase, and GLS2, the liver-type glutaminase [34]. In cancer, GLS1 is expressed more often than GLS2, and thus, more frequently targeted for therapy. The most common GLS1 pharmacological inhibitors are bis-2-(5-phenylacetamido-1,2,4-thiadiazol-2-yl) ethyl sulfide (BPTES) and CB-839, discussed in depth in Chap. 2, which have shown tumor suppression ability in breast cancer, leukemia, and lymphoma cells [35-39]. The inhibition of GLS1 expression has been extensively studied in cancer researches for cancer treatment [40-43]. As discussed in the previous section, GLS1 expression is also upregulated in PAH and has been targeted for PAH treatment. Specifically, pharmacological inhibition of GLS1 using C968 or CB-839 in rats exposed to monocrotaline, a pneumotoxic liveractivated compound commonly used to induce pulmonary hypertension, significantly decreased pulmonary arteriolar and right ventricular remodeling and ameliorated pulmonary hypertension [44]. Thus, the use of GLS1-inhibiting drugs can target both cancer and PAH.

On the other hand, the role of GLS2 in cancer has been quite controversial and needs further studies to gain a comprehensive understanding [10]. A study by Martin-Rufian et al. suggests that GLS2 overexpression in cancer cells inhibits $c-M Y C$ expression $[45,46]$ and prevents the proliferation of several types of glioma cells [47]. In contrast, a study by Xiang et al. showed that knocking down GLS2 increases the sensitivity of cervical tumors to radiotherapy [48]. In hyperglycemia, GLS2 has been identified as a treatment target [49]. Specifically, in type 2 diabetic patients, dysregulation of glucagon causes an increase in gluconeogenesis in the liver and skeletal muscles, resulting in hyperglycemia. Inhibition of GLS2 can block glutaminolysis, decrease the hepatic metabolic flux from glutamine to glucose, decrease the initial blood glucose level, and increase insulin sensitivity [49]. Despite some attempts to study the selective inhibition of GLS1 and GLS2 [35, 49, 50], there has not been a defined pharmacological inhibition of GLS2 that is qualified for general use in research. As a promising therapeutic target, research on the role of GLS2 in cancer and cardiovascular diseases should be further expanded.

\subsection{Glutamine Supplementation Is Implemented for Treatment of Cancer and a Variety of Cardiovascular Diseases}

In addition to blocking glutamate production from glutamine, glutamine supplementation has also been proven effective in cardiovascular disease prevention and sickle cell disease treatment and is used in combination with chemotherapy for cancer treatment.

In cancer treatment, the use of dietary glutamine supplementation is to make up for the tumor's "nitrogen trap" behavior that aggressively consumes dietary amino acids as well as the amino acids synthesized by the host [12]. Despite the suspicion that increasing glutamine availability may support tumor growth, a study by Austgen et al. shows that dietary supplementation of glutamine leads to no significant change in tumor growth in vivo [51]. The additional source of glutamine helps replenish glutathione (GSH) level, a crucial intracellular antioxidant, and hepatic detoxifier, in natural killer cells. Experimental data supports that glutamine supplementation impedes tumor growth by restoring the function of natural killer cells and increasing immunoregulation [52, 53]. The restored GSH level also protects patients from oxidative damages as well as increases the selectivity and decreases the cardiotoxicity and neurotoxicity effects of chemotherapeutic drugs, such as doxorubicin, methotrexate, cyclophosphamide, Eloxatin, and 5-fluorouracil, to name a few [54]. In combination with chemotherapy, supplemented glutamine can potentially help increase treatment efficacy and improve patient outcomes [55]. 
However, despite the promising experimental data, we still do not have enough evidence to recommend its regular use in cancer treatment. More experimental and clinical studies should be done to investigate the use of glutamine supplementation in cancer treatment.

On the other hand, the use of glutamine supplementation to protect against cardiometabolic diseases and its benefits have been widely established [11]. In diabetic patients, chronic oral administration of glutamine decreases blood glucose concentration for patients with type 1 and 2 diabetes, increases circulating insulin levels, and reduces systolic blood pressure in type 2 diabetic patients [56]. In mice, glutamine supplementations increase glucose tolerance and decrease mean arterial blood pressure [11]. For mice on a high-fat diet, studies show that glutamine intake helps lower body weight, decreases hyperglycemia, and improves insulin sensitivity [57]. In patients with coronary artery diseases, glutamine supplementation enhances myocardial repair and prevents damage to the cardiovascular system during surgical interventions or cardiac injuries $[9,58,59]$. The protective role of glutamine in ischemia-reperfusion injury is especially well investigated [60-64]. Glutamine reduces oxidative stress, ameliorates inflammation, and inhibits apoptosis, necrosis, and lipid peroxidation following ischemia-reperfusion. Its tissue protection effects reach a variety of organs throughout the body, including skeletal muscles, intestine, liver, kidney, brain, and heart [11]. Especially, dietary glutamine supplementation has been approved by the United States Food and Drug Administration as a treatment for sickle cell disease and is now being prescribed for patients to reduce the rate of complications $[11,65,66]$. This is a result of a successful phase 3 clinical trial, which demonstrated a significantly lower number of pain crises in patients with sickle cell disease when taking oral pharmaceutical grade glutamine. The precise mechanism of the glutamine treatment for sickle cell disease is unknown. Nevertheless, glutamine may serve as an energy source for endothelial cells and early stage reticulocytes, or premature red blood cells (RBCs) that still contain mitochondria. The established role of glutamine as a protective treatment against cardiovascular diseases reinforces the need for more clinical trials to determine its pharmacological efficacy.

Compared to cardiovascular diseases, the implementation of glutamine supplements in cancer treatment is still in its early stages of the investigation. Researchers should take advantage of the established work done on cardiovascular diseases and explore their potential integration into cancer therapy. Building upon previous knowledge can pave the way towards many groundbreaking discoveries.

\section{$3 \quad$ Lipid Metabolism Plays an Important Role in Cancer Proliferation and Cardiovascular Disease Progression (Fig. 2)}

\subsection{Fatty Acid Oxidation in Cancer and Cardiovascular Diseases}

Mitochondria fatty acid $\beta$-oxidation (FAO) involves the breakdown of energy-rich lipid molecules and plays a crucial role in the bioenergy metabolism exploited by cancer cells. The upregulation of FAO in cancer is relatively less thoroughly examined than glucose or glutamine metabolism and has been discussed in detail in Chap. 3 [67]. A variety of FAO enzymes has been shown to be overexpressed in many cancers [68]. For example, in chronic lymphocytic leukemia (CLL) cells, Liu et al. recorded a drastic increase in carnitine palmitoyltransferase 1A (CPT1A), CPT1B, and CPT2 expression [69]. High expression of CPT1A has also been shown to be indicative of poor patient outcomes in both ovarian cancer and acute myeloid leukemia (AML) [70, 71]. In prostate cancer, FAO has been identified as a dominant bioenergetics pathway and can potentially serve as a biomarker for prostate cancer diagnosis [72]. The association between FAO upregulation and metastasis has also been established in breast cancer [73, 74], colorectal cancer [75], and glioblastoma [76, 77], in which an increased FAO helps cancer cells overcome anoi- 


\section{Cardiovascular diseases}

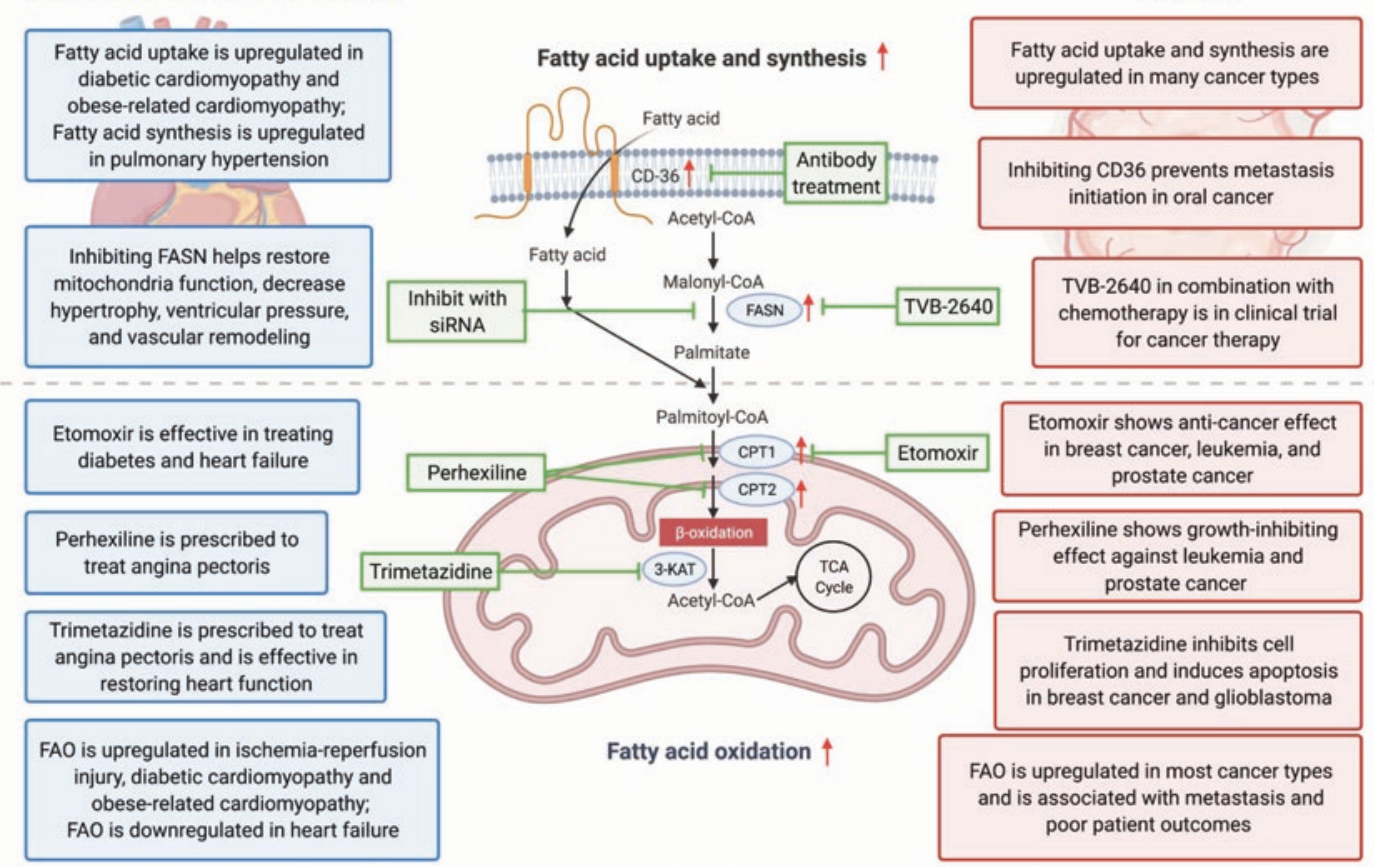

Fig. 2 Lipid metabolism dysregulation and corresponding therapeutic targets in cancer and cardiovascular diseases. Similar metabolic dysregulations in fatty acid uptake, synthesis, and oxidation have been observed in both cancer and cardiovascular diseases, thus highlighting several common treatment targets. The "repurposing" of pharmacological inhibitors used in cardiovascular diseases to cancer therapy has shown promising experimental results. $C D 36$ cluster of differentiation 36, FASN fatty acid synthase, CPT carnitine palmitoyltransferase, 3-KAT 3-ketoacyl-CoA thiolase

kis, a type of programmed cell death that occurs when anchorage-dependent cells detach from the extracellular matrix (ECM) [68]. Other studies suggest that FAO affects metastasis via cancer stem cell regulation $[8,78]$. Despite the difference in its underlying mechanistic benefits towards cancer proliferation, there is an upregulation of FAO for most cancer types.

However, the alterations in FAO vary depending on the specific cardiovascular disease and the stage of the disease. The role of FAO in cardiovascular diseases is especially important, considering that it is the predominant pathway utilized by myocardiocytes for energy production [79]. In heart failure patients, many independent studies have shown an increase in the level of circulating free fatty acids and a significant decrease in the rate of FAO in the heart [80]. This downregulation of FAO is primarily due to mitochondria dys- function and leads to an upregulated glycolysis pathway, signifying the shift back to what is known as "the fetal energy metabolism" of the failing heart. On the other hand, during ischemiareperfusion injury, an elevated circulating level of free fatty acids increases FAO in the heart [7]. For diabetic cardiomyopathy and obese-related cardiomyopathy, there are consistent reports stating that cardiac FAO is upregulated in these conditions, along with an increase in circulating free FA and FA uptake [80]. Despite FAO upregulation, the high influx of FA overwhelms the consumption capacity, resulting in myocellular lipid accumulation and lipid toxicity in the heart, an extremely common phenomenon in these cardiomyopathy conditions [81]. The changes in FAO in these cardiovascular diseases all resulted in higher oxygen consumption per ATP molecule and an overall lower cardiac efficiency [80]. 


\subsection{Pharmacological Inhibition of Fatty Acid Oxidation Has Proven Effective in Slowing Cancer Progression and Treating Cardiovascular Diseases}

The importance of FAO on cardiovascular diseases and cancer progression has made FAO a promising target for treatment. CPT1 is one of the most common and well-investigated targets for FAO inhibition in cancer therapy. Pharmacological inhibition of CPT1 using etomoxir has shown promising anticancer results in breast cancer, leukemia, and prostate cancer [68]. In cardiovascular diseases, etomoxir was used to treat diabetes and heart failure and showed great results in vivo. However, due to toxicity effects in the liver and the heart, phase II clinical trials with etomoxir were terminated [68]. A revision of etomoxir dosage might be able to improve the clinical outcome and pave the way for effective cancer and cardiovascular disease treatment. Other CPT1 and CPT2 inhibitors such as oxfenicine, aminocarnitine, ST1326, and avocatin B, all show anticancer effects in vitro and in vivo [68].

Perhexiline is another pharmacological inhibitor of CPT1 and CPT2, currently being prescribed in Asia, Australia, and New Zealand as medicine to treat angina pectoris [13, 14], a symptom of coronary artery disease characterized by chest pain or discomfort. Studies done on chronic lymphocytic leukemia and prostate cancer cells have shown that perhexiline has a growth-inhibiting effect against cancer cells [6, 69]. Similarly used in the treatment of angina pectoris in Europe and Asia, trimetazidine is a competitive inhibitor of 3-ketoacyl-CoA thiolase (3-KAT), a component of the trifunctional protein (TFP) in FAO [68, 82]. In many studies, trimetazidine has shown positive effects in restoring heart function in hypertrophied hearts in vivo or heart failure patients [80]. In cancer, trimetazidine was also found to inhibit cell proliferation and induce apoptosis in breast cancer and glioblastoma cells in vitro [83, 84]. With the safe usage of perhexiline and trimetazidine established in cardiovascular disease, the potential clinical application of these drugs in cancer treatment should be carefully examined.

Despite the positive effect of FAO inhibition in many cardiovascular conditions, adverse cardiac consequences can arise when FAO is downregulated. Specifically, inhibited FAO can cause a buildup of lipids in cardiovascular tissues and increase lipid toxicity in the already damaged tissues. Drugs targeting FAO that lack specificity can cause damage to neighboring organs and may worsen the condition. Thus, it is crucial to take this into account when using these drugs for cancer treatment, as they might cause negative impacts on the cardiovascular system.

\subsection{Inhibition of Fatty Acid Synthesis Has Proven Both Effective in Cancer Treatment and Protective Against Pulmonary Hypertension}

As discussed in Chap. 3, fatty acid synthesis and fatty acid elongation are upregulated in many cancer types, including lung, breast, and bladder cancer. Its upregulation is also associated with cancer metastasis [85-87]. Antibody treatments targeting CD36, a fatty acid receptor, in the mouse model of human oral cancer have shown strong evidence in preventing metastasis initiation [88]. Another promising treatment targeting fatty acid synthesis for cancer therapy is TVB2640, a fatty acid synthase (FASN) inhibitor that is currently involved in a variety of clinical trials in combination with chemotherapy [89]. FASN activity is also shown to increase in pulmonary hypertension. A study by Singh et al. shows that inhibition of FAS using siRNA in pulmonary hypertension-induced mice helps restore mitochondria function, and decrease hypertrophy, ventricular pressure, and vascular remodeling [5]. These promising results show potential for FAS inhibition to be applied to both cancer and pulmonary hypertension therapy. 
4

\section{Upregulated Tryptophan Catabolism Has Been Linked to the Progression of Cardiovascular Diseases and Enhanced Immune System Evasion in Cancer} (Fig. 3)

Tryptophan is one of the nine essential amino acids that play key roles in protein synthesis and participate in the synthesis of a spectrum of crucial molecules. In mammals, the kynurenine metabolic pathway is tryptophan's main catabolic route, resulting in $95 \%$ of peripheral tryptophan catabolism in mammals [90]. The kynurenine pathway is a complex metabolic route that is driven by the enzymes indoleamine 2,3-dioxygenase (IDO) and, to a lesser extent, tryptophan 2,3-dioxygenase (TDO). IDO has two isoenzymes, IDO1 and IDO2, with IDO1 being the primary one controlling tryptophan degradation [91]. In this pathway, tryptophan is converted to kynurenine, and the regulation is primarily associated with IDO1, the rate-limiting enzyme.

In the cardiovascular system, IDO and kynurenine are known as cardiovascular relaxing factors, which bring down pressure during systemic inflammation [92]. Thus, the expression of IDO and kynurenine levels is upregulated postinflammation and correlated with onsets of stroke events [93]. The increase in tryptophan degradation accompanied by an increased kynurenine/

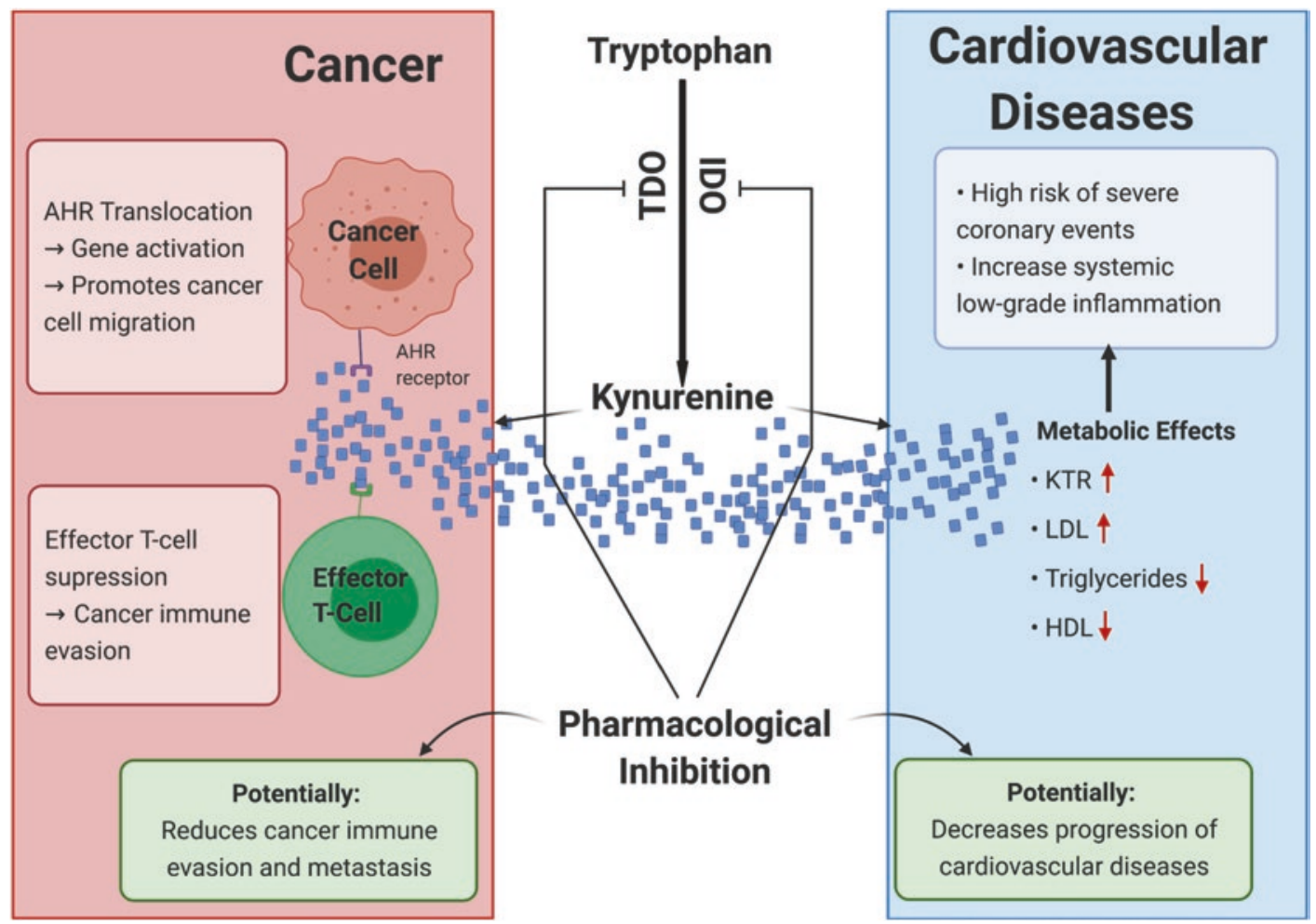

Fig. 3 The upregulation of tryptophan catabolism is linked to the progression of cardiovascular diseases and enhanced immune system evasion in cancer. The upregulation of tryptophan 2,3-dioxygenase (TDO) and indolamine 2,3-dioxygenase (IDO) and increase in kynurenine-to-tryptophan ratio (KTR) are positively associated with risks of severe coronary events and systemic low-grade inflammation. In tumors, kynurenine binds to aryl hydrocarbon receptor (AHR), which causes the receptors to translocate into the cell's nucleus, activating genes that help cell migration. In addition, kynurenine acts as an endogenous ligand that inhibits T cells via a variety of mechanisms to enhance immune evasion in tumors. Pharmacological inhibition of the kynurenine pathway could be applied to the treatment of cancer and cardiovascular diseases. $L D L$ low-density lipoproteins, $H D L$ high-density lipoproteins 
tryptophan ratio (KTR) in the blood plasma is indicative of the body's counteraction to an inflammation response, and can thus be predictive of cardiovascular events [93]. Increased KTR is also positively associated with low-density lipoproteins (LDL) and body mass index (BMI) and is negatively associated with HDL and triglycerides [94]. In individuals suffering from coronary heart disease, low tryptophan plasma concentration in combination with high KTR is prevalent [4]. The upregulation of IDO is also identified in the core of atherosclerotic plaques in humans [95].

For cancer, an elevated level of IDO expression is correlated with advanced-stage breast cancer [96]. Moreover, tryptophan degradation via the kynurenine metabolic pathway has also been linked to tumoral immune resistance [97]. Opitz et al. shows that many cancers upregulate TDO to boost tryptophan consumption [98]. When IDO and TDO are upregulated, a high level of kynurenine is synthesized, which binds to the aryl hydrocarbon receptor (AHR), inducing gene expressions that help tumor cells proliferate and metastasize. High kynurenine concentration also suppresses effector $\mathrm{T}$ cells, thus aiding cancer cells to evade immune responses [98]. Overexpression of IDO also occurs in different classes of immune cells, specifically antigen-presenting cells, which increases immune suppression and impedes the immune system's ability to recognize and attack malignant cells [99].

In general, IDO upregulation in tryptophan catabolism is associated with both cardiovascular diseases and cancer and supports tumor progression via immune suppression and inflammatory tumor carcinogenesis [98]. The pharmacological administering of 1-methyl tryptophan, an IDO inhibitor, could prove to be a novel therapeutic solution for future investigation [93]. The concerted use of IDO and TDO pharmacological inhibitors could also be a potential treatment candidate for cancer therapy.

\section{$5 \quad$ Pyruvate Metabolism Abnormality Is Associated with Cardiovascular Diseases and Chemoresistance in Cancer}

The dysregulation of pyruvate metabolism is observed in myocardial ischemia, hypertrophy, and heart failure [3], and contributes to cancer chemoresistance [2, 100]. Pyruvate dehydrogenase complex (PDHC) is responsible for the conversion of pyruvate to acetyl-CoA, a key enzyme of aerobic cellular oxidation, and the connector between glycolysis and the TCA cycle [101, 102]. Previous studies on ischemia and reperfusion reveals a decreased flux of pyruvate through the PDHC, leading to a metabolic shift towards myocardial lactate production and a large rate of glycolysis despite high oxygen consumption [3]. PDHC activity has also been shown to decrease in hypertrophied and diabetic hearts [3]. Moreover, the upregulation of pyruvate dehydrogenase kinase (PDK), an enzyme that inactivates pyruvate dehydrogenase (the first enzyme in the PDHC), has been linked to cardiomyopathy as well as poor prognosis in patients after major cardiovascular accidents [3]. Consequentially, PDHC and PDK have become treatment targets for many cardiovascular diseases. Studies have shown that infusing pyruvate or stimulating PDHC can help recover the contractile function of the damaged heart [3]. Especially, activating PDC using the PDK inhibitor, dichloroacetate (DCA), has shown protective effects against heart failure, ischemia, and reperfusion [103] (Fig. 4).

The upregulation of PDK is also seen in many cancers and is consistently associated with invasion, metastasis, and drug resistance [104]. In bladder cancer, PDK upregulation is connected to aerobic glycolysis and chemotherapy resistance $[2,105]$. A study by Woolbright et al. showed that by using DCA to inhibit PDK, bladder cancer cells showed a decrease in growth and a G0-G1 cell cycle arrest [2]. Other studies also reveal the 


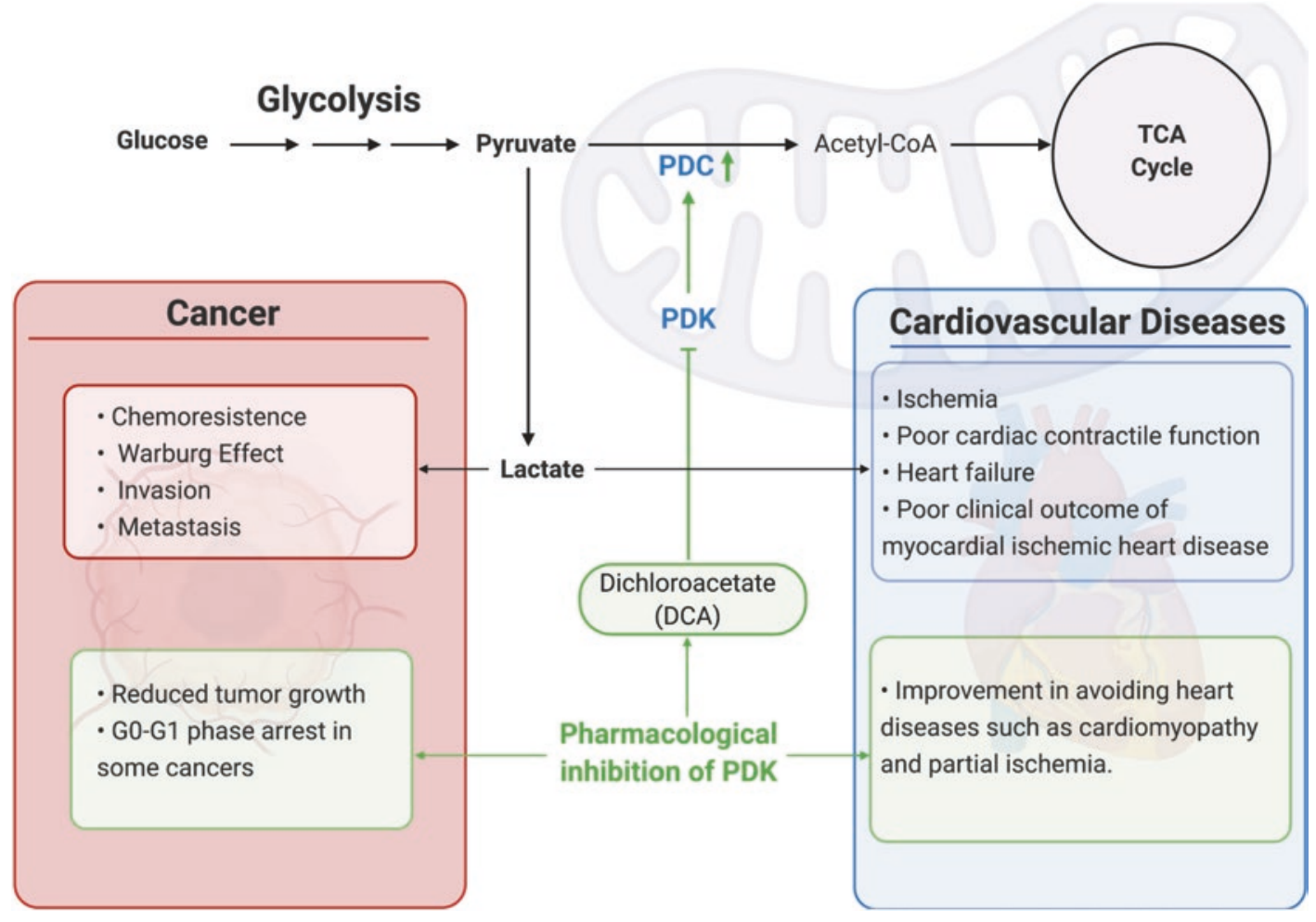

Fig. 4 The effects of dichloroacetate (DCA) on cancer and cardiovascular diseases. DCA inhibits pyruvate dehydrogenase kinase (PDK) resulting in the upregulation of the pyruvate dehydrogenase complex (PDC)

significant role of PDK overexpression in contributing to drug resistance and the resistance to chemotherapy [105, 106]. However, despite the promising anticancer and cardioprotective effects, the use of DCA has shown many drawbacks due to its short half-life, low potency, and many toxic side effects, preventing it from being successfully incorporated into a clinical setting [107].

The shortcomings regarding DCA should not discourage researchers from identifying pyruvate metabolism as a potential treatment target for cancer and cardiovascular diseases. Promisingly, a list of small PDK-inhibitor molecules has been recently proposed, which can serve as the starting point for many investigations targeting this pathway [104]. Aside from PDK, pharmacological inhibitors of other key regulators of pyruvate metabolism and glucose oxidation should also be explored.

\section{Conclusion}

Altogether, many studies have pointed out similar trends in metabolic abnormalities in cancer and various cardiovascular diseases. The common metabolic dysregulations have served as overlapping treatment targets, allowing researchers and clinicians to expand their studies and treatment options. Some pharmacological inhibitors used to treat cardiovascular conditions have gone through different phases of clinical trials. However, despite also targeting problems exhibited in cancer, these drugs have not yet reached similar phases in cancer treatment. With the use of metabolomics technologies [108], researchers have continued to identify the metabolic similarities of these two diseases, paving the way for the next steps in the investigation to improve cancer therapy. 


\section{References}

1. World Health Organization (2010). Chapter 1: Burden: Mortality, morbidity and risk factors. In Global status report on noncommunicable diseases. Geneva: WHO.

2. Woolbright, B. L., et al. (2018). The role of pyruvate dehydrogenase kinase-4 (PDK4) in bladder cancer and chemoresistance. Molecular Cancer Therapeutics, 17(9), 2004-2012.

3. Sun, W., et al. (2015). The role of pyruvate dehydrogenase complex in cardiovascular diseases. Life Sciences, 121, 97-103.

4. Wirleitner, B., et al. (2003). Immune activation and degradation of tryptophan in coronary heart disease. European Journal of Clinical Investigation, 33(7), 550-554.

5. Singh, N., et al. (2016). Inhibition of fatty acid synthase is protective in pulmonary hypertension. British Journal of Pharmacology, 173(12), 2030-2045.

6. Itkonen, H. M., et al. (2017). Lipid degradation promotes prostate cancer cell survival. Oncotarget, 8(24), 38264-38275.

7. Folmes, C. D., et al. (2009). High rates of residual fatty acid oxidation during mild ischemia decrease cardiac work and efficiency. Journal of Molecular and Cellular Cardiology, 47(1), 142-148.

8. Ito, K., et al. (2012). A PML-PPAR-delta pathway for fatty acid oxidation regulates hematopoietic stem cell maintenance. Nature Medicine, 18(9), 1350-1358.

9. Chavez-Tostado, M., et al. (2017). Oral glutamine reduces myocardial damage after coronary revascularization under cardiopulmonary bypass. A randomized clinical trial. Nutrición Hospitalaria, 34(2), 277-283.

10. Jin, L., Alesi, G. N., \& Kang, S. (2016). Glutaminolysis as a target for cancer therapy. Oncogene, 35(28), 3619-3625.

11. Durante, W. (2019). The emerging role of l-glutamine in cardiovascular health and disease. Nutrients, 11,9 .

12. Medina, M. A. (2001). Glutamine and cancer. The Journal of Nutrition, 131(9 Suppl), 2539S-2542S; discussion 2550S-1S.

13. Ashrafian, H., Horowitz, J. D., \& Frenneaux, M. P. (2007). Perhexiline. Cardiovascular Drug Reviews, 25(1), 76-97.

14. Kennedy, J. A., et al. (2000). Effect of perhexiline and oxfenicine on myocardial function and metabolism during low-flow ischemia/reperfusion in the isolated rat heart. Journal of Cardiovascular Pharmacology, 36(6), 794-801.

15. Li, T., Copeland, C., \& Le, A. (2021). Glutamine metabolism in cancer. Advances in Experimental Medicine and Biology, 1311, https://doi. org/10.1007/978-3-030-65768-0_2.

16. Le, A., et al. (2012). Glucose-independent glutamine metabolism via TCA cycling for proliferation and survival in B cells. Cell Metabolism, 15(1), 110-121.
17. Souba, W. W. (1993). Glutamine and cancer. Annals of Surgery, 218(6), 715-728.

18. Medina, M. A., et al. (1992). Relevance of glutamine metabolism to tumor cell growth. Molecular and Cellular Biochemistry, 113(1), 1-15.

19. Espat, N. J., et al. (1995). Normalization of tumorinduced increases in hepatic amino acid transport after surgical resection. Annals of Surgery, 221(1), 50-58.

20. Medina, M. A., Quesada, A. R., \& Nunez, I. (1991). de Castro, L-glutamine transport in native vesicles isolated from Ehrlich ascites tumor cell membranes. Journal of Bioenergetics and Biomembranes, 23(4), 689-697.

21. Xi, P., et al. (2011). Regulation of protein metabolism by glutamine: Implications for nutrition and health. Frontiers in Bioscience (Landmark Ed), 16, 578-597.

22. Cruzat, V., et al. (2018). Glutamine: Metabolism and immune function, supplementation and clinical translation. Nutrients, 10, 11.

23. Zheng, Y., et al. (2016). Metabolites of glutamate metabolism are associated with incident cardiovascular events in the PREDIMED PREvencion con DIeta MEDiterranea (PREDIMED) Trial. Journal of the American Heart Association, 5, 9.

24. Morris, C. R., et al. (2008). Erythrocyte glutamine depletion, altered redox environment, and pulmonary hypertension in sickle cell disease. Blood, 111(1), 402-410.

25. Dornier, E., et al. (2017). Glutaminolysis drives membrane trafficking to promote invasiveness of breast cancer cells. Nature Communications, 8(1), 2255

26. Budczies, J., et al. (2015). Glutamate enrichment as new diagnostic opportunity in breast cancer. International Journal of Cancer, 136(7), 1619-1628.

27. Koochekpour, S., et al. (2012). Serum glutamate levels correlate with Gleason score and glutamate blockade decreases proliferation, migration, and invasion and induces apoptosis in prostate cancer cells. Clinical Cancer Research, 18(21), 5888-5901.

28. Vangipurapu, J., et al. (2019). Nine amino acids are associated with decreased insulin secretion and elevated glucose levels in a 7.4-year follow-up study of 5,181 Finnish men. Diabetes, 68(6), 1353-1358.

29. Bodi, V., et al. (2012). Metabolomic profile of human myocardial ischemia by nuclear magnetic resonance spectroscopy of peripheral blood serum: A translational study based on transient coronary occlusion models. Journal of the American College of Cardiology, 59(18), 1629-1641.

30. Xiang, L., et al. (2019). Glutaminase 1 expression in colorectal cancer cells is induced by hypoxia and required for tumor growth, invasion, and metastatic colonization. Cell Death \& Disease, 10(2), 40.

31. Chan, S. Y., \& Loscalzo, J. (2008). Pathogenic mechanisms of pulmonary arterial hypertension. Journal of Molecular and Cellular Cardiology, 44(1), 14-30.

32. Piao, L., et al. (2013). Cardiac glutaminolysis: A maladaptive cancer metabolism pathway in the right 
ventricle in pulmonary hypertension. Journal of Molecular Medicine (Berlin), 91(10), 1185-1197.

33. Ge, J., et al. (2018). Glutaminolysis promotes collagen translation and stability via alphaketoglutarate-mediated mTOR activation and proline hydroxylation. American Journal of Respiratory Cell and Molecular Biology, 58(3), 378-390.

34. Saha, S. K., et al. (2019). Multiomics analysis reveals that GLS and GLS2 differentially modulate the clinical outcomes of cancer. Journal of Clinical Medicine, 8, 3 .

35. Matre, P., et al. (2016). Inhibiting glutaminase in acute myeloid leukemia: Metabolic dependency of selected AML subtypes. Oncotarget, 7(48), 79722-79735.

36. Elgogary, A., et al. (2016). Combination therapy with BPTES nanoparticles and metformin targets the metabolic heterogeneity of pancreatic cancer. Proceedings of the National Academy of Sciences of the United States of America, 113(36), E5328-E5336.

37. Wang, J. B., et al. (2010). Targeting mitochondrial glutaminase activity inhibits oncogenic transformation. Cancer Cell, 18(3), 207-219.

38. Robinson, M. M., et al. (2007). Novel mechanism of inhibition of rat kidney-type glutaminase by bis2-(5-phenylacetamido-1,2,4-thiadiazol-2-yl)ethyl sulfide (BPTES). The Biochemical Journal, 406(3), 407-414.

39. Gross, M. I., et al. (2014). Antitumor activity of the glutaminase inhibitor CB-839 in triple-negative breast cancer. Molecular Cancer Therapeutics, 13(4), 890-901.

40. Zimmermann, S. C., et al. (2016). Allosteric glutaminase inhibitors based on a 1,4-di(5-amino-1,3,4thiadiazol-2-yl)butane scaffold. ACS Medicinal Chemistry Letters, 7(5), 520-524.

41. Xiang, Y., et al. (2015). Targeted inhibition of tumorspecific glutaminase diminishes cell-autonomous tumorigenesis. The Journal of Clinical Investigation, 125(6), 2293-2306.

42. Dang, C. V., et al. (2011). Therapeutic targeting of cancer cell metabolism. Journal of Molecular Medicine (Berlin), 89(3), 205-212.

43. Hirschey, M. D., et al. (2015). Dysregulated metabolism contributes to oncogenesis. Seminars in Cancer Biology, 35(Suppl), S129-S150.

44. Bertero, T., et al. (2016). Vascular stiffness mechanoactivates YAP/TAZ-dependent glutaminolysis to drive pulmonary hypertension. The Journal of Clinical Investigation, 126(9), 3313-3335.

45. Dang, C. V., Le, A., \& Gao, P. (2009). MYC-induced cancer cell energy metabolism and therapeutic opportunities. Clinical Cancer Research, 15(21), 6479-6483.

46. Le, A., \& Dang, C. V. (2013). Studying Myc's role in metabolism regulation. Methods in Molecular Biology, 1012, 213-219.

47. Martin-Rufian, M., et al. (2014). Both GLS silencing and GLS2 overexpression synergize with oxidative stress against proliferation of glioma cells. Journal of Molecular Medicine (Berlin), 92(3), 277-290.

48. Xiang, L., et al. (2013). Knock-down of glutaminase 2 expression decreases glutathione, NADH, and sensitizes cervical cancer to ionizing radiation. Biochimica et Biophysica Acta, 1833(12), 2996-3005.

49. Miller, R. A., et al. (2018). Targeting hepatic glutaminase activity to ameliorate hyperglycemia. Nature Medicine, 24(4), 518-524.

50. Lee, Y. Z., et al. (2014). Discovery of selective inhibitors of Glutaminase-2, which inhibit mTORC1, activate autophagy and inhibit proliferation in cancer cells. Oncotarget, 5(15), 6087-6101.

51. Austgen, T. R., et al. (1992). The effects of glutamine-enriched total parenteral nutrition on tumor growth and host tissues. Annals of Surgery, 215(2), 107-113.

52. Fahr, M. J., et al. (1994). Harry M. Vars Research Award. Glutamine enhances immunoregulation of tumor growth. JPEN Journal of Parenteral and Enteral Nutrition, 18(6), 471-476.

53. Yoshida, S., et al. (1995). Effect of glutamine supplementation on protein metabolism and glutathione in tumor-bearing rats. JPEN Journal of Parenteral and Enteral Nutrition, 19(6), 492-497.

54. Gaurav, K., et al. (2012). Glutamine: A novel approach to chemotherapy-induced toxicity. Indian Journal of Medical and Paediatric Oncology, 33(1), 13-20.

55. Savarese, D. M., et al. (2003). Prevention of chemotherapy and radiation toxicity with glutamine. Cancer Treatment Reviews, 29(6), 501-513.

56. Greenfield, J. R., et al. (2009). Oral glutamine increases circulating glucagon-like peptide 1, glucagon, and insulin concentrations in lean, obese, and type 2 diabetic subjects. The American Journal of Clinical Nutrition, 89(1), 106-113.

57. Opara, E. C., et al. (1996). L-glutamine supplementation of a high fat diet reduces body weight and attenuates hyperglycemia and hyperinsulinemia in C57BL/6J mice. The Journal of Nutrition, 126(1), 273-279.

58. Khogali, S. E., et al. (2002). Is glutamine beneficial in ischemic heart disease? Nutrition, 18(2), 123-126.

59. Sufit, A., et al. (2012). Pharmacologically dosed oral glutamine reduces myocardial injury in patients undergoing cardiac surgery: A randomized pilot feasibility trial. JPEN Journal of Parenteral and Enteral Nutrition, 36(5), 556-561.

60. Zabot, G. P., et al. (2014). Glutamine prevents oxidative stress in a model of mesenteric ischemia and reperfusion. World Journal of Gastroenterology, 20(32), 11406-11414.

61. Kim, K. S., et al. (2013). The effect of glutamine on cerebral ischaemic injury after cardiac arrest. Resuscitation, 84(9), 1285-1290.

62. Prem, J. T., et al. (1999). The role of glutamine in skeletal muscle ischemia/reperfusion injury in the rat hind limb model. American Journal of Surgery, 178(2), 147-150. 
63. Esposito, E., et al. (2011). Glutamine contributes to ameliorate inflammation after renal ischemia/ reperfusion injury in rats. Naunyn-Schmiedeberg's Archives of Pharmacology, 383(5), 493-508.

64. Stangl, R., et al. (2011). Reduction of liver ischemiareperfusion injury via glutamine pretreatment. The Journal of Surgical Research, 166(1), 95-103.

65. Niihara, Y., et al. (2005). L-glutamine therapy reduces endothelial adhesion of sickle red blood cells to human umbilical vein endothelial cells. $B M C$ Blood Disorder, 5, 4.

66. Niihara, Y., et al. (2018). A Phase 3 Trial of 1-Glutamine in Sickle Cell Disease. The New England Journal of Medicine, 379(3), 226-235.

67. Park, J. K., et al. (2021). The heterogeneity of lipid metabolism in cancer. Advances in Experimental Medicine and Biology, 1311, https://doi. org/10.1007/978-3-030-65768-0_3.

68. Ma, Y., et al. (2018). Fatty acid oxidation: An emerging facet of metabolic transformation in cancer. Cancer Letters, 435, 92-100.

69. Liu, P. P., et al. (2016). Elimination of chronic lymphocytic leukemia cells in stromal microenvironment by targeting CPT with an antiangina drug perhexiline. Oncogene, 35(43), 5663-5673.

70. Shao, H., et al. (2016). Carnitine palmitoyltransferase 1A functions to repress FoxO transcription factors to allow cell cycle progression in ovarian cancer. Oncotarget, 7(4), 3832-3846.

71. Shi, J., et al. (2016). High expression of CPT1A predicts adverse outcomes: A potential therapeutic target for acute myeloid leukemia. eBioMedicine, 14, 55-64.

72. Liu, Y. (2006). Fatty acid oxidation is a dominant bioenergetic pathway in prostate cancer. Prostate Cancer and Prostatic Diseases, 9(3), 230-234.

73. Tan, J., \& Le, A. (2021). The heterogeneity of breast cancer metabolism. Advances in Experimental Medicine and Biology, 1311, https:// doi.org/10.1007/978-3-030-65768-0_6.

74. Carracedo, A., et al. (2012). A metabolic prosurvival role for PML in breast cancer. The Journal of Clinical Investigation, 122(9), 3088-3100.

75. Wang, Y. N., et al. (2018). CPT1A-mediated fatty acid oxidation promotes colorectal cancer cell metastasis by inhibiting anoikis. Oncogene, 37(46), 6025-6040.

76. Quinones, A., \& Le, A. (2021). The multifaceted glioblastoma: From genomic alterations to metabolic adaptations. Advances in Experimental Medicine and Biology, 1311, https://doi. org/10.1007/978-3-030-65768-0_4.

77. Buzzai, M., et al. (2005). The glucose dependence of Akt-transformed cells can be reversed by pharmacologic activation of fatty acid beta-oxidation. Oncogene, 24(26), 4165-4173.

78. Xie, Z., et al. (2016). Inborn errors of long-chain fatty acid beta-oxidation link neural stem cell selfrenewal to autism. Cell Reports, 14(5), 991-999.
79. Lopaschuk, G. D., et al. (2010). Myocardial fatty acid metabolism in health and disease. Physiological Reviews, 90(1), 207-258.

80. Fillmore, N., Mori, J., \& Lopaschuk, G. D. (2014). Mitochondrial fatty acid oxidation alterations in heart failure, ischaemic heart disease and diabetic cardiomyopathy. British Journal of Pharmacology, 171(8), 2080-2090.

81. Schulze, P. C., Drosatos, K., \& Goldberg, I. J. (2016). Lipid use and misuse by the heart. Circulation Research, 118(11), 1736-1751.

82. Ma, Y., et al. (2020). Functional analysis of molecular and pharmacological modulators of mitochondrial fatty acid oxidation. Scientific Reports, 10(1), 1450 .

83. Bensaad, K., et al. (2014). Fatty acid uptake and lipid storage induced by HIF-1alpha contribute to cell growth and survival after hypoxia-reoxygenation. Cell Reports, 9(1), 349-365.

84. Halama, A., et al. (2018). Accelerated lipid catabolism and autophagy are cancer survival mechanisms under inhibited glutaminolysis. Cancer Letters, 430, 133-147.

85. Jung, Y. Y., Kim, H. M., \& Koo, J. S. (2015). Expression of lipid metabolism-related proteins in metastatic breast cancer. PLoS One, 10(9), e0137204.

86. Hua, Y., et al. (2011). Dynamic metabolic transformation in tumor invasion and metastasis in mice with LM-8 osteosarcoma cell transplantation. Journal of Proteome Research, 10(8), 3513-3521.

87. Luo, X., et al. (2017). Emerging roles of lipid metabolism in cancer metastasis. Molecular Cancer, 16(1), 76.

88. Pascual, G., et al. (2017). Targeting metastasisinitiating cells through the fatty acid receptor CD36. Nature, 541(7635), 41-45.

89. Dean, E. J., et al. (2016). Preliminary activity in the first in human study of the first-in-class fatty acid synthase (FASN) inhibitor, TVB-2640. Journal of Clinical Oncology, 34(15_suppl), 2512-2512.

90. Takikawa, O. (2005). Biochemical and medical aspects of the indoleamine 2,3-dioxygenaseinitiated L-tryptophan metabolism. Biochemical and Biophysical Research Communications, 338(1), 12-19.

91. Zhai, L., et al. (2015). Molecular pathways: Targeting IDO1 and other tryptophan dioxygenases for cancer immunotherapy. Clinical Cancer Research, 21(24), 5427-5433.

92. Hofmann, F. (2010). Ido brings down the pressure in systemic inflammation. Nature Medicine, 16(3), 265-267.

93. Mangge, H., et al. (2014). Disturbed tryptophan metabolism in cardiovascular disease. Current Medicinal Chemistry, 21(17), 1931-1937.

94. Pertovaara, M., et al. (2007). Indoleamine 2,3-dioxygenase enzyme activity correlates with risk factors for atherosclerosis: The cardiovascular risk 
in Young Finns study. Clinical and Experimental Immunology, 148(1), 106-111.

95. Niinisalo, P., et al. (2010). Activation of indoleamine 2,3-dioxygenase-induced tryptophan degradation in advanced atherosclerotic plaques: Tampere vascular study. Annals of Medicine, 42(1), 55-63.

96. Sakurai, K., et al. (2005). Study of indoleamine 2,3-dioxygenase expression in patients with breast cancer. Gan to Kagaku Ryoho, 32(11), 1546-1549.

97. Uyttenhove, C., et al. (2003). Evidence for a tumoral immune resistance mechanism based on tryptophan degradation by indoleamine 2,3-dioxygenase. Nature Medicine, 9(10), 1269-1274.

98. Opitz, C. A., et al. (2011). An endogenous tumourpromoting ligand of the human aryl hydrocarbon receptor. Nature, 478(7368), 197-203.

99. Katz, J. B., Muller, A. J., \& Prendergast, G. C. (2008). Indoleamine 2,3-dioxygenase in T-cell tolerance and tumoral immune escape. Immunological Reviews, 222, 206-221.

100. Le, A., Udupa, S., \& Zhang, C. (2019). The metabolic interplay between cancer and other diseases. Trends Cancer, 5(12), 809-821.

101. Calvani, M., Reda, E., \& Arrigoni-Martelli, E. (2000). Regulation by carnitine of myocardial fatty acid and carbohydrate metabolism under normal and pathological conditions. Basic Research in Cardiology, 95(2), 75-83.
102. Bose, S., Zhang, C., \& Le, A. (2021). Glucose metabolism in cancer: The Warburg effect and beyond. Advances in Experimental Medicine and Biology, 1311, https://doi. org/10.1007/978-3-030-65768-0_1.

103. Bersin, R. M., \& Stacpoole, P. W. (1997). Dichloroacetate as metabolic therapy for myocardial ischemia and failure. American Heart Journal, 134(5 Pt 1), 841-855.

104. Stacpoole, P. W. (2017). Therapeutic targeting of the pyruvate dehydrogenase complex/pyruvate dehydrogenase kinase (PDC/PDK) axis in cancer. Journal of the National Cancer Institute, 109, 11.

105. Sradhanjali, S., \& Reddy, M. M. (2018). Inhibition of pyruvate dehydrogenase kinase as a therapeutic strategy against cancer. Current Topics in Medicinal Chemistry, 18(6), 444-453.

106. Lu, C. W., et al. (2011). Overexpression of pyruvate dehydrogenase kinase 3 increases drug resistance and early recurrence in colon cancer. The American Journal of Pathology, 179(3), 1405-1414.

107. Stacpoole, P. W., et al. (1998). Clinical pharmacology and toxicology of dichloroacetate. Environmental Health Perspectives, 106(Suppl 4), 989-994.

108. Hoang, G., Udupa, S., \& Le, A. (2019). Application of metabolomics technologies toward cancer prognosis and therapy. International Review of Cell and Molecular Biology, 347, 191-223.

Open Access This chapter is licensed under the terms of the Creative Commons Attribution 4.0 International License (http://creativecommons.org/licenses/by/4.0/), which permits use, sharing, adaptation, distribution and reproduction in any medium or format, as long as you give appropriate credit to the original author(s) and the source, provide a link to the Creative Commons license and indicate if changes were made.

The images or other third party material in this chapter are included in the chapter's Creative Commons license, unless indicated otherwise in a credit line to the material. If material is not included in the chapter's Creative Commons license and your intended use is not permitted by statutory regulation or exceeds the permitted use, you will need to obtain permission directly from the copyright holder. 


\section{Index}

A

Acetyl-CoA carboxylase (ACC), 41, 42

Acetyl-CoA carboxylase 1 (ACC1), 48

Acetyl-CoA via acetyl-CoA synthetase (ACSS2), 45

Adipocytes, 210

Aerobic glycolysis, 6, 7, 107, 109

Alanine, 190

Aldehyde dehydrogenase 1 family member A1

(ALDH1A1), 72

Alpha-ketoglutaramate (KGM), 236

Alpha-ketoglutarate (aKG), 67, 68

Amino acid metabolism, 221

dysregulation, 221

glucose, 222

Aminooxetane-3-carboxylate (AOC), 27

Aminotransferases, 28

AMP-activated protein kinase (AMPK), 42, 48, 119

Amyotrophic lateral sclerosis (ALS), 241

Angiogenesis, 69, 153, 154

Antitumor cytokines, 178

Argininosuccinate synthetase 1 (ASS1), 28

Aryl hydrocarbon receptor (AHR), 257, 258

Asparagine synthase (ASNS), 27

ATP citrate lyase (ACLY), 41

Autoimmune disorders, 240

\section{B}

$\beta$-hydroxybutyrate ( $\beta \mathrm{OHB}), 209$

B-cell lymphoma, 44

B-cell receptor (BCR), 109

Benzene-tricarboxylate analog (BTA), 41

Benzylserine, 27

Brain tumors, 72, 73

Breast cancer susceptibility gene 1 (BRCA1), 42

C

CAF-cancer cell interactions, 206

CAF-derived exosomes (CDEs), 195

amino acids, 195

glucose, 196

glutamine, 197

lactate, 197
miRNAs, 195

mitochondrial activity and oxygen consumption, 197

mitochondrial function, 197

normoxic and hypoxic conditions, 197

SIRT1/PGC-1 $\alpha, 197$

TCA cycle, 197

TCA metabolites, 196

Canavaninosuccinate (CSA), 133

Cancer cell metabolism, 40

Cancer cells, 19, 41, 78

Cancer-associated adipocytes (CAAs), 206, 210

Cancer-associated fibroblasts (CAFs), 31, 92, 190, 206

AMPK pathway, 208

AZD3965, 208

cancer cell-related counterpart, 191

cancer cells, 190

cancer metabolism, 206

gene expression profiles, 206

heterocellular communication, 200

in solid tumors, 207

in vitro experiments, 199

in Warburg effect, 191

ketolysis and ketogenesis, 210

ketone, 210

ketone bodies, 210

lipidomic reprogramming, 200

MCT1 and MCT4 transporters, 208

MCT4 transporter, 191

metabolic alterations, 206

metabolic coupling, 191

metabolic pathways, 190

metabolites, 208

mitochondria, 199

OXPHOS metabolism, 199

pathological analysis, 206

properties and characteristics, 190

proteomics, 191

reciprocal stimulation, 200

secrete ketone bodies, 209

TCA cycle, 199

TME, 191

tumor cell growth, 206

Cancer-friendly fibroblasts, 206

Cancer glucose metabolism, 8 
Cancer metabolism heterogeneity, 78

Cancer stem cells (CSCs), 7, 62, 83, 162, 163 glutamine, 165 glycolytic enzymes, 162-164

Cancer therapy, 30, 32, 49, 208, 210

Carbonic anhydrase 9 (CA9), 191

Cardiovascular diseases, 250

Carnitine palmitoyltransferase 1 (CPT1), 42, 48

Catechol- $O$-methyl transferase (COMT), 93

Cellular lipid metabolism, 40

Cerebrospinal fluid (CSF), 63

Chemotherapy, 104

Chimeric antigen receptor (CAR), 175

Chimeric antigen receptor (CAR) T cells cancer therapy, 180

cytokine, 181 engineered, 180, 181 HPSE-expressing, 180 interleukins, 180 therapeutic effects, 180

Chloroquine (CQ), 82

Cholesterol synthesis, 46

Choline kinase (CK), 93

Choline metabolism, 92, 93

Chronic lymphocytic leukemia (CLL), 110, 254

Citrate transporter protein (CTP), 41

Clear-cell renal cell carcinoma (ccRCC), 31, 48, 119

Coenzyme A (CoA), 68

Colorectal cancer metastasis, 43

Combination therapy, 84,85

BPTES, 25 metabolic pathways, 25

Cyclin-dependent kinase inhibitor 2A (CDKN2A), 61 Cysteine catabolism, 72

Cysteine dioxygenase-1 (CDO1), 73

Cysteine sulfinic acid (CSA), 73

Cystine, 72

\section{D}

de novo fatty acid synthesis (DNLS), 41

De novo lipogenesis, 169

Diabetes, 48

blood glucose levels, 219

and cancer, 218, 219

glucose levels, 219

hyperglycemia, 219

T2D, 219

Diabetes drugs, 225

Diabetes mellitus, 218

6-diazo-5-oxo-1-norleucine (DON), 27

Diffuse large B-cell lymphoma (DLBCL), 109

E

Electron transport chain (ETC), 18, 110

Epidermal growth factor receptor (EGFR), 44, 61

Epigallocatechin gallate (EGCG), 26

Epithelial-to-mesenchymal transition (EMT), 42, 97

Estrogen metabolism, 93
Estrogen receptors (ER), 90

Extracellular matrix (ECM), 97, 174

Extracellular signal-regulated kinase (ERK), 82

F

Fatty acid

cholesterol synthesis, 46

elongation, 45

metastasis, 47

molecular mechanisms

clinical trials, CPT1, 48, 49

CPT1, 48

FAO, 47, 49, 50

lipids, 47

tumor microenvironment, 47

Fatty acid metabolism, 106, 107

Fatty acid oxidation (FAO), 47, 63, 64, 108, 139, 141,250

cancer, 254, 255

cardiovascular disease, 254, 255

Fatty acid synthase (FASN), 256

Fatty acid synthesis

ACC, 41, 42

ACLY, 41

carbons, 41

clinical trials, cancer, 42, 43

CTP, 41

glucose, 41

intracellular signaling pathways, 43

PI3K, 43

tumor microenvironment, 45

Fatty acid-binding protein (FABP), 47

Fatty acid-synthesizing enzyme (FASN), 109

Fatty acid uptake, 45, 47

FDG PET/CT, 110-112

${ }^{18} \mathrm{~F}$-fluorodeoxyglucose positron-emission tomography (FDG PET), 21, 105

Fibroblasts, 31

Flavopiridol, 11

Fluoro-2-deoxy-d-glucose (FDG), 6

Focused ultrasound (FUS), 63

Follicular lymphoma (FL), 104

G

Gamma-aminobutyric acid (GABA), 230, 237

ADHD, 237, 238

autoimmune disorders, 240

GAD, 237

receptors, 239, 240

Gamma-aminobutyric acid type A receptor alpha-1 (GABRA1), 62

Gene expression, 105, 108

Genetic markers, 44

Genomic alterations

EGFR mutations, 66

GBM, 67

IDH1 mutations, 68-71

lipid metabolism dysregulation, 68 
p53 mutations, 66

PTEN mutations, 65, 66

reductants, 68

TCA cycle, 68

Glial cells, 60

Glioblastoma cancer stem-like cells (GSCs), 63

Glioblastoma multiforme (GBM), 20, 45

benefits, combined therapy, 71, 72

brain cancer, 60

brain tumors, 72,73

cancer development, 60

diversity, 60

gliomas, 60

subtype classification, 60-62

temporal and spatial heterogeneity, 60

Glioblastomas, 69

Gluconeogenesis, 9

Glucose, 41

Glucose metabolism, 10, 66, 79, 121, 131, 219

CSC subpopulation, 7

HeLa cells, 7

tumor microenvironment, 7

Glucose transporter (GLUT), 92, 175

Glucose transporter-1 (GLUT1), 79

Glucose transporter-4 (GLUT1-4), 9

Glucose-6-phosphate (G6P), 6

Glutamate, 21, 22, 231, 233-235, 241-244

Glutamate carboxypeptidase II (GCP II), 81, 230, 241,242

Glutamate dehydrogenase (GDH), 67, 68, 222

Glutamate dehydrogenase 1 (GLUD1), 78

Glutamic-oxaloacetic transaminase (GOT), 28

Glutamic-oxaloacetic transaminase 1 (GOT1), 78

Glutamic-pyruvate transaminase (GPT), 28

Glutaminase (GLS), 21, 67, 231

Glutamine, 72, 197, 230, 231

AMPAR, 235

elevated level, 231, 232

glutamate, 232

KGM, 236

MYC, 235

neurodegenerative diseases, 234

NMDA, 234

Glutamine addiction, 21, 31, 67, 209

bloodstream, 20

cancer cells, 20

IDH1, 21

$\alpha-\mathrm{KG}$ fuels, 21

Glutamine deprivation, 106

Glutamine metabolism, 26, 78, 109, 119, 122, 128, 129, $164,165,201,222,250,251$

antitumor immune responses, 31

CAFs, 31

cancer/hypertension/hyperglycemia, 253

characteristic features, 18

cytotoxic T cells, 30

fibroblasts, 31

GLS1 expression, 23

glutamine/glutamate, 251, 252

glutamine-deprived microenvironments, 31 glutaminolysis, 23, 252

inhibition, 22

macrophages, 31

mechanism, 24

microenvironment, 31

supplementation, 253, 254

$\mathrm{T}$ cells and NK cells, 30

TCA cycle, 18

TP53 gene codes, 23

tumor microenvironment, 31

TXNIP, 30

Glutamine mimetics

DON, 28

Glutamine synthase (GS), 67, 232, 233

Glutamine transaminase K (GTK) expression, 82

Glutamine-addicted cancer therapy, 25

Glutaminolysis, 28, 31, 40, 165, 252

Glutathione (GSH), 21, 122, 223

Glutathione disulfide (GSSG), 122

Glutathione via glutathione S-transferase P (GSTP), 94

Glyceraldehyde 3-phosphate dehydrogenase

Glycine, 29 (GAPDH), 10

Glycogen metabolism, 8 HIF- $1 \alpha$ induction, 9 tumor genetics, 8

Glycogen signaling pathway, 222

Glycolysis, 8, 40, 207

Glycolysis pathway, 219

Glycolytic cancer cells, 44

Glycolytic enzymes, 192

Glycolytic inhibition, 71

Glycolytic upregulation, 92

Growth factor signaling pathways, 66

\section{$\mathbf{H}$}

Heparanase (HPSE), 180

Hepatocellular carcinoma (HCC), 47, 141

Hepatocytes, 44

Heterogeneity, 71

Heterogeneity of cancer, 138, 141, 144, 145

Heterogeneous malignant lymphomas, 104, 112

Heterogeneous nuclear ribonucleoprotein (hnRNP), 66

Hexokinase 2 (HK2), 9, 65, 79

Hexosamine biosynthetic pathway (HBP), 79, 219

diabetes and cancer, 220

dNTP pool, 220

glucose levels, 219

$O$-GlcNAcylation, 220

oncogenes, 220

UDP-GlcNAc, 220

Human growth hormone (HGH) secretion, 207

Huntington's disease (HD), 233

Hydroxy-3-methylglutaryl-CoA reductase

(HMGCR), 46

Hydroxychloroquine (HCQ), 82, 85

2-hydroxyglutarate (2HG), 68

Hyperammonemia, 237 
Hyperinsulinemia, 220-222

MAPK pathways, 221

PI3K pathway, 221

Hypernephroma, 118

Hypoxia, 153-156

Hypoxia-induced apoptosis, 66

Hypoxia-inducible factor (HIF), 119, 154

Hypoxia-inducible factor 1-alpha (HIF-1 $\alpha$ ), 45, 81

Hypoxia-inducible factor-1 (HIF-1), 106, 191

Hypoxic cancer cells, 45

Immune cells

Akt signaling, 178

glucose deprivation, 178

immune response, 177

metabolic reprogramming, 177

Immunometabolism, 175

Immunotherapy, 175

In silico modeling, 97

Indoleamine-2,3-dioxygenase (IDO)

immunohistochemical staining, 29

immunosuppressive enzyme, 29

INCB024360, 29

inhibitors, 29

Inter- and intra-tumoral heterogeneities, 40

International Agency for Research on Cancer (IARC), 218

Intracellular glucose flux, 79

Intratumoral heterogeneity, 97, 98, 123, 150, 157

advantage, 151

cancer cells, 62

cancer metabolism, 153

cancer stem cells, 62

clinical outcomes, 156, 157

epigenetic alterations, 152,153

factors, 158

FAO, 63, 64

genetic alterations, 151,152

genotype and epigenetic states, 62

in vivo tumor, 156

liquid biopsy, 62, 63

mitochondrial activity, 63, 64

subclones, 151

temporal, 155

tumor microenvironment, 153, 154

tumors, 154, 155

Invasion, 69

Isocitrate dehydrogenase $3 \alpha$ (IDH3 $\alpha$ ), 194

Isocitrate dehydrogenase-1 (IDH1), 61

\section{K}

Kirsten rat sarcoma viral oncogene homolog (KRAS) glucose metabolism, 79 glutamine metabolism, 78

lipid scavenging, 79, 80

macropinocytosis, 79, 80

Kynurenine-to-tryptophan ratio (KTR), 257
L

Lactate dehydrogenase (LDH), 105

Lactate dehydrogenase A (LDHA), 79

Lactic acidosis, 106

Leucine, 222

Leucine metabolism, 222

Limbic encephalitis (LE), 240

Lipid anabolic metabolism, 168, 169

Lipid metabolism, 40, 41, 131, 167, 255

FAO, 254, 255

pharmacological inhibition, 256

pulmonary hypertension, 256, 259

Lipid scavenging, 79, 80

Lipid synthesis, 40, 43-45

Lipid synthesis regulation, 43

Lipidomic technologies, 40

Lipidomics, 40

Lipids, 47

Lipogenic cancer cells, 44

Lipoprotein lipase (LPL), 47

Liquid biopsy, 62-64

Liquid chromatography-mass spectrometry (LC-MS), 96

Liver receptor homolog 1 (LRH-1), 129

Low-density lipoprotein receptor (LDLR), 80

Lung cancer, 44

M

Macropinocytosis, 79, 80

Malignant cells, 105

Mammalian target of rapamycin (mTOR) pathway, 43

Mammalian target of rapamycin complex 1 (mTORC1), 106,222

Mantle cell lymphoma (MCL), 110

Mass spectroscopy (MS), 95

Matrix metalloproteinase (MMP), 239

Mechanistic target of rapamycin (mTOR), 67

Metabolic adaptivity, 90, 95, 98

Metabolic barrier, 181

Metabolic competition, 175

Metabolic fingerprint, 95, 96

Metabolic heterogeneity (MH), 91, 96, 98, 111

Metabolic networks, 82, 83

Metabolic nutrients, 45

Metabolic pathways, 71

Metabolic phenotypes, 118, 120, 129, 139-141

Metabolic processes, 144, 145

Metabolic profile, 44, 62, 63, 65, 71, 95

Metabolic tumor volume (MTV), 111

Metabolism, 18

Metabolism heterogeneity, 84, 85

Metabolomics analysis, 69

Metabotropic glutamate (mGlu), 241

Metastasis, 42, 43, 46, 47

Metformin, 25, 83, 84, 208, 223, 224

Methionine, 222

Methylthioadenosine phosphorylase (MTAP), 122

MicroRNAs (miRNAs), 194

IDH3 $\alpha, 194$

inhibit OXPHOS, 194 
miR-155, 194

pancreatic cancer cells, 194

SDH and FH, 194

TP53INP1, 194

Microvesicles (MV), 194

Mitochondrial activity, 63, 64

Mitochondrial metabolism, 165, 166

CSC, 168

OXPHOS, 166-168

ROS, 168

Mitochondrial $\beta$-oxidation, 40

Mitogen-activated protein kinase (MAPK) pathways, 43, 82,221

Mitostemness, 165

Monocarboxylate transporter 1 (MCT1), 82, 208

Monocarboxylate transporter 2 (MCT2), 49

Monocarboxylate transporter 4 (MCT4), 82

mTOR activation, 109

mTOR signaling, 70, 71, 222

Mucin 1 (MUC1), 80, 81

Multidrug resistance (MDR), 110

$M Y C$ expression, 26

N

$\mathrm{N}$-acetyl-aspartyl-glutamate (NAAG), 23, 63, 81, 112, 230, 231, 240, 244

GABA-ergic, 238

GABA-ergic system, 243

GCP II, 242, 243

glutamate, 241, 242

mGlu, 241

NADH dehydrogenase, 84

Neoadjuvant chemotherapy-resistant breast cancer, 46

Neurodegenerative diseases, 234

Neurofibromatosis type 1 (NF1), 61

Neurofilament light (NEFL), 62

Neurological diseases, 230-232, 234, 235, 237, 239, 240

Nicotinamide adenine dinucleotide phosphate (NADPH), 97

Nitric oxide synthase (NOS), 234

Non-Hodgkin lymphomas (NHLs), 104

FAO, 108

FAS, 107

HIF-1 activity, 110

lymphomas, 109

metabolic phenotypes, 108

mTOR, 109

MYC, 109

p53 target genes, 106

$\mathrm{PI} 3 \mathrm{~K} / \mathrm{AKT} / \mathrm{mTOR}$ pathway, 110

Non-small cell lung cancer (NSCLC), 43

Normal fibroblasts (NF), 190, 193

Nuclear magnetic resonance (NMR), 95

\section{O}

Obesity, 221

Oncogenic heterogeneity, 118

Oncogenic pathways, 7
Organic anion transporter protein-1B1 (OATP1B1), 46

Oxidative phosphorylation (OXPHOS), 6, 105, 109, 176, 192, 206

Oxidative stress, 72, 133

Oxygen-rich environments, 5

$\mathbf{P}$

Palmitic acid, 43

Pancreatic cancer (PDAC) cells, 199

metabolite tracing, 200

nutrient-deprived conditions, 200

proliferation rate, 200

PSCs, 200

TCA cycle, 199

Pancreatic cancers

antidiabetic drug, 83, 84

autophagy, 82

autophagy inhibition, 85

cancer cells, 78

cancer metabolism, 78

CPI-613, 83

fibroblasts, 82

gene expression, 78

genetics, 78

glutaminase II pathway, 81, 82

homeostasis, 83

hypovascular, 82

immune cells, 82

metabolism heterogeneity, 84,85

metformin, 83, 84

MUC1 overexpression, 80, 81

p53 functions, 81

PDAC pathogenesis, 83

PDAC tumor microenvironment, 85

PDAC tumors, 83

stromal interactions, 82,83

tumor microenvironment, 78

tumor progression, 78

Pancreatic ductal adenocarcinoma (PDAC), 28, 47, 78, 82,85

Pancreatic-stellate cells (PSC), 83

Pentose phosphate pathway (PPP), 6, 43, 78, 79, 97, 119

Perifusion assays, 26

Peripheral blood supply, 63

Peroxisome proliferator-activated receptors (PPARs), 49,50

Peroxisome proliferator-responsive element (PPRE), 225

Peroxisomes, 49

Phenylbutyrate, 27

Phosphatase and tensin homolog (PTEN), 43, 61

Phosphatidylinositol (3,4,5)-trisphosphate (PIP3), 43

Phosphatidylinositol-4,5-bisphosphate 3-kinase catalytic subunit alpha (PIK3CA), 43

Phosphoenolpyruvate carboxykinase 2 (PCK2), 9

Phosphofructokinase (PFK), 10

Phosphoglycerate dehydrogenase (PHGDH), 29, 94

Phosphoglycerate kinase 1 (PGK, 130

Phosphoinositide 3-kinase (PI3K) pathway, 67

Phosphoprotein 53 (p53), 61 
Phosphoserine, 22

Phosphoserine phosphatase (PSPH), 94

PI3K/AKT/mTOR pathway, 110

Platelet-derived growth factor receptor alpha (PDGFRA), 61

Polyphenols, 223

Positron-emission tomography (PET) imaging, 6

Primary effusion lymphoma (PEL), 105, 107

Primary liver cancer

carcinoma metabolism, 129

glucose metabolism, 132

lipid metabolism, 133

metabolic signatures

HCC, 131, 132

oxidative stress, 133

mutations, 128

LCSC, 130

LRH-1, 129

PGK1, 130

MYC, 128, 129

therapeutic investigations, 134

Progesterone receptors (PR), 90

Proliferation-activated receptor gamma coactivator-1a (PGC-1a), 97

Prolyl 4-hydroxylases (PHDs), 20

Prolyl-hydroxylase (PHD) proteins, 178

Prostate cancer cells, 199

Protein phosphatase 2A (PP2A), 42

Pyruvate dehydrogenase (PDH), 73, 163

Pyruvate dehydrogenase complex (PDC), 258

Pyruvate dehydrogenase kinase (PDK), 258, 259

Pyruvate dehydrogenase kinase 1 (PDK1), 69

Pyruvate metabolism, 258

\section{R}

Radiation therapy, 104

Reactive oxygen species (ROS), 6, 48, 70, 78

Receptor tyrosine kinases (RTKs), 43, 66

Renal cell carcinoma (RCC), 20, 118

gene dependence, 123

gene independence, 122, 123

glycolysis, 120

intratumoral heterogeneity, 121

metabolic phenotypes, 119

metabolic signature, 121

temporal impact, 121

therapy, 123

Restricting enzyme movement, 26

Reverse Warburg effect, 191

Richter's transformation (RT), 111

\section{$\mathbf{S}$}

$S$-adenosylmethionine (SAM) production, 223

Serine and glycine biosynthesis pathways, 29

Serine biosynthesis, 94

Serine hydroxymethyltransferase (SHMT), 29

Single-nucleotide polymorphism (SNP), 46

Sirtuins (SIRTs), 197
Small cell lung cancer (SCLC), 240

Solute carrier family 12 member 5 (SLC12A5) neural markers, 62

Sorafenib, 134

Spare respiratory capacity (SRC), 181

Spatial heterogeneity, 62

Stable isotope-resolved metabolomics (SIRM), 23

Standardized uptake value (SUV), 111, 157

Sterol regulatory element-binding protein 1 (SREBP-1) cleavage, 68

Stiff-person syndrome (SPS), 240

Stoichiometric analysis, 94

Stromal Cav-1

fibroblasts, 192

IкBK, 193

oxidative stress and hypoxia, 194

transcription factor, 193

vascularization, 193

Synaptotagmin 1 (SYT1), 62

Targeting amino acid synthesis, 28

Targeting glutamine metabolism

BPTES, 24

c-MYC expression, 25

combination therapy, 25

energy regulation and biosynthesis, 24

GDH, 26

GLS and hypoxia, 23

GLS2 gene, 24

glutamine dependency, 25

glutaminolysis, 24

mammalian cells, 23

MYC, 26

SIRM, 23

trigger cell cycle arrest, 23

T-cell antitumor activity, 181

T-cell function, 177

Temozolomide (TMZ), 66, 71

Thiamine, 106, 107

Thiazolidinediones, 224

Thioredoxin-interacting protein (TXNIP), 30

T lymphocytes, 177

Total lesion glycolysis (TLG), 111

Total metabolic tumor volume (TMTV), 111

Transaminase upregulation, 28

Transaminases, 28

Tricarboxylic acid (TCA)

cycle, 4, 94, 208

dysfunction, 20

FH mutations, 20

membrane-bound enzyme, 20

mutations, 18

steps, 18

Triple-negative breast cancer (TNBC), 49, 90

Troglitazone, 225

Tryptophan, 29, 257

Tryptophan catabolism, 250, 257, 258

Tuberous sclerosis protein (TSC), 43 
Tumor-associated macrophages (TAMs), 174

Tumor growth, 69

Tumor immunity

adipocytes, 175

aerobic glycolysis, 175

amino acid, 175

antitumor activity, 174

cancer cells, 175

immune cell functions, 175

Tumor microenvironment (TME), 45, 61, 82, 138, 139, 141,206

apoptosis, 176

cancer cells and immune cells, 174

checkpoint inhibitors, 174

FAO, 141

glutamine, 30

immunometabolism-targeting strategy, 178

lipid scavenging, 142

macrophages, 30

MCT1 and MCT4, 176

metabolic symbiosis, 144, 145

mitochondrial genes, 143

mTORC, 143

nutrient utilization, 142, 143

nutrient/oxygen availability, 139, 140

OXPHOS and FAO, 176

single tumor, 144

T lymphocyte proliferation, 176

TCA, 142

T-cell dysfunction, 177

T-cell function, 177

Tumor microenvironment-related obstacles, 179
Tumor progression, 82

Tumorigenesis, 28

Tumor-initiating cells (TICs), 162

Type 2 diabetes (T2D), 218, 220

V

Vascular endothelial growth factor (VEGF), 69, 109

von Hippel-Lindau (VHL) tumor-suppressor gene, 119

W

Warburg effect, 4-6, 66, 92, 191, 219

aerobic glycolysis, 5

application, 6

ATP production, 6

biochemical processes, 6

cellular glucose metabolism, 4

dietary changes, 11

GAPDH, 10

genetic alterations, 6

glycolytic pathway, 9

HK2, 10

LDHA inhibitors, 11

metabolic reprogramming, 6

NADPH and nucleotide components, 6

oxidative phosphorylation, 5

PFK, 10

respiration and fermentation, 4

ROS production, 6

TCA cycle, 4 